$\frac{18}{3} z^{2} \cdot 958+1$

UCRL-ID-108175

\title{
ASCOT \\ Meteorological Towers
}

\author{
K. Patrick Ellis
}

September 1991

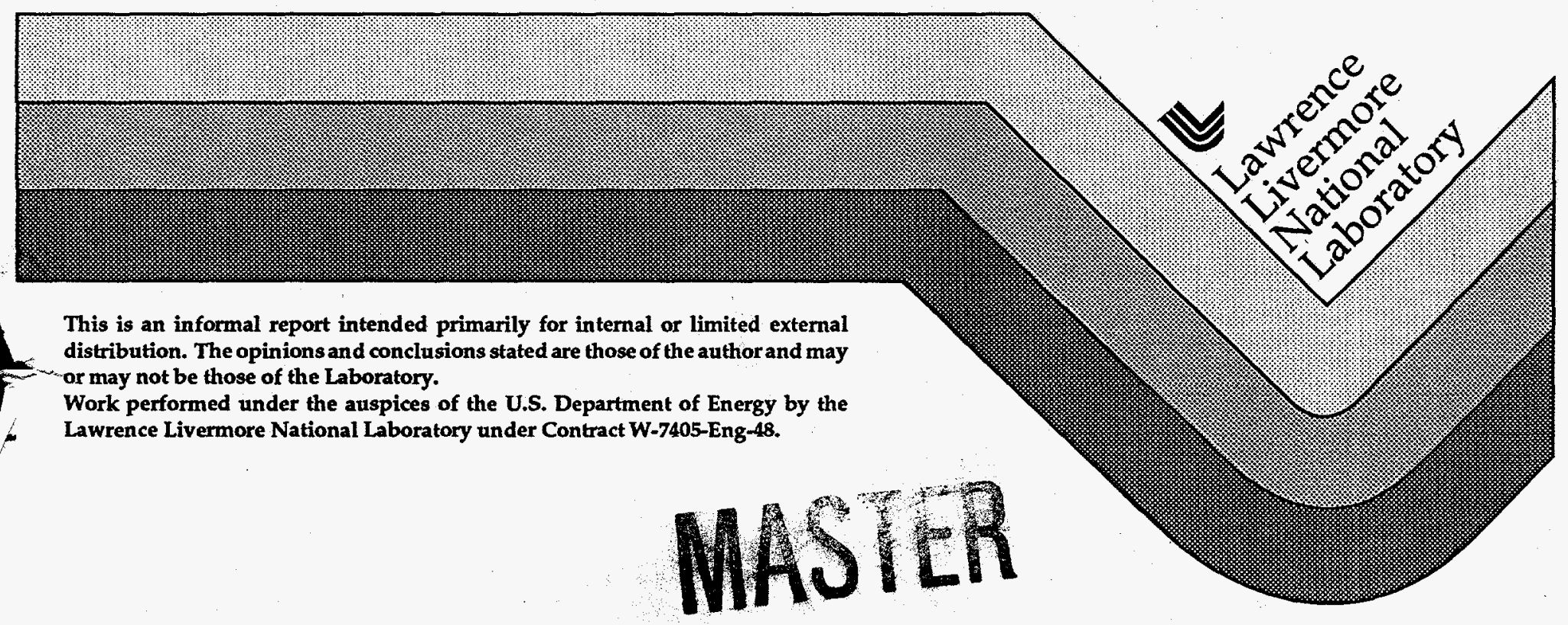




\section{DISCLAIMER}

This document was prepared as an account of work sponsored by an agency of the United States Government. Neither the United States Government nor the University of California nor any of their employees, makes any warranty, express or implied, or assumes any legal liability or responsibility for the accuracy, completeness, or usefulness of any information, apparatus, product, or process disclosed, or represents that its use would not infringe privately owned rights. Reference herein to any specific commercial products, process, or service by trade name, trademark, manufacturer, or otherwise, does not necessarily constitute or imply its endorsement, recommendation, or favoring by the United States Government or the University of California. The views and opinions of authors expressed herein do not necessarily state or reffect those of the United States Government or the University of California, and shall not be used for advertising or product endorsement purposes.

This report has been reproduced

directly from the best available copy.

Available to DOE and DOE contractors from the Office of Scientific and Technical Information

P.O. Box 62, Oak Ridge, TN 37831

Prices available from (615) 576-8401, FTS 626-8401

Available to the public from the

National Technical Information Service

U.S. Department of Commerce

5285 Port Royal Rd.

Springfield, VA 22161 


\section{DISCLAIMER}

Portions of this document may be illegible in electronic image products. Images are produced from the best available original document. 


\section{ASCOT \\ (Atmospheric Studies in Complex Terrain) METEOROLOGICAL TOWERS}

Review of LLNL's Participation in 1991 ASCOT/Winter Validation Study

K. Patrick Ellis

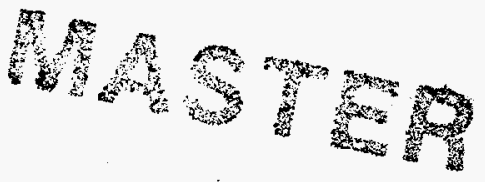

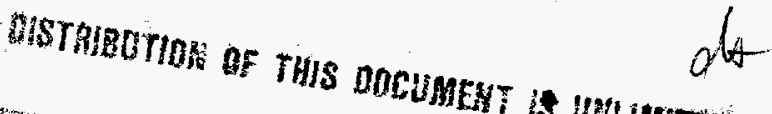




\title{
Review of LLNL's Participation in 1991 ASCOT/Winter Validation Study*
}

\author{
K. Patrick Ellis \\ Lawrence Livermore National Laboratory \\ Livermore, California 94550
}

\section{Introduction}

During the winter of 1991, LLNL participated in a series of field experiments near the Rocky Flats Plant south of Boulder, Colorado. These experiments were made in conjunction with the winter validation studies being managed by Rocky Flats personnel. This is a review of the tethersonde data taken during the period of January 28, 1991 through February 8, 1991.

\section{Location}

The LLNL Tethersonde experimental site was located at the Coal Creek Canyon outlet of the front range of the Rockies. The site was approximately 2.5 kilometers west of Highway 93, and 150 meters south of Highway 72. UTM coordinates are $476.305 \mathrm{E}$, and $4413.984 \mathrm{~N}$, Meridian is $105 \mathrm{~W}$, magnetic declanation of 12 degrees east at an altitude of 2003.75 meters MSL.

\section{Tethersonde}

A.I.R. Tethersonde equipment was used. The ADAS was an A.I.R. 3C. The sonde was equipped with an anemometer set, wet and dry bulb thermistors, an altimeter, and was attached to a $3.25 \mathrm{~m}^{3}$ tethered balloon. The assigned sonde frequency was $404.2 \mathrm{MHz}$. All systems had been calibrated in January 1991 by A.I.R. The compass error corrections are shown in Fig. 1. The only notable problem is on the night of February $6 / 7$. The relative humidities are too high, probably caused by a dried out wet bulb. (See Appendix A for instrument specifications and Appendix B for Tethersonde data.)

\section{Towers}

Meteorological data were collected by a network of eight towers. Seven of them with two levels of instrumentation. Four of these, in addition to wind speed, wind direction, and temperature sensors, were equipped with net radiometers. One tower was instrumented at only one level. Five of the towers were located along the front range near Rocky Flats, one was located up Coal Creek Canyon, and one west of Standley Lake (see Fig. 2 for these 7 locations); one tower was located at Tolland, west of the Town of Rollinsville. Table 1 summarizes the locations of these towers and their instrumentation. (See Appendix $\mathrm{C}$ and D.)

* Work performed under the auspices of the U.S. Department of Energy by the Lawrence Livermore National Laboratory under Contract No. W-7405-Eng-48. 


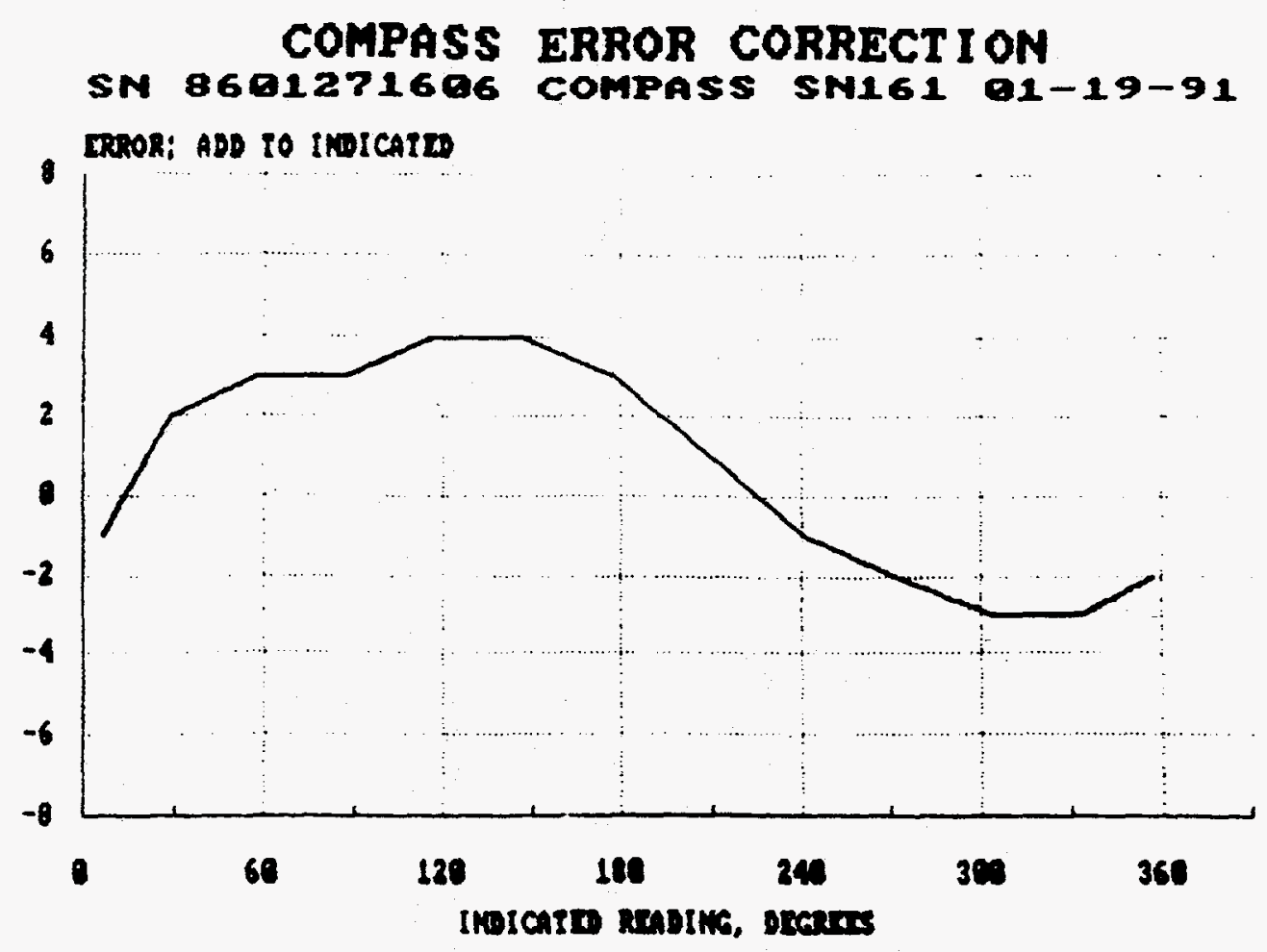

Figure 1.

404.2 MHZ

\#2 SONDE

Tower Instrumentation

Each tower is instrumented with a Handar 540 Data Collection Platform (DCP), Wind Speed (w/s), Wind Direction (w/d), and Temperature Sensors. Four of the towers have Net Radiation Sensors. Each tower has two solar panels to provide power and a Yagi antenna for data transmission.

\section{Data Collection Platform}

The DCP is manufactured by Handar Corporation in Sunnyvale, California. It has a specially designed Panel for ASCOT operations. The firmware (4.Q) was developed for ASCOT use. There is a met card for each level of instrumentation. These DCPs are mounted on the tower at varying heights. The criteria for determining the mounting location is a projection of snow depth during the winter months, or, if an anti-climbing device is installed. Programming of the DCP is done with a handar 545C programing set in the field. The specific commands for each sensor as well as other criteria is shown. 


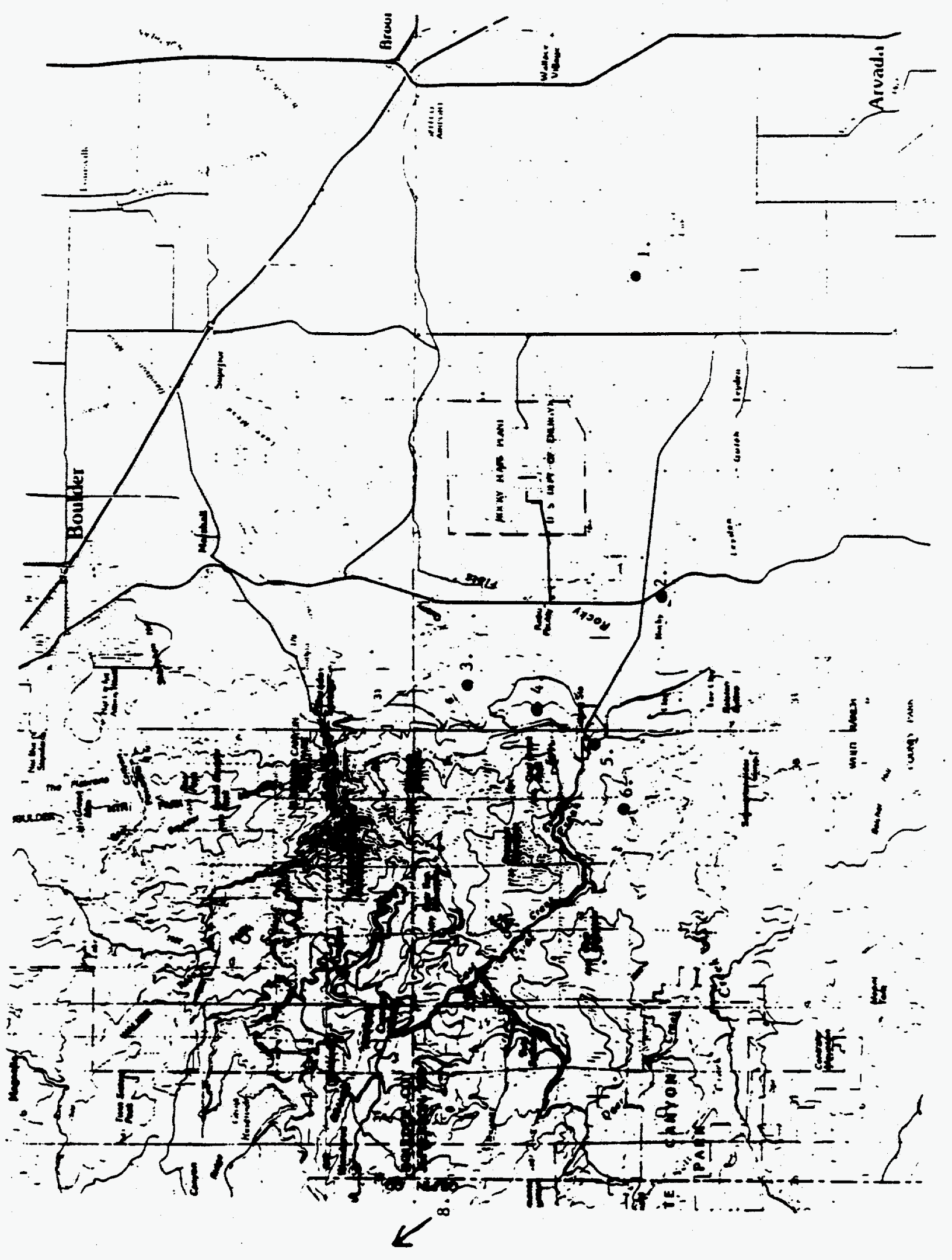

Figure 2. 


\section{Sensors}

The wind speed/wind direction (ws/wd) crossarm assembly is mounted on the tower above ground level as shown. This is also true of the temperature sensors. The cables from these sensors are then run down the tower and attached to the bottom of the DCP to the panel as shown in Fig. 3. All sensors have been refurbished and calibrated at the Bureau of Land Management (BLM) Maintenance Depot in Boise, Idaho. Their standards meet or exceed the manufacturer's design specifications. (See Appendix A.)

A. The $w / s$ sensor is a Handar $430 \mathrm{~A}$. Two sizes of ice skirts were manufactured, we have both in use. The 2 " sensor has a frequency velocity factor of 00.586 . The $1-3 / 4$ " sensor has a frequency velocity factor of 00.626 (see Fig. 4 for which tower has which sensor). After the $\mathrm{w} / \mathrm{s}$ sensor is inserted in the ws/wd cross arm assembly and attached to DCP, it is checked against a hand-held Davis Turbo Wind Meter for comparison.

B. The $w / d$ sensor is a Handar $431 \mathrm{~A}$. After inserting this sensor in the ws/wd cross arm assembly, it is aligned by two individuals, one on the tower to make adjustments, and one on the ground sighting in the alignment. A hand-held SUUNTO KB-14/360 compass is used. The alignment is to True North, and the magnetic declanation is arrived at by using the GEOMAG computer program. By adding or-substracting the declanation, the vane is aligned and tightened by set screws. Several scans using the $545 \mathrm{C}$ program set are done to confirm readings. The vane alignment is accurate to $\pm 2^{\circ}$.

C. The temperature sensor is a Handar 432B. It is attached to the tower just below the ws/wd crossarm. After connecting the sensor to the DCP, its readings are checked against a thermometer.

D. Two solar panels are attached to the tower and aimed in a southerly direction at approximately $45^{\circ}$ above the horizon. The larger panel is an ARCO M-65 and is connected to the DCP to keep the internal DCP battery charged. An ARCO M-86 solar panel is attached to the tower in the same manner but is connected to a 12-volt battery in a insulated box; then, in turn, this battery is attached to the DCP to provide backup power in case of extended cloud cover or damage to the other panel.

E. A Yagi antenna is mounted within 3 feet of the DCP, and is attached to it by a coaxial cable. It is aimed at about $35^{\circ}$ above the horizon and at an angle of approximately $204^{\circ}$ magnetic (towards the GOES satellite).

F. A Radiation and Energy Balance $Q^{*} 6$ Net Radiometer is attached to the tower 10 feet above ground level on four of the towers. These units are used to measure the net radiation (this is the energy retained by the surface for heating soil and air). These radiometers are calibrated by the manufacturer, and we, in turn, program other calculations that will give us the $\mathrm{W} / \mathrm{m}^{2}$ that we desire in our computer programs (see Fig. 5). 


\section{ASCOT}

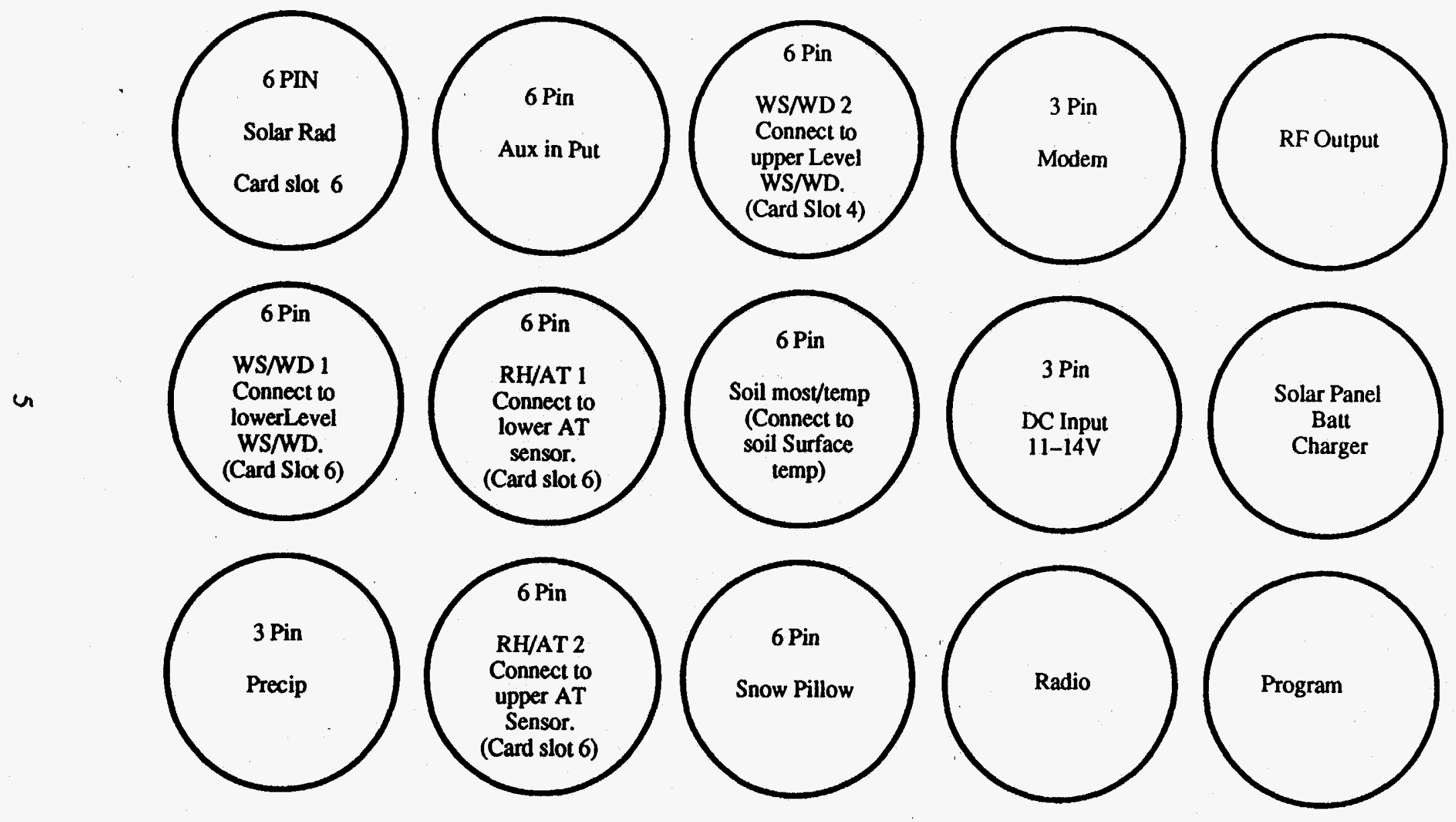

Figure 3. 


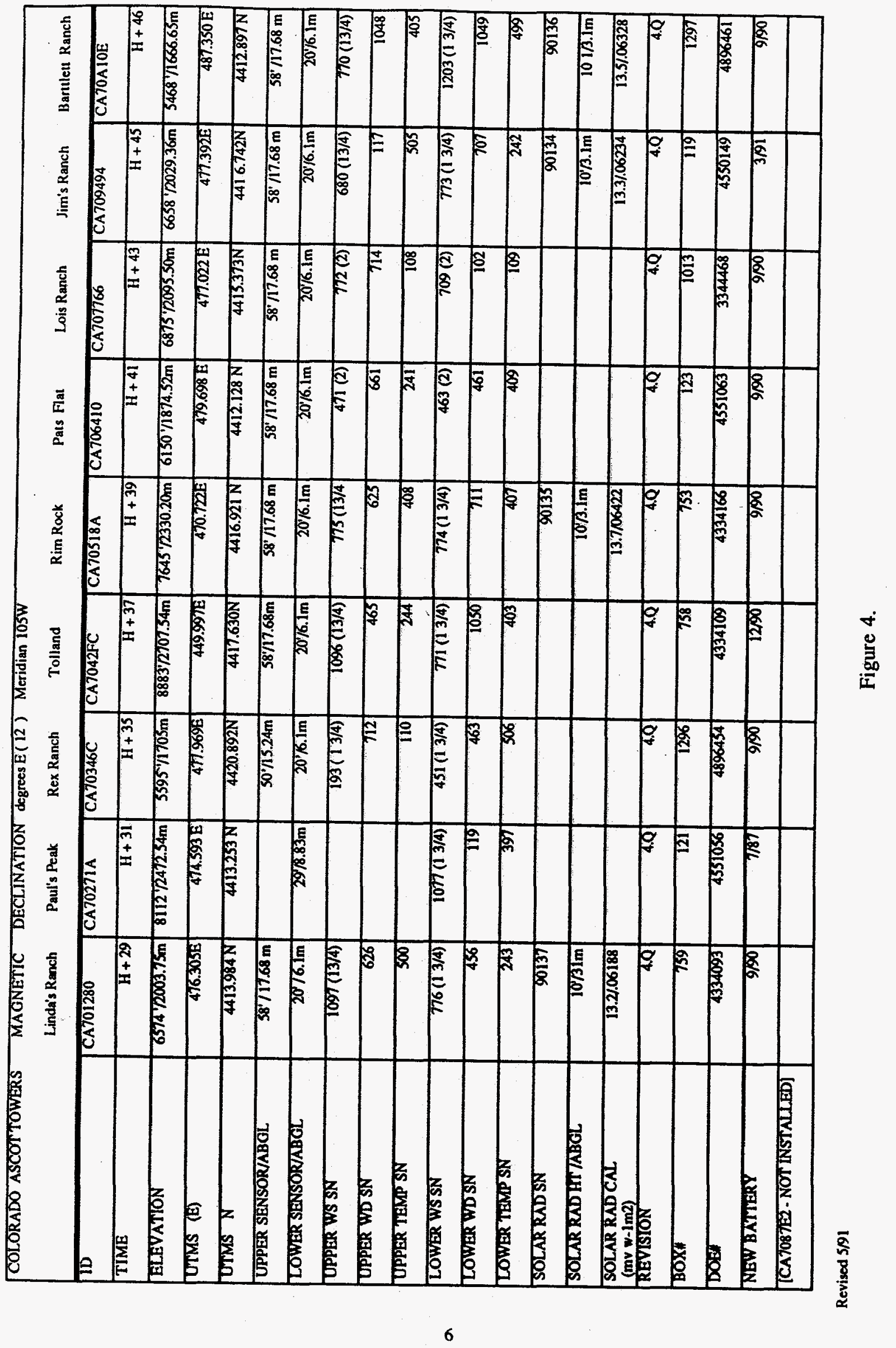




\section{Net Radiation Calibrations}

To determine the $\mathrm{W} / \mathrm{m}^{2}$ that is the designed parameters for data reporting. The Radiation and Energy Balance Systems, Inc. (REBS) net radiometer is designed to work on a system that has a 10 microvolt resolution and range of -10 to +75 millivolts. This signal must be reduced as the Handar 540 is designed to operate at a $-25 \mathrm{mV}$ to a $+25 \mathrm{mV}$. To do this three $100,000 \mathrm{ohm}$ resistors in series are installed. See Fig 1.

To compute the net radiation information $\mathrm{W} / \mathrm{m}^{2}$ it is necessary to do the following, example REBS gives a calibration factor of $13.5 \mathrm{~W} / \mathrm{m}^{2} \mathrm{mV}$, it must be multiplied by three (because that is a result of the three $100 \mathrm{kilohm}$ registers) this equals 40.5 multiply this number by $25 \mathrm{mV}$ (full scale) $=1012.5$ then divide by 16000 counts (This is 0 scale) so one count is number $.06328 \mathrm{~W} / \mathrm{m}^{2}$ to find the $\mathrm{W} / \mathrm{m}^{2}$ for any particular time of day it takes the counts reported by the Handar subtract 16000 and multiply by the calibration factor of .06328 .

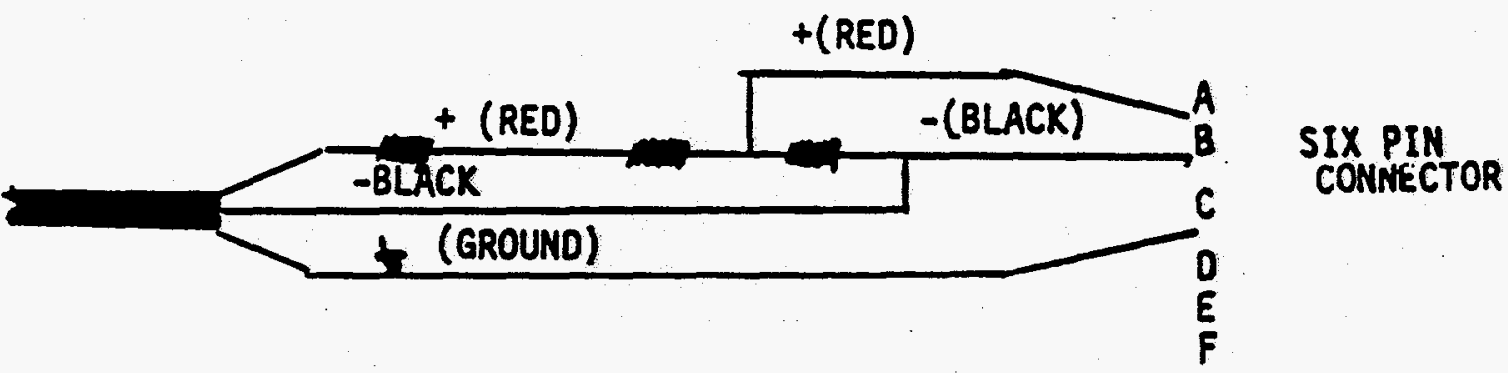

Figure 5. 
Table 1

\begin{tabular}{lccl}
\hline \multicolumn{1}{c}{ Site } & Instrumentation & UTM Coordinates & Elevation \\
1. Barttlett Ranch & 1,3 & $487.350 \mathrm{E} 4412.897 \mathrm{~N}$ & $1666.65 \mathrm{~m}$ \\
2. Pat's Flat & 1 & $479.698 \mathrm{E} 4412.128 \mathrm{~N}$ & $1874.52 \mathrm{~m}$ \\
3. Jim's Ranch & 1,3 & $477.392 \mathrm{E} 4415.742 \mathrm{~N}$ & $2029.36 \mathrm{~m}$ \\
4. Lois' Ranch & 1 & $477.002 \mathrm{E} 4415.373 \mathrm{~N}$ & $2095.50 \mathrm{~m}$ \\
5. Linda's Ranch & 1,3 & $476.305 \mathrm{E} 4413.984 \mathrm{~N}$ & $2003.75 \mathrm{~m}$ \\
6. Paul's Peak & 2 & $474.593 \mathrm{E} 4413.253 \mathrm{~N}$ & $2472.54 \mathrm{~m}$ \\
7. Rim Rock & 1,3 & $470.722 \mathrm{E} 4416.921 \mathrm{~N}$ & $2330.20 \mathrm{~m}$ \\
8. Tolland & 1 & $449.997 \mathrm{E} 4417.630 \mathrm{~N}$ & $2707.54 \mathrm{~m}$ \\
\hline
\end{tabular}

(1) 2 levels wind speed, wind direction, $\sigma_{\theta}$, temperature and $\Delta \mathrm{T}$. Instrument levels are $6.1 \mathrm{~m}$ and $17.68 \mathrm{~m}$.

(2) 1 level wind speed, wind direction, $\sigma_{\theta}$, and temperature. Instrument level is $8.83 \mathrm{~m}$

(3) Net radiometer

These towers provided a continuous set of hourly averaged data for the experimental period except for the following: all data for all towers was lost from February 6, 1991, 1100 LST until February 8, 1991, 1100 LST due to data collection problems at LLNL where transmissions from the tower instruments through the satellite and satellite receiver at Whollups Island, Virginia, to LLNL were temporarily interrupted. In addition to the above data loss, data from Jim's Ranch is missing from January 28 until January 31, 1991, 1400 LST and from February 6, 1991, 1100 LST on. See Appendix A. for instrument specifications.

The quality, of all data is very good.

\section{The Experiments}

The experimental plan called for balloon ascents to start on the hour commencing at $2000(8: 00 \mathrm{pm})$ local standard time and continuing each hour with the final launch at 0500 local standard time. This schedule was changed when weather or equipment malfunctions made it unsafe or impossible to launch. 


\section{Summary for Each Evening \\ January 28}

No experiment scheduled this evening. Equipment was set up and tested.

\section{January 29}

The weather did not cooperate at all this evening. Although the sky was clear to start with, the temperature was cold and winds were $5-10 \mathrm{~m} / \mathrm{s}$ at ground level. As evening progressed the sky became more clouded and winds became stronger. No runs were attempted. Decided to terminate at approximately 2300 (11:00 pm L.S.T.)

\section{January 30}

No experiment scheduled this evening.

\section{January 31}

Temperature cold and the sky to the west was clear; however, above and to the east, the clouds were scattered with about $50 \%$ coverage. The surface winds, 0-10 meters ABGL, varied from about $3 \mathrm{~m} / \mathrm{s}$ to $6 \mathrm{~m} / \mathrm{s}$ down the canyon, approximately $270^{\circ}$. Above that elevation, the wind increased to speeds of up to $15 \mathrm{~m} / \mathrm{s}$. On the early runs, the winds were from the west to southwest but later on they became quite variable coming from all directions.

\begin{tabular}{|c|c|c|c|c|}
\hline $\begin{array}{l}\text { MST } \\
\text { Time }\end{array}$ & $\begin{array}{l}\text { Max. W/S } \\
\text { in } M / S\end{array}$ & $\begin{array}{l}\text { Max. Height } \\
\text { in M. ABGL }\end{array}$ & $\begin{array}{l}\text { Height of } \\
\text { Winds at } \\
\text { Max. Speed } \\
\text { in M. ABGL }\end{array}$ & $\begin{array}{l}\text { Height of } \\
\text { Inversion } \\
\text { in M. ABGL }\end{array}$ \\
\hline 2000 & \multicolumn{4}{|c|}{ Did not run-Too windy } \\
\hline 2100 & 8.3 & 268 & 45 & 172 \\
\hline 2200 & 8.3 & 510 & 45 & 180 \\
\hline 2300 & 16.0 & 260 & 260 & 200 \\
\hline 0000 & 15.8 & 211 & 200 & 180 \\
\hline 0100 & \multicolumn{4}{|c|}{ Did not run-Too windy } \\
\hline 0200 & \multicolumn{4}{|c|}{ Did not run-Too windy } \\
\hline 0300 & \multicolumn{4}{|c|}{ Did not run-Too windy } \\
\hline 0400 & \multirow{2}{*}{\multicolumn{4}{|c|}{ Did not run-Too windy }} \\
\hline 0500 & & & & Did not run-Too windy \\
\hline
\end{tabular}




\section{February 1}

The temperatures were a little warmer this evening, the sky was about $80 \%$ covered above and to the west. As evening progressed, sky cover reduced to about $10 \%$, then increased again to about $40-50 \%$. The surface winds 0-10 m ABGL were 2-6 m/s; then a shallow drainage setup about 50-60 m deep. A full moon was evident as the evening progressed. The wind speed increased enough to cause launching problems. The sonde was blown off and the balloon was punctured. Did not attempt any runs after 2:00 am.

\begin{tabular}{|c|c|c|c|c|}
\hline $\begin{array}{l}\text { MST } \\
\text { Time }\end{array}$ & $\begin{array}{l}\text { Max. W/S } \\
\text { in } M / S\end{array}$ & $\begin{array}{l}\text { Max. Height } \\
\text { in M. ABGL }\end{array}$ & $\begin{array}{l}\text { Height of } \\
\text { Winds at } \\
\text { Max. Speed } \\
\text { in M. ABGL }\end{array}$ & $\begin{array}{l}\text { Height of } \\
\text { Inversion } \\
\text { in } M . A B G L\end{array}$ \\
\hline 1800 & 6.9 & 589 & 60 & 170 \\
\hline 1900 & 11.5 & 589 & 45 & 170 \\
\hline 2000 & 10.6 & 621 & 45 & 160 \\
\hline 2100 & 10.7 & 621 & 50 & 140 \\
\hline 2200 & \multicolumn{4}{|c|}{ Did not run-Tether problems } \\
\hline 2300 & 11.4 & \multicolumn{2}{|c|}{ Sonde blew off } & \\
\hline 0000 & 10.0 & 474 & 70 & 205 \\
\hline 0100 & 12.2 & 321 & 70 & 200 \\
\hline 0200 & \multirow{2}{*}{\multicolumn{4}{|c|}{$\begin{array}{l}\text { Did not run rest of night-Too windy } \\
\text { and attempted to patch balloon }\end{array}$}} \\
\hline 0300 & & & & \\
\hline \multicolumn{5}{|l|}{0400} \\
\hline 0500 & & & & \\
\hline
\end{tabular}

\section{February 2}

No experiment scheduled for this evening

\section{February 3}

This evening's experiments were hampered greatly by strong surface winds in the evening and early night; however, around $2300(11: 00 \mathrm{pm})$ everything seemed to quiet down and we were able to start our experiments. The winds remained uniform all the way up during each run; however, during each run the wind speed increased until it was unsafe to make the $0300(3: 00 \mathrm{am})$ run-no drainage was observed, and winds were steady out of the west to the northwest. 


\begin{tabular}{|c|c|c|c|c|}
\hline $\begin{array}{l}\text { MST } \\
\text { Time }\end{array}$ & $\begin{array}{l}\text { Max. W/S } \\
\text { in } M / S\end{array}$ & $\begin{array}{l}\text { Max. Height } \\
\text { in M. ABGL }\end{array}$ & $\begin{array}{l}\text { Height of } \\
\text { Winds at } \\
\text { Max. Speed } \\
\text { in M. ABGL }\end{array}$ & $\begin{array}{l}\text { Height of } \\
\text { Inversion } \\
\text { in } M . A B G L\end{array}$ \\
\hline 2000 & \multicolumn{4}{|c|}{ No run-Too windy } \\
\hline 2100 & \multicolumn{4}{|c|}{ No run-Too windy } \\
\hline 2200 & \multicolumn{4}{|c|}{ No run-Too windy } \\
\hline 2300 & 3.5 & 608 & 40 & 95 \\
\hline 0000 & 4.5 & 610 & 65 & 100 \\
\hline 0100 & 8.5 & 433 & 100 & 170 \\
\hline 0200 & 9.6 & 181 & 110 & 105 \\
\hline 0300 & \multicolumn{4}{|c|}{ Did not run-Too windy } \\
\hline 0400 & \multicolumn{4}{|c|}{ Did not run-Too windy } \\
\hline 0500 & \multicolumn{4}{|c|}{ Did not run-Too windy } \\
\hline
\end{tabular}

\section{February 4}

Early indications such as temperature changes and low-surface wind speeds indicated that this night would provide drainages of a sort. This happened-drainages set up early and stayed all evening. They varied in depth. The jets were in the 6-7 m/s range.

\begin{tabular}{|c|c|c|c|c|}
\hline $\begin{array}{l}\text { MST } \\
\text { Time }\end{array}$ & $\begin{array}{l}\text { Max. W/S } \\
\text { in } M / S\end{array}$ & $\begin{array}{l}\text { Max. Height } \\
\text { in M. ABGL }\end{array}$ & $\begin{array}{l}\text { Height of } \\
\text { Winds at } \\
\text { Max. Speed } \\
\text { in M. ABGL }\end{array}$ & $\begin{array}{l}\text { Height of } \\
\text { Inversion } \\
\text { in M. ABGL }\end{array}$ \\
\hline 2000 & 6.4 & 590 & 60 & 125 \\
\hline 2100 & 7.0 & 630 & 55 & 155 \\
\hline 2200 & 6.8 & 698 & 55 & 135 \\
\hline 2300 & 7.0 & 601 & 50 & 185 \\
\hline 0000 & 7.6 & 631 & 65 & 140 \\
\hline 0100 & 7.3 & 656 & 70 & 160 \\
\hline 0200 & 8.3 & 591 & 60 & 155 \\
\hline 0300 & 7.4 & 580 & 80 & 205 \\
\hline 0400 & 7.2 & 419 & 55 & 170 \\
\hline 0500 & \multicolumn{4}{|c|}{ Did not run-Tether line destroyed } \\
\hline
\end{tabular}

February 5

No experiments scheduled this evening. 


\section{February 6}

The chance for drainages this experimental evening again looked good as the sky was clear, temperature was cold, and surface wind speeds were calm. Drainage did indeed set up early and lasted all night with a jet of $6-8 \mathrm{~m} / \mathrm{s}$ at 60 to 90 meters above the ground. A nice temperature inversion appeared at about the 15-meter Height. All the profiles were very similar.

\begin{tabular}{|c|c|c|c|c|}
\hline $\begin{array}{l}\text { MST } \\
\text { Time }\end{array}$ & $\begin{array}{l}\text { Max. W/S } \\
\text { in } M / S\end{array}$ & $\begin{array}{l}\text { Max. Height } \\
\text { in M. ABGL }\end{array}$ & $\begin{array}{l}\text { Height of } \\
\text { Winds at } \\
\text { Max. Speed } \\
\text { in M. ABGL }\end{array}$ & $\begin{array}{l}\text { Height of } \\
\text { Inversion } \\
\text { in M. ABGL }\end{array}$ \\
\hline 2000 & \multicolumn{4}{|c|}{ No run-Winch broke } \\
\hline 2100 & \multicolumn{4}{|c|}{ No run-Winch repair } \\
\hline 2200 & 6.8 & 509 & 60 & 180 \\
\hline 2300 & 7.8 & 506 & 55 & 150 \\
\hline 0000 & 8.1 & 523 & 60 & 130 \\
\hline 0100 & 7.3 & 559 & 55 & 120 \\
\hline 0200 & 7.6 & 555 & 50 & 120 \\
\hline 0300 & 7.8 & 493 & 50 & 125 \\
\hline 0400 & 6.7 & 572 & 50 & 140 \\
\hline 0500 & 7.4 & 539 & 55 & 200 \\
\hline
\end{tabular}

\section{February 7}

This night's experiments and tethersonde profiles were almost a duplicate of the previous night's experiments. The same wind speeds, directions, jets, inversions, and drainage flows occurred at approximately the same elevations. The sky was clear and the temperatures were somewhat warmer. No problems occurred and all runs were completed.

\begin{tabular}{|c|c|c|c|c|}
\hline $\begin{array}{l}\text { MST } \\
\text { Time }\end{array}$ & $\begin{array}{l}\text { Max. W/S } \\
\text { in } M / S\end{array}$ & $\begin{array}{l}\text { Max. Height } \\
\text { in M. ABGL }\end{array}$ & $\begin{array}{l}\text { Height of } \\
\text { Winds at } \\
\text { Max. Speed } \\
\text { in M. ABGL }\end{array}$ & $\begin{array}{l}\text { Height of } \\
\text { Inversion } \\
\text { in M. ABGL }\end{array}$ \\
\hline 2000 & 7.7 & 352 & $60 / 350$ & 160 \\
\hline 2100 & 8.7 & 471 & 80 & 225 \\
\hline 2200 & 7.4 & 414 & 55 & 195 \\
\hline 2300 & 7.0 & 574 & 45 & 250 \\
\hline 0000 & 8.6 & 538 & 40 & 255 \\
\hline 0100 & 6.5 & 456 & 45 & 375 \\
\hline 0200 & 8.4 & 525 & 50 & 270 \\
\hline 0300 & 7.2 & 424 & 45 & 200 \\
\hline 0400 & 6.9 & 516 & 55 & 450 \\
\hline 0500 & 7.1 & 419 & 65 & 410 \\
\hline
\end{tabular}




\section{Summary}

The early experiments were of great value as the winds were strong $(5-6 \mathrm{~m} / \mathrm{s})$ near ground level and got stronger with Height. There were changes in wind directions as the balloon arose above the influence of the surrounding hills. At the latter part of the experimental period, good drainages set up and remained throughout the night. The jets were well defined as were the temperature inversions. The data from the meterological tower, Rich Coulters SODAR, and the Dopler Lidar all seem to be consistent with the Tethersonde Data.

Filename: KPE.Ip.8/21/91 
APPENDIX A 


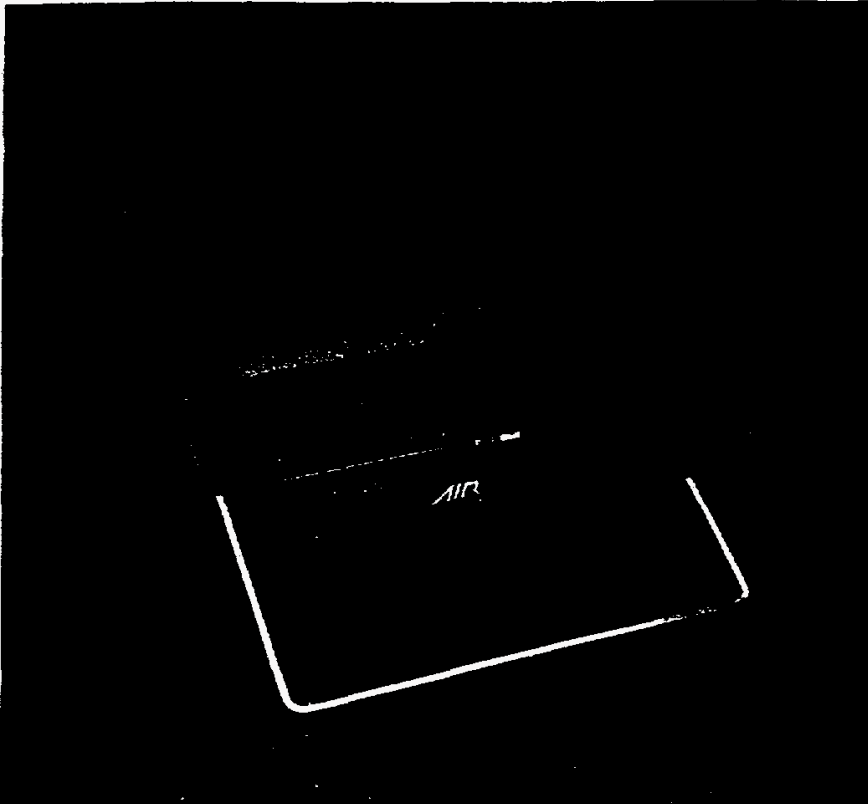

\section{9 \\ ADAS $^{\text {TM }}$}

ATMOSPHERIC DATA ACQUISITION SYSTEM

MODEL AIR-3C (MULTISONDE ${ }^{\text {TW }}$ ADAS)

Multiple Alrsonde /Tethersonde Ground Station. The AIR Atmospheric Data Acquisition System (ADAS) is a complete upper air ground station for receiving and processing telemetered data from balloon borne meteorological sensor packages. Model AIR-3C is designed to receive and process data from up to 3 separate sensor packages at the same time. It can aiso perform all the functions of the Model AIR-3A for upper air soundings with automatic wind computation. It can be used with both free and tethered balloon systems. This model is capable of computing over 50 meteorological variables in real-time from telemetered Tethersonde or Airsonde data.

The AIR-3C ADAS consists of a $400-410 \mathrm{Mhz}$ crystal controlled receiver, a Z-80 microcomputer, sottware in ROM, LED displays, a key pad, a paper tape reader, a theodolite angle interface, an IEEE-488 (GPIB) interface, a serial output port, a cassette tape recorder interface, and rechargeable internal battery pack.

Real-time digltal data. The AIR-3C receives data sequentially from up to three Airsondes and Tethersondes in any combination. The internal microcomputer converts it into digital meteorological data. The data is then sent in real-time to the output ports in digital format where it can be printed. recorded on cassette, or used by additional data processing equipment. Cassette recorded data can be read back through the AIR-3C at a later time and directed to the display, serial port or GPIB.

Automatic upper air soundings are possible when the AIR-3C is used with an AIR Airsonde and optical or radio theodolite. The AIR-3C can receive angle data through the theodolite interface. The interface works with AlP designed shaft encoder assemblies that can be attached to optical or radio theodolites. The AIR-3C generates an audible tone to signal the optical theodolite operator when the angles are being read.

When making upper air soundings, the AIR-3C can compute time, pressure, height, temperatures, humidity (from wet/drybulb or carbon hygristor), balloon speed and direction, and dozens of additional meteorological variables. A complete set of Airsonde data is output every 5-6 seconds; balloon speed and direction can be automatically averaged over a specified number of readings before output.

\section{FEATURES}

Recoives and procesees tolemetered data from up to three Areondes ${ }^{\circ}$ and Tethersondes ${ }^{\circ}$ with a single unit

Reah-time digital data output in meteorological units

Computes over 50 meteorological variables in realtime

$\square$ Interfaces to radlo or optical theodolite for automatic wind computation

Optional internal 400-410 Mhz recelver

$\square$ Outputs to serial port, IEEE-488, cassette recorder, and intogral dispiay

$\square$ Koy ped and papor tape reader for command and date entry

$\square$ Automatic operation reduces operator training, Aliminates analog data reduction, and improves data quality

$\square$ Portable-emaller than a briefcase, internal battery powner

$\square$ Intemal bottery automatically takes over during power fallure

Onhy syotem capable of multiple sonde data acquitition 


\section{APPLICATIONS}

\section{Simultaneous data from 3 levels in the atmosphere}

Measurements from up to 3 Tethersondes separated horizontally by up to $20 \mathrm{~km}$

Research and operational rawinsonde soundings

Boundary layer research

Air pollution studies - yields accurate wind and stabillty profiles

Noise propagation studies

Sea breeze studies

Mountain valley studles

Comparison data for acoustic and FM-CW boundary layer radars
For the Tethersonde, the AIR-3C can compute time. pressure, height, temperature, humidity, wind speed and direction, plus many more meteorological variables. All in a variety of selectable units. A complete set of Tethersonde data is output every 10-12 seconds.

The AIR-3C performs nearly automatically from the time the power is turned on. Commands and data are entered through the labeled key pad and paper tape reader. The paper tape reader is used primarily to enter calibration data from the tape that accompanies each AIR sonde.

Completely portable. Small size, together with internal battery power, makes the AIR-3C a completely portable unit. When used with a battery powered cassette recorder. the system can acquire and record data in the field without external power

\section{ADAS SPECIFICATIONS MODEL AIR-3C (MULTISONDE ${ }^{m}$ ADAS)}

\section{Receiver}

Frequencies:

Bandwidth:

AFC Capture Range:

AFC Tracking:

Sensitivity:

Audio Frequency Input (From external receiver)

Connector:

Signal Level:

\section{Data Processing}

Microprocessor:

Computed Values Include (each sonde):

Ory-bulb Temperature
Wet-bulb Temperature
Wet-bulb Depression
Relative Humidity
Dew Point
Mixing Ratio
Saturation Vapor Pressure

Theodolite Interface

Shaft Encoder Assemblies:

\section{Key Pad}

\section{Paper Tape Reader}

Tape type:

Punch format:
BNC

$1 \mathrm{Vpp}$ (nominal)

Crystal controlled, 3 frequencies within $400-410 \mathrm{Mhz}$ range

$15 \mathrm{Khz}$

$\pm 50 \mathrm{Khz}$

$\pm 60 \mathrm{Khz}$

$0.25 \mu \mathrm{V}$

Frequency:

Z-80 with ROM firmware

$\begin{array}{ll}\text { Time } & \text { Azimuth Angle } \\ \text { Date } & \text { Elevation Angle } \\ \text { Pressure } & \text { Wind Speed } \\ \text { Height ASL } & \text { Wind Direction } \\ \text { Height AGL } & \text { Potential Temperature } \\ \text { Ascent Pate } & \text { Enthalpy } \\ \text { Distance } & \text { Retractivity }\end{array}$

GMD uses AIR-3A-1EG RD-65 uses AIR-3A-1ER Optical theodolite uses AIR-3A-2E

25-keys, labeled \& color coded

Black ISO-standardized 1 in. wide 8 track ISO standard, ASCII

Output

Ports:

Length:

Width:

Height:

Mass:

Power

Line:
Operating Temperature: $\quad 5^{\circ} \mathrm{C}$ to $40^{\circ} \mathrm{C}$

Displays (LED):

5 character data units 10 bar tuning meter

IEEE-488 (GPIB)

RS-232C/20 ma TTY

Baud rates: $150,300,600$,

$1200,2400,4800$, or 9600

Format: ASCIl character

Cassette Tape Recorder (Send/

Receive)

Baud rates: 300 or 1200

Airsonde-New data every 5-6 sec.

Tethersonde-New data every 10-12 sec.

Dimenslons and Mass

$31.1 \mathrm{~cm}(12.25 \mathrm{in}$.

$22.2 \mathrm{~cm}$ (8.75 in.)

$8.9 \mathrm{~cm}$ (3.5 in.)

$5.3 \mathrm{~kg}(11.7 \mathrm{lb}$.

Internal Battery (Std.)

115/220VAC $47-63 \mathrm{~Hz} 12 \mathrm{~W}$

Automatic switch-over if line voltage falls

Type: Rechargeable Operating time: 3 hours Recharge time: 18 hours

External Battery (Opt.): $\quad$ 12V DC $10 \mathrm{~W}$ 


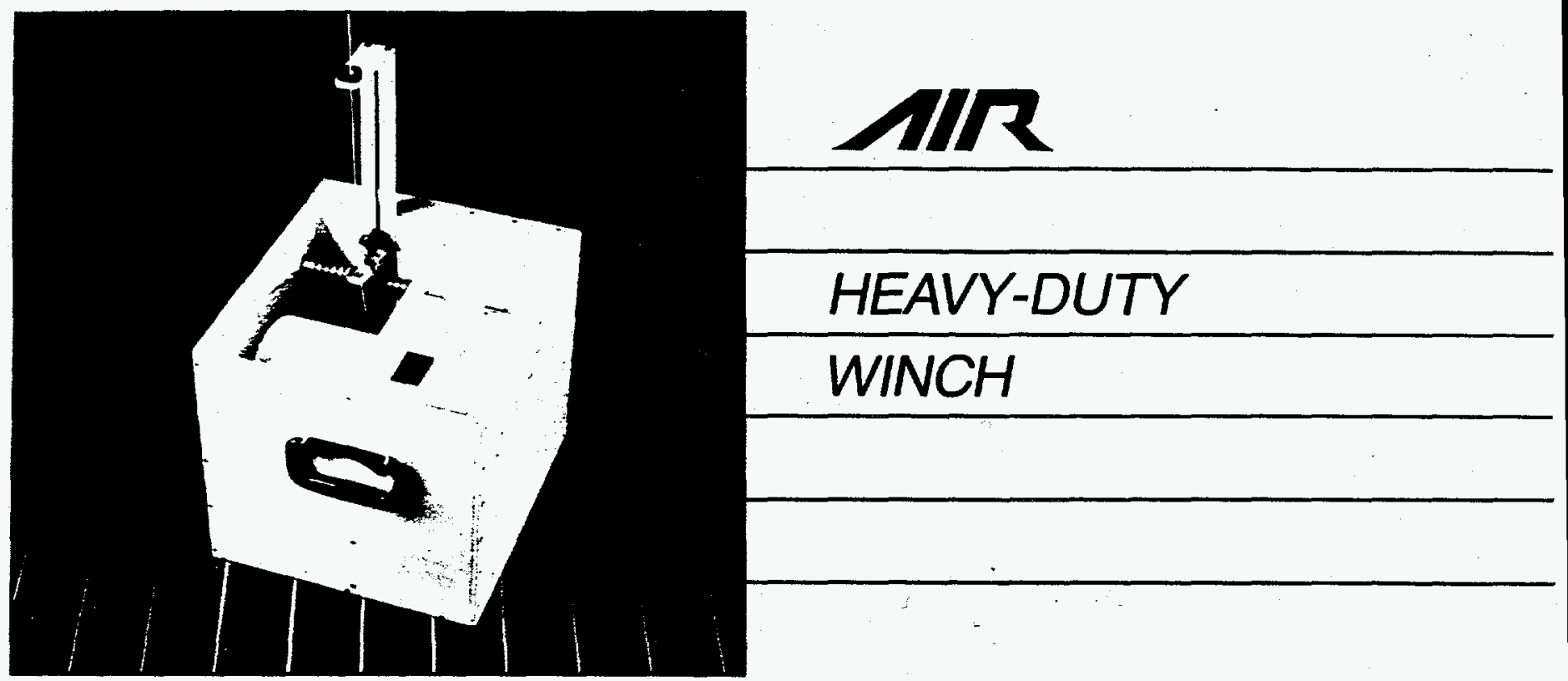

For rapid retrieval. The TS-3AW heavy-duty winch is designed for rapid retrieval of a tethered balloon or kite under heavy wind loading. The winch features reversible, variable speeds controlled through a hand-held selector. The hightorque drive train guarantees minimal speed variations under changing loads.

Long-term reliability and ruggedness. The Winch is constructed of quality materials for rugged use and long-term reliability in severe environments. Machined parts are made from stainless steel and aluminum using precision N/C machines. Wear is minimized through the use of ceramic and hardened steel line guides and an all ball bearing drive train.

The winch drum and level-wind are driven by a single $1 / 2 \mathrm{HP}$ high-torque industrial motor. The drum is rotated through a toothed belt drive and worm gear transmission.

Safety and ease of use. An emergency stop switch immediately halts the winch operation. A level-winding mechanism causes even line distribution on the drum preventing line abrasion and assuring smooth line feed. Drum flange guards prevent line fouling. Overload and overheat circuitry protects the winch. The self-braking worm gear drive assures line feed control even during loss of power. External drive take-off permits retrieval during electrical power failure.

The compact design with no protrusions permits easy transporting. The winch reduces to a volume of about one cubic foot. The remote control and power cord are stored in an integral compartment. The line guides slide into the body of the winch.
High torque at high speeds for fast retrieval under heavy loads

$\square 1 / 2 \mathrm{HP}$ industrial motor and control circuitry provide long-term reliability

$\square$ Stainless steel and anodized aluminum construction resists corrosion

Variable speed

Reversible

Remote control for ease of use

Self-braking worm gear drive for safe and rellable control

Automatic level-winding system for even line distribution on drum

Clean, compact design for easy transport 


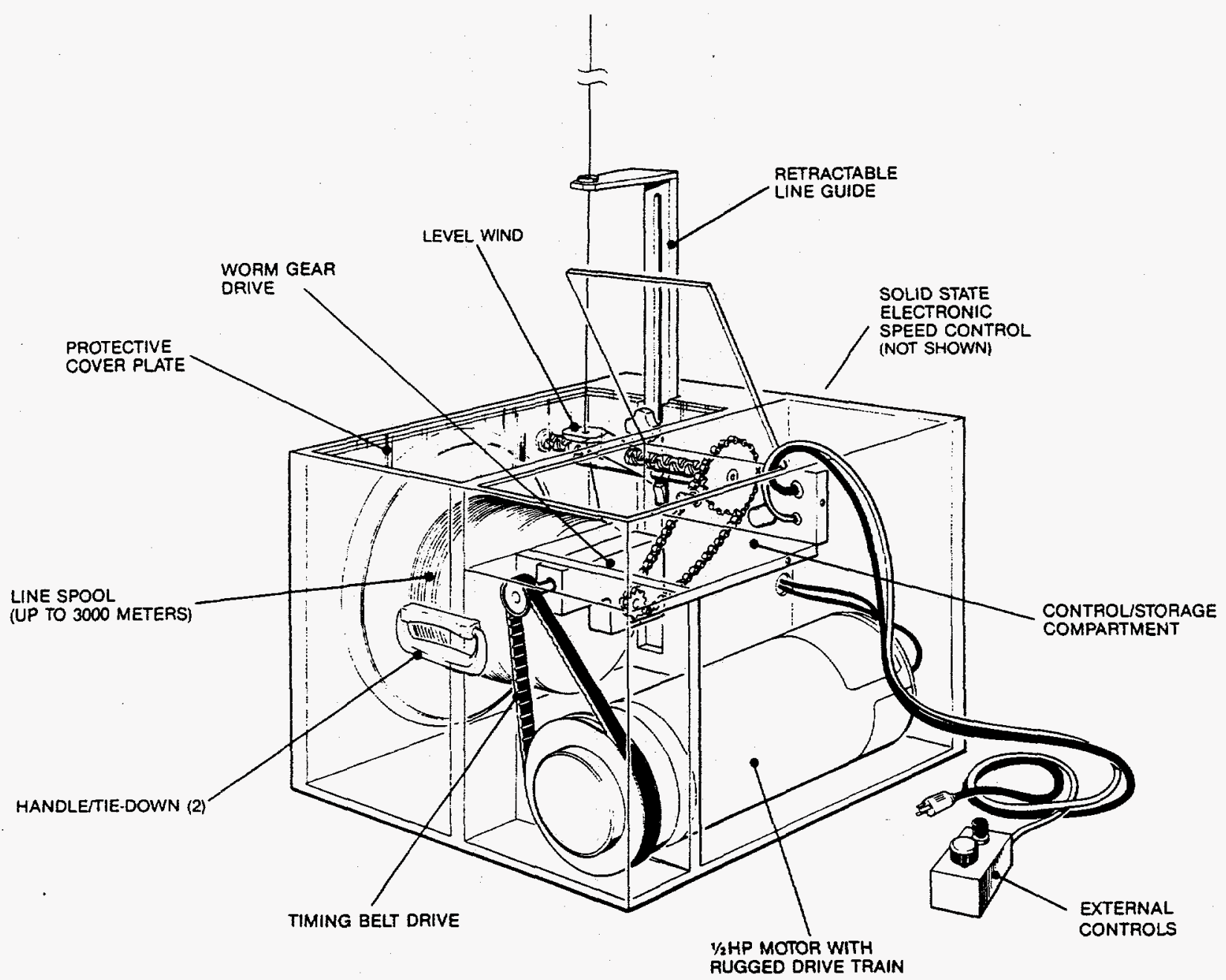

\section{HEAVY-DUTY WINCH TS-3AW SPECIFCATONS}

Speed: Variable 0-5.8 ft/s $(0-1.8 \mathrm{~m} / \mathrm{s}) @ 75 \mathrm{lbs} .(34 \mathrm{~kg}) \mathrm{load}$

Maximum Load: $100 \mathrm{lbs} .(45 \mathrm{~kg}) \quad$ (Higher torque available with optional gearing)

Drum Capacity: 6 in. $(15.2 \mathrm{~cm})$ diameter, $5.5 \mathrm{in} .(14.0 \mathrm{~cm})$ wide drum with $9 \mathrm{in} .(22.9 \mathrm{~cm})$ diameter flange

Max. Line Lengths: $\quad 10,000 \mathrm{ft}(3 \mathrm{~km}) 160 \mathrm{lbs} .(74 \mathrm{~kg})$ test line

$7,000 \mathrm{ft}(2 \mathrm{~km}) 240 \mathrm{lbs} .(110 \mathrm{~kg})$ test line

Drive Train: $\quad 1 / 2 \mathrm{HP}$ dc motor with $20: 1$ worm drive

Ambient Operating Temperature: $\quad+32^{\circ} \mathrm{F}$ to $+122^{\circ} \mathrm{F}\left(0^{\circ} \mathrm{C}\right.$ to $\left.+50^{\circ} \mathrm{C}\right)(\mathrm{Std})$

$-22^{\circ} \mathrm{F}$ to $+122^{\circ} \mathrm{F}\left(-30^{\circ} \mathrm{C}\right.$ to $\left.+50^{\circ} \mathrm{C}\right)(\mathrm{Opt})$

Power Requirements: $\quad 95-135$ VAC $\quad 50-60 \mathrm{~Hz} 10$ Amps max. (Std) 195-260 VAC $\quad 50-60 \mathrm{~Hz} \quad 5$ Amps max. (Opt)

Dimensions: $13.7 \mathrm{in}$. width, $13.5 \mathrm{in}$. depth, $11.75 \mathrm{in}$. height ( $34.8 \mathrm{~cm}$ width, $34.3 \mathrm{~cm}$ depth, $29.8 \mathrm{~cm}$ height)

Weight: $61 \mathrm{lbs} .(27.7 \mathrm{~kg})$ 

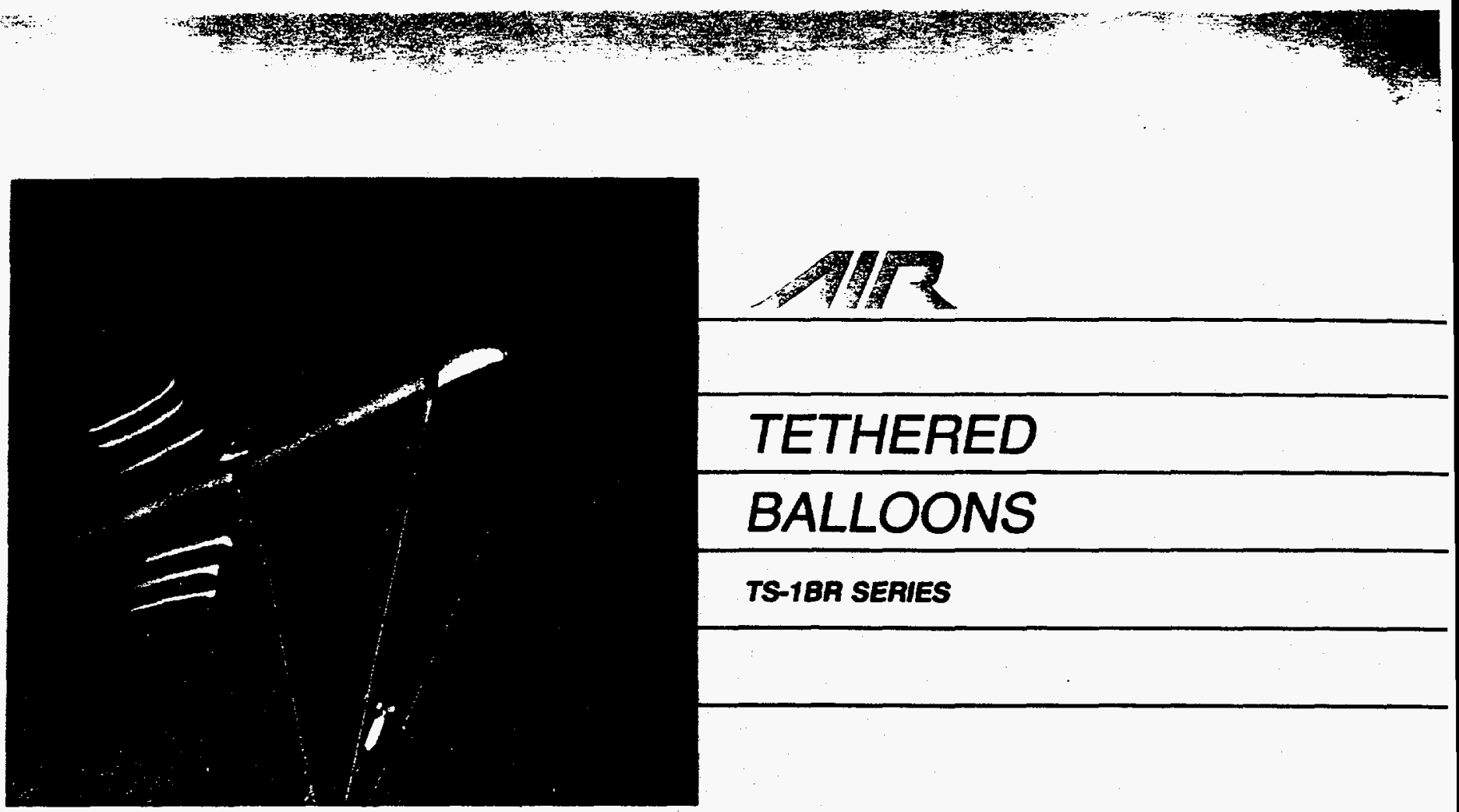

\section{TETHERED}

\section{BALLOONS}

TS-1BR SERIES

Proven reflabillty. The AIR Tethered Balloon was developed specifically to serve as a vehicle for the AIR Tethersonde atmospheric sounding probe. With years of proven field research, from the Arctic to the Antarctic, from the Sahara Desert to tropical rain forests, the AIR Tethered Balloon has proven its reliability and endurance.

It is used in a variety of applications including portable meteorological towers, air pollution sensor platforms, boundary layer research, radar target platforms, and point source (stack) aerosol simulation.

Superior payload to volume ratto. The Tethered Balloon is fabricated with $1.5 \mathrm{mil}(0.038 \mathrm{~mm})$ urethane plastic. This material has several time the shear and tear strength of standard: balloon polyethylene. Very thin and strong, this material givegs AIR's Tethered Balloon its high lift to fohme ratio: Paytoad to

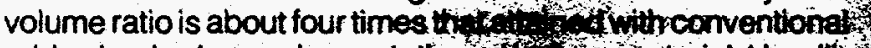

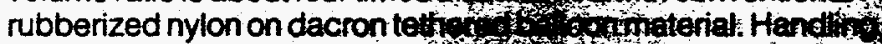

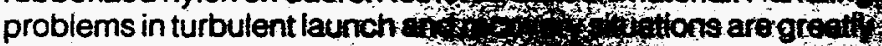
diminished. The Tethered Bets of pay for ho reduced helium or hydrogent costs of heavy cylindarest

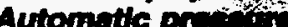

designed to autorem on on pressure during $n^{2}$

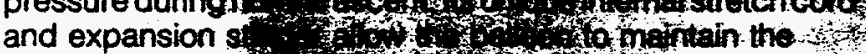
aerodynamic she rated altitude and 30 to

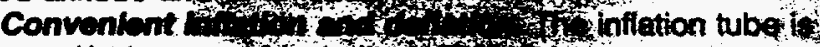
mounted in the now of the bitos, of, of anoperator to

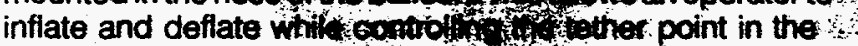

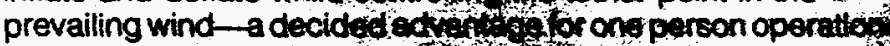
in gusty turbulent winds:

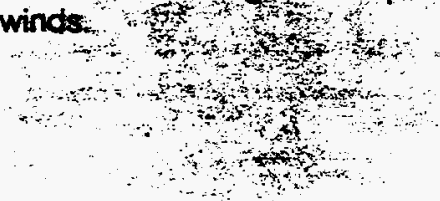

\section{FEATURES}

Economiced

Rugoed-Durable

Wide operuting temperature range

Vory high payloed tin to volume ratio

Portibla-1-2 Ko maco

Anodymanle ablithy

Hot ditude $-1.5 \mathrm{KM}$

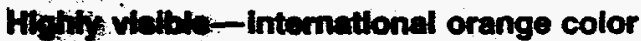
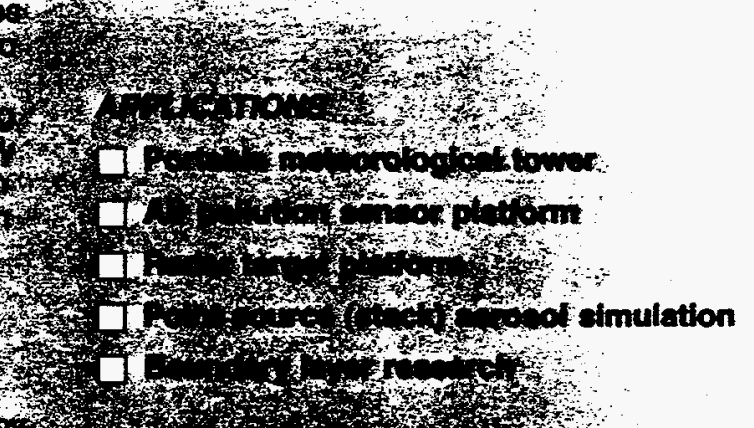


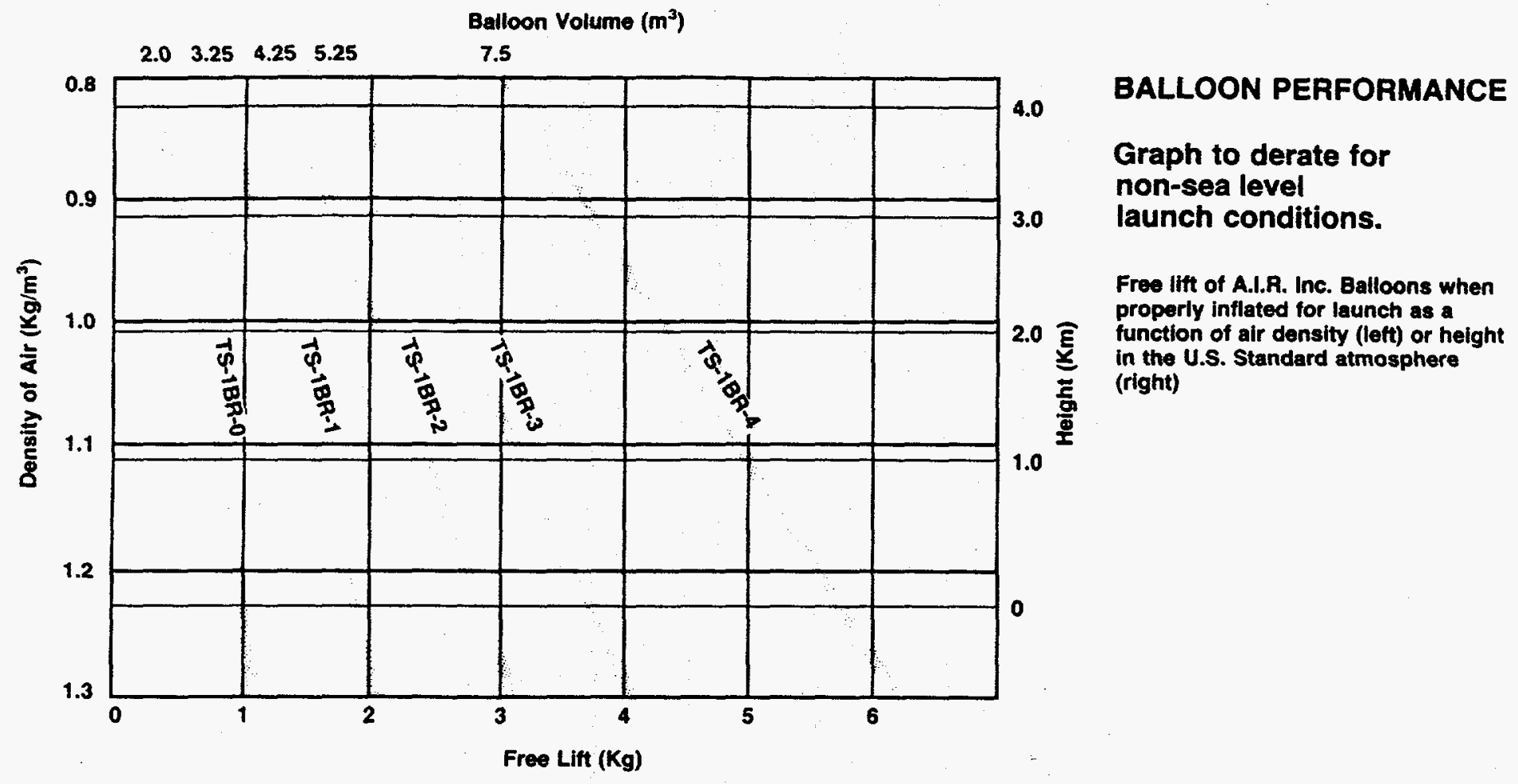

BALLOON DATA TS-1BR-SERIES

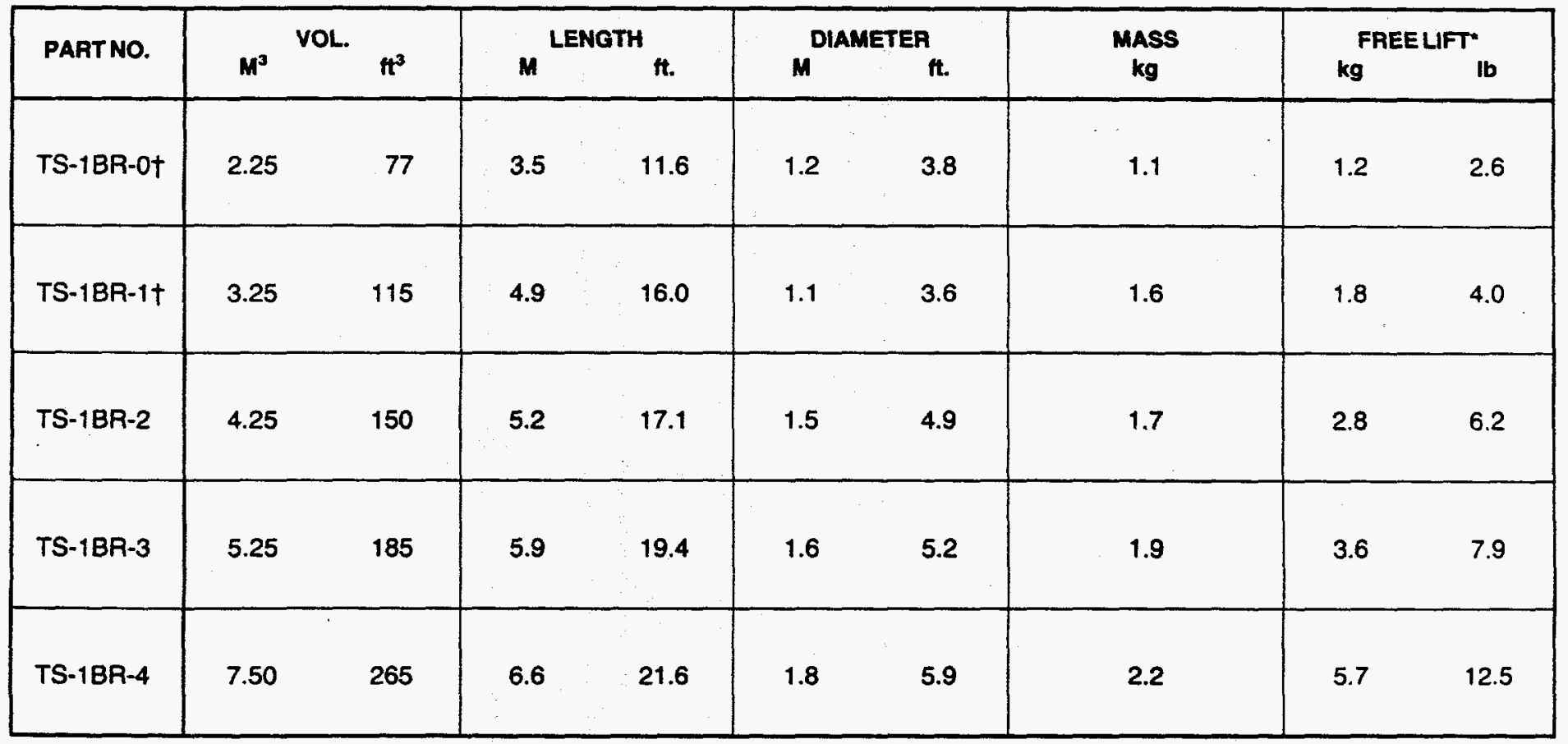

- The free lift is the mass, exclusive of the balfoon, that the balloon will litt. The free lift values in this table are for seal level in the U.S. Standard atmosphere,

1962. + These balloons are below $115 \mathrm{lt}^{3}$ in volume and 6t. in diameter; therefore federal Aviation Regulation, Part 101 is not applicable, according to

Anticle 101.1 of Part 101. 

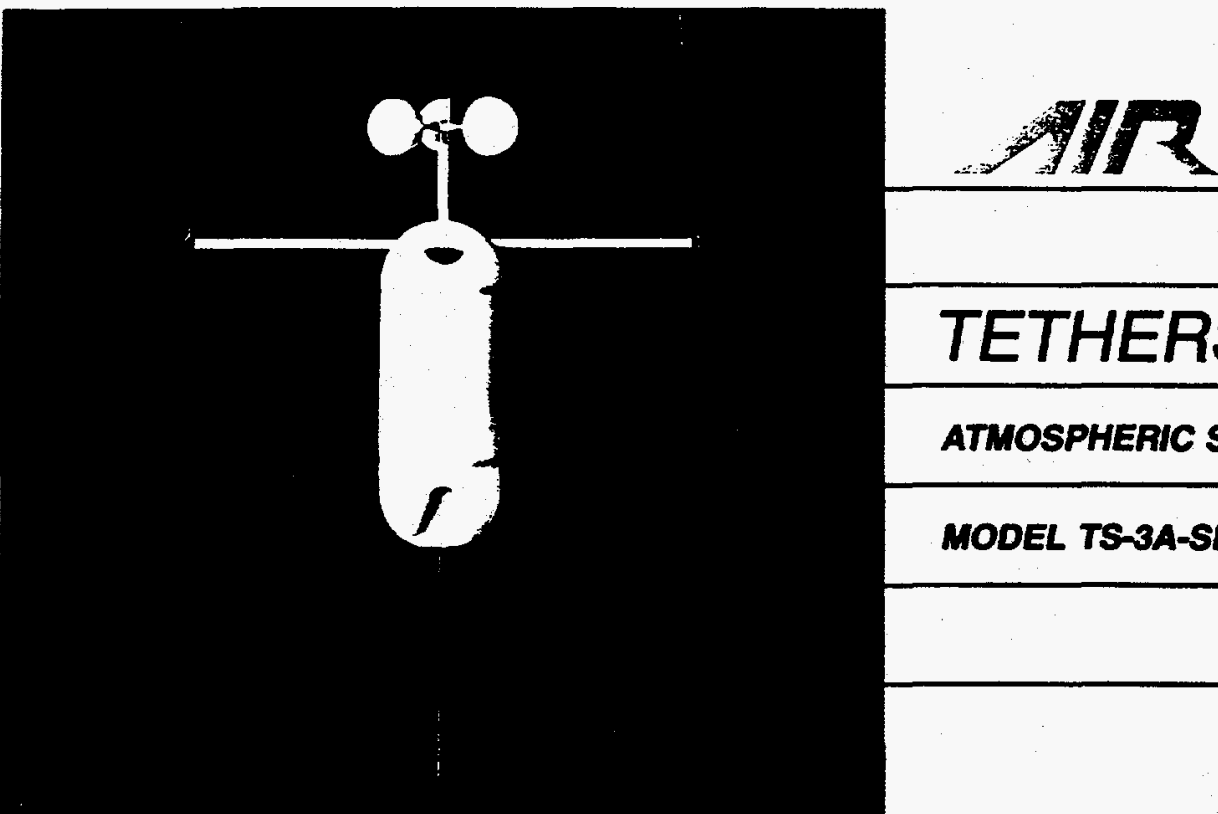

TETHERSONDE ${ }^{\circledR}$

ATMOSPHERIC SOUNDING PROBE

MODEL TS-3A-SP SERIES

A unique boundary layer probe. The AIR Tethersonde meteorological sounding sensor package is designed for use in boundary layer measurements. If accurately measures pressure, temperature, humidity, wind speed and direction.

While suspended below a gas-filled, blimp-shaped tethered balloon (TS-1BR) or specially designed kite, the Tethersonde system operates effectively at altitudes up to $3 \mathrm{~km}$ above the surface. Altitude of the tethered balloon or kite is controlled by AIR's Heavy-Duty Winch (TS-3AW)

All calibration information is contained on a punched paper tape-no baseline is required. All electronics are integrated on a single printed circuit board. The housing is lightweight molded polystyrene. Power is provided by a sealed $9 \mathrm{~V}$ alkaline battery.

Nine data channols overy 8 to 10 soconde. In addition to five standard sensors, the Tethersonde has four spare analog channels available for additional sensors. The Tethersonde's CMOS Integrated circuitry time-multiplexes data transmission. Five standard data channels (T, Tw or RH, P, Ws, Wd) plus 4 spere channels are scanned and transmitted every 8-10 seconds. The transmitted information permits automatic synchronization and callbration by the ADAS ground station.

Dry and wot butb tempernturesenemeesured by model AS-3A-SP. Two carefully matched thermistore aenury isortormance comparable to. a good Assmann psychrometer. A shicido inents radiation errors and protects the thermistore from demege. Aepiration is produced by a small fan and the watesenoir is sufficient for several hours of operation. At tempen ures below $0^{\circ} \mathrm{C}$, the frozen wick continues to provide humidity inteif tion by moseuning the evaporation with respect to ice to as low as $=30 \mathrm{c}$.

Humblty is computed by the ADS ground station from the dry and wet bulb temperatures for modd AS-3A-SP or from the dry bulb temperature and resistance of the carbon mygristor for model AS-3A$\mathrm{SPH}$. The carbon hygristor replaces the wet bulb in this model.

Preaver is measured by a peitented electronic aneroid capacitance pressure sensor. The sensor is mounted intemally for protection against thermal transients and mochenical strese. Temperature data for compensation is provided by a themistor attached to the sensor.

Mind speed is meesured with a patented three-cup anemometer and a light-chopper tachometer. The design features very low starting speed and excellent sensitivity. The individual cups are easily replaced and are manufactured from a single moid for near perfect balance and match.

Wind direction is measured with a small electronically controlled magnetic compass. Wind direction detection relies on the aerodynamic balloon or kite serving as a wind vane to orient the Tethersonde and compass.

\section{FEATURES}

$\square$ Precielon sensors:

Thermistor dry \& wot bulbe $\left( \pm 0.5^{\circ} \mathrm{C}\right)$

Humldity ( $\pm 5 \%)$

Aneroid preasure cell $( \pm 1 \mathrm{mb})$

Wind Speved $( \pm 0.25 \mathrm{~m} / \mathrm{s})$

Wind Direction ( \pm 5 deg)

$\square$ High resolution: (0.01* C, 0.1\% RH, $0.1 \mathrm{mb}$, $0.1 \mathrm{~m} / \mathrm{a}, 1 \mathrm{ceg}$ )

Faet reapones

Extenative Incthidual beaing and callbration

Uohtumigit $(205,8 \times)$ allowe use of small baloon (2.25 int)

\section{Rellable, rugand condruction}

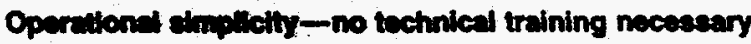

Crybal conturend trenemither-no RF tuning

Economicant it?<smiles>C[As]1[As]=[As]1</smiles><smiles></smiles>

Appucatronis,

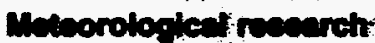

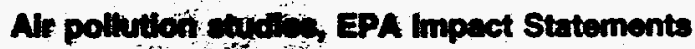

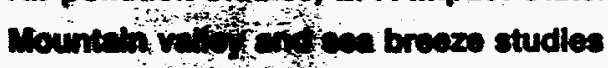

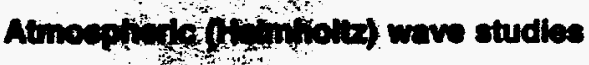

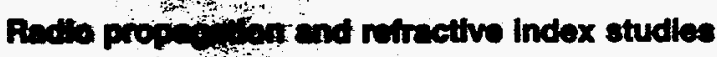

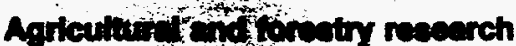

Sound popongtion pudies 


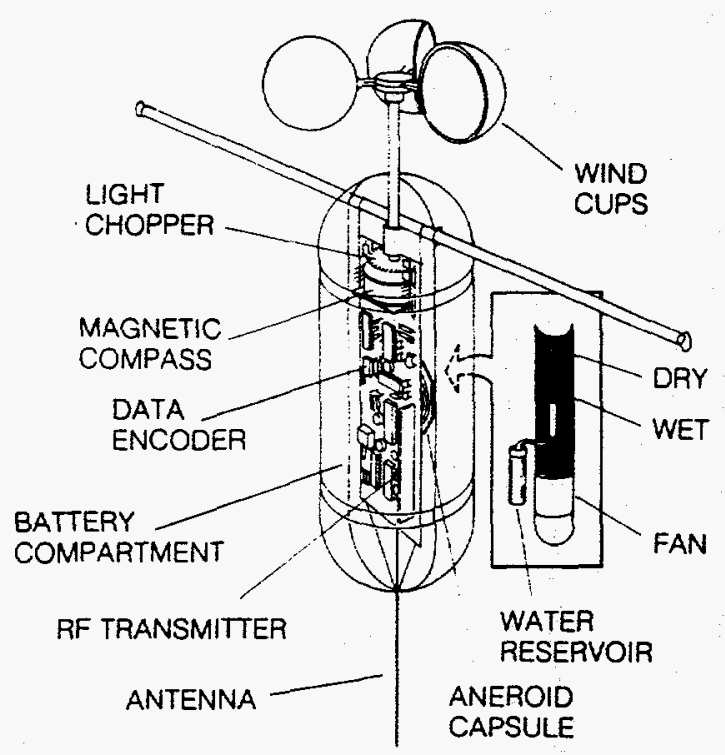

High stability, narrow band transmisaion. The Tethersonde telemeters its data to the AIR Atmospheric Data Acquisition System (ADAS), an automatic, digital output ground station. A crystal controlled FM transmitter produces high stability, narrow band transmissions which allow communication at distances up to 20 $\mathrm{km}$ with only $10 \mathrm{mw}$ of power

Crystal control eliminates RF tuning problems and insures reliable noise-free data. It aiso permits a number of Tethersondes to operate in close proximity without interference.

\section{GENEAAL DESCRIPTION}

Construction:

Body molded of expanded polystyrene. A single printed circuit card contains

Power: $\quad$ Sealed $9 \mathrm{~V}$ alkaline transistor all electronics.

Operating time:

Size: 6.8 hours

Weight:

Fits into $30 \mathrm{~cm} \times 30 \mathrm{~cm} \times 10 \mathrm{~cm}$ volume 225 grams ( $8 \mathrm{oz}$.)

\section{ORDERING INFORMATION}

Specify transmission frequency if other than $403.5 \mathrm{Mhz}$ Model TS-3A-SP Drybulb and wet bulb humidity measurement

Model TS-3A-SPH Drybulb and carbon hygristor humidity measurement

\section{TETHERSONDE SPECIFICATIONS MODEL TS-3A-SP and TS-3A-SPH}

\begin{tabular}{|c|c|c|c|}
\hline \multicolumn{4}{|l|}{ SENSORS } \\
\hline \multicolumn{2}{|c|}{ Temperature (Wet and Dry Bulb) } & \multicolumn{2}{|c|}{ Pressure (Absolute Barometric) } \\
\hline $\begin{array}{l}\text { Range: } \\
\text { Precision: }\end{array}$ & $\begin{array}{l}+50^{\circ} \mathrm{C} \text { to }-70^{\circ} \mathrm{C} \\
0.5^{\circ} \mathrm{C} \text { for }+40^{\circ} \mathrm{C} \text { to }-40^{\circ} \mathrm{C} \\
1.0^{\circ} \mathrm{C} \text { for }+50^{\circ} \mathrm{C} \text { to }-70^{\circ} \mathrm{C}\end{array}$ & $\begin{array}{l}\text { Range: } \\
\text { Accuracy: } \\
\text { Precision: }\end{array}$ & $\begin{array}{l}1050 \text { to } 600 \mathrm{mb} \\
5 \mathrm{mb} \\
1 \mathrm{mb}\end{array}$ \\
\hline Thermistor Match: & $0.1^{\circ} \mathrm{C}$ for $+35^{\circ} \mathrm{C}$ to $-20^{\circ} \mathrm{C}$ & Resolution: & $0.1 \mathrm{mb}$ \\
\hline Resolution: & $0.01^{\circ} \mathrm{C}$ & Temperature Compensation: & Computed by AlA ground station \\
\hline RMS Noise Equivalent: & $0.04^{\circ} \mathrm{C}$ & Wind Speed & \\
\hline $\begin{array}{l}\text { Response Time: } \\
\text { Humidity (TS-3A-SP, }\end{array}$ & $\begin{array}{l}\text { Dry bulb: } 3 \text { sec., Wet bulb: } 12 \mathrm{sec} \text {. } \\
\text { chrometric equation) }\end{array}$ & $\begin{array}{l}\text { Range: } \\
\text { Precision: }\end{array}$ & $\begin{array}{l}0.20 \mathrm{~m} / \mathrm{s} \\
0.25 \mathrm{~m} / \mathrm{s}\end{array}$ \\
\hline Range: & $3 \%$ to $100 \%$ & Resolution: & $0.1 \mathrm{~m} / \mathrm{s}$ \\
\hline Precision: & $\begin{array}{l}3 \% \text { for } 0^{\circ} \mathrm{C} \text { to } 50^{\circ} \mathrm{C} \\
5 \% \text { for }-10^{\circ} \mathrm{C} \text { to } 0^{\circ} \mathrm{C} \\
10 \% \text { for }-25^{\circ} \mathrm{C} \text { to }-10^{\circ} \mathrm{C}\end{array}$ & $\begin{array}{l}\text { Wind Direction } \\
\text { Range: } \\
\text { Precision. }\end{array}$ & $\begin{array}{l}2-358 \text { (4 deadband) } \\
5\end{array}$ \\
\hline Resolution: & $0.1 \% \mathrm{RH}$ & Resolution: & 1 \\
\hline Humidity (TS-3A-SPH, & arbon hygristor) & Spare Inputs (4 each) & \\
\hline Range: & $20 \%$ to $100 \% \mathrm{RH}$ & Range: & $0-100 \%$ \\
\hline Precision: & $5 \% \mathrm{RH},+40^{\circ} \mathrm{C}$ to $-40^{\circ} \mathrm{C}$ & Precision: & $1 \%$ \\
\hline Resolution: & $0.1 \% \mathrm{RH}$ & Resolution: & $0.1 \%$ \\
\hline
\end{tabular}

\section{TRANSMITTER}

Carrier Frequency:

Modulation:

Audio Modulation:

Transmitter Type:

Stability:

Deviation:

\author{
403.5 Mhz (std), \\ 400-410 Mhz (optional) \\ FM, narrow band \\ $1.5 \mathrm{Khz}$ to $3.5 \mathrm{Khz}$ \\ Crystal VCXO with $9 \times$ multiplication \\ $0.02 \%\left(+50^{\circ} \mathrm{C}\right.$ to $\left.-70^{\circ} \mathrm{C}\right)$ \\ $\pm 5 \mathrm{Khz}$
}

Tuning:

Sidebands:

Transmitter Power:

Telemetry Range (nominal):

Antenna:

RFPolarization: $\pm 20 \mathrm{Khz}$

$35 \mathrm{~dB}$ down

10 milliwatts

$20 \mathrm{~km}$

$1 / 4$ wave vertical monopole

linear, vertical 


\section{A Specifications}

The Handar 431A Wind Direction Sensor is designed for remote and unattended applications where extremes in environmental conditions are encountered. Precision heavy-duty construction using corrosion resistant aluminum and stainless steel ensure operation in high winds and heavy icing conditions. The sensor is finished with mattblack epoxy paint which provides de-icing in the presence of solar radiation.

The 431A uses a precision wire-wound potentiometer with a narrow gap. The wire-wound potentiometer has excellent linearity and low rotational torque. The vane is supported with a stainless steel shaft turning in shielded instrument grade, stainless steel grade bearings. Once the instrument is mounted and aligned, it may be removed and replaced in the field while maintaining its original orientation using an alignment sleeve.

SENSOR TYPE

VANE MATERIAL

TURNING RADIUS

SLEEVE DIAMETER

WEIGHT

RANGE

THRESHOLD

DISTANT CONSTANT

MAXIMUM WIND SPEED

ACCURACY

ICE LOAD

ELECTRICAL

Resistance

POT Gap

MATING CONNECTOR

MOUNTING
Vane/Potentiometer

Aluminum

13.5 Inches

1.315 Inches

2 Pounds

$0.360^{\circ}$

1 mph

15 Feet

$150 \mathrm{mph}$

$2^{\circ}$

2 Inches

1000 Ohms

$10^{\circ}$

MS 3106A.14S.6S

$3 / 2$ Inch IPS Nipple

Fig. 6 


\section{AVB TEMPERATURE SENSOR}

The HANDAR 432 AB is a temperature sensor designed for use with HANDAR Data Loggers and Data Logging Systems. The sensor is provided in two configurations. The " $A$ " model uses a single thermister type sensor and the " $B$ " model uses a composite thermolinear network to provide a linear voltage vs temperature output. The resistor compensation network for the $B$ model is included within the sensor housing.
The 432AVB is a waterproof sensor and is intended for use in measuring air and water temperatures. For air temperatures the 434A Vane Aspirated Shield or $442 \mathrm{~A}$ naturally aspirated parallel plate shield should be used. Water temperatures can be measured by immersing the sensor directly in the liquid.

\section{SPECIFICATIONS}

432A

Maximum operating temp: $-50^{\circ} \mathrm{C}$ to $+50^{\circ} \mathrm{C}$

Storage temperature: $-80^{\circ} \mathrm{C}$ to $+120^{\circ} \mathrm{C}$

Maximum error from $-20^{\circ} \mathrm{C}$ to $+50^{\circ} \mathrm{C}$ is $0.1^{\circ} \mathrm{C}$

Maximum error from $-50^{\circ} \mathrm{C}$ to $-20^{\circ} \mathrm{C}$ is $0.3^{\circ} \mathrm{C}$ $432 B$

Maximum operating temp: $-50^{\circ} \mathrm{C}$ to $+50^{\circ} \mathrm{C}$

Time Constant: 15 seconds

Storage temperature: $75^{\circ} \mathrm{C}$ maximum
Maximum error from $-50^{\circ} \mathrm{C}$ to $+50^{\circ} \mathrm{C}$ is $0.1^{\circ} \mathrm{C}$

Size:

Sensor element: $6^{\prime \prime}$ in length $\times 0.5^{\prime \prime}$ diameter

Cable: $10^{\prime}$ length submersable, w/rodent shield

Connector: 520-0086 connector $520-0091$ cable clamp HPN520-0092 Boot

Or Pigtail

Weight: $0.9 \mathrm{lbs}$ (including cable)
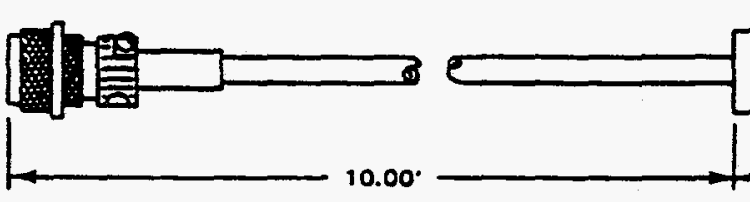

Fig. 7 


\section{A Specifications}

The Handar 430A Wind Speed Sensor is designed for remote and unattended applications where extremes in environmental conditions are encountered. Precision heavy.duty construction from aluminum and stainless steel ensure operation in high winds and heavy icing conditions. The sensor is finished with matt-black epoxy paint which provides de-icing in the presence of solar radiation.

The 430A uses a rotating magnetic field in proximity to a solid-state Hall Effect device to provide a pulse output proportional to wind speed. The Hall Effect Transducer provides stable operation over large temperature and voltage variations and consumes less than 2.0 MA current. The electrical characteristics of the 430A are ideal for remote solar and battery powered installations where low-current consumption is a necessity.

SENSOR TYPE

CUP SIZE

CUP MATERIAL

MATERIAL HOUSING

FINISH

TURNING RADIUS

WEIGHT

TRANSDUCER TYPE

Input Voltage

Current

Output Voltage (10K Load)

Pulse Type

RANGE

STARTING THRESHOLD

ACCURACY

DISTANCE CONSTANT

TEMPERATURE/OPERATING

ICE LOAD

OUTPUT FREOUENCY

VELOCITY CONSTANT

MATING CONNECTOR

MOUNTING
3. Cup Annemometer

2.130 Inches

Aluminum

Aluminum

Black Epoxy Paint

3.920 Inches

16 Ounces

Hall Effect

10.15 Volts

2.0 MA @ 12.5V Typ

$10 \mathrm{~V}-7.0 / 65$ Typ

$15 \mathrm{~V}-11.5 / .65$ Typ

$0.150 \mathrm{mph}$

$1 \mathrm{mph}$

\pm .25 mph or $2 \%$

15 Feet

$-40^{\circ} \mathrm{C}$ to $+70^{\circ} \mathrm{C}$

2 Inches

$0.279 \mathrm{~Hz} / \mathrm{mph}$

MS 3106A 14S.6S

Y: Inch IPS Nipple

Fig. 8 
APPENDIX B 


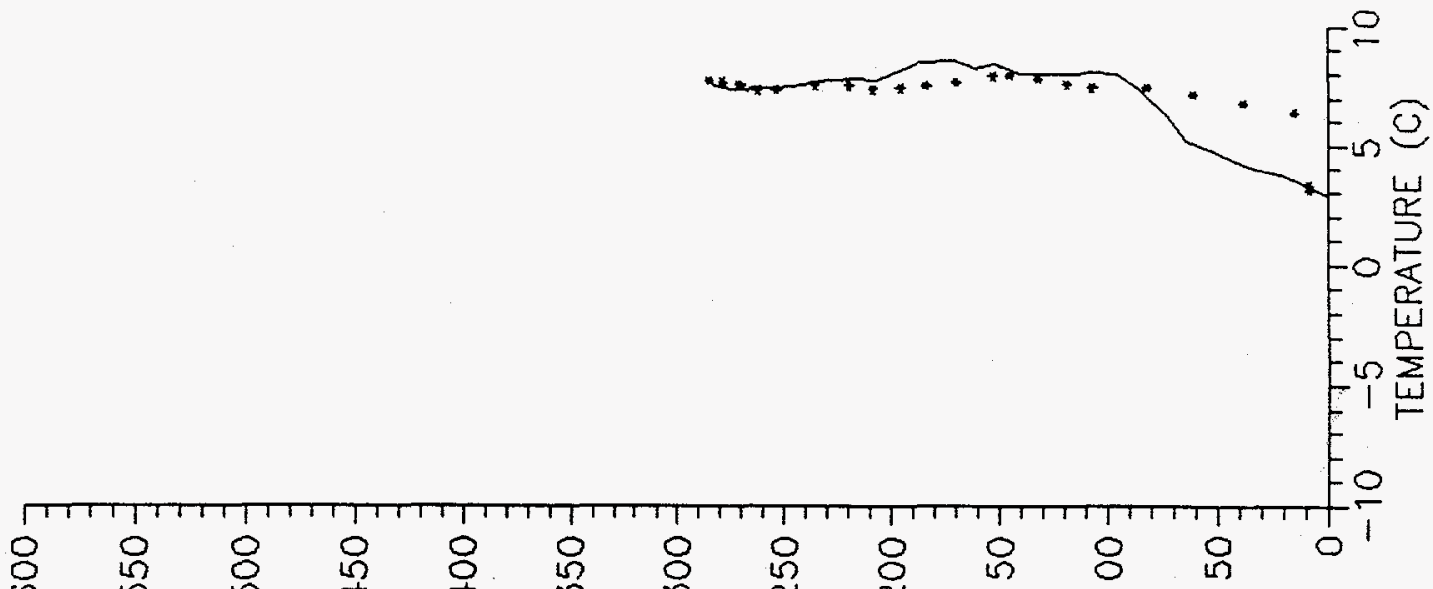

$\frac{5}{0} \mid:$

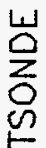

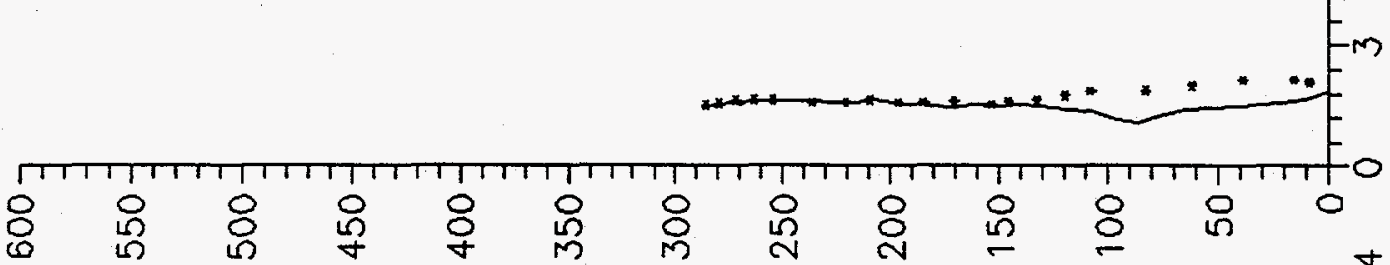

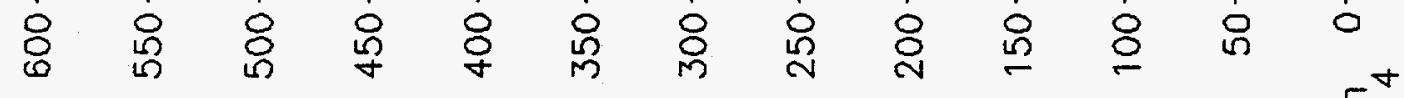

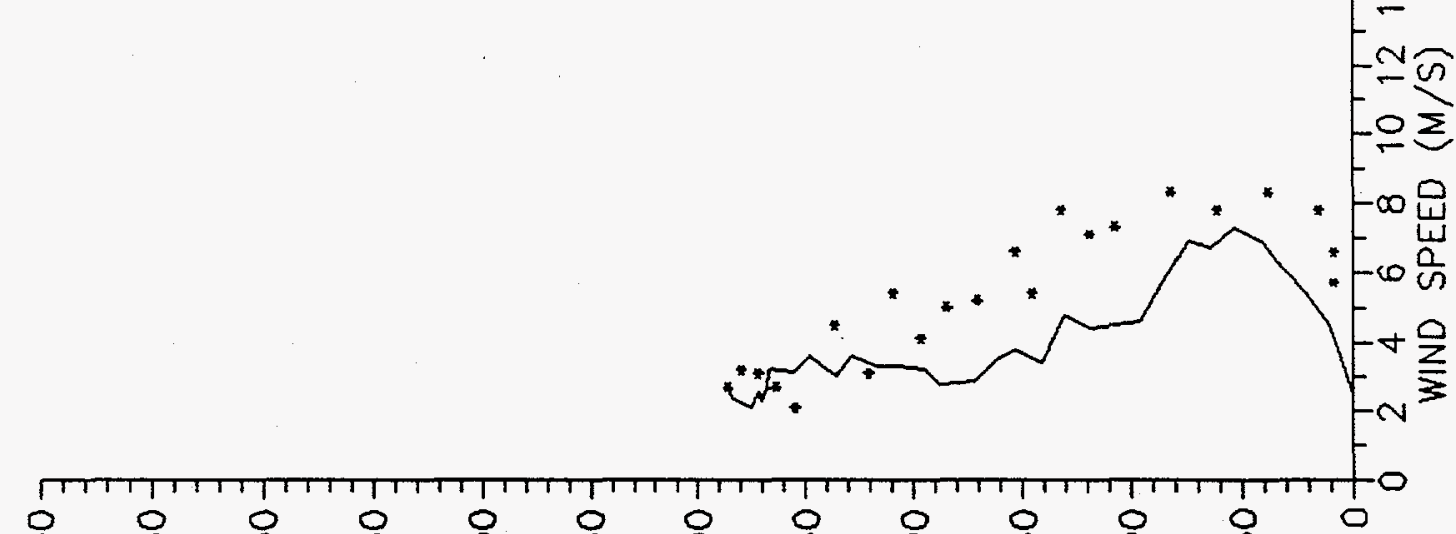
용

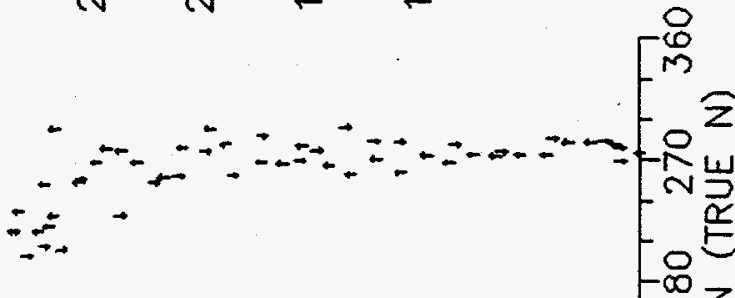

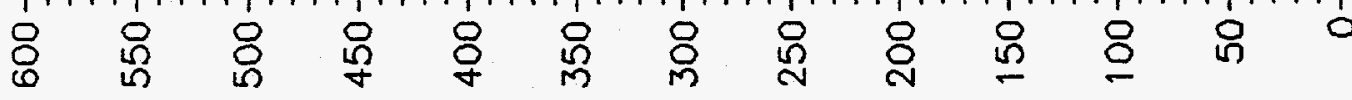
$(75 \forall$ w) $1 \mathrm{HOIJH}$ 


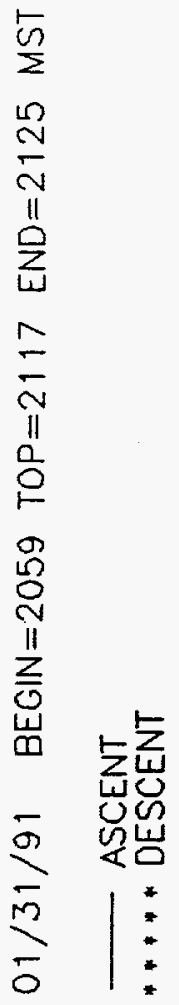

U.
Z
号

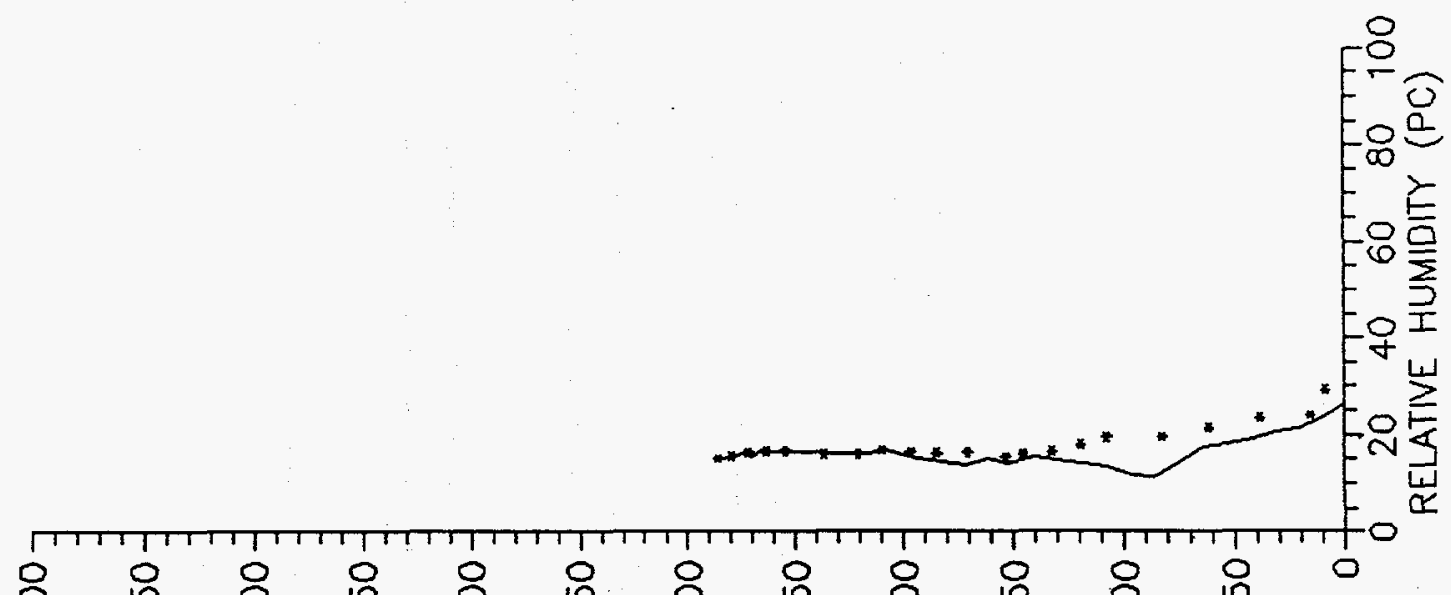

$z$
$\vdots$
$z$
3

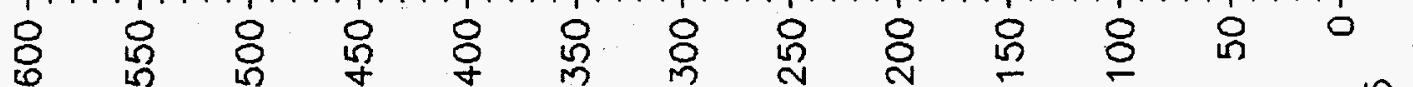

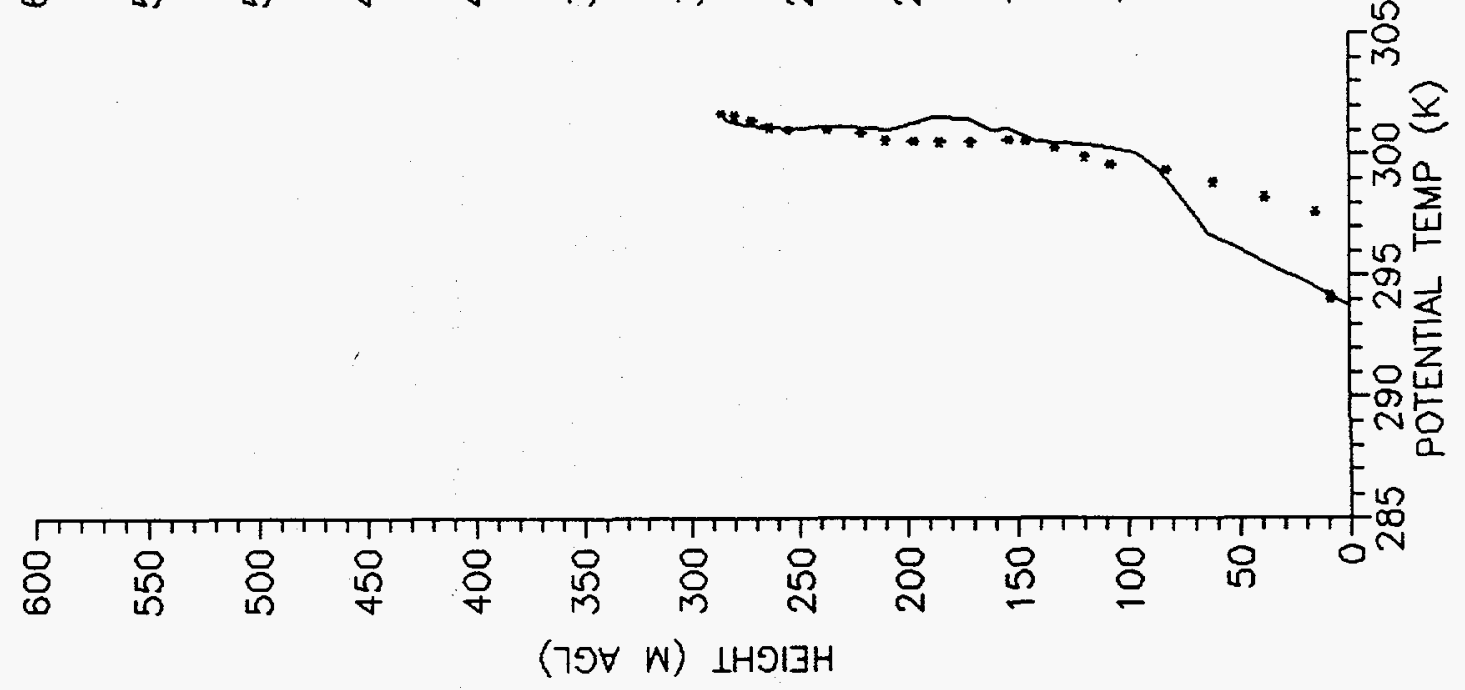


COAL CREEK CANYON TSONDE $01 / 31 / 91 \quad B E G I N=2202 \quad$ TOP $=2240 \quad$ END $=2244 \quad$ MST

ASCENT
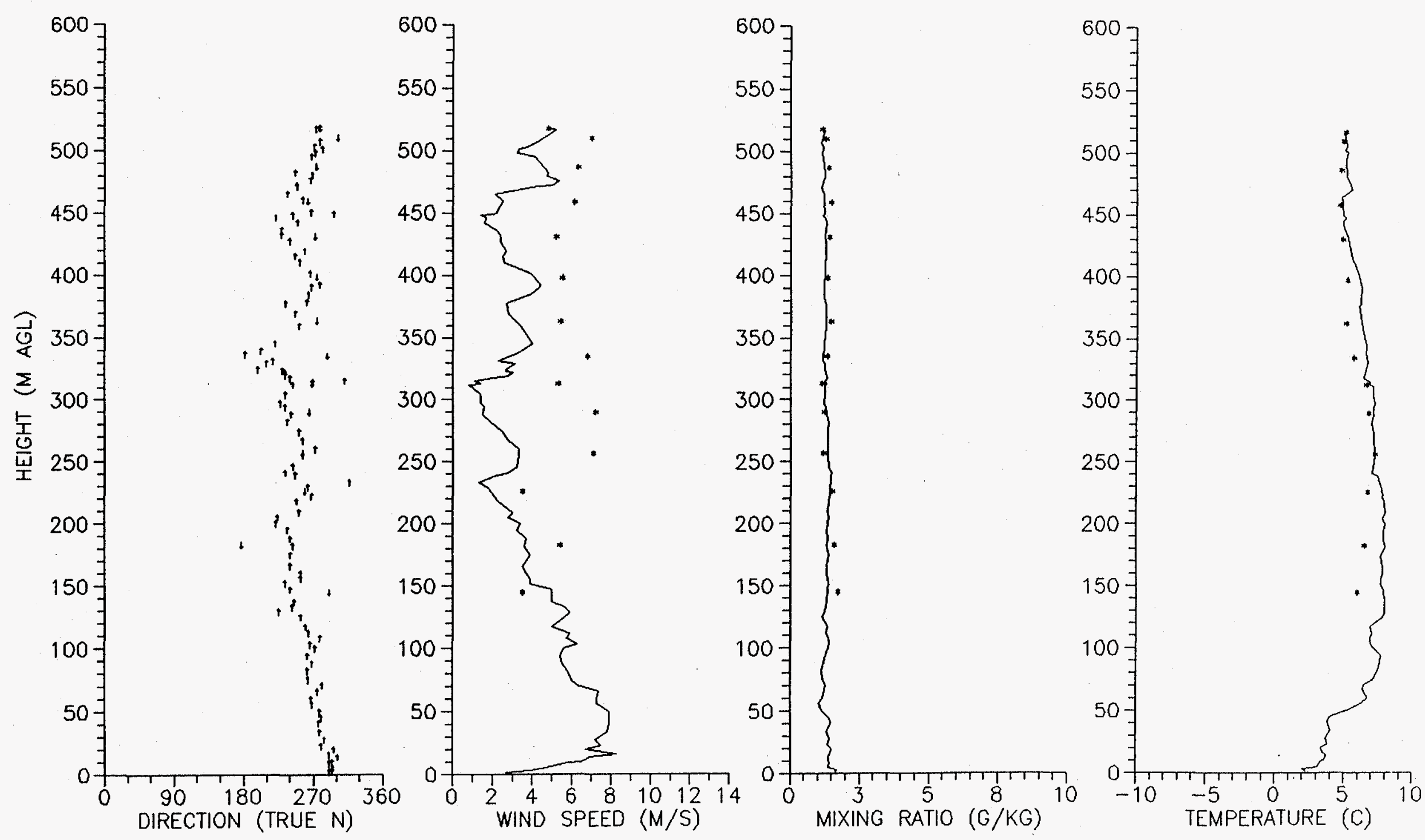

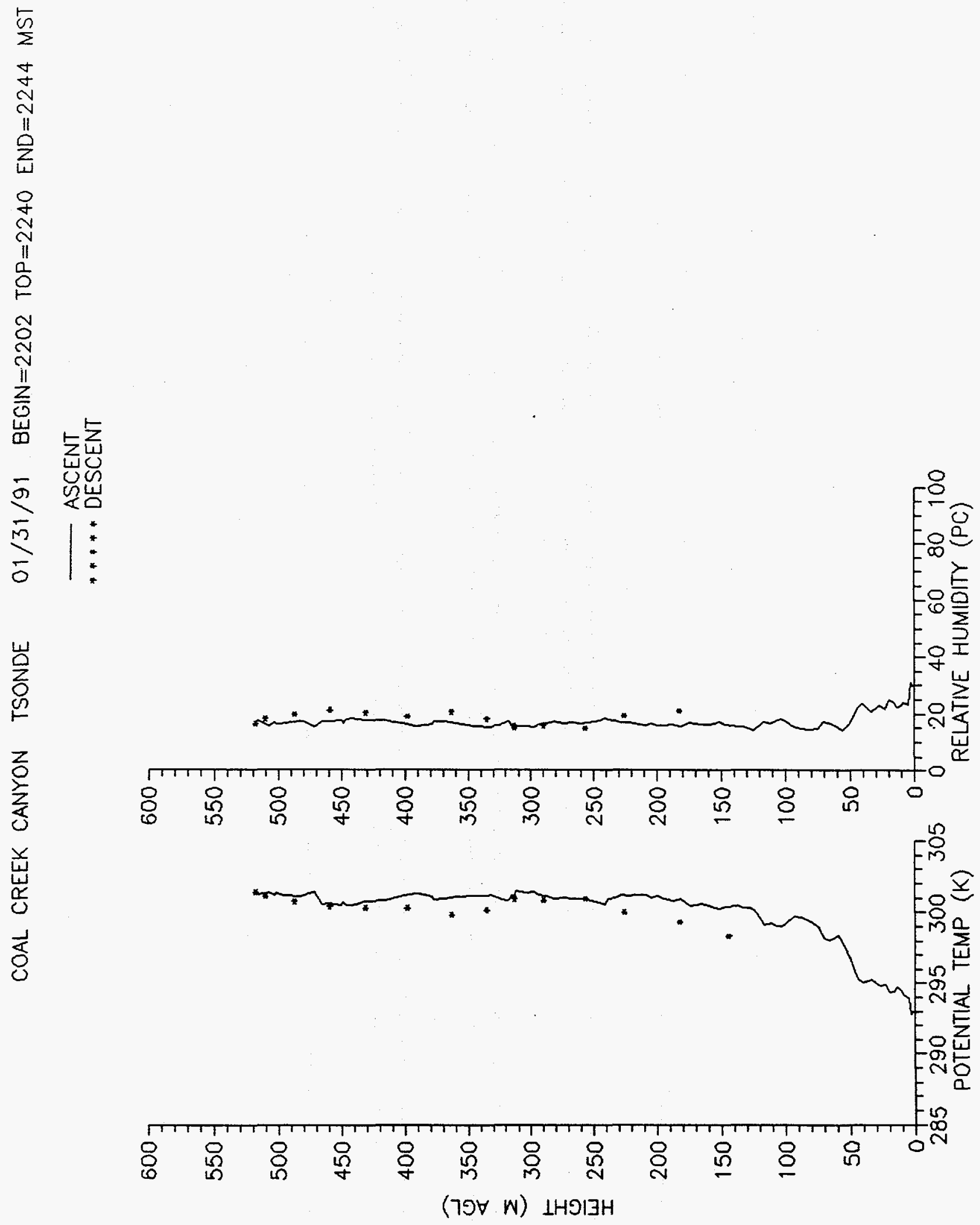
COAL CREEK CANYON TSONDE $01 / 31 / 91 \quad B E G I N=2301 \quad$ TOP $=2316 \quad$ END $=2317 \quad \mathrm{MST}$

- ASCENT

**** DESCENT
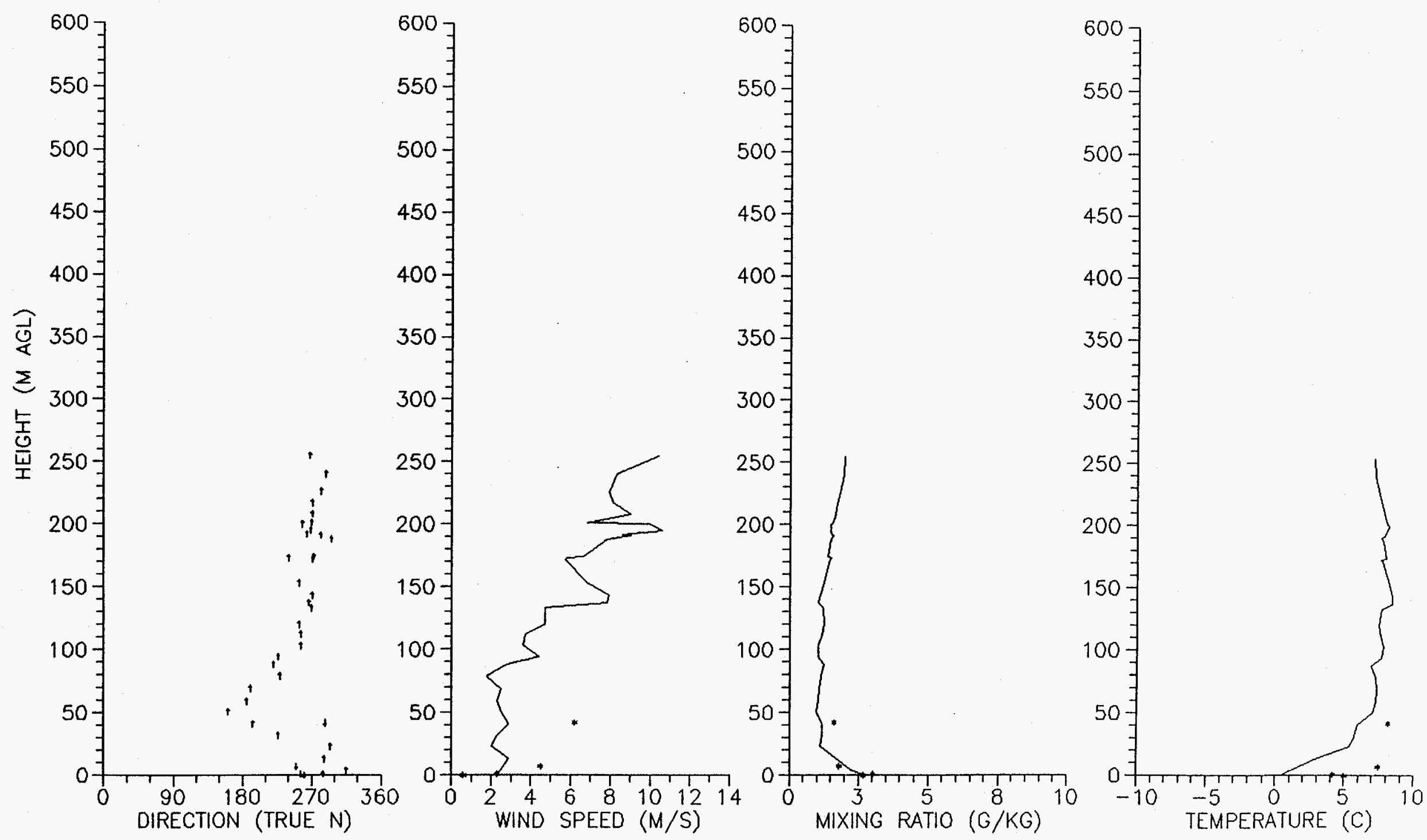


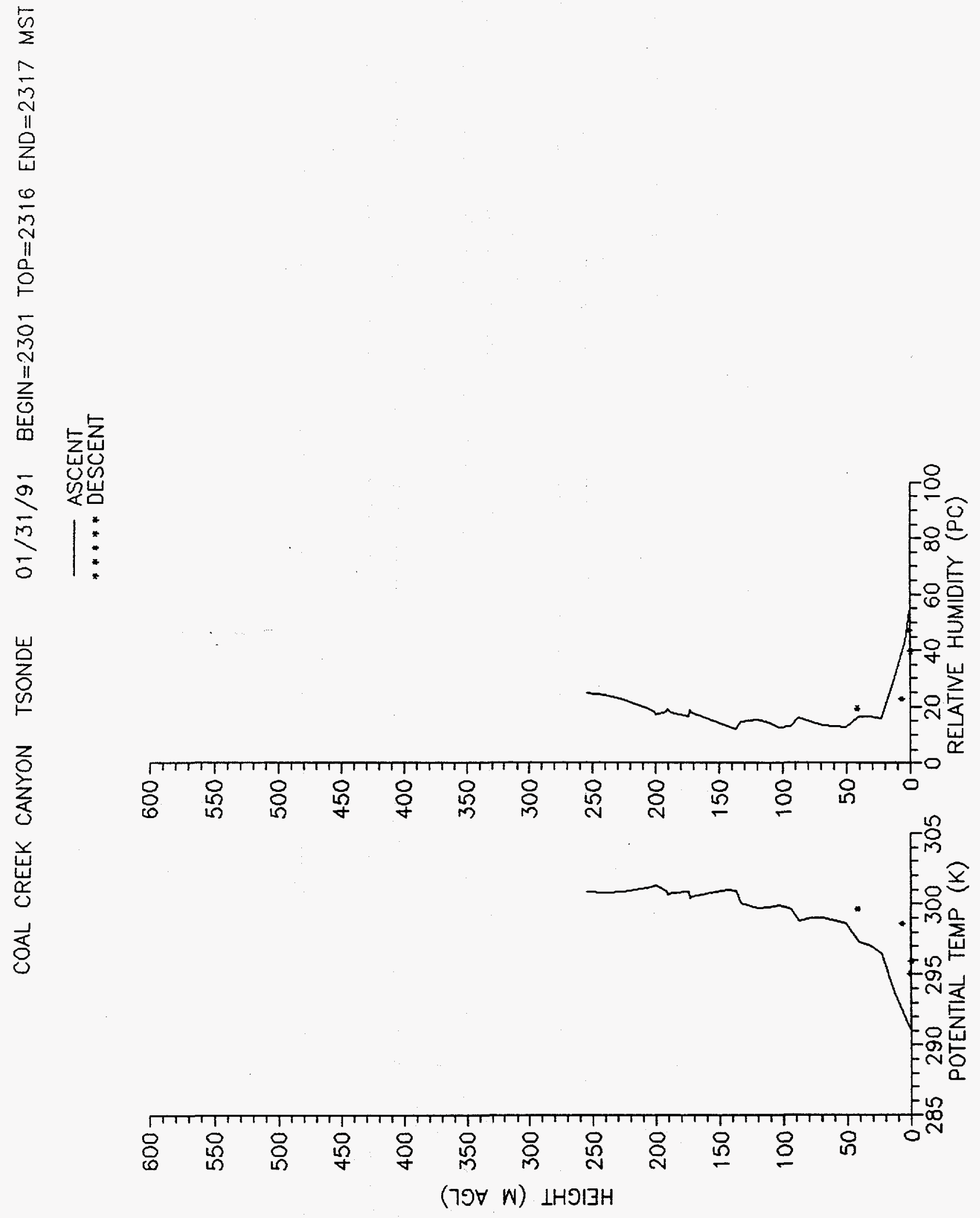


COAL CREEK CANYON TSONDE $01 / 31 / 91 \quad B E G I N=2358 \quad$ TOP $=0007 \quad$ END $=0021 \quad \mathrm{MST}$

ASCENT
$* * * *$ DESCENT
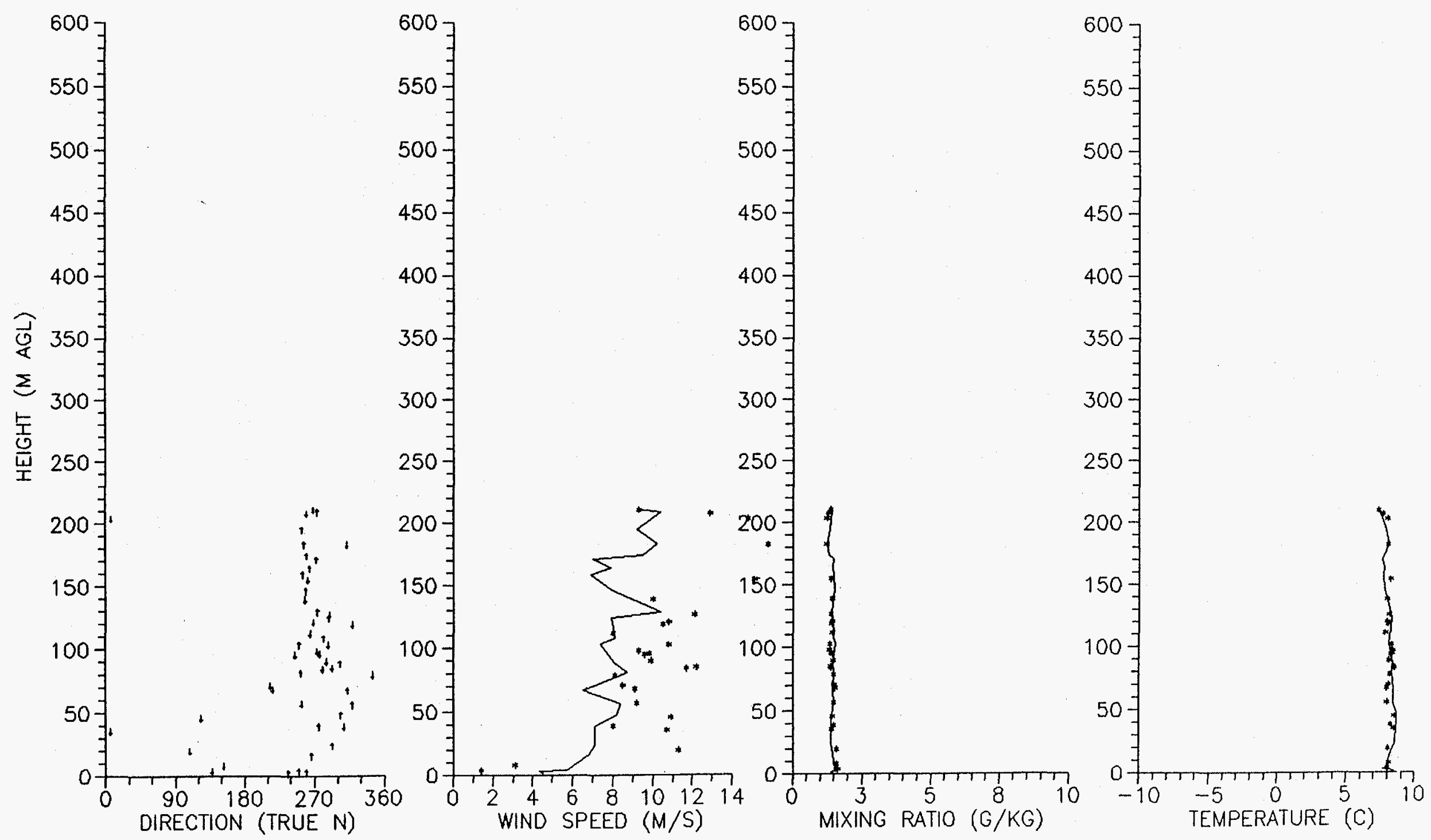

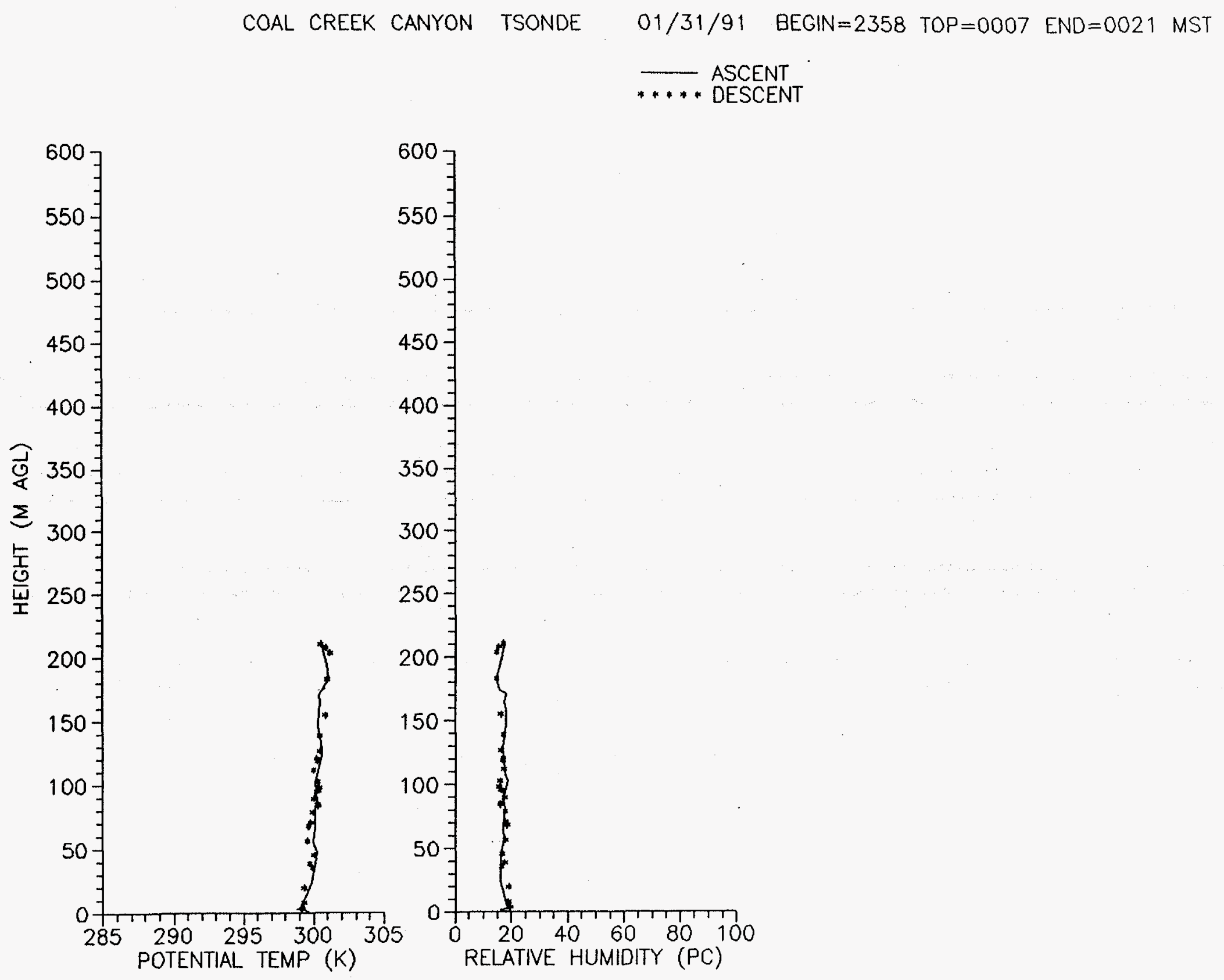
COAL CREEK CANYON TSONDE $02 / 01 / 91 \quad$ BEGIN $=1806 \quad$ TOP $=1833 \quad$ END $=1845 \quad$ MST

ASCENT
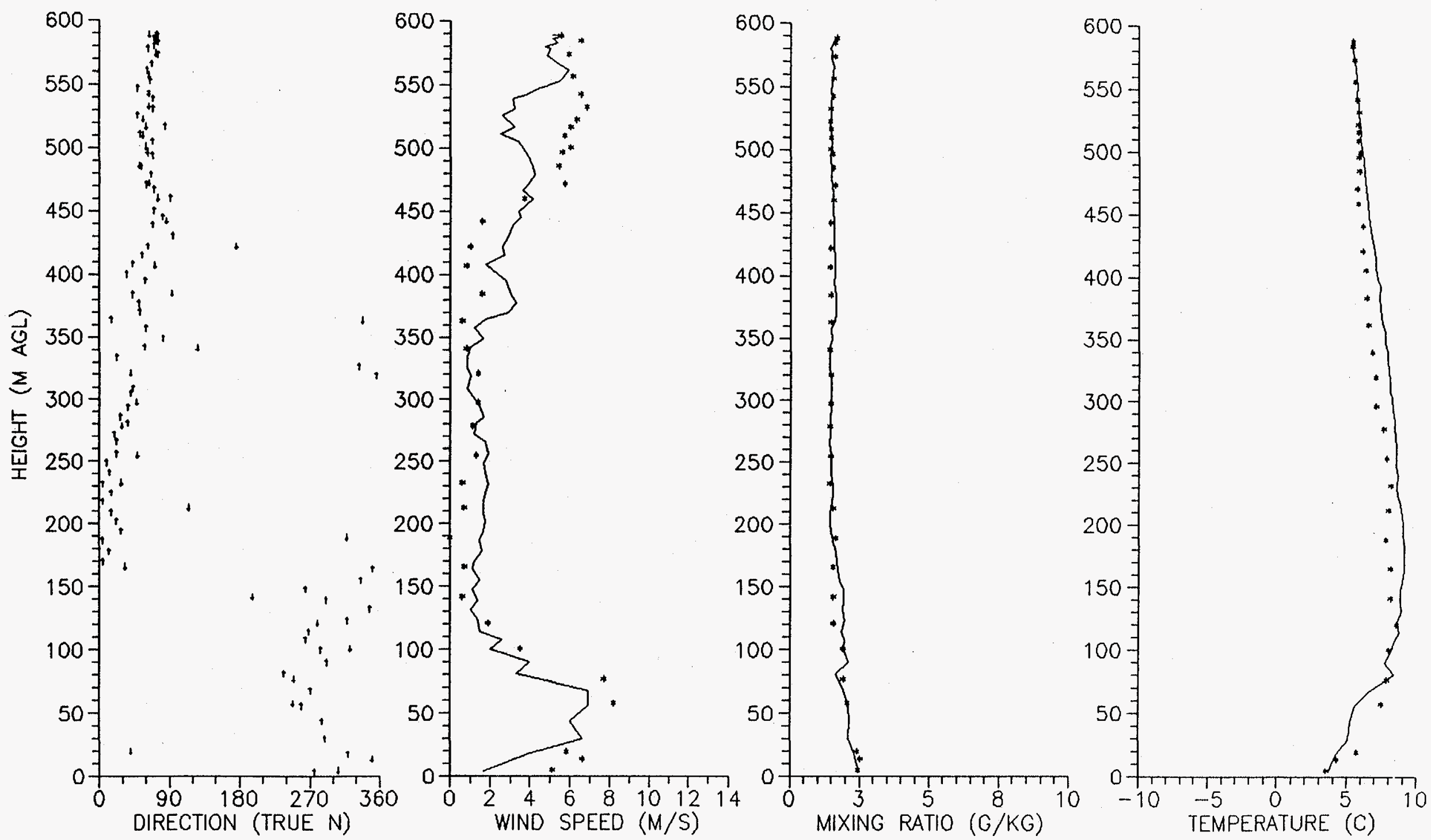


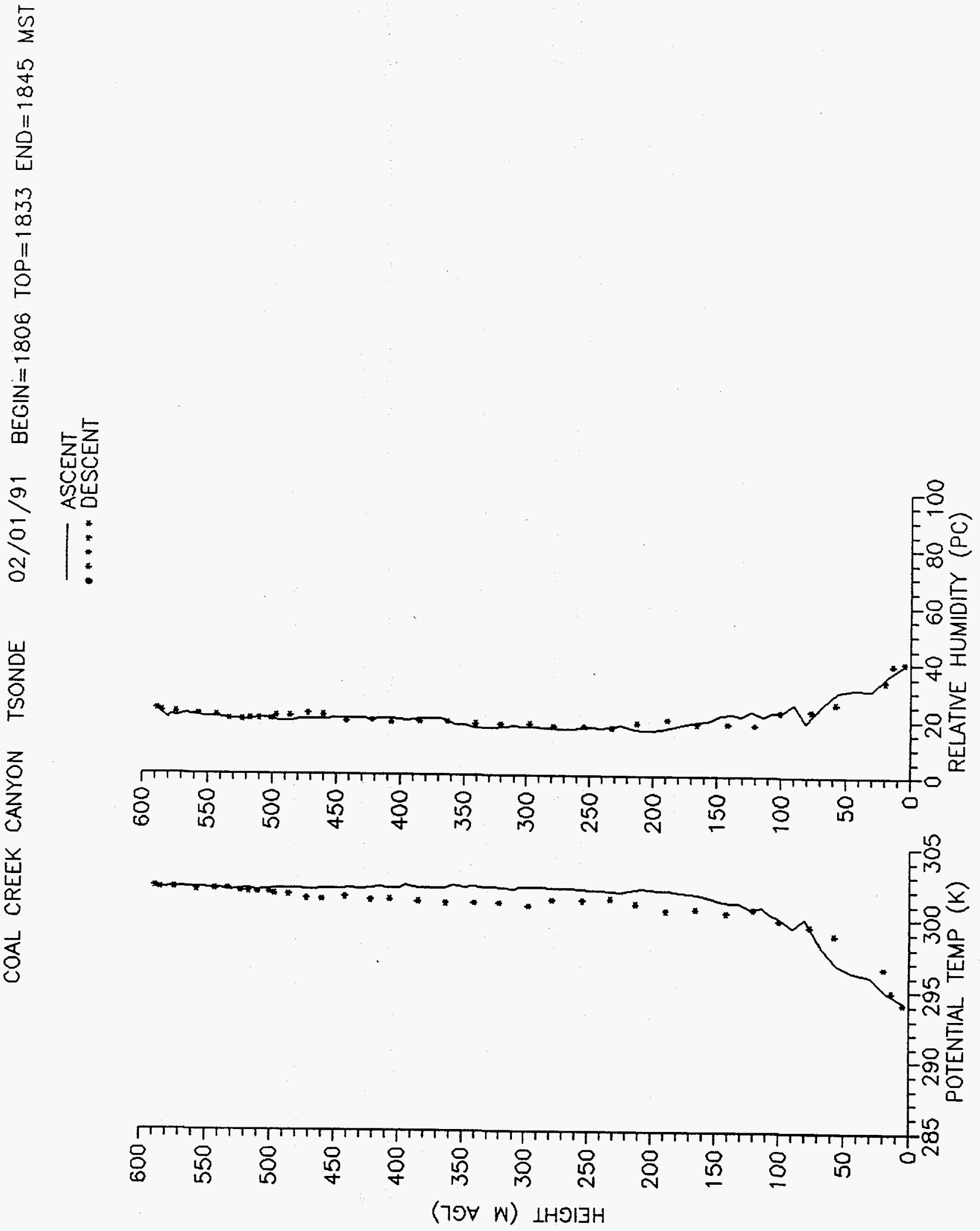


COAL CREEK CANYON TSONDE 02/01/91 BEGIN=1859 TOP $=1922 \quad$ END $=1932 \quad \mathrm{MST}$

$*$ ASCENT
$* *$ DESCENT
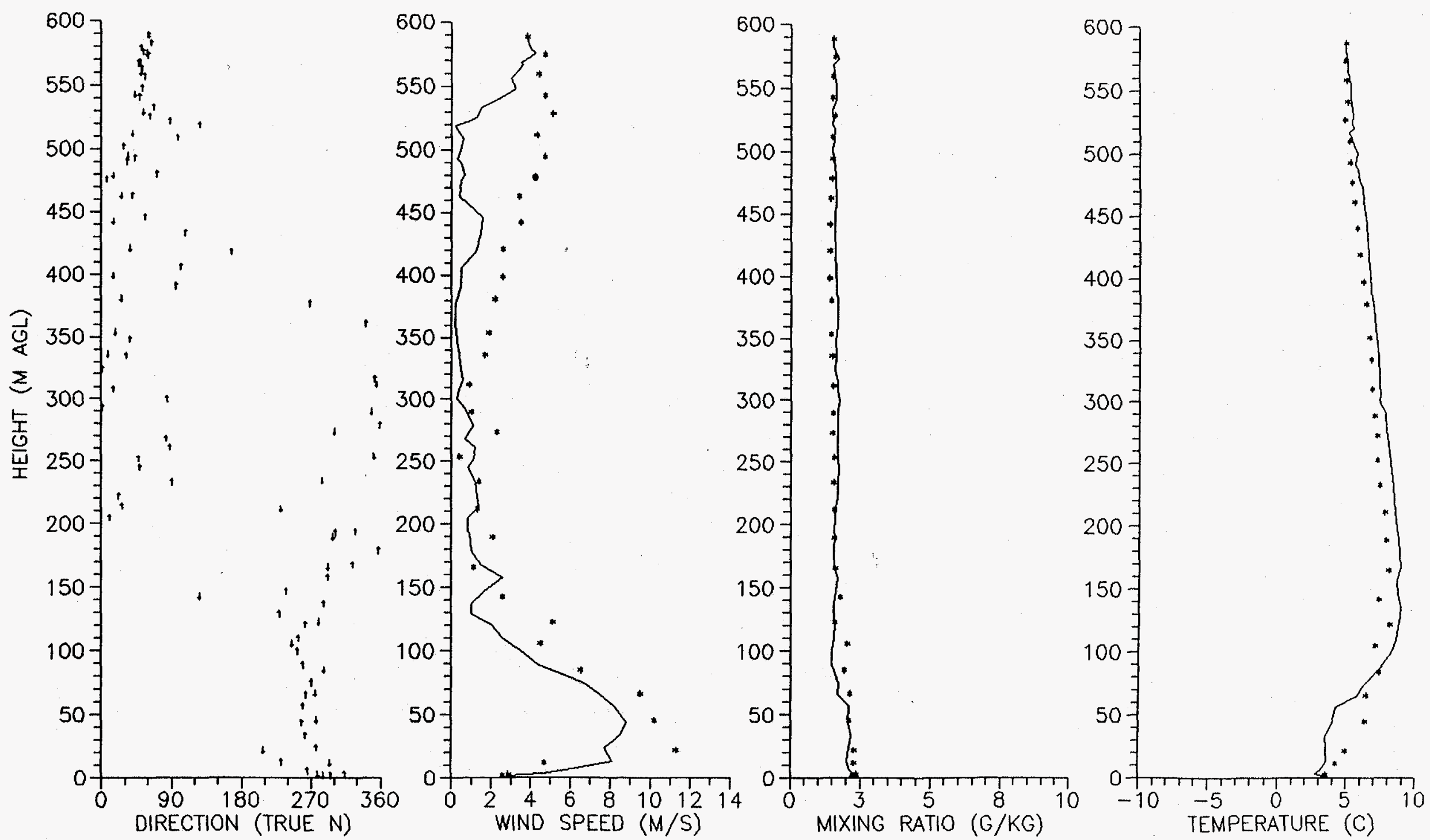
COAL CREEK CANYON TSONDE 02/01/91 BEGIN=1859 TOP=1922 END=1932 MST ASCENT
$\cdots * *$ DESCENT

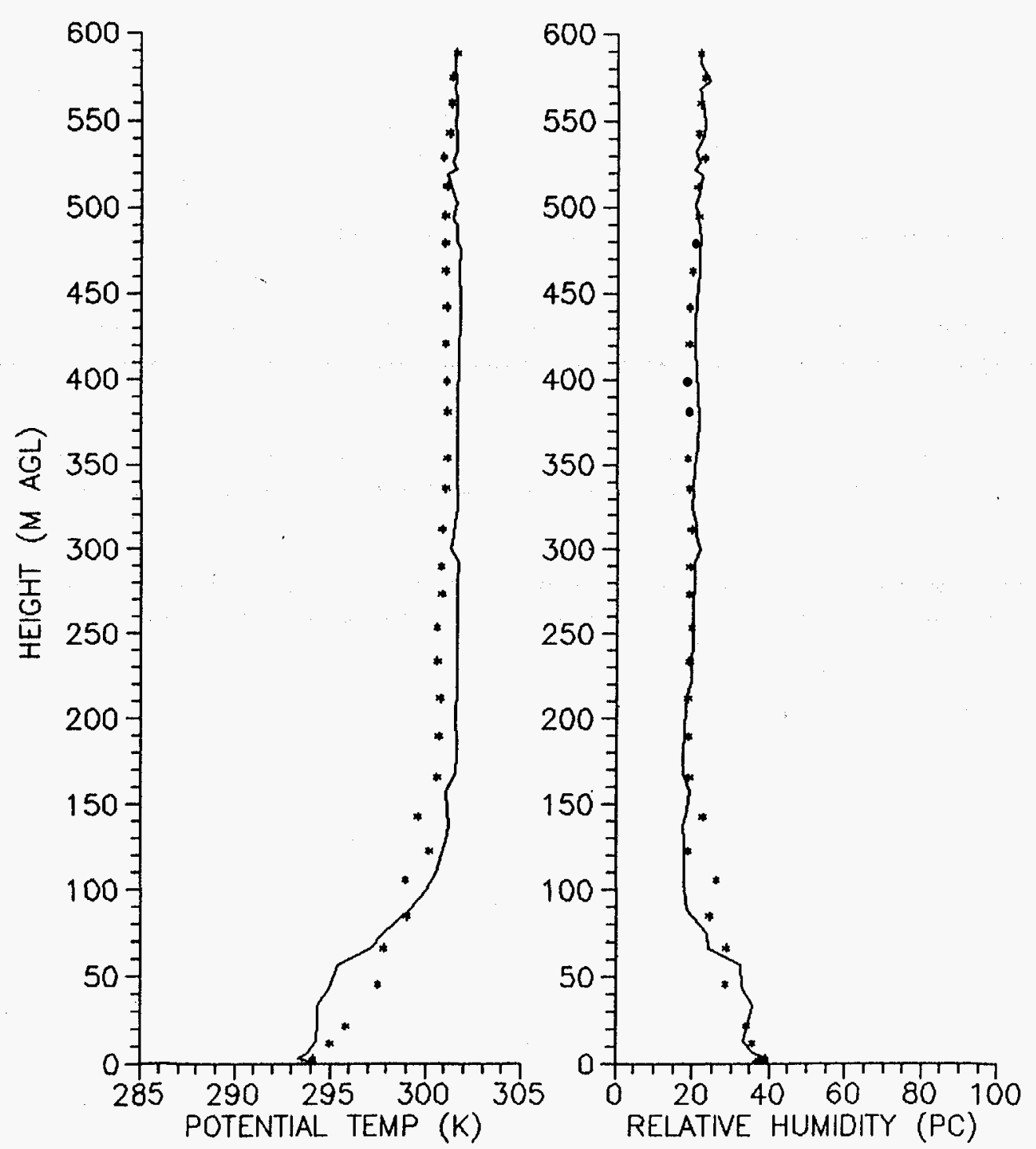




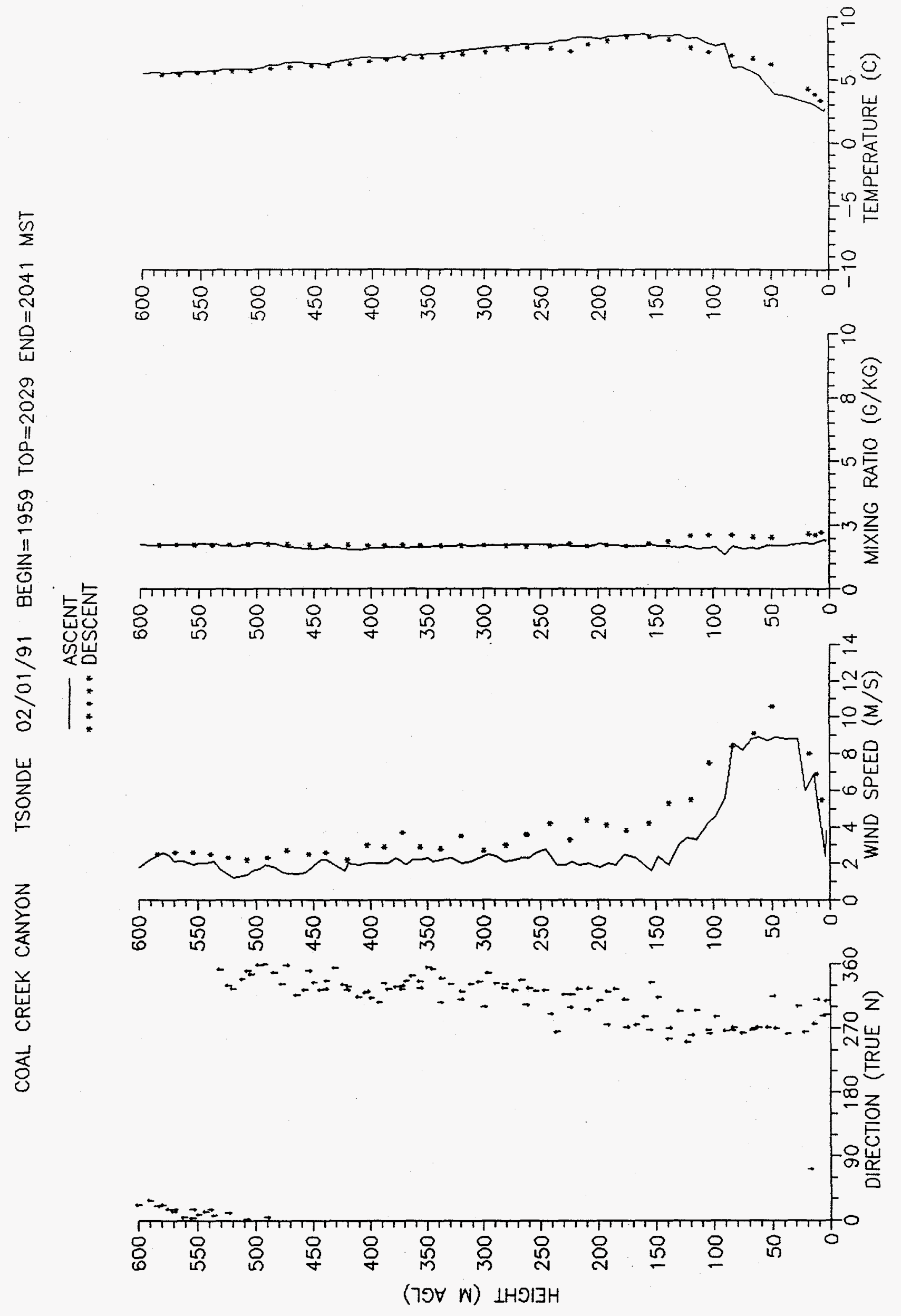


$\stackrel{n}{\Sigma}$

¿্ঠ

$\stackrel{\text { II }}{\text { II }}$

$\stackrel{\mathbb{N}}{\stackrel{N}{O}}$

II

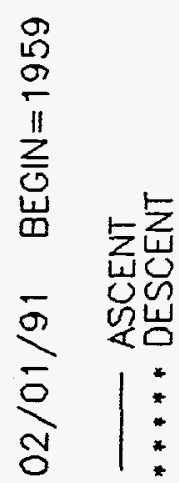

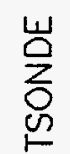

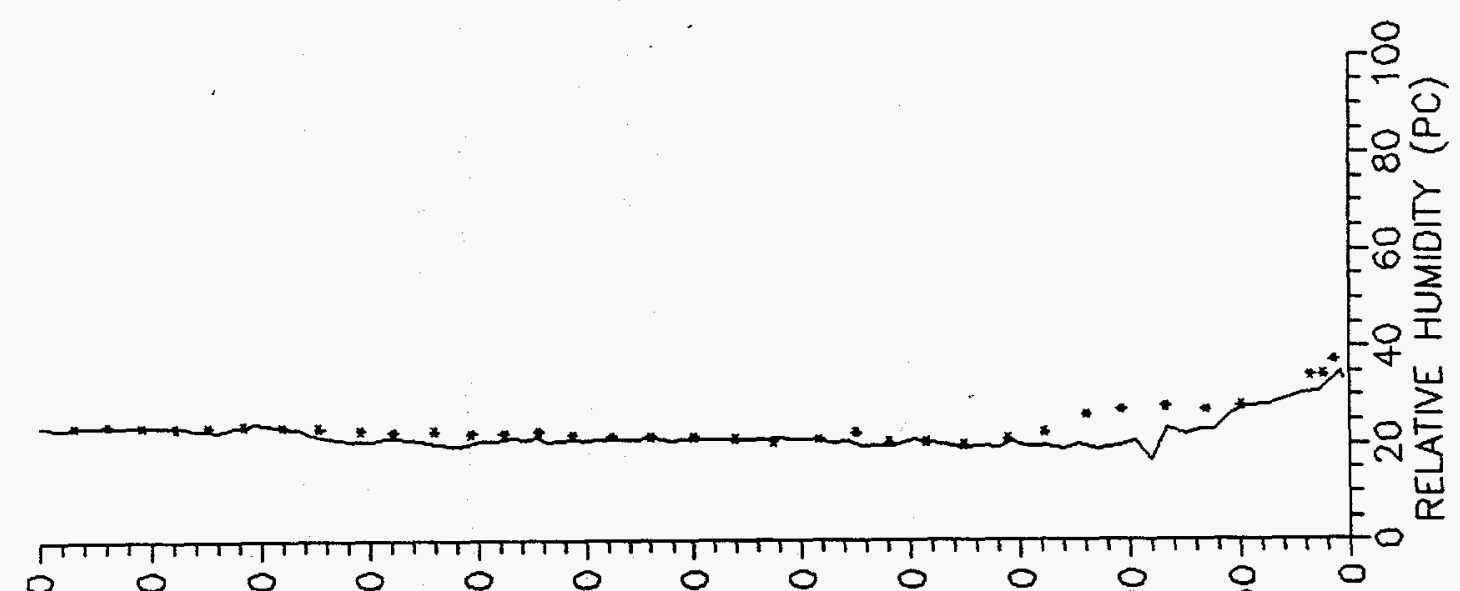

主

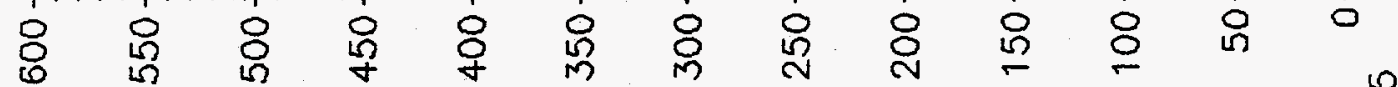

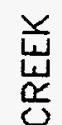

\section{(n)}

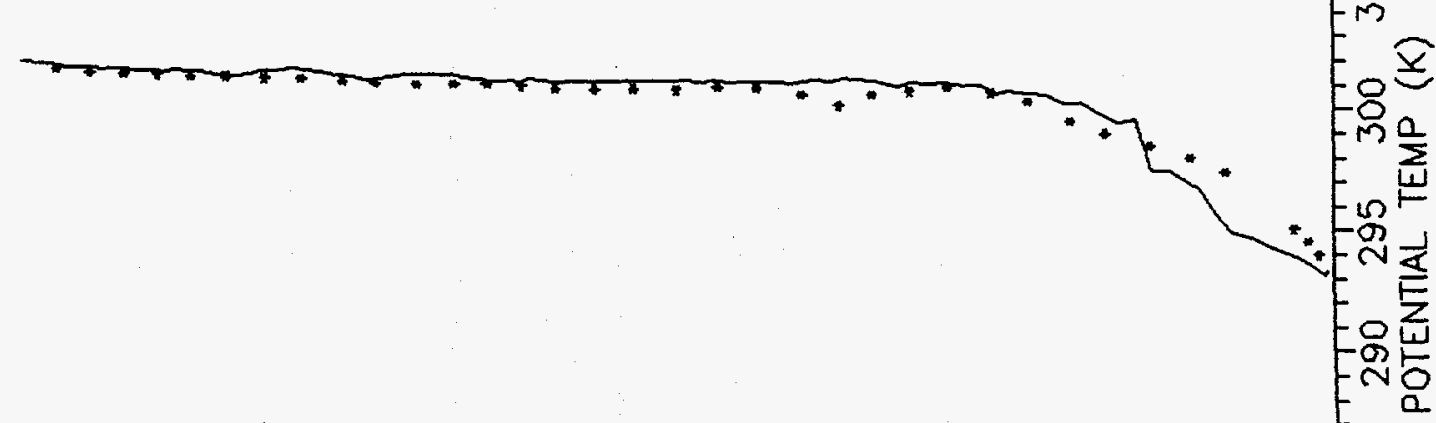

응

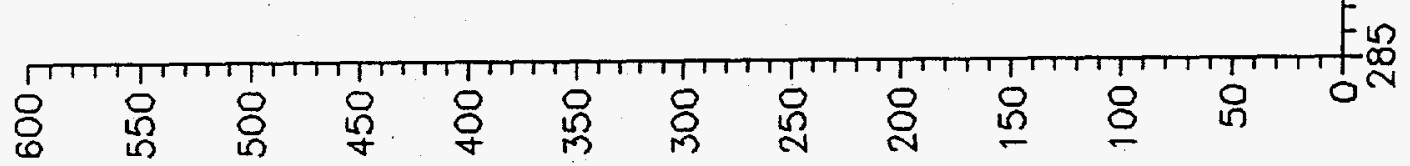

(7פ* W) $\mathrm{LH}$ 
COAL CREEK CANYON TSONDE 02/01/91 BEGIN=2101 TOP=2135 END $=2145 \quad \mathrm{MST}$

ASCENT
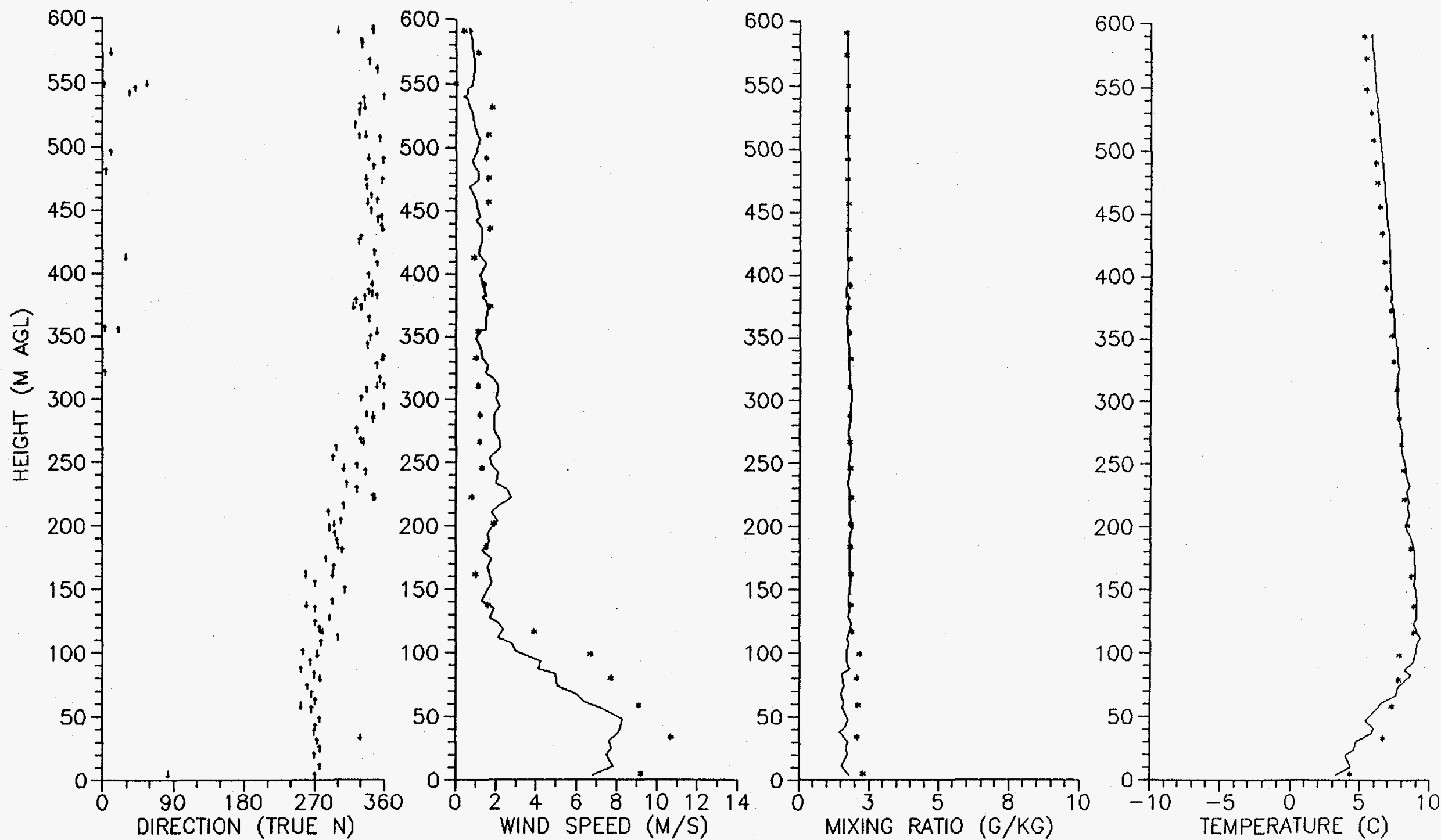


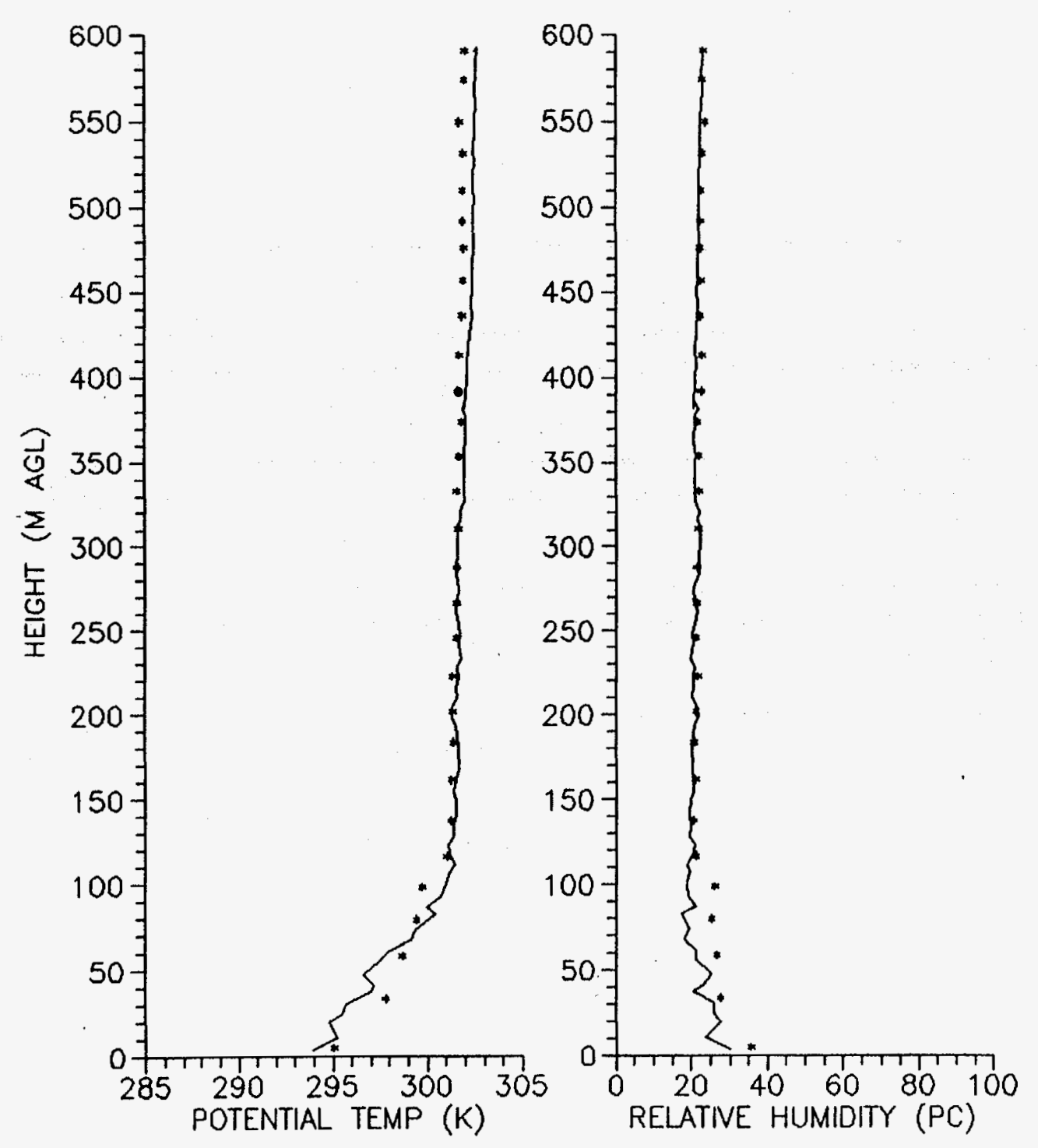




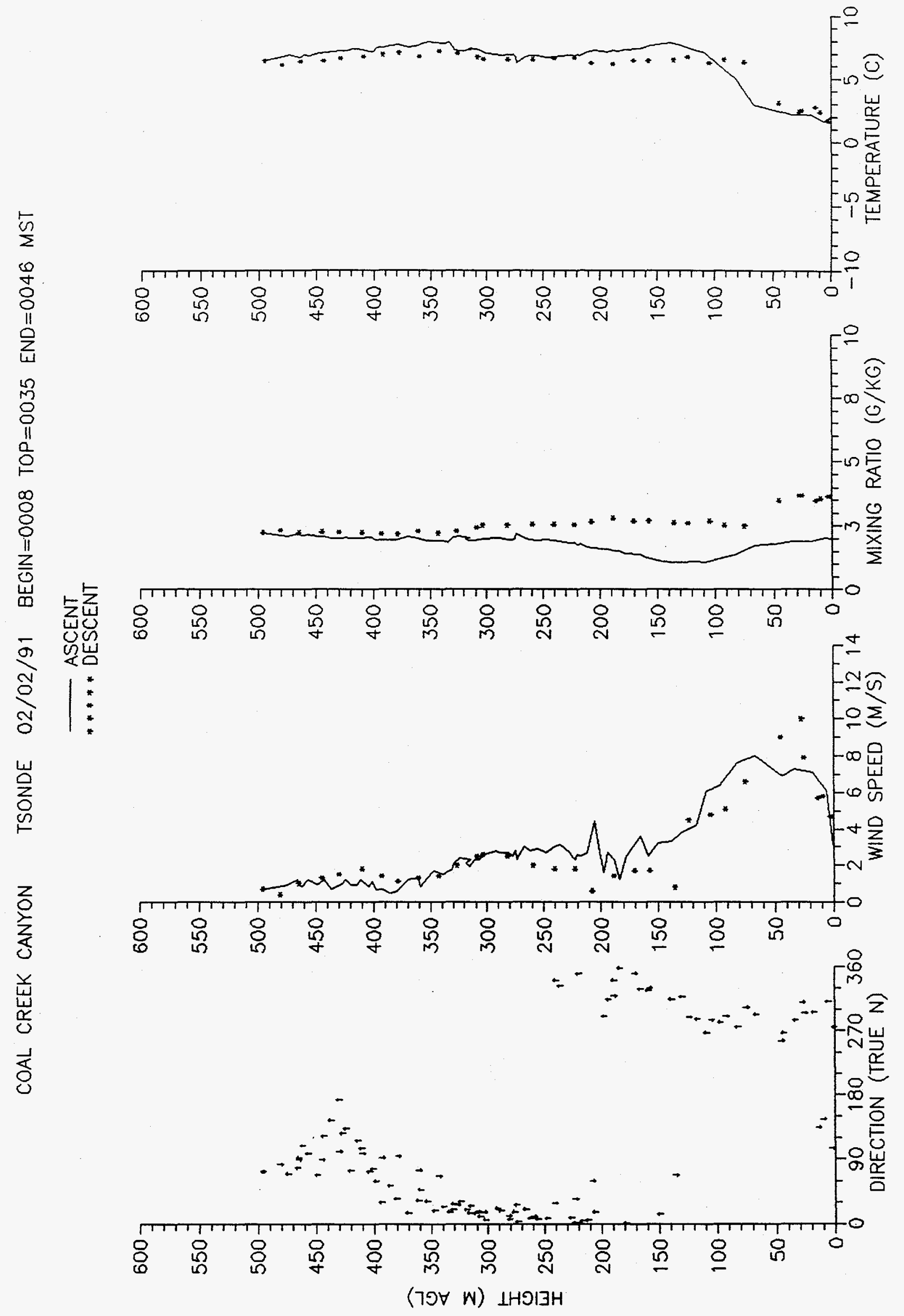


COAL CREEK CANYON TSONDE 02/02/91. BEGIN=0008 TOP $=0035 \quad$ END $=0046 \quad \mathrm{MST}$ $\cdots *$ ASCENT
$\cdots *$ DESCENT

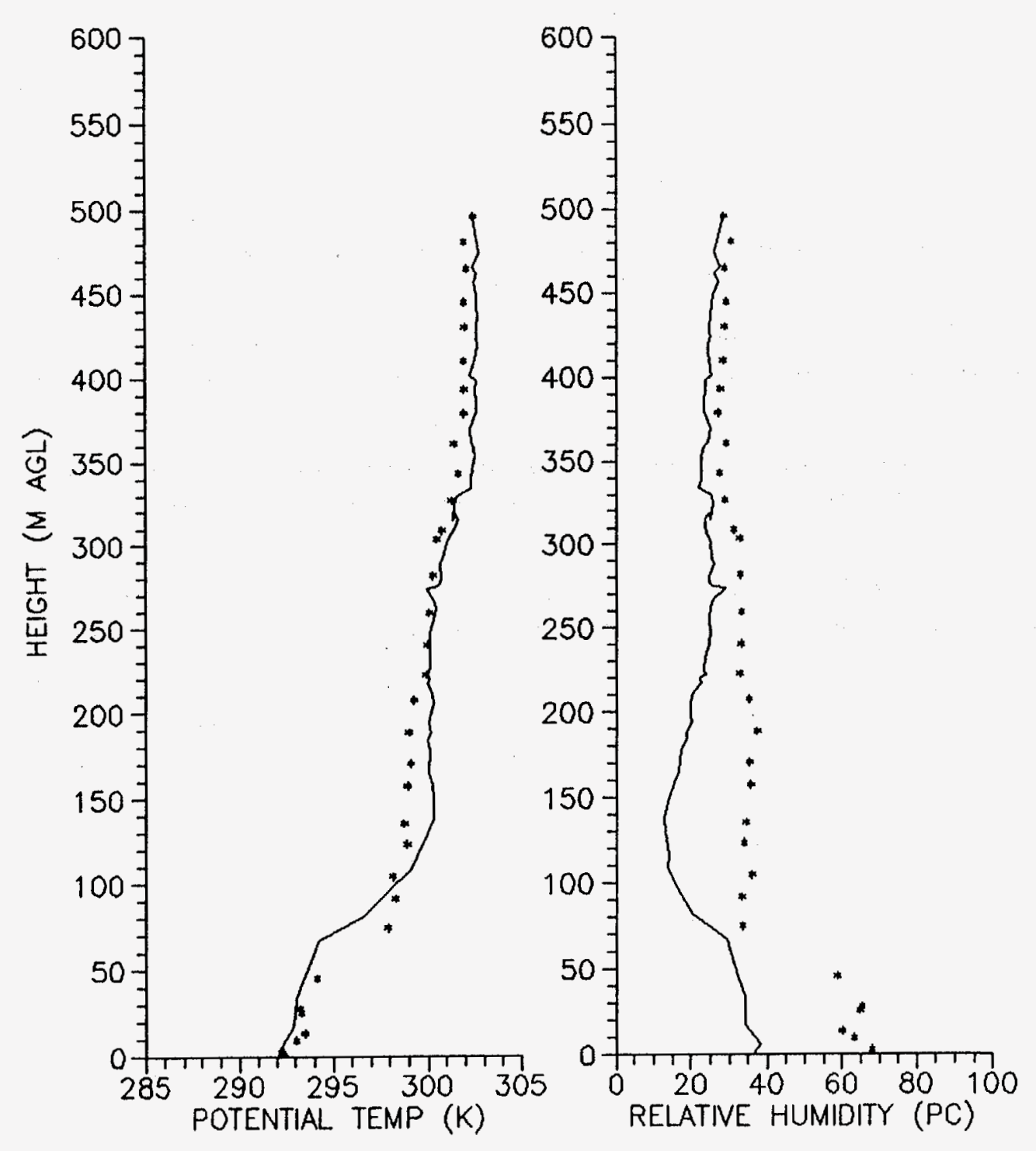




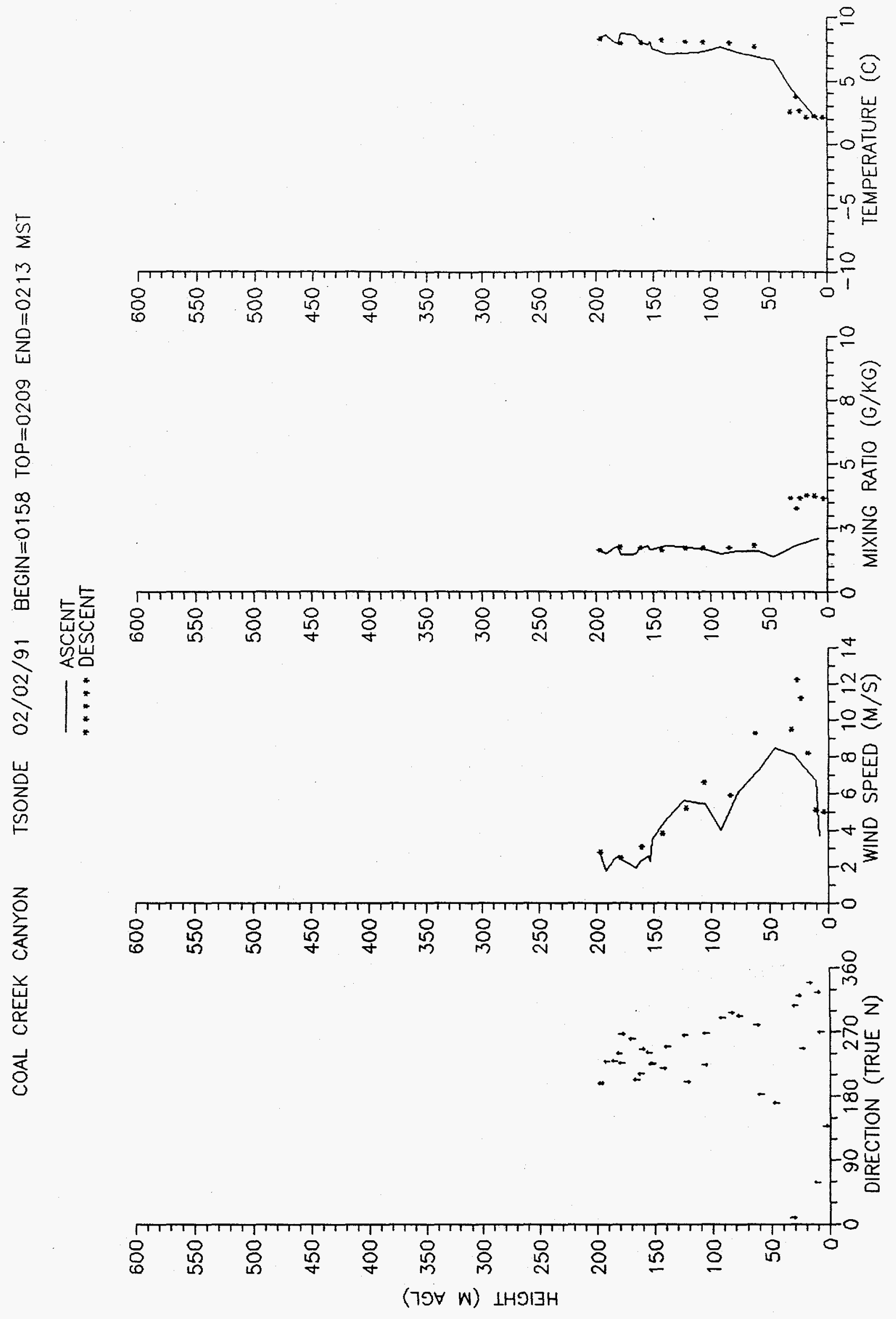




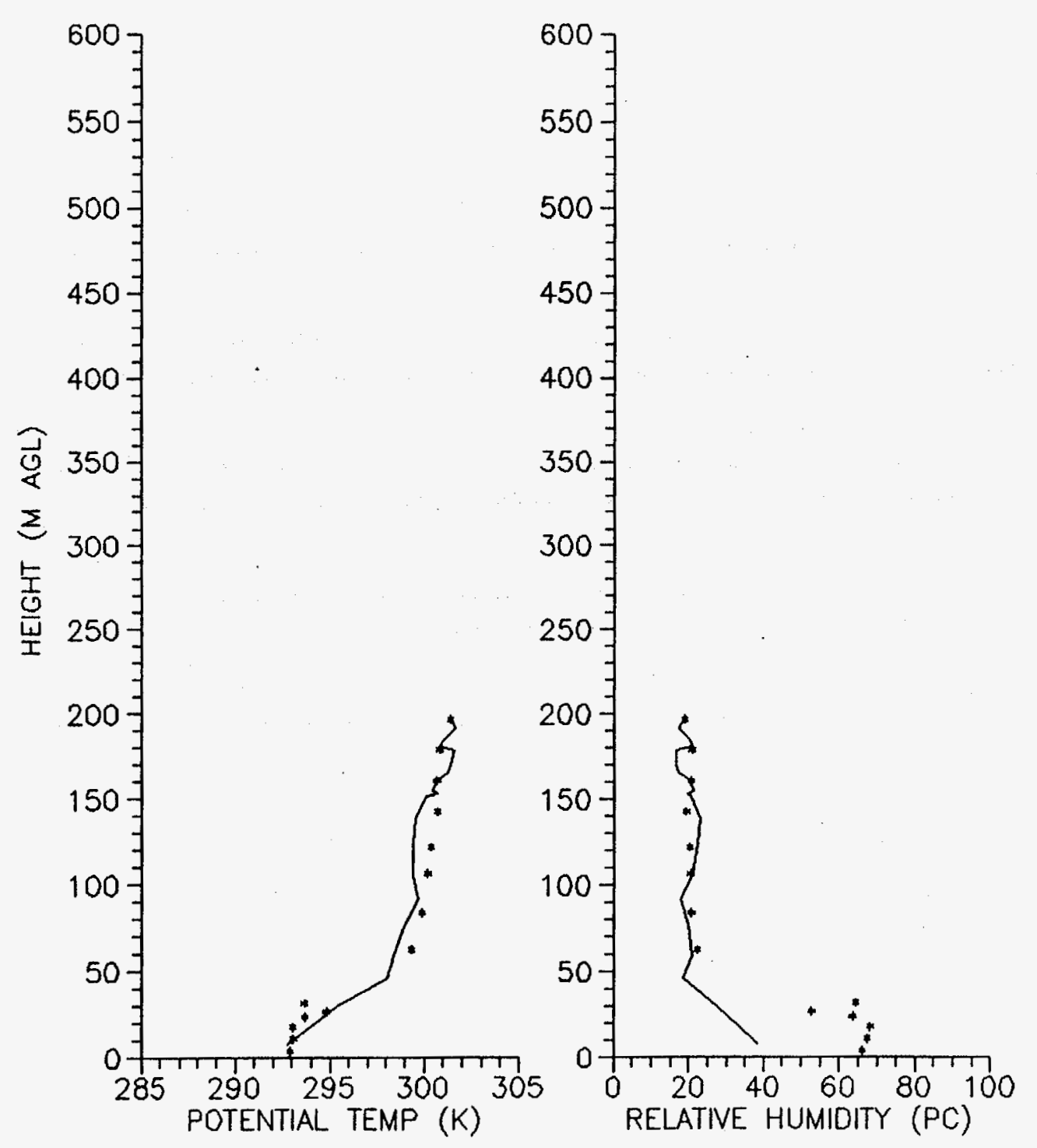




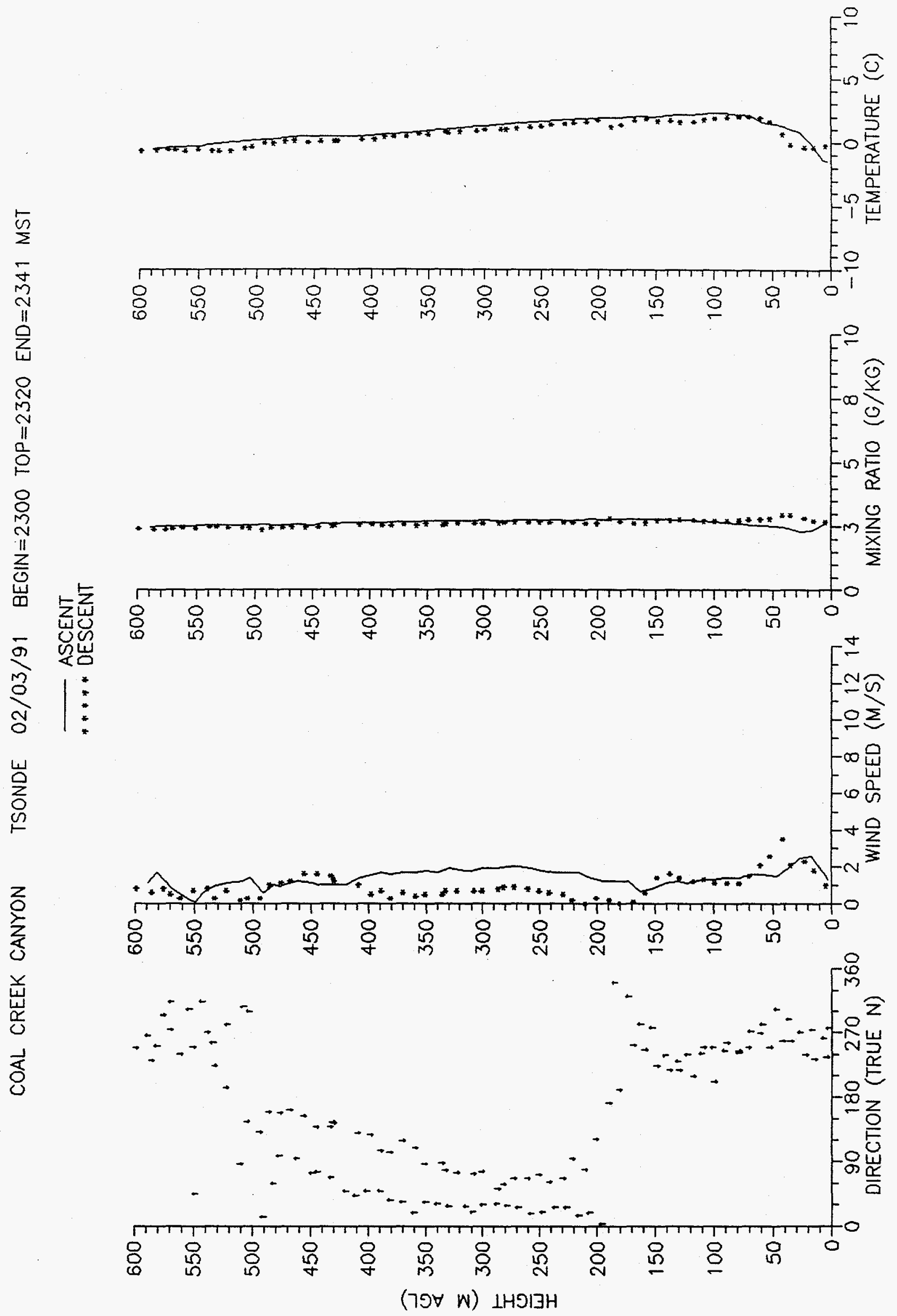


COAL CREEK CANYON TSONDE 02/03/91 BEGIN=2300 TOP $=2320 \quad$ END $=2341 \quad \mathrm{MST}$

$$
\begin{aligned}
& -\ldots \text { ASCENT } \\
& * * * \text { DESCENT }
\end{aligned}
$$
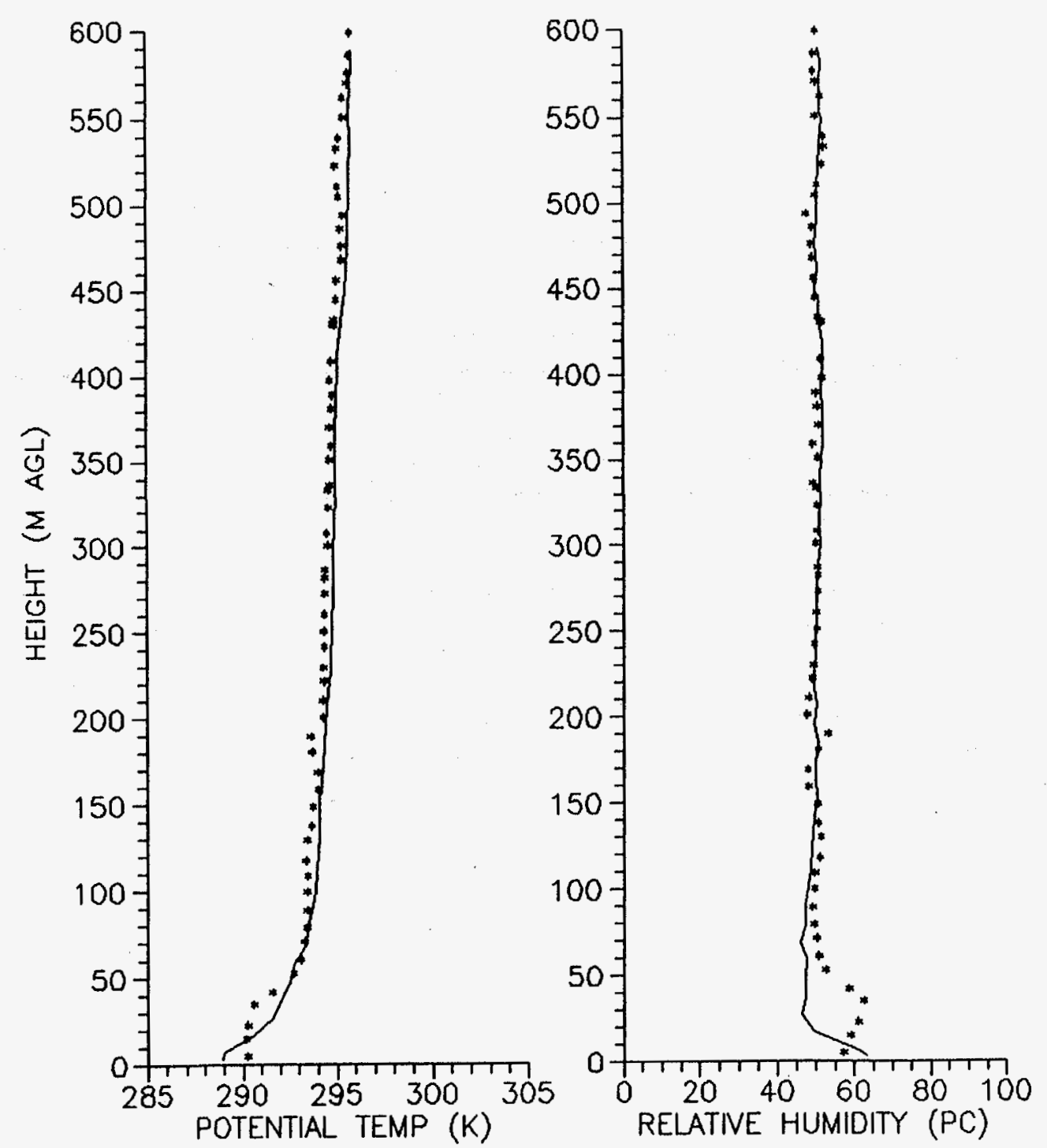
COAL CREEK CANYON TSONDE 02/03/91 BEGIN=2359 TOP=0023 END=0049 MST

ASCENT
$\cdots *$ DESCENT
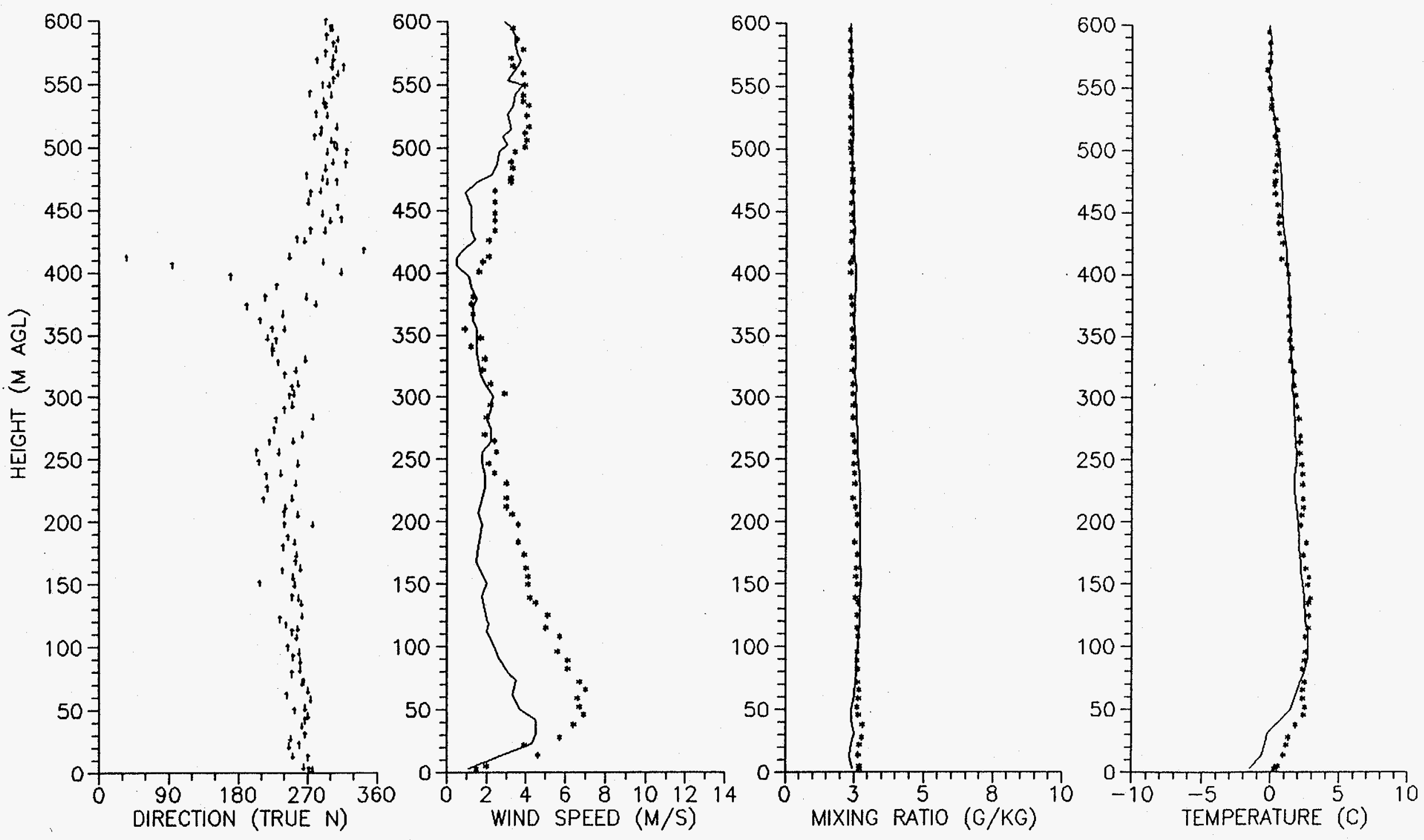
上文

넌

-

$\sum_{0}^{\infty}$

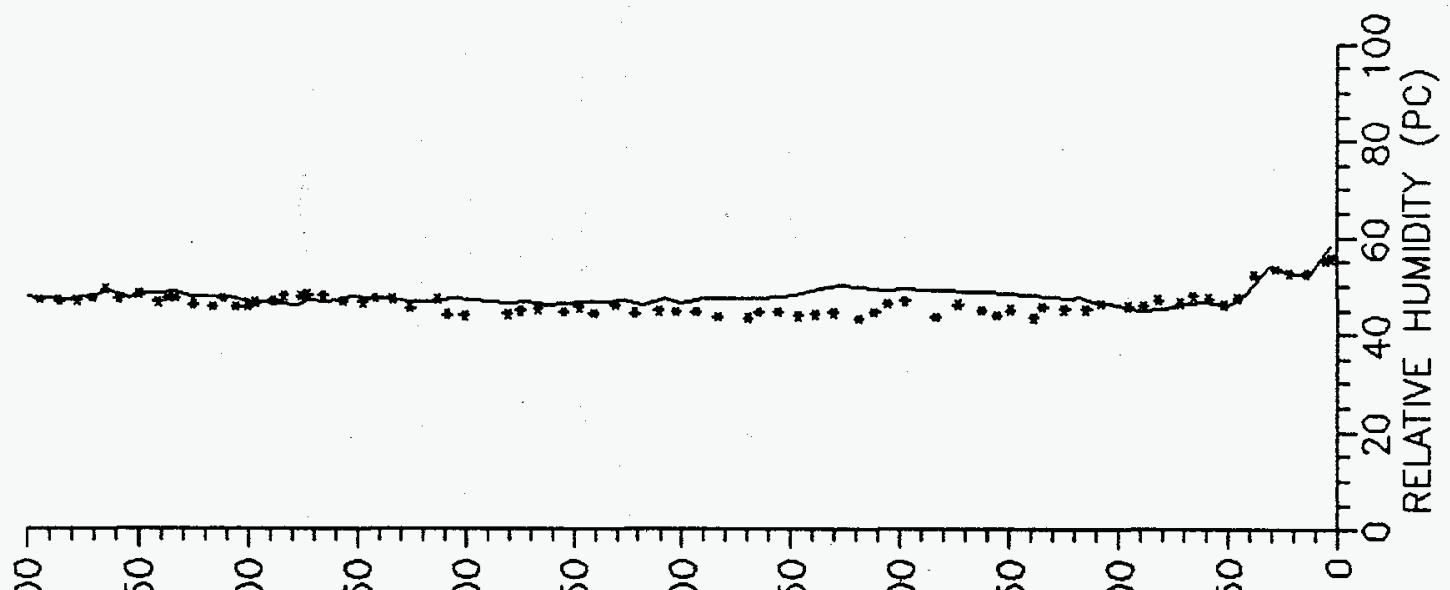

ㄴ.

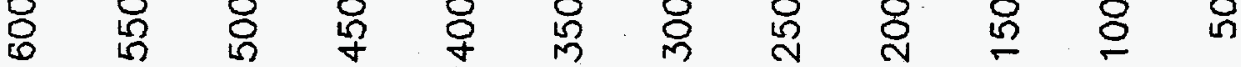

$\frac{w}{u}$

$\frac{1}{6}$

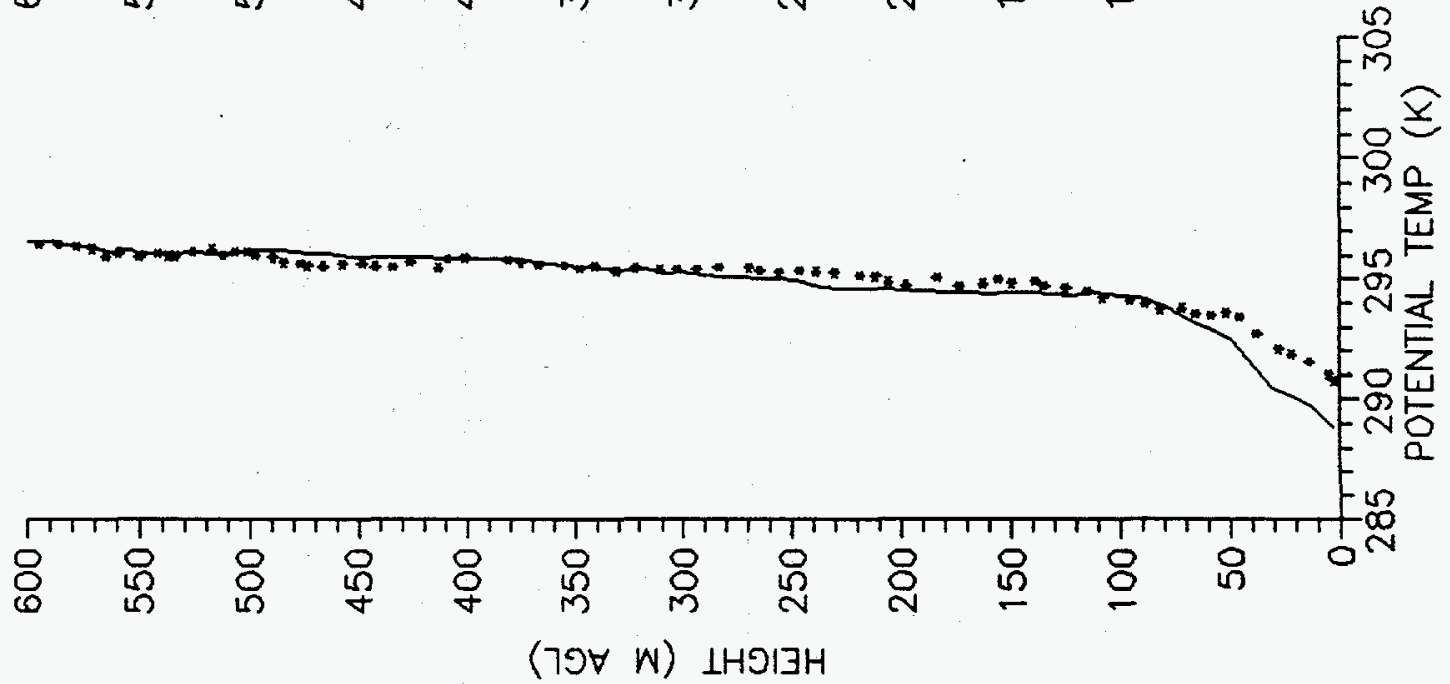




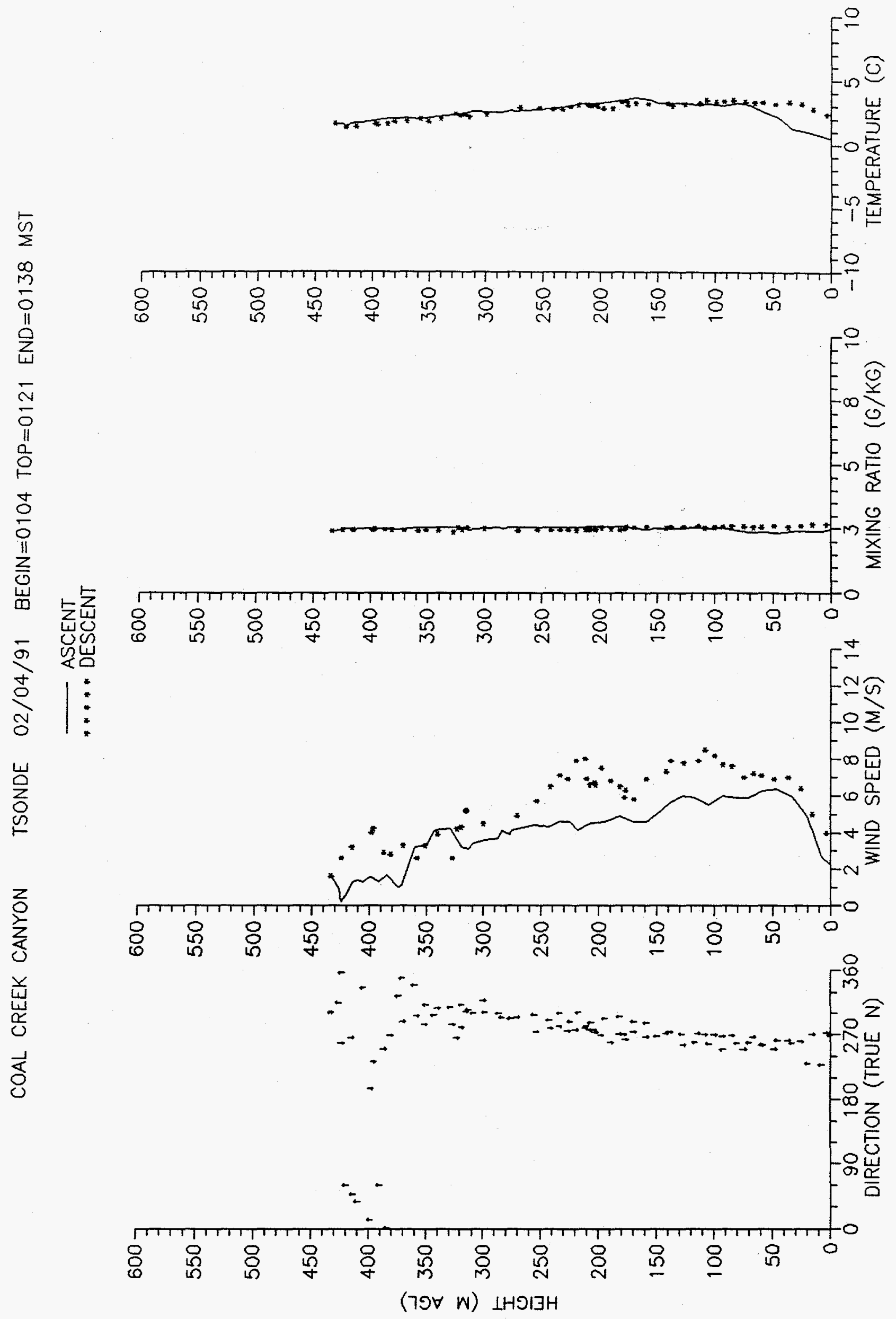




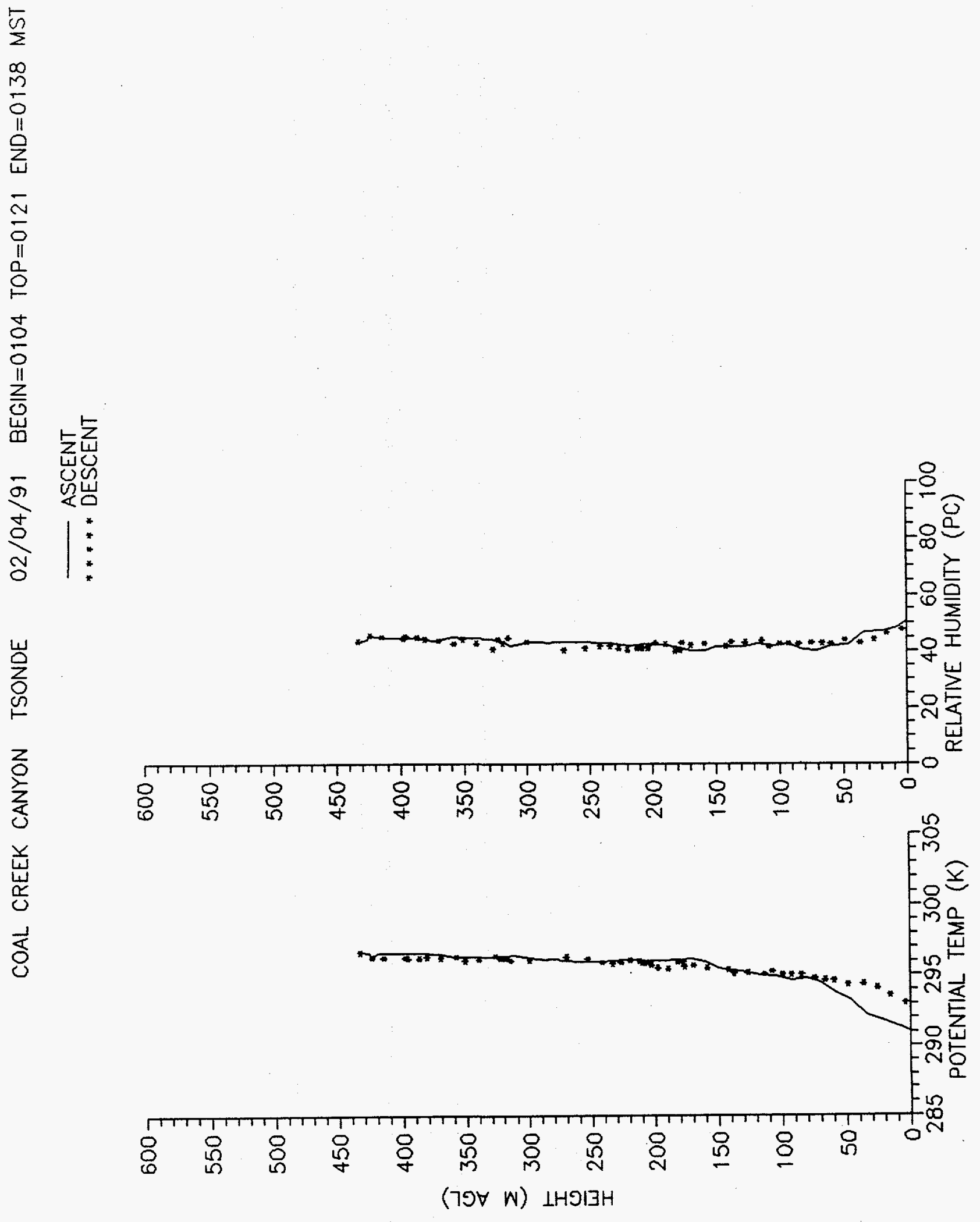




\section{COAL CREEK CANYON TSONDE 02/04/91 BEGIN=0205 TOP $=0215$ END=0222 MST}

ASCENT
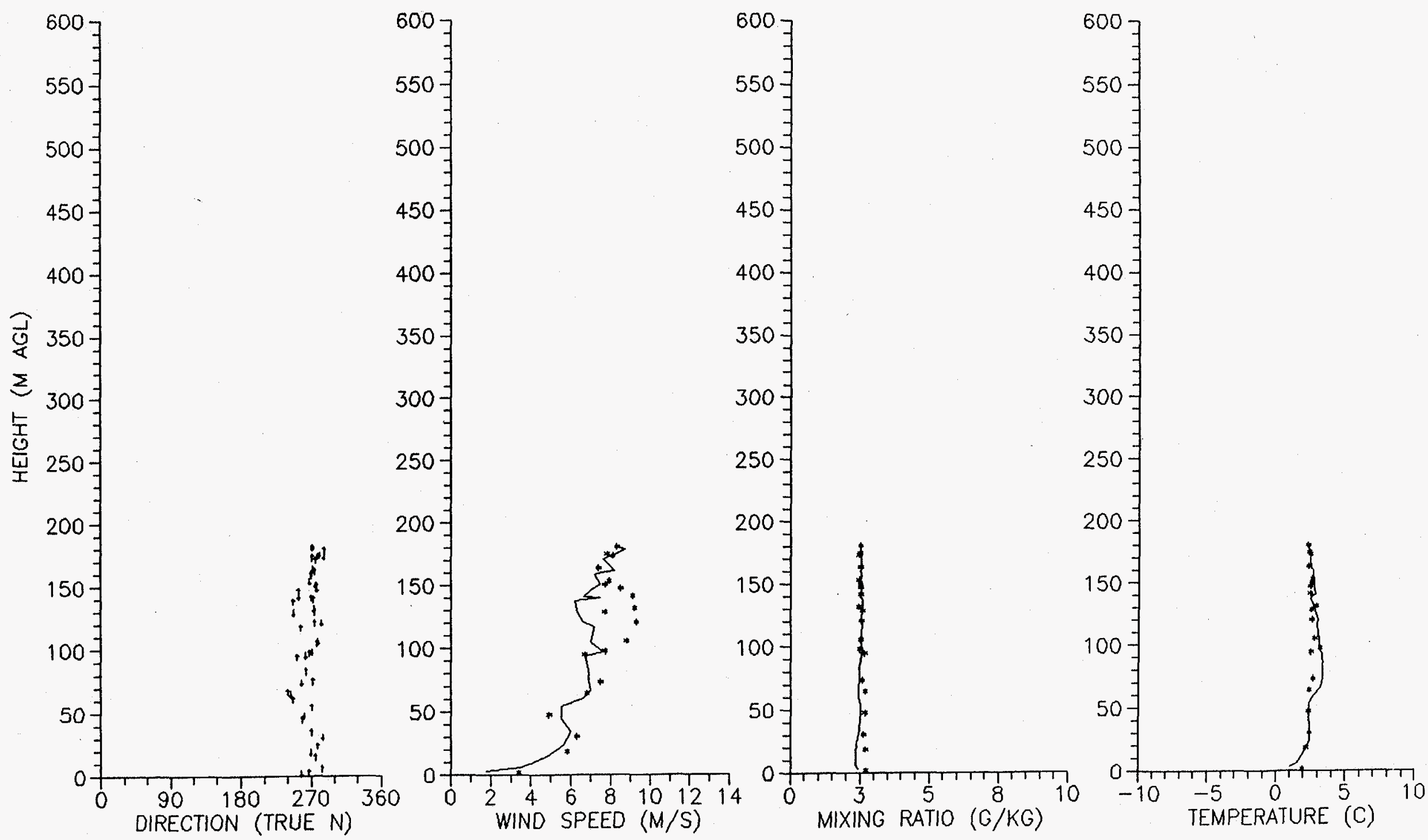
COAL CREEK CANYON TSONDE
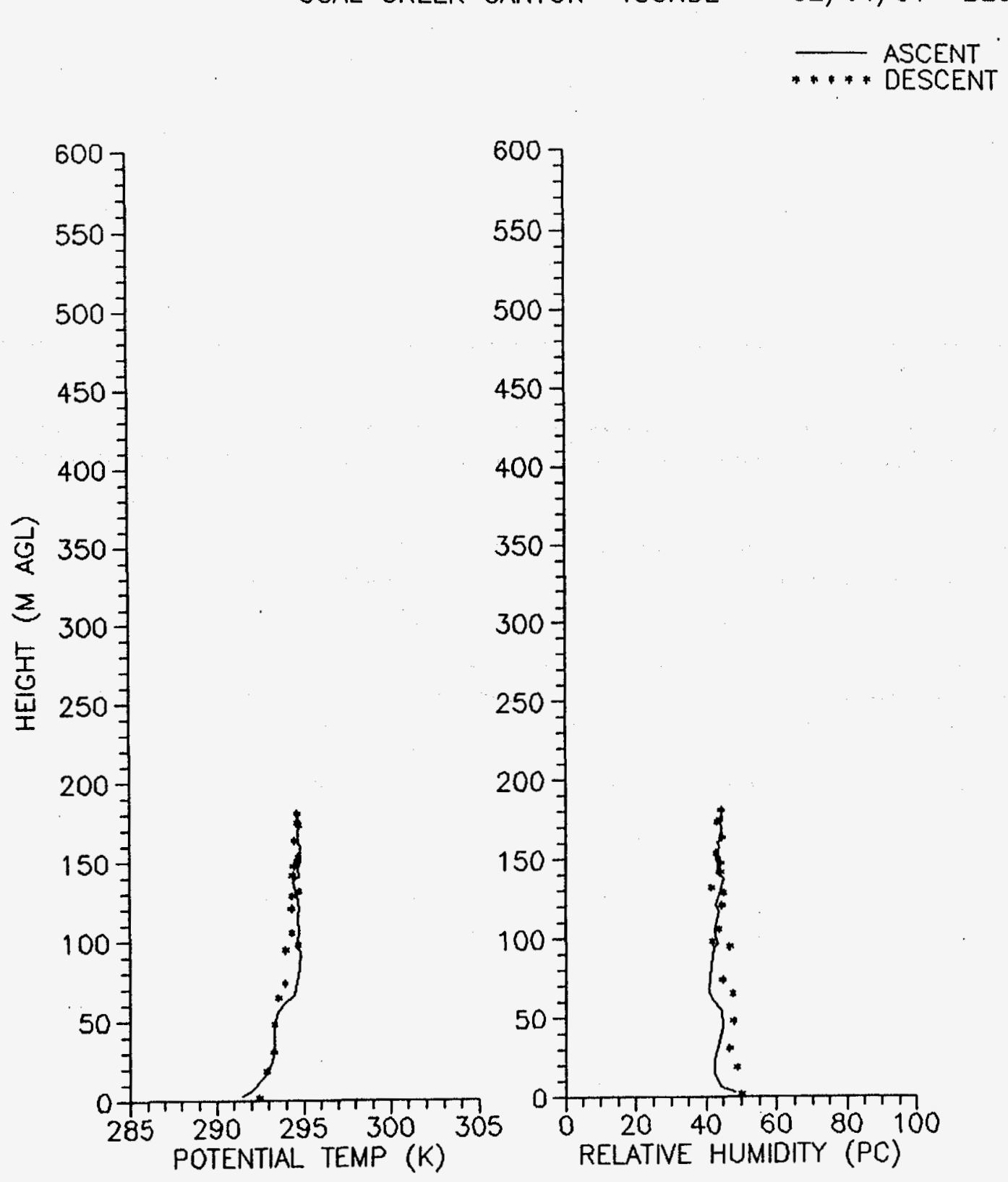
COAL CREEK CANYON

**** ASCENT
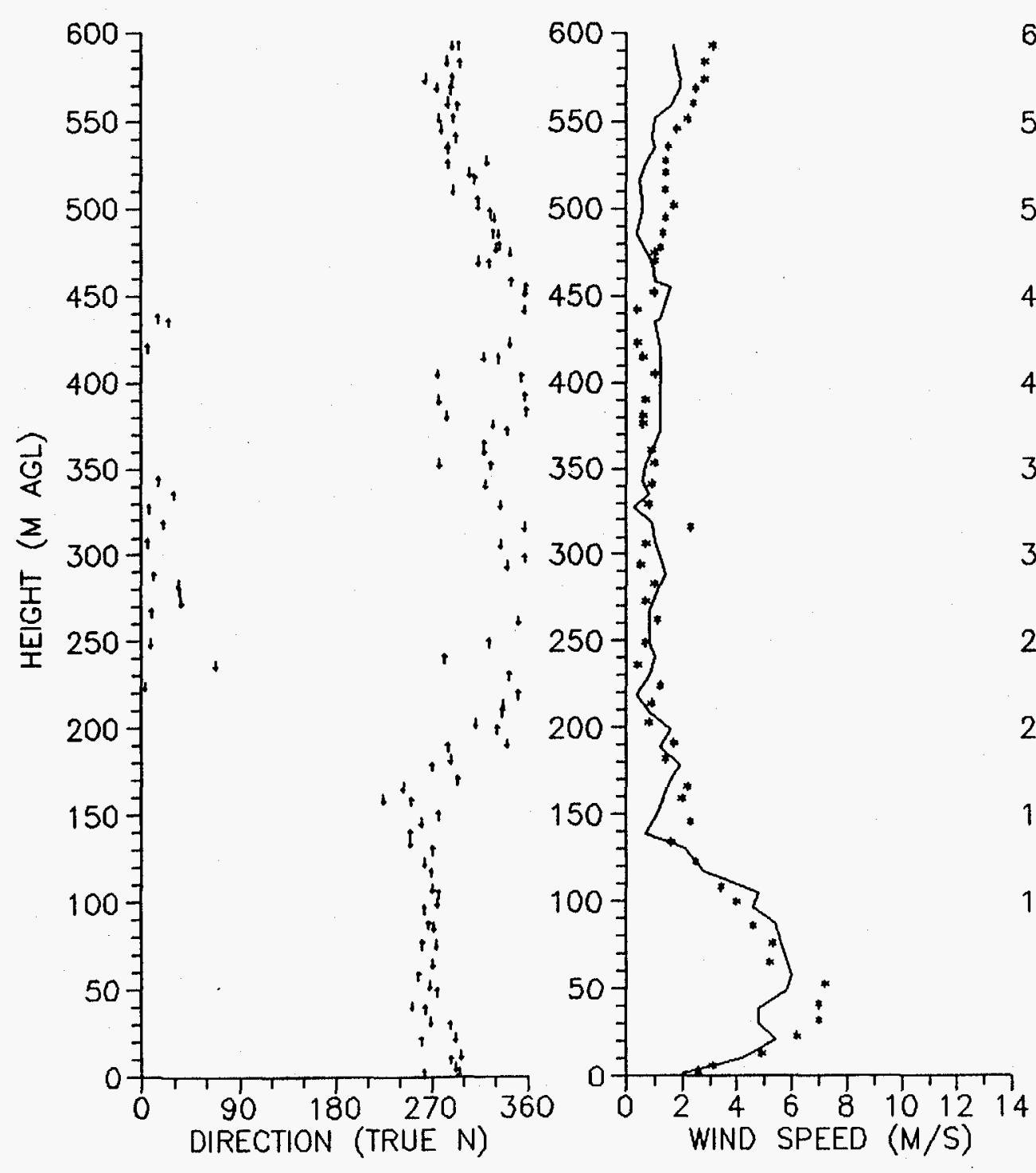
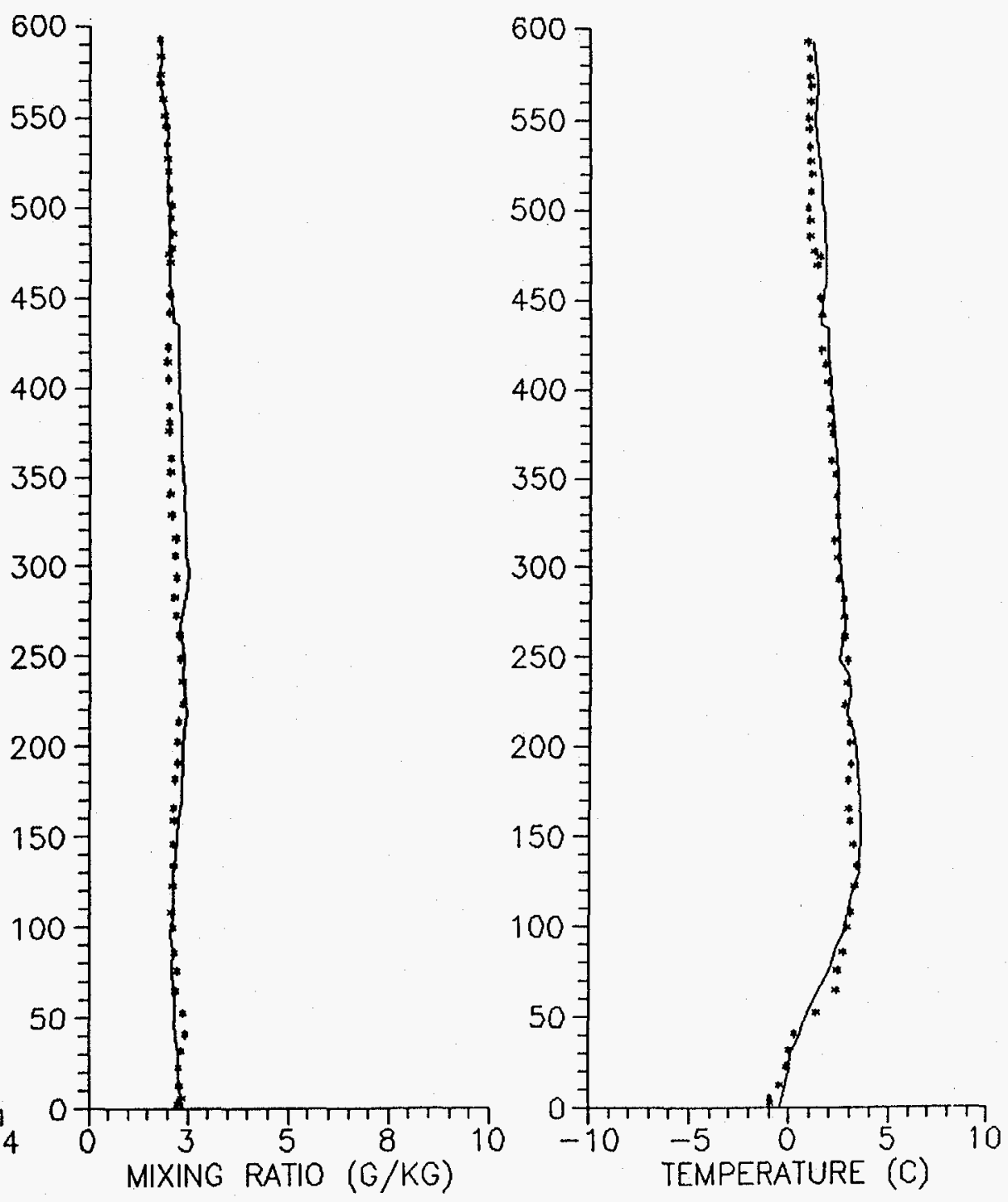


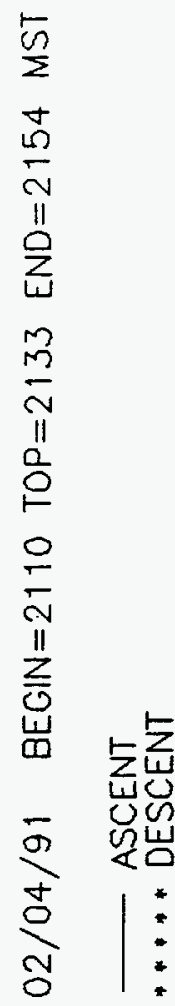

$\stackrel{3}{3}$

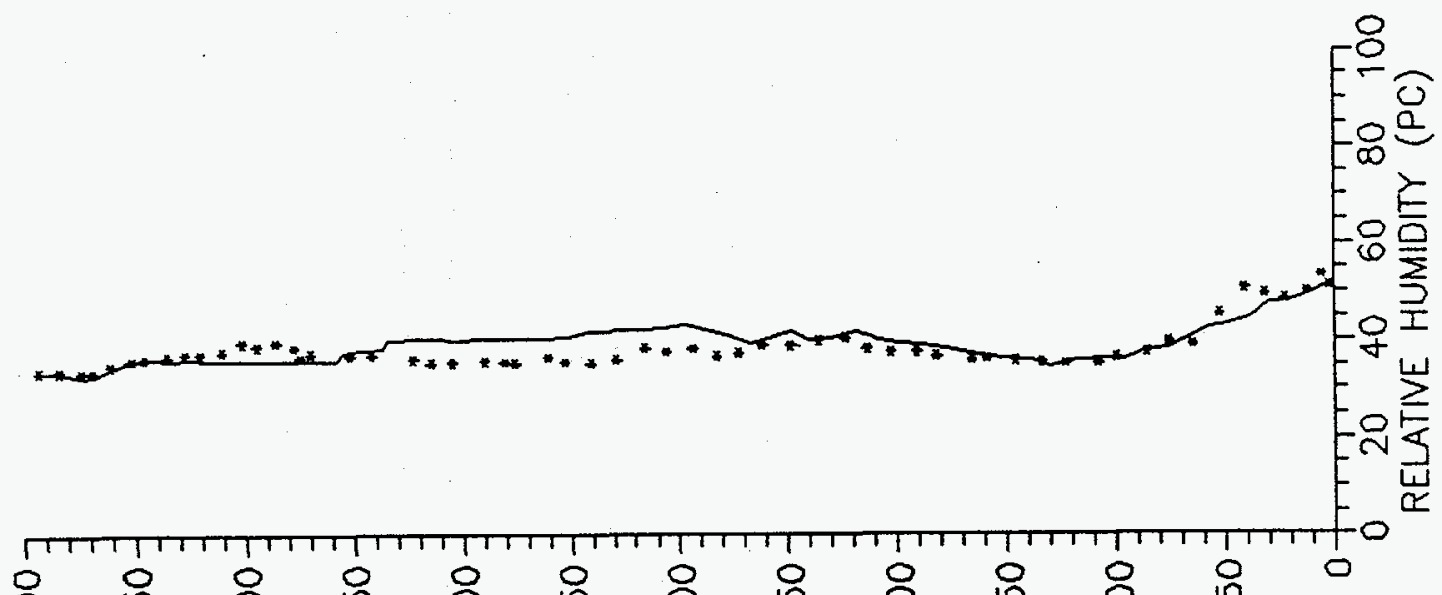

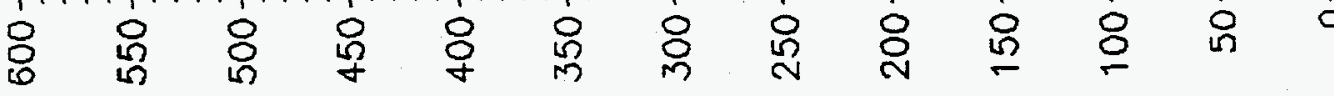

$\frac{1}{2}$

崖

8

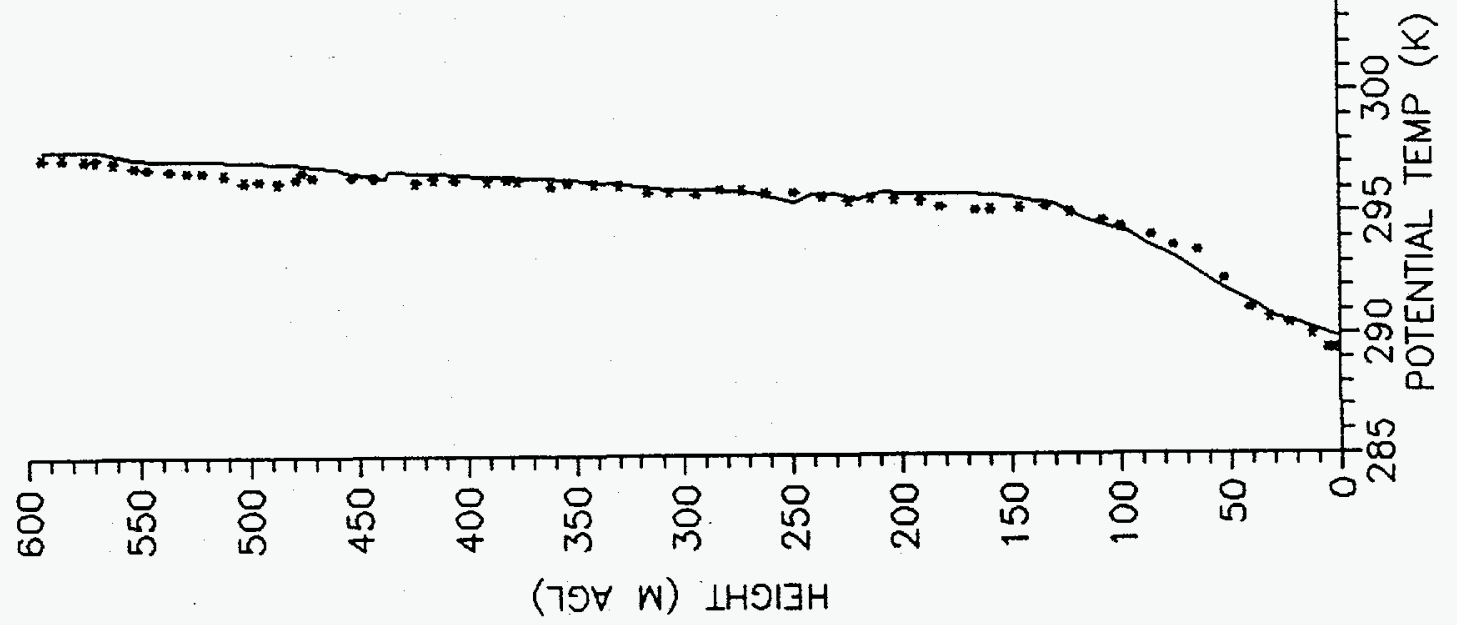




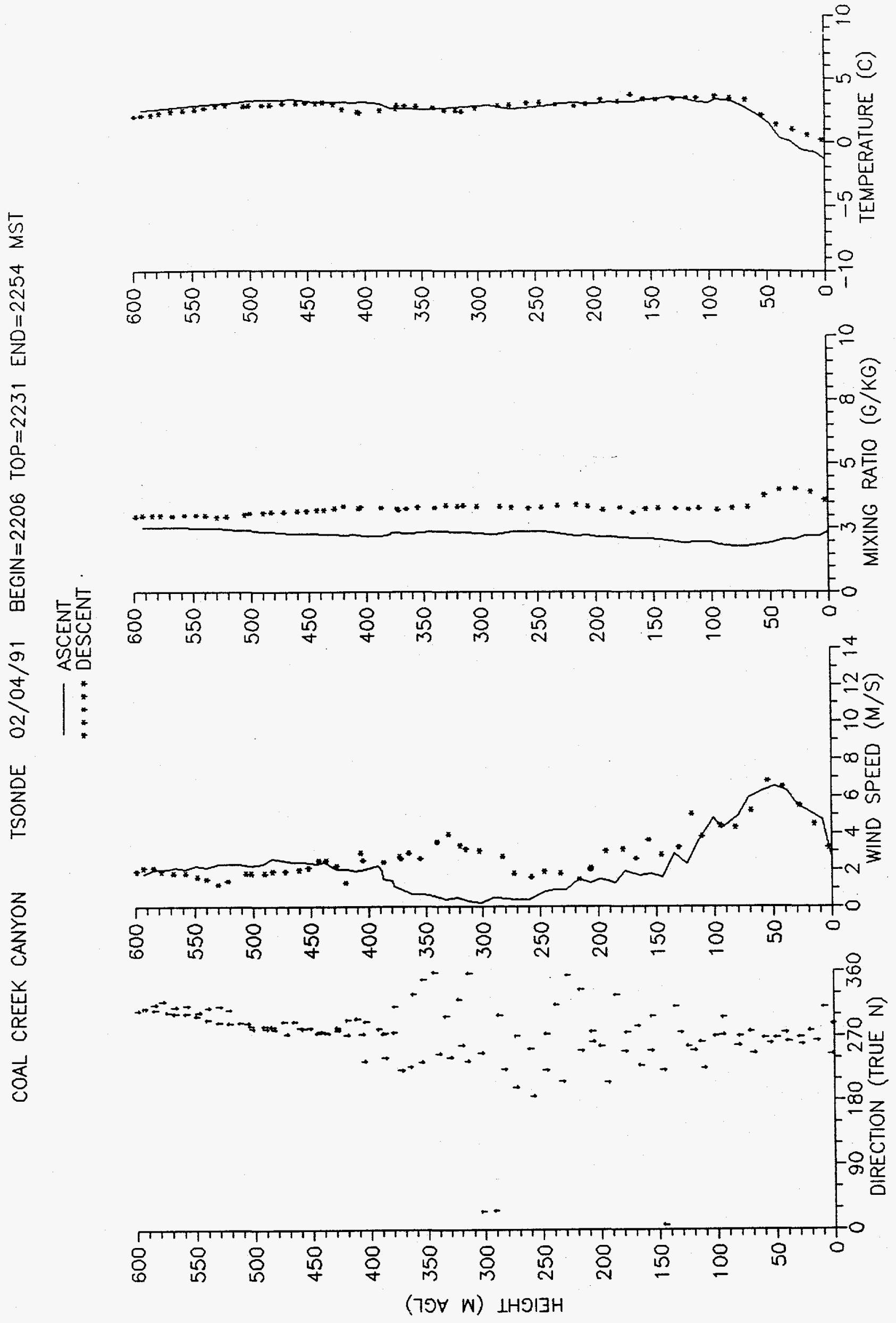


COAL CREEK CANYON TSONDE

$02 / 04 / 91 \quad B E G I N=2206 \quad T O P=2231 \quad E N D=2254 \quad M S T$

*** ASCENT
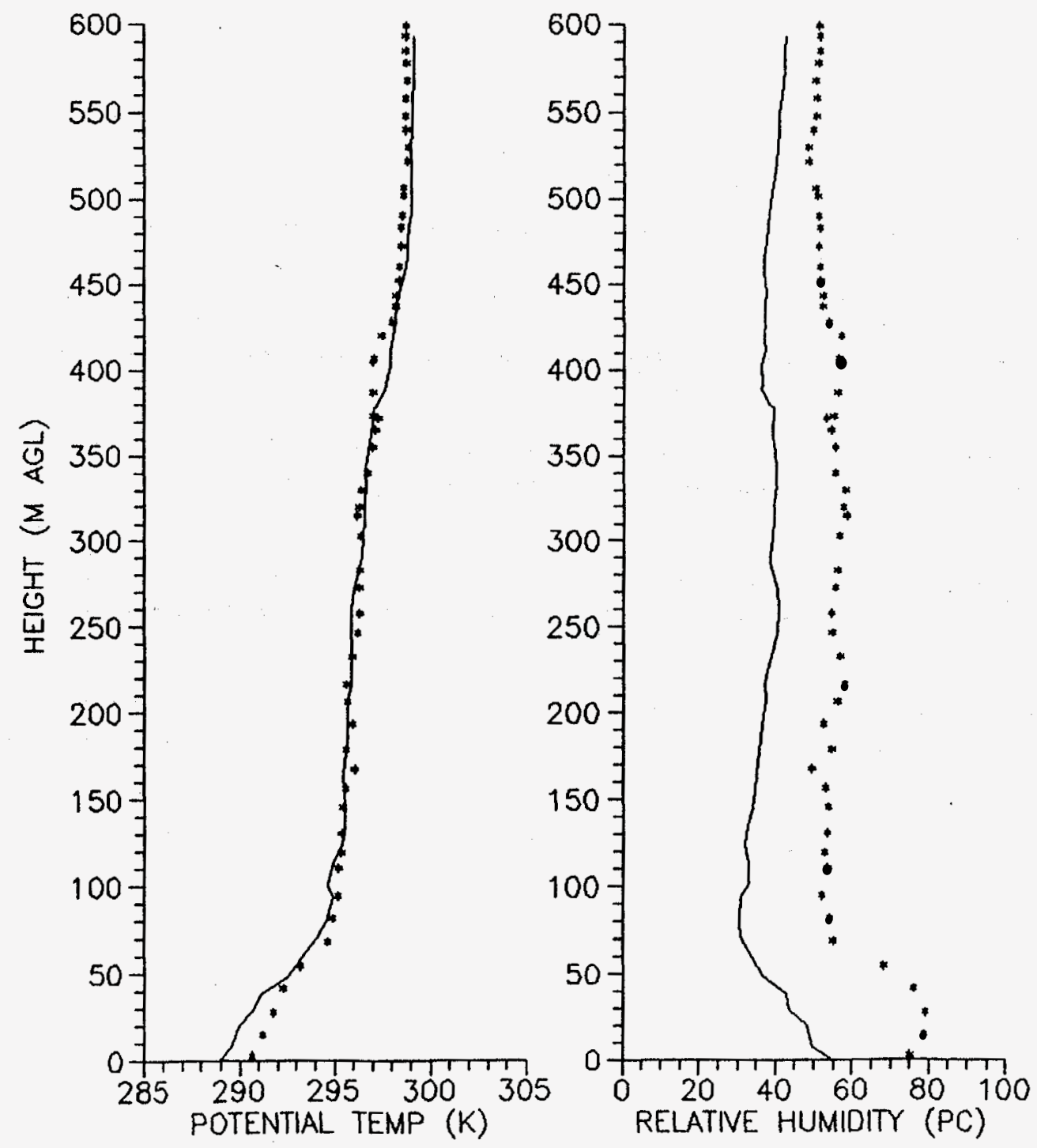


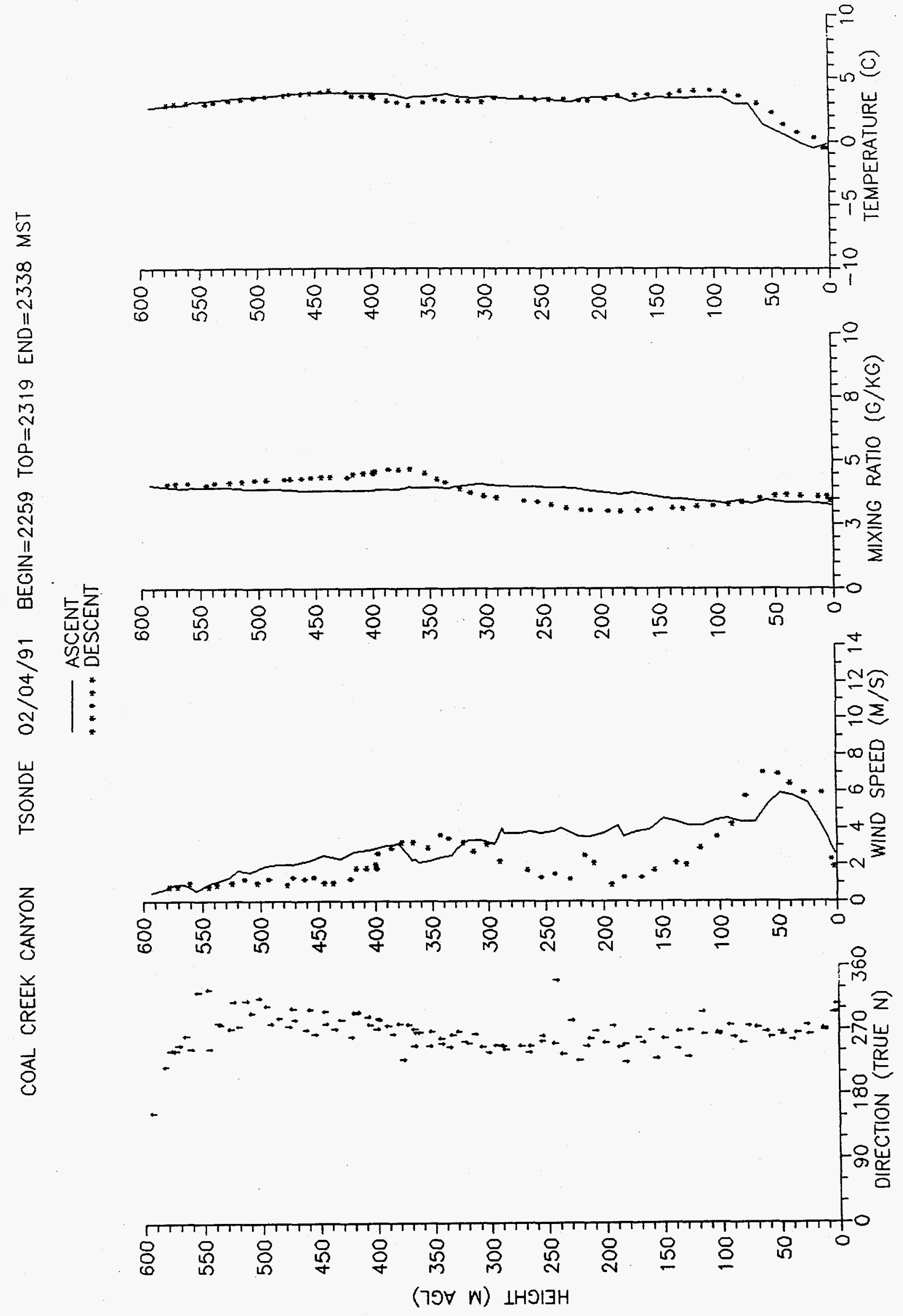




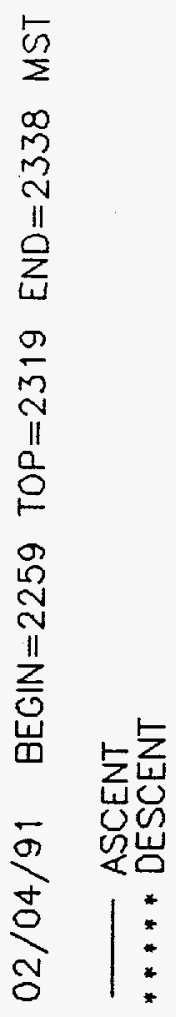

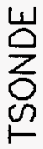

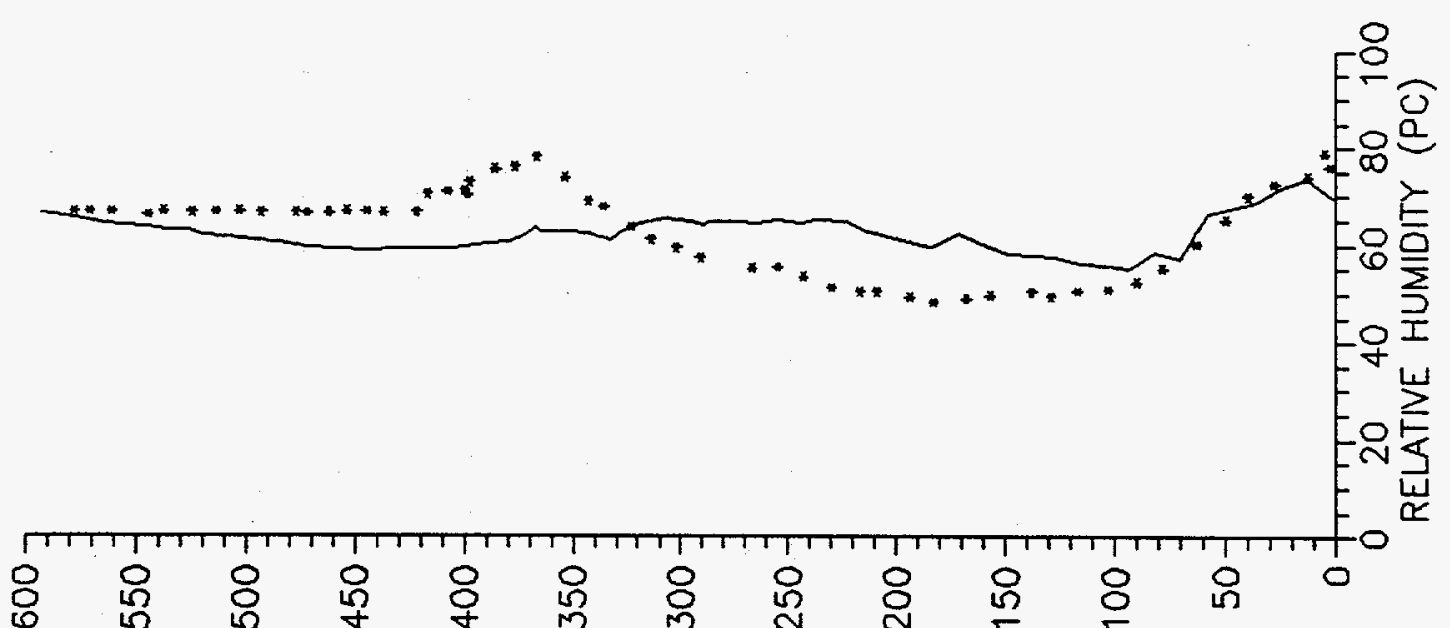

$\frac{z}{2}$

$\frac{\checkmark}{u}$
㭊
$\frac{\delta}{\delta}$

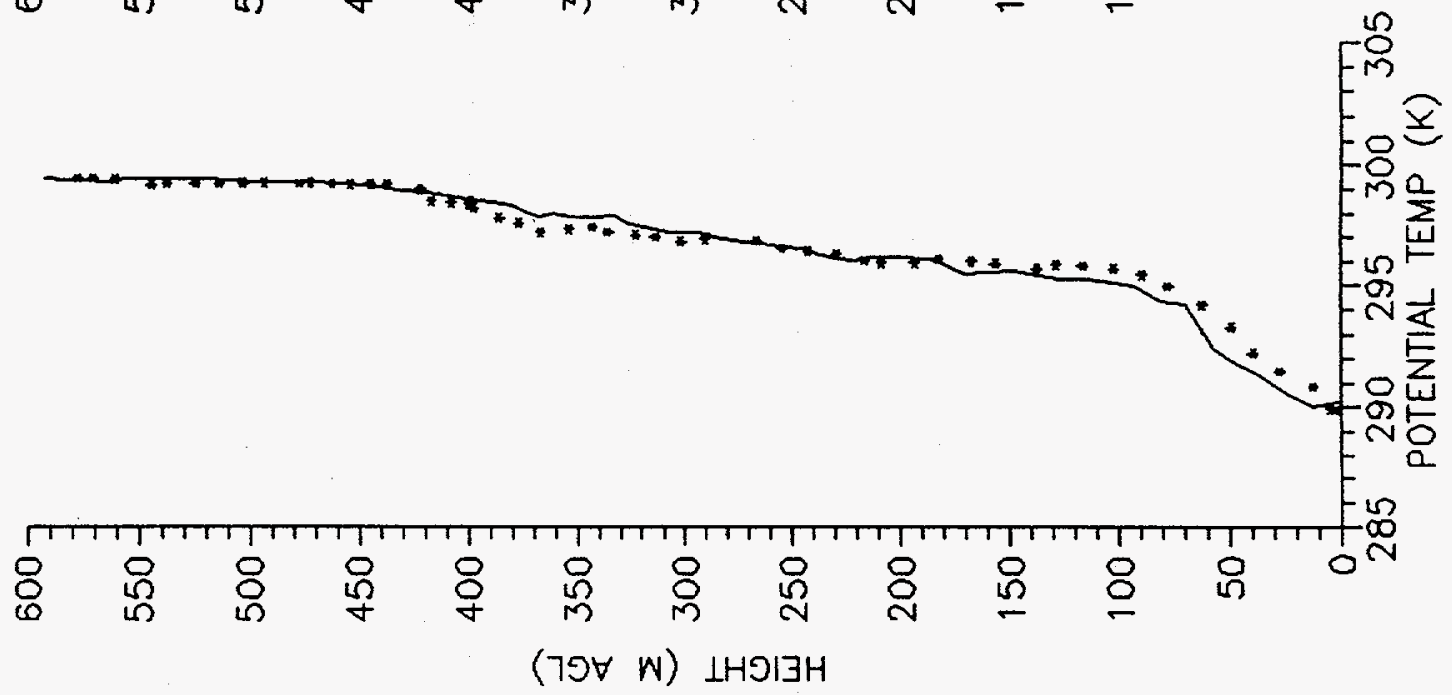


COAL CREEK CANYON TSONDE 02/05/91 BEGIN=0000 TOP $=0020 \quad$ END $=0041 \quad \mathrm{MST}$
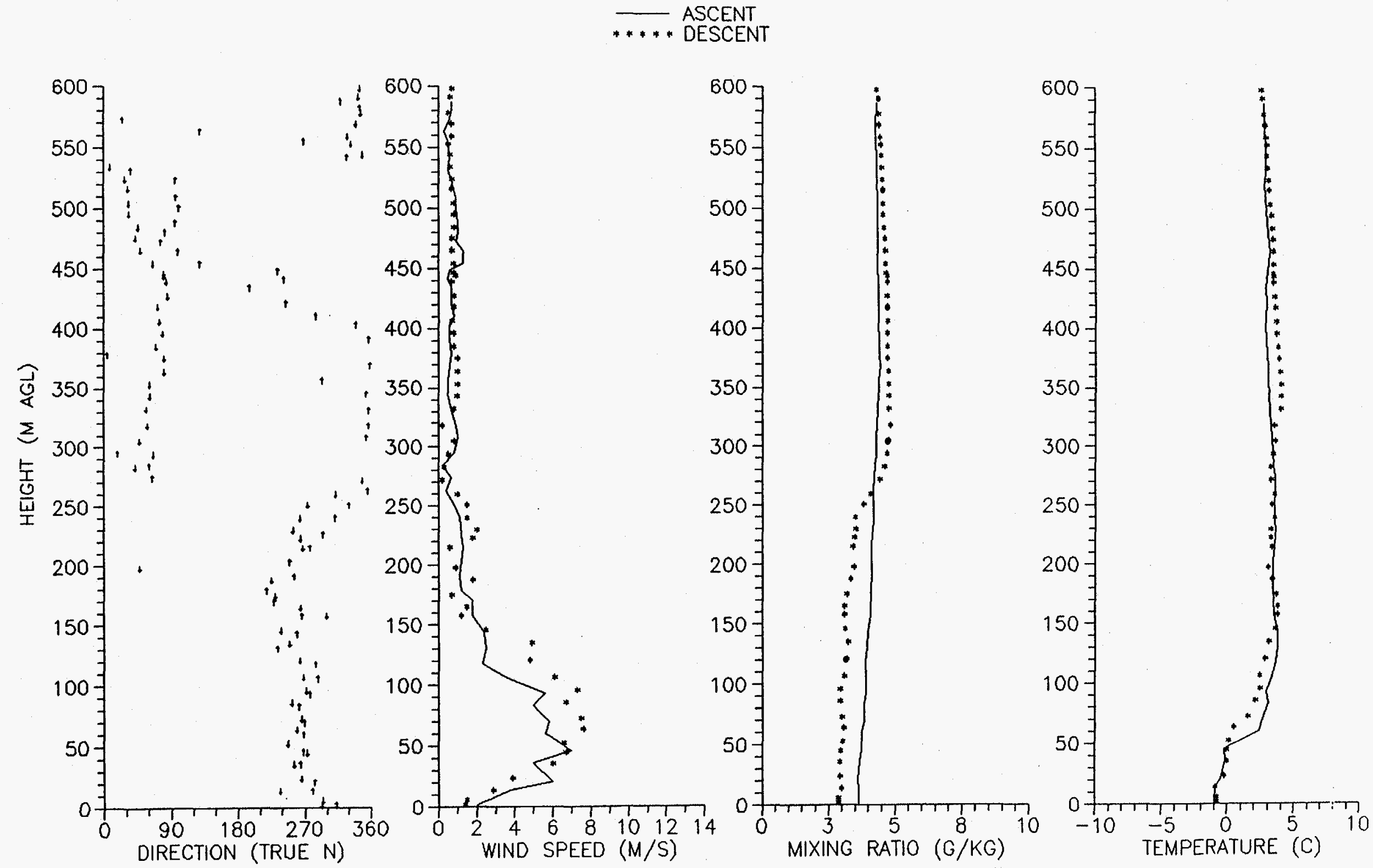


\section{.... ASCENT}
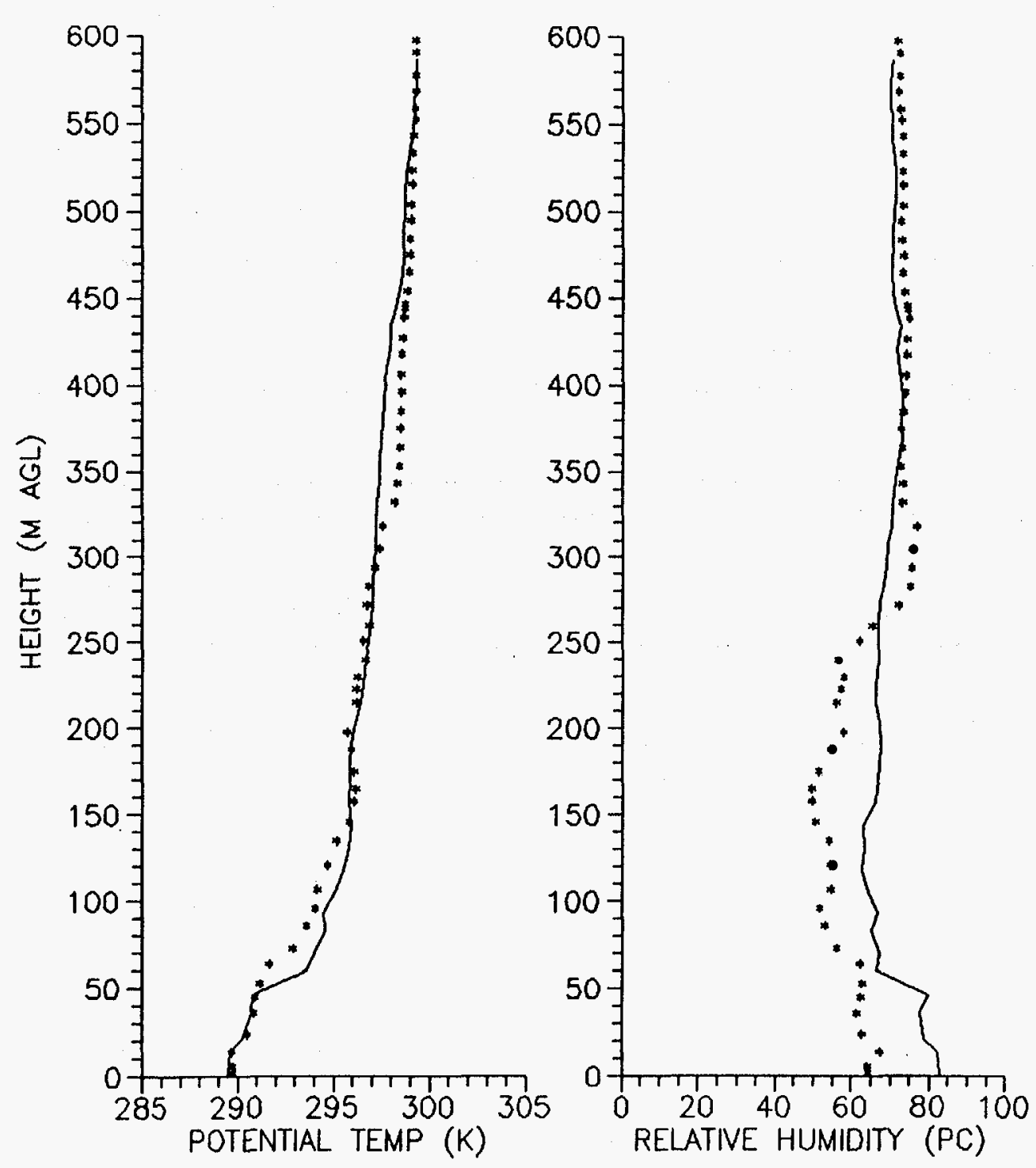


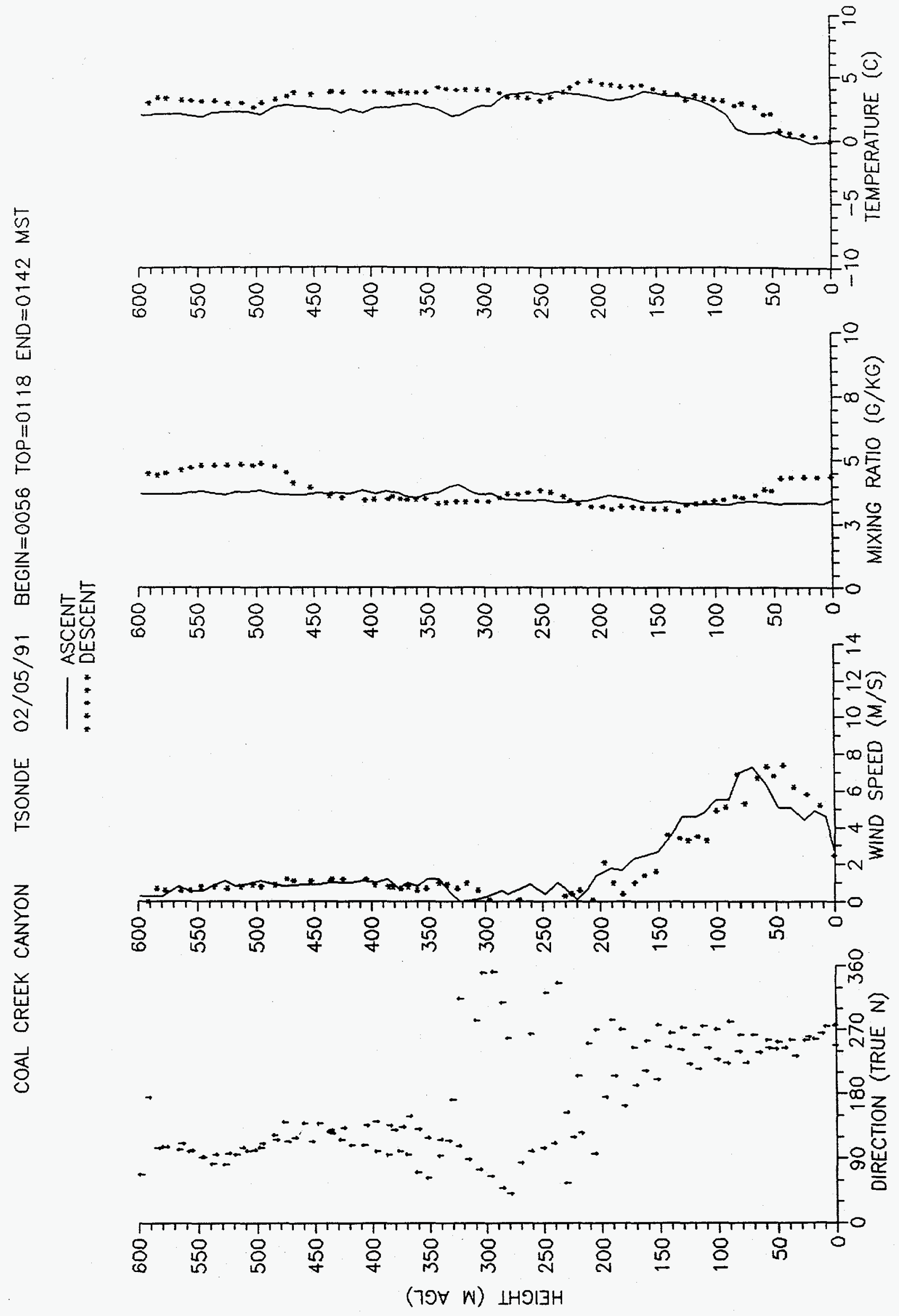




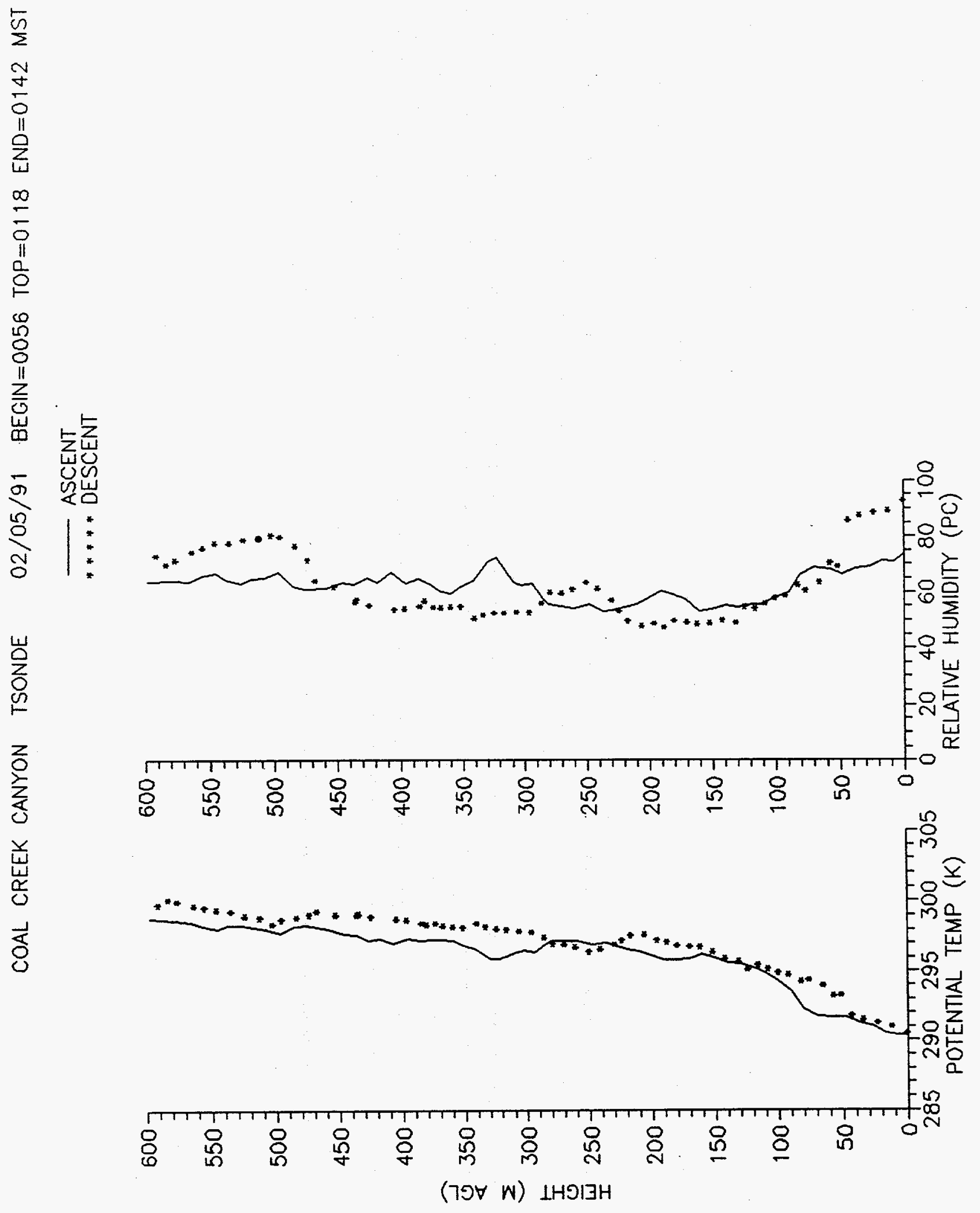




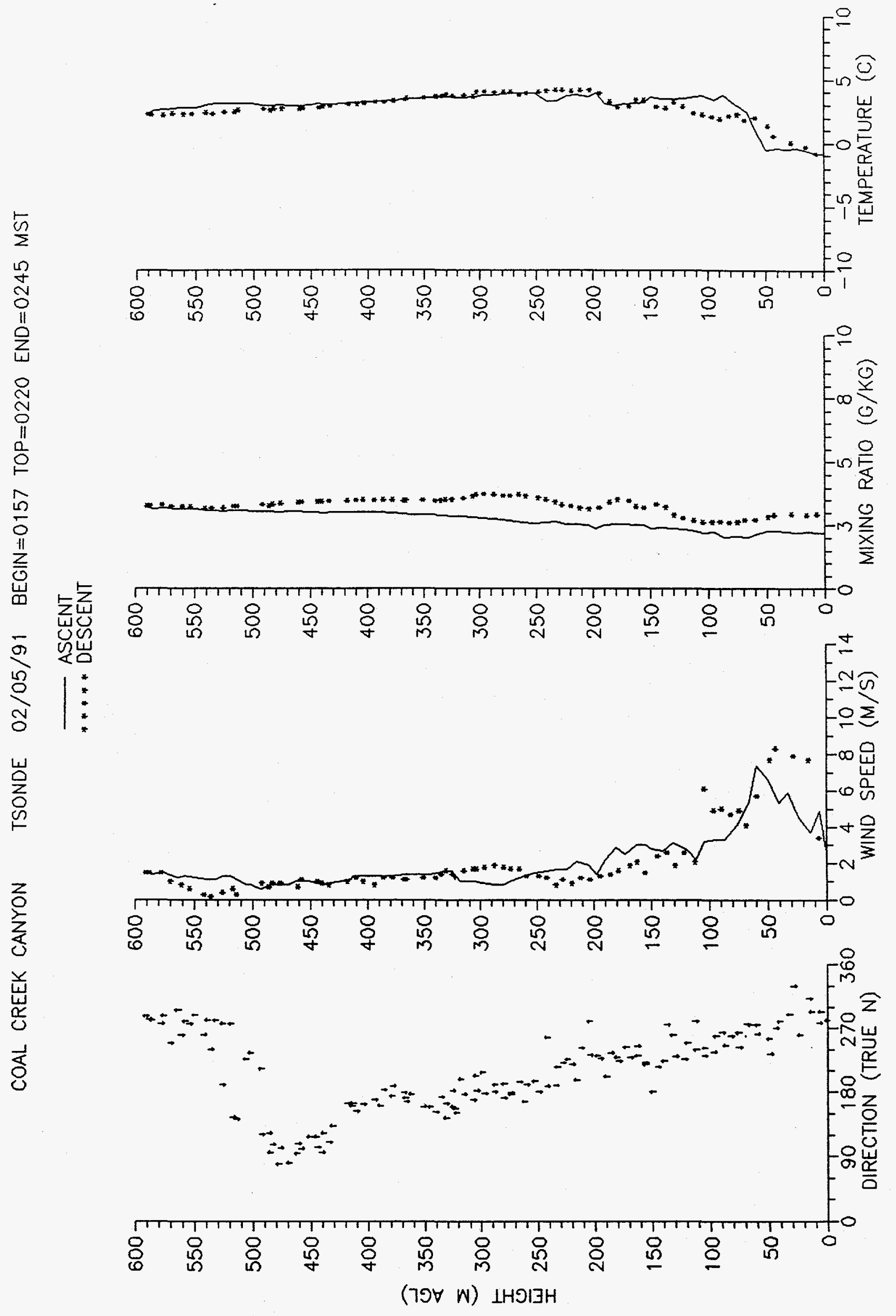




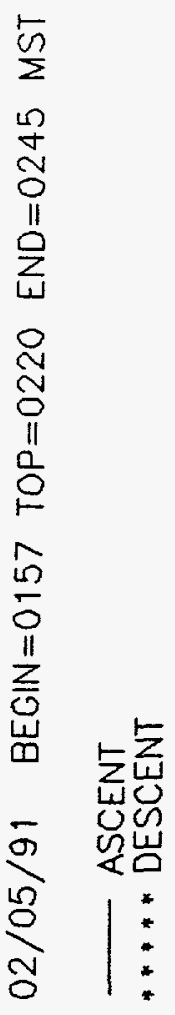

峁

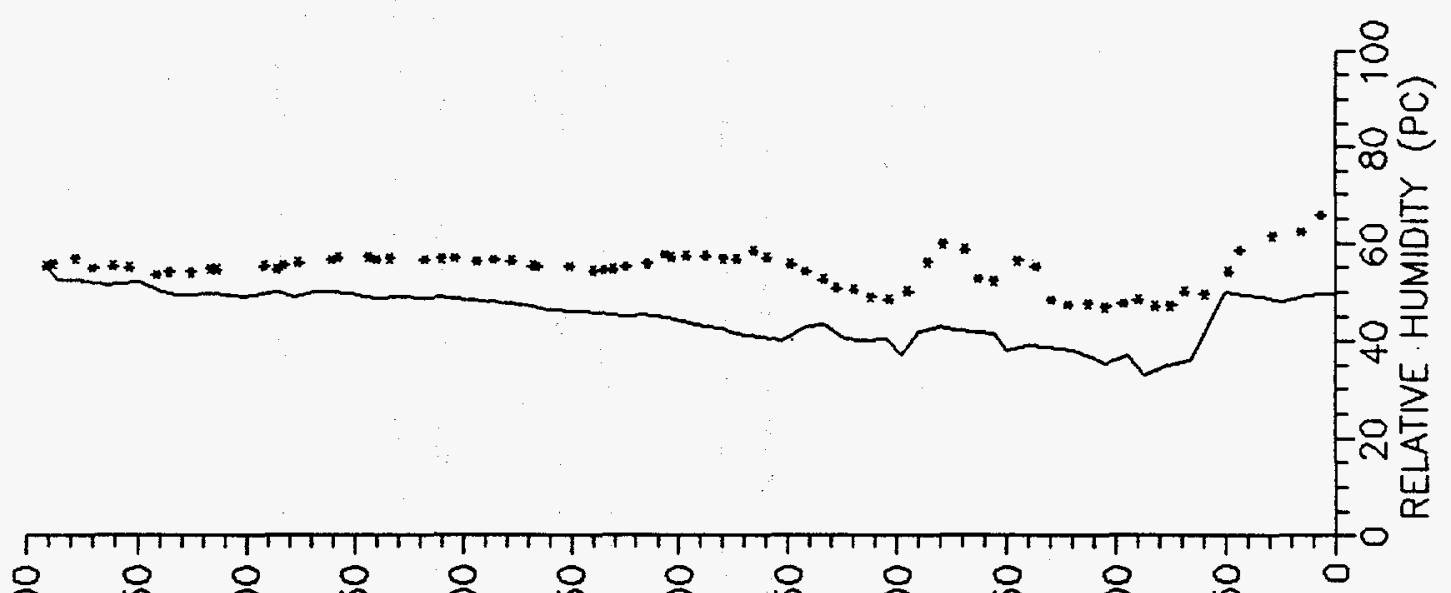

方

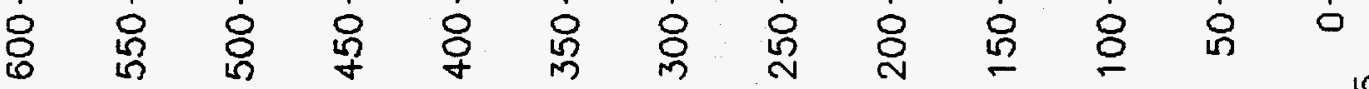

岀
峞
安

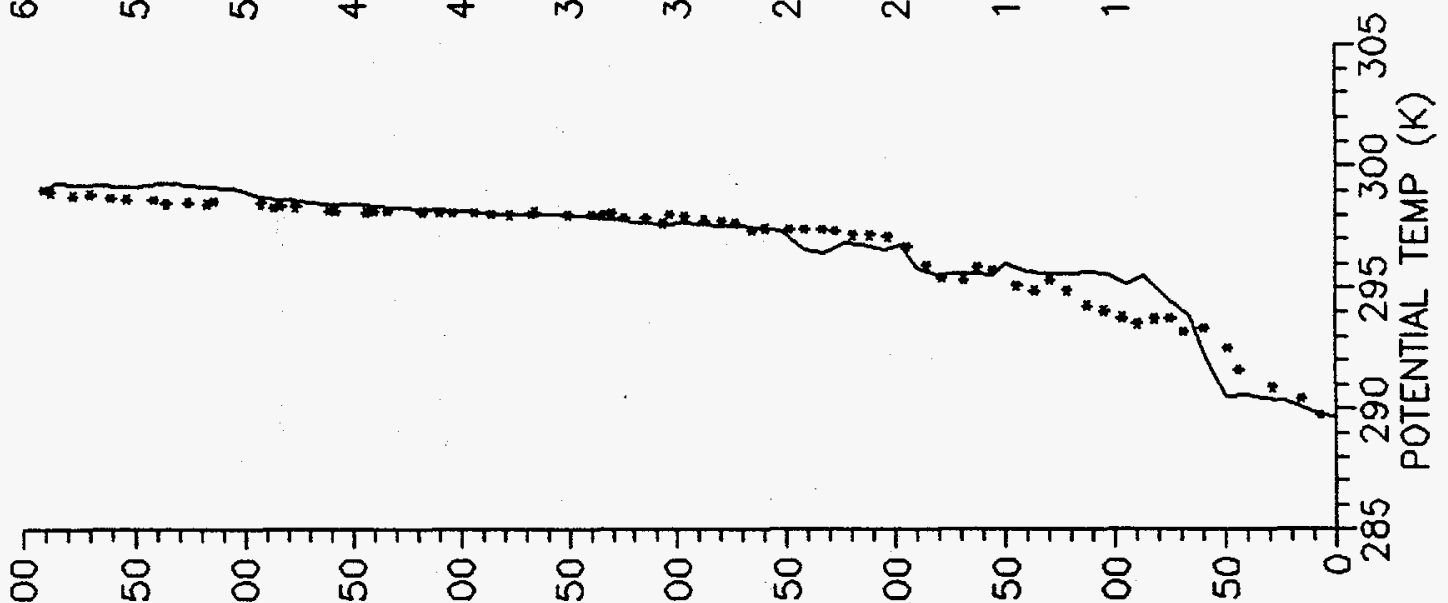

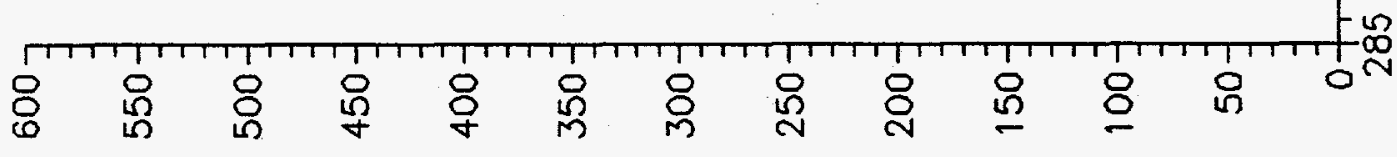

(าפ十 W) $\mathrm{LHOIJH}$ 


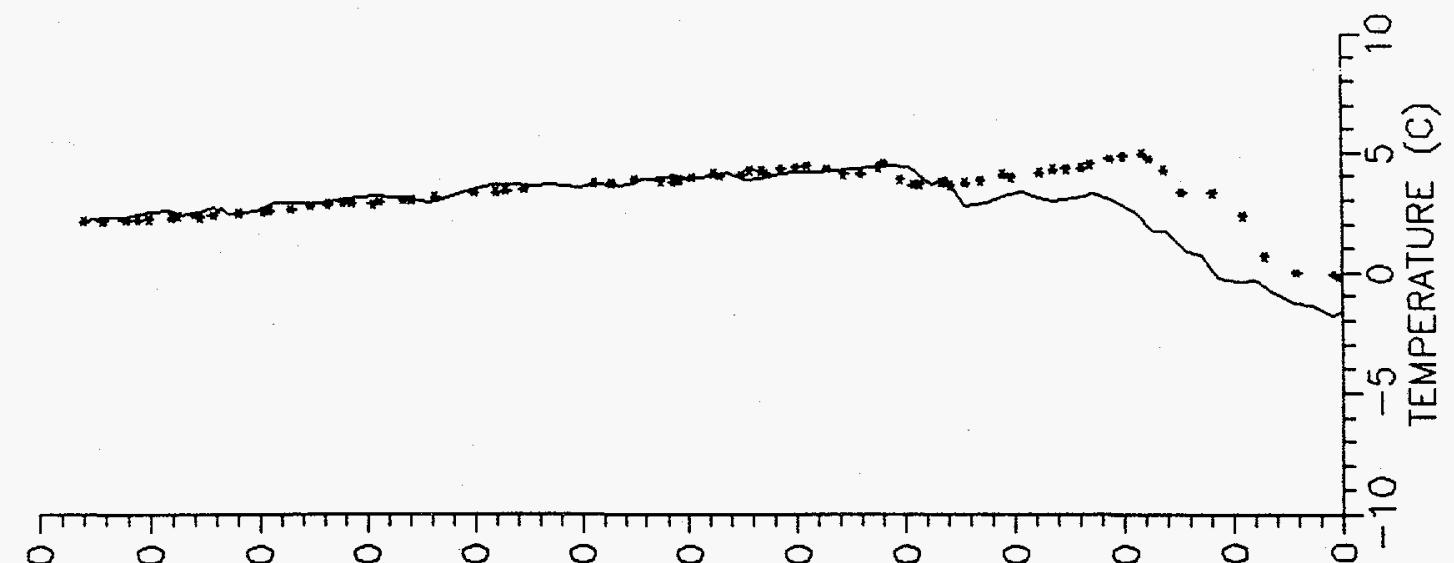

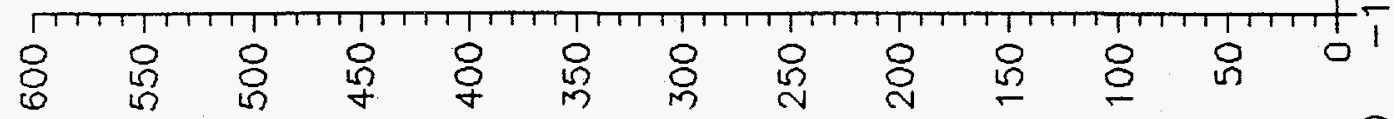

일

कृ

充至 U仓仓

क

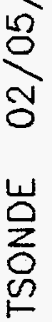

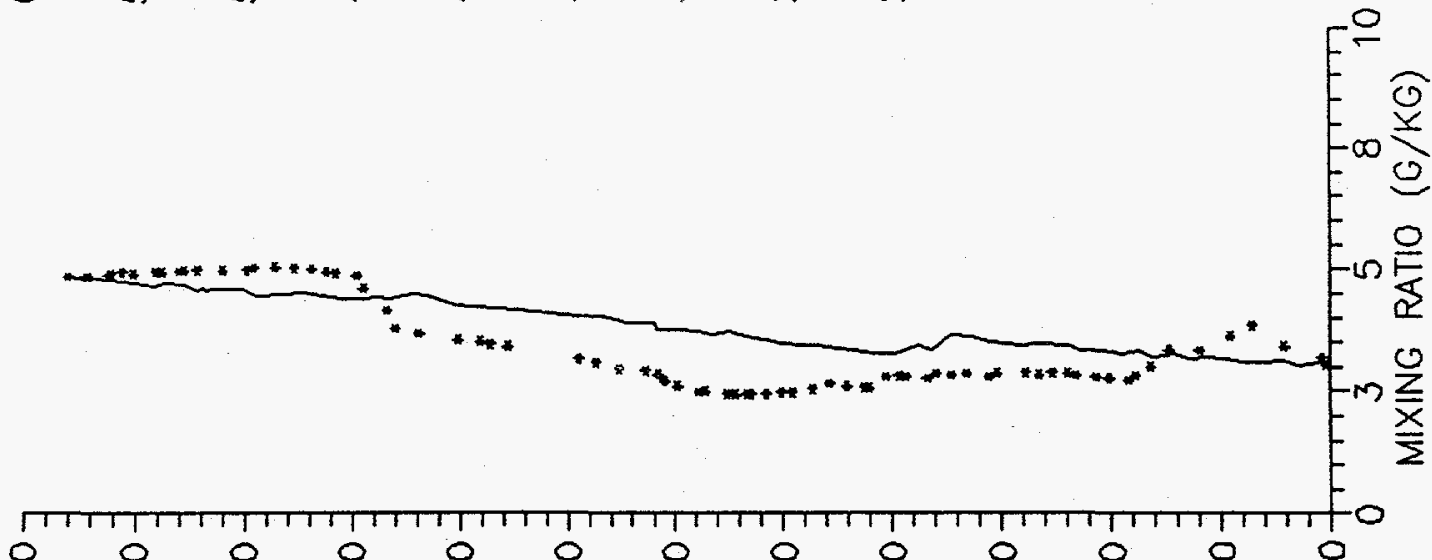

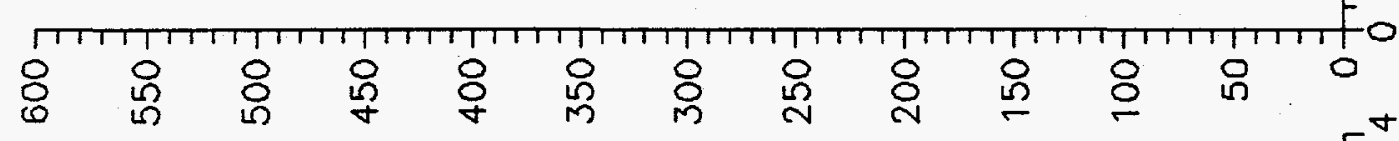

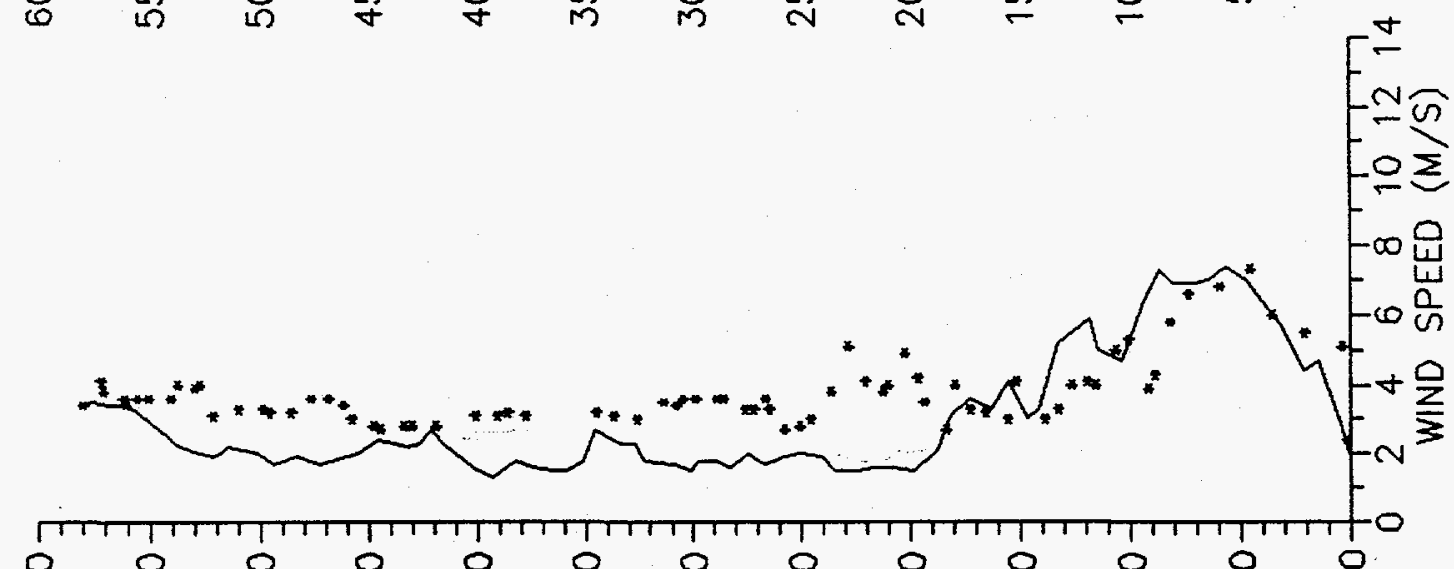

용

$\sum_{\substack{c \\ 0}}^{z}$

象量

嵌
号
$\frac{1}{8}$

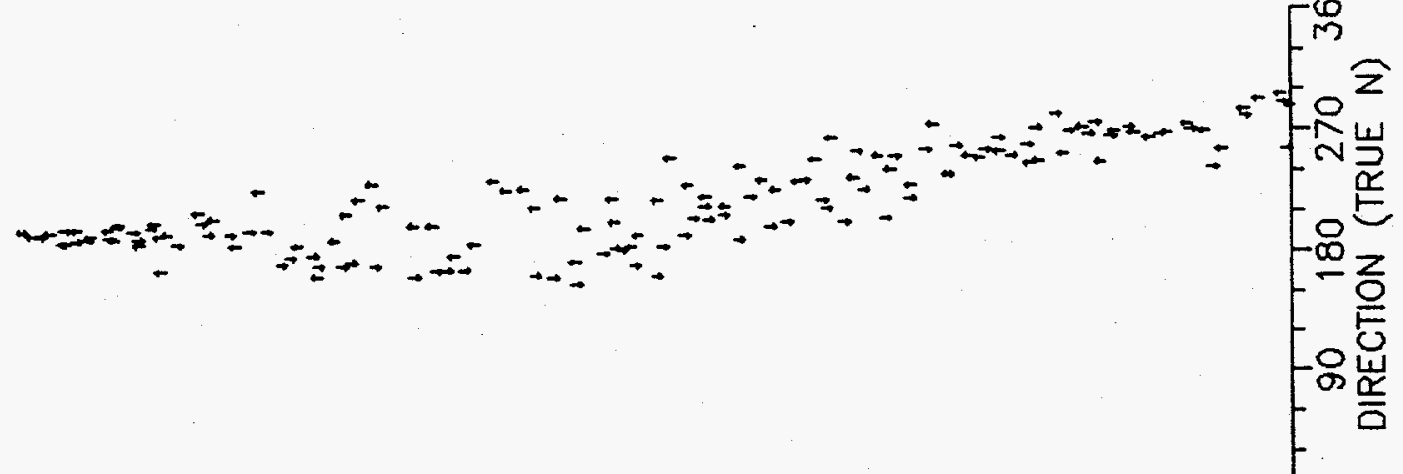

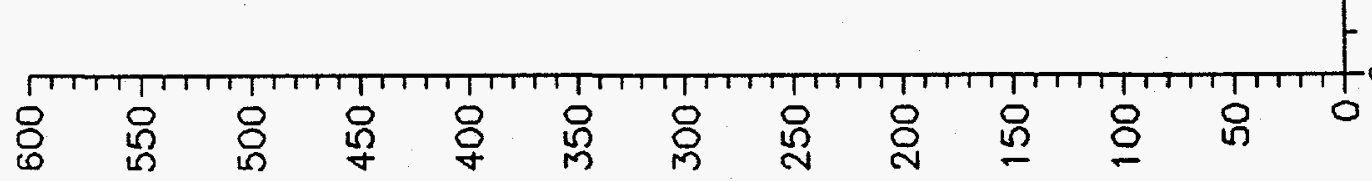

(7פ十 W) $\mathrm{LH}$ HIJH 
COAL CREEK CANYON TSONDE 02/05/91 BEGIN=0301 TOP=0326 END=0353 MST

*** ASCENT
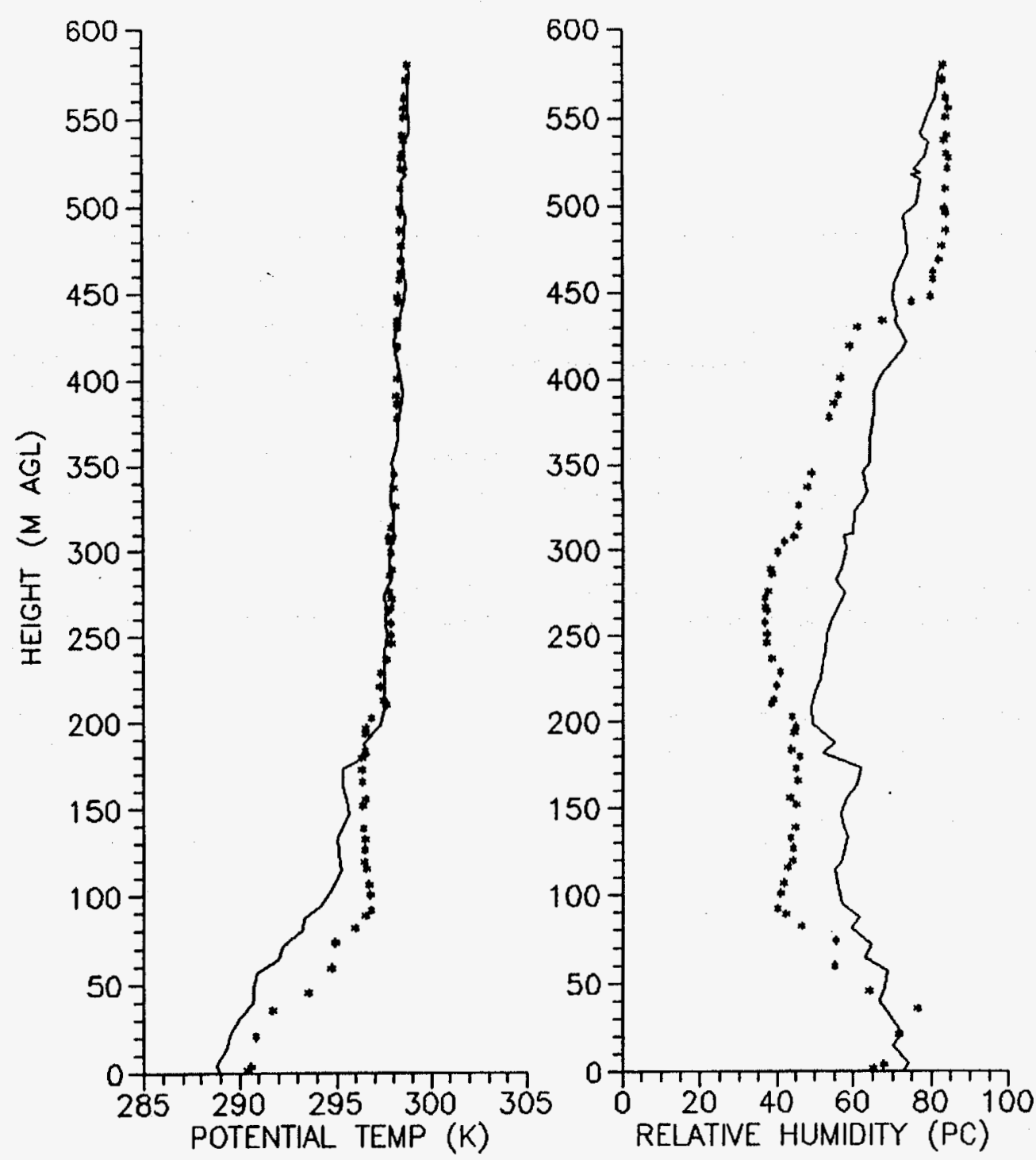
COAL CREEK CANYON TSONDE 02/05/91 BEGIN=0359 TOP=0416 END=0453 MST

- ASCENT
$* * * *$ DESCENT
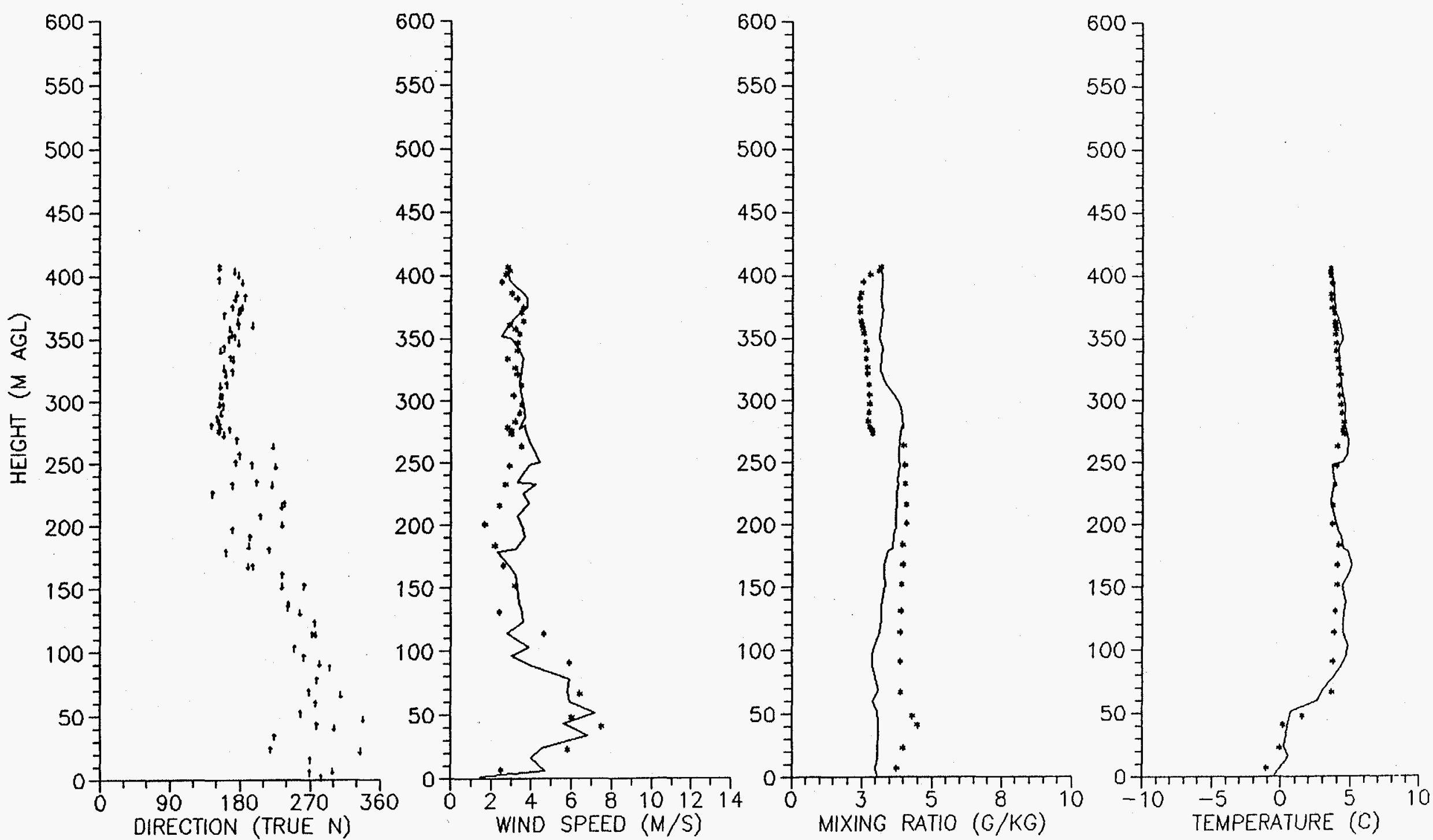
COAL CREEK CANYON TSONDE 02/05/91 BEGIN $=0359$ TOP $=0416 \quad$ END $=0453 \quad$ MST ASCENT
$* * * *$ DESCENT

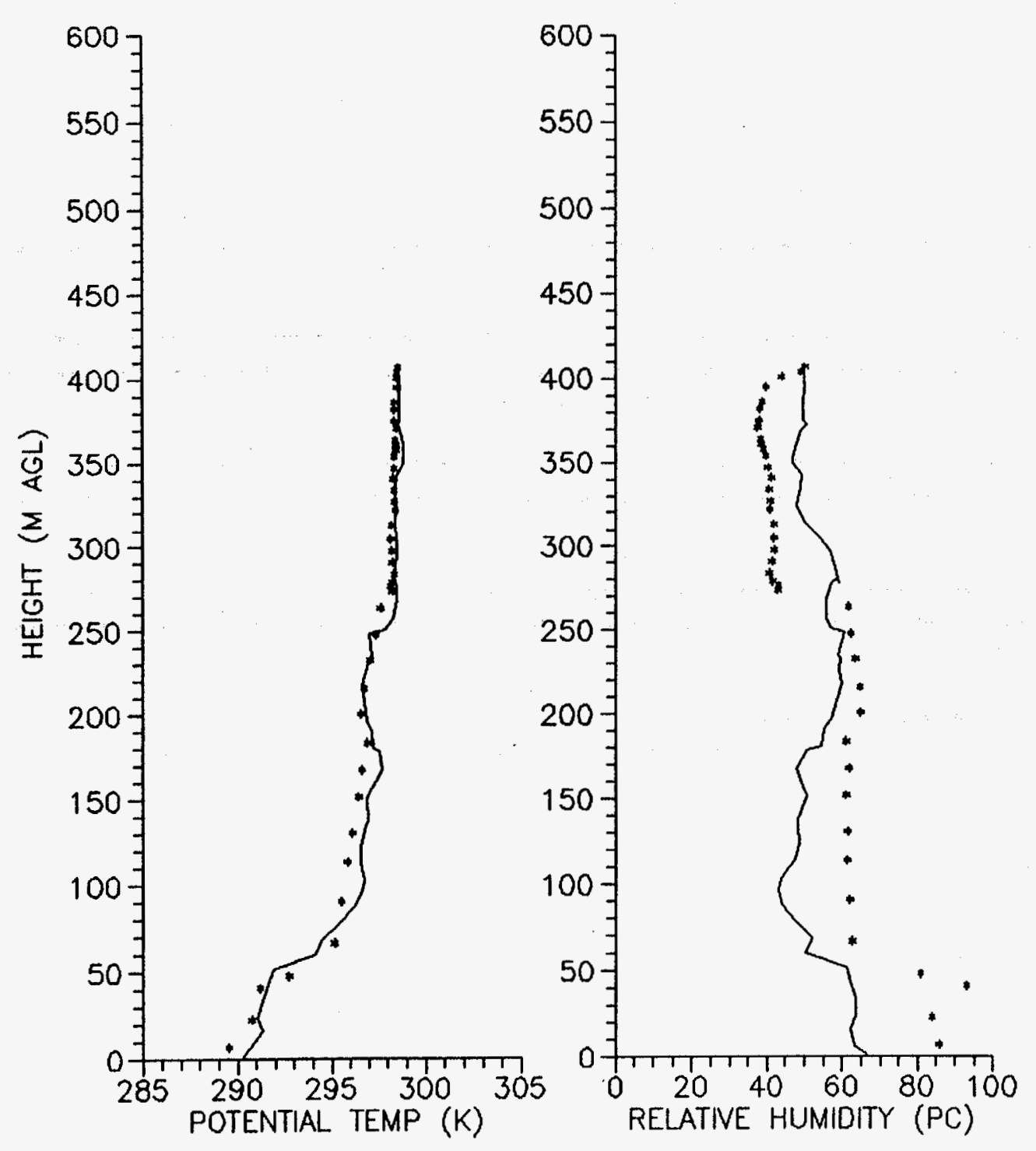


COAL CREEK CANYON TSONDE 02/06/91 BEGIN=1959 TOP=2005 END=2009 MST ASCENT
$* * * *$ DESCENT
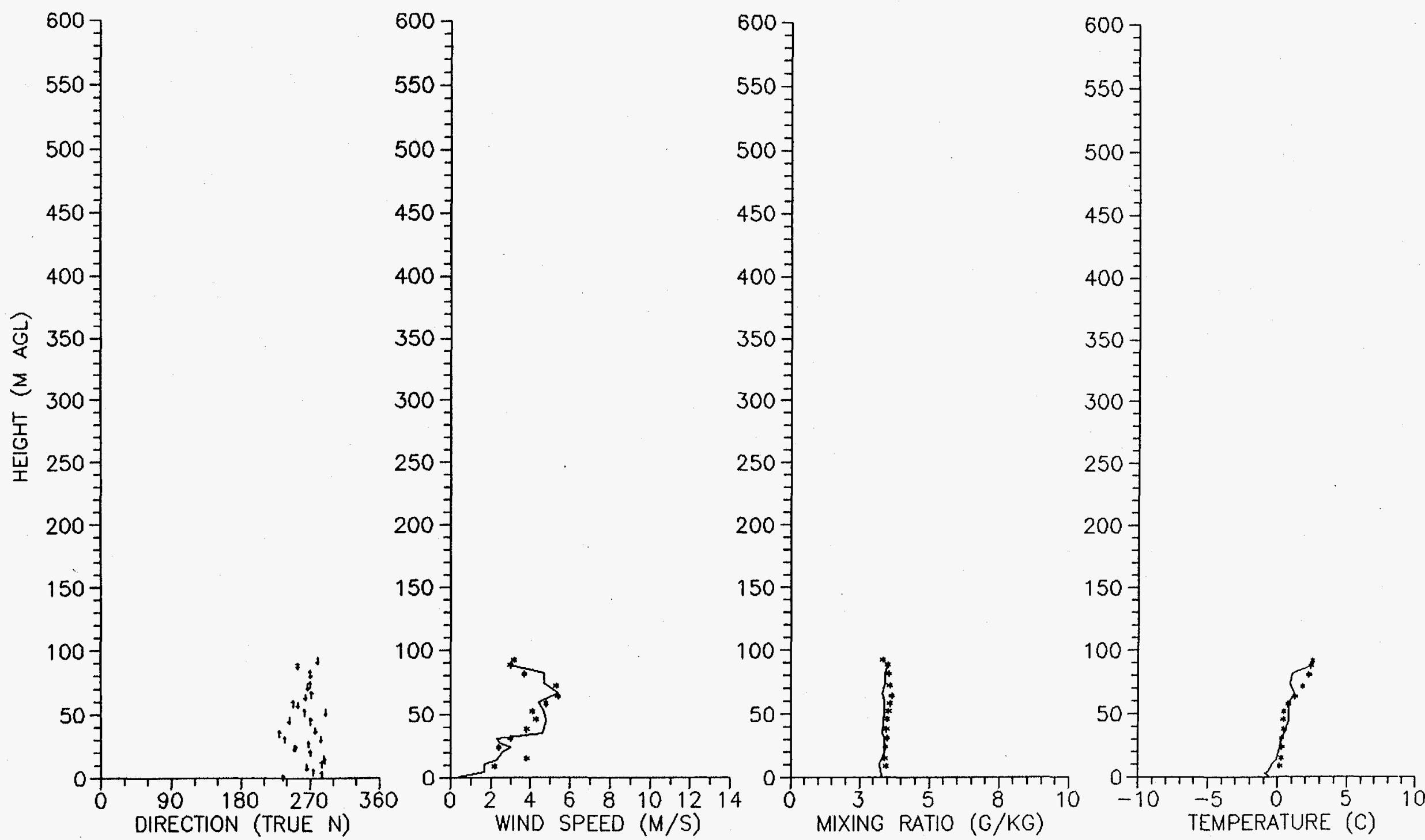


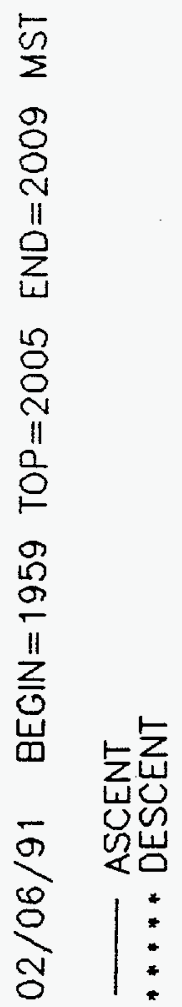

$\stackrel{\text { 岁 }}{Z}$

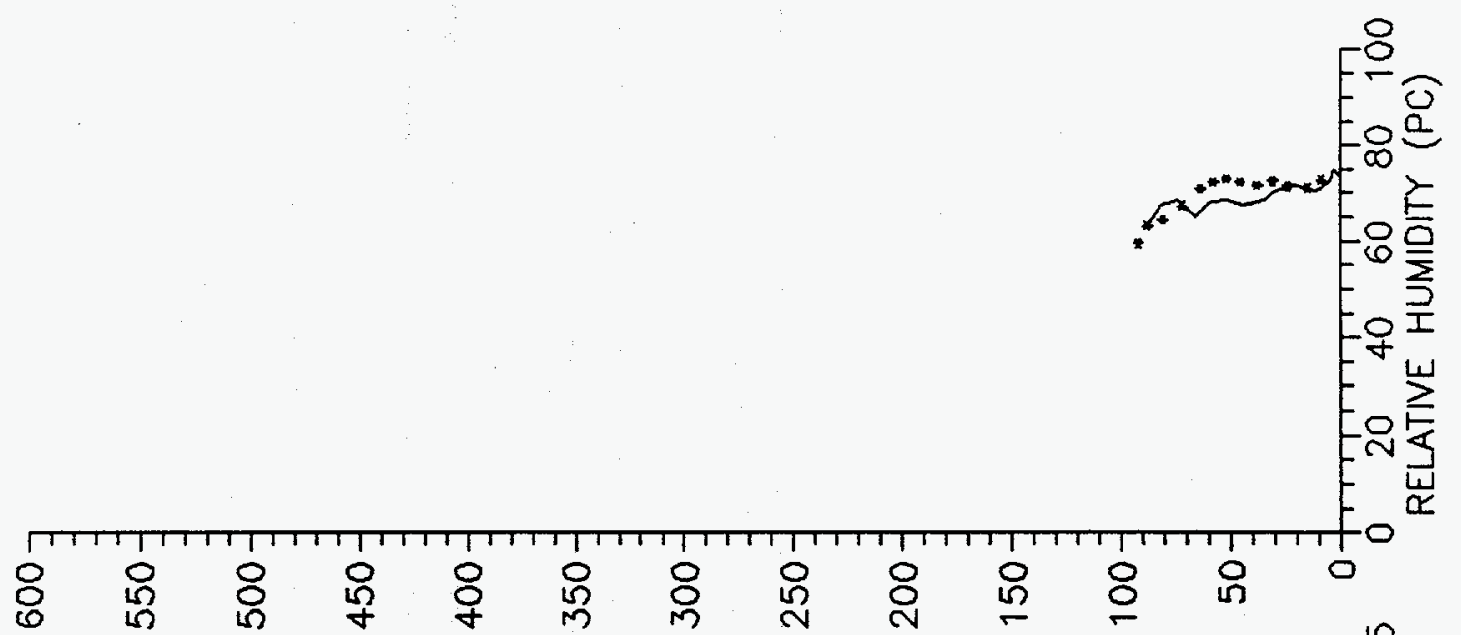

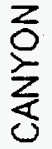

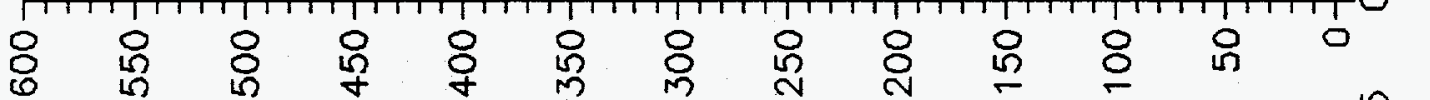

$\frac{x}{\frac{w}{0}}$

8

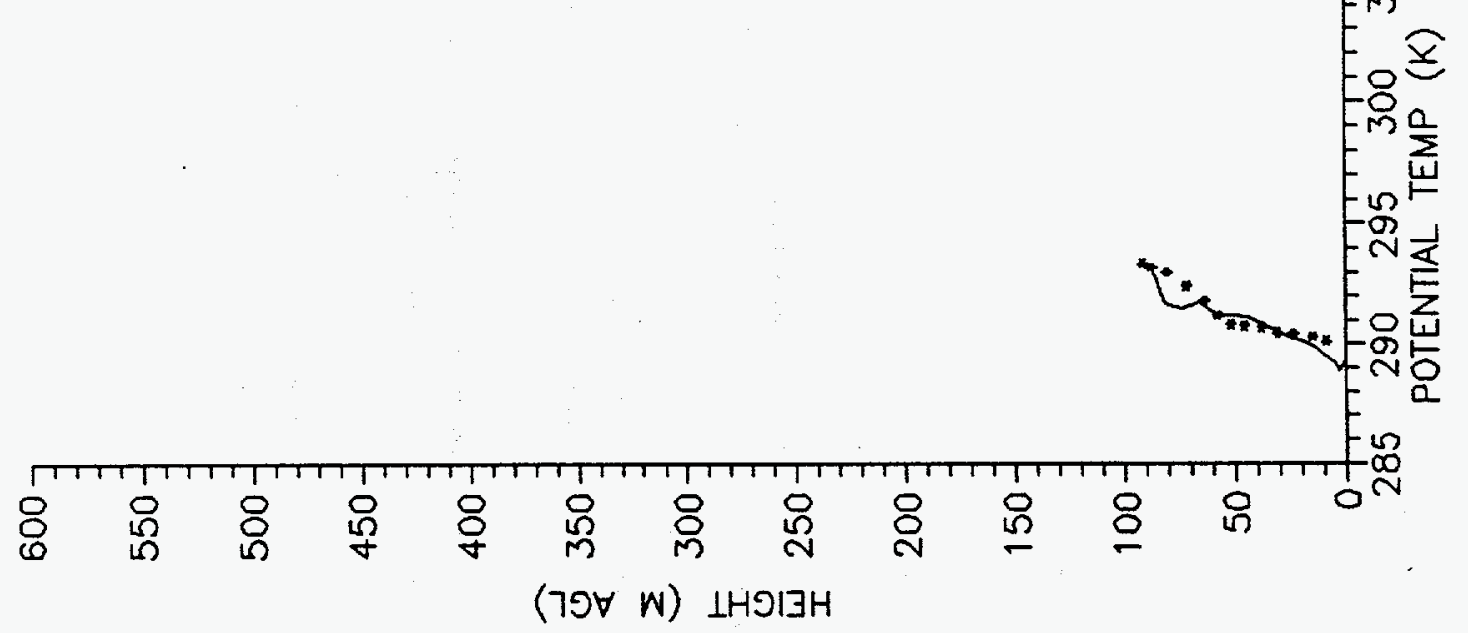


COAL CREEK CANYON TSONDE 02/06/91 BEGIN $=2159$ TOP $=2221 \quad$ END $=2233$ MST

- ASCENT
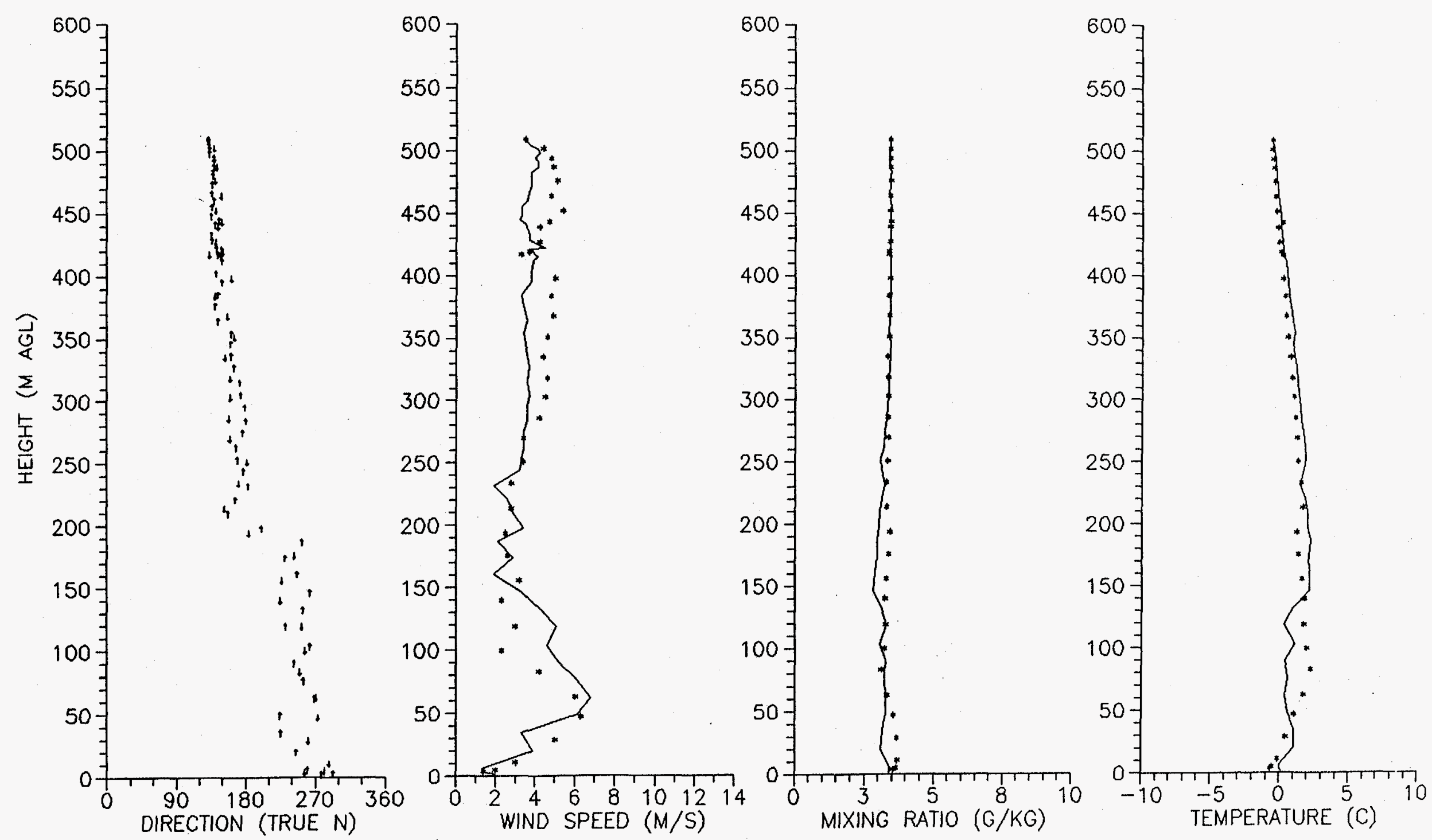
COAL CREEK CANYON TSONDE 02/06/91 BEGIN=2159 TOP=2221 END=2233 MST

$$
\text { -** ASCENT }
$$
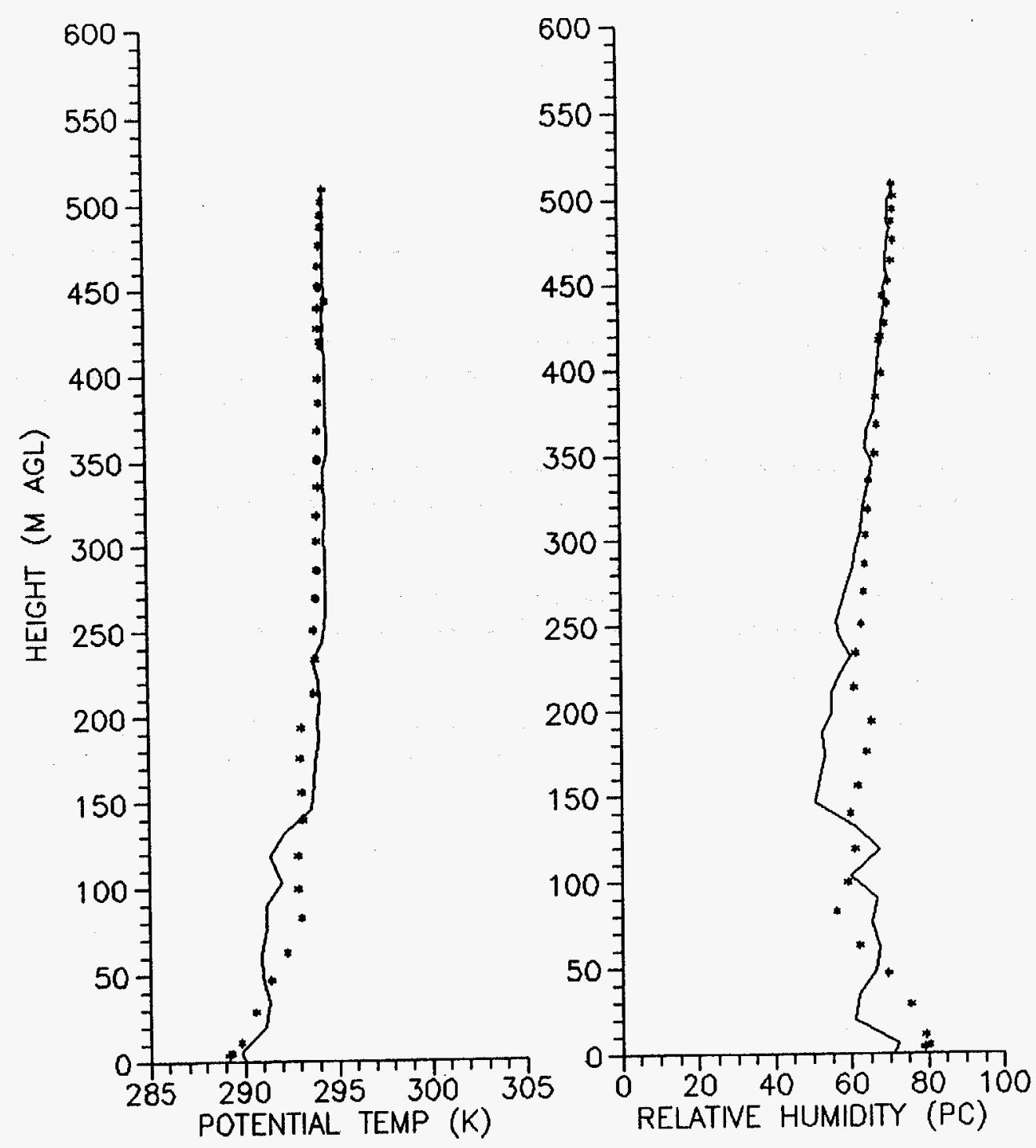
COAL CREEK CANYON TSONDE $02 / 06 / 91 \quad B E G I N=2303 \quad$ TOP $=2324 \quad E N D=2336 \quad M S T$

- ASCENT
$* * *$ DESCENT
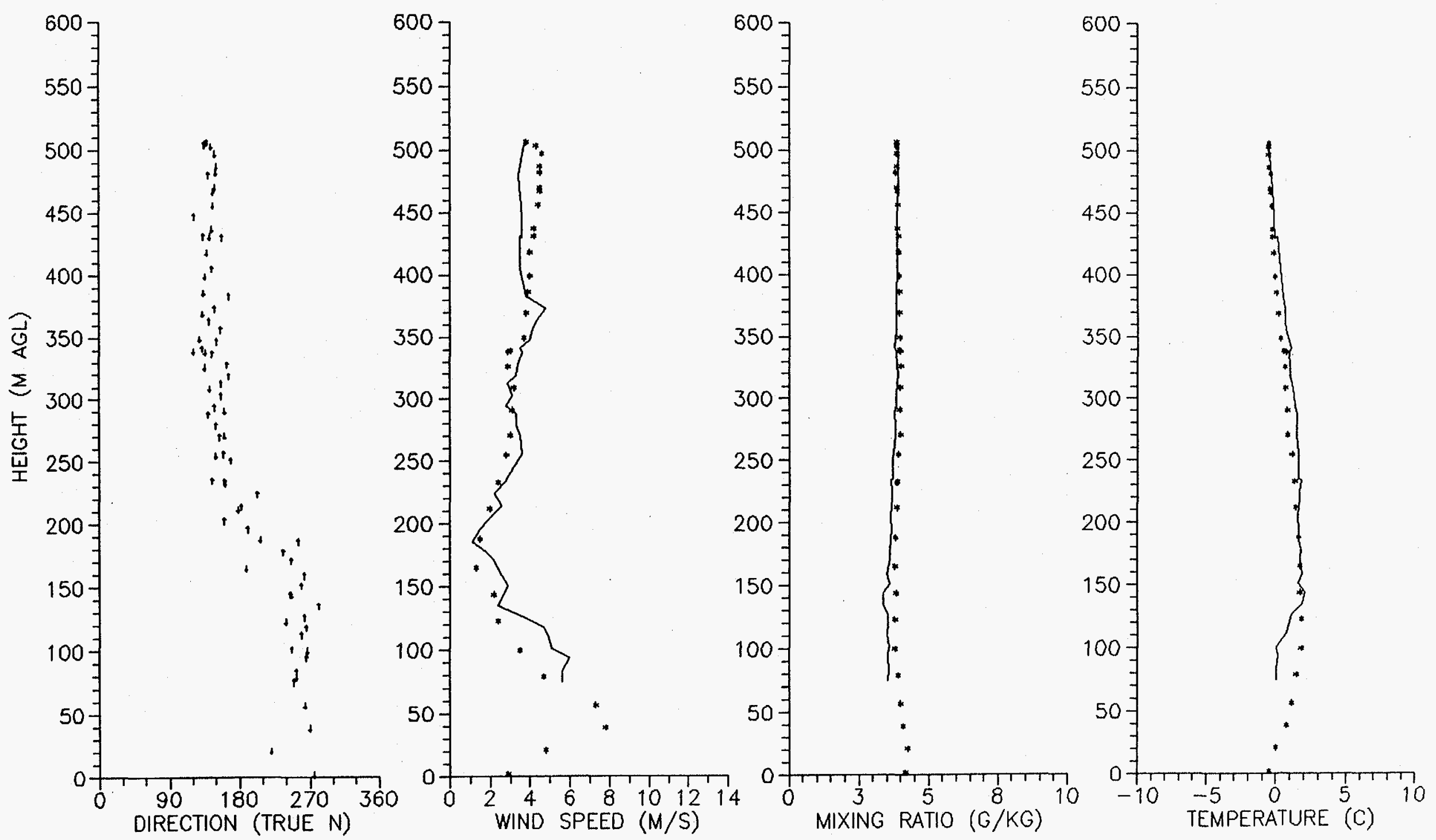


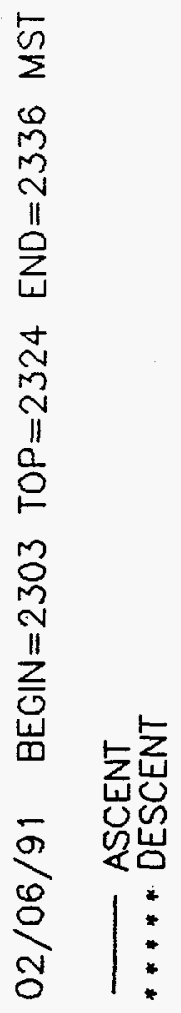

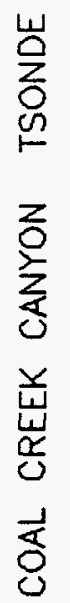

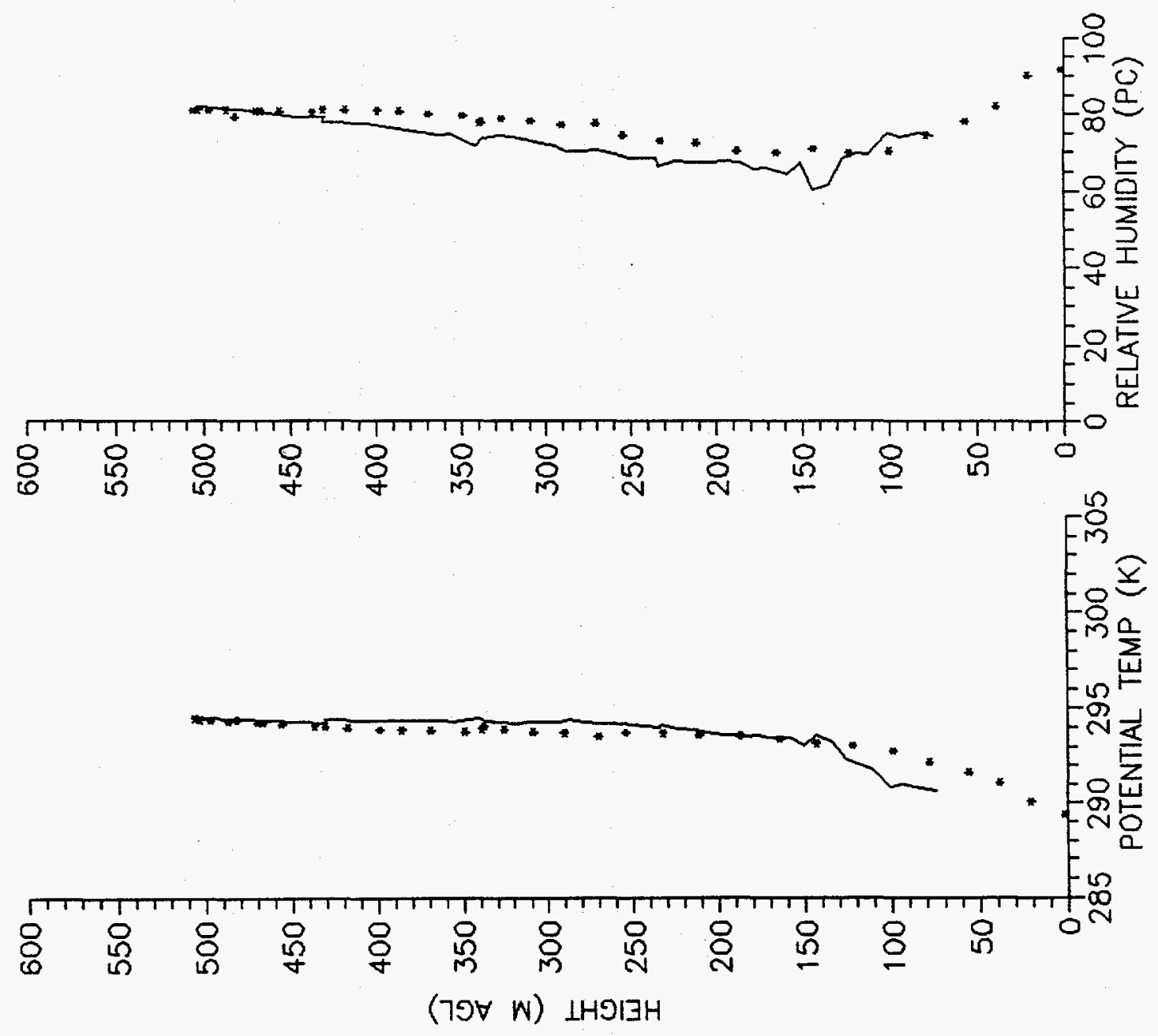




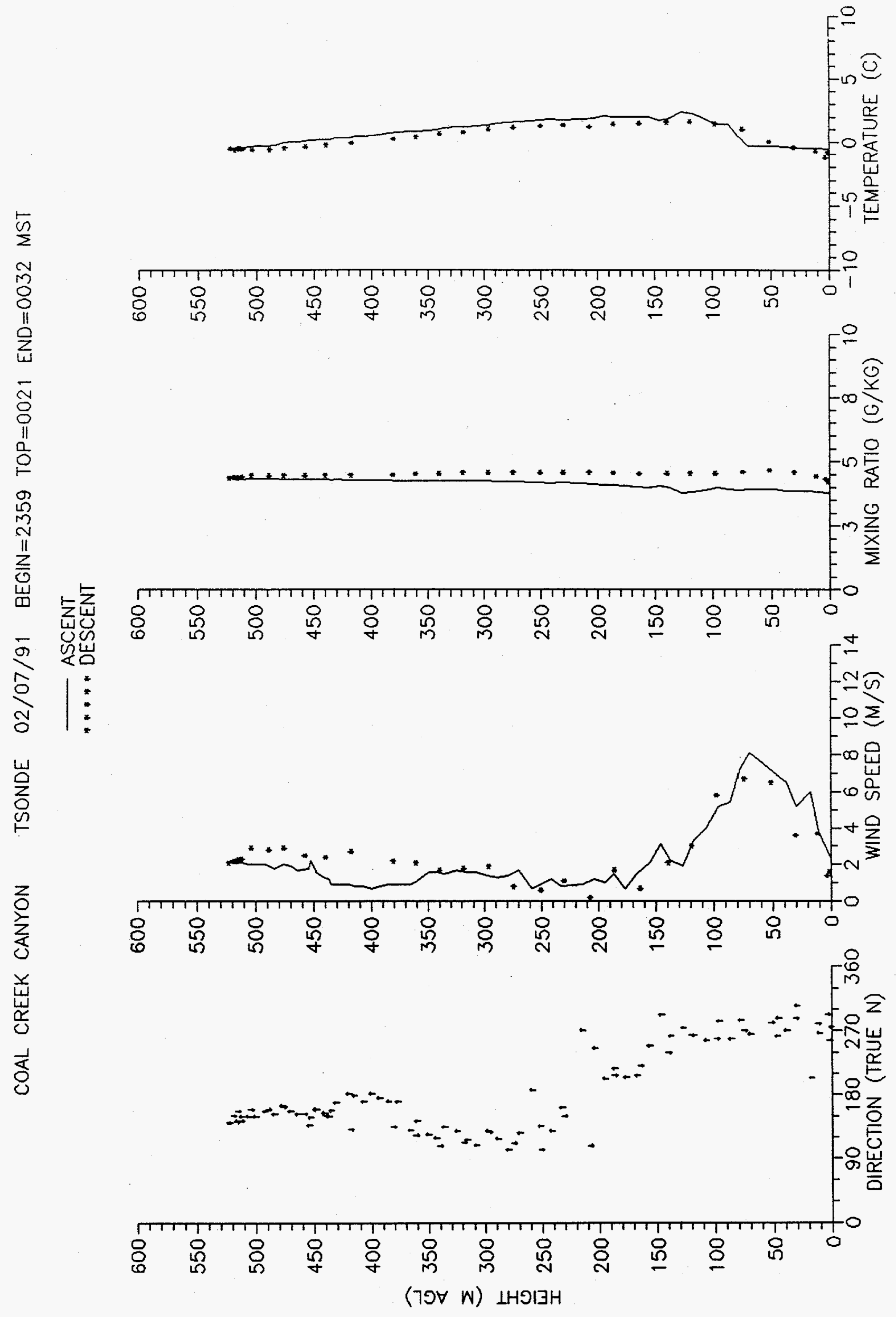


COAL CREEK CANYON TSONDE 02/07/91 BEGIN $=2359$ TOP $=0021 \quad$ END $=0032$ MST *... ASCENT DESENT
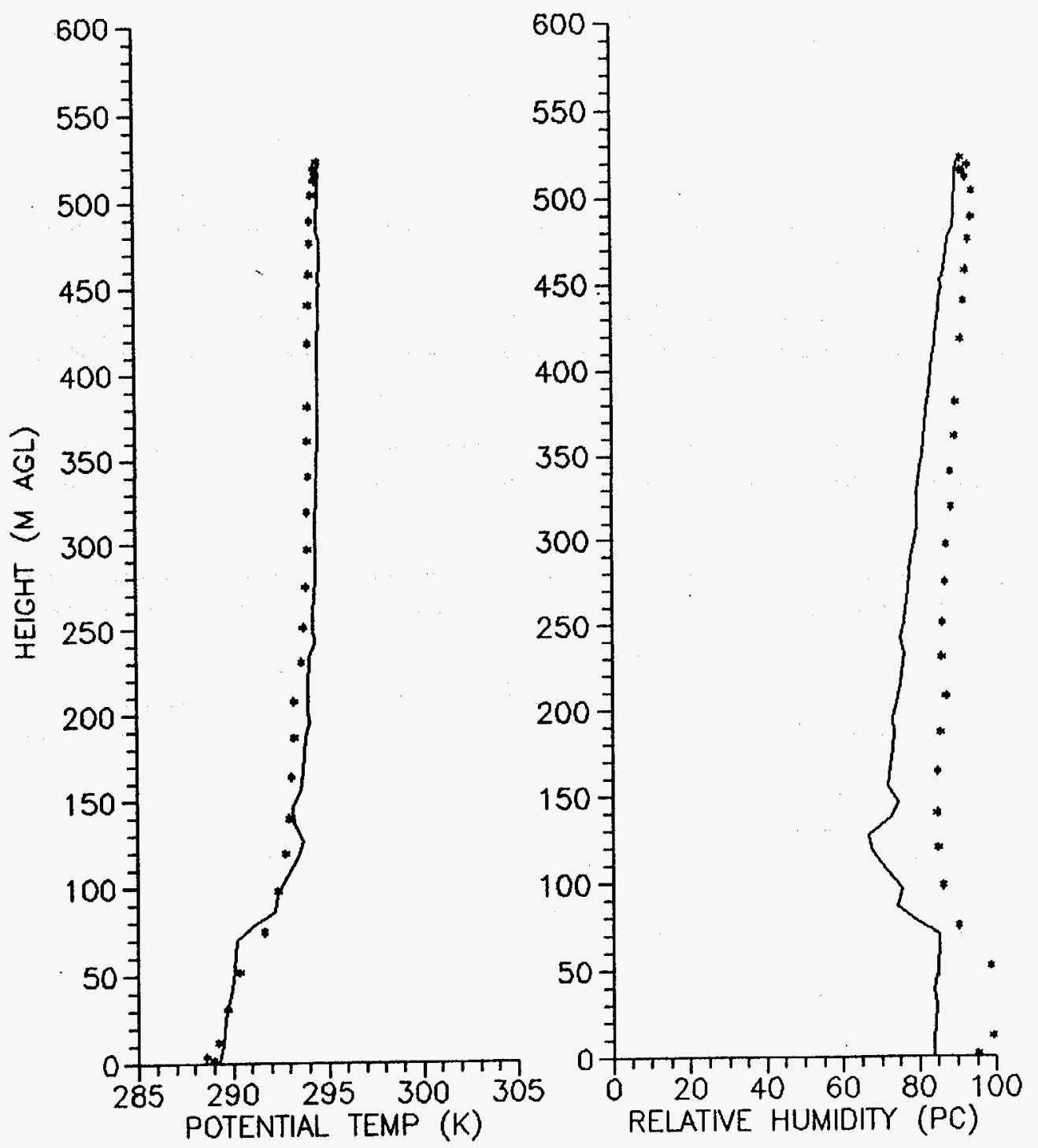
COAL CREEK CANYON TSONDE 02/07/91 BEGIN=0059 TOP=0117 END=0127 MST

\#... ASCENT
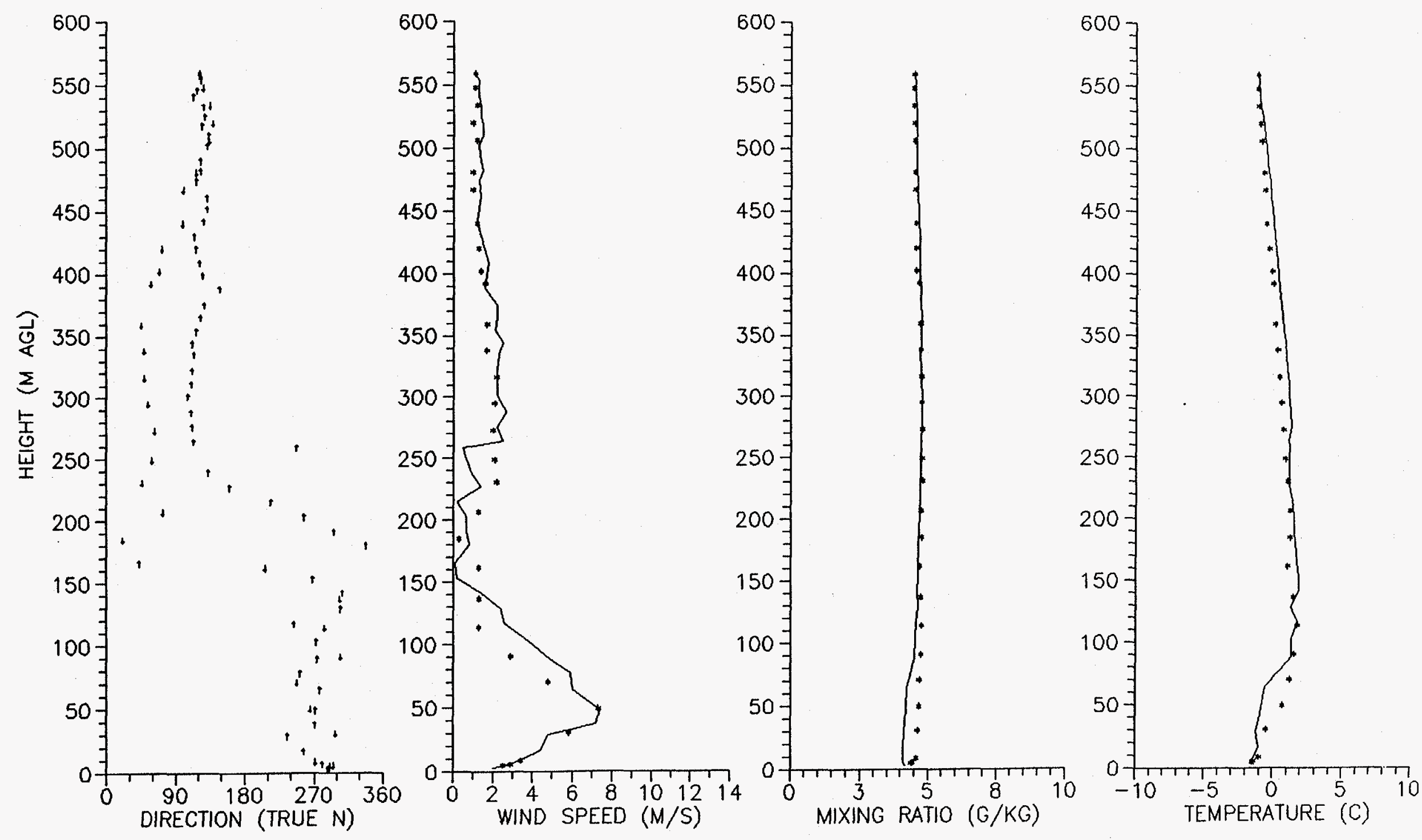


$$
\text { *..* ASCENT }
$$
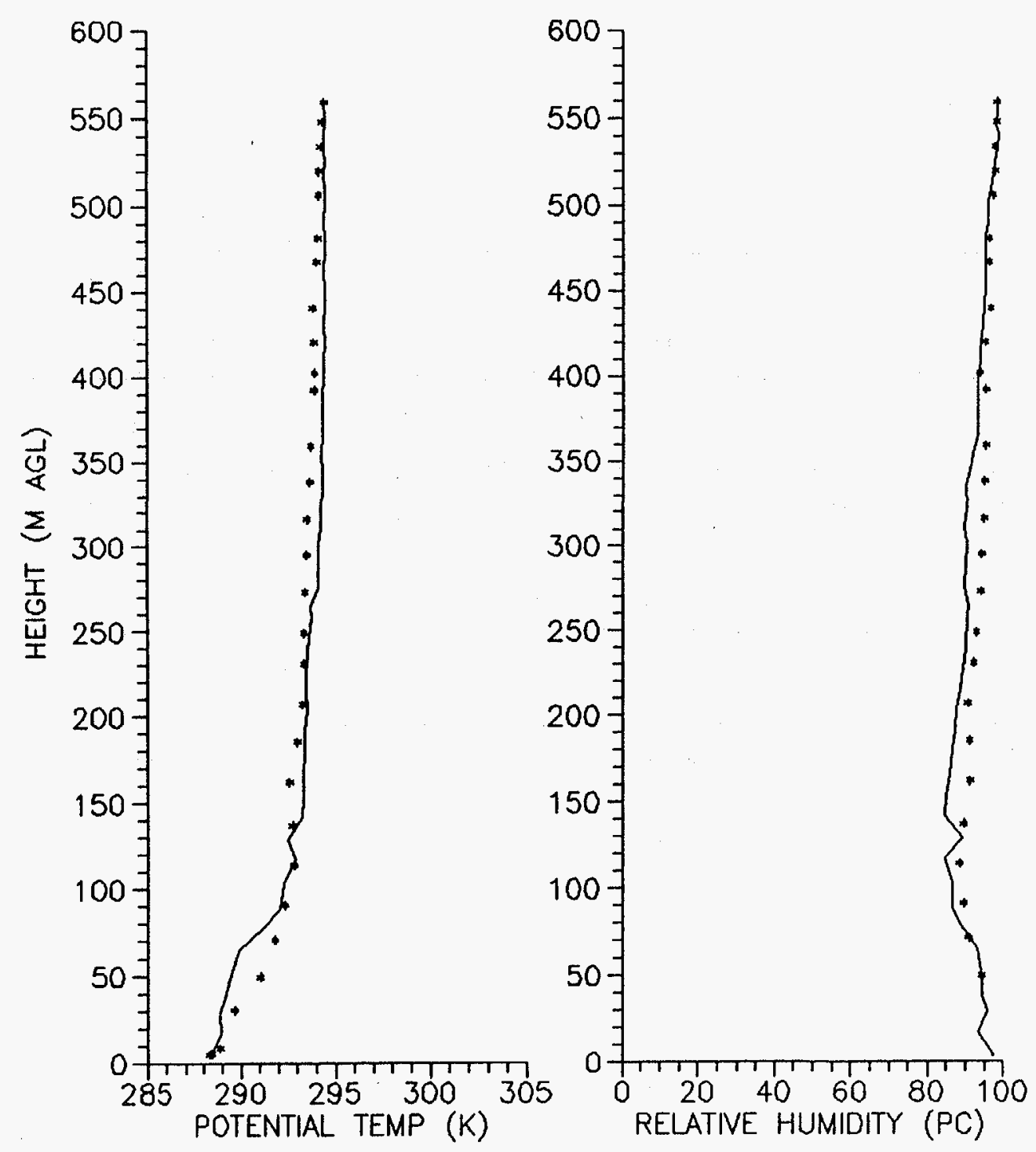
COAL CREEK CANYON TSONDE 02/07/91 BEGIN=0200 TOP $=0219 \quad$ END $=0230$ MST

ASCENT
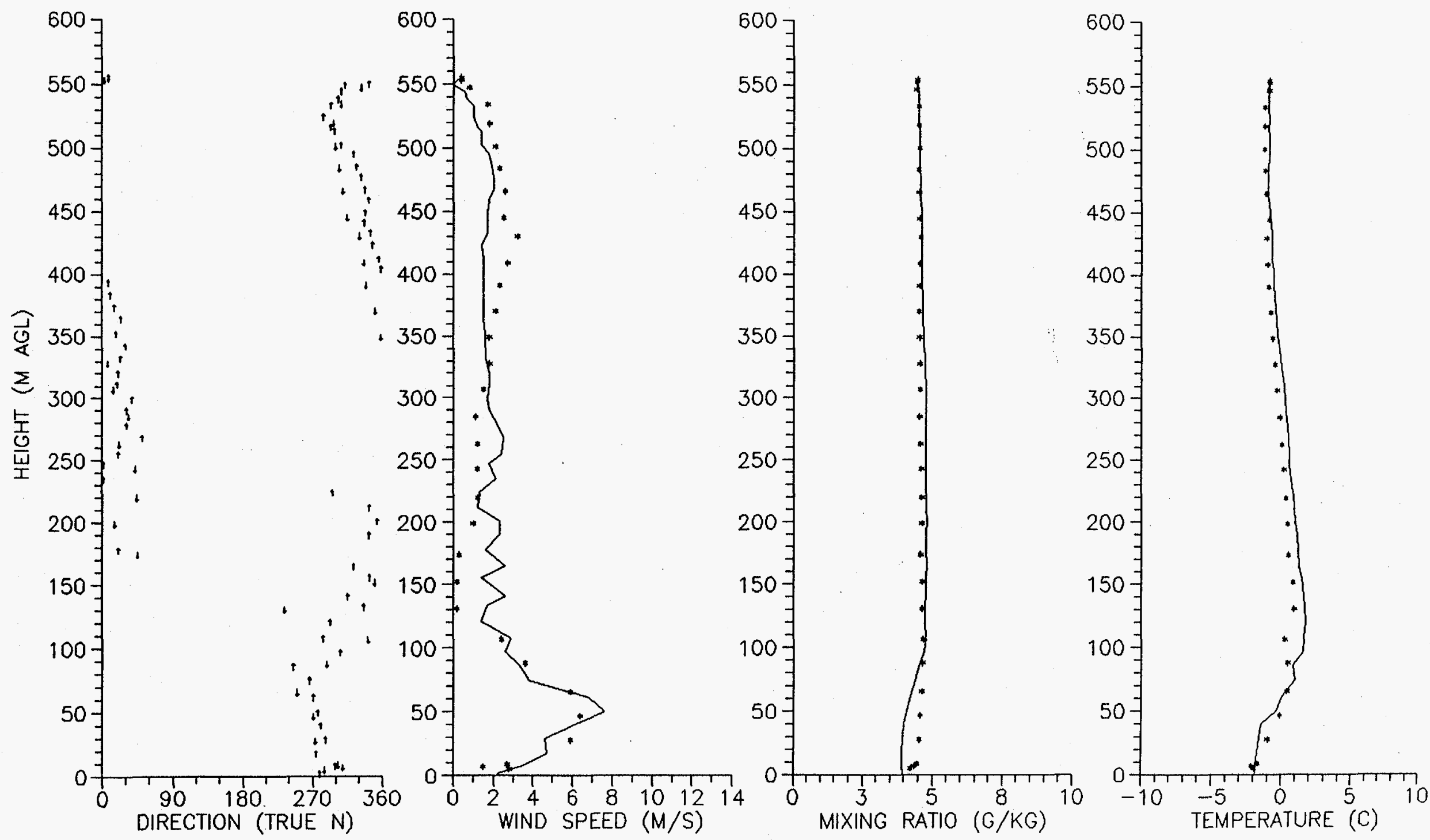
COAL CREEK CANYON

TSONDE

$02 / 07 / 91$

ASCENT

**** DESCENT
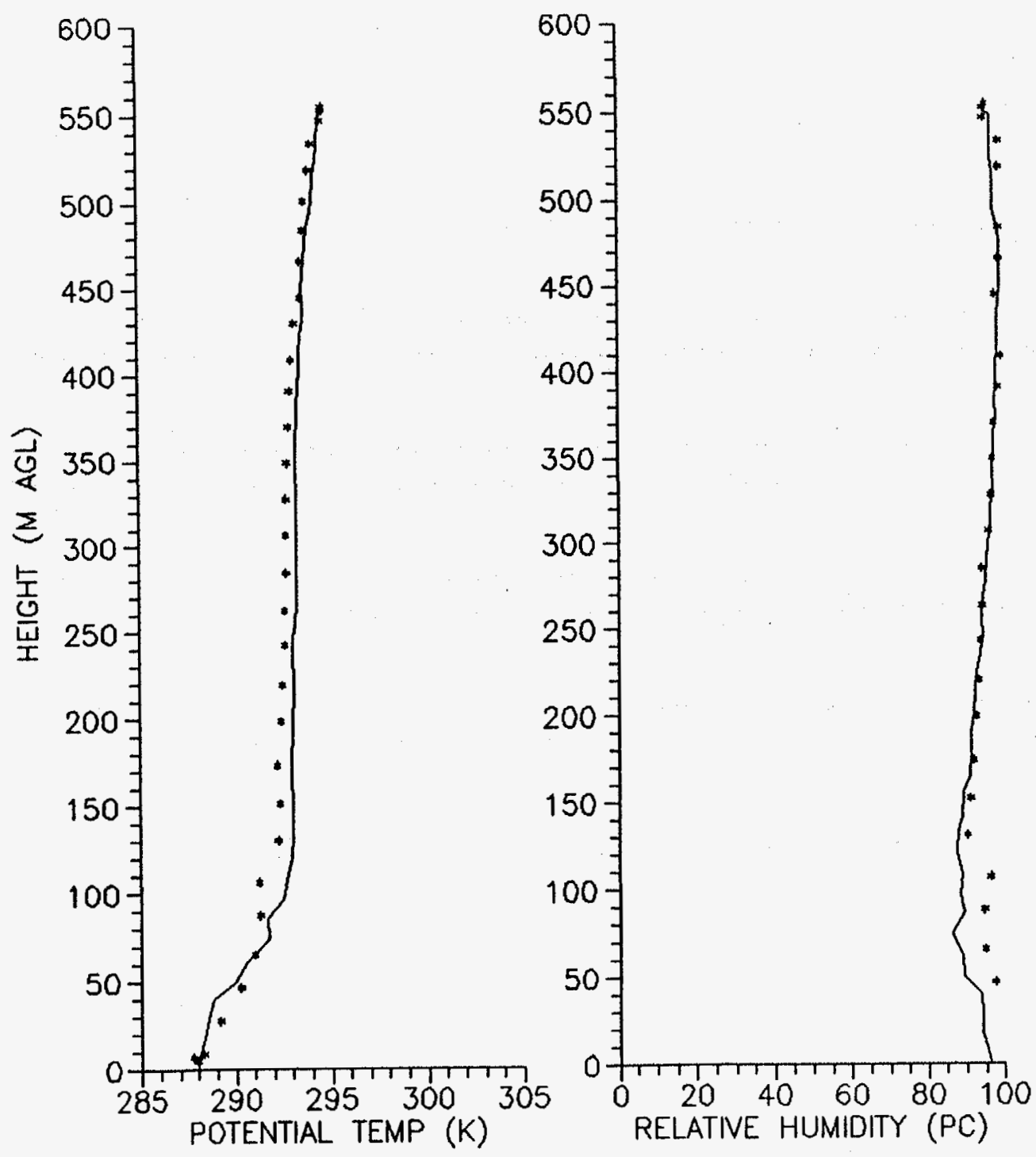
COAL CREEK CANYON TSONDE 02/07/91 BEGIN=0300 TOP=0322 END=0331 MST

ASCENT
$* * * *$ DESCENT

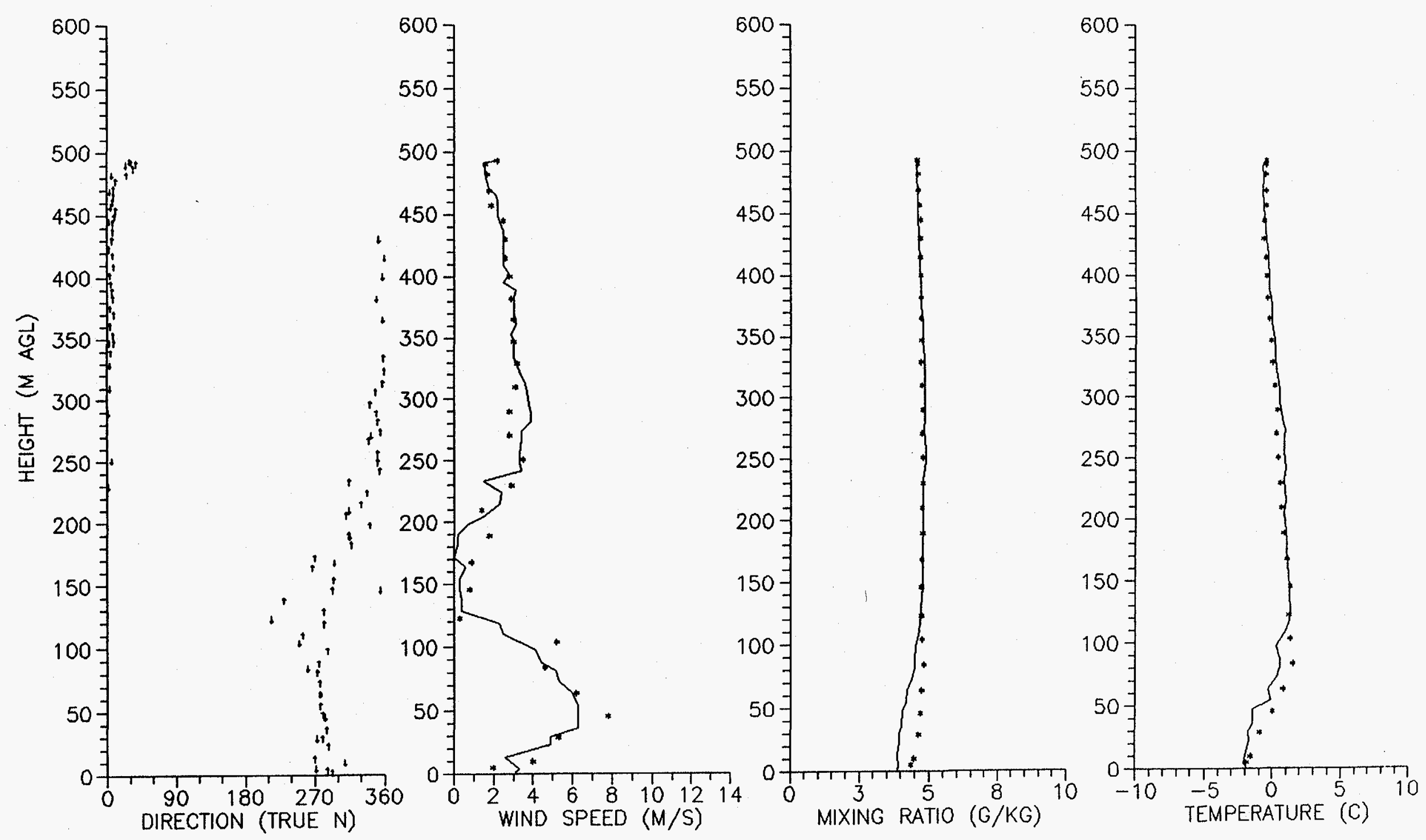


COAL CREEK CANYON TSONDE 02/07/91 BEGIN=0300 TOP $=0322 \quad$ END $=0331$ MST

$$
\text { **** ASCENT }
$$
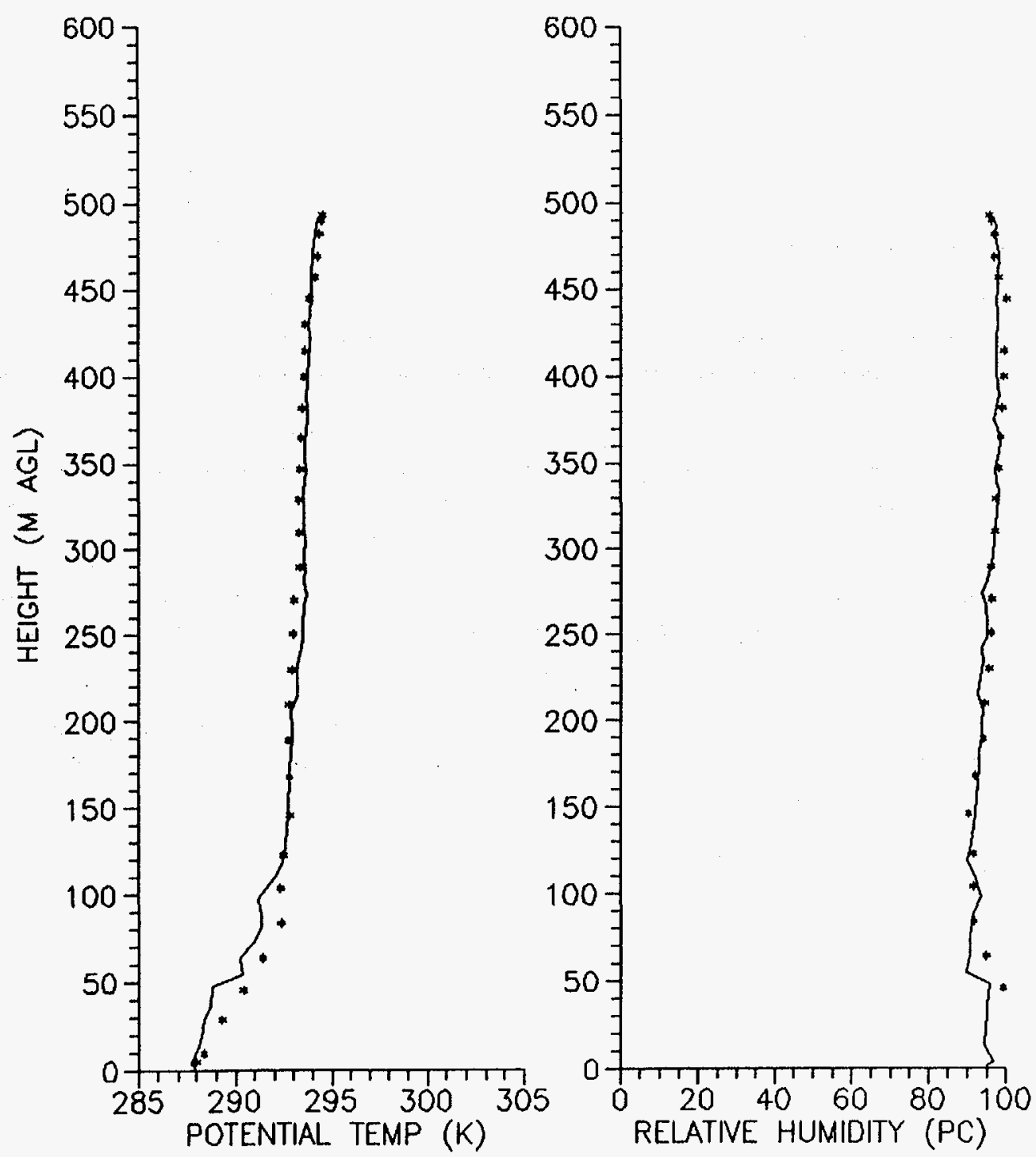
COAL CREEK CANYON TSONDE 02/07/91 BEGIN=0400 TOP $=0424 \quad$ END $=0436$ MST

ASCENT
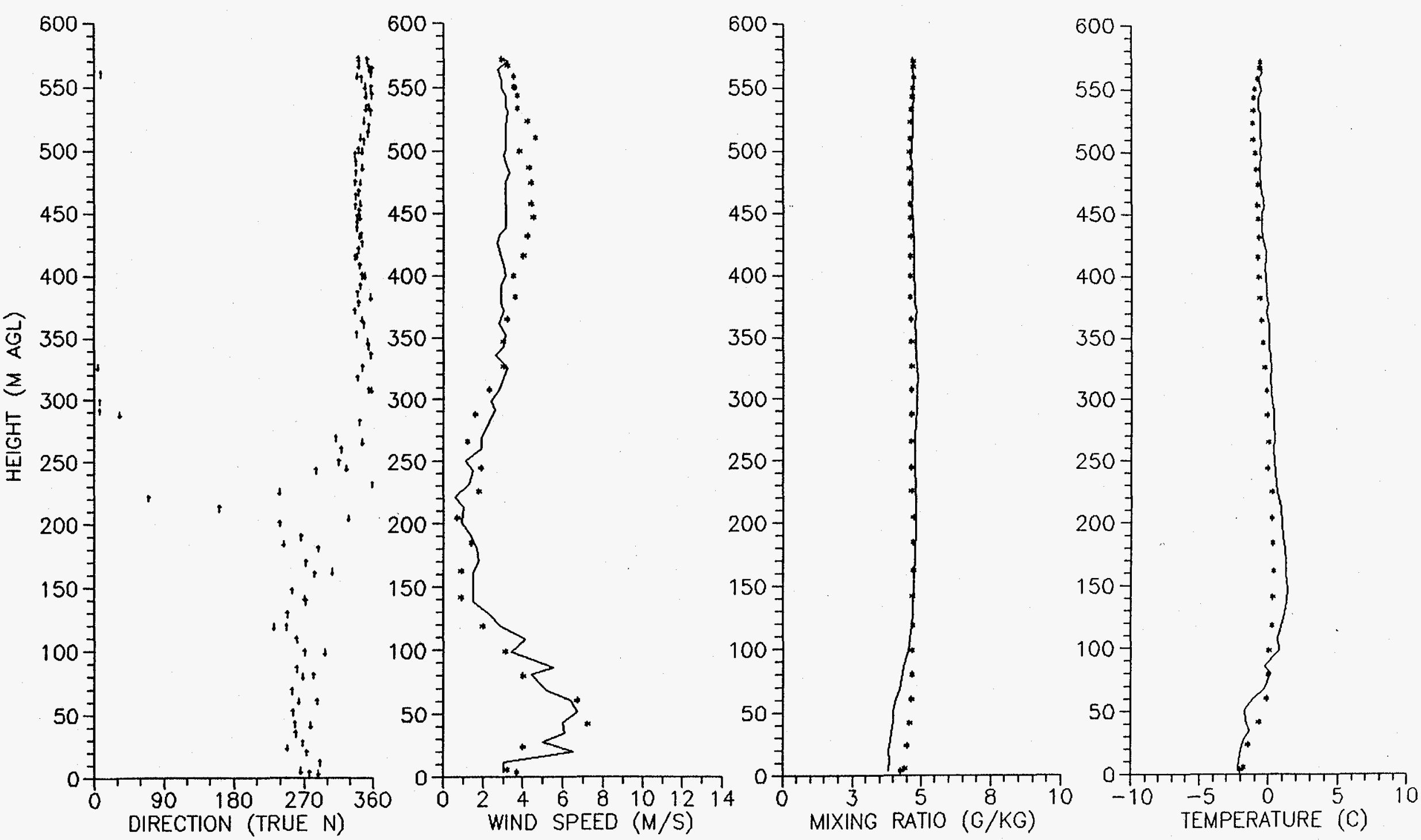
COAL CREEK CANYON TSONDE 02/07/91 BEGIN=0400 TOP $=0424$ END $=0436$ MST - ASCENT
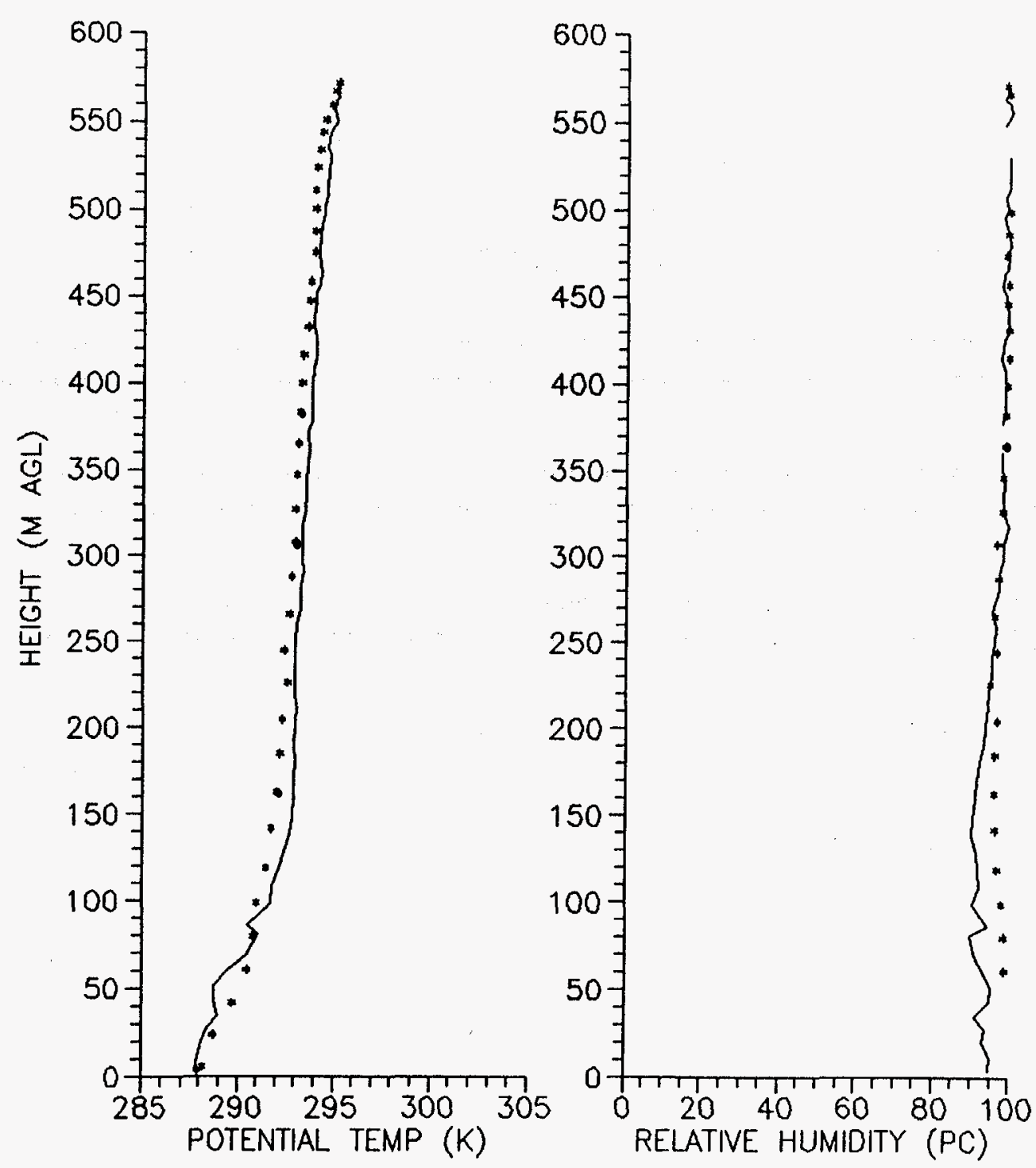
COAL CREEK CANYON TSONDE $02 / 07 / 91 \quad B E G I N=0459 \quad$ TOP $=0517 \quad$ END $=0527 \quad M S T$

*** ASCENT
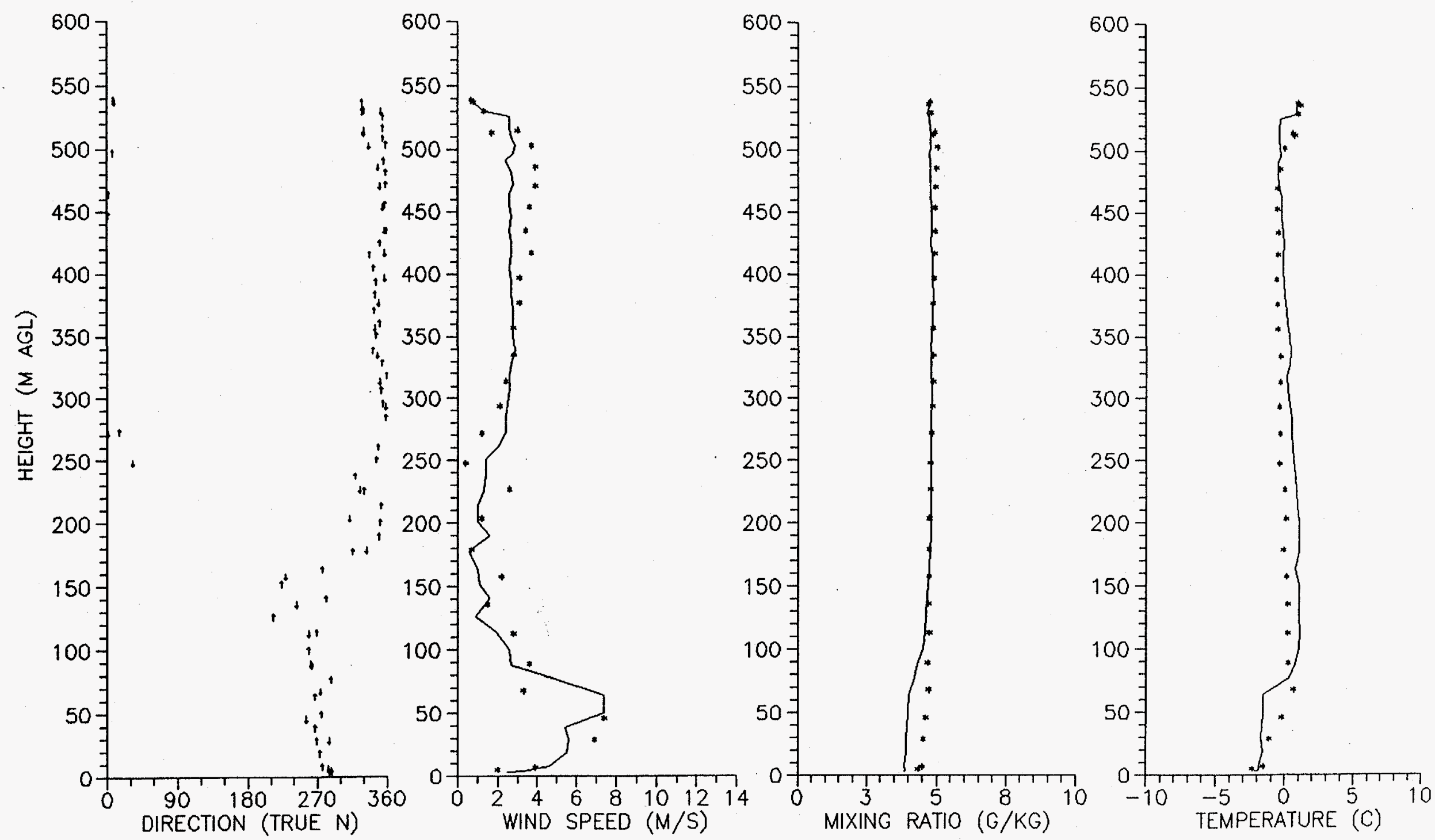
COAL CREEK CANYON TSONDE 02/07/91 BEGIN $=0459$ TOP $=0517 \quad$ END $=0527$ MST
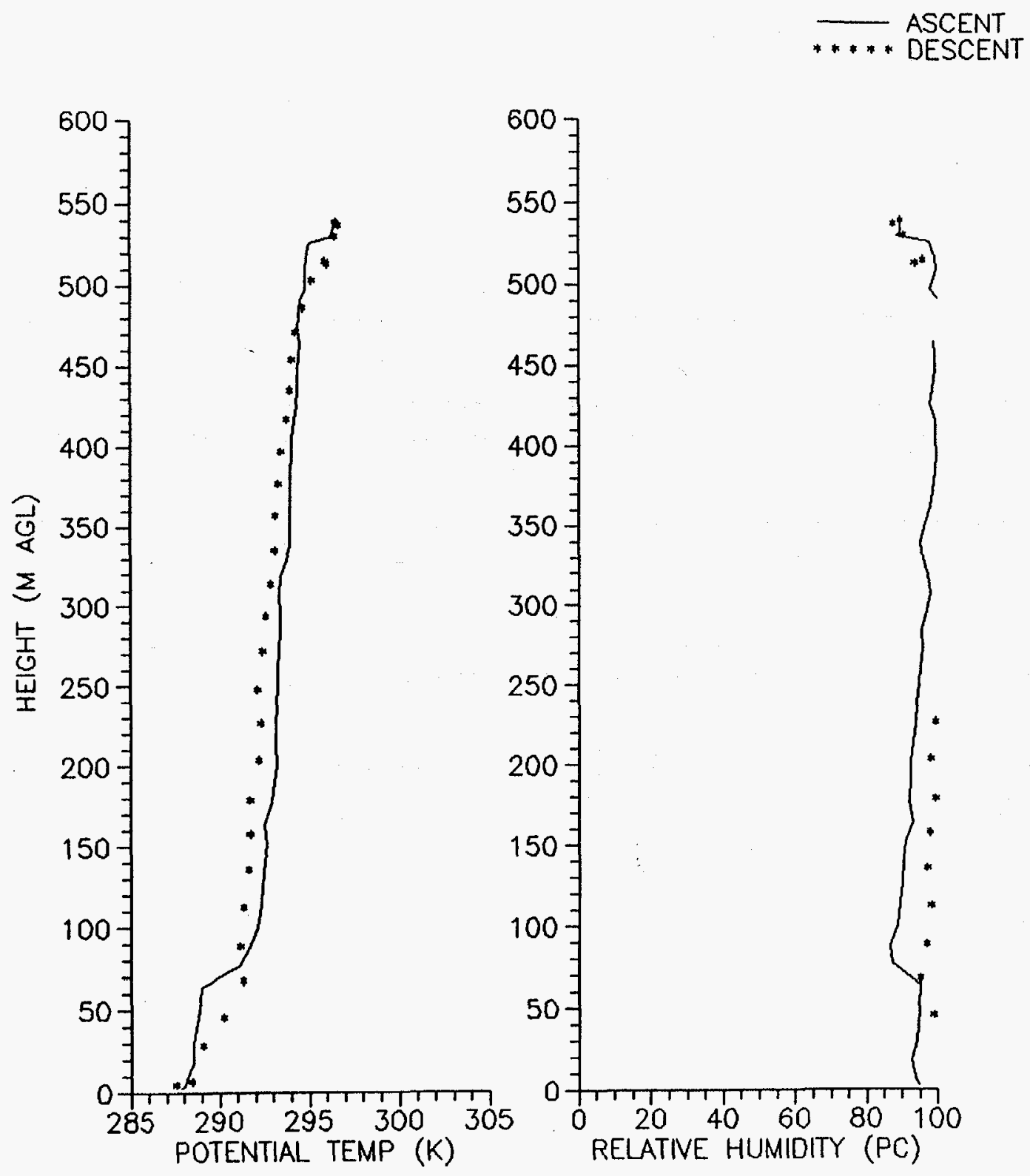
ASCENT
$* * *$ DESCENT
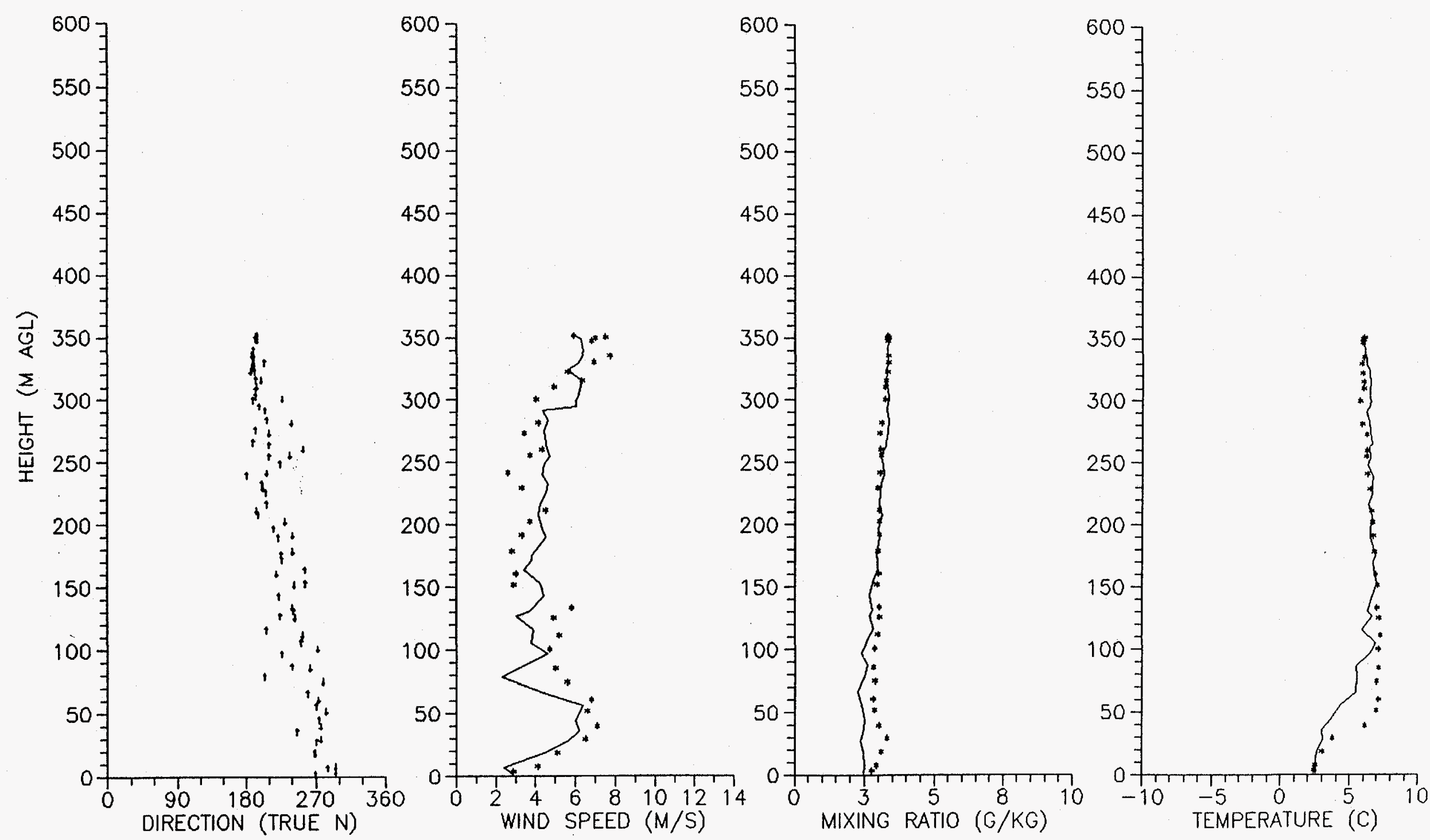

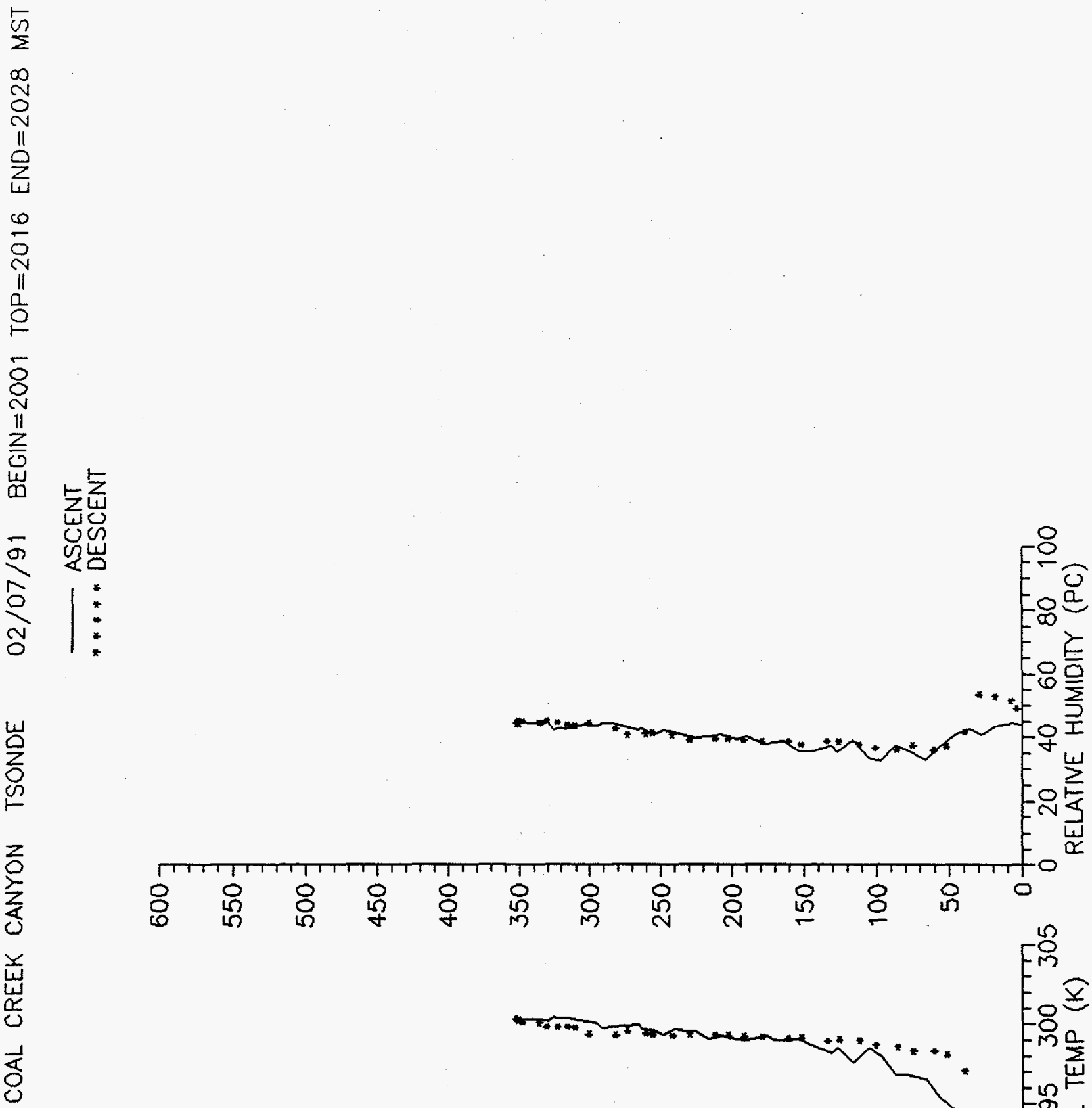
m $N$ N

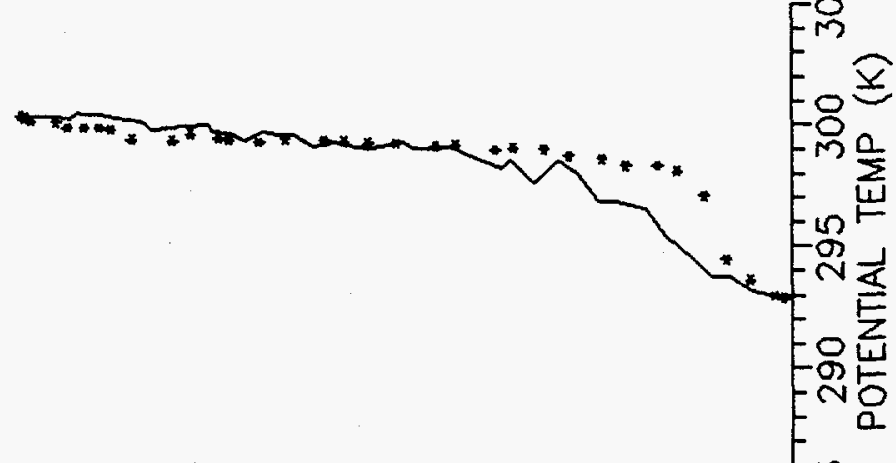

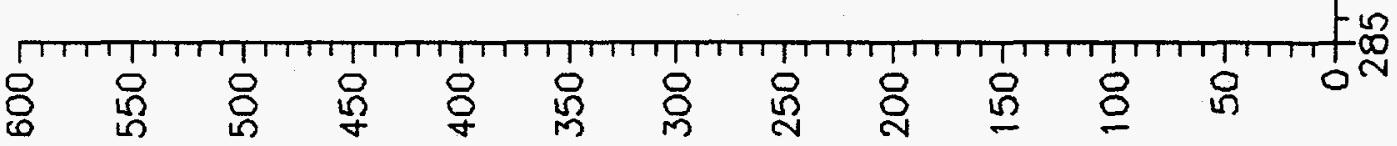

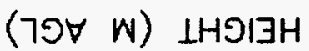




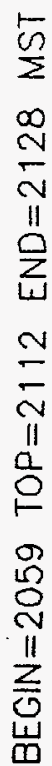
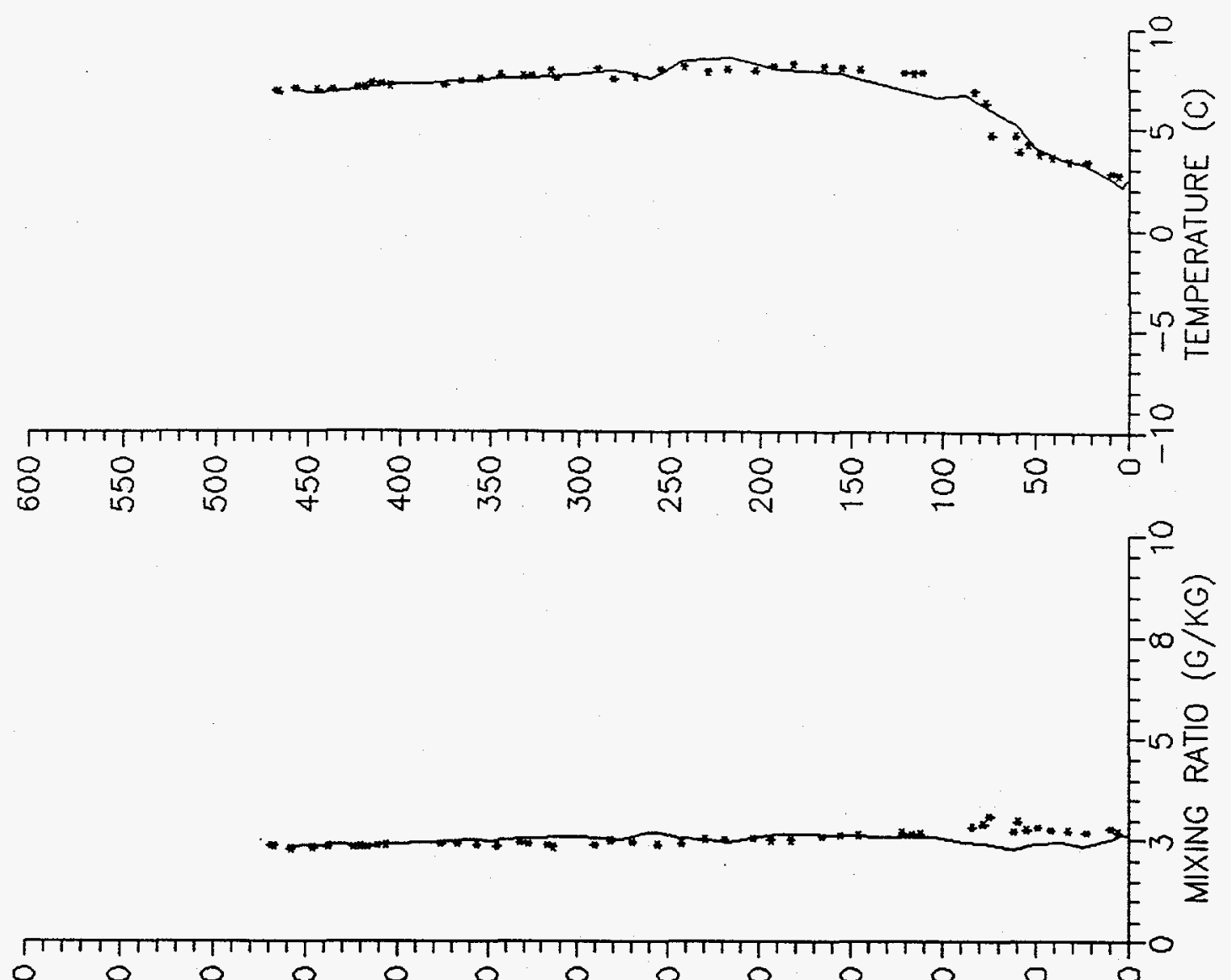

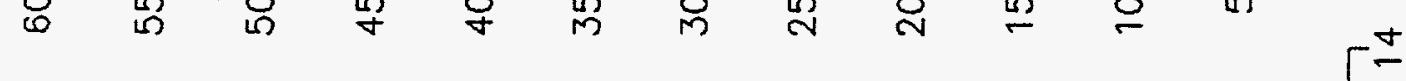

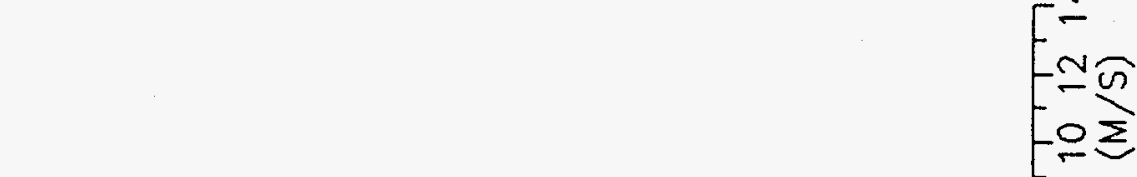

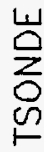

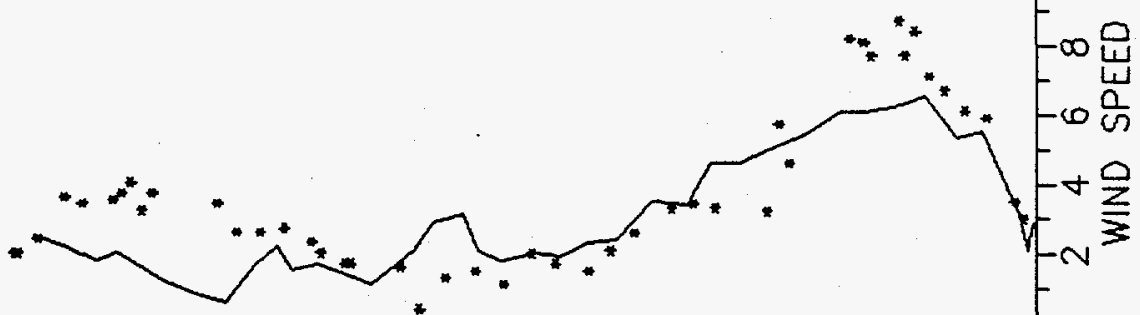

$\sum_{\substack{0 \\ 0}}^{2}$

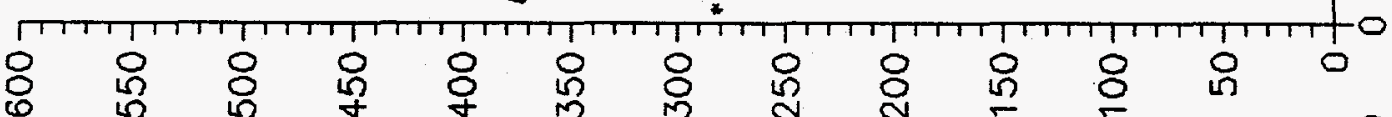

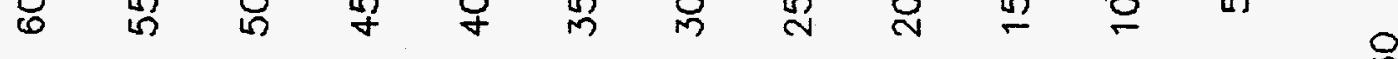

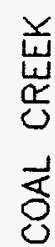

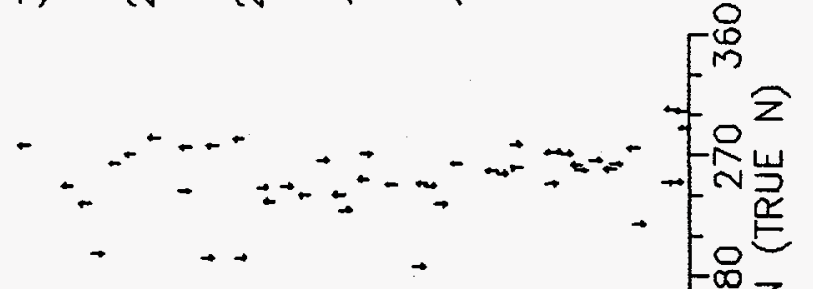

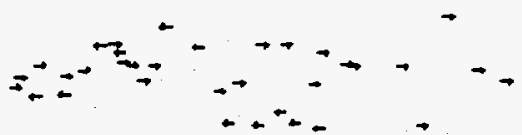

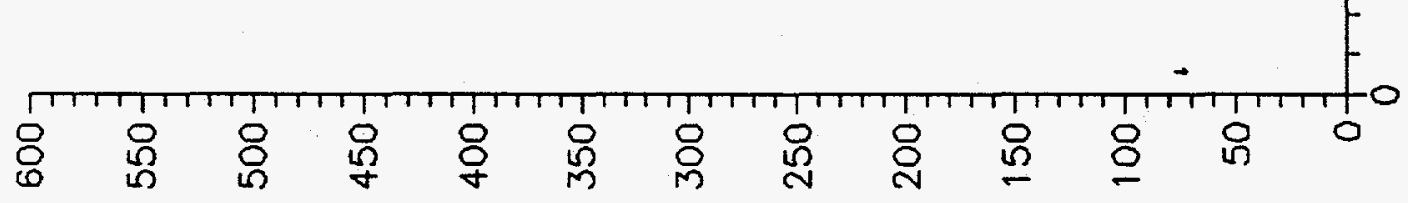

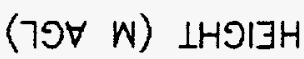




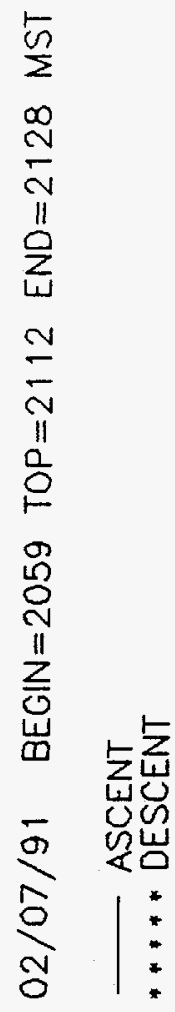

岁

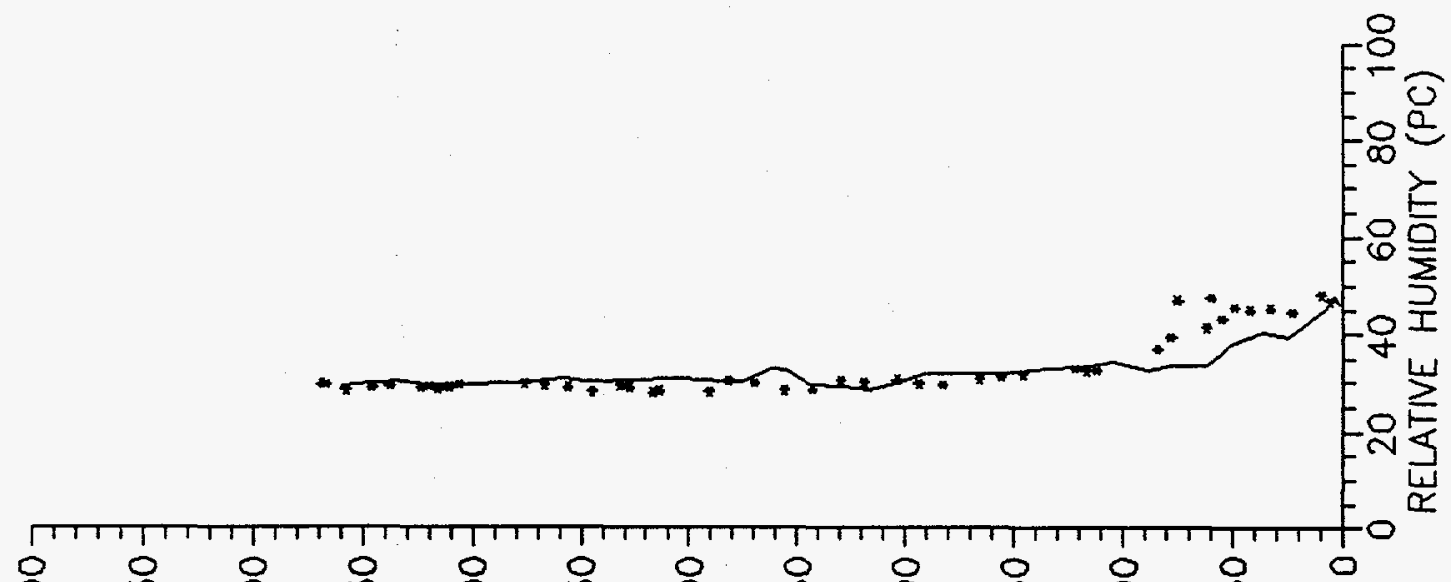

$\frac{z}{3}$

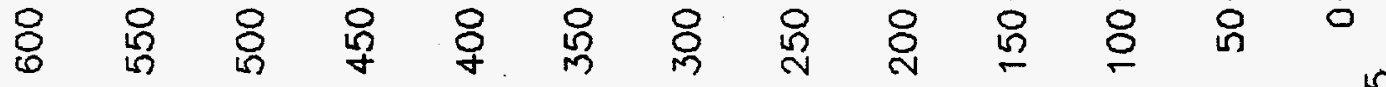

嵌

8

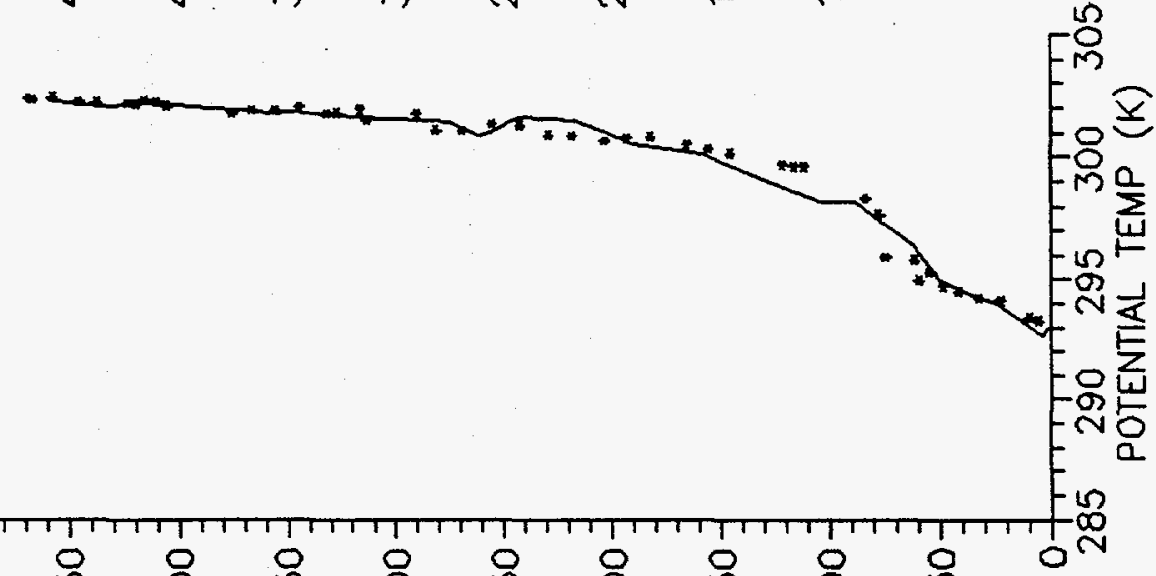

ठํ.

(7פV W) $\mathrm{LHOIJH}$ 
COAL CREEK CANYON TSONDE $02 / 07 / 91 \quad$ BEGIN $=2159 \quad$ TOP $=2213 \quad$ END $=2227 \quad M S T$

\section{ASCENT}
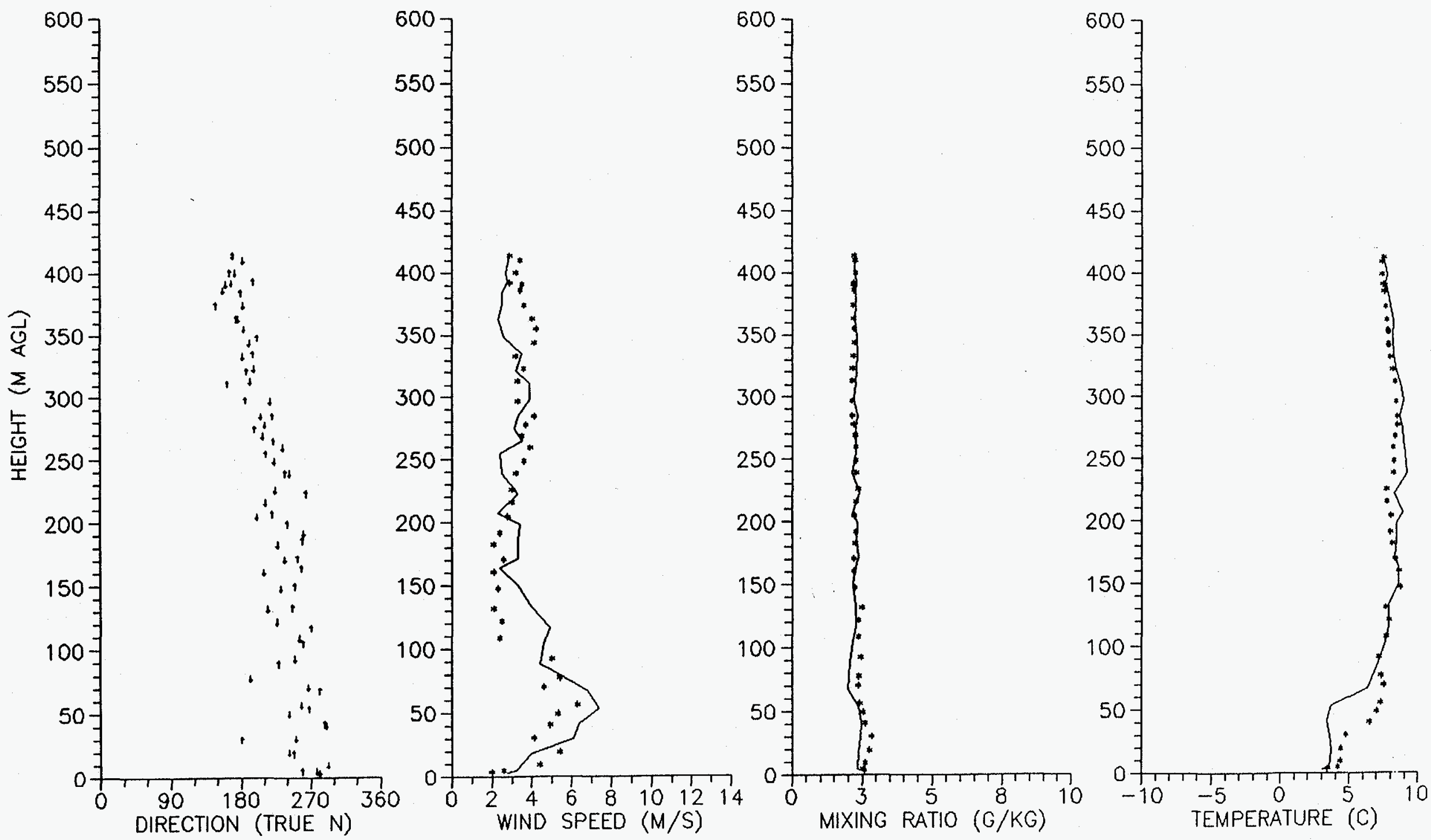


\section{ASCENT}
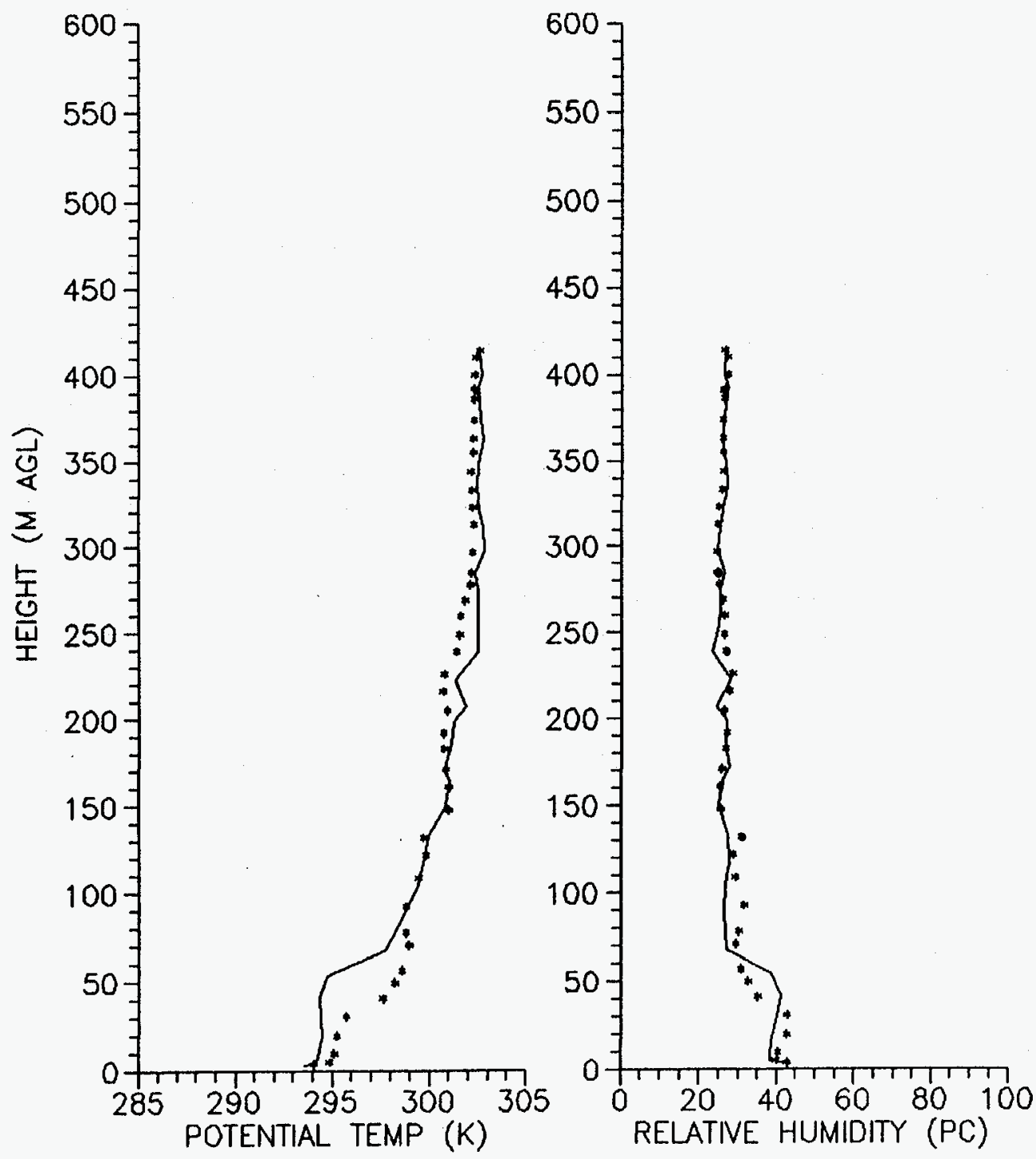
COAL CREEK CANYON TSONDE 02/07/91 BEGIN=2259 TOP=2318 END=2334 MST

$\begin{array}{ll} & \text { ASCENT } \\ * * * * & \text { DESCENT }\end{array}$
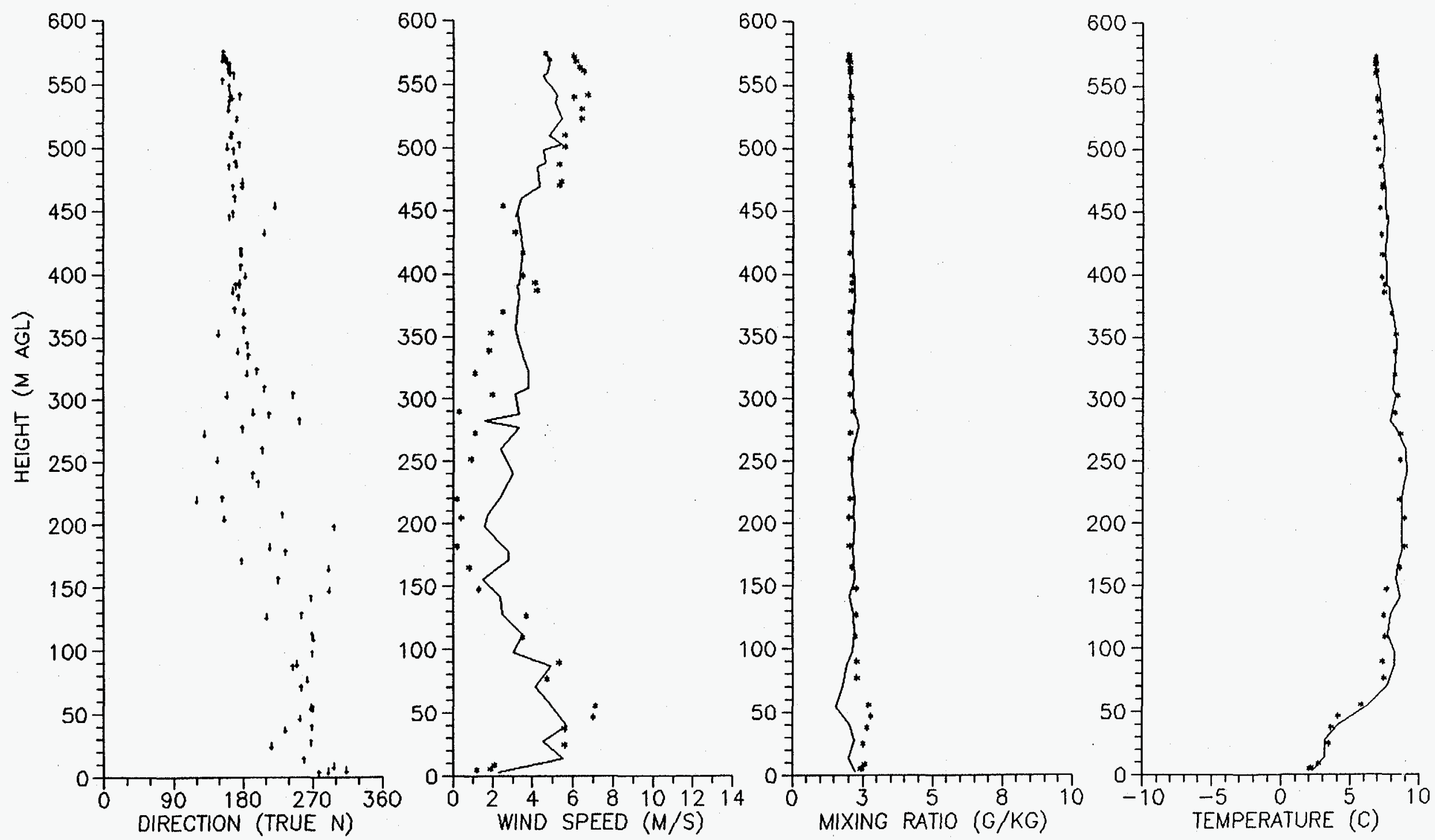


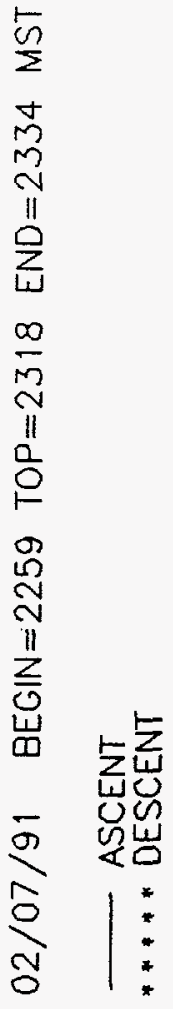

$\stackrel{\text { U }}{\text { 岁 }}$

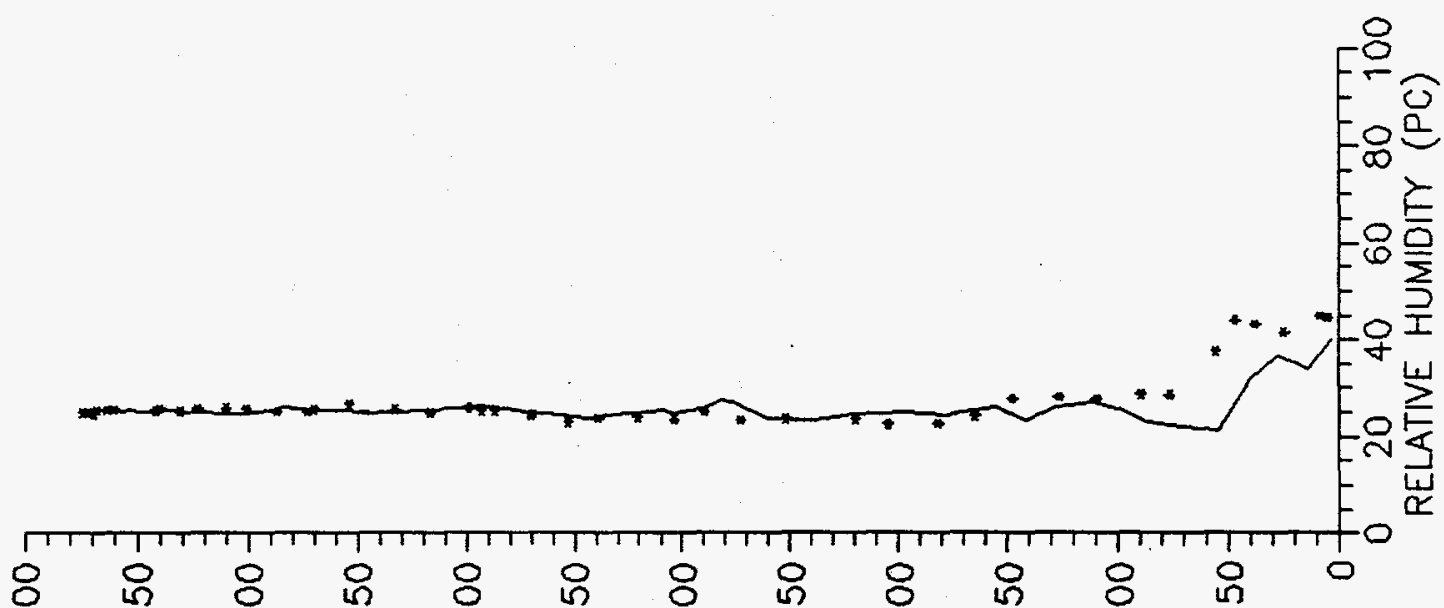

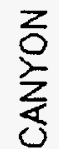

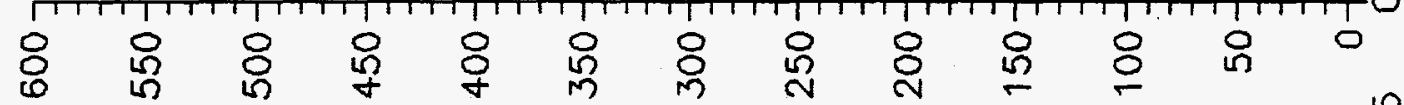

$x$

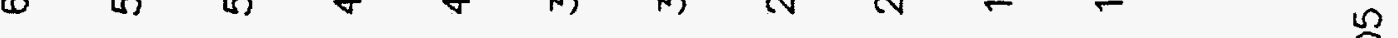

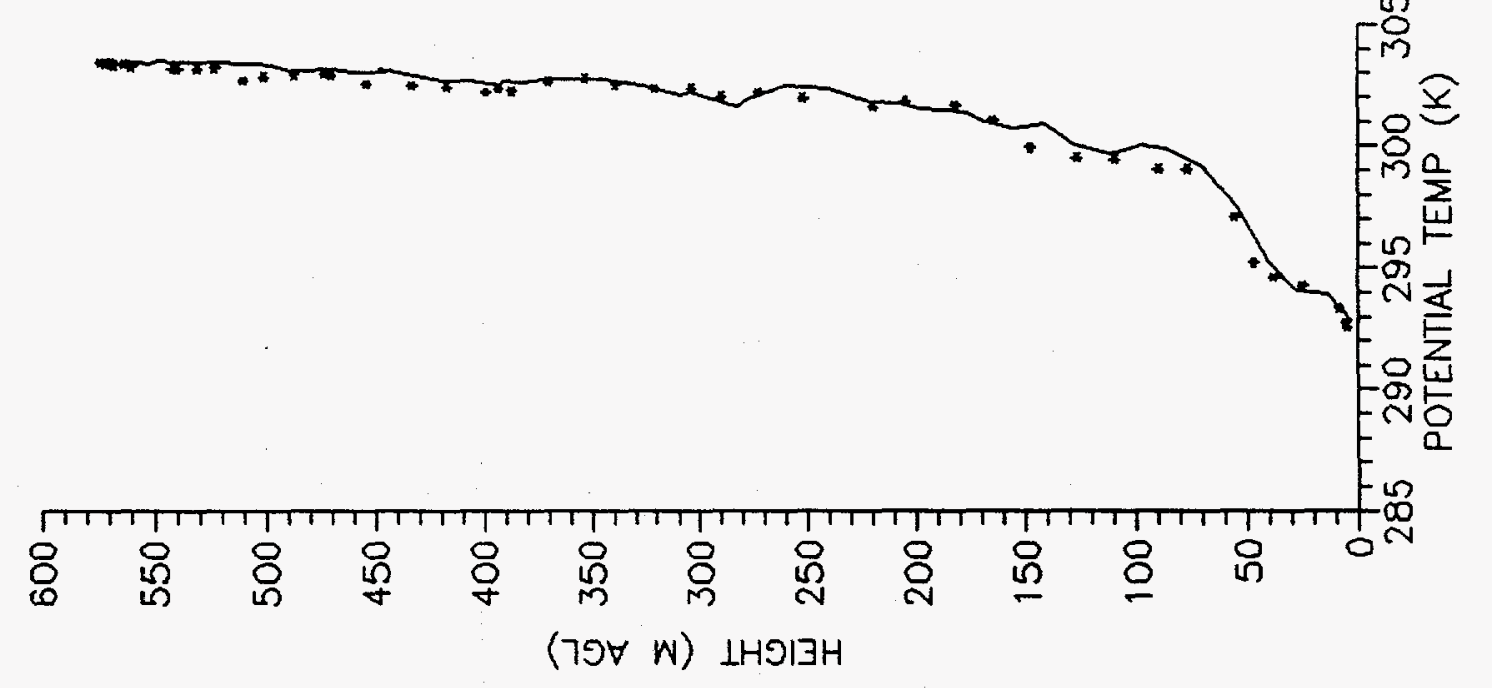

Fim

岩

$\frac{1}{5}$ 


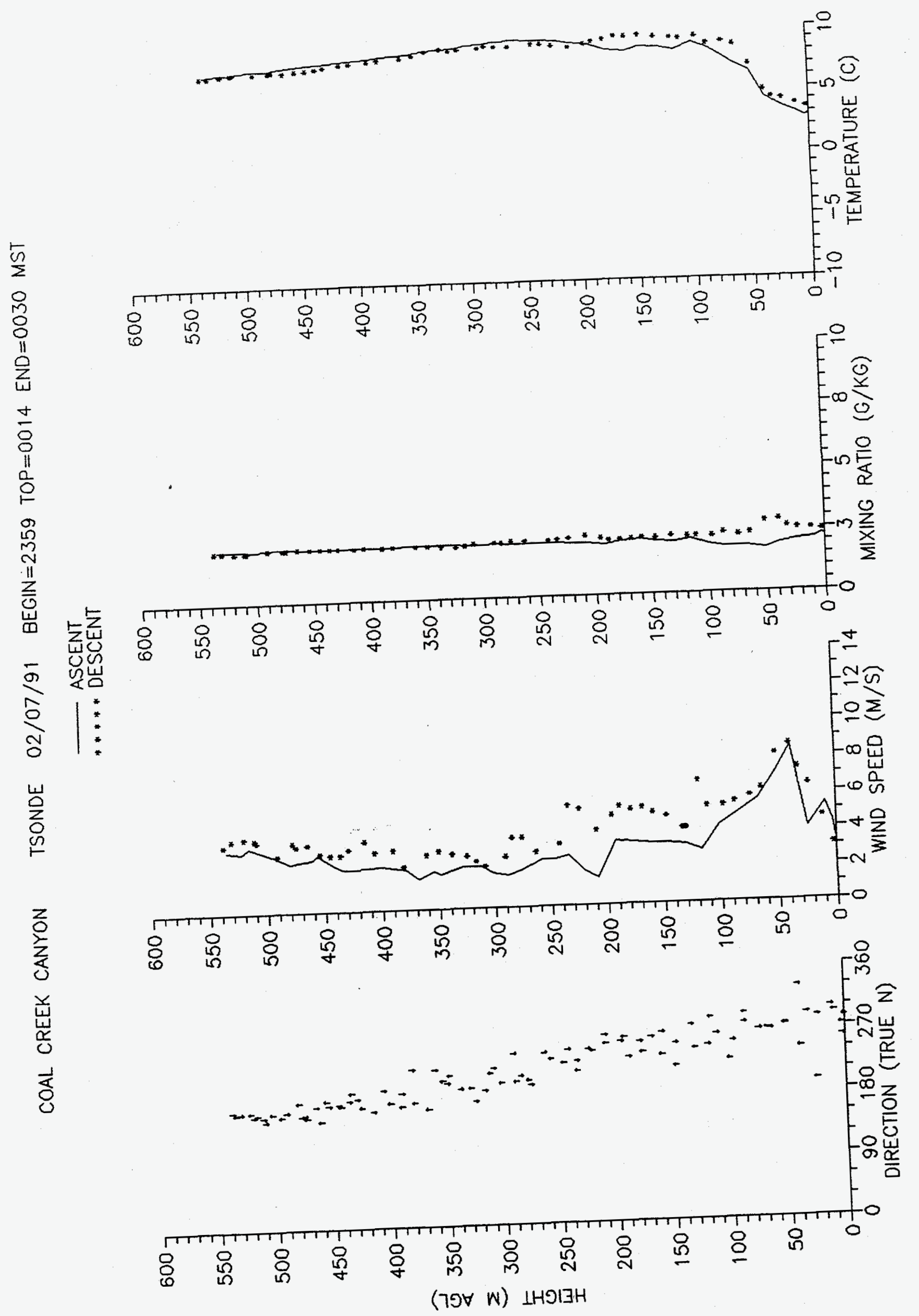



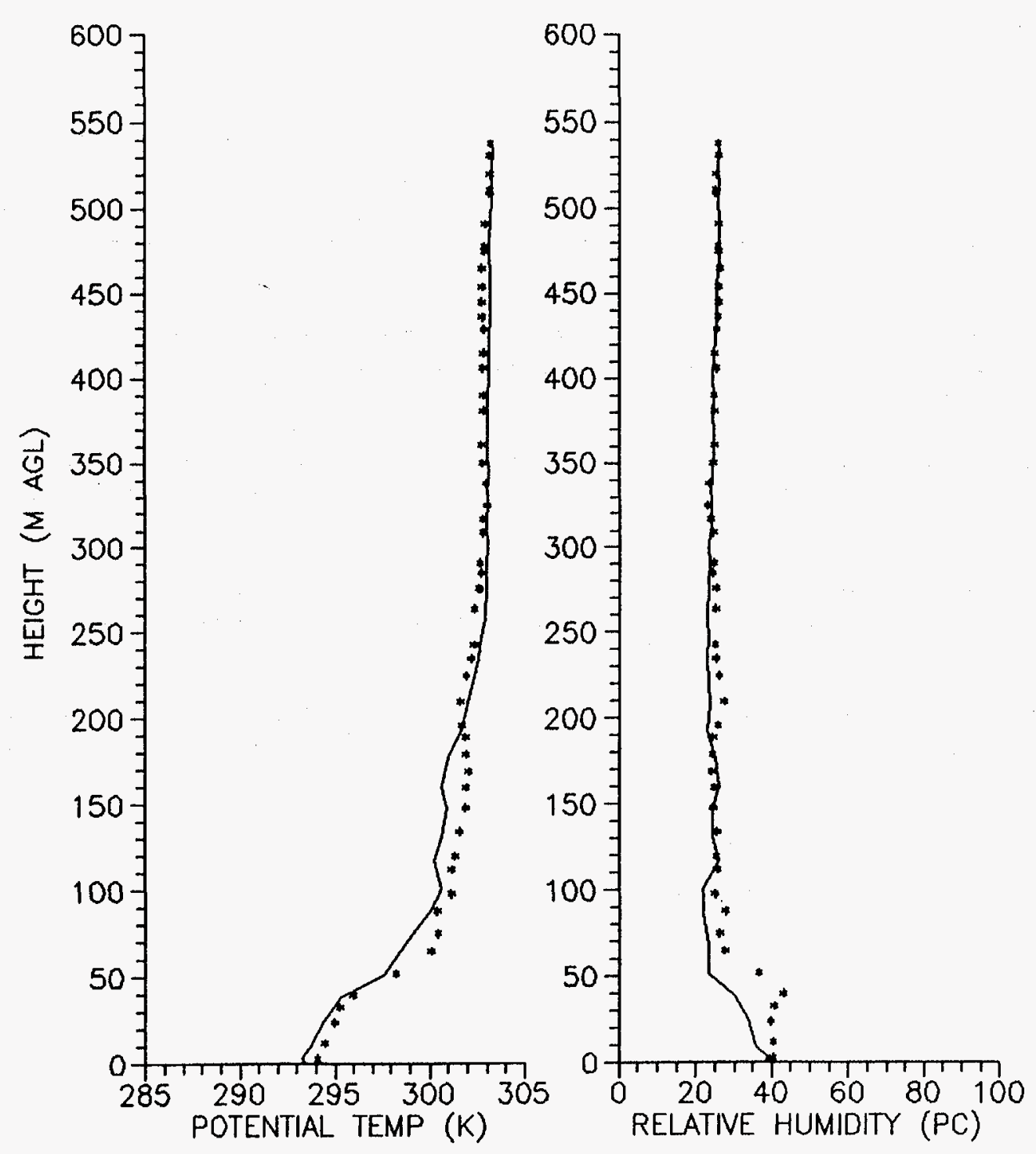
COAL CREEK CANYON TSONDE 02/08/91 BEGIN=0059 TOP=0113 END=0128 MST

ASCENT
$* * * *$ DESCENT
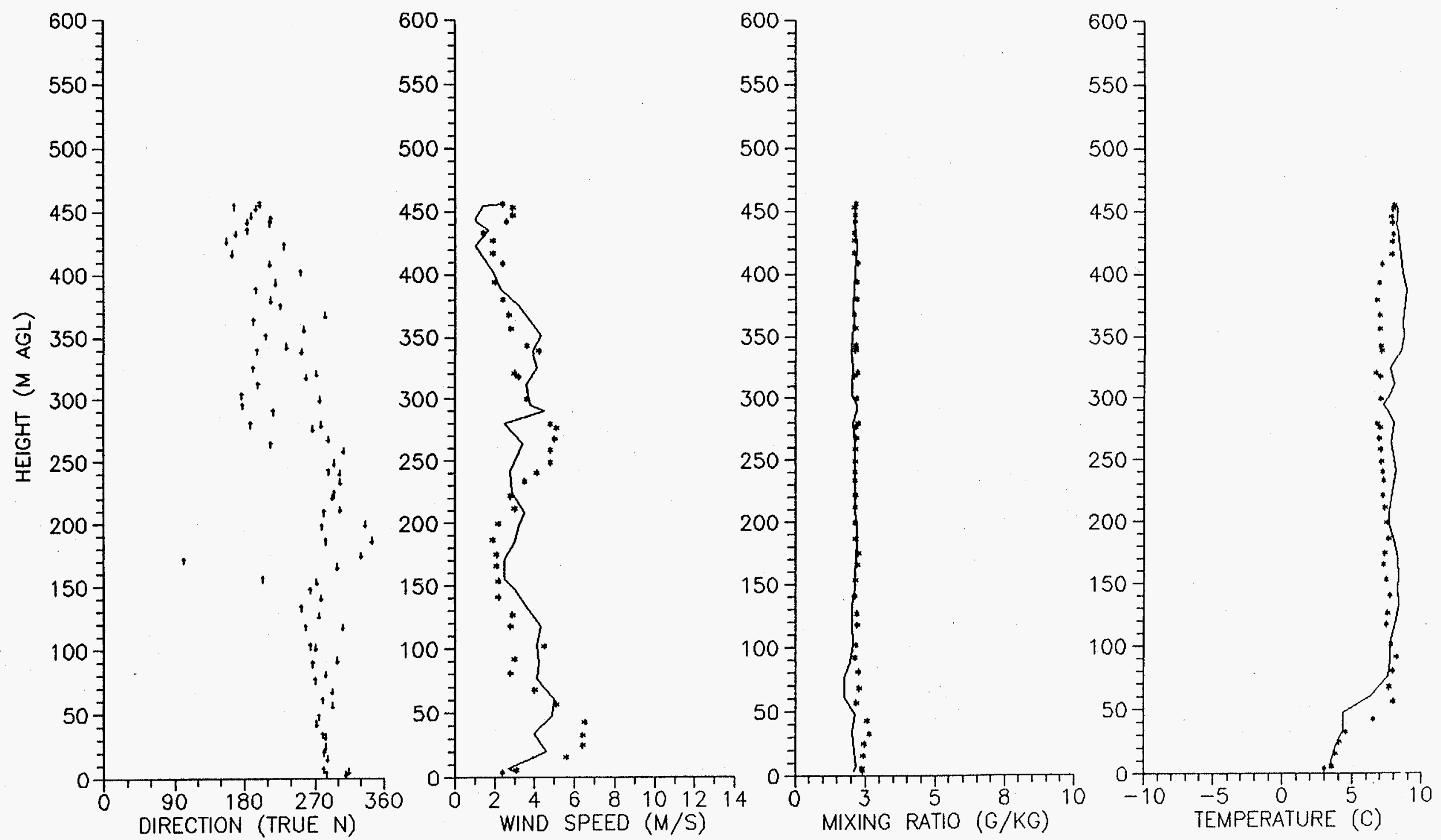


\section{COAL CREEK CANYON TSONDE}

$02 / 08 / 91 \quad B E G I N=0059 \quad T O P=0113 \quad E N D=0128 M S T$

ASCENT
$* * * *$ DESCENT
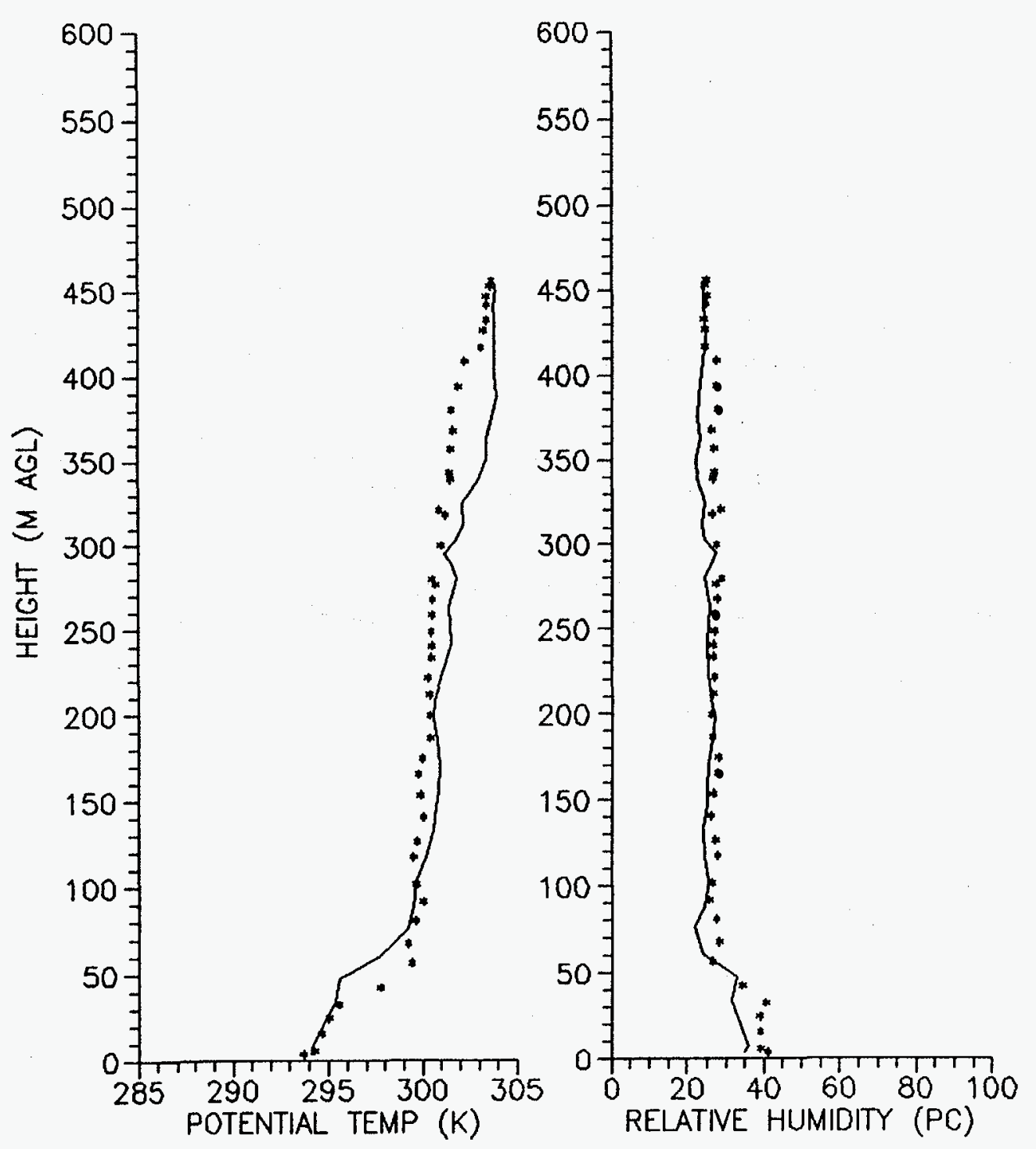


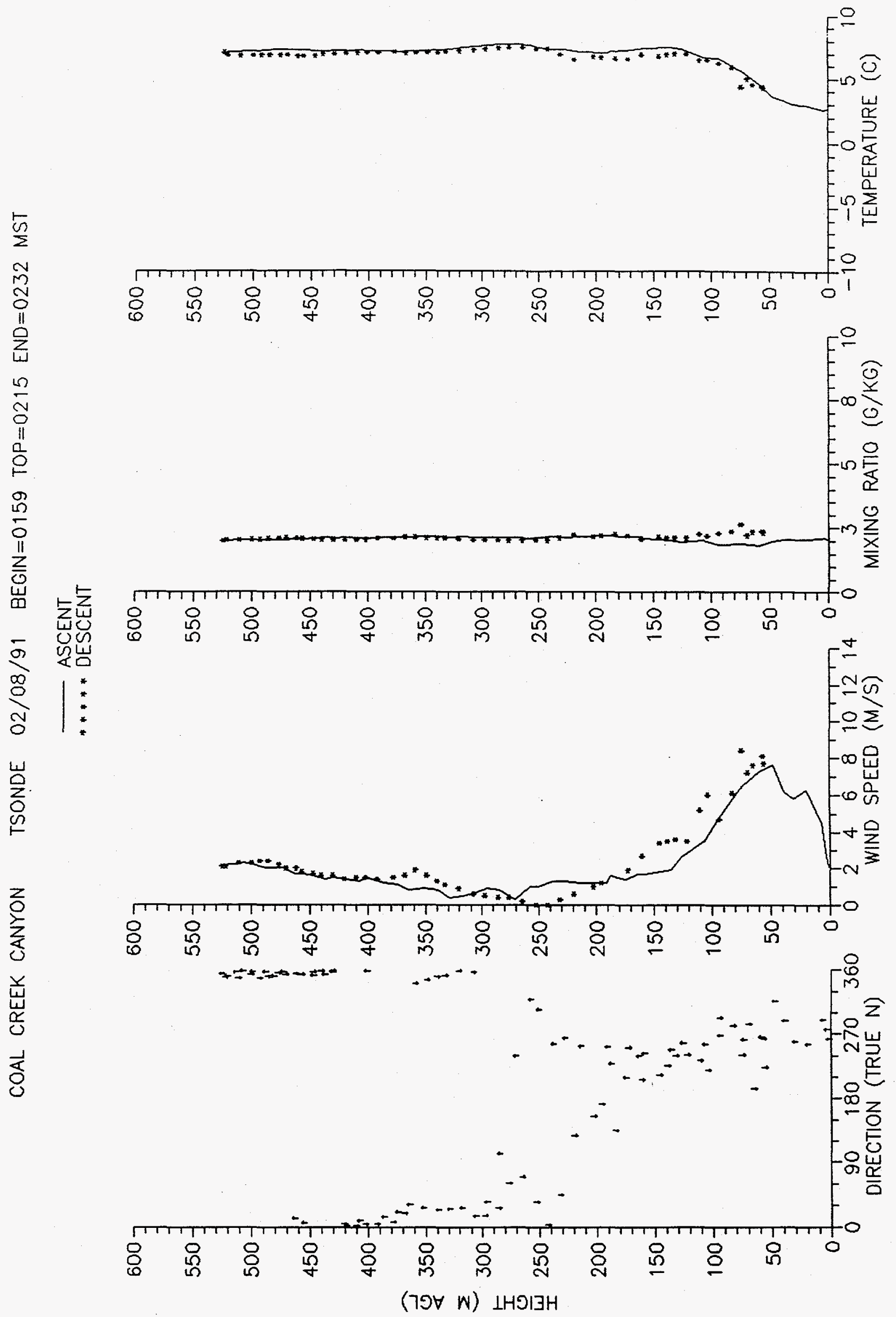




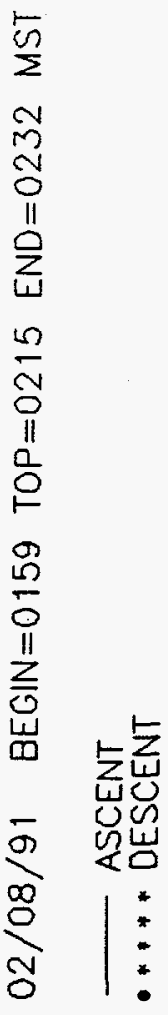

$\frac{1}{8}$

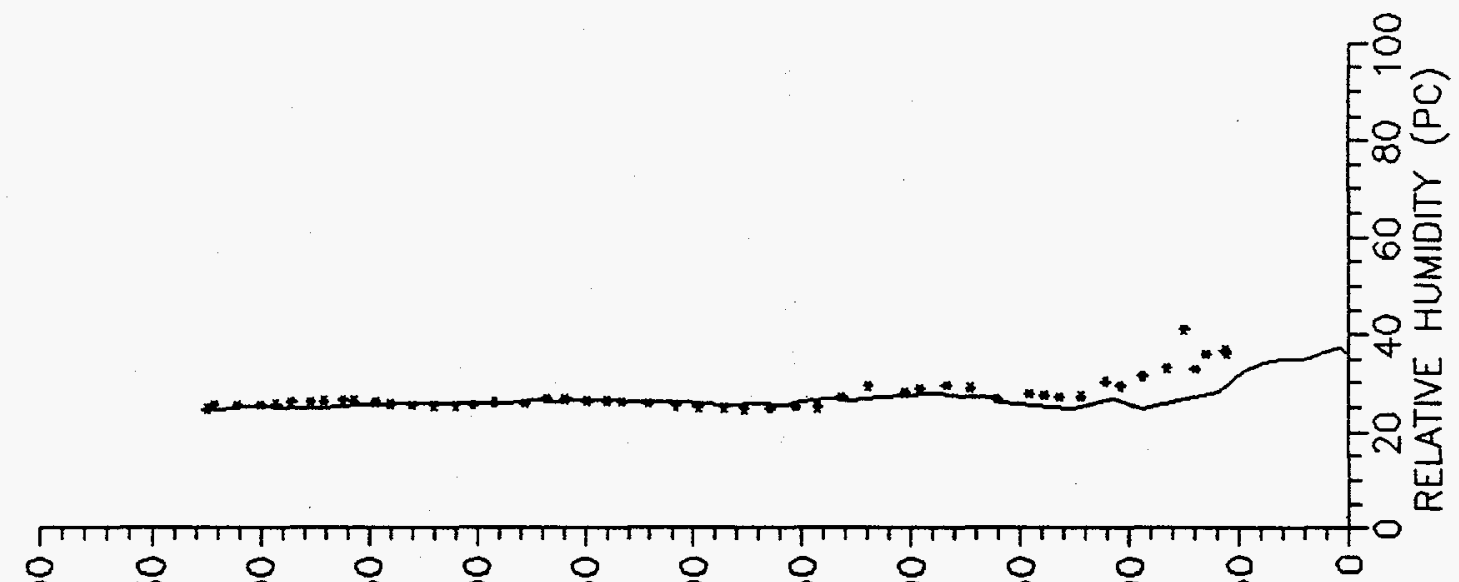

$\frac{2}{2}$

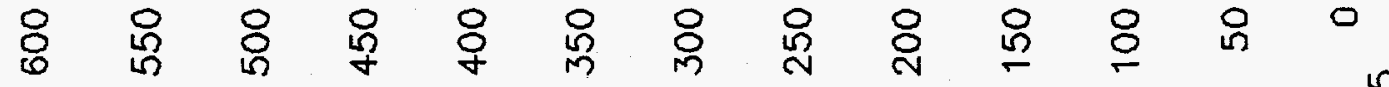

$\frac{y}{\frac{u}{\alpha}}$

8

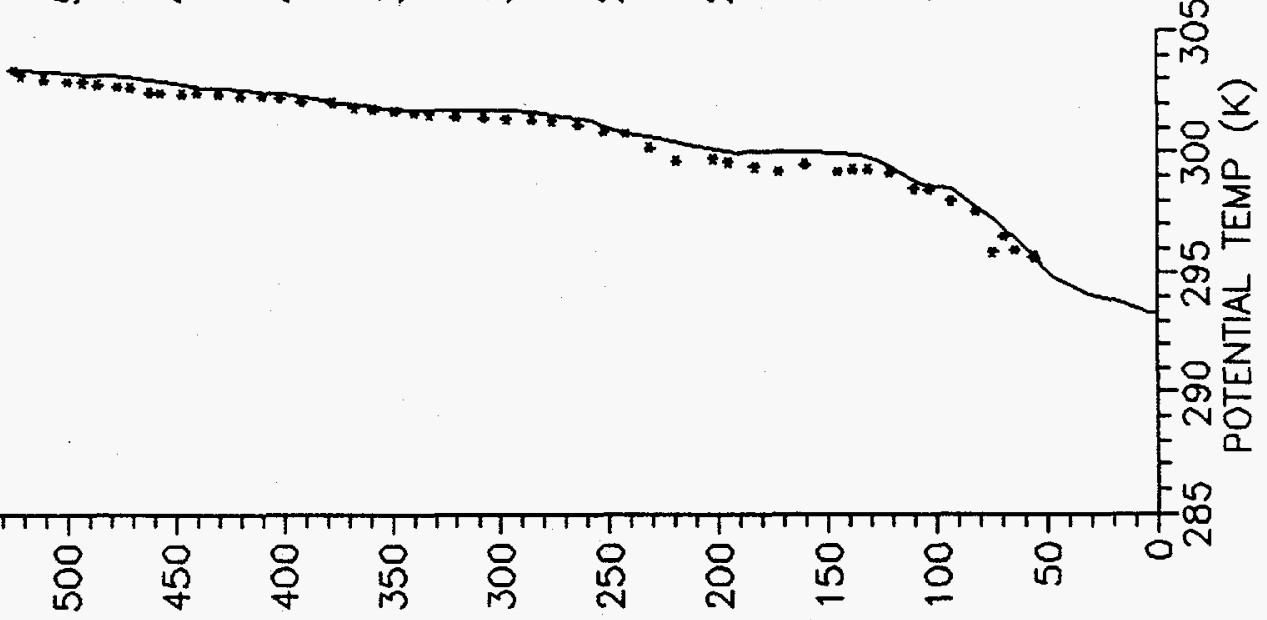

( 
ASCENT
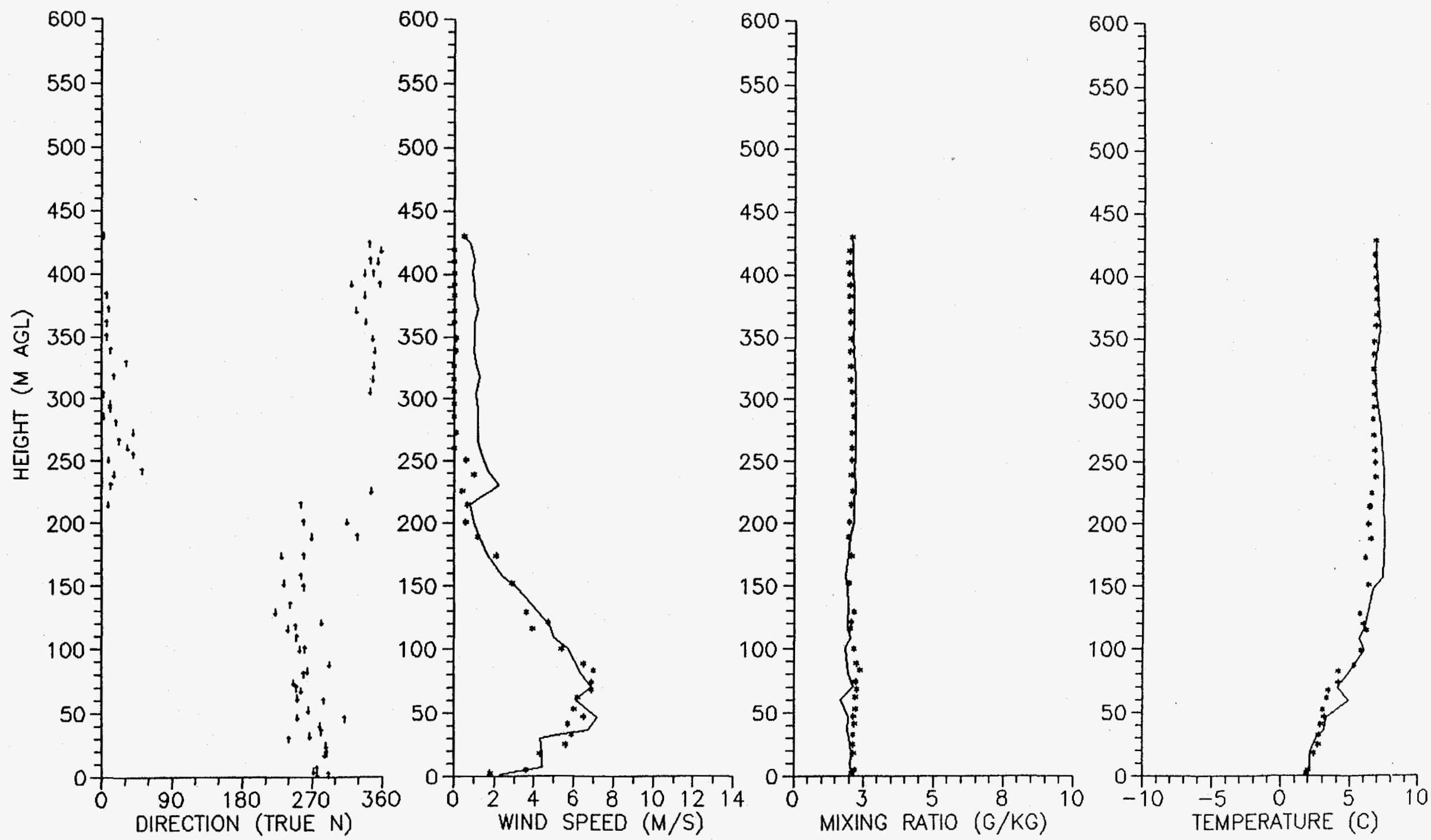

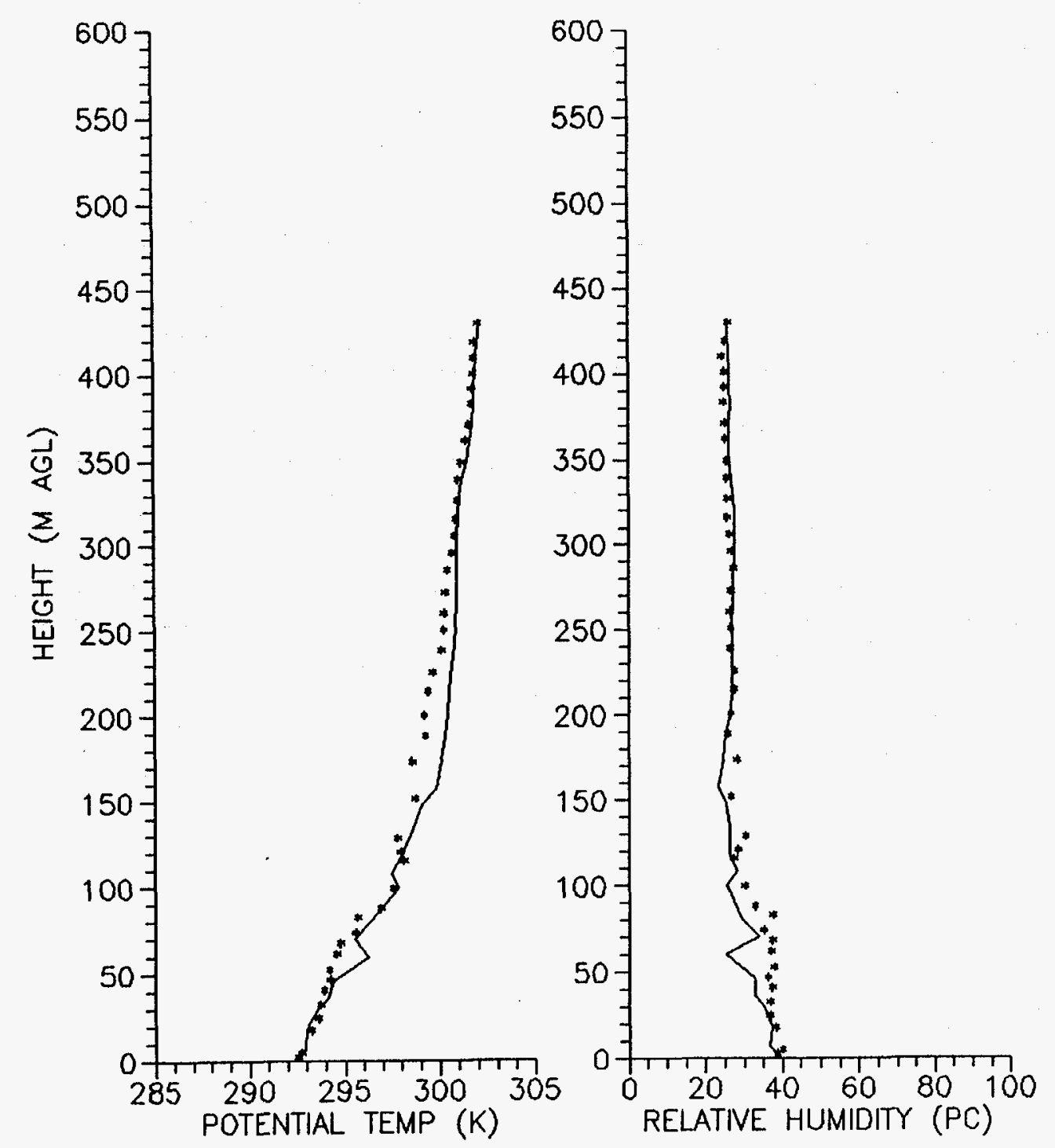
COAL CREEK CANYON TSONDE 02/08/91 BEGIN $=0359$ TOP $=0413 \quad$ END $=0429$ MST

ASCENT
$\cdots * *$ DESCENT

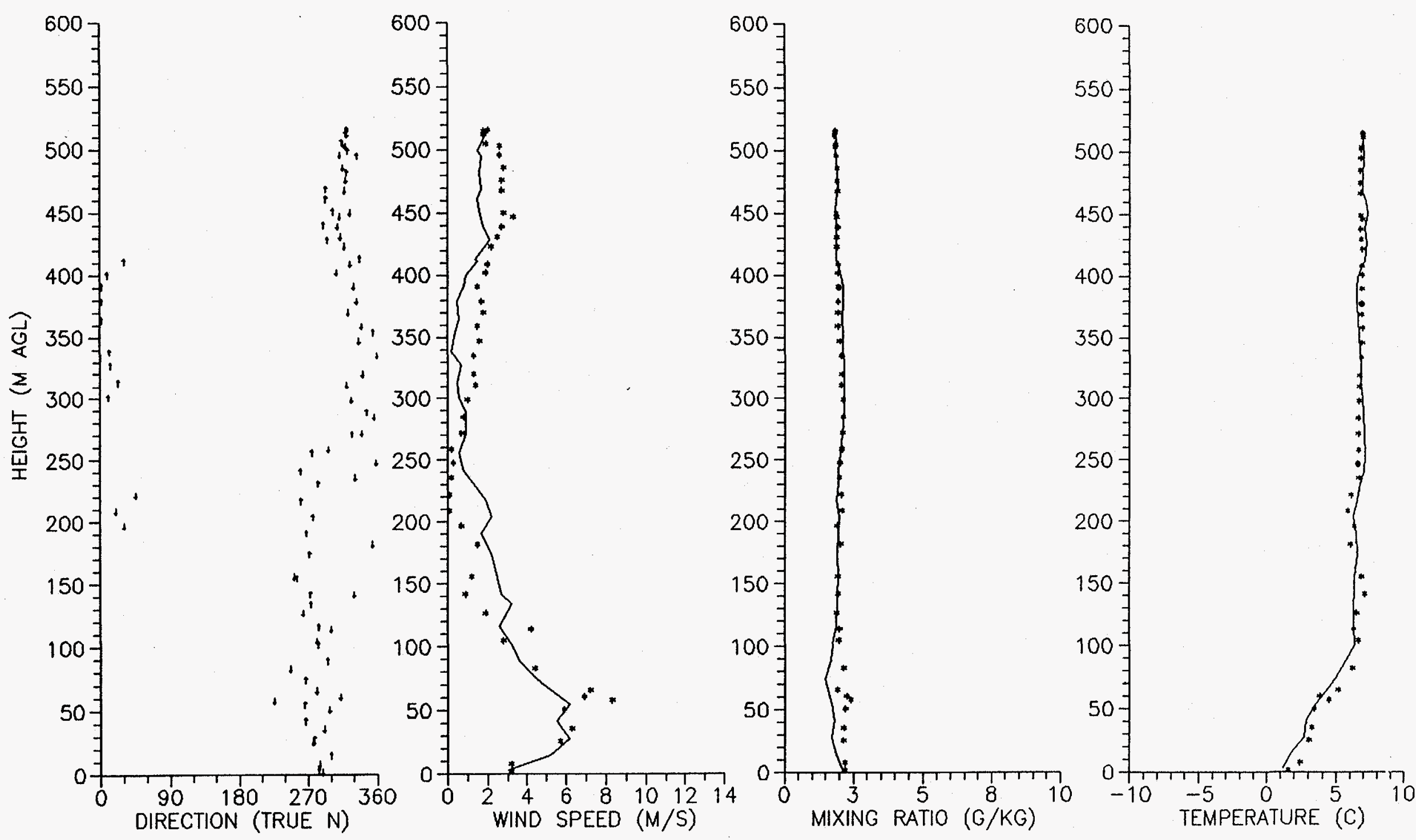




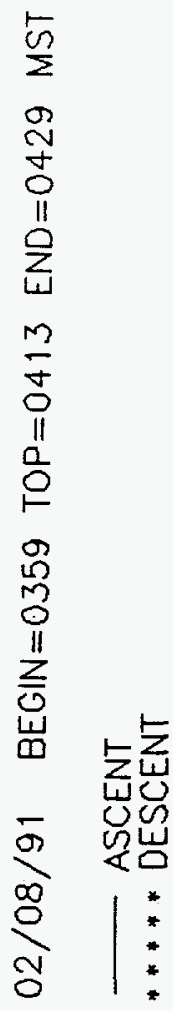

$\sum_{0}^{4}$

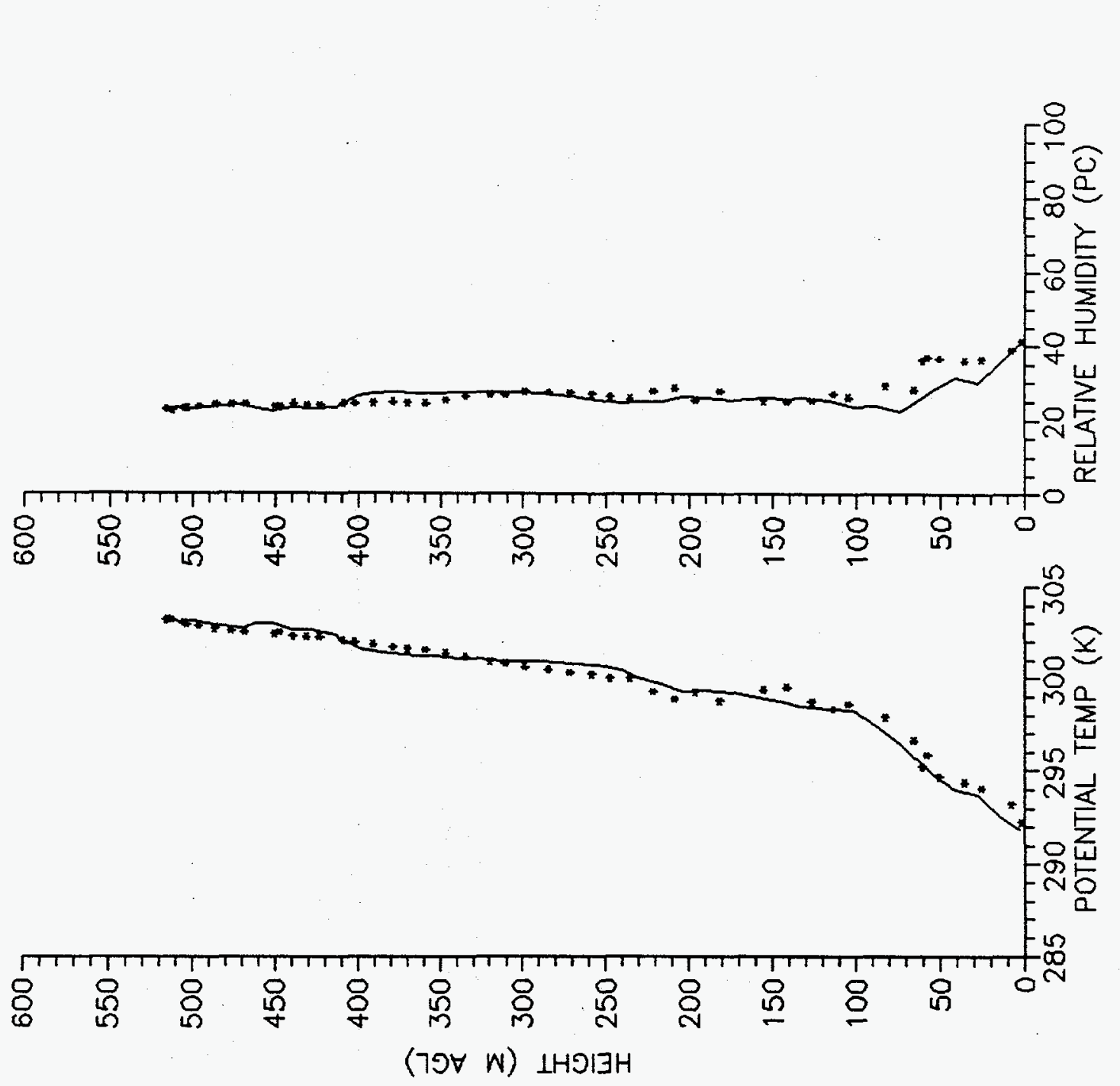

$\frac{2}{5}$

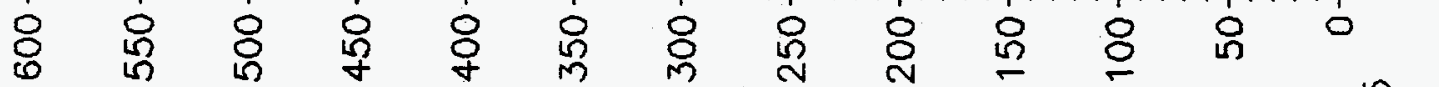

嵌

8 
COAL CREEK CANYON TSONDE 02/08/91 BEGIN=0459 TOP=0510 END=0518 MST

- ASCENT
$\cdots * *$ DESCENT
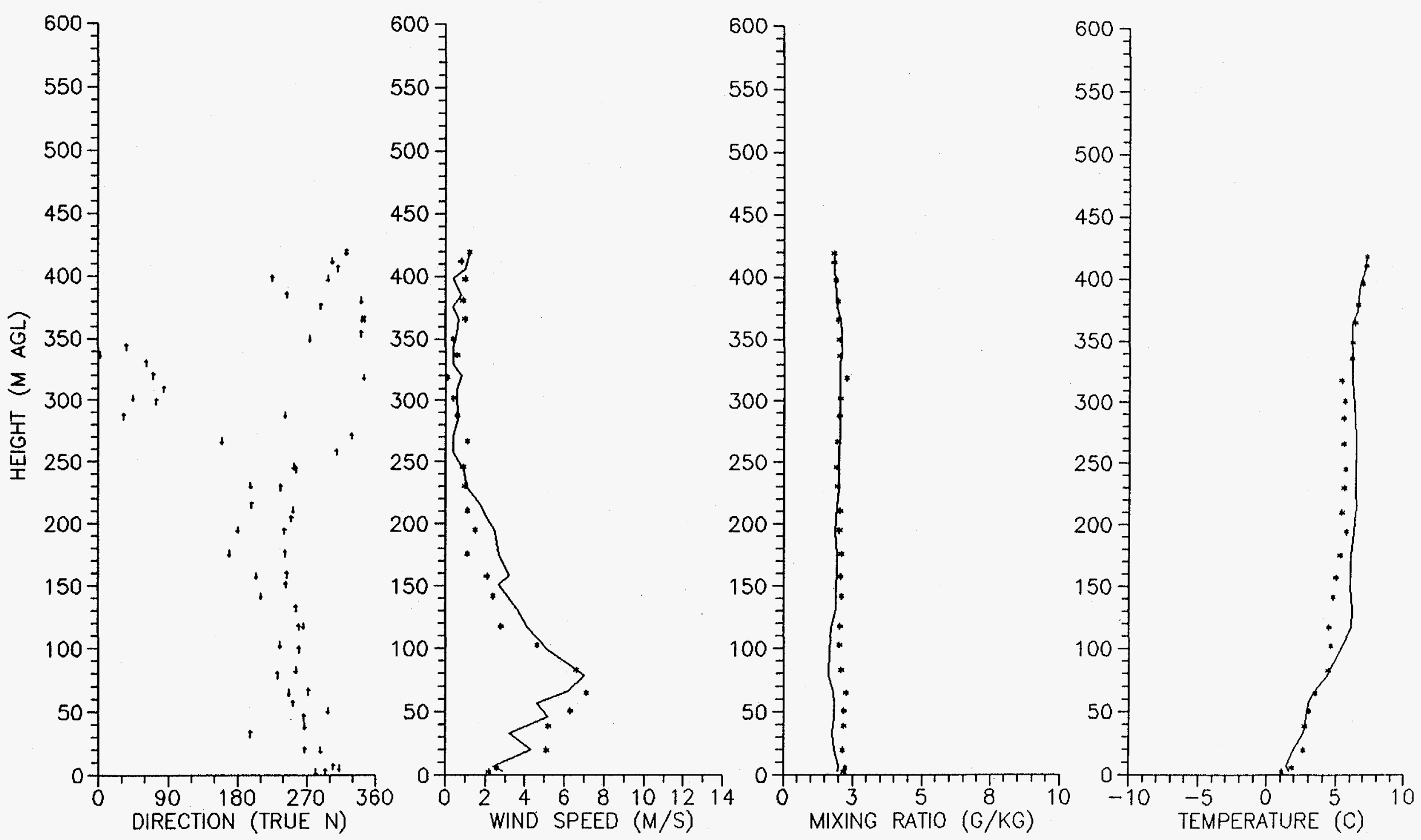


\section{ASCENT}

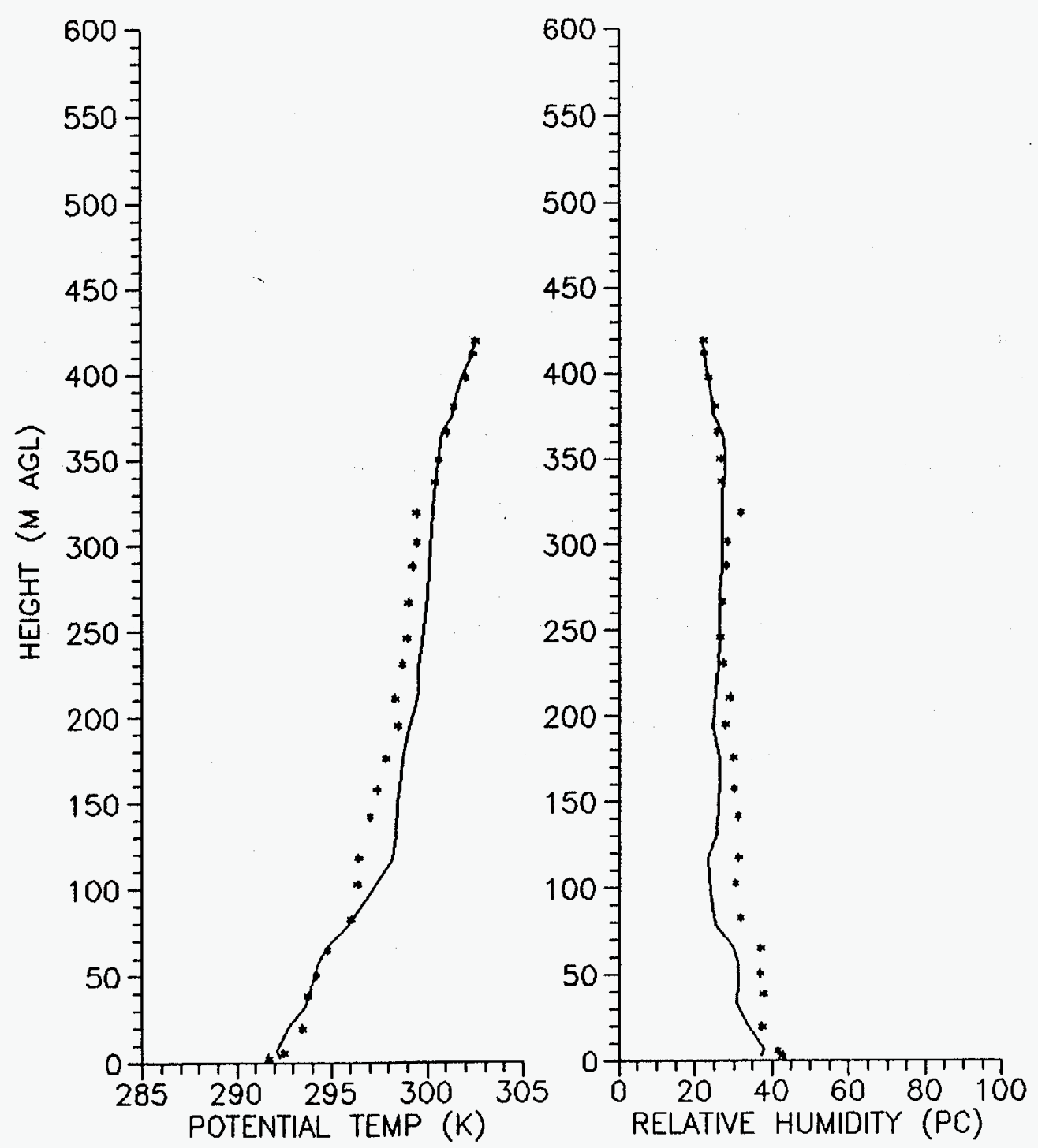


APPENDIX C 


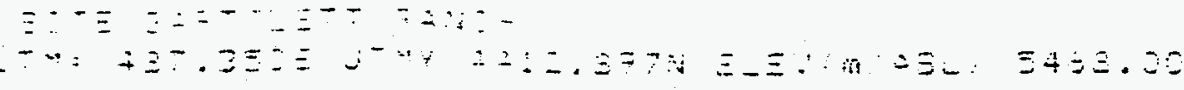

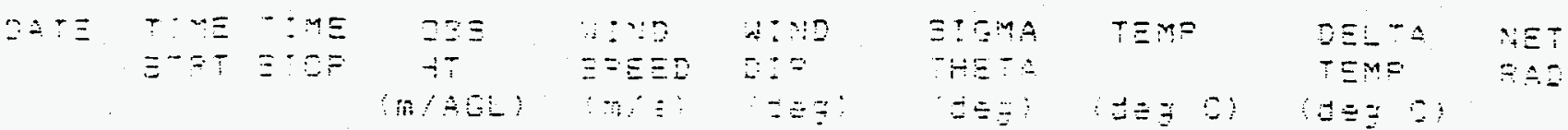

\begin{tabular}{|c|c|c|c|c|c|c|c|c|c|}
\hline$\therefore$ & $\because$ & 03 & $\Xi .: Z$ & 3.7 & $\therefore 3.9$ & $\Xi \div .7$ & -1.3 & & \\
\hline$\therefore:$ & $\because$ & $\because 8$ & 17.53 & $\div . \equiv$ & $\therefore=$ & -3.7 & $-3=$ & $\therefore:$ & $-=2.4$ \\
\hline-2 & $\therefore$ & 20 & $=.10$ & $\Xi . i$ & $\therefore$ & $8:$ & 2.1 & & \\
\hline$\because \vdots$ & 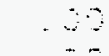 & $=0$ & $:^{7}, \pm 3$ & 3. & 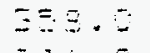 & 75.4 & 2.5 & $0 . \Xi$ & -49. \\
\hline 2 & $\because$ & $\because$ & 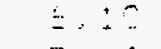 & $\therefore .4$ & 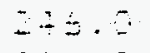 & $4=$. & $\therefore 2$ & & \\
\hline$\therefore$ & $=$ & $\therefore$ & $=\vdots$ & 3.9 & $-\div 9$, & $=7.9$ & $\therefore$ & $\therefore 3$ & -30.5 \\
\hline$\ddot{-}$ & $\therefore$ & $\because 2$ & $\leq . \therefore$ & $\therefore$ & $2=4,0$ & $85: 7$ & 3.7 & & \\
\hline$\therefore$ & $\therefore$ & $\because=9$ & 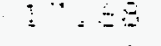 & $\because=$ & $\div 73=0$ & 31,9 & $1=$ & 0.5 & -49.2 \\
\hline 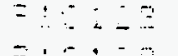 & $\therefore \because$ & $\because: 9$ & 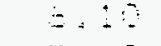 & $\therefore=5$ & $=43.0$ & 13.2 & 2.5 & & \\
\hline$\because:-3$ & $\therefore 2$ & $\because 0$ & 17,53 & $\bar{z}=\bar{Z}$ & $24: .0$ & $\therefore 6=7$ & $i=5$ & 0.7 & -40.3 \\
\hline$\therefore: 2$ & $=\therefore$ & $=2$ & $\leq 10$ & 2.4 & -98.0 & $? 2.2$ & $-6=6$ & & \\
\hline $21: 5$ & $\because$ & $\therefore=$ & $: 7=8$ & $\because .1$ & $20 t .0$ & 63,2 & $\because 2$ & 0.5 & -45.8 \\
\hline 818 & $=-0$ & 0 & $5 .: 3$ & $\Xi \ldots$ & 203.0 & 31.5 & 2. & & \\
\hline $0: \div$ & $=0$ & 3 & $7=3$ & $\doteq . !$ & 105.0 & 30. & 1.4 & 0.5 & $-5-1$ \\
\hline 942 & $\ldots$ & $\because 8$ & $=.8$ & 4. & 192.0 & 42.5 & 0.5 & & \\
\hline $8:=3$ & $=8$ & $=0$ & 17.50 & 4.7 & 126.0 & 44.5 & $\therefore 0$ & 0.4 & $-43 \cdot 6$ \\
\hline $\begin{array}{l}1+25 \\
8+23\end{array}$ & $\because 0$ & 702 & 5.10 & 3. & 271.0 & 11.5 & $\therefore$ & & \\
\hline $\begin{array}{l}123 \\
123\end{array}$ & $\begin{array}{l}200 \\
=-0\end{array}$ & $=0$ & $17=59$ & 7.4 & 207.0 & 10.0 & -9 & 0.2 & 20.2 \\
\hline $\begin{array}{l}125 \\
925\end{array}$ & $\begin{array}{l}=20 \\
=-0\end{array}$ & 00 & 3.10 & 10.4 & 276.0 & 13.4 & 3.0 & & \\
\hline $\begin{array}{l}1010 \\
101=0\end{array}$ & $\div 2$ & 20 & 17.58 & $: 2 .:$ & 271.0 & 12.3 & 3.3 & 0.3 & 35.2 \\
\hline $\begin{array}{l}701-6 \\
=10120\end{array}$ & 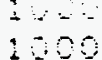 & $\because 20$ & 6.10 & 7.6 & 312.0 & .1 & $4 \cdot \frac{2}{3}$ & & \\
\hline $\begin{array}{l}7101=3 \\
7101=9\end{array}$ & $\begin{array}{l}200 \\
1: 00\end{array}$ & $\therefore: 0$ & 17.58 & 10.6 & 303. & 109 & $\begin{array}{l}4 \cdot 3 \\
4 \cdot 3\end{array}$ & 0.1 & 145.0 \\
\hline $9+10 \div=0$ & $\begin{array}{l}0 \\
1\end{array}=90$ & $=-80$ & $\therefore 12$ & 8.4 & 334.0 & $\begin{array}{r}10.8 \\
7.3\end{array}$ & $\begin{array}{l}4.3 \\
4.7\end{array}$ & 0.4 & \\
\hline $\begin{array}{l}10 \div 0 \\
7: 0:=3\end{array}$ & $i=30$ & $=00$ & $17=5$ & 7.2 & 323.0 & $\begin{array}{r}7.3 \\
25.9\end{array}$ & $\begin{array}{l}4.7 \\
1.3\end{array}$ & $0 . i$ & -43.3 \\
\hline 910129 & $1=00$ & $=200$ & $\begin{array}{r}0 \\
7 \\
7\end{array}$ & $=5$ & .0 & 28.3 & 1.8 & 0.0 & 27 \\
\hline $9102=9$ & 1300 & 140 & 5.10 & 1.9 & 31.0 & 38.9 & 1.8 & & \\
\hline$=10128$ & 130 & 1400 & $17: 60$ & 2.3 & 30.0 & 33.0 & 1.8 & 0.0 & 152.4 \\
\hline$=10128$ & 1400 & $\$ 00$ & 0.10 & 2.5 & 2.0 & $17: 2$ & 0.4 & & \\
\hline $9: 0225$ & 140 & $=000$ & 17.53 & 2.6 & 354.0 & 17.7 & 0.5 & 0.1 & 111.8 \\
\hline 910128 & $1 \equiv 0$ & $1 \pm 00$ & 6.10 & 2.5 & 20.0 & 11.2 & -0.5 & & \\
\hline 710128 & 1500 & $1 \leq 00$ & 17.53 & 2.5 & 14.0 & 12.0 & -0.8 & 0.0 & 36.2 \\
\hline 910128 & 160 & 1700 & $6=10$ & 5.6 & 349.0 & 11.5 & -1.5 & & \\
\hline $010: 20$ & $1=00$ & 1700 & 17.68 & 6.4 & 339.0 & 11.0 & -1.4 & 0.1 & -10.9 \\
\hline 710123 & 1700 & 1300 & 6.10 & 8.9 & 355.0 & 12.3 & -4.1 & & \\
\hline 910120 & 1700 & 1500 & 17.68 & 10.0 & 345.0 & 6.9 & -4.2 & -0.1 & -14.2 \\
\hline 28 & 1300 & 1900 & 6.10 & 3.4 & 8.0 & 10.8 & -5.8 & & \\
\hline 910129 & 1800 & 1900 & 17.69 & 8.4 & 2.0 & 11.9 & -5.9 & -0.1 & -6.5 \\
\hline 710128 & 1900 & 2000 & 0.10 & 7.5 & 29.0 & 6.5 & -7.9 & & \\
\hline$=10128$ & 1900 & 2000 & 17.63 & 8.1 & 25.0 & 5.8 & -3.0 & -0.1 & -4.2 \\
\hline 910129 & 2000 & 2100 & 6.10 & 6.2 & 26.0 & 9.2 & -10.2 & & \\
\hline 910128 & 2000 & 2100 & 17.68 & 6.3 & 22.0 & 8.7 & -10.3 & -0.1 & -0.7 \\
\hline 911 & 2100 & 2200 & 6.10 & 6.2 & 19.0 & $\overline{8} . \overline{8}$ & -12.2 & & \\
\hline 910 & 2100 & 2200 & 17.68 & 6.2 & 15.0 & 9.4 & -12.3 & -0.1 & 0.1 \\
\hline 91 & 2200 & 2300 & 6.10 & 6. & 11.0 & 7.0 & -13.5 & & \\
\hline 710 & 2200 & 2300 & 17.68 & 5. & 6.0 & 7.8 & -13.6 & -0.1 & 0.0 \\
\hline & 2300 & 0 & 6.10 & & 13.0 & 8.8 & -14.2 & & \\
\hline 910 & 2300 & 0 & 17.68 & 5. & 8.0 & 10.5 & $-14 \cdot 3$ & -0.1 & 0.3 \\
\hline
\end{tabular}




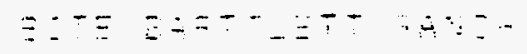

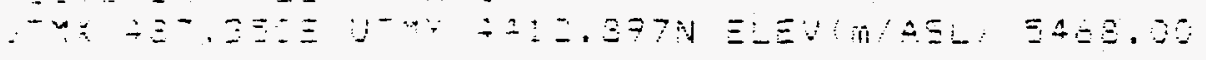

\begin{tabular}{|c|c|c|c|c|c|c|c|c|c|}
\hline $5: \pm \equiv$ & $\begin{array}{l}\because n \equiv \\
\vdots:=T\end{array}$ & $\begin{array}{l}\because n \equiv \\
\because 9=\end{array}$ & 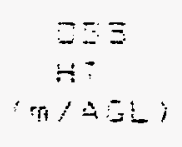 & $\begin{array}{c}\text { W I D } \\
\text { SFED } \\
m:\end{array}$ & $\begin{array}{l}4: 40 \\
=:= \\
4=9\end{array}$ & 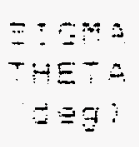 & $I \equiv 3 \quad \vdots$ & 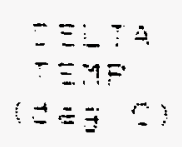 & $\begin{array}{l}A: D \\
B A D\end{array}$ \\
\hline$=:::=$ & $=$ & 00 & $E=10$ & $=:$ & $=5,5$ & $=.7$ & $-i=1 \equiv$ & & \\
\hline$\because: \therefore::=$ & $\therefore$ & $: 0$ & $17 . \pm 9$ & $4=$ & 24.0 & $\because .5$ & $-: E .5$ & $-a$ & $-1 .=$ \\
\hline$\therefore::::=$ & $\because=$ & $\therefore=$ & $5 .: 0$ & 2. & $\because \approx 0$ & 10.4 & $-\div \div .=$ & & \\
\hline$\because: \because:: \because$ & $\because 8$ & $\therefore 2$ & $\because ? \leq 0$ & 2.5 & $: 9.0$ & $\therefore \% ?$ & $-1 \pm=4$ & -0. & $-5: 2$ \\
\hline$=:-\therefore$ & $\therefore$ & $\therefore$ & $=\therefore$ & $\therefore .4$ & 30.0 & 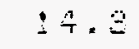 & $-i 7=i$ & & \\
\hline$=::: \vdots=$ & $\because \cdots$ & $\therefore \because$ & $\therefore \because=\bar{E}$ & $\therefore 1$ & 56.0 & 9. & $-: 7$. & $\because 6$ & -8.5 \\
\hline$=:-:=9$ & $\therefore$ & -5 & $\therefore: \because 9$ & $\therefore=$ & -2.0 & $\because 2 . \bar{z}$ & $-i \leq .3$ & & \\
\hline$=: 2:=$ & $\because: 3$ & $\therefore$ & $: 7=0$ & $\because 7$ & $\therefore 5.0$ & $\therefore 2$. & $-i \bar{i}$ & -5.2 & $-1 .:$ \\
\hline$\nabla: 2:-9$ & $\therefore \because$ & $\because \because$ & 6.2 & $\therefore 7$ & $=0$ & 13.5 & $-i \vec{i} \equiv$ & & \\
\hline$=: 91=$ & $-\because$ & $\because c$ & $: \because=2$ & 2.1 & $\Xi=.0$ & $: 4.3$ & $-\div 7 .=$ & $-2 .:$ & 0.3 \\
\hline$=101=$ & $\equiv$ & $=-5$ & $b .:$ & $\because 3$ & 5.5 .0 & 17.3 & -13.0 & & \\
\hline$=: 9129$ & $\because 20$ & $=0$ & $: 7.68$ & $\therefore 2$ & 70.0 & 7.5 & $-: \Xi .:$ & -0.1 & -2.8 \\
\hline $7101=7$ & $=20$ & 98 & $\leq .10$ & 2.0 & 59.0 & 21.9 & -18.4 & & \\
\hline$=: 0: \div$ & $=\infty$ & -93 & $47: \leq 5$ & 5.5 & 45.0 & 9.4 & -13.4 & 0.0 & -9.2 \\
\hline $710: 5$ & -9 & 300 & $=.2$ & 0.2 & $14: .0$ & 81.3 & -19.7 & & \\
\hline $9: 10127$ & $\because 0$ & 39 & $17 .=2$ & 2.2 & 39.0 & 27.5 & -13.7 & 0.8 & -17.5 \\
\hline 710127 & $\equiv 2$. & $=00$ & $5 .: 0$ & 0.1 & 249.0 & 27.8 & -10.2 & & \\
\hline $7101=7$ & $\Xi 20$ & -0 & $17=-3$ & 0.5 & 309.0 & 1.4 & -17.0 & 1.2 & -5.5 \\
\hline 910125 & $=9$ & 85 & 0.10 & 0.0 & $23 \omega=0$ & 46.1 & -12.7 & & \\
\hline 910127 & $\mp: 0$ & 0 & 17.5 & 0.4 & 315.0 & 12.2 & -13.6 & -0.9 & 6.1 \\
\hline $9: 0429$ & 100 & $: \because 0$ & $=0.10$ & 0.2 & 317.0 & 26.3 & -12.6 & & \\
\hline 710127 & 100 & 100 & $17 .=5$ & 0.6 & 318.0 & 15.8 & -12.3 & 0.3 & 15.8 \\
\hline $9101=7$ & $1: 0$ & $: 00$ & 5.10 & 0.1 & 7.0 & 38.1 & -7.9 & & \\
\hline 910127 & $1: 00$ & .200 & 17.99 & 0.5 & 359.0 & 42.7 & -7.3 & 0.6 & 36.5 \\
\hline 710127 & 1200 & 1300 & 0.20 & 0.1 & 7.0 & 14.2 & -12.4 & & \\
\hline 910127 & $1=0$ & 230 & 17.43 & 1.5 & 5.0 & 13.2 & -11.5 & 0.8 & 44.4 \\
\hline 910127 & 1300 & $: 40$ & $5=10$ & $1=1$ & 10.0 & 17.4 & -12.2 & & \\
\hline 910127 & 1200 & 1400 & 17.68 & $1 \cdot 1$ & 4.0 & b & -11.6 & 0.6 & 40.9 \\
\hline 710127 & 140 & 3500 & $b=10$ & 0.0 & 14.0 & 4 & -5.3 & & \\
\hline $7: 0127$ & 1400 & $: 500$ & 17.58 & 0.0 & 25.0 & 14.0 & -4.6 & 2.2 & 19.0 \\
\hline 710127 & $i \equiv 00$ & 1500 & $5=10$ & $\cdot 5$ & $194: 0$ & 20 & -10.0 & & \\
\hline 710127 & $1 \equiv 0$ & $1 \leq 00$ & 17.53 & 2.0 & 181.0 & 1.4 & -9.8 & 0.2 & -9.0 \\
\hline 910129 & $1=0$ & 1700 & 6.10 & 0.9 & 199.0 & 23.6 & -10.1 & & \\
\hline 910129 & $1 \leq 00$ & 1700 & 17.38 & 1.5 & 183.0 & 23.3 & -10.2 & -0.1 & -36 \\
\hline 710129 & $1=90$ & .900 & 6.10 & 1.1 & 236.0 & 19.8 & -14.5 & & \\
\hline 910129 & 1700 & 1300 & 17.68 & 1.2 & 229.0 & 24.4 & -12.6 & 1.9 & -36.4 \\
\hline 910127 & 1300 & 1900 & 6.10 & 0.3 & 233.0 & 38.2 & -16.0 & & \\
\hline 710129 & 1900 & 1900 & 17.68 & 1.2 & 251.0 & 34.2 & -13.3 & 2.7 & -29.3 \\
\hline 910129 & 1700 & 2000 & 6.10 & 0.3 & 197.0 & 58.0 & -15.9 & & \\
\hline 910129 & 1700 & 2000 & 17.68 & 0.5 & 145.0 & 65.1 & -14.7 & 1.2 & -23.8 \\
\hline 710129 & 2000 & 2100 & 6.10 & 0.8 & 237.0 & 41.0 & -14.3 & & \\
\hline 910127 & 2000 & 2100 & 17.68 & 1.1 & 232.0 & 44.6 & -12.6 & 1.7 & -3.5 \\
\hline 910127 & 2100 & 2200 & 6.10 & 0.9 & 154.0 & & -11.3 & & \\
\hline 710127 & 2100 & 2200 & 17.68 & 1.4 & 179.0 & & -9.9 & 1.4 & -5.1 \\
\hline 910127 & 2200 & 2300 & 6.10 & 0.9 & 234.0 & & -10.8 & & \\
\hline 910129 & 2200 & 2300 & 17.68 & 0.9 & 325.0 & & -7.9 & 2.9 & -13.5 \\
\hline 910129 & 2300 & 0 & 6.10 & 2.8 & 235.0 & 56.1 & -3.6 & & \\
\hline 9 & 20 & 0 & 17.63 & 3.6 & 288.0 & 6 & -2.2 & 1.4 & \\
\hline
\end{tabular}


三-

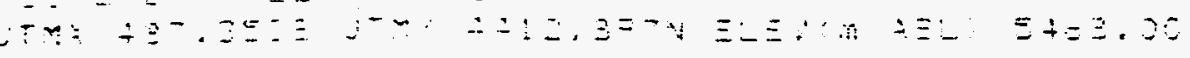

\begin{tabular}{|c|c|c|c|c|c|c|c|c|c|}
\hline$=\therefore T \equiv$ & $\equiv-$ & $\begin{array}{l}\because \vdots \equiv \\
\because \div=\end{array}$ & $\begin{array}{c}5=\Xi \\
y \\
m-6 L\end{array}$ & $\begin{array}{l}410 \\
0=0 \\
3: \vdots\end{array}$ & $\begin{array}{l}200 \\
0:= \\
=8\end{array}$ & 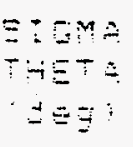 & 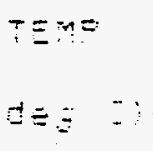 & 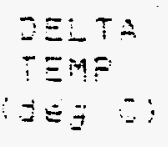 & $\begin{array}{l}n E= \\
\therefore+1\end{array}$ \\
\hline$=: \because \vdots:$ & 3 & .90 & $\Delta .: 0$ & $\therefore .0$ & $-3: 5$ & $-\vdots . \quad \vdots$ & $-\vdots . .=$ & & \\
\hline$=\because \div=$ & 2 & $: 00$ & $17=8$ & 7.5 & $\Xi-2.0$ & $: \leq .7$ & $-\therefore 2$ & 1.5 & -17.5 \\
\hline$\because \cdots: \because$ & $\therefore$ & $\therefore 0$ & 5.10 & 7.2 & $3-3,8$ & $\because z .7$ & $-\vdots=\vdots$ & & \\
\hline$\because:::$ & $\vdots \because$ & $-\because$ & $: 7 .=0$ & $9 .=$ & $\equiv: \square=\because$ & $: 9.9$ & $\therefore$ & 1.0 & $-\bar{j} \mathrm{O} .3$ \\
\hline$=: 0: 2$ & $\because:$ & 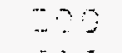 & 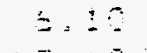 & 2.4 & $157, \because$ & $45 . \bar{Y}$ & $-\vdots . \Xi$ & & \\
\hline$\therefore: \therefore:=$ & $\because \because$ & $\therefore:$ & $\therefore \quad=3$ & $\therefore=$ & $: 985$ & -2.5 & -3.5 & 6.7 & -45.4 \\
\hline$=: \because: \div$ & $\because \because$ & $\because 2$ & 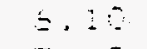 & $\therefore$ & 90.0 & $=-\Delta$ & $-\therefore . \dot{=}$ & & \\
\hline$=: 2:: \because$ & $\because \because$ & 40 & $17:=3$ & $3=-$ & $7 \div .5$ & 35.0 & $-:=7$ & 9 & -47.3 \\
\hline$=: 0::$ & $\therefore \therefore$ & $\because=$ & $5=10$ & $z=$ & $3: 7.0$ & 5.9 & $-\vdots .3$ & & \\
\hline$=: \because: Z$ & $3=$ & $=20$ & $\therefore \because=3$ & 3.5 & $=1: 0$ & $4: .1$ & -9.7 & 0.9 & -43.1 \\
\hline$= \pm \div \div$ & $\equiv$ & $=03$ & $=.10$ & $\Xi$. & $\because 57.0$ & $4 \div .1$ & $-1,0$ & & \\
\hline$=3-1=$ & $\because \therefore$ & $=0$ & $: 7==8$ & $=8$ & 203.0 & 35.3 & -0.5 & 1.3 & $-4 \leq .5$ \\
\hline$=10$ & $=0$ & $\because 0$ & $5=: 5$ & $7=3$ & 279.0 & 30.1 & -6.7 & & \\
\hline$=10 \pm=3$ & $=\because \because$ & 3 & $\vdots \bar{z}:=3$ & $E$ & -59.0 & 23.8 & $-0 \cdot 2$ & $0 . \Xi$ & -54.1 \\
\hline$=1019$ & 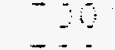 & $=10$ & $8: 10$ & 7.5 & 2530 & 10.8 & -9.9 & & \\
\hline $9: 01=0$ & $\therefore$ & $=0$ & 7.5 & 7.1 & 230.0 & 5.5 & -0.5 & 0.6 & -55.2 \\
\hline$=10190$ & 20 & 80 & $\leq .0$ & 3.5 & -91.0 & 2.5 & 0.7 & & \\
\hline$=10 \mathrm{i}$ & 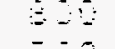 & $=0$ & 37.93 & $0 . \because$ & 270.0 & 23.6 & 1.3 & 0.5 & -30.9 \\
\hline 910130 & $=-3$ & 200 & 6.10 & $\Xi .7$ & 259.0 & 36.0 & 3.6 & & \\
\hline 910120 & $=-0$ & $:-00$ & 17.08 & $5=5$ & $2=5.0$ & 33.3 & 3.7 & 0.1 & 14.2 \\
\hline 710120 & 280 & $: 100$ & $5: 10$ & 5.9 & 274.0 & 12.7 & 4.1 & & \\
\hline 910130 & 100 & $\therefore 200$ & $17:=3$ & $8=4$ & 271.0 & 10.7 & $4 \cdot 2$ & 0.1 & 49.5 \\
\hline $7: 0190$ & $1: 00$ & $1=0$ & 5.10 & 5.3 & 239.0 & 54.8 & 5.9 & & \\
\hline 710130 & $1: 00$ & 200 & $17: 28$ & 5.5 & 279.0 & 56.7 & $s .0$ & 0.1 & 77.7 \\
\hline $7101=0$ & $1=00$ & 300 & $\varepsilon=: 0$ & 3.4 & 95.0 & 72.0 & 7.7 & & \\
\hline 710130 & $2=00$ & $: 200$ & $17: \leq 8$ & 4.0 & 92.0 & 50.6 & 7.8 & -0.1 & 102.8 \\
\hline $0101=0$ & 1200 & .400 & 6.20 & 5.7 & 09.0 & 11.8 & 6.8 & & \\
\hline 1019 & 1200 & $: 200$ & $17 . \leq 8$ & 7.5 & 102.0 & 10.3 & 3.9 & 0.1 & 121.2 \\
\hline 710130 & 140 & 150 & 5.10 & 7.6 & 297.0 & 15.7 & 7.9 & & \\
\hline 90020 & $1+30$ & 1500 & $17 .=8$ & 8.4 & 281.0 & 13.9 & 8.0 & 0.1 & 95.9 \\
\hline$=10130$ & $1=0$ & $i=00$ & 5.10 & 5.2 & 273.0 & 12.4 & 7.7 & & \\
\hline 910130 & $1 \equiv 0$ & $:=00$ & 17.68 & 7.2 & 269.0 & 10.5 & 7.9 & 0.2 & 24.4 \\
\hline 710130 & 1500 & 1700 & $=10$ & 5.8 & 280.0 & 11.2 & 5.9 & & \\
\hline 710130 & 150 & 1790 & 17.63 & 7.0 & 278.0 & 9.1 & 6.7 & 0.8 & -41.7 \\
\hline 7101.0 & 1720 & 1900 & 6.10 & 7.6 & 279.0 & 10.7 & 5.2 & & \\
\hline 910130 & 1700 & 1300 & 17.68 & 9.1 & 276.0 & 9.9 & 5.9 & 0.7 & -51.2 \\
\hline 910120 & 1300 & 1700 & 6.10 & 7.6 & 286.0 & 12.8 & 5.5 & & \\
\hline 910130 & 1300 & 1900 & 17.68 & 8.7 & 281.0 & 10.7 & 6.2 & 0.7 & -52.6 \\
\hline 910139 & 1900 & 2000 & 6.10 & 5.3 & 294.0 & 13.1 & 5.2 & & \\
\hline 910130 & 1000 & 2000 & 17.68 & 6.2 & 288.0 & 16.6 & 6.2 & 1.0 & -50.9 \\
\hline 910130 & 2000 & 2100 & 6.10 & 7.4 & 264.0 & 10.4 & 5.4 & & \\
\hline 910130 & 2000 & 2100 & 17.68 & 9.0 & 260.0 & 7.4 & 6.1 & 0.7 & -47.7 \\
\hline .10130 & 2100 & 2200 & 6.10 & 10.1 & 265.0 & 8.9 & 6.2 & & \\
\hline 910130 & 2100 & 2200 & 17.68 & 11.7 & 261.0 & 7.3 & 6.7 & 0.5 & -40.2 \\
\hline 710130 & 2200 & 2300 & 6.10 & 5.7 & 263.0 & 14.1 & 6.4 & & \\
\hline 10130 & 2200 & 2300 & 17.68 & 7.0 & 261.0 & 13.0 & 6.9 & 0.5 & -42.5 \\
\hline & 2300 & 0 & 6.10 & 5.2 & 2.0 & & 6.3 & & \\
\hline 16 & 2300 & 0 & 17.68 & 6.0 & 285.0 & 32.3 & 7.1 & 0.8 & -53.6 \\
\hline
\end{tabular}




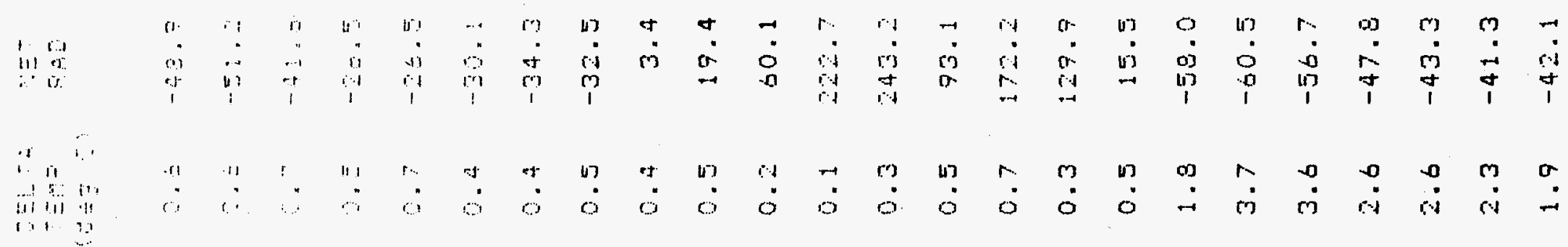

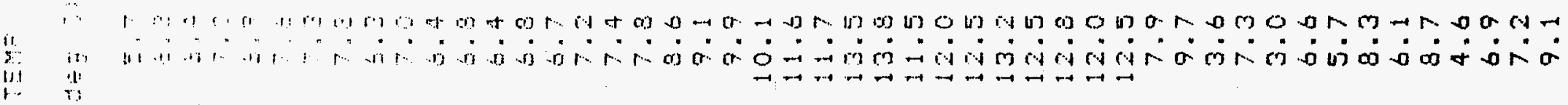

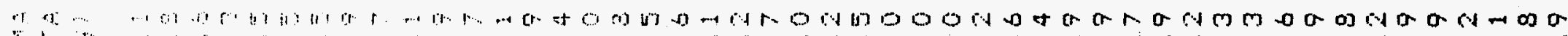
in

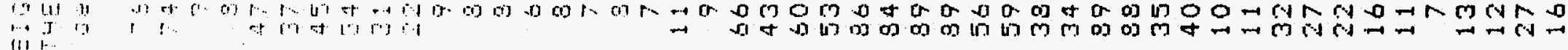

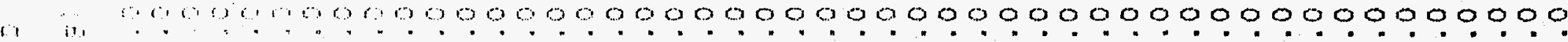

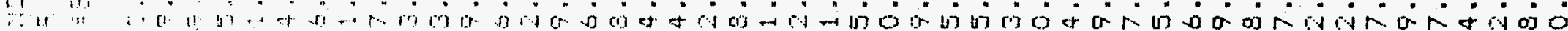
*

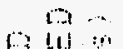

$\rightarrow$ li. in

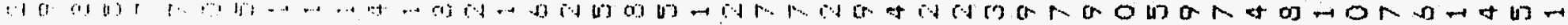
ति

6 $1.1+3$

III

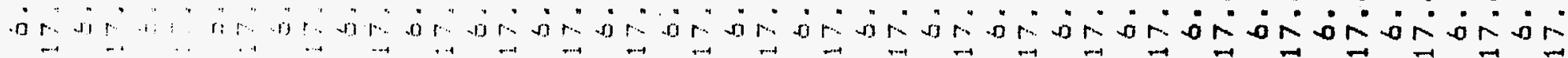

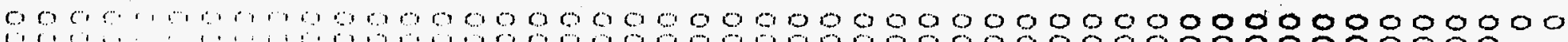
1.

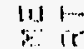

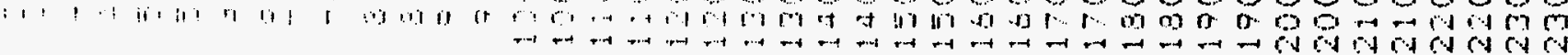

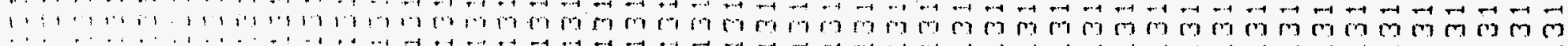
, , i, i,

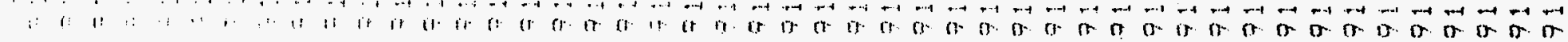




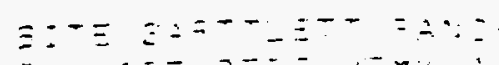

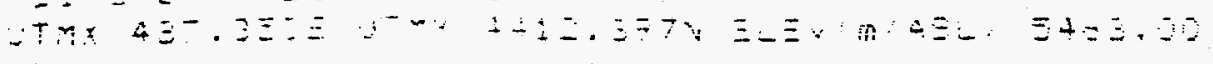

\begin{tabular}{|c|c|c|c|c|c|c|c|c|c|}
\hline 24:三 & $\begin{array}{l}\cdots E \\
a^{\prime}\end{array}$ & $\begin{array}{l}\because+5 \\
\vdots \div F\end{array}$ & 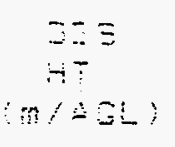 & 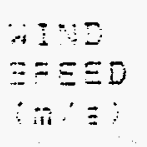 & $\begin{array}{l}0: 30 \\
=2=0 \\
-=8\end{array}$ & 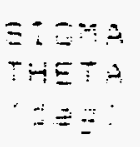 & $1=\Xi \div$ & $\begin{array}{l}z=-2 \\
=-1= \\
=\equiv-\end{array}$ & $\begin{array}{l}\because=5 \\
\because=5\end{array}$ \\
\hline$=$ & 8 & $\therefore 0$ & 5.10 & a. $=$ & $z=-\because$ & $\therefore: \Xi$ & $=\equiv$ & & \\
\hline & $=$ & $: 00$ & $17 . \leq 2$ & 10. & 205.0 & 9. & $\therefore .2$ & $\therefore=$ & $-4+2=$ \\
\hline$\because: \ldots$ & $\because$ & -20 & 5.19 & 3. & $=30$ & $\therefore:$. & $=.3$ & & \\
\hline$\because \therefore z$ & & .0 & $17 .=0$ & $20=$ & $=75.5$ & $9 .=$ & $\therefore:$. & $\therefore 2$ & $-4: . \overline{7}$ \\
\hline$=:-\therefore$ & $\because \because$ & $=0$ & $\therefore \therefore$ & $?: 4$ & $\therefore \because$ & $\therefore:=$ & $\because=$ & & \\
\hline$=$ & & $\cdots 2$ & $\because:=$ & $=:$ & $5+8$ & $\because$ & $\therefore, \Xi$ & $\therefore 7$ & $-93=$ \\
\hline$=: Z$ & $\cdots$ & $\therefore:-$ & 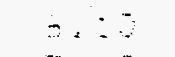 & $\because:$ & $=-\sigma, \sigma$ & 15 & $\overline{7} .3$ & & \\
\hline$\because: 2=$ & $\because \therefore$ & $\therefore$ & $\because \because:=\Xi$ & $\Xi:=$ & $=1.0$ & $\therefore=$ & $\therefore=U$ & $\therefore .4$ & $-3^{-}$. \\
\hline 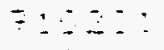 & $\therefore:$ & $=9$ & $=\therefore 2$ & 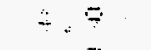 & $20 \div .0$ & 20.7 & 9.7 & & \\
\hline $715=$ & 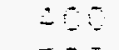 & $=00$ & $1=: \leq$ & $\therefore=$ & 257.0 & i5. & 10.2 & $\therefore$ & $-5=\overrightarrow{7}$ \\
\hline$=1 \div 2=$ & $=: 8$ & $=0$ & $=:-$ & $\Xi=4$ & $\because 7-9$ & $00=4$ & $7=1$ & & \\
\hline 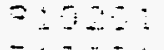 & $\because 2$ & $=0$ & $i 7=5$ & 4.2 & 27.3 .0 & 22.2 & 9.7 & 9.3 & -35.6 \\
\hline $719=$ & $=0$ & 20 & $5=20$ & $\therefore 3$ & $2-0.0$ & 93.1 & 7.4 & & \\
\hline $\begin{array}{l}718201 \\
=19\end{array}$ & $=28$ & 20 & $17=5$ & $3=0$ & $39=0$ & $95=7$ & 7.7 & 0.5 & $-34 \div-$ \\
\hline $\begin{array}{l}10 \\
=19\end{array}$ & $\therefore$ & $\$ 0$ & $=: \square$ & $4=0$ & 27.5 & $14=4$ & 7.5 & & \\
\hline $\begin{array}{ll}710 \\
7102\end{array}$ & $=$ & $\begin{array}{l}200 \\
=00\end{array}$ & $7=5$ & $\begin{array}{l}5.1 \\
-10\end{array}$ & $27-6$ & $7=1$ & $\begin{array}{l}16.7 \\
7.9\end{array}$ & 1.2 & -23.2 \\
\hline $7: 021$ & 30 & $=00$ & $17=58$ & $\therefore$. & $: 8.0$ & 67.5 & 9.8 & $\because$ & -1.9 \\
\hline$=: 0: 21$ & $=0$ & $=200$ & $=.10$ & 2.4 & 172.0 & 67.2 & 5.4 & & \\
\hline $3: 0191$ & $=-0$ & $: 20$ & 17.58 & 2.0 & 127.0 & 54.4 & 6.3 & 0.7 & 44.2 \\
\hline $0102-1$ & 100 & 1100 & \pm .10 & 2.6 & 103.0 & 32.1 & 6.3 & & \\
\hline $71020:$ & 1900 & $1: 00$ & 17.68 & 3.3 & 165.0 & 20.6 & 7.1 & 0.8 & 132.8 \\
\hline $71020:$ & $1: 00$ & 200 & 6.10 & 2.0 & 174.0 & 46.4 & 7.4 & & \\
\hline 71020 & $1: 00$ & $: 200$ & $17=03$ & 2.7 & 151.0 & 39.3 & 9.3 & 0.4 & 171.4 \\
\hline $71020:$ & 1200 & 1300 & 5.10 & 1.0 & 41.0 & 70.1 & 12.3 & & \\
\hline $71020:$ & 1200 & $: 200$ & 17,58 & 1.5 & 55.0 & 51.5 & 12.7 & 0.4 & 308.7 \\
\hline $7: 020:$ & 1300 & 1400 & 5.10 & 1.9 & 157.0 & 17.8 & 15.5 & & \\
\hline 71020 & 1300 & 1400 & 17.53 & 2.4 & 159.0 & 13.9 & 15.4 & -0.1 & 303.5 \\
\hline $71020:$ & 1420 & 1500 & 5.10 & 2.4 & 85.0 & 77.8 & 14.7 & & \\
\hline $940:$ & 14.90 & $: 500$ & 17.63 & 2.9 & 90.0 & 77.8 & 14.3 & 0.1 & 206.1 \\
\hline 71920 & 1500 & 1500 & 5.10 & 3.0 & 1.0 & 17.0 & 11.4 & & \\
\hline 71920 & $1=00$ & 1500 & 17.68 & 3.3 & 352.0 & 18.0 & 11.5 & 0.2 & 113.1 \\
\hline$=10201$ & 1500 & 1700 & 6.10 & 3.0 & 319.0 & 11.0 & 3.7 & & \\
\hline 710201 & $2=00$ & 1700 & 17.59 & 4.2 & 313.0 & 7.4 & 9.5 & 0.5 & $-\overline{5} .7$ \\
\hline $71020:$ & 1700 & 1300 & 5.10 & 2.2 & 275.0 & 26.9 & 5.4 & & \\
\hline $9102=1$ & 1700 & 1300 & 17.58 & 4.0 & 293.0 & 10.9 & 7.5 & 2.1 & -53.0 \\
\hline 910201 & 1300 & 1700 & 6.10 & 1.8 & 247.0 & 12.6 & 4.0 & & \\
\hline $\begin{array}{l}710201 \\
710201\end{array}$ & $\begin{array}{l}1.900 \\
1900\end{array}$ & $\begin{array}{l}1900 \\
2000\end{array}$ & $\begin{array}{r}17.68 \\
6.10\end{array}$ & $\begin{array}{l}2.8 \\
1.9\end{array}$ & $\begin{array}{l}272.0 \\
245.0\end{array}$ & $\begin{array}{l}16.8 \\
12.0\end{array}$ & $\begin{array}{l}6.1 \\
3.9\end{array}$ & 2.1 & $-53 . q$ \\
\hline 910201 & 1700 & 2000 & 17.68 & 2.3 & 258.0 & 9.6 & 4.8 & 0.9 & -56.2 \\
\hline 710201 & 2000 & 2100 & 6.10 & 2.3 & 244.0 & 12.4 & 3.8 & & \\
\hline $\begin{array}{l}910201 \\
910201\end{array}$ & 2000 & 2100 & 17.68 & 3.1 & 256.0 & 10.5 & 4.9 & 1.1 & -50.2 \\
\hline $\begin{array}{l}910201 \\
71020:\end{array}$ & $\begin{array}{l}2100 \\
2100\end{array}$ & $\begin{array}{l}2200 \\
2200\end{array}$ & $\begin{array}{r}6.10 \\
17.63\end{array}$ & $\begin{array}{l}1.9 \\
3.0\end{array}$ & $\begin{array}{l}248.0 \\
259.0\end{array}$ & $\begin{array}{l}16.6 \\
10.2\end{array}$ & $\begin{array}{l}3.7 \\
5.0\end{array}$ & 1.3 & -50.1 \\
\hline $91020 !$ & 2200 & 2300 & 6.10 & 0.8 & 213.0 & 20.4 & 2.4 & & \\
\hline 710201 & 2200 & 2300 & 17.68 & 1.9 & 235.0 & 16.0 & 4.0 & 1.6 & -58.9 \\
\hline 910201 & 2300 & 0 & 6.10 & 0.7 & 226.0 & 15.1 & 2.5 & & \\
\hline 910201 & 2300 & 0 & 17.68 & 1.5 & 237.0 & 16.4 & 4.4 & 1.9 & -59.7 \\
\hline
\end{tabular}




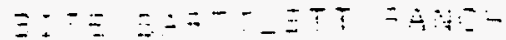

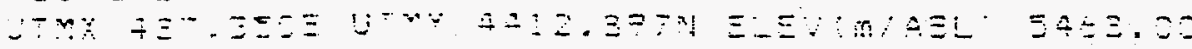

\begin{tabular}{|c|c|c|c|c|c|c|c|}
\hline $\begin{array}{l}\because: E \\
=-5 T\end{array}$ & $\begin{array}{r}*-1 \Xi \\
\Xi O F\end{array}$ & $\begin{array}{c}\text { UE } \\
H \vdots \\
\text { (ด) }\end{array}$ & $\begin{array}{l}\therefore 50 \\
\equiv=\Xi \Xi 0 \\
\therefore: \Xi\end{array}$ & 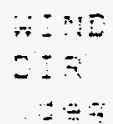 & 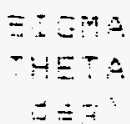 & 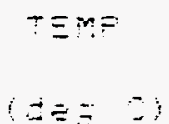 & $\begin{array}{l}5 \equiv-\div \\
Y \equiv M= \\
B \equiv=\end{array}$ \\
\hline
\end{tabular}

\begin{tabular}{|c|c|c|c|c|c|c|c|c|c|}
\hline$=\vdots \cdots$ & 3 & $\therefore 0$ & 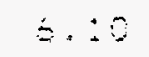 & $\therefore=1$ & $\because-\therefore,-5$ & $=3 . \div$ & -2.2 & & \\
\hline$\because:-\cdots$ & $\because$ & $\because 0$ & $17: \leq 2$ & 2.5 & $\because=5.0$ & $30+\therefore$ & $\therefore=$ & 2.3 & $-5=-$ \\
\hline$=:-1$ & $\therefore \because$ & $\because$ & $5: 3$ & 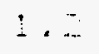 & 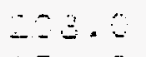 & $\because \therefore$ & $\therefore . \rightarrow$ & & \\
\hline$=:-\cdots$ & $\because \because$ & $\because: 0$ & $\therefore 7$. & $\therefore ?$ & $: \because: .2$ & $\because 2=$ & 3.5 & $2 .=$ & $-5 ?$ \\
\hline$=\cdots=\cdots$ & $-\cdots$ & $\because \because$ & $=\because$ & $\because \because$ & -72.0 & $=5:$ & $\therefore=3$ & & \\
\hline$=\vdots \ldots \ldots$ & $\therefore 3$ & $\because \because$ & $\therefore-3$ & $\vdots \because$ & 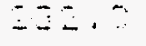 & $\div 7=$ & 2.3 & 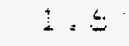 & $-\equiv=$ \\
\hline$=a-\cdots$ & $\because \because=$ & $\therefore \div$ & $\therefore \therefore$ & $\vdots:=$ & $=+2,3$ & -6.5 & -5.8 & & \\
\hline$\because:-y_{0}$ & $=\because 0$ & $\because 3$ & $\therefore \because=5$ & $\vdots=$ & 237.0 & \pm 5.5 & $\therefore$. & $\therefore 5$ & $-4=0$ \\
\hline$=\vdots=\cdots$ & 290 & $\because 0$ & $5: \vdots$ & $\therefore=$ & 240.5 & 11.5 & 2.5 & & \\
\hline$=\cdot \cdots$ & $\therefore \because$ & $\because \because$ & $\because \because=3$ & $1: 7$ & -59.5 & $\vdots: \leq \dot{I}$ & 3.3 & $\because 27$ & $-32,3$ \\
\hline$=: 2: 23$ & $=20$ & $=50$ & $b . \therefore$ & $\vdots=$ & 235.0 & 10.0 & 0.2 & & \\
\hline 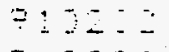 & $=0$ & $=0$ & $17:=3$ & $1: \div$ & $\therefore 4.0$ & $5 \div 5$ & 3.1 & $\therefore .9$ & $-4:=0$ \\
\hline 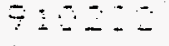 & $=-5$ & 5 & 5.5 & 1.3 & 289.0 & 27.3 & -1.0 & & \\
\hline $9102=$ & $=0$ & $\because 8$ & $17=0$ & 1.5 & $2=0.0$ & 10.5 & 3.2 & $4: 2$ & -47.5 \\
\hline$i=1$ & $-\bar{j}$ & $\ddot{=0}$ & $5 .: 0$ & $i \cdot \vdots$ & $2-7.5$ & 20.7 & $-6 \cdot 3$ & & \\
\hline $715 \div 2$ & $=0$ & $=06$ & 17.53 & 1.5 & $\because 40.6$ & 16.3 & 3.3 & 3.6 & -27.7 \\
\hline $710=-9$ & $=0$ & 700 & 5.10 & 0.5 & $\because 2=0$ & 43.0 & $\because .6$ & & \\
\hline $7: 0=2$ & 30 & 90 & 17.58 & $0=5$ & 227.0 & 10.9 & 5.3 & 2.7 & 40.2 \\
\hline $719=2$ & 720 & $\div 03$ & 6.10 & 9.9 & 1.55 .0 & 70.9 & 8.2 & & \\
\hline$=10202$ & 9.90 & 1200 & $17: 68$ & 0.4 & 192.0 & 22.4 & 7.3 & $1 . i$ & 126 \\
\hline 71020 & 100 & 1100 & 5.10 & 9.6 & 189.0 & 18.7 & 7.3 & & \\
\hline 910232 & 1000 & $\$ 100$ & 17.58 & 1.0 & 180.0 & 10.3 & 7.8 & 0.5 & 256.0 \\
\hline $01020=$ & 1100 & 1200 & $s=10$ & $1 . i$ & 130.0 & 33.0 & 12.3 & & \\
\hline $910-2$ & 1100 & $\div-90$ & $17: 50$ & $1 \times 4$ & $15 b .0$ & 27.9 & $12 \times 2$ & -0.1 & 347 \\
\hline 910292 & 1.00 & $: 300$ & 6.10 & 0.7 & 178.0 & 26.9 & 14.1 & & \\
\hline $910=9$ & 1200 & -300 & $17 . \leq 9$ & 1.4 & 172.0 & 17.9 & 13.7 & -0.2 & 357.3 \\
\hline 910202 & 1300 & 1400 & 6.10 & 1.4 & 94.0 & 13.5 & 14.5 & & \\
\hline $710=0$ & 1300 & 1400 & 17.63 & 1.9 & 63.0 & 21.0 & 14.2 & -0.5 & 30.0 \\
\hline $7+0<-2$ & 1400 & $:=00$ & 6.10 & 1.7 & 32.0 & 13.8 & 13.0 & & \\
\hline 910202 & 140 & $: 500$ & 17.69 & 2.1 & 71.0 & 12.3 & $12=5$ & -0.5 & 928.6 \\
\hline 910292 & 1500 & 160 & 3.10 & 1.2 & 84.0 & $13 \cdot 2$ & 11.3 & & \\
\hline $71090=$ & $1=00$ & $i 500$ & 17.53 & 1.3 & 63.0 & 14.7 & 11.2 & -0.1 & 115.7 \\
\hline 790203 & 1500 & 1700 & 6.10 & 0.3 & 89.0 & 17.2 & 11.2 & & \\
\hline ว10こ2 & 1600 & 1700 & $17=63$ & 1.1 & 64.0 & 13.0 & 11.3 & 0.1 & 1.5 \\
\hline 910202 & 1700 & 1900 & 6.10 & 1.3 & 243.0 & 41.6 & 5.1 & & \\
\hline 710202 & 1700 & 1300 & 17.58 & 1.6 & 274.0 & 53.1 & 7.4 & 2.3 & -59.7 \\
\hline 910202 & 1300 & 1900 & 6.10 & 1.8 & 232.0 & $11 \cdot 3$ & 3.9 & & \\
\hline 91023 & 1900 & 1900 & 17.68 & 2.1 & 252.0 & 11.9 & 6.5 & 2.6 & -65.6 \\
\hline $9: 0202$ & 1900 & 2000 & 6.10 & 1.1 & 210.0 & 70.5 & 2.6 & & \\
\hline 910202 & 1900 & 2000 & 17.68 & 1.5 & 201.0 & 25.9 & 6.2 & 3.6 & -57.1 \\
\hline 910202 & 2000 & 2100 & 6.10 & 1.5 & 231.0 & 32.7 & 1.1 & & \\
\hline 910202 & 2000 & 2100 & 17.68 & 1.5 & 232.0 & 41.4 & 4.5 & 3.4 & -69.0 \\
\hline $71020=$ & 2100 & 2200 & 6.10 & 1.1 & 250.0 & 38.5 & 0.2 & & \\
\hline 91020 & 2100 & 2200 & 17.68 & $1 \cdot 2$ & 243.0 & 58.5 & 3.5 & 3.3 & -67.8 \\
\hline 910202 & 2200 & 2300 & 6.10 & 1.6 & 232.0 & 17.8 & 1.0 & & \\
\hline $91020=$ & 2200 & 2300 & 17.68 & 1.8 & 243.0 & 20.2 & 5.1 & 4.1 & -64.0 \\
\hline $91020=$ & $\begin{array}{l}2300 \\
-700\end{array}$ & 0 & 6.10 & 3.7 & 266.0 & 27.4 & 7.4 & & \\
\hline 910202 & 2300 & 0 & 17.68 & 4.9 & 269.0 & 17.5 & 9.2 & 1.8 & -68.6 \\
\hline
\end{tabular}




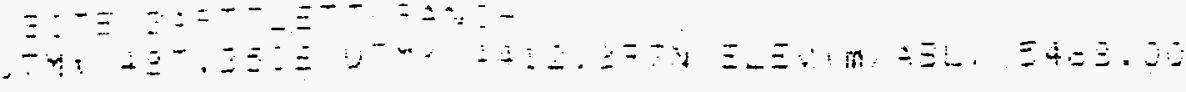

\begin{tabular}{|c|c|c|c|c|c|c|c|c|c|}
\hline 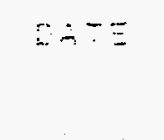 & $\begin{array}{l}\because: \because \equiv \\
\Xi-: T\end{array}$ & $\begin{array}{l}-95 \\
=95\end{array}$ & $\begin{array}{c}=E \equiv \\
47 \\
4: 6 \leq:\end{array}$ & $\begin{array}{l}\cdots \\
\vdots \Xi \equiv 0 \\
\vdots \vdots \vdots\end{array}$ & $\begin{array}{l}3 \\
D \\
\vdots \\
\vdots\end{array}$ & 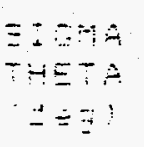 & $\exists \equiv 5 \quad-:$ & 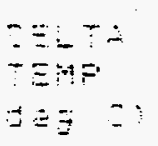 & $\begin{array}{l}\because \equiv \\
\because \pm Z\end{array}$ \\
\hline$\because:-\bar{z}$ & 2 & $\therefore 00$ & 5.10 & 2.5 & $x=4.0$ & $23: 5$ & $\Xi .2$ & & \\
\hline$=2=\bar{z}$ & 5 & 50 & $: 7.03$ & 3.2 & $\Xi \Xi \pm, 0$ & $\therefore \therefore=$ & $\Xi .=$ & $\therefore$. & $-\dot{\Delta}=0$ \\
\hline$=: \cdots$ & $\because \because$ & 50 & $5 . ; 0$ & 0.5 & $z \therefore 0$ & $-\div . \div$ & $\therefore 7$ & & \\
\hline$\because: \div:=$ & $\therefore$ & $a$ & $\therefore y, \leq 3$ & 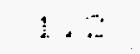 & $=\Xi .8$ & $\because=2$ & $\Xi . \Xi$ & $\therefore=$ & $-5 \dot{s}$ \\
\hline 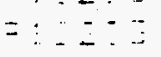 & $\therefore$ & $\because 2$ & $\therefore .8$ & $\therefore$. & $2 a+6$ & $=5.2$ & 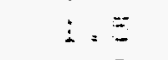 & & \\
\hline$\because 2=2$ & $\therefore$ & $\ldots$ & $-\quad-\vdots$ & i. & $\because 7=0$ & 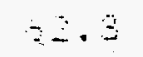 & $\therefore=$ & $\therefore$ & -64 \\
\hline 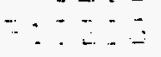 & $\because \cdots$ & $\ldots$ & $=\therefore$ & $\therefore:=$ & $\because=$ & $7=5$ & $\therefore:$ & & \\
\hline 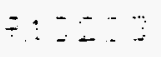 & $\therefore$ & $\therefore-$ & $: 7==8$ & $i=3$ & 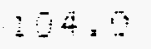 & 73.5 & 0.3 & $\therefore=$ & $-=$ \\
\hline 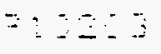 & $\therefore$ & $=8$ & $\div . \therefore$ & $\therefore \Xi$ & $=5.5$ & $\therefore E=$ & $\therefore=$ & & \\
\hline 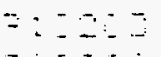 & $\therefore$ & $\because 3$ & $\therefore 7 .=3$ & $\therefore \equiv$ & $=9.9$ & 39.0 & $\therefore .3$ & $\therefore=$ & $-\Xi 7.7$ \\
\hline 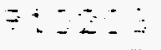 & $\because \cdots$ & $\therefore \because$ & 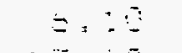 & 3.5 & 23.0 & $=9.0$ & .3 & & \\
\hline$\because \div 9=$ & $\Xi 20$ & $=3$ & $17=68$ & 1.2 & $228 \cdot 0$ & 61.3 & 5.3 & $2 . \equiv$ & $-\equiv 7.3$ \\
\hline $7: 208$ & $=20$ & -9 & b. & $\therefore 2$ & 200,0 & 35.2 & 1.7 & & \\
\hline 61020 & $=90$ & 0 & $17=08$ & 5.9 & 247.0 & 13.9 & 4.5 & 2.6 & $-5=4$ \\
\hline $712=2$ & 0 & 0 & $=.20$ & 1.5 & $=14.0$ & 34.2 & 5.3 & & \\
\hline 710203 & 90 & $\equiv 00$ & $i^{7} .=3$ & 2.0 & 214.0 & 25.2 & 7.3 & 2.0 & -39.0 \\
\hline 910203 & 302 & 700 & $\$ .20$ & \pm .5 & $=0.0$ & 24.4 & $\leq .2$ & & \\
\hline $9: 0202$ & 850 & 90 & $17 \times 3$ & $\therefore$. & $7: .0$ & $2: .5$ & 7.5 & 1.4 & 58.2 \\
\hline$=15292$ & $=89$ & 1200 & 5.10 & 1.3 & 92.0 & 35.9 & 3.4 & & \\
\hline$=10203$ & $=90$ & $: .00$ & 17.58 & 1.7 & 94.0 & 21.3 & 8.2 & -0.2 & 198.6 \\
\hline$=152 \mathrm{Z}$ & 1950 & 1.00 & 5.10 & 0.5 & 84.0 & 18.4 & 10.5 & & \\
\hline 910293 & $1=0$ & 150 & $17 .=0$ & 1.2 & 87.0 & 17.1 & 10.3 & -0.5 & 300.2 \\
\hline $719-3$ & $1=-0$ & 200 & 5.10 & $i .2$ & 124.0 & 57.4 & 13.1 & & \\
\hline 710203 & $1: 0$ & -20 & 17.03 & 1.7 & 114.0 & 55.0 & $12 . \overline{7}$ & -0.2 & 345.7 \\
\hline 915203 & 1250 & $: 200$ & 6.10 & 1.6 & 171.0 & 25.6 & 13.3 & & \\
\hline 919203 & $1 \div 0$ & 1300 & 17.58 & 2.0 & 153.0 & 17.1 & 13.1 & -0.2 & 323.7 \\
\hline 710903 & 1290 & 400 & 5.10 & 1.0 & 54.0 & 42.6 & 14.5 & & \\
\hline 710203 & 1200 & 1400 & 17.69 & 1.3 & 92.0 & 34.3 & 14.3 & -0.2 & 242.5 \\
\hline 910203 & 1400 & $: 500$ & 6.10 & 4.7 & 319.0 & 13.5 & 13.8 & & \\
\hline 010203 & $: 400$ & $:=00$ & 17.63 & 5.1 & 310.0 & 11.6 & 13.7 & -0.1 & 257.4 \\
\hline 910203 & $1 \equiv 00$ & 1500 & 5.10 & 5.3 & 305.0 & 10.5 & 12.3 & & \\
\hline 710203 & 1500 & 1500 & 17.68 & 5.5 & 297.0 & 9.1 & 12.4 & 0.2 & 103.6 \\
\hline 910203 & 1500 & 1700 & 3.10 & 5.1 & 305.0 & 7.1 & 7.3 & & \\
\hline 710203 & 1500 & 1700 & 17.60 & 5.4 & 299.0 & 5.0 & 10.3 & 0.5 & 10.4 \\
\hline 910203 & 1700 & 1800 & 5.10 & 4.0 & 272.0 & 38.4 & 7.8 & & \\
\hline 910203 & 1700 & 1900 & 17.68 & 5.0 & 272.0 & 37.2 & 3.6 & 0.8 & -45.1 \\
\hline 910203 & 1300 & 1900 & 5.10 & 2.0 & 208.0 & 34.5 & 7.0 & & \\
\hline 910203 & 1800 & 1900 & 17.68 & 2.3 & 215.0 & 41.4 & 7.4 & 0.4 & -52.5 \\
\hline 910203 & 1700 & 2000 & 6.10 & 2.0 & 195.0 & 28.1 & 6.6 & & \\
\hline 710203 & 1700 & 2000 & 17.68 & 2.4 & 193.0 & 24.5 & 6.9 & 0.3 & -53.7 \\
\hline 10203 & 2000 & 2100 & 6.10 & 1.9 & 1.37 .0 & 56.5 & 4.1 & & \\
\hline 710203 & 2000 & 2100 & 17.68 & 3.1 & 127.0 & 45.4 & 4.7 & 0.6 & -46.0 \\
\hline 910203 & 2100 & 2200 & 6.10 & 0.2 & 254.0 & 37.9 & 1.8 & & \\
\hline 910203 & 2100 & 2200 & 17.68 & 0.5 & 199.0 & 53.2 & 2.7 & 0.9 & -39.1 \\
\hline 910203 & $2=00$ & 2300 & 6.10 & 0.6 & 253.0 & 32.8 & 0.7 & & \\
\hline 910203 & 2200 & 2300 & 17.68 & 1.0 & 217.0 & 72.3 & 2.3 & 1.6 & $-3 t .4$ \\
\hline 910203 & 2300 & 0 & 6.10 & 1.6 & 243.0 & 12.9 & 0.3 & & \\
\hline 910203 & 2300 & 0 & 17.38 & 2.3 & 248.0 & 14.0 & 1.8 & 1.5 & -43.0 \\
\hline
\end{tabular}




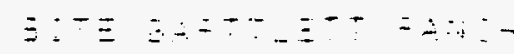

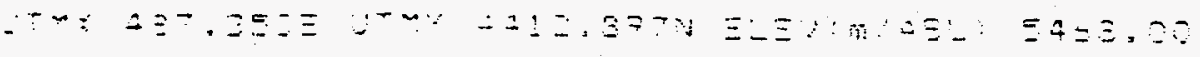

\begin{tabular}{|c|c|c|c|c|c|c|c|c|c|}
\hline$\because \because+\equiv$ & $\begin{array}{l}-\cdots \\
\vdots=\overline{1}\end{array}$ & $\begin{array}{l}-9 E \\
3+0 F\end{array}$ & 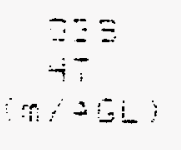 & 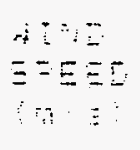 & $\begin{array}{c}\because \vdots \\
\vdots \vdots= \\
\vdots \vdots\end{array}$ & 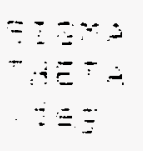 & 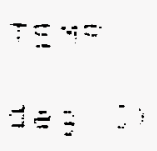 & 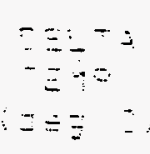 & $\begin{array}{l}\because \equiv- \\
\therefore \Delta D\end{array}$ \\
\hline$=\cdots:$ & $?$ & $: 23$ & 6.10 & $\therefore$ & $\because:=$, & $\because \approx$ & -2. & & \\
\hline 等 & - & $\therefore$ & $17=3$ & $\therefore \dot{1}$ & $\because 2=.9$ & $\therefore::$ & $\therefore 7$ & $\therefore$ & $-1=$ \\
\hline$\because \because 4$ & - & $\because \because$ & $\Delta .: 0$ & $\vdots$ & $\because z-\therefore$ & $\because a=$ & $\vdots .5$ & & \\
\hline$\therefore \cdots-$ & $\therefore$ & $\because$ & $\vdots 7 .=9$ & $\therefore=$ & $\div \therefore, \square$ & $\therefore 5.5$ & $\because$ & 3.7 & $-78=$ \\
\hline 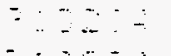 & $\because$ & & $=\ldots$ & $\therefore$ & $-23 \cdot 0$ & $\therefore-$ & $2: 4$ & & \\
\hline$\therefore$ & & $\because:$ & $\vdots^{\cdots} \cdot=\Xi$ & $\therefore$ & $=43=$ & $\therefore E$ & $=2$ & $\therefore .4$ & $-\equiv 2.4$ \\
\hline $7: 9: \div$ & $=\therefore$ & $\therefore$ & $=\therefore$ & 2 & $5: 25$ & $34 .=$ & $\therefore=7$ & & \\
\hline$=: 9-2: 2$ & $\because$ & $\therefore \because$ & 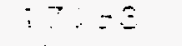 & $\therefore=$ & $=0=0$ & $5: 3$ & 2.9 & $2=9$ & $=\therefore .=$ \\
\hline$=: 202$ & $\therefore-\therefore$ & $=2$ & $=: \therefore$ & $\therefore$ & $\therefore=2.0$ & $\because 2.2$ & $\therefore=$ & & \\
\hline$=: 025$ & 29 & $=20$ & $: \because \ldots$ & $\therefore$. & 7.0 & 92.9 & 2.9 & $a$ & -52.5 \\
\hline$=192 \%$ & $=2$ & $=0$ & $5 .: 8$ & $\therefore$ & $1=1.3$ & 47.3 & 0.5 & & \\
\hline$=29 \%$ & $\Xi \because$ & $=8$ & $17 . \pm 9$ & $\therefore$ & 104.9 & $\therefore \leq .2$ & $\therefore 2$ & $\therefore .5$ & $-\Xi \pm .5$ \\
\hline$=182$ & $=90$ & 200 & $=.: 0$ & $\therefore$ & $\because 2=0$ & 30.0 & $-1=0$ & & \\
\hline 71801 & $=8$ & 90 & $\div 7=43$ & $\therefore=3$ & $157=0$ & $\because 5.2$ & $1=1$ & $2 . !$ & $-50=2$ \\
\hline$=: 024$ & $\because$ & 300 & 5.10 & $\because .3$ & 295.6 & 15.5 & -0.9 & & \\
\hline$\because: 8=$ & -20 & 800 & $17, \leq 8$ & \pm .5 & 219.0 & 25.7 & $\therefore 2$ & 2.1 & $-3: 4$ \\
\hline$=102 A$ & $\equiv$ & 700 & $=.50$ & $0=$ & 293.0 & $61 \times 1$ & 4.2 & & \\
\hline $718=4$ & 300 & 700 & 17.08 & $1=0$ & -57.0 & 49.3 & 4.5 & 0.3 & 76.4 \\
\hline 71024 & $=90$ & $: 90$ &. .10 & 9.9 & $\$ 7.0$ & 27.7 & 3.4 & & \\
\hline 710244 & 70 & $: 00$ & $17=88$ & $0 .=$ & 52.0 & 27.4 & 3.1 & -0.3 & 202.6 \\
\hline 71024 & $1 \because 20$ & $\therefore 20$ & $5 .: 0$ & 1.0 & 173.0 & 29.1 & 9.3 & & \\
\hline $7: 0204$ & 1000 & $: 100$ & 17.58 & 1.4 & 162.0 & 35.5 & 9.2 & -0.1 & 296.4 \\
\hline$=102-4$ & $1: 20$ & $: 200$ & 6.10 & 2.0 & 31.0 & 53.7 & 9.4 & & \\
\hline $9: 9294$ & $1: 50$ & -200 & 17.68 & $=.3$ & 32.0 & 67.8 & 9.2 & -0.2 & 348.1 \\
\hline $710=04$ & 1200 & $\therefore=00$ & 6.10 & 3.2 & 48.0 & 10.3 & 8.7 & & \\
\hline 710254 & $1=0$ & 1300 & 17.68 & 3.5 & 44.0 & 9.3 & 3.4 & -0.3 & 347.4 \\
\hline 710204 & 1300 & $: 200$ & $b=10$ & 3.3 & 27.0 & 21.6 & 3.8 & & \\
\hline 910204 & $: 700$ & 1400 & 17.58 & 3.5 & 23.0 & 21.4 & 3.5 & -0.3 & 310.5 \\
\hline 710204 & 1400 & 5500 & $\leq 10$ & 3.3 & 14.0 & 11.8 & 3.4 & & \\
\hline 710204 & 1450 & $: 500$ & 17.58 & 3.2 & 10.0 & 13.5 & 8.4 & 0.0 & 230.1 \\
\hline 710204 & $1=0$ & $: 500$ & 6.10 & $3 . \overline{3}$ & 3.0 & 9.1 & 8.3 & & \\
\hline 710204 & 1500 & $i \leq 00$ & 17.69 & 3.2 & 3.0 & 11.5 & 3.4 & 0.1 & 121.1 \\
\hline$=10204$ & 1500 & 1700 & 6.10 & 2.2 & 347.0 & 10.3 & 7.5 & & \\
\hline 710204 & 1500 & 1700 & 17.58 & 2.8 & 338.0 & 9.0 & 7.6 & 0.1 & 8.7 \\
\hline 919204 & 1700 & 1300 & 6.10 & 1.2 & 250.0 & 34.6 & 4.0 & & \\
\hline 710204 & 1700 & 1000 & 17.68 & 1.8 & 280.0 & 32.9 & 4.5 & 0.5 & -50.5 \\
\hline$=10204$ & 1300 & 1700 & 6.10 & 1.3 & 237.0 & 10.1 & 1.0 & & \\
\hline 710204 & 1300 & 1900 & 17.38 & 1.7 & 254.0 & 8.8 & 2.6 & 1.6 & -53.3 \\
\hline 910204 & 1900 & 2000 & 6.10 & 0.7 & 235.0 & 7.1 & -0.4 & & \\
\hline $710=4$ & 1900 & 2000 & 17.68 & 0.8 & 225.0 & 8.9 & 3.1 & 3.5 & -53.2 \\
\hline 710204 & 2000 & 2100 & 6.10 & 0.9 & 242.0 & 12.8 & -0.7 & & \\
\hline 910204 & 2000 & 2100 & 17.68 & 0.9 & 256.0 & 13.5 & 1.9 & 2.6 & -52.5 \\
\hline 910204 & 2100 & 2200 & 6.10 & 1.4 & 236.0 & 6.7 & -1.3 & & \\
\hline 710204 & 2100 & 2200 & 17.68 & 1.2 & 254.0 & 5.9 & 1.6 & 2.9 & -50.9 \\
\hline 910204 & 2200 & 2300 & 6.10 & 1.7 & 234.0 & 6.6 & -1.6 & & \\
\hline 910204 & 2000 & 2300 & 17.68 & 1.5 & 251.0 & 6.4 & 1.6 & 3.2 & -51.7 \\
\hline 910204 & 2300 & 0 & 6.10 & 0.7 & 224.0 & 14.2 & -1.8 & & \\
\hline 910204 & 2300 & 0 & 17.88 & 1.1 & 225.0 & 16.3 & 0.1 & 1.9 & -55.1 \\
\hline
\end{tabular}




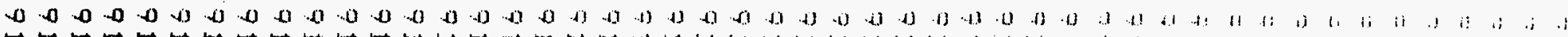

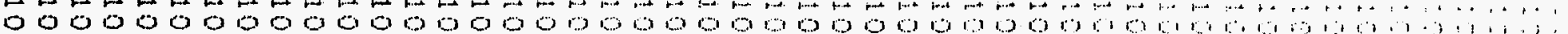

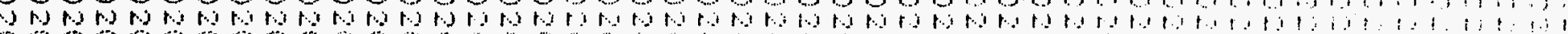

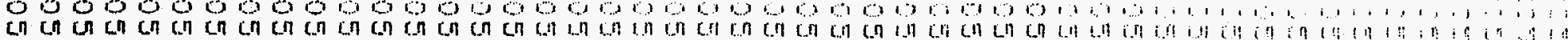

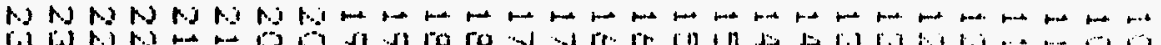

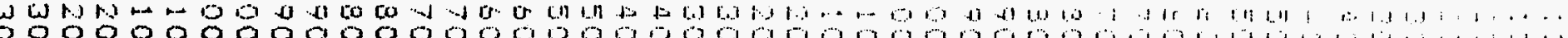

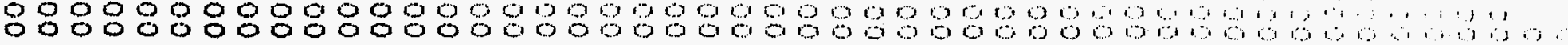

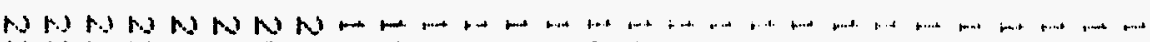

W0

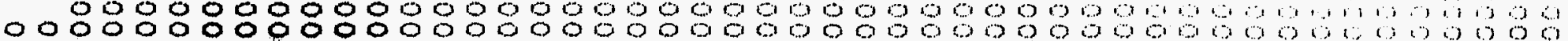

-

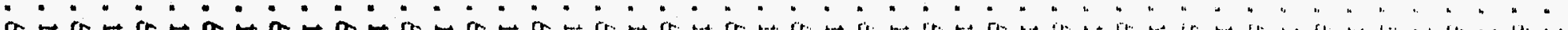

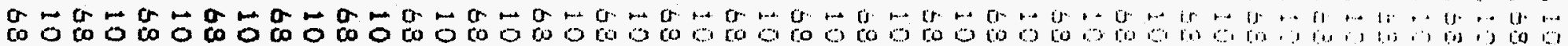

מOMOMONH ova $\begin{gathered}0 \\ 0\end{gathered}$

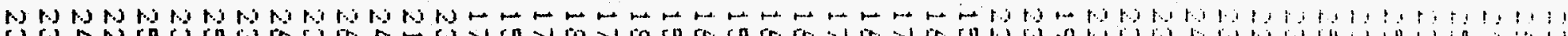

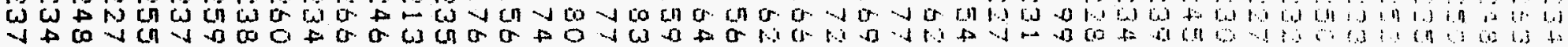

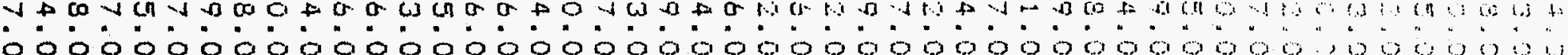

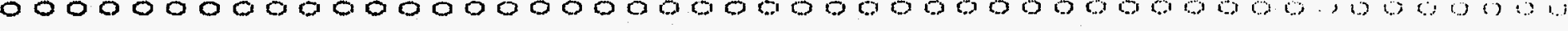

0 ค

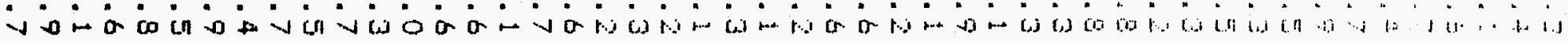

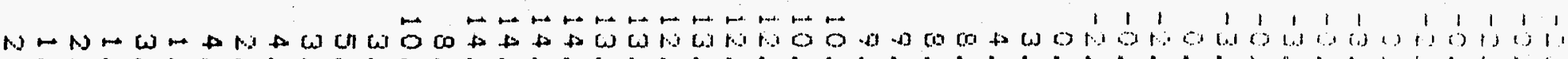

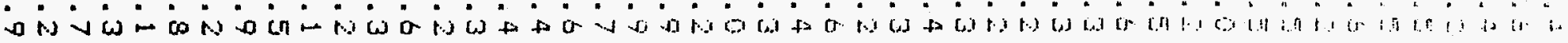

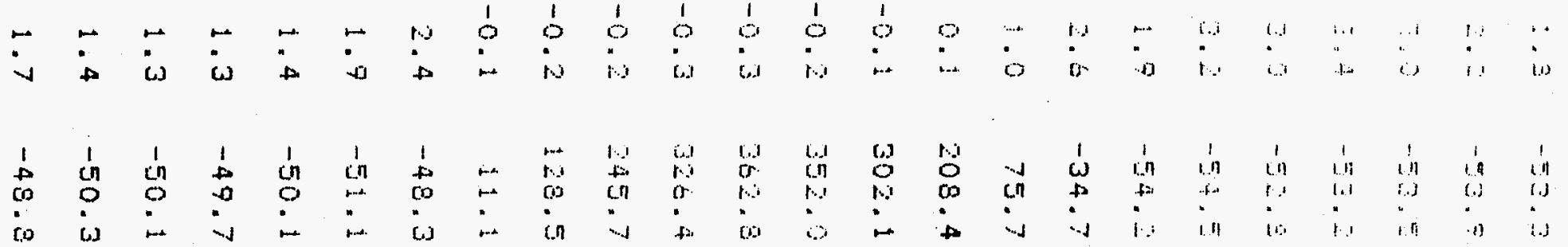




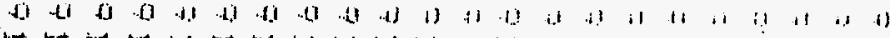
ब1

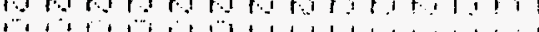

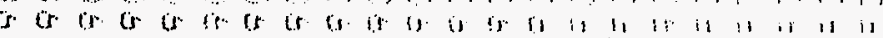

$\cdots$

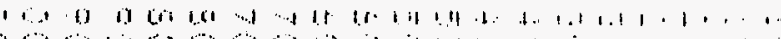

86000

- w.........

a कа

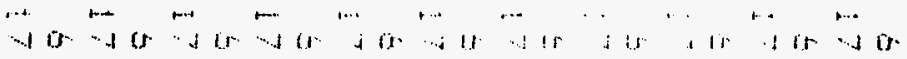

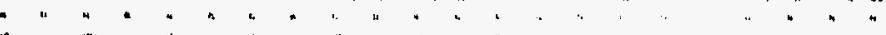
o. 0 o

tnit "

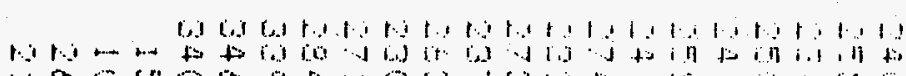
$\div$ -

\%

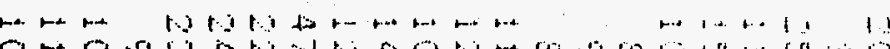

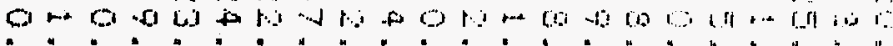

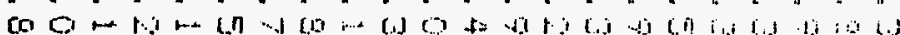

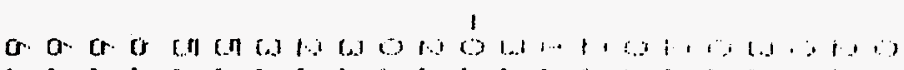

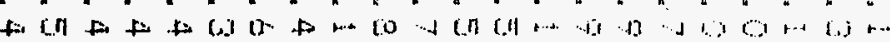

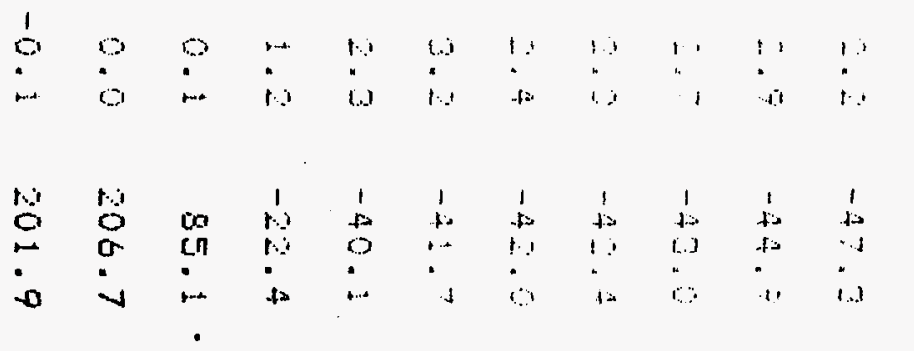




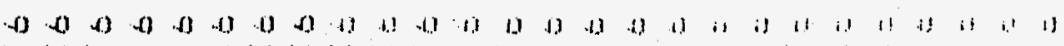
क

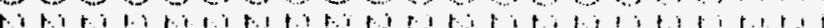

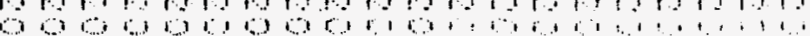

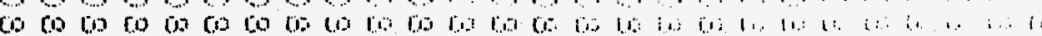

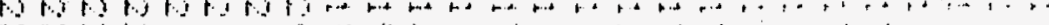
(3)

क

, $, \because, 1,3,2$

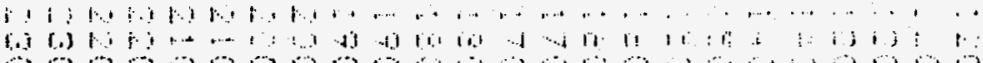

о69

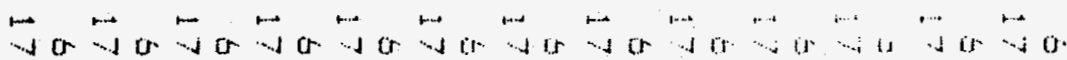

a $\begin{gathered}0 \\ 0\end{gathered}$

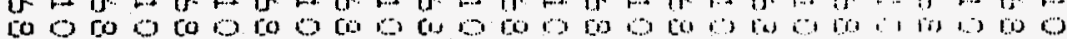

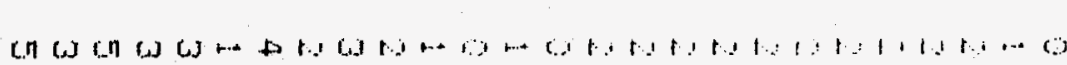

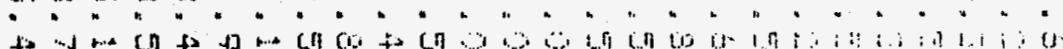

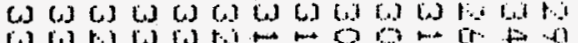

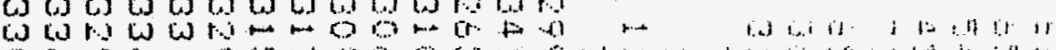

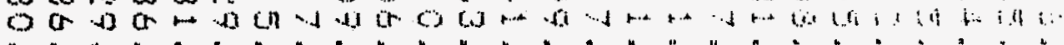

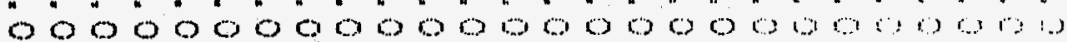

an $\cos \pi-40$

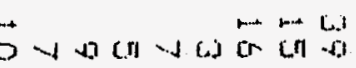

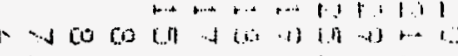

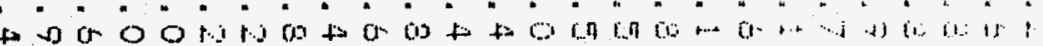

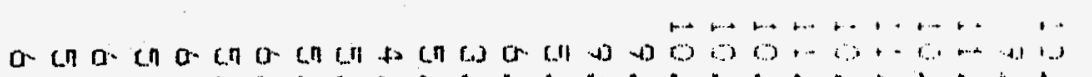

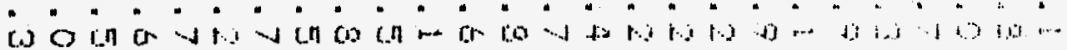


$\Xi:-\because: \cdots \equiv=2 \because \vdots-$

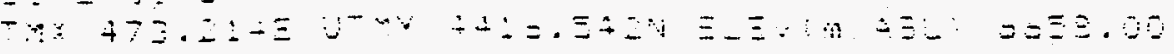

\begin{tabular}{|c|c|c|c|c|c|c|c|c|c|}
\hline$\because-5$ & $\begin{array}{l}T: n E \\
\equiv T E T\end{array}$ & $\begin{array}{l}-:= \\
=10\end{array}$ & $\begin{array}{c}2 E= \\
+4 \\
\operatorname{mol}\end{array}$ & 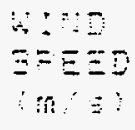 & $\begin{array}{l}\therefore= \\
\therefore i= \\
z=\Xi\end{array}$ & 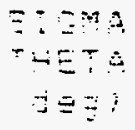 & $\because \Xi \Xi Z=$ & 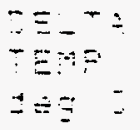 & $\begin{array}{l}\because \Xi 5 \\
\because \div 9\end{array}$ \\
\hline$=$ & $\therefore=-0$ & $:=00$ & $\dot{0} \cdot 10$ & $I=$ & 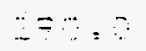 & $\because z=:$ & $\vdots \Xi=\overline{3}$ & & \\
\hline$\because \because$ & $\therefore:-0$ & $: 50$ & 17.50 & 4.4 & $\therefore \theta-3$ & 49.5 & $1 \overline{3}=1$ & -8.4 & 我 \\
\hline 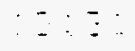 & $\because: \because$ & $\vdots=-2$ & 5.15 & 4.4 & $30=5$ & 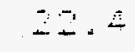 & $\vdots \ddot{-} .4$ & & \\
\hline$\because \therefore$ & $\because \because$ & $:=:$ & $\therefore \because \div: 3$ & $\exists=\stackrel{3}{-}$ & $\ddot{z}=\ddot{\square}$ & $24=$ & $1=2$ & $\because \because$ & $\because \vdots=\vdots$ \\
\hline$\therefore:$ & 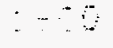 & $\therefore \quad \therefore$ & $=\vdots$ & 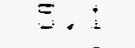 & $\because 2.2$ & 19.5 & 10,7 & & \\
\hline 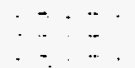 & & & 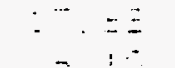 & $\begin{array}{l}=- \\
=-\end{array}$ & $\begin{array}{l}\because=-5 \\
\therefore=-3\end{array}$ & $\begin{array}{c}1= \\
z\end{array}$ & $\begin{array}{r}15= \\
7=\end{array}$ & $\because-i$ & $-\xi 4, \equiv$ \\
\hline 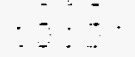 & & . : - & $\because . \pm$ & $=\quad \because$ & -91.5 & 7.7 & 7.5 & $\therefore=:$ & $-5 \div, \overline{3}$ \\
\hline$y:=$ & $\therefore \therefore \div$ & $=\because$ & $=\square$ & $=\therefore$ & -53.5 & 5.5 & 15.7 & & \\
\hline$\therefore::$ & $: \vdots 2$ & $:=-\because$ & $\vdots^{-}=\Xi$ & \pm .2 & -72.0 & 3.0 & 10.2 & 5.5 & -95.4 \\
\hline$\because: \because$ & $:=8$ & 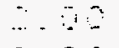 & $=29$ & $\therefore 5$ & $28=0$ & 10.7 & 9.5 & & \\
\hline$\therefore:=$ & $:=8$ & $\therefore-5$ & $0^{7}:=2$ & $=.5$ & $=7+9$ & 7.9 & 9.6 & 0.1 & $-7:=:$ \\
\hline & $2 \because 5$ & $2: 09$ & $\dot{B}:$ & $\equiv$. & $=97.0$ & 7.3 & 3.9 & & \\
\hline$\therefore=i$ & $=-1$ & $\because=0$ & $\because \because, 63$ & $\because: 3$ & $-35=0$ & 9.7 & 7.2 & 6.3 & -93.7 \\
\hline$: 2:$ & $\because: \because 0$ & $\therefore \therefore$ & $8, \therefore$ & $4:-$ & 236.0 & 12.2 & 0.8 & & \\
\hline $13 !$ & 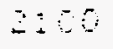 & -200 & $\because 7.53$ & 4.4 & -53.0 & 13.0 & 7.1 & 0.3 & -76.6 \\
\hline$i=!$ & $2-0$ & $\therefore=90$ & $5,: 0$ & $3=3$ & 285.0 & 20.7 & 3.4 & & \\
\hline$\div 2 !$ & $\because=90$ & $\therefore=00$ & 17.63 & 9.7 & 230.0 & 22.1 & 9.0 & 3.6 & -57.4 \\
\hline$\therefore:$ : & $\because 300$ & 5 & 0.20 & 4.4 & $\ddot{2} 53.0$ & 13.3 & 9.2 & & \\
\hline $13:$ & $\because 300$ & 3 & $17: 53$ & 4.9 & 201.0 & 18.6 & 7.2 & 0.0 & -72.8 \\
\hline
\end{tabular}




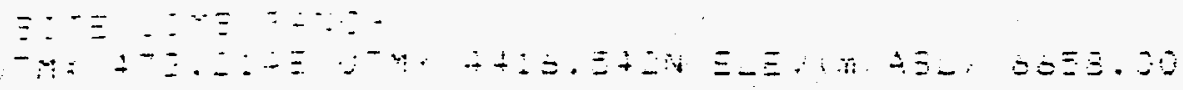

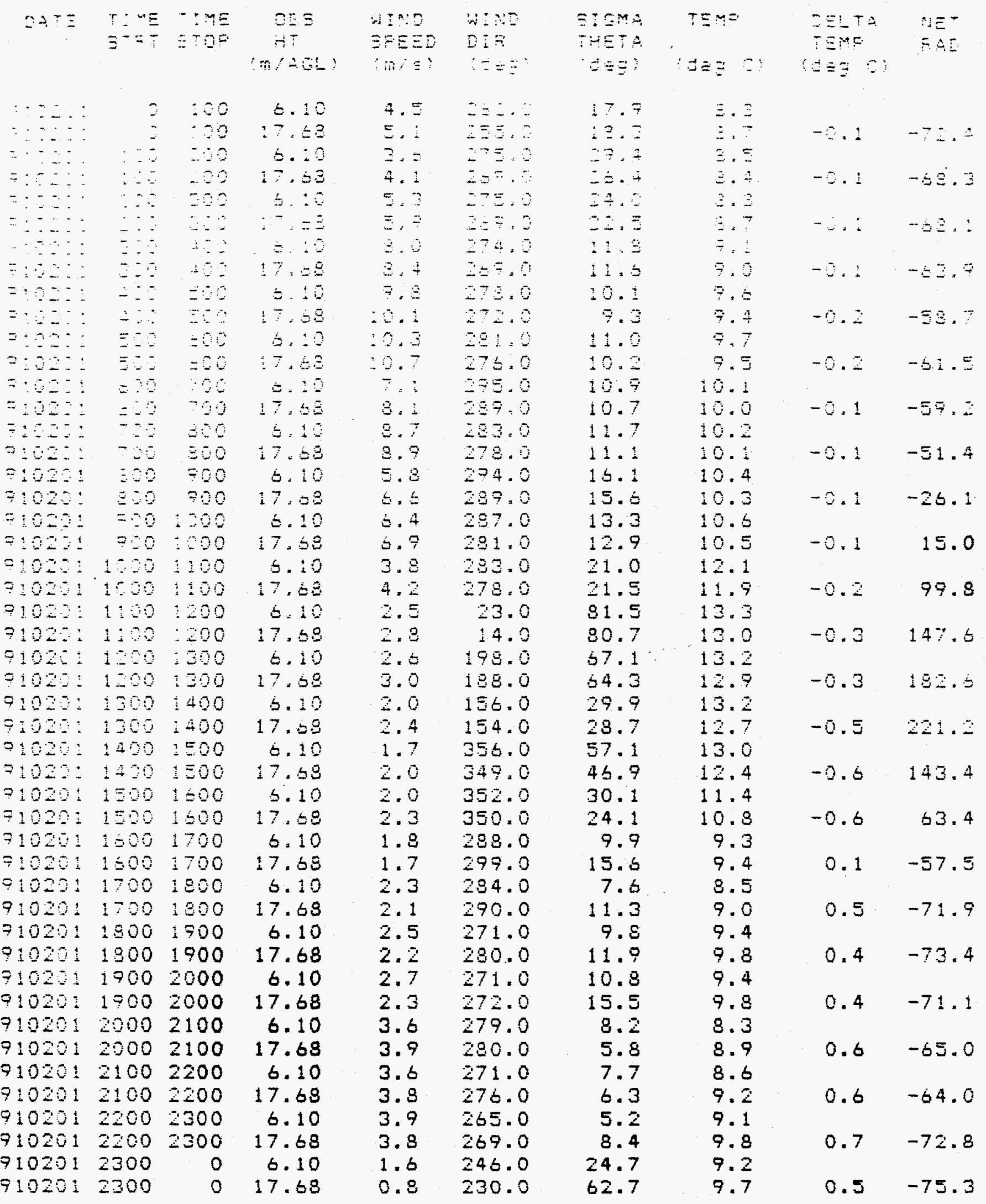




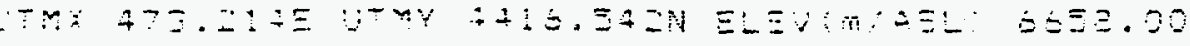

\begin{tabular}{|c|c|c|c|c|c|c|c|c|c|}
\hline DATE & $\begin{array}{l}5: \equiv \\
\Xi-7 T\end{array}$ & $\begin{array}{l}\text { TIE } \\
\text { ETOF }\end{array}$ & $\begin{array}{c}205 \\
-17 \\
\text { की }\end{array}$ & $\begin{array}{l}110 \\
\equiv=E \equiv 0 \\
n \\
n\end{array}$ & $\begin{array}{r}\cdots \\
\because:= \\
\therefore:\end{array}$ & 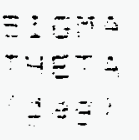 & 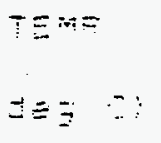 & 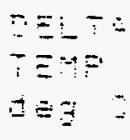 & $\begin{array}{l}\because \equiv- \\
\because \Xi Z\end{array}$ \\
\hline$\because: \div:=$ & 3 & 100 & 6.10 & $\vdots . \leq$ & 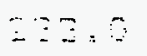 & $10=2$ & $\Xi=$ & & \\
\hline$\because: \because \cdots$ & 8 & $: 00$ & $17, \leq 3$ & 0.7 & $\therefore=0$ & 43. & $2: 3$ & $\therefore$. & $-7 \leq .:$ \\
\hline$\because: \because$ & $\therefore 2$ & $\therefore 20$ & $\leq+19$ & $\therefore .=$ & $-\because=\because$ & $\therefore .9$ & $\therefore=$ & & \\
\hline$\because: \cdots$ & 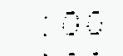 & 80 & $\div 7.58$ & 5.5 & $2 \leq .9$ & $2=5$ & $E=$ & & $-7 \because, 4$ \\
\hline$\because \because \therefore \because$ & $\because \theta$ & $\because 8$ & $=\therefore$ & $\therefore=$ & $\because-2.0$ & $y=:$ & $\because=$ & & \\
\hline$=:-z:$. & $\because 2$ & 800 & $: \because .50$ & $\therefore$ & $\because 2.0$ & $=3.4$ & $\Sigma$. & $\therefore$ & $-7=\vdots$ \\
\hline$=:-2$ & $\because 8$ & -50 & 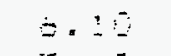 & $\vdots=\vdots$ & $\therefore=5.0$ & $\therefore 9$ & 5.5 & & \\
\hline$\because: \because 2:-$ & 3 & -00 & $i 7, \leq 3$ & $\therefore=$ & $\because 4.0$ & 73.4 & $\leq .9$ & $\therefore 5$ & -5. \\
\hline$\because 10$ & $\therefore 3$ & $=0$ & $=.5$ & $\therefore .7$ & $25 \leq 0$ & 30.1 & $\therefore$. & & \\
\hline $7: 52=$ & 5 & $=50$ & $: 7=\leq 3$ & $\because=$ & 29.9 & 54.7 & 6.7 & 5.3 & -55.5 \\
\hline$\because: 2=$ & $\Xi 0$ & $=00$ & $=.: 0$ & $\because 9$ & 250.0 & $22=4$ & 7.3 & & \\
\hline$=2$ & $\Xi 20$ & $=00$ & $\leq 7 . \leq 8$ & $\therefore 0$ & $\therefore=7.0$ & 54.0 & $\therefore 4$ & 2.1 & -51.5 \\
\hline $9102=2$ & $=0$ & 70 & $\leqslant .10$ & 1.4 & $=49.0$ & $33 \cdot 1$ & $3 \cdot 3$ & & \\
\hline $\begin{array}{l}=.020 \\
=.60\end{array}$ & $=00$ & 702 & $17,=2$ & $\because 1$ & $-20 \cdot 0$ & 75.5 & 3.9 & 0.3 & -67.4 \\
\hline $\begin{array}{l}71920 \\
7100-\end{array}$ & 80 & $\therefore 00$ & $=: 10$ & 3.7 & $\angle E=0$ & 57.1 & 10.7 & & \\
\hline $\begin{array}{l}71020 \\
71020\end{array}$ & $\begin{array}{l}700 \\
300\end{array}$ & 200 & 17.68 & $4=4$ & -7.6 & 56.4 & 11.2 & 0.3 & -57.3 \\
\hline $\begin{array}{l}71020 \% \\
9+020\end{array}$ & $\begin{array}{l}300 \\
890\end{array}$ & 700 & 5.10 & 4.2 & $3 \leq .0$ & $51: 2$ & 11.4 & & \\
\hline $\begin{array}{l}310202 \\
710202\end{array}$ & $\begin{array}{l}820 \\
950\end{array}$ & 700 & $17 . \leq 3$ & $=2$ & -98.0 & 45.5 & $\begin{array}{ll}11=5 \\
13\end{array}$ & 0.1 & 18.5 \\
\hline $\begin{array}{l}10202 \\
=10202\end{array}$ & $=00$ & 100 & 0.10 & 3.7 & 305.0 & 15.5 & 13.5 & & \\
\hline & $\begin{array}{r}-20 \\
100\end{array}$ & 1.00 & 17.63 & 4.7 & 300.0 & 14.2 & 13.3 & -0.2 & 90.1 \\
\hline $\begin{array}{l}71020= \\
710202\end{array}$ & & 1100 & $\therefore .10$ & 2.0 & 121.0 & 74.8 & 13.8 & & \\
\hline $\begin{array}{l}710202 \\
910202\end{array}$ & $\begin{array}{l}1.00 \\
1.00\end{array}$ & 1100 & 17.68 & 2.3 & 47.0 & 80.1 & 13.6 & -0.2 & 240.0 \\
\hline 71020 & $1: 00$ & $: 200$ & $\leq 10$ & 2.4 & 172.0 & 20.2 & 13.6 & & \\
\hline $71020=$ & 1100 & 200 & 17.68 & 2.7 & is 8.0 & 17.1 & 13.1 & -0.5 & 234.7 \\
\hline 90202 & 1200 & 1300 & 5.10 & 2.2 & 131.0 & 23.1 & 15.0 & & \\
\hline 710202 & 1200 & 1300 & 17.53 & 2.4 & 131.0 & 25.5 & 14.1 & -0.7 & 315.5 \\
\hline$\because 1020$ & 1300 & 1400 & 5.10 & 2.0 & 73.0 & $43 \cdot 1$ & 14.5 & & \\
\hline 910202 & 1300 & 1400 & 17.58 & 2.2 & 57.0 & 45.7 & 13.4 & -1.1 & 270.4 \\
\hline 710292 & 1400 & 1500 & 6.10 & 1.0 & 101.0 & 40.3 & 15.2 & & \\
\hline 71020 & 1400 & 1500 & 17.68 & i. 1 & 103.0 & 45.3 & 14.7 & -0.5 & 180.8 \\
\hline 710202 & $1=00$ & 1500 & 6.10 & $i \cdot i$ & 107.0 & 67.5 & 13.0 & & \\
\hline$=10202$ & $i=00$ & 1500 & 17.63 & 1.0 & 112.0 & 73.2 & 12.7 & -0.3 & 45.4 \\
\hline 710202 & 1600 & 1700 & 6.10 & 1.5 & 273.0 & 41.1 & 10.1 & & \\
\hline 710202 & 1600 & 1700 & 17.68 & 1.5 & 271.0 & 57.5 & 10.7 & 0.6 & -77.7 \\
\hline 910202 & 1700 & 1500 & 6.10 & 1.5 & 250.0 & 75.8 & 10.0 & & \\
\hline 910202 & 1700 & $\$ 900$ & 17.68 & 1.9 & 242.0 & 97.6 & 10.6 & 0.6 & -73.1 \\
\hline 910202 & 1300 & 1700 & 6.10 & 4.9 & 297.0 & 20.0 & 11.1 & & \\
\hline 710202 & 1800 & 1900 & 17.68 & 6.2 & 254.0 & 21.9 & 11.2 & 0.1 & -102.5 \\
\hline 710202 & 1700 & 2000 & 6.10 & 5.7 & 274.0 & 41.2 & 11.4 & & \\
\hline 710202 & 1900 & 2000 & 17.68 & 6.3 & 289.0 & 36.5 & 11.3 & -0.1 & -110.6 \\
\hline 910202 & 2000 & 2100 & 6.10 & 5.7 & 294.0 & 17.3 & 10.8 & & \\
\hline 910202 & 2000 & 2100 & 17.68 & 6.7 & 287.0 & 15.5 & 10.8 & 0.0 & -105.7 \\
\hline 910202 & 2100 & 2200 & 6.10 & 5.9 & 297.0 & 11.7 & 10.0 & & \\
\hline 910202 & 2100 & 2200 & 17.63 & 7.1 & 291.0 & 10.7 & 10.0 & 0.0 & -102.4 \\
\hline 910202 & 2200 & 2300 & 6.10 & 8.3 & 292.0 & 14.3 & 9.9 & & \\
\hline 910202 & 2200 & 2300 & 17.68 & 9.2 & 237.0 & 13.8 & 9.8 & -0.1 & -105.7 \\
\hline 910202 & 2300 & 0 & 6.10 & 10.1 & 88.0 & 14.1 & 10.2 & & \\
\hline 910202 & 2300 & 0 & 17.68 & 10.9 & 283.0 & 13.3 & 10.0 & -0.2 & -111.7 \\
\hline
\end{tabular}




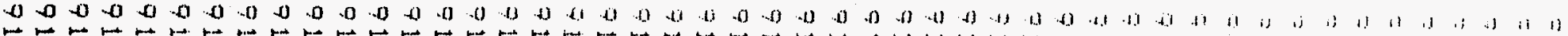
боб ond

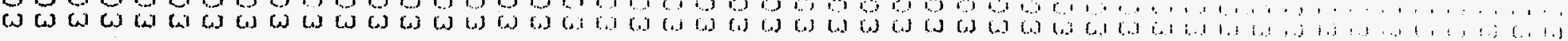
w

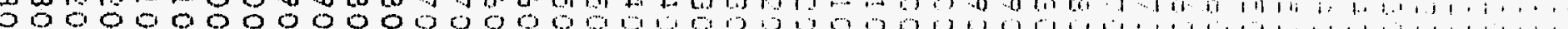

$98989898909890960 \%$

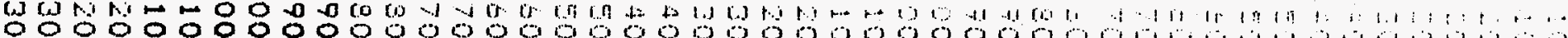
о

Ta o

- - - -

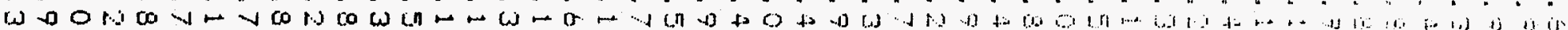

a d w

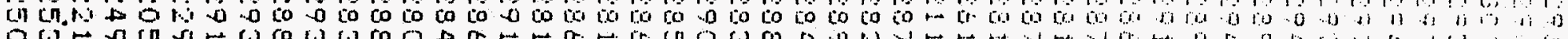

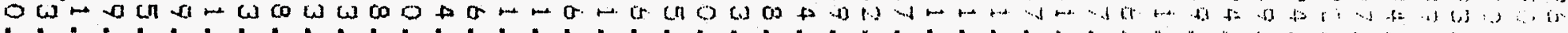

00000000000000000000000000000000 क

W由 م

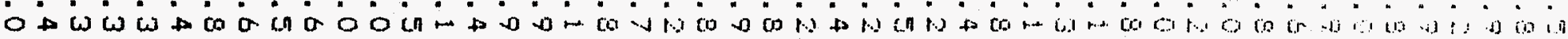

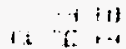

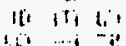
a.

NWNAw

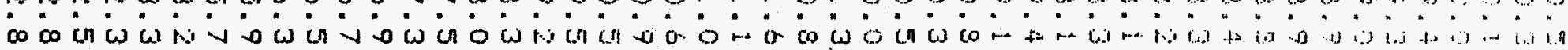

\begin{tabular}{|c|c|c|c|c|c|c|c|c|c|c|c|c|c|c|c|c|c|c|c|c|c|c|c|}
\hline & & & 1 & 1 & 1 & 1 & 1 & 1 & 1 & 1 & 1 & 1 & 1 & 1 & 1 & 1 & 1 & 1 & 1 & 1 & 1 & 1 & $i$ \\
\hline 0 & 0 & 0 & 0 & 0 & 0 & 0 & 0 & $O$ & $\infty$ & 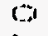 & 0 & 0 & 0 & 0 & 0 & $\%$ & 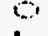 & \% & $\therefore$ & $\therefore j$ & 0 & (1) & $\vdots$ \\
\hline 0 & $M$ & m & 10 & i.) & $m$ & $\omega$ & $\omega$ & $\omega$ & 5 & 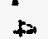 & un & in & in & in & (1) & $\cdots$ & $\leftrightarrow$ & ד. & $\ldots$ & $\therefore$ & $\ldots$ & $\ldots$ & $i$ \\
\hline 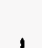 & 1 & t & I & 1 & t & t & t & & mo & t. & f. & 1. & $t .1$ & $m$ & & 1 & 1 & 1 & 1 & 1 & 1 & 1 & 1 \\
\hline en & in & in & 10 & -4 & 60 & $\because$ & + & in & $-\sqrt{0} t$ & 0 & 0 & co & in & $+\infty$ & 10 & co & $\delta$ & $\therefore$ & $\Leftrightarrow$ & $\therefore$ & ' & \&) & ... \\
\hline & + & $\mathrm{cn}$ & +2 & co & 0 & +2 & $f^{2}$ & $c n$ & 60 & t.t & (U) & ris & (.n) & $w$ & -1 & is & -4.1 & $\therefore$ & 1) & , & $\therefore$ & 6 & $\therefore$ \\
\hline & • & . & a & . & • & $\cdot$ & - & : & . & 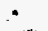 & 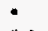 & . & - & • & - & : & • & 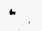 & " & . & " & . & . \\
\hline 1,1 & $\infty$ & 0 & or. & (S) & $\checkmark d$ & -1 & 0 & tr. & 4 & -1.1 & (C) & 0 & $\boldsymbol{\alpha}$ & N & 10 & 6. & $f=1$ & $f: 1$ & is & $1 r$ & $\cdot 1$ & if. & 11 \\
\hline
\end{tabular}$$
\text { ( }
$$ 


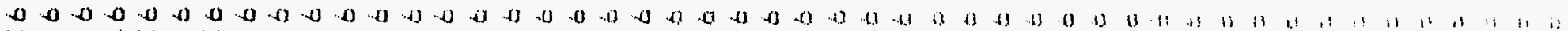
боб .

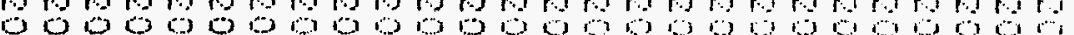

1010 M

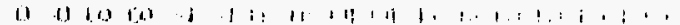

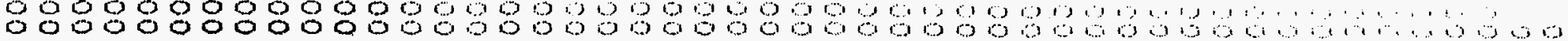

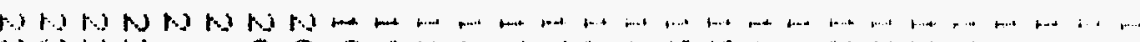

W6

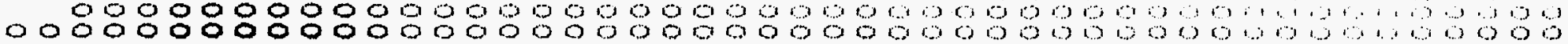

to

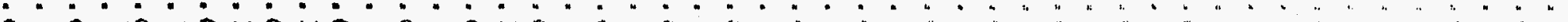

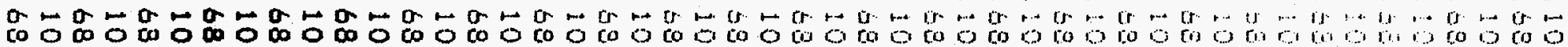

O-OH- jo

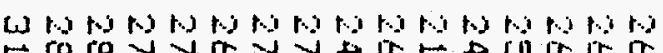

to 0 d

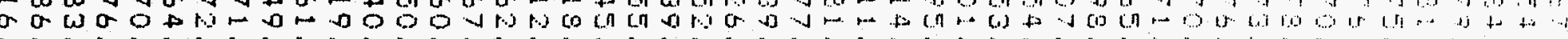

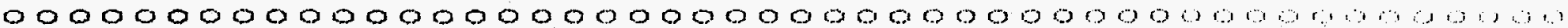

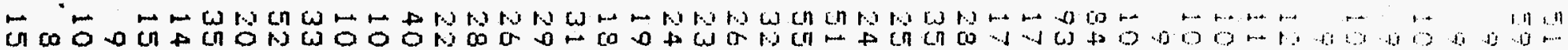
o to

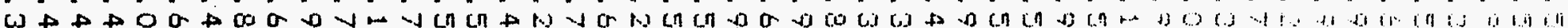

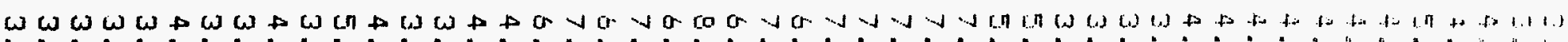

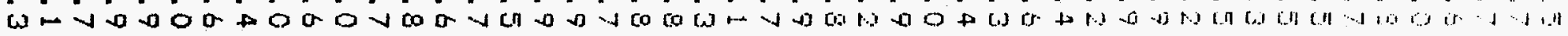


$\equiv:-\equiv \ldots \equiv= \pm \because:$

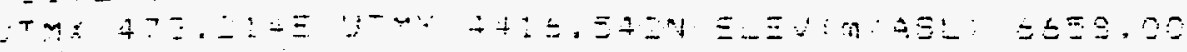

\begin{tabular}{|c|c|c|c|c|c|c|c|c|}
\hline $\begin{array}{l}\because \because \bar{E} \\
\vdots-\bar{T}\end{array}$ & 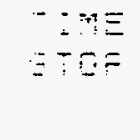 & $\begin{array}{c}2= \\
67 \\
n=0\end{array}$ & $\begin{array}{l}\therefore= \\
a=E \equiv E \\
\therefore . \vdots\end{array}$ & $\begin{array}{l}x y= \\
9= \\
\vdots=\end{array}$ & 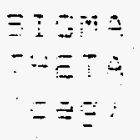 & 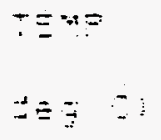 & 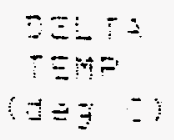 & $\begin{array}{l}\because=- \\
\because \therefore=\end{array}$ \\
\hline 7 & $\therefore 20$ & $=.10$ & $\therefore: \equiv$ & $\because \Xi \because$ & $=-3$ & $=$. & & \\
\hline$\therefore \equiv$ & $: 00$ & $17= \pm E$ & $\therefore:$ & $\bar{z}: \therefore$ & $5: .4$ & 2. & $\because .9$ & $-7 z=$ \\
\hline$\therefore$ & $\therefore$ & $=\ldots$ & 2.7 & $\because=\therefore$ & $\therefore$ & $\because{ }^{-}$ & & \\
\hline$\because$ & $\therefore$ & $\therefore: \Delta ?$ & $\therefore=$ & $\therefore=0$ & $=8.7$ & $\Xi$ & -34 & $-7 \equiv$ \\
\hline$\because$ & 0 & $=: \therefore$ & $\vdots:-$ & $\therefore a$ & $\therefore$ & 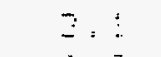 & & \\
\hline$\therefore=$ & & 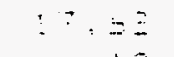 & $\therefore \therefore$ & 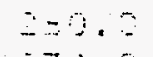 & 43.3 & $\therefore=$ & $-\therefore 3$ & $-74=\Xi$ \\
\hline$\therefore 2$ & $\therefore$ & $=.: 0$ & $\therefore 3$ & $=4.0$ & 17.2 & 4.2 & & \\
\hline$\cdots 2$ & $\because$ & $: 7:=8$ & $\therefore 4$ & $-\Xi=4.0$ & 27.7 & 4.2 & $\therefore=$ & -74. \\
\hline$\because \because 2$ & 30 & $5: 20$ & $\therefore 4$ & $\therefore=2: 0$ & $2 \pm .0$ & 3.5 & & \\
\hline$\therefore \because$ & $=0$ & $17 .=3$ & $\therefore .5$ & 231.0 & 25.3 & 5.1 & $\therefore .5$ & .74 .3 \\
\hline$\because \therefore$ & $\therefore 8$ & $s=10$ & $\therefore=$ & $23 \leq .5$ & 5.7 & 4.4 & & \\
\hline$\equiv \therefore$ & $=0$ & $: 7 .: 5$ & $\therefore$ & 275.0 & 3.6 & $\equiv$. & 5.7 & $-74 .=$ \\
\hline$=\because$ & $\because 6$ & $=.19$ & $\therefore:$ & $=3.0$ & 29.5 & $=.7$ & & \\
\hline$=z$ & 8 & $\therefore 7=3$ & $\because=$ & $=47.0$ & 52.4 & $\equiv .7$ & 0.0 & -73. \\
\hline$\because$ & .0 & 0.10 & : : & $\therefore 7.0$ & 12.2 & 5.0 & & \\
\hline 30 & $=0$ & 17.53 & 3. & $-91=0$ & 37.7 & 5.1 & 0.1 & -30.7 \\
\hline 3.0 & 30 & $\doteq .50$ & 2.4 & 50.0 & 95.4 & 7.4 & & \\
\hline$=8$ & $=00$ & $17 .=8$ & 2.5 & $\equiv 2.0$ & 32.3 & 9.7 & $0 . \overline{3}$ & 37.2 \\
\hline$=50$ & .00 & i. : & i. $!$ & 145.0 & 30.4 & 10.7 & & \\
\hline$=-0$ & 100 & $: 7.63$ & 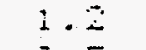 & 145.0 & 32.1 & 10.3 & -0.4 & 157.5 \\
\hline $5 \quad 203$ & $1: 00$ & 6.10 & $\therefore 5$ & 153.0 & 15.3 & 10.5 & & \\
\hline $5 \quad 100$ & $\because 100$ & 17.53 & 2.7 & $152 \cdot 0$ & $12 \cdot 5$ & 10.1 & -0.7 & 254.8 \\
\hline$=1: 00$ & $=0$ & 5.10 & 3.5 & 147.0 & 15.5 & 10.3 & & \\
\hline $\begin{array}{lll}E & 1 & -10 \\
E & 1\end{array}$ & $=0$ & 17.48 & 4.2 & 148.0 & $12 \cdot 1$ & 10.0 & -0.8 & 310.7 \\
\hline $\begin{array}{ll}5 & 1-20 \\
1 & 1-20\end{array}$ & 200 & $\begin{array}{r}5=10 \\
17-3\end{array}$ & $4 \cdot 2$ & 140.0 & 13.1 & 11.2 & -1 & \\
\hline$=130$ & 400 & $\begin{array}{r}1.58 \\
b .10\end{array}$ & $\begin{array}{l}4.3 \\
3.7\end{array}$ & $\begin{array}{l}140.0 \\
158.0\end{array}$ & $\begin{array}{l}10.7 \\
14.7\end{array}$ & $\begin{array}{l}10.1 \\
11.8\end{array}$ & -1.1 & 317.7 \\
\hline $5 \quad 1900$ & $: 400$ & $: 7.09$ & 4.4 & 156.0 & 11.3 & 10.5 & -1.0 & 231.0 \\
\hline$\Xi 1+9$ & $: 500$ & 5.10 & 3.1 & 152.0 & 14.4 & 12.4 & & \\
\hline 5140 & $==00$ & 17.68 & 3.7 & $1 \equiv 1.0$ & $1 \pm .3$ & 11.7 & -0.7 & 199.4 \\
\hline$\equiv 1=00$ & $i 500$ & 5.10 & 2.4 & 145.0 & 15.6 & 12.4 & & \\
\hline $5: i=00$ & $i \leq 00$ & 17.63 & 2.9 & 145.0 & 12.5 & 11.3 & -0.6 & 76.4 \\
\hline $51=00$ & 1700 & 5.10 & 2.1 & 234.0 & 7.1 & 7.7 & & \\
\hline$=1500$ & 1700 & 17.68 & 2.1 & 205.0 & 9.1 & 7.9 & 0.2 & -59.4 \\
\hline$\equiv 1700$ & 1300 & 6.10 & 2.7 & 242.0 & 4.8 & 7.1 & & \\
\hline $\begin{array}{l}\equiv 1700 \\
E 1800\end{array}$ & $\begin{array}{l}1500 \\
1900\end{array}$ & $\begin{array}{r}17.68 \\
6.10\end{array}$ & $\begin{array}{l}2.6 \\
1.9\end{array}$ & $\begin{array}{l}217.0 \\
255.0\end{array}$ & $\begin{array}{r}6.6 \\
27.2\end{array}$ & $\begin{array}{l}9.6 \\
8.6\end{array}$ & 0.5 & -75.4 \\
\hline 51300 & 1900 & 17.68 & 1.6 & 233.0 & 36.6 & 9.1 & 0.5 & -73.5 \\
\hline$\equiv 1700$ & 2000 & 6.10 & 1.1 & 253.0 & 19.7 & 7.2 & & \\
\hline $5 \quad 1700$ & 2000 & 17.68 & 0.4 & 301.0 & 26.7 & 7.0 & -0.2 & -59.5 \\
\hline$\Xi 2000$ & 2100 & 6.10 & 1.2 & 258.0 & 19.7 & 6.7 & & \\
\hline 52000 & 2100 & 17.68 & 0.7 & 270.0 & 28.8 & 7.3 & 0.4 & -57.5 \\
\hline 52100 & 2200 & 6.10 & 1.4 & 258.0 & 13.7 & 7.6 & & \\
\hline$\equiv 2100$ & 2200 & 17.68 & 0.8 & 261.0 & 16.4 & 7.4 & -0.2 & -64.6 \\
\hline 2200 & 2300 & 6.10 & 1.7 & 268.0 & 18.4 & 7.6 & & \\
\hline 2200 & 2300 & 17.58 & 0.9 & 264.0 & 30.0 & 7.5 & -0.1 & -64.2 \\
\hline$\overline{5} 2300$ & 0 & 6.10 & 1.2 & 242.0 & 28.9 & 6.8 & & \\
\hline 2300 & & 17.68 & 0.5 & 248.0 & 48.1 & 6.8 & 0.0 & 4.1 \\
\hline
\end{tabular}




$\begin{array}{lllllll} & 0\end{array}$




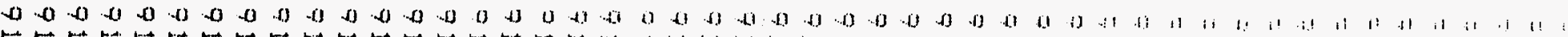

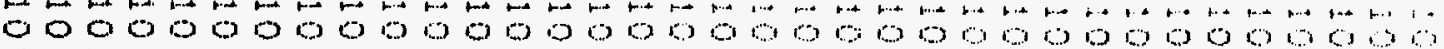

n

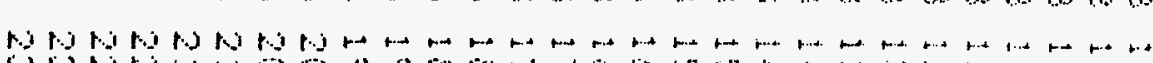

$\omega \omega \mathrm{\omega}$

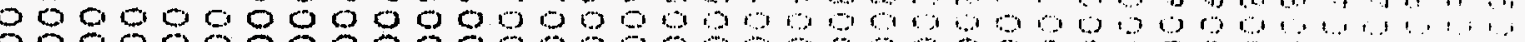

\section{HWHWWMW H}

wL1

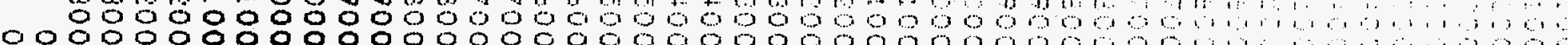

जo

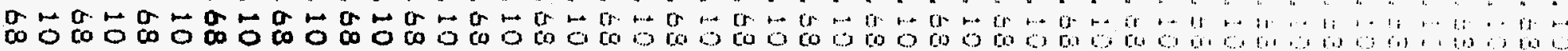

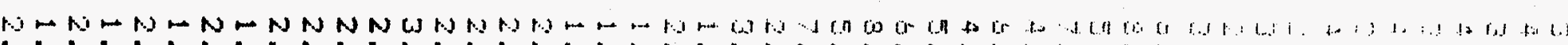

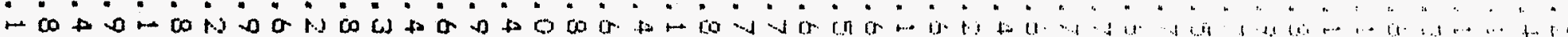

WW WH

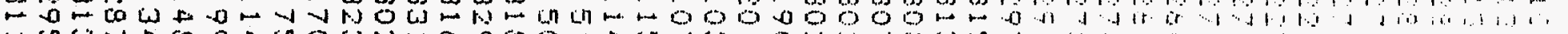
- Cn W

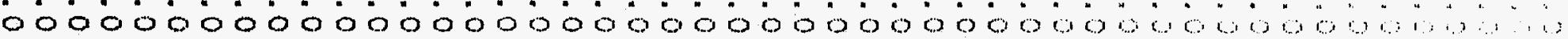

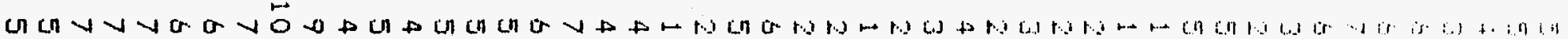

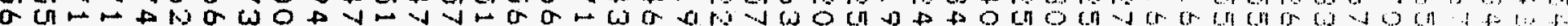

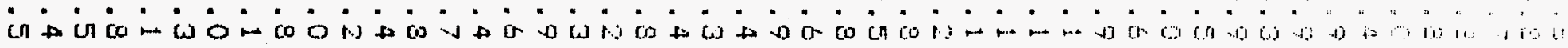

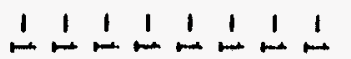

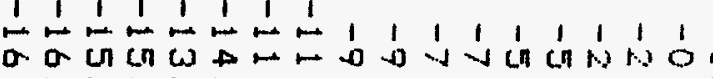

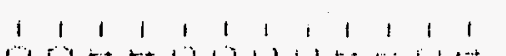

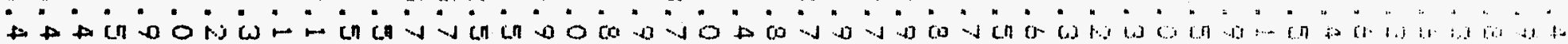

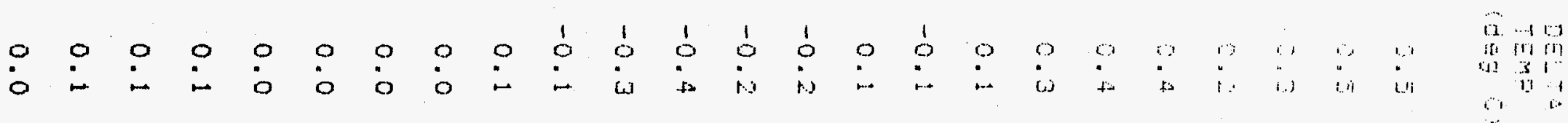

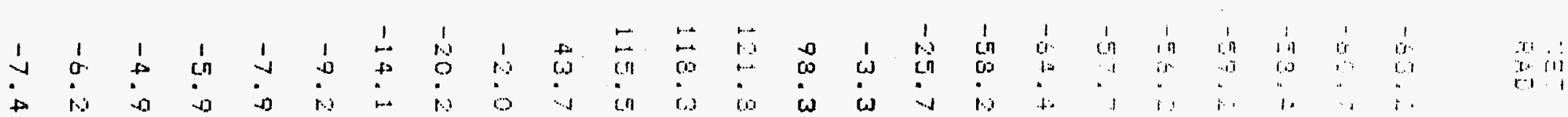




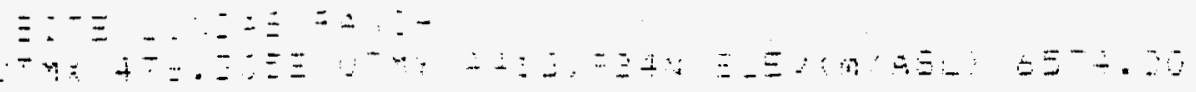

\begin{tabular}{|c|c|c|c|c|c|c|c|c|c|}
\hline$\because \div \square$ & $\begin{array}{l}\because: \cdots \\
\equiv-Y\end{array}$ & $\begin{array}{l}-n E \\
\because 70 F\end{array}$ & $\begin{array}{l}z= \\
y-5 \\
0\end{array}$ & 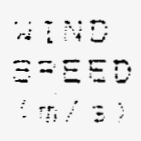 & $\begin{array}{l}\therefore y= \\
3=9 \\
3=4\end{array}$ & 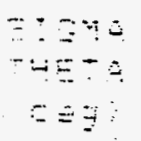 & $\Xi \Xi Z \quad \therefore$ & 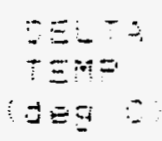 & 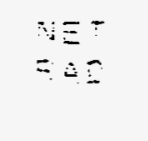 \\
\hline$=\cdots, z=$ & & $\therefore 2$ & $\approx .: 0$ & $\therefore \therefore$ & $.0 \pm 0$ & $\Xi 2$. & $-i=.7$ & & \\
\hline$=$ & $\therefore$ & $: 9$ & $17 .=\Xi$ & $\therefore 3$ & $7 \leq$ & $\because 7$ & $-\vdots \leq . \geq$ & $\because:$ & $-7 .=$ \\
\hline$\because \therefore-$ & & $\therefore 8$ & $\leq \ldots$ & $\therefore=$ & $: \because z$ & $\Xi 2 . \pm$ & $-: 3.2$ & & \\
\hline 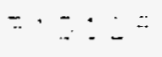 & & $\therefore$ & 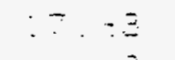 & $\therefore 2$ & $: \because=$ & $\angle A .2$ & $-: 2 \cdot \cdots$ & $\because$. & $-:=. \pm$ \\
\hline$=:-:=$ & & & $=\quad=$ & $\therefore \Rightarrow$ & $2: 0,=$ & $\therefore \quad:$ & $-: \because,=$ & & \\
\hline$\because: \because: \therefore$ & & $\ldots$ & $\therefore \ldots$ & $\therefore-$ & $\therefore \therefore$. & $= \pm .4$ & $-:=$. & $\therefore=$ & $-\because \therefore=$ \\
\hline$=\because \vdots \because$ & & $\because$ & $\therefore \quad \therefore$ & $\ldots$ & $: \div, 5$ & $5 \% .9$ & $-\vdots=\vdots$ & & \\
\hline$\therefore \because:=$ & $\because \because$ & -5 & $\because \quad=5$ & $\therefore:$ & $x=0$ & $=7.7$ & -17.5 & o: & -5.9 \\
\hline$=: \therefore:=$ & $\div \because$ & $\because 2$ & $\therefore \therefore$ & $\therefore=$ & 97.5 & 23.3 & $-:=:=$ & & \\
\hline$\because: 0:=$ & $\therefore \therefore$ & 80 & $: \because=8$ & $\therefore \therefore$ & $=5.5$ & $\because \pm . .7$ & -19.4 & 3.1 & -7.5 \\
\hline$=: 5:=$ & $=-$ & 50 & 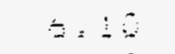 & $\therefore 3$ & $70=9$ & $\therefore i=7$ & $-20=1$ & & \\
\hline 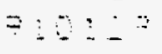 & $\because \because$ & $=9$ & $\vdots 7: \therefore \vdots$ & $\therefore i$ & 90.0 & 20.6 & $-20,5$ & $0 .:$ & $-1: .4$ \\
\hline $9: 01: 7$ & $=-0$ & 80 & 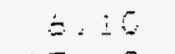 & $\therefore$. & $\because 22.0$ & 27.0 & -20.7 & & \\
\hline$=: 3: 29$ & $\leq 20$ & 90 & $\therefore:=3$ & $\therefore$ & $113=0$ & $\because 2.5$ & -20.5 & 0.2 & $-\mathrm{i} \uparrow .7$ \\
\hline$=20:=$ & $\because=$ & 200 & $5 . i 0$ & 2.4 & 200.0 & 12.3 & -21.0 & & \\
\hline$=: 0: 27$ & $7=0$ & $=00$ & $17: \leq 3$ & 3.7 & $\therefore 91.0$ & 15.0 & -20.7 & 0.7 & -30.0 \\
\hline$=10129$ & $\Xi 20$ & 700 & $5=10$ & 0.3 & 320.0 & 56.3 & -13.5 & & \\
\hline $715:=$ & $\therefore$ & 70 & 17.53 & 0.5 & 320.0 & 75.9 & -18.7 & -0.4 & -64.1 \\
\hline $5101 \div \%$ & $=-0$ & 120 & $\theta=10$ & 2.0 & 79.0 & 26.8 & -16.0 & & \\
\hline $91019=$ & $=20$ & $: 200$ & $17=3$ & $: .0$ & 109.0 & 23.1 & $-1 \leq .2$ & -0.2 & -46.8 \\
\hline $719: 29$ & \pm 90 & $: 100$ & 6.10 & $1: 7$ & 80.0 & 16.7 & -15.0 & & \\
\hline 510125 & 1000 & 1.00 & $1 \vec{i} .53$ & 1.5 & 93.0 & 14.5 & -16.3 & -0.3 & 16.5 \\
\hline $9: 01=$ & $1: 00$ & .200 & $5 .: 0$ & 1.7 & 57.0 & 20.2 & -15.2 & & \\
\hline 910127 & $1: 0$ & $: 200$ & $17=0$ & 2.0 & 74.0 & 17.1 & $-1=6$ & -0.4 & 46.4 \\
\hline $71012=$ & 1200 & 1200 & 0.10 & 1.5 & 74.0 & 22.6 & -13.6 & & \\
\hline$=19129$ & 1.90 & $: 200$ & 17.53 & $\therefore .7$ & 101.0 & 20.1 & -14.3 & -0.7 & 55.9 \\
\hline 710129 & 1300 & 140 & 5.10 & $i .5$ & 102.0 & 27.1 & -13.1 & & \\
\hline 910127 & 1300 & 1400 & 17.68 & 1.7 & 109.0 & $22: 0$ & 713.5 & -0.5 & 55.7 \\
\hline $71010=$ & 1400 & $: 500$ & $=10$ & 1.3 & 75.0 & 41.1 & -12.7 & & \\
\hline$=101:=$ & $1+0$ & $: 500$ & $17: \leq 3$ & 1.5 & 112.0 & 46.1 & -13.1 & -0.2 & 37.3 \\
\hline 710127 & $i=00$ & $:=00$ & 5.10 & 1.3 & 104.0 & 71.8 & -11.0 & & \\
\hline 710127 & $1=00$ & 1500 & $17 . \leq 3$ & 2.5 & 97.0 & 74.1 & -10.9 & 0.1 & 7.9 \\
\hline 910127 & 1500 & $1: 00$ & 5.10 & 3.8 & 260.0 & .8 & -3.5 & & \\
\hline 700127 & 1500 & 1700 & 17.68 & 4.9 & 266.0 & 20.2 & -3.0 & 0.5 & -60.3 \\
\hline 910127 & 1700 & $: 200$ & 6.10 & 4.1 & 283.0 & 20.5 & -3.1 & & \\
\hline $910: 29$ & 1700 & $: 300$ & 17.68 & 6.1 & 288.0 & 17.5 & -2.7 & 0.4 & -63.1 \\
\hline 910127 & 1300 & 1700 & 6.10 & 5.9 & 289.0 & 18.2 & -2.8 & & \\
\hline 910129 & 1300 & 1900 & 17.63 & 8.9 & 293.0 & 15.4 & -2.5 & 0.3 & -55.0 \\
\hline 710127 & 1900 & 2000 & 6.10 & 5.6 & 270.0 & 20.4 & -2.4 & & \\
\hline 910129 & 1900 & 2000 & 17.68 & 7.7 & 276.0 & 20.2 & -2.1 & 0.3 & -31.4 \\
\hline 910129 & 2000 & 2100 & 6.10 & 6.5 & 259.0 & 26.3 & $-2 \cdot 2$ & & \\
\hline 710129 & 2000 & 2100 & 17.68 & 8.9 & 270.0 & 27.1 & -1.7 & 0.3 & -40.3 \\
\hline 910129 & 2100 & 2200 & 6.10 & 7.7 & 298.0 & .0 & -2.0 & & \\
\hline 910129 & 2100 & 2200 & 17.68 & 11.1 & 293.0 & .0 & -1.7 & 0.3 & -29.9 \\
\hline 910129 & 2200 & 2300 & 6.10 & & 294.0 & & -1.4 & & \\
\hline 910127 & 2200 & 2300 & 17.68 & 10.6 & 295.0 & & -1.1 & 0.3 & -34.4 \\
\hline $\begin{array}{l}910129 \\
710127\end{array}$ & 2300 & 0 & 6.10 & & 292.0 & & -2.0 & & \\
\hline 710127 & 2300 & 0 & 17.63 & 11.0 & 293.0 & 15.2 & -1.8 & 0.2 & -43.8 \\
\hline
\end{tabular}




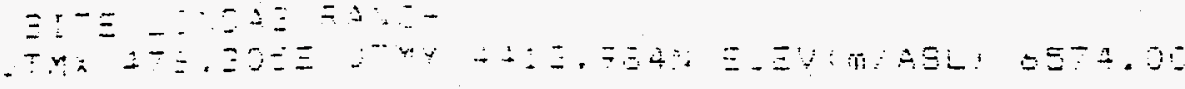

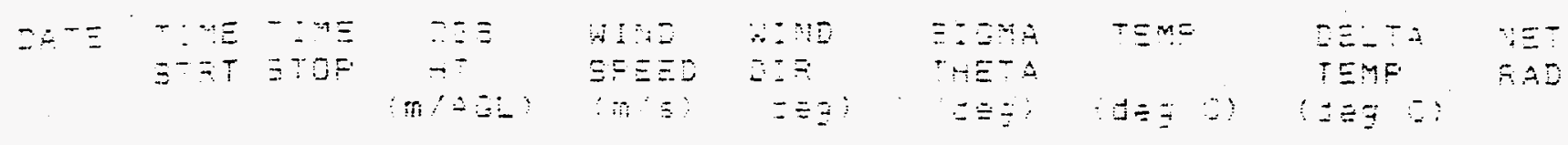

\begin{tabular}{|c|c|c|c|c|c|c|c|c|c|}
\hline$\because$ & 0 & $\because: 0$ & $\therefore: 5$ & 7.5 & $\therefore=4.5$ & $i=a$ & $-\therefore, \vdots$ & & \\
\hline$=$ & 9 & 00 & $\because \because=3$ & \pm 2.2 & $\Rightarrow z$ & $\therefore \leq z$ & $-\therefore \because$ & 5.3 & $-5 \pm .7$ \\
\hline - & & 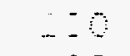 & $=:-$ & $0:$ & $\because \div 129$ & 12.2 & $-2=5$ & & \\
\hline$\because:$ & - & $\therefore 3$ & $:-\quad: \Xi$ & $\because \cdots$ & $\therefore=2.8$ & $: 4 . \equiv$ & -1.7 & 0.3 & $-5 \div .+$ \\
\hline$\because::$ & $\cdots$ & $\because \because$ & $\therefore \quad \therefore$ & $\because$. & 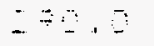 & $\therefore \equiv$. & $-\vdots, ?$ & & \\
\hline$\because 9: 3$ & $\therefore a$ & $\because 0$ & $\vdots \quad=\Xi$ & $\therefore \therefore$ & $\because 72.2$ & $1 \div, 2$ & $-\because: \vdots$ & $\therefore .7$ & -74.7 \\
\hline$\because: \because:=$ & $\because \because$ & $\therefore 0$ & $\Delta:$ & $\because .4$ & $\therefore 74.0$ & 15.4 & $-\therefore .7$ & & \\
\hline$\because: \because:=$ & $\because \because$ & $\therefore$ & $1:=0$ & 14.2 & $\because \square$ & 14.0 & $-: .4$ & 0.3 & -79.4 \\
\hline$\because: \vdots: \vdots$ & $\therefore$ & $\because \because 2$ & 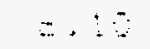 & -.4 & $\because 90.0$ & 20.3 & -8.7 & & \\
\hline$=: 50$ & $\therefore \%$ & 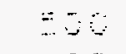 & 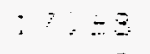 & 20.8 & 291.0 & 15.4 & -3.4 & 0.3 & -77.3 \\
\hline$=1 .: 2$ & $=90$ & $=5$ & $\pm \therefore=$ & $7 .=$ & 292.0 & 19.2 & -1.4 & & \\
\hline$=: 015$ & $=-0$ & $=8$ & $:^{-7}=0$ & $\therefore .4$ & 292.0 & 15.5 & -1.2 & 2.2 & -7.6 .7 \\
\hline$=10: 20$ & $=20$ & 90 & $=.10$ & $\Xi .4$ & $=92.0$ & 13.4 & -2.3 & & \\
\hline$=: 0: 30$ & 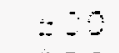 & $\because 9$ & $: \because .=3$ & 12.3 & 293.0 & 14.6 & $-: .7$ & 0.3 & -74.5 \\
\hline$F: \div:$ & -5 & $\therefore 0$ & $\therefore \quad:$ & 3.4 & $\because 2.0$ & 20.4 & -1.3 & & \\
\hline 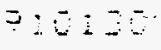 & $\because$ & $\because 0$ & 17.35 & $\therefore \ldots$ & 205.0 & $1 \leq .3$ & -1.5 & 5.3 & -69.3 \\
\hline $9: 0179$ & 55 & $=00$ & $\Delta=10$ & 3.7 & 290.0 & 21.1 & 0.7 & & \\
\hline $7: 0150$ & 525 & $=0$ & $: 7.55$ & $\therefore 2.5$ & 292.0 & 16.8 & 5.7 & 0.0 & -35.4 \\
\hline $9: 0130$ & $=-0$ & 150 & $\therefore .10$ & $\overline{7} .2$ & 254.0 & 13.7 & 1.1 & & \\
\hline$=10: 30$ & $=5$ & 100 & 17.50 & 13.3 & 293.0 & 15.2 & 1.1 & 0.0 & 25.9 \\
\hline $7: 0130$ & 1.20 & 1100 & \pm .15 & 5.4 & $\because 72.0$ & 18.9 & 2.5 & & \\
\hline $710 \mathrm{i} 3$ & 1.00 & $1: 00$ & 17.53 & 12.0 & 293.0 & 15.3 & 2.4 & -0.1 & 85.0 \\
\hline 710130 & $1: 50$ & 2200 & $s=10$ & 5.7 & 274.0 & 13.7 & 3.4 & & \\
\hline 9191.70 & $1: 20$ & $:=0$ & 17.58 & 12.4 & 295.0 & 14.7 & 3.4 & 0.0 & 124.6 \\
\hline 710130 & $1=00$ & 1300 & 5.10 & 7.9 & 288.0 & 21.2 & 4.4 & & \\
\hline 710130 & $1=20$ & $: 300$ & $\pm 7=59$ & 11.4 & 291.0 & 17.9 & 4.3 & -0.1 & 143.3 \\
\hline $710 \pm 70$ & 1.00 & 1400 & $\pm=10$ & 5.4 & 294.0 & 43.0 & 5.7 & & \\
\hline $7: 0130$ & 130 & 1400 & $17=03$ & 7.5 & 293.0 & 38.5 & 5.6 & -0.1 & 154.2 \\
\hline $7: 0:=0$ & 1400 & 1500 & 5.10 & 2.9 & 259.0 & 54.2 & 6.1 & & \\
\hline $7101=$ & 1400 & 1500 & 17.53 & 3.7 & 272.0 & 58.7 & 5.9 & -0.2 & $101 .=$ \\
\hline 710150 & 1500 & 1500 & $\$ .10$ & 3.6 & 301.0 & 45.3 & 4.9 & & \\
\hline $9: 0130$ & 1500 & 1500 & 17.68 & 4.7 & 291.0 & $\$ 1.0$ & 5.1 & 0.2 & -0.6 \\
\hline $910: 30$ & 1500 & 1700 & 6.10 & 6.0 & 293.0 & 28.1 & 4.0 & & \\
\hline 910130 & 1500 & 1700 & 17.63 & 8.4 & 294.0 & 22.2 & 4.2 & 0.2 & -56.1 \\
\hline 710130 & 1700 & 1300 & 6.10 & 7.7 & 276.0 & 20.7 & 3.4 & & \\
\hline 710130 & 1700 & 1300 & 17.68 & 10.7 & 279.0 & 18.0 & 3.6 & 0.2 & -62.3 \\
\hline 910130 & 1800 & 1900 & 6.10 & 6.5 & 293.0 & 27.8 & 3.6 & & \\
\hline 710130 & 1300 & 1900 & 17.68 & 9.1 & 295.0 & 23.2 & 3.9 & 0.3 & -64.4 \\
\hline 910130 & 1700 & 2000 & 6.10 & 3.2 & 259.0 & 76.1 & 3.8 & & \\
\hline 910130 & 1900 & 2000 & 17.68 & 4.1 & 260.0 & 67.9 & 4.0 & 0.2 & -62.5 \\
\hline 910130 & 2000 & 2100 & 6.10 & 4.8 & 264.0 & 40.3 & 3.3 & & \\
\hline 910130 & 2000 & 2100 & 17.68 & 6.4 & 263.0 & 36.5 & 4.0 & 0.2 & -46.4 \\
\hline 710130 & 2100 & 2200 & 6.10 & 3.8 & 273.0 & 65.8 & 3.7 & & \\
\hline 910130 & 2100 & 2200 & 17.68 & 4.9 & 269.0 & $\$ 1.0$ & 3.9 & 0.2 & -36.2 \\
\hline 910 & 2200 & 2300 & 5.10 & 5.6 & 273.0 & 32.9 & 4.4 & & \\
\hline 7101 & 2200 & 2300 & 17.68 & 7.7 & 276.0 & 27.4 & 4.6 & 0.2 & -44.5 \\
\hline 9101 & 2300 & 0 & 6.10 & 9.7 & 296.0 & 22.0 & 5.2 & & \\
\hline 910 & 2300 & 0 & 17.68 & 12.2 & 297.0 & 18.2 & 5.4 & 0.2 & -56.7 \\
\hline
\end{tabular}




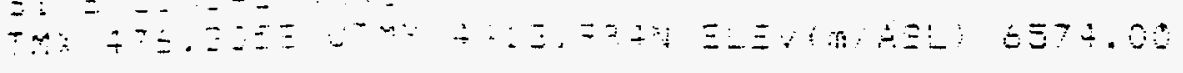

\begin{tabular}{|c|c|c|c|c|c|c|c|c|c|}
\hline$\because \therefore T \equiv$ & $\begin{array}{l}\because \cdots \equiv \\
\Xi=\bar{T}\end{array}$ & $\begin{array}{l}\cdots m E \\
\equiv E\end{array}$ & $\begin{array}{c}a z \equiv \\
y- \\
n+3\end{array}$ & $\begin{array}{c}\because \because \\
\Xi=\Xi \\
\vdots\end{array}$ & $\begin{array}{l}4: 00 \\
\therefore:= \\
\therefore=5\end{array}$ & 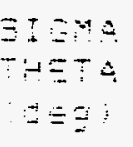 & $\Xi \equiv \Xi \quad \therefore$ & 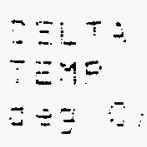 & \begin{tabular}{l}
$\because \equiv-$ \\
\hdashline$=5$
\end{tabular} \\
\hline$\because:$ & 2 & $: 20$ & $\dot{c} .: 0$ & $=.3$ & $\Xi 3.3$ & $\therefore 7.5$ & $\div .9$ & & \\
\hline$=\because \vdots \vdots$ & $?$ & 20 & $17 . \leq 3$ & $3 . \div$ & $z=3,2$ & $i \because \Xi$ & $=.2$ & $\therefore 2$ & $-5-. .3$ \\
\hline$\because: \cdots$ & $\therefore=$ & $\therefore 2$ & $\div . \vdots 9$ & $3 . \equiv$ & $=3 . \because$ & $\therefore 2.7$ & $=.1$ & & \\
\hline 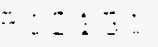 & 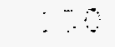 & $\because 0$ & $i^{-7}=E$ & $\therefore \approx .5$ & $=75.8$ & $\therefore \div$ & $\Xi$. & 5.3 & $-\equiv E . i$ \\
\hline$\therefore: \because: \square$ & $\therefore \%$ & $\because 2$ & $=\quad-$ & 7.0 & $\because=0$. & $-\ldots$ & $\because E$ & & \\
\hline$=: 2: z$ & $\therefore$ & 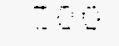 & $: \because$. & $\vdots 29$ & 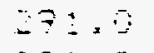 & $:^{-}=$ & $\approx \equiv$ & $2=3$ & -43.9 \\
\hline$=: 9:=$ & $=0$ & -9 & $\therefore \quad \therefore$ & $3=$ & $235=0$ & 25.5 & $\equiv . \Xi$ & & \\
\hline$\because: \because:$ & $\therefore 8$ & $\because 9$ & $\therefore \vec{Z}=\dot{3}$ & 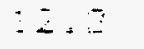 & $\because 91,0$ & $\therefore .5$ & $\equiv .7$ & 0.2 & -37. \\
\hline$=: 5:=$ & $\because \because 8$ & $\because 5$ & 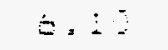 & 7.3 & $5 E .0$ & $\because 2$ & 4.5 & & \\
\hline$=: \therefore$ & $\because \because 8$ & $\because 8$ & $\because 7.5$ & $: \div .5$ & $\because 7.0$ & 15.5 & 4.3 & 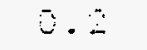 & $-45 .=$ \\
\hline$=: 8: 2$ & $\equiv$ & $\because \because$ & $\doteq=$ & 5.5 & 27.5 & 23.5 & 4.5 & & \\
\hline$=: \div: \div$ & $=5$ & $=90$ & $i^{7}=-8$ & $5=$ & $\because 75.0$ & 18.9 & 4.5 & 5.2 & -46.5 \\
\hline $7: 51: 2$ & $=0$ & -5 & $\theta \therefore$ & $=13$ & 234.0 & 23.5 & $\Xi$. & & \\
\hline$=012:$ & $=9$ & 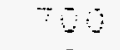 & $: 7=28$ & $8=7$ & $230 \times 5$ & 19.2 & $\equiv .3$ & 0.2 & -42.5 \\
\hline $9: 9: 5:$ & -0 & $=8$ & $\doteq .19$ & 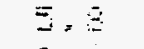 & 291.0 & 19.7 & $\Xi$. & & \\
\hline $7: 15:$ & 80 & $\Xi 00$ & 17.98 & 9.5 & 206.0 & $1=.7$ & $\Xi .4$ & 0.3 & -41.7 \\
\hline 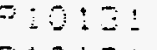 & 50 & 700 & $\Leftrightarrow=10$ & $5=$ & 234.0 & 17.7 & 5.7 & & \\
\hline $710 \pm=$ & 300 & $=00$ & 17.63 & 7.2 & 289.0 & 15.2 & 5.7 & 0.2 & -11.7 \\
\hline $9: 013:$ & 700 & $: 00$ & 5.10 & 4.2 & 277.0 & 18.5 & 7.3 & & \\
\hline$=: 013:$ & $=-0$ & 1.00 & 17.58 & 6.2 & 232.0 & 15.9 & 7.6 & 0.3 & 17.2 \\
\hline$=1013:$ & 1900 & $: 100$ & $5 .: 10$ & 5.7 & 293.0 & 21.5 & 3.2 & & \\
\hline $7: 0 i=:$ & 1200 & $: 100$ & 17.63 & 3.1 & 293.0 & 17.1 & 3.3 & 0.1 & 53.0 \\
\hline $7101=:$ & $:: 00$ & $=00$ & 6.10 & 6. 1 & 294.0 & 20.2 & 7.3 & & \\
\hline $7: 015:$ & $1: 00$ & $: 200$ & $17 . \leq 8$ & 8.5 & 295.0 & 15.6 & 9.8 & 0.0 & 229.7 \\
\hline $91015:$ & $: 200$ & 1300 & 6.10 & 5.5 & 274.0 & 13.6 & 11.3 & & \\
\hline $910: 3:$ & 120 & 1200 & 17.58 & 3.3 & 295.0 & 14.3 & 11.3 & 0.0 & 254.5 \\
\hline $71010 !$ & 1300 & $\therefore 00$ & 6.10 & 4.0 & 310.0 & 23.4 & 11.9 & & \\
\hline $7 \pm 013$ & $: 300$ & 1400 & 17.58 & 5.4 & 309.0 & 22.3 & 11.9 & 0.0 & 240.2 \\
\hline $7: 0: 21$ & 1400 & 1500 & 5.10 & 4.4 & 284.0 & 25.1 & 13.0 & & \\
\hline 71013 & 1400 & 1500 & 37.68 & 5.1 & 287.0 & 21.3 & 13.0 & 0.0 & 291.1 \\
\hline $710: 31$ & 1500 & 1500 & $=.10$ & 2.5 & 288.0 & 24.3 & 12.6 & & \\
\hline 710131 & 1300 & 1000 & 17.58 & 4.2 & 293.0 & 19.9 & 12.7 & 0.1 & 104.6 \\
\hline $910: 3 i$ & 1500 & 1700 & 5.10 & 2.4 & 277.0 & 20.3 & 10.6 & & \\
\hline 710131 & 1500 & 1700 & 17.68 & 3.6 & 288.0 & 16.5 & 11.1 & 0.5 & -33.8 \\
\hline $710: 31$ & 1700 & 1300 & 6.10 & 2.9 & 278.0 & 11.2 & 7.0 & & \\
\hline 910131 & 1700 & 1300 & 17.68 & 5.3 & 281.0 & 7.7 & 7.9 & 0.9 & -75.1 \\
\hline $91013:$ & 1900 & 1900 & 6.10 & 3.8 & 283.0 & 12.4 & 5.6 & & \\
\hline 910131 & 1900 & 1900 & 17.68 & 6.5 & 286.0 & 8.9 & 6.3 & 0.7 & -73.1 \\
\hline $910 \pm 31$ & 1700 & 2000 & 6.10 & 3.9 & 281.0 & 12.1 & 4.7 & & \\
\hline 710131 & 1900 & 2000 & 17.68 & 6.8 & 284.0 & 8.4 & 5.5 & 0.8 & -73.6 \\
\hline 910131 & 2000 & 2100 & 6.10 & 3.7 & 282.0 & 13.0 & 3.4 & & \\
\hline 910131 & 2000 & 2100 & 17.68 & 6.5 & 283.0 & 9.1 & 4.0 & 0.6 & -74.4 \\
\hline 710131 & 2100 & 2200 & 6.10 & 3.1 & 280.0 & 15.2 & 3.3 & & \\
\hline 910131 & 2100 & 2200 & 17.68 & 5.6 & 282.0 & 10.2 & 3.9 & 0.6 & -69.7 \\
\hline 910131 & 2200 & 2300 & 6.10 & 2.3 & 280.0 & 17.8 & 3.0 & & \\
\hline 910131 & 2200 & 2300 & 17.68 & 4.0 & 281.0 & 13.0 & 4.1 & 1.1 & -60.9 \\
\hline 91013 & 2300 & 0 & 6.10 & 2.5 & 284.0 & 19.8 & 5.8 & & \\
\hline $9101:$ & 2300 & 0 & 17.68 & 3.9 & 288.0 & 19.4 & 7.1 & 1.3 & -63.4 \\
\hline
\end{tabular}




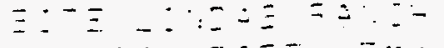

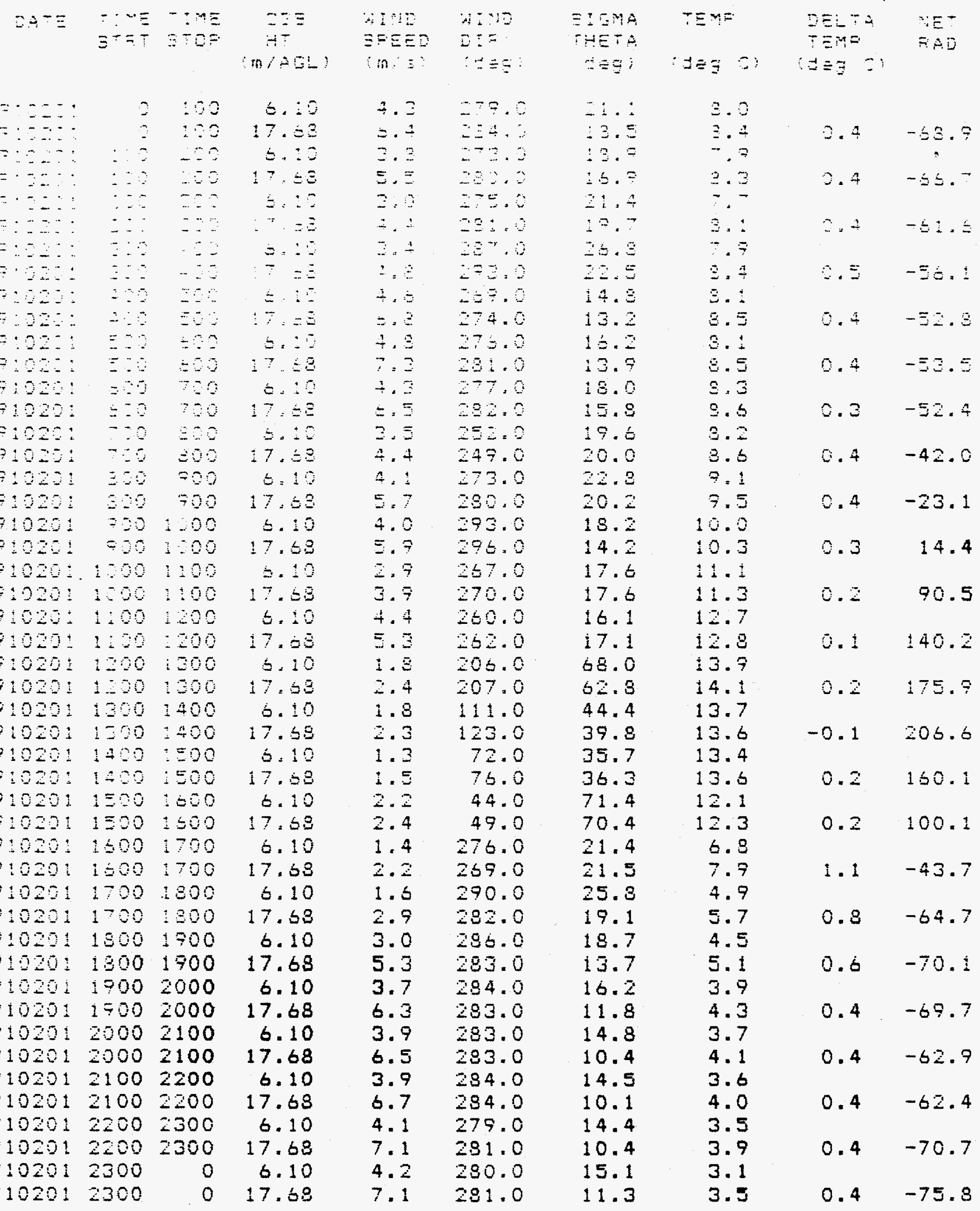




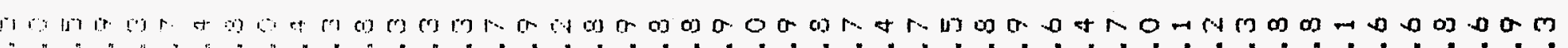

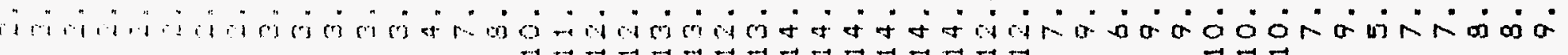

(1) i., in Ji:

1 1.

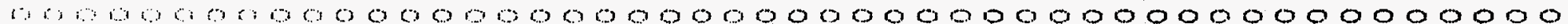
(1) $\therefore$ से ablat

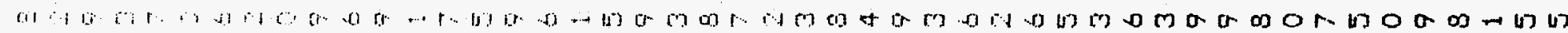

(i) (n)

a

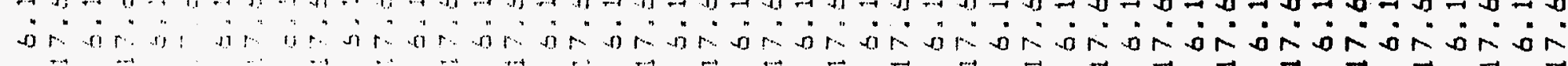
in:

1.1. it. in

का का \%

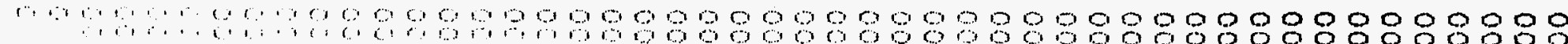
-.. 1. (1) (4)

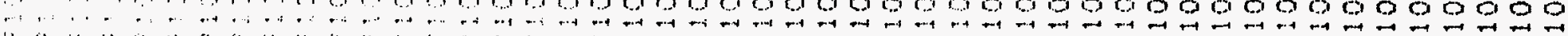




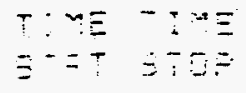

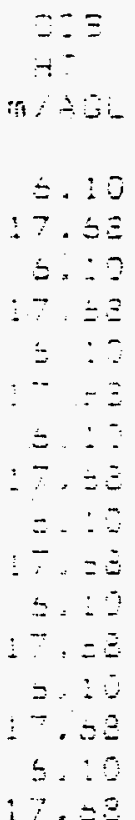

$=$
$=$
$=$
$=$
$=$
$=$
$=$
$=$
$=$

.

.

列

\begin{tabular}{|c|c|c|c|}
\hline & $1: 20$ & -10 & 17.03 \\
\hline 710203 & 1200 & 1300 & $\$ .10$ \\
\hline 1020 & $1=10$ & 1300 & $17=3$ \\
\hline 020 & 1200 & 1400 & 5.15 \\
\hline 10203 & 1300 & 1400 & 17.08 \\
\hline 223 & 1450 & $: \equiv 00$ & 5.10 \\
\hline 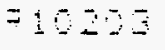 & $1-10$ & $:=0$ & 17.53 \\
\hline 1020 & $1 \equiv 20$ & 1500 & 0.10 \\
\hline $02: 2$ & $1=00$ & $i=00$ & 17.53 \\
\hline 10203 & $1 \leq 0$ & 1700 & 5.10 \\
\hline 910263 & $1=00$ & 1700 & 17.58 \\
\hline 710203 & 1700 & 1900 & 5.10 \\
\hline 710203 & 1700 & 1300 & 17.68 \\
\hline 710203 & 1900 & 1900 & 6.10 \\
\hline 910203 & 1300 & 1900 & 17.68 \\
\hline 710203 & 1900 & 2000 & 6.10 \\
\hline 710203 & 1700 & 2000 & 17.63 \\
\hline 910203 & 2000 & 2100 & 6.10 \\
\hline 910203 & 2000 & 2100 & 17.68 \\
\hline 710203 & 2100 & 2200 & 6.10 \\
\hline 910203 & 2100 & 2200 & 17.53 \\
\hline 910203 & 2200 & 2300 & 5.10 \\
\hline 91025 & 2200 & 2300 & 17.68 \\
\hline 910 & 2300 & 0 & 6.10 \\
\hline 710 & 2300 & 0 & 17.53 \\
\hline
\end{tabular}

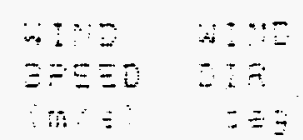

$3:-34$
$\square=52$

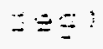

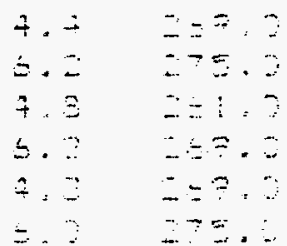

$\div$.

$\therefore$

$+$

$5=$

$4=$

$\div=1$

$+4$

5,7

4.2

s. 1

A. :

5.

4.0

5.:

4. 2

5.9

2.5

4.7

$\because .5$

3.5

3.5

5.

4.

5. 4

5.0

7.7

4. 2

5. 3

4.7

5. 4

4. 1

5.7

4.6

6.1

3.4

4.7

0.8

1. 2

1.0

1.4

1. 2

2.2

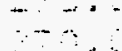

$-3$.

$\therefore \pm=$

$\because \vec{B}$

$\because \pm 1$.

$\div=0$

253.0

271.0

23.0

290.0

259.0

$-40.0$

260.0

263.0

$-70.0$

234.0

275.0

281.0

2ङ. 0

271,0

-91.0

285.0

294.0

287.0

202.0

286.0

277.0

282.0

268.0

274.0

-66. 0

272.0

256.0

259.0

277.0

275.0

285.0

270.0

289.0

292.0

281.0

276.0

\section{$\div 7.3$}

$\therefore 2 .=$

$\therefore=$

$: \overrightarrow{:}=$

$\because=$

$\vdots . \div$

$\div=$

$\div ;=$

10

$1=$.

$1=9$

16.7

17.4

17.6

13.5

17.

19.5

19.3

20.2

21.5

17.7

17.1

$16 . \overline{7}$

23.7

18. 3

17.5

14.1

18.2

14.4

13.2

14.3

19.5

15.9

17.6

15.5

15.3

14.9

15.3

14.6

47.2

46.9

34.5

45.9

42.7

52.2

13.3

16.8
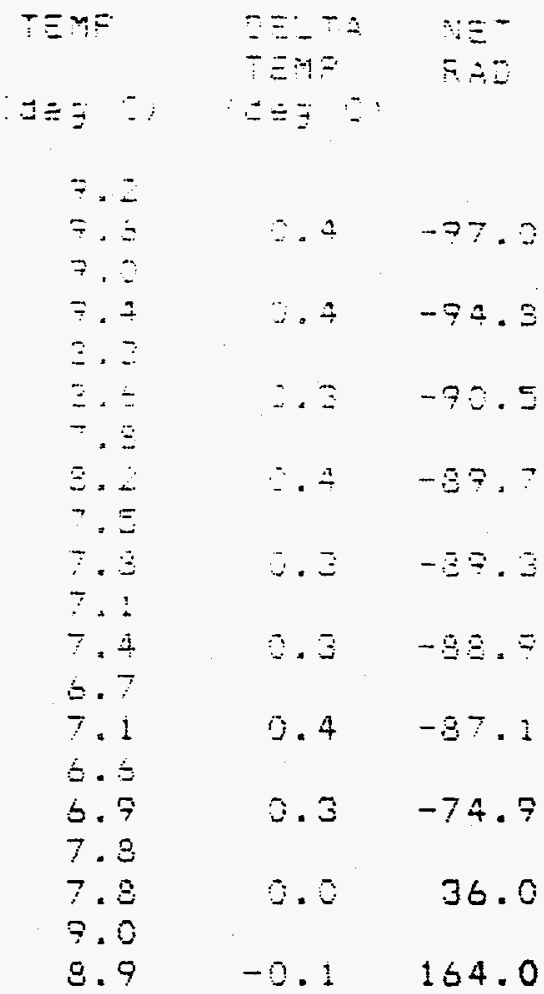

10.0

7.8

1. 5.5

10.7

11.6

11.4

10.5

10.7

10.7

10.5

7.2

9.2

7.7

7.9

5.6

6. 8

5.8

6.0

5.2

5. 4

4. 4

4.7

1. 3

2.0

1.0

2.0

$-0.7$

0.2
$-0.2 \quad 268.7$

$-6.1 \quad 297.1$

$-0.2 \quad 24.7$

$-0.1 \quad 213.4$

$-0.2 \quad 2.4 .2$

0.076 .1

$0.2-23.3$

$0.2-62.0$

$0.2-70.4$

$0.2-68.5$

$0.3 \quad-57.2$

$0.7-46.4$

1. $0-46.8$

$0.9-51.4$ 


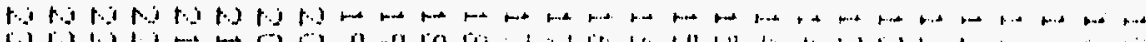

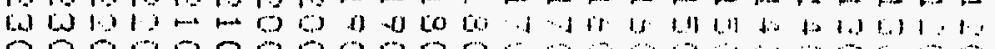

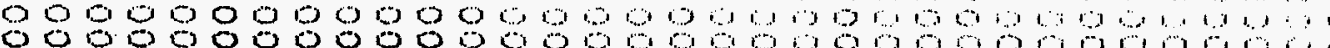

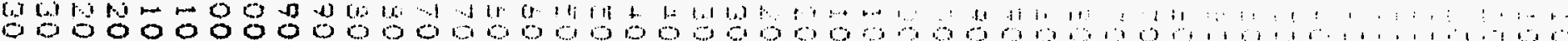

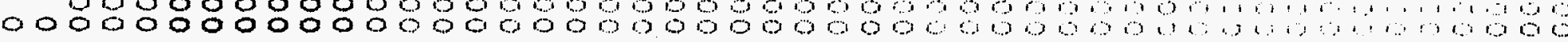

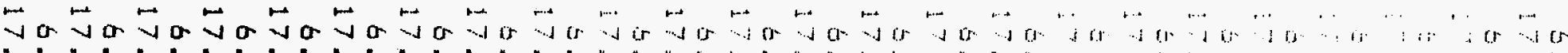

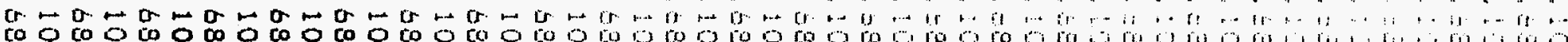

WNWWWNW W a

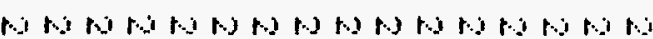

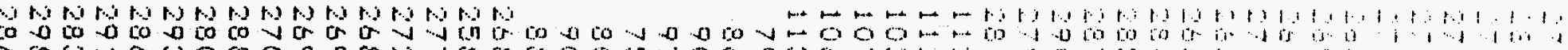

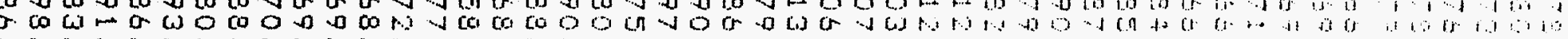

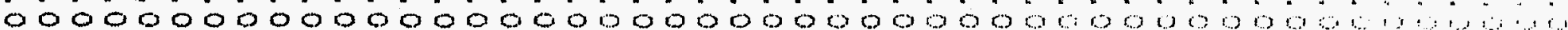
๑ o

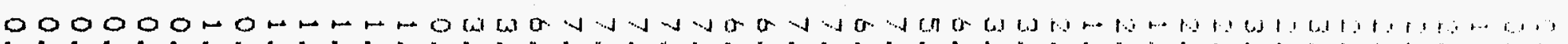

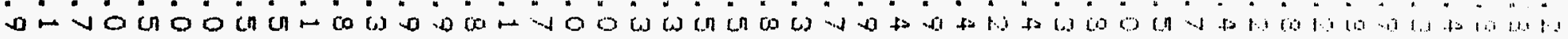

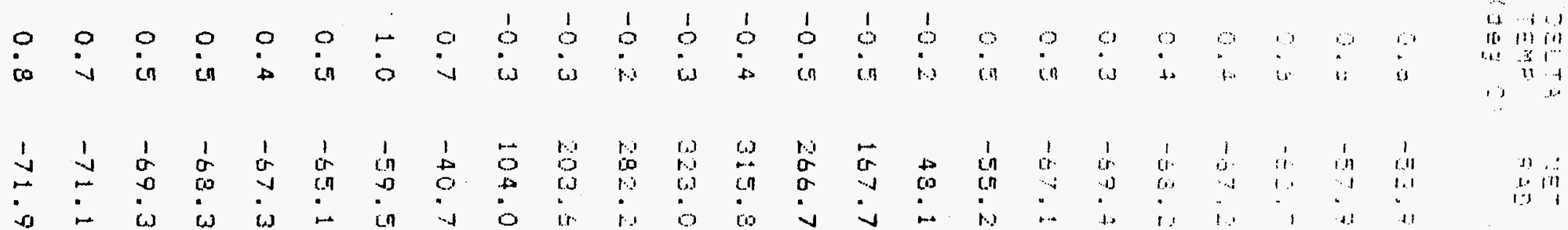




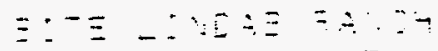

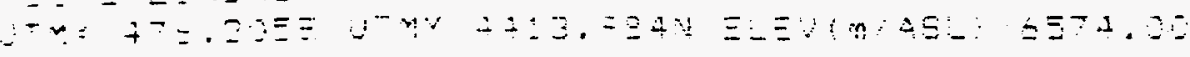

\begin{tabular}{|c|c|c|c|c|c|c|c|c|c|}
\hline$\because \because \vdots$ & $\begin{array}{l}-\because \equiv \\
\vdots:=T\end{array}$ & $\begin{array}{c}91 \\
975\end{array}$ & $\begin{array}{c}2 E \\
45 \\
m+B=\end{array}$ & $\begin{array}{c}\square \\
5=5 \equiv \\
n=\vdots\end{array}$ & $\begin{array}{l}\therefore:= \\
\therefore \therefore \\
\because=5\end{array}$ & 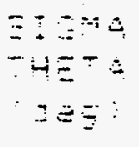 & $d \equiv z \quad \because$ & 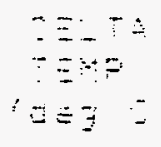 & 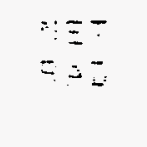 \\
\hline$=$ & $=$ & 10 & $\therefore .: 0$ & 2.3 & $\therefore \therefore$, & $=2$. & -2. & & \\
\hline & & $\because 2$ & 17.53 & $3 .=$ & 282.2 & $\therefore 5 \div$ & $2 \div$ & $\therefore=$ & -73.5 \\
\hline$\because:: \div$ & $\therefore$ & $\therefore:=$ & $=:: 0$ & $\therefore$. & $\because ? 2$. & $: 3$. & $-2=\vdots$ & & \\
\hline$\because: \cdots=$ & $\because$ & $\because 0$ & $7 .=\Xi$ & $=$. & $\therefore=0$ & $\therefore \doteq .2$ & 2.3 & $\because .4$ & -73.4 \\
\hline$\because: \because$ & $\because 2$ & $\therefore 2$ & $\therefore \therefore$ & $\therefore \therefore$ & 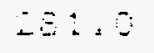 & $20 . \div$ & $-2 . \div$ & & \\
\hline$\because: \because: \because z$ & $\because \because$ & $2: 0$ & $\therefore \because \doteq \equiv$ & $3 . \div$ & $\therefore 7.3$ & $\therefore \div . \Xi$ & 2. & $\therefore \Xi$ & -7.3 \\
\hline$\because: \because:=$ & $\cdots: 5$ & $\therefore=$ & $\doteq: \therefore$ & $\therefore$. & $=7.0$ & $: \Xi .5$ & $-0 .:$ & & \\
\hline$=: 6=0$ & $=.0$ & $\div 2$ & 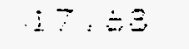 & $\Xi$. & $=74.0$ & 15.5 & 2.2 & 2.4 & -74.3 \\
\hline$=: 2: 05$ & $\therefore 20$ & $\because \because 2$ & $\leq:: 0$ & $\Xi \therefore$ & $=73.0$ & $1 \leq .7$ & $\therefore=$ & & \\
\hline$=: 8:=$ & $=20$ & $\Xi 20$ & $\dot{L}:=8$ & +.9 & $\therefore 74.0$ & $: 3.2$ & 2.5 & 2.4 & $-7 E .:$ \\
\hline$=: \div-\overline{3}$ & $=20$ & $=00$ & $\sigma: \therefore$ & 2.3 & $\because 01.0$ & 17.9 & -0.1 & & \\
\hline$\because: 9: 25$ & $\Xi \because 0$ & $\therefore 0$ & $17:=5$ & $=E$ & 285.0 & $: 3.0$ & 0.4 & $\therefore .5$ & $-7 E .1$ \\
\hline$=102=$ & $=30$ & -9 & $=.5$ & 3.4 & 239.0 & 17.3 & $0 .:$ & & \\
\hline$=1-25$ & $=20$ & 70 & $17=0$ & $\Xi .9$ & 285.0 & 12.1 & 0.5 & 5.5 & -74.0 \\
\hline $9: 220$ & -8 & $=00$ & $\Delta .20$ & $\therefore 9$ & $\because 96,0$ & 13.0 & 0.5 & & \\
\hline$=:-2=$ & 500 & 30 & 17.58 & $\equiv .2$ & -34.0 & 13.1 & $\therefore$ & 5.5 & -59.0 \\
\hline$=12205$ & 800 & $=00$ & 5.10 & $=.7$ & 201.5 & 15.4 & 3.1 & & \\
\hline$=10205$ & 50 & 700 & 17.68 & 4.5 & 231.0 & 11.9 & 3.1 & 0.0 & 52.7 \\
\hline $7: 0205$ & 700 & $: .00$ & 0.10 & 1.2 & 248.0 & 58.9 & 7.9 & & \\
\hline$=10205$ & 300 & 1.00 & $17=68$ & 2.7 & 249.0 & 58.3 & 7.8 & -0.1 & 173.1 \\
\hline$=1025$ & 1500 & $1: 00$ & 5.10 & 2.3 & 114.0 & 17.8 & 10.3 & & \\
\hline 910205 & 100 & $1: 00$ & 17.53 & 2.7 & \pm 21.0 & 14.2 & 7.9 & -0.4 & 272.0 \\
\hline $71020=$ & $1: 00$ & $: 200$ & $=10$ & $3=1$ & 124.0 & 15.1 & 10.7 & & \\
\hline 710203 & $1: 00$ & 1200 & 17.63 & 3.9 & 132.0 & 11.7 & 10.2 & -0.5 & 324.7 \\
\hline 710205 & 1200 & 1200 & 5.10 & 3.4 & 120.0 & 15.9 & 10.9 & & \\
\hline $7: 2205$ & 1200 & 1200 & $17=08$ & 4.3 & 129.0 & 12.4 & 10.4 & -0.5 & 334.5 \\
\hline$=10205$ & 1300 & 1400 & 5.10 & 3.1 & 120.0 & 10.4 & 11.5 & & \\
\hline 710205 & 1350 & 1400 & 17.58 & 3.7 & 135.0 & 12.9 & 11.0 & -0.5 & 301.5 \\
\hline$=305$ & 1450 & 1500 & 0.10 & 2.7 & 125.0 & 14.2 & 12.1 & & \\
\hline 91005 & 1400 & $=500$ & 17.58 & 3.4 & 133.0 & 10.7 & 11.7 & -0.4 & 217.4 \\
\hline 910205 & 1500 & $1=00$ & 6.10 & 1.7 & 163.0 & 58.9 & 11.6 & & \\
\hline$=10205$ & 1500 & $\$ 500$ & 17.68 & 2.3 & 1.66 .0 & 47.8 & 11.5 & -0.1 & 114.5 \\
\hline$=10205$ & 1500 & 1700 & 5.10 & 1.3 & 285.0 & 13.1 & 5.7 & & \\
\hline$=10205$ & 1500 & 1700 & 17.68 & 1.8 & 264.0 & 14.4 & 7.3 & 1.4 & -42.1 \\
\hline 710205 & 1700 & 1300 & 6.10 & 1.9 & .0 & 14.9 & 4.4 & & \\
\hline 910205 & 1700 & 1300 & 17.68 & 3.3 & 270.0 & 12.4 & 5.1 & 0.7 & -64.1 \\
\hline 710205 & 1300 & 1900 & 6.10 & 2.5 & 272.0 & 17.4 & 4.8 & & \\
\hline 910205 & 1800 & 1700 & 17.68 & 3.9 & 274.0 & 15.2 & 5.2 & 0.4 & -68.9 \\
\hline 910205 & 1900 & 2000 & 6.10 & 2.7 & 284.0 & 21.6 & 3.8 & & \\
\hline $\begin{array}{l}710205 \\
910205\end{array}$ & $\begin{array}{l}1700 \\
2000\end{array}$ & $\begin{array}{l}2000 \\
2100\end{array}$ & $\begin{array}{r}17.68 \\
6.10\end{array}$ & $\begin{array}{l}4.5 \\
2.7\end{array}$ & $\begin{array}{l}292.0 \\
297.0\end{array}$ & $\begin{array}{l}15.5 \\
19.4\end{array}$ & $\begin{array}{l}4.2 \\
2.9\end{array}$ & 0.4 & -65.6 \\
\hline 710205 & 2000 & 2100 & 17.68 & 4.6 & 284.0 & 14.9 & 3.4 & 0.5 & -67.4 \\
\hline 710205 & 2100 & 2200 & 6.10 & 2.8 & 284.0 & 19.3 & 2.6 & & \\
\hline 910205 & 2100 & 2200 & 17.68 & 4.8 & 282.0 & 13.7 & 2.9 & 0.3 & -66.4 \\
\hline 910205 & 2200 & 2300 & 6.10 & 3.3 & 277.0 & 18.3 & 2.6 & & \\
\hline $\begin{array}{l}910205 \\
710205\end{array}$ & $\begin{array}{l}2200 \\
2300\end{array}$ & $\begin{array}{r}2300 \\
0\end{array}$ & $\begin{array}{r}17.63 \\
6.10\end{array}$ & $\begin{array}{l}5.4 \\
2.9\end{array}$ & $\begin{array}{l}278.0 \\
277.0\end{array}$ & 13.9 & $\begin{array}{l}2.9 \\
2.4\end{array}$ & 0.3 & -6 \\
\hline 710205 & 2300 & 0 & 17.68 & 4.9 & 278.0 & 15.5 & 2.7 & 0.3 & -66.1 \\
\hline
\end{tabular}




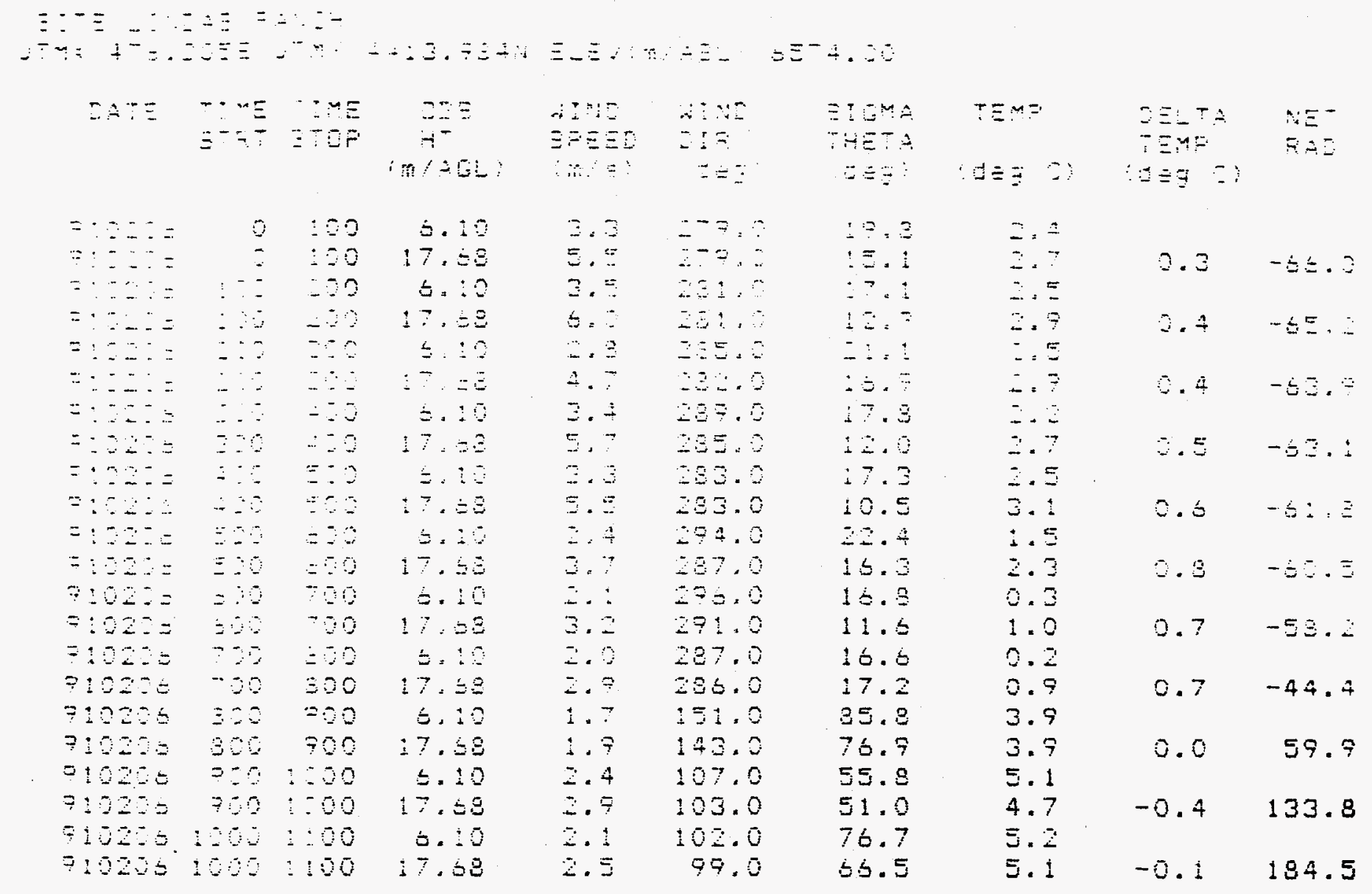




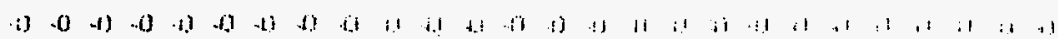
$\therefore$.

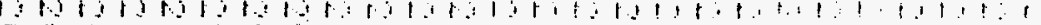

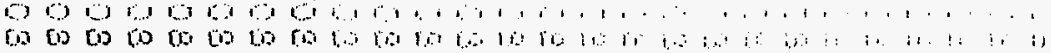

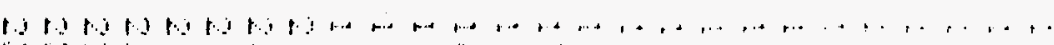

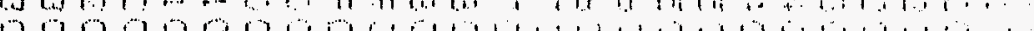
0000006060

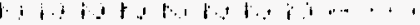

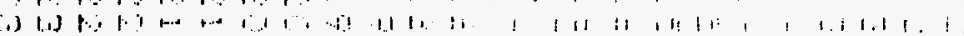
(1) 000000000 का

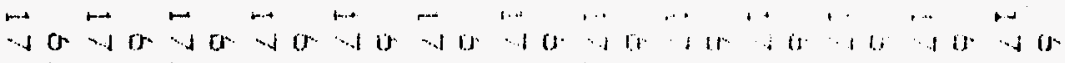

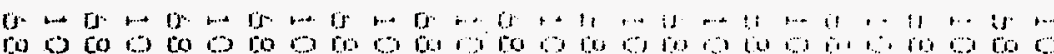

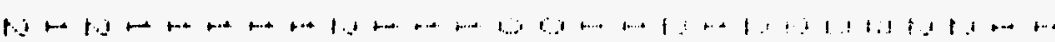
on

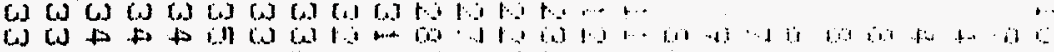

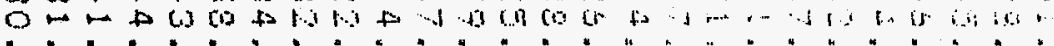

000000\%

(N)

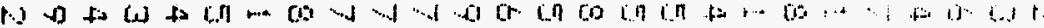

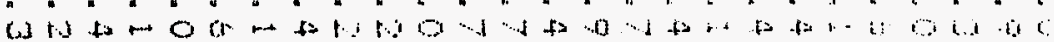

$\cos \begin{gathered}0 \\ 0\end{gathered}$

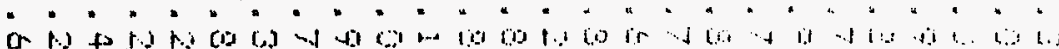

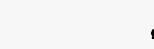

$$
\begin{aligned}
& \begin{array}{lllllllllllll}
0 & 0 & 0 & 0 & 0 & \cdots & 0 & 0 & 1 & 1 & 1 & 1 & 1 \\
+\infty & 0 & 0 & 0 & 0 & 0 & 0 & i & \cdots & 1 & \cdots & 0 & 0
\end{array}
\end{aligned}
$$

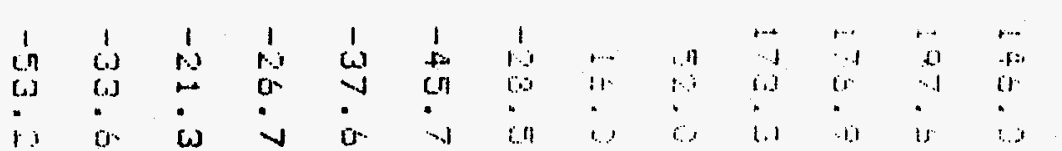$$
\text { ii }+1, y
$$$$
\text { 1.1 } 35
$$ 


\begin{tabular}{|c|c|c|c|c|c|c|c|c|}
\hline$E \therefore T$ & $\begin{array}{l}r= \\
\vdots Y=T\end{array}$ & $\begin{array}{l}\cdots \\
\vdots 1\end{array}$ & \begin{tabular}{c}
$\because \Xi \Xi$ \\
\hdashline$\vdots$ \\
01
\end{tabular} & 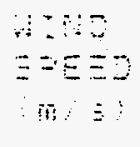 & $\begin{array}{l}180 \\
\vdots \because 8 \\
\vdots=\end{array}$ & 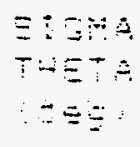 & $d=9$ & 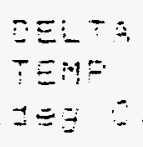 \\
\hline$\therefore \ldots$ & $\because$ & $\therefore 0$ & 5.10 & $\therefore$. & $-\therefore$. & 53.5 & $-\because .7$ & \\
\hline$\therefore=$ & 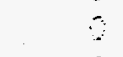 & 80 & $17 .=0$ & $\because$. & $\div \quad 5$ & $=\vdots .=$ & $\therefore 5$ & $5=$ \\
\hline$\because:-\ldots \vdots$ & $\because$ & $\because 2$ & 5.2 & $\Xi$ & $\therefore-$ & $\therefore-2$ & $-\vdots \vdots$ & \\
\hline$=: \because::$ & $\therefore$ & $=$ & $\div: \div 3$ & 3.2 & $\because 2.5$ & $5: 1$ & $-\vdots=2$ & 3.2 \\
\hline$\because \therefore=$ & $\because \because$ & $\because$ & $=\therefore$ & $\because=?$ & $=$. & $=2$ & $-3 \cdot \vdots$ & \\
\hline $7:-2=$ & $\therefore$ & $\because \therefore$ & $=$ & $=$ & 7. & $7=7$ & $-\cdots$ & 1 \\
\hline$=\div \div$ & $\because \therefore$ & $\because$ & $\therefore \therefore$ & $\therefore:$ & $\because \cdots$ & $43 \cdot 3$ & -1.2 & \\
\hline$=: \because: 28$ & 2 & $\therefore$ & $\because=: \because=$ & $\because:$ & $2: 0$ & $+8 \cdot 2$ & $-\vdots . \vdots$ & 0 \\
\hline$\because: \because 2=$ & - & $\because$ & $=\ldots$ & 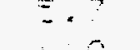 & $=120$ & $=3.3$ & -0.7 & \\
\hline$=: 9:=$ & $\therefore$ & - & $\therefore 7,-5$ & $=.8$ & $=-3.6$ & 43.7 & -6.7 & 0 \\
\hline 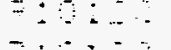 & $=\because$ & $=\therefore$ & $\dot{\Delta}=: 0$ & $=20$ & $\begin{array}{l}23.5 \\
-17\end{array}$ & $37 \cdot 4$ & $-6 \cdot 5$ & \\
\hline$=\because \div=$ & $\because \therefore$ & $\because$ & $\therefore=-3$ & $\begin{array}{l}=.9 \\
=1\end{array}$ & $\begin{array}{l}-4.0 \\
-=-9\end{array}$ & $\begin{array}{l}36 \cdot 0 \\
-2=\end{array}$ & $-5 \cdot 5$ & 2.1 \\
\hline$=-5$ & $\begin{array}{l}=-8 \\
=-0\end{array}$ & $\because$ & $: 7.5$ & 87 & $\begin{array}{l}20 \\
260\end{array}$ & -3.2 & $3 \times 4$ & \\
\hline $715: 0$ & -5 & $=8$ & 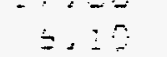 & 4.9 & -93.5 & $\begin{array}{l}-8.4 \\
77.9\end{array}$ & 0.5 & .1 \\
\hline 710128 & $\because 58$ & $\equiv 25$ & $17=50$ & 4.5 & 290.0 & 73.2 & 0.4 & 61 \\
\hline$\div 10: 23$ & 50 & $=0$ & 5.10 & $=.5$ & 239.0 & 36.2 & 0.7 & U. 1 \\
\hline 70123 & 800 & 900 & $\because .53$ & 0.3 & 278.0 & 33.4 & 0.9 & \\
\hline $9: 0: 2 \varepsilon$ & $=20$ & 100 & $=.50$ & $\equiv .4$ & 287.0 & 36.5 & 0.7 & 0.8 \\
\hline$=10123$ & $=0$ & 1000 & 17.68 & 5.7 & 293.0 & 31.5 & 0.7 & \\
\hline 210123 & 1000 & 1100 & 5.10 & $\because .0$ & $=92.0$ & 28.6 & 3.1 & \\
\hline $9: 0: 23$ & 1000 & $: 100$ & 17.68 & 7.2 & 301.0 & 24.1 & $3 . i$ & 0.0 \\
\hline $7: 0 i 29$ & $1: 00$ & $\because 00$ & $5=10$ & 5.7 & 278.0 & 26.2 & 4.1 & \\
\hline$=10123$ & $1: 00$ & $: 200$ & 17.53 & 5.0 & 305.0 & 22.4 & 4.1 & 0.0 \\
\hline 910128 & 1200 & 1300 & 6.10 & $3 \cdot 3$ & 179.0 & 71.5 & 4.6 & \\
\hline$=10: 28$ & 1200 & 1200 & $17=08$ & 4.2 & 185.0 & 73.5 & 4.6 & 0.0 \\
\hline $71012=$ & 1300 & 1400 & 6.10 & 1.3 & 146.0 & 62.9 & 3.5 & \\
\hline $710: 23$ & 1300 & 1400 & 17.53 & 2.0 & 158.0 & 60.9 & 3.3 & 0.3 \\
\hline$=10128$ & $: 400$ & 1500 & 0.10 & 1.1 & 22.0 & 49.8 & 1.3 & \\
\hline$=19123$ & 140 & $:=00$ & 17.68 & 0.0 & 32.0 & 54.8 & 1.7 & 0.4 \\
\hline 710123 & $1=00$ & $:=00$ & 0.10 & 2.8 & 354.0 & 17.2 & -2.1 & \\
\hline 710123 & 1700 & 1600 & 17.68 & 1.7 & 0.0 & 15.7 & -2.1 & 0.0 \\
\hline 910122 & 1500 & 1700 & 6.10 & 5.2 & 352.0 & 11.9 & -3.1 & \\
\hline 910123 & $1 \leq 00$ & 1700 & 17.63 & 6.4 & 0.0 & 11.0 & -3.1 & 0.0 \\
\hline $9101=8$ & 1700 & 1800 & 6.10 & 7.1 & 0.0 & 11.5 & -6.2 & \\
\hline $710: 23$ & 1700 & 1300 & 17.53 & 7.2 & 8.0 & 9.8 & -6.3 & -0.1 \\
\hline $710: 28$ & 1300 & 1700 & 6.10 & 6.3 & 1.0 & 10.1 & -7.9 & \\
\hline 910123 & 1300 & 1900 & 17.68 & 6.4 & 9.0 & 9.8 & -3.0 & -0.1 \\
\hline 910123 & 1700 & 2000 & 6.10 & 5.6 & 13.0 & 10.0 & -9.3 & \\
\hline 910128 & 1900 & 2000 & 17.68 & 5.3 & 21.0 & 9.4 & -9.4 & -0.1 \\
\hline 910123 & 2000 & 2100 & 6.10 & 5.1 & 10.0 & 7.6 & -11.6 & \\
\hline 710128 & 2000 & 2100 & 17.68 & 5.2 & 19.0 & 7.4 & -11.6 & 0.0 \\
\hline 910128 & 2100 & 2200 & 6.10 & 5.5 & 7.0 & 7.4 & -14.2 & \\
\hline 910128 & 2100 & 2200 & 17.68 & 5.6 & 15.0 & 7.2 & -14.2 & 0.0 \\
\hline 710128 & 2200 & 2300 & 6.10 & 5.4 & 5.0 & 8.3 & -15.6 & \\
\hline 910128 & 2200 & 2300 & 17.68 & 5.5 & 13.0 & 7.7 & -15.7 & -0.1 \\
\hline 710128 & 2300 & 0 & 6.10 & 4.5 & 355.0 & 9.4 & -16.5 & \\
\hline 9101 & 2300 & & 17.68 & 4 & 0 & 8 & -16.6 & \\
\hline
\end{tabular}




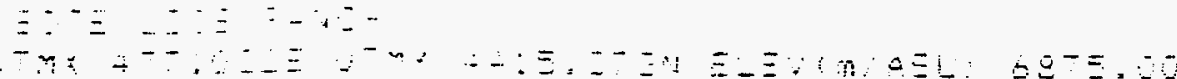

\begin{tabular}{|c|c|c|c|c|c|c|c|c|}
\hline$E \div=\Xi$ & $\begin{array}{l}\because \because E \\
\vdots \div=\bar{I}\end{array}$ & $\begin{array}{l}-: D \\
\equiv: D F\end{array}$ & $\begin{array}{l}=Z \equiv \\
-\because \div\end{array}$ & $\begin{array}{l}\vdots \\
\Xi \equiv \equiv \equiv D\end{array}$ & $\begin{array}{l}x: \cdots \\
\vdots \vdots 2\end{array}$ & $\begin{array}{l}\equiv:-4 \\
-1 E-4\end{array}$ & 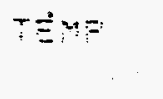 & $\begin{array}{l}F=\square \\
T E:=\end{array}$ \\
\hline & & & $\sin i \leq 1$ & $\therefore n^{\circ}=$ & $\therefore \leq=$ & $\therefore \equiv$ & 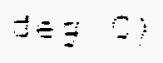 & : dez \\
\hline$\because: \because \div=$ & 5 & $\bigcirc \mathrm{O}$ & 6.10 & 3.7 & $: 3: \overline{3}$ & $\because z \therefore$ & $-\vdots \therefore, 7$ & \\
\hline$\because:-:=$ & 9 & $\because \because 3$ & $17 . \vdots 3$ & 3,2 & $\ddot{-}=.2$ & 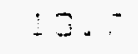 & $-\vdots .2$ & $-\infty \cdot 1$ \\
\hline$\because=2 \div=$ & $\because$ & $\because \because 0$ & 5.29 & $3 . \div$ & $: 1, \because$ & 7. & $-\vdots z . \Xi$ & \\
\hline$\because \div \div=$ & $\therefore$ & $\begin{array}{l}-34 \\
-20\end{array}$ & $17=53$ & $3 .=$ & $=3.2$ & 3.9 & $-: 2 . \div$ & -0.1 \\
\hline $\begin{array}{l}-1 \\
=\cdots\end{array}$ & 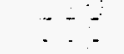 & $\therefore-$ & $\begin{array}{l}=2 \\
=-1\end{array}$ & $\therefore=5$ & $\begin{array}{r}3=0 \\
-30\end{array}$ & $\leq$ & $-!: .0$ & \\
\hline$=$ & 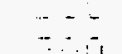 & $\begin{array}{l}-\cdots \\
-\because\end{array}$ & $\begin{array}{r}== \\
-=\end{array}$ & $\begin{array}{l}\therefore=\ddot{0} \\
\therefore \quad \because\end{array}$ & $\begin{aligned}-3 & =0 \\
& =0\end{aligned}$ & $\because 2$ & $-1 \div$ & $\therefore$ \\
\hline 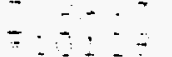 & $\begin{array}{l}\because \because 1 \\
\because \because 3\end{array}$ & $\begin{array}{l}-=8 \\
-\therefore-5\end{array}$ & $\begin{array}{l}3 .-3 \\
-7 \quad-3\end{array}$ & $\because 7$ & 7.5 & $y=5$ & $\begin{array}{l}-17.0 \\
-19\end{array}$ & \\
\hline$=:-1=$ & $\therefore=$ & $=1$ & 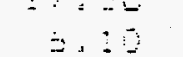 & $\begin{array}{l}-2= \\
1.5\end{array}$ & $\begin{array}{l}+0.0 \\
-1.0\end{array}$ & $\begin{array}{r}23 \\
-6.3\end{array}$ & $\begin{array}{l}-17.1 \\
-17.5\end{array}$ & $\because$ \\
\hline$\because:-: \leq=$ & $\therefore$ & -5 & $\therefore \because=\Xi$ & 1. & 30.5 & 27.2 & -17.5 & 5.5 \\
\hline$=\therefore: 3=$ & $\because \because$ & $=: 0$ & 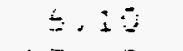 & 0.7 & $11:=0$ & $42 .=$ & $-20 . i$ & \\
\hline$=: 2:=$ & $=-2$ & $=0$ & 17.5 & $=0$ & $: \pm \div .0$ & 45.3 & $-20 . i$ & 0.5 \\
\hline$=101-3$ & $=2$ & 90 & $5 .: 0$ & $\therefore=3$ & $: 79.0$ & $8 \cdot 1$ & -23.4 & \\
\hline$=101=$ & $=20$ & 0 & $\therefore y=53$ & $\because \therefore$ & 179.0 & 8.0 & -20.4 & 0.0 \\
\hline$\because 21=7$ & -0 & $=20$ & 5,10 & $2=1$ & 133.0 & 10.7 & -20.4 & \\
\hline $9101=$ & $7: 0$ & 200 & $: 7, \leq 9$ & 0.0 & 133.0 & $\varepsilon .3$ & -20.2 & 0.2 \\
\hline 9010 & 50 & $=00$ & $5 .: 0$ & 1.5 & 103.0 & 11.9 & -13.4 & \\
\hline $9101-7$ & 30 & 700 & $17, \pm 0$ & 0.0 & 155.0 & \pm 1.5 & -18.3 & 0.1 \\
\hline 710127 & 50 & 190 & $=10$ & 1.5 & 134.0 & 12.8 & -15.2 & \\
\hline $7121=9$ & $=0$ & $: 200$ & $17=53$ & 0.3 & 192.0 & 17.1 & $-1 \leq .3$ & -0.1 \\
\hline 910197 & 10 & $\div: 00$ & 5.10 & 1.4 & 149.0 & 20.7 & -14.9 & \\
\hline $7: 01=7$ & 139 & 150 & $17 . \leq 3$ & 6.9 & 158.0 & 15.5 & -15.0 & -0.1 \\
\hline$= \pm 01 \div 7$ & $2 \div 00$ & -00 & 5.10 & 1.0 & $1 \pm 1.0$ & 17.6 & -15.3 & \\
\hline 710127 & $1:-0$ & 20 & 17.58 & 9.9 & 155.0 & 13.0 & -13.5 & -0.2 \\
\hline 710129 & 150 & 159 & 0.10 & $i=1$ & 154.0 & 13.1 & -13.0 & \\
\hline $7101=7$ & $1-00$ & 500 & 17.63 & 0.0 & 162.0 & 12.4 & -13.1 & -0.1 \\
\hline $710 \div=9$ & 1200 & 190 & 5.10 & $1 * 2$ & 150.0 & 14.7 & -12.3 & \\
\hline 910127 & 1300 & 400 & $17=58$ & 0.0 & 150.0 & 14.5 & -12.4 & -0.1 \\
\hline$\div 10: 27$ & 1450 & 1500 & $b=10$ & 3.4 & 175.0 & 12.1 & -15.2 & \\
\hline$=: 01: 7$ & 1400 & $:=00$ & 17.58 & 0.0 & 191.0 & $12=1$ & -15.1 & 0.1 \\
\hline $9101=9$ & $1 \leq 00$ & 1503 & 6.10 & 2.3 & 157.0 & 42.6 & $-12 \cdot 5$ & \\
\hline 910127 & 1500 & 1500 & \pm 7.58 & 2.4 & 177.0 & 54.5 & -11.3 & $1 \cdot 2$ \\
\hline 710129 & 1500 & 1700 & $5 . \pm 0$ & 3.9 & 286.0 & 89.9 & -4.6 & \\
\hline 910179 & 1500 & 1700 & 17.58 & 4.2 & 282.0 & 80.6 & -4.2 & 0.4 \\
\hline $7101 \div 7$ & 1700 & 1300 & 6.10 & 7.7 & 271.0 & 13.7 & & \\
\hline 910129 & 1700 & $: 300$ & 17.68 & 8.1 & 281.0 & 12.8 & -2.6 & 0.3 \\
\hline $7101=9$ & $1 \Xi 00$ & 1700 & 6.10 & 10.9 & 272.0 & 10.8 & -2.6 & \\
\hline $91012 \overline{4}$ & 1300 & 1700 & 17.68 & 11.4 & 292.0 & 10.0 & $-2 \cdot 4$ & 0.2 \\
\hline $7101 \div 9$ & 1900 & 2000 & 6.10 & 8.2 & 275.0 & 17.7 & -2.0 & \\
\hline 410197 & 1900 & 2000 & 17.68 & 8.7 & 284.0 & 14.6 & -1.8 & 0.2 \\
\hline $9+0129$ & 2000 & 2100 & 6.10 & 10.1 & 272.0 & 12.6 & -1.5 & \\
\hline 910127 & 2000 & 2100 & 17.68 & 10.7 & 281.0 & 11.7 & -1.3 & 0.2 \\
\hline 710129 & 2100 & 2200 & 6.10 & 12.5 & 270.0 & 10.9 & -1.9 & \\
\hline 910129 & 2100 & 2200 & 17.68 & 13.0 & 280.0 & 9.6 & -1.7 & 0.2 \\
\hline 910129 & 2200 & 2300 & 6.10 & 12.4 & 269.0 & 11.5 & $-1 \cdot 1$ & \\
\hline 910127 & 2200 & 2300 & 17.68 & 13.0 & 278.0 & 10.3 & -0.8 & 0.3 \\
\hline 910129 & 2300 & 0 & 6.10 & 13.8 & 276.0 & 11.7 & -2.4 & \\
\hline $910 \div 27$ & 2300 & 0 & 17.38 & 14.4 & 285.0 & 10.3 & -2.2 & 0.2 \\
\hline
\end{tabular}




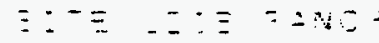

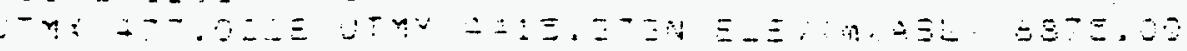

\begin{tabular}{|c|c|c|c|c|c|c|c|c|}
\hline$\because \div \div$ & $\begin{array}{l}\cdots \\
\vdots=\bar{Y}\end{array}$ & $\begin{array}{l}715 \\
\because O F\end{array}$ & $\begin{array}{c}z=\Xi \\
-1 \\
\therefore=-1\end{array}$ & 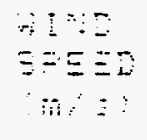 & $\begin{array}{l}10 \\
0:= \\
=3\end{array}$ & 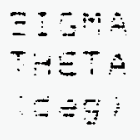 & 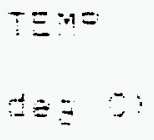 & 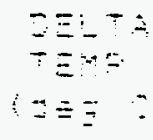 \\
\hline$\because: \because$ & 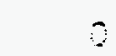 & .00 & $\$ .10$ & 13.3 & 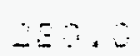 & $\therefore 2.3$ & $-i .3$ & \\
\hline$\therefore$ & & $: 5$ & $\because 7=58$ & 27.2 & $\therefore \equiv .0$ & $12: 2$ & $-1: 5$ & $0: 2$ \\
\hline$\therefore \ldots$ & $\cdots$ & -1 & $=10$ & $: 4.2$ & $-9=0$ & $\therefore 2.0$ & $-\ldots 4$ & \\
\hline$\therefore \therefore=$ & & O & 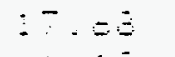 & $\therefore \equiv$ & $-\because y^{\prime}$ & 20.7 & $-\vdots$. & 5.5 \\
\hline$\because: \because$ & & $\therefore 8$ & $\therefore \therefore$ & $: \div, 9$ & $\because 77.9$ & $\therefore \div 9$ & $-\therefore=$ & \\
\hline$\because \because 7:$ & 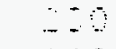 & 8 & $=0$ & $: E$. & -35.2 & $\therefore \leq$ & $-\because=8$ & 2.4 \\
\hline$=: \because 2:$ & & $\therefore 8$ & $=\therefore$ & $\because=$ & $=-5$ & 12.4 & -5.5 & \\
\hline$-9:=$ & 80 & $: 92$ & $\because+=3$ &.$=$ & $=0.9$ & 12.2 & -0.3 & 0.3 \\
\hline$\because: 0: 20$ & $\therefore \because 2$ & $=-9$ & $\doteq:$ & $\%$ & 279.0 & 23.5 & -6.5 & \\
\hline$=: 2: 2$ & $\therefore 8$ & $\approx 5$ & $\therefore \because=3$ & $=.5$ & 225.0 & 21.2 & -0.3 & $=$ \\
\hline$\because: 2:-1$ & $\because \because$ & $=\therefore 8$ & 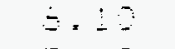 & $\therefore \therefore$ & $-7 \equiv .0$ & $: 7 . \vdots$ & $-1 \cdot 1$ & \\
\hline$=: 0: 3$ & $=0$ & $=-0$ & $\because 7 .=3$ & $:: . \div$ & $2=3.0$ & $15=$ & -0.9 & 0.2 \\
\hline 72030 & $=0$ & -8 & $\leq 20$ & $\therefore=\vdots$ & 267.0 & 15.4 & -1.4 & \\
\hline$=: 15=0$ & $\div 0$ & -8 & $: 7:=8$ & 12. & -76. & 14.5 & -1.3 & $0 . i$ \\
\hline $9: 0128$ & 700 & $\leq 00$ & $=-10$ & $\therefore=3$ & 276.0 & 17.5 & -1.5 & \\
\hline$=10150$ & -80 & $\therefore 00$ & $: 7 x \leq 8$ & $: 3.7$ & 230.0 & 13.3 & -1.5 & 0.1 \\
\hline 910190 & 30 & $=00$ & $5: 10$ & 12.4 & $23 \equiv .0$ & 19.9 & -0.2 & \\
\hline$=015$ & 300 & 700 & 17.58 & 12.9 & -93.0 & 18.2 & -0.1 & $0 . i$ \\
\hline 910150 & $=00$ & $=900$ & 5.10 & 13.4 & 259.0 & 13.0 & 1.1 & \\
\hline $9: 0150$ & $=20$ & $=80$ & 17.63 & 14.1 & $27 \leq 0$ & 16.7 & 1.3 & 0.2 \\
\hline$=: 0 \pm 30$ & 100 & 1100 & 5.10 & $2=8$ & $2 \leq 6.0$ & 18.9 & 2.6 & \\
\hline 710150 & 1000 & 1100 & 17.58 & 13.6 & 274.0 & 17.5 & 2.8 & 0.2 \\
\hline 710130 & 1100 & 1200 & 5.10 & 13.8 & 250.0 & 18.3 & 3.4 & \\
\hline $9: 0130$ & 1100 & 200 & 17.98 & $14=4$ & 259.0 & 17.1 & 3.5 & 0.1 \\
\hline 910130 & 1200 & 1300 & 5.10 & 11.2 & 270.0 & 19.2 & 3.8 & \\
\hline 910130 & 1200 & 1500 & $17=58$ & $11=9$ & 279.0 & 18.2 & 3.9 & 0.1 \\
\hline 710130 & 1300 & 1400 & 6.10 & 5.4 & 278.0 & 73.6 & 5.2 & \\
\hline$=10: 30$ & 1300 & 1400 & $17=58$ & 6.0 & 255.0 & 73.3 & 5.3 & 0.1 \\
\hline $7: 0 \pm 30$ & 1400 & 1500 & $=10$ & 4.5 & 220.0 & 65.1 & 5.3 & \\
\hline$=1913$ & $1+00$ & 1500 & 17.63 & 4.7 & 230.0 & 60.4 & 5.5 & 0.2 \\
\hline $910: 30$ & $1 \equiv 00$ & 1500 & 6.10 & 2.7 & 236.0 & 100.1 & 4.6 & \\
\hline 710530 & 1500 & $i=00$ & 17.59 & 2.3 & 203.0 & 95.2 & 4.6 & 0.0 \\
\hline 710130 & 1500 & 1700 & 5.10 & 5.4 & 287.0 & 63.8 & 3.6 & \\
\hline 710130 & 1500 & 1700 & 17.53 & 5.6 & 293.0 & 59.5 & 3.7 & 0.1 \\
\hline 910130 & 1700 & 1300 & 6.10 & 10.7 & 262.0 & 18.1 & 3.5 & \\
\hline 710130 & 1700 & 1300 & 17.68 & 11.3 & 270.0 & 16.1 & 3.6 & 0.1 \\
\hline 710130 & 1300 & 1900 & 6.10 & 8.2 & 282.0 & 28.6 & 3.5 & \\
\hline 710130 & 1300 & 1700 & 17.68 & 8.7 & 289.0 & 27.4 & 3.6 & 0.1 \\
\hline 710130 & 1900 & 2000 & 6.10 & 4.7 & 310.0 & 89.7 & 3.4 & \\
\hline 710130 & 1700 & 2000 & 17.68 & 4.7 & 312.0 & 90.6 & 3.6 & 0.2 \\
\hline 710130 & 2000 & 2100 & 6.10 & 3.9 & 195.0 & 73.7 & 3.5 & \\
\hline 910130 & 2000 & 2100 & 17.68 & 4.1 & 209.0 & 74.0 & 3.7 & 0.2 \\
\hline 710130 & 2100 & 2200 & 6.10 & 4.1 & 159.0 & 75.3 & 3.6 & \\
\hline 910130 & 2100 & 2200 & 17.68 & 4.1 & 174.0 & 71.7 & 3.6 & 0.0 \\
\hline 910130 & 2200 & 2300 & 6.10 & 7.8 & 259.0 & 40.0 & 4.2 & \\
\hline 710130 & 2200 & 2300 & 17.68 & 3.2 & 268.0 & 38.8 & 4.4 & 0.2 \\
\hline 910130 & 2300 & 0 & 5.10 & 12.3 & 258.0 & 19.3 & 4.7 & \\
\hline 710130 & 2300 & 0 & 17.68 & 12.9 & 267.0 & 17.6 & 4.9 & 0.2 \\
\hline
\end{tabular}




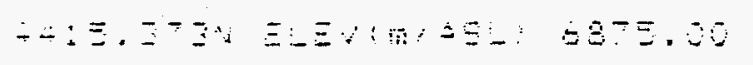

\begin{tabular}{|c|c|c|c|c|c|c|c|}
\hline こニミミ & $\begin{array}{l}\cdots \\
\because: 0\end{array}$ & 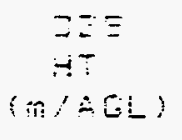 & $\begin{array}{l}\therefore \\
\Xi \Xi \Xi \equiv \\
\therefore \therefore: \equiv\end{array}$ & $\begin{aligned} \because & = \\
\vdots & = \\
\because & =\end{aligned}$ & 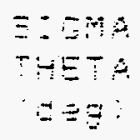 & 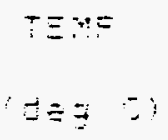 & 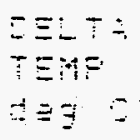 \\
\hline$\therefore:=$ & $\therefore 0$ & $5 .: 0$ & 17 & $\ddot{-}=z$ & $i \Delta .=$ & $=$. & \\
\hline- & $\therefore: 0$ & $: 7.53$ & $\therefore ?$. & $\div 2.3$ & $\therefore \leq:$ & $\Xi$. & 2.2 \\
\hline$\because:$ & $\therefore 2$ & $\leq .: 0$ & 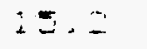 & $200,=$ & $\therefore 7:$ & $=2$ & \\
\hline$[:=$ & 200 & $17 .=3$ & 15.7 & $2 \leq 7.0$ & 15.7 & $\approx .3$ & 2.1 \\
\hline$\therefore-8$ & $\because$ & $5 . \therefore$ & 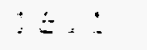 & $\because=0.2$ & $\therefore 5.4$ & $= \pm$ & \\
\hline$\therefore=$ & $\because 8$ & $\therefore=0$ & $\therefore=: 7$ & $2=7$, & $14=3$ & 5.5 & 3.2 \\
\hline$\therefore \because$ & $\because 9$ & $=. \vdots$ & $\div 2.2$ & 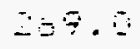 & 17.5 & $=.3$ & \\
\hline$\because \because$ & $\because$ & $\because \div . \pm$ & $: 2.7$ & $\because 77=0$ & 10.9 & 5.5 & 0.2 \\
\hline$\therefore \therefore$ & $\therefore 8$ & 5.25 & 10.3 & 235.5 & 20.5 & 4.4 & \\
\hline$\therefore$ & $=80$ & $17 .=9$ & 12.3 & -93.0 & 19. & 4.5 & 0.1 \\
\hline$\equiv$ & $=-0$ & 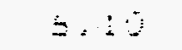 & 7.9 & $27 \leq .5$ & 26.3 & 4.5 & \\
\hline$\equiv 0$ & $\therefore$ & $: 7.68$ & $3=2$ & $25 b .5$ & $24=8$ & $\dot{4} .5$ & $a=1$ \\
\hline C: & -80 & $=.10$ & $\overline{7}, \overline{7}$ & 207.0 & $1 \overline{1} 1$ & $\equiv .0$ & \\
\hline $013:$ & 0 & $17: 58$ & $\therefore \therefore, 2$ & -75.0 & 16.7 & 5.1 & 0.1 \\
\hline $01:$ & $=80$ & $=.10$ & 10.2 & 257,0 & 12.9 & 5.4 & \\
\hline-5 & $\equiv 0$ & $17=08$ & 10.6 & 257.0 & 11.9 & 5.5 & 0.1 \\
\hline 380 & $=00$ & $\leq .: 0$ & 10.1 & $2 \div 20$ & 11.3 & 6.0 & \\
\hline $215:$ & $=0$ & $17 . \leq 3$ & 10.7 & $=69.0$ & 10.5 & 6.2 & 0.2 \\
\hline 5151 & 100 & 6.10 & 2. 1 & 233.0 & 11.6 & 7.5 & \\
\hline $015:$ & .00 & 17.53 & 8.4 & 272.0 & 10.8 & 7.8 & 0.2 \\
\hline $0131+190$ & 1100 & $6=10$ & $=.8$ & 280.0 & 14.5 & 3.4 & \\
\hline$: 3: 1000$ & $: 100$ & 17.58 & 7.1 & 208.0 & 13.2 & 8.6 & 0.2 \\
\hline $13: 1:-0$ & $: 200$ & 4.10 & 7.4 & 259.0 & 17.6 & 7.3 & \\
\hline $1311: 00$ & -200 & 17.53 & 7.7 & 270.0 & 16.5 & 7.9 & 0.1 \\
\hline $12: \quad 1200$ & 1300 & 5.10 & 3.8 & 250.0 & 16.4 & 10.9 & \\
\hline $13: 1200$ & $: 300$ & 17.53 & 7.2 & 269.0 & 15.1 & 11.0 & 0.1 \\
\hline 1300 & $: 400$ & 6.10 & 5.4 & 304.0 & 47.0 & 11.4 & \\
\hline 1350 & 1400 & 17.63 & 5.0 & 312.0 & 46.0 & 11.4 & 0.0 \\
\hline $1 \div 30$ & $: 500$ & $6 .: 0$ & 3.7 & 233.0 & 50.1 & 13.0 & \\
\hline$:=20$ & $: 500$ & 17.58 & $\begin{array}{l}4.1 \\
=\end{array}$ & 291.0 & 56.1 & 13.0 & 0.0 \\
\hline 1500 & 1000 & 6.10 & 5.4 & 301.0 & 14.9 & 11.8 & \\
\hline 1500 & 1600 & 17.63 & 5.7 & 308.0 & 13.9 & 11.7 & -0.1 \\
\hline $1=00$ & 1700 & 5.10 & 5.0 & 297.0 & 11.6 & 10.1 & \\
\hline 1311000 & 1700 & 17.58 & 5.4 & 306.0 & 9.5 & 10.4 & 0.3 \\
\hline 1700 & 1800 & 6.10 & 4.9 & 278.0 & 8.6 & 7.3 & \\
\hline 1311700 & 1300 & 17.68 & 5.5 & 287.0 & 7.5 & 10.0 & 0.7 \\
\hline 1311900 & 1900 & 6.10 & 5.1 & 273.0 & 8.5 & 9.6 & \\
\hline 311800 & 1900 & 17.68 & 5.5 & 282.0 & 7.1 & 10.2 & 0.6 \\
\hline 311900 & 2000 & 6.10 & 4.7 & 264.0 & 9.5 & 9.7 & \\
\hline 1311700 & 2000 & 17.68 & 5.2 & 271.0 & 9.1 & 10.2 & 0.5 \\
\hline $3: 2000$ & 2100 & 6.10 & 4.3 & 272.0 & 8.2 & 9.0 & \\
\hline 312000 & 2100 & 17.68 & 4.7 & 281.0 & 7.0 & 9.6 & 0.6 \\
\hline 312100 & 2200 & 6.10 & 3.4 & 265.0 & 16.6 & 8.7 & \\
\hline 312100 & 2200 & 17.68 & 3.4 & 276.0 & 14.9 & 9.3 & 0.6 \\
\hline 312200 & 2300 & 6.10 & 2.5 & 264.0 & 31.3 & 8.4 & \\
\hline 312200 & 2300 & 17.68 & 2.5 & 275.0 & 34.5 & 8.9 & 0.5 \\
\hline 312300 & 0 & 6.10 & 5.4 & 258.0 & 18.3 & 9.1 & \\
\hline 12300 & 0 & 17.69 & 6.7 & 268.0 & 16.3 & 9.2 & 0.1 \\
\hline
\end{tabular}




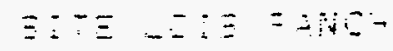

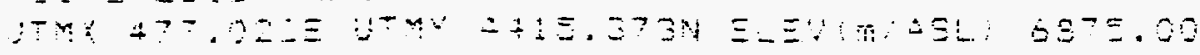

\begin{tabular}{|c|c|c|c|c|c|c|c|}
\hline $\begin{array}{l}\text { T:NE } \\
S: S T\end{array}$ & $\begin{array}{l}Y: D \\
\text { STOF }\end{array}$ & $\begin{array}{c}-0 \equiv \\
r T \\
m / 20 \leq\end{array}$ & $\begin{array}{l}\therefore:= \\
S E \Xi \equiv 0 \\
\therefore: \equiv\end{array}$ & $\begin{array}{l}2=0 \\
2:= \\
=5\end{array}$ & 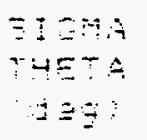 & $E=z \quad 2$ & $\begin{array}{l}E E- \\
Y E Y= \\
\square=Z\end{array}$ \\
\hline$\because-\cdots$ & $: 00$ & $\Delta=: 0$ & 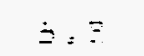 & $\ldots=-9$ & 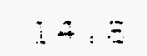 & $\equiv=\Xi$ & \\
\hline$\therefore$ & $: 00$ & $17 . \leq 8$ & $7, \cdots$ & $\therefore==\therefore$ & $+7:=$ & \pm .5 & $\because:$ \\
\hline$\because:$ & $2: 2$ & 5.10 & $\therefore$ & 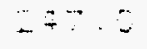 & $\therefore:=?$ & 3.2 & \\
\hline$\therefore: 9$ & $\cdots=$ & $\therefore-5$ & $\because:$ & $\because=5$ & $:=$ & $E$. & $S=:$ \\
\hline$\therefore \therefore$ & $\because \because:$ & $=. .0$ & $\therefore \therefore$ & $\therefore=3,0$ & -2.2 & 3.2 & \\
\hline$\therefore \because$ & $\therefore$ & $-a=\vdots$ & $=: 4$ & 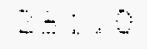 & : $: 7$ & $\because+\div$ & $\therefore$ \\
\hline 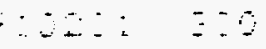 & $\because 58$ & $\theta=i$ & $\cdots$ & $\Xi \approx$ & $: 2.7$ & 2.5 & \\
\hline$\because 2:$ & $\because 3$ & $4^{\cdots}=5$ &.$- \div$ & $2=7.3$ & 13.1 & 5.5 & $\therefore:$ \\
\hline$=2:$ & $=0$ & 5.5 & $\therefore=$ & -9.5 & 11.4 & 3.7 & \\
\hline $2:$ & -8 & 17.58 & $\Xi$. & $\ddot{-}=\overline{7} .0$ & 10.5 & $\Xi .3$ & $\cdots$ \\
\hline IZ: & $=00$ & $5:: 0$ & $\Xi . \%$ & -56.0 & 11.0 & 9.7 & \\
\hline $2:$ & $=90$ & $1:-60$ & 7.3 & 2750 & 10.3 & 3.3 & 0.1 \\
\hline $32 \div$ & 90 & 6.10 & $=.5$ & $2=0.0$ & 11.7 & 3.9 & \\
\hline $90:$ & -00 & 17.58 & $7 .:$ & $z=0$ & 11.0 & 7.1 & 0.2 \\
\hline 3.0 & 800 & $5 .: 0$ & 7.5 & $2=0.0$ & 13.5 & 9.4 & \\
\hline-20 & 300 & $17 . \leq 3$ & 7.3 & $2 \leq 0.0$ & 12.3 & 7.5 & 0.1 \\
\hline 120: & $=00$ & 5.10 & 5.4 & 273.0 & 22.2 & 9.5 & \\
\hline 025 & 900 & 17.69 & $=.5$ & 297.0 & 22.7 & 7.3 & 0.2 \\
\hline$=00$ & 100 & 5.10 & 5.7 & 285.0 & 15.7 & 10.1 & \\
\hline 1221 & 1200 & $17=08$ & 0.2 & $2=4.0$ & 14.6 & 10.3 & 9.2 \\
\hline 5201950 & $1: 00$ & $5 .: 0$ & 3.9 & 267.0 & 17.4 & 11.0 & \\
\hline 20150 & 1100 & 17.55 & 4.2 & $27 \leq 0$ & 1.5 & 11.7 & 0.1 \\
\hline $0: 1: 00$ & 1200 & 5.10 & 4.5 & 250.0 & 27.2 & 13.3 & \\
\hline 201150 & $: 200$ & 17.68 & 4.3 & 257.0 & 24.9 & \pm 3.2 & -0.1 \\
\hline $201 \quad 130$ & $: 200$ & 6.10 & 3.5 & 192.0 & 35.1 & 13.4 & \\
\hline $1520: 1200$ & 1200 & 17.68 & 3.4 & 202.0 & 32.0 & 13.3 & -0.1 \\
\hline 2021300 & 1400 & 6.10 & 2.4 & 148.0 & $22 \cdot 2$ & 12.5 & \\
\hline$\because 1300$ & 1400 & 17.68 & 2.5 & 157.0 & 23.5 & 12.5 & -0.1 \\
\hline 011400 & 1500 & 6.10 & 1.7 & 33.0 & 57.9 & 12.7 & \\
\hline 011400 & $: 500$ & 17.68 & 1.5 & $\begin{array}{r}33.0 \\
2=0\end{array}$ & 66.4 & 12.7 & 0.0 \\
\hline $\begin{array}{ll}1 & 1500 \\
1 & 1500\end{array}$ & is $\leq 00$ & $\begin{array}{r}6.10 \\
7.43\end{array}$ & $2 \cdot 3$ & $\begin{array}{l}350.0 \\
350.0\end{array}$ & $\begin{array}{l}28.3 \\
24.3\end{array}$ & 11.0 & -0 \\
\hline $\begin{array}{l}11500 \\
11600\end{array}$ & $\begin{array}{l}1500 \\
1700\end{array}$ & 17.63 & $\therefore=4$ & 353.0 & 24.3 & 10.3 & -0.2 \\
\hline $\begin{array}{ll}-1000 \\
201 & 1090\end{array}$ & $\begin{array}{l}1.00 \\
1700\end{array}$ & $\begin{array}{r}0.10 \\
17.68\end{array}$ & $\begin{array}{l}1.8 \\
1.6\end{array}$ & $\begin{array}{l}306.0 \\
335.0\end{array}$ & 38.3 & 7.2 & \\
\hline $20: 1700$ & 1000 & 6.10 & 1.6 & 303.0 & 21.4 & $\begin{array}{l}4.8 \\
9.1\end{array}$ & 0.6 \\
\hline 0011700 & 1800 & 17.68 & 1.6 & 330.0 & 26.9 & 9.7 & 0.6 \\
\hline $201 \quad 1300$ & 1900 & 6.10 & 1.7 & 272.0 & 26.0 & 8.9 & \\
\hline 2011900 & 1900 & 17.68 & 1.0 & 297.0 & 36.7 & 9.3 & 0.4 \\
\hline $20: 1700$ & 2000 & 6.10 & 2.2 & 230.0 & 10.0 & 3.9 & \\
\hline 2011900 & 2000 & 17.68 & 1.3 & 305.0 & 17.2 & 9.2 & 0.3 \\
\hline 2012000 & 2100 & 6.10 & 3.7 & 277.0 & 5.7 & 8.1 & \\
\hline 2012000 & 2100 & 17.68 & 3.6 & 297.0 & 8.8 & 9.3 & 1.2 \\
\hline $20: 2100$ & 2200 & 6.10 & 3.4 & 275.0 & 6.3 & 3.3 & \\
\hline 2012100 & 2200 & 17.68 & 3.4 & 293.0 & 7.7 & 7.8 & 1.0 \\
\hline 2012200 & 2300 & 6.10 & 2.3 & 273.0 & 6.5 & 9.1 & \\
\hline 2012200 & 2300 & 17.68 & 2.1 & 276.0 & 20.0 & 9.8 & 0.7 \\
\hline 2012300 & 0 & 6.10 & 0.9 & 287.0 & 21.6 & 3.9 & \\
\hline 2012300 & 0 & 17.68 & 0.2 & 345.0 & 43.7 & 8.9 & 0.0 \\
\hline
\end{tabular}




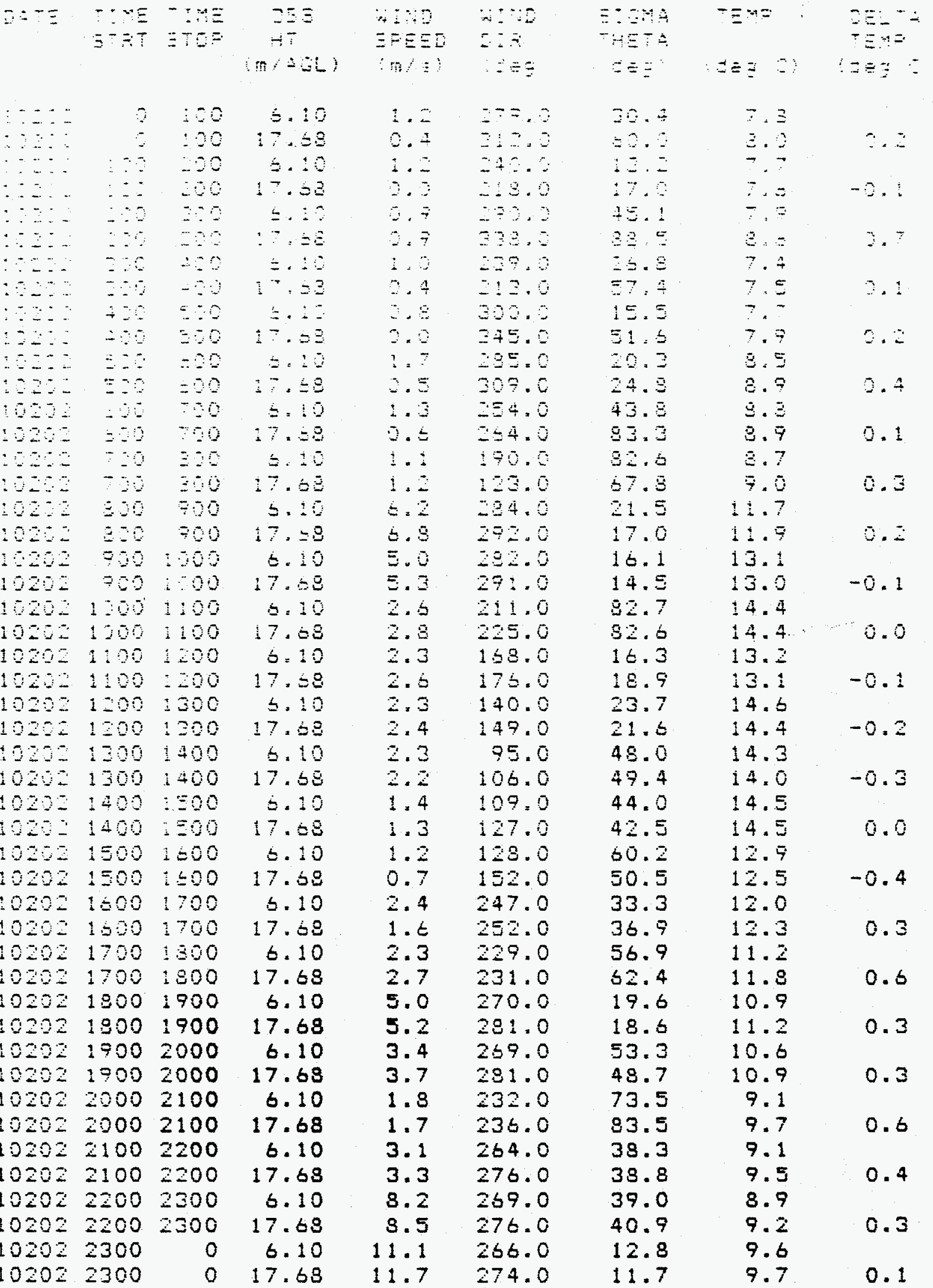


$\equiv:-\overline{-} \equiv=\therefore \therefore-$

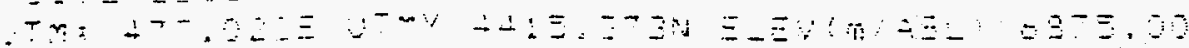

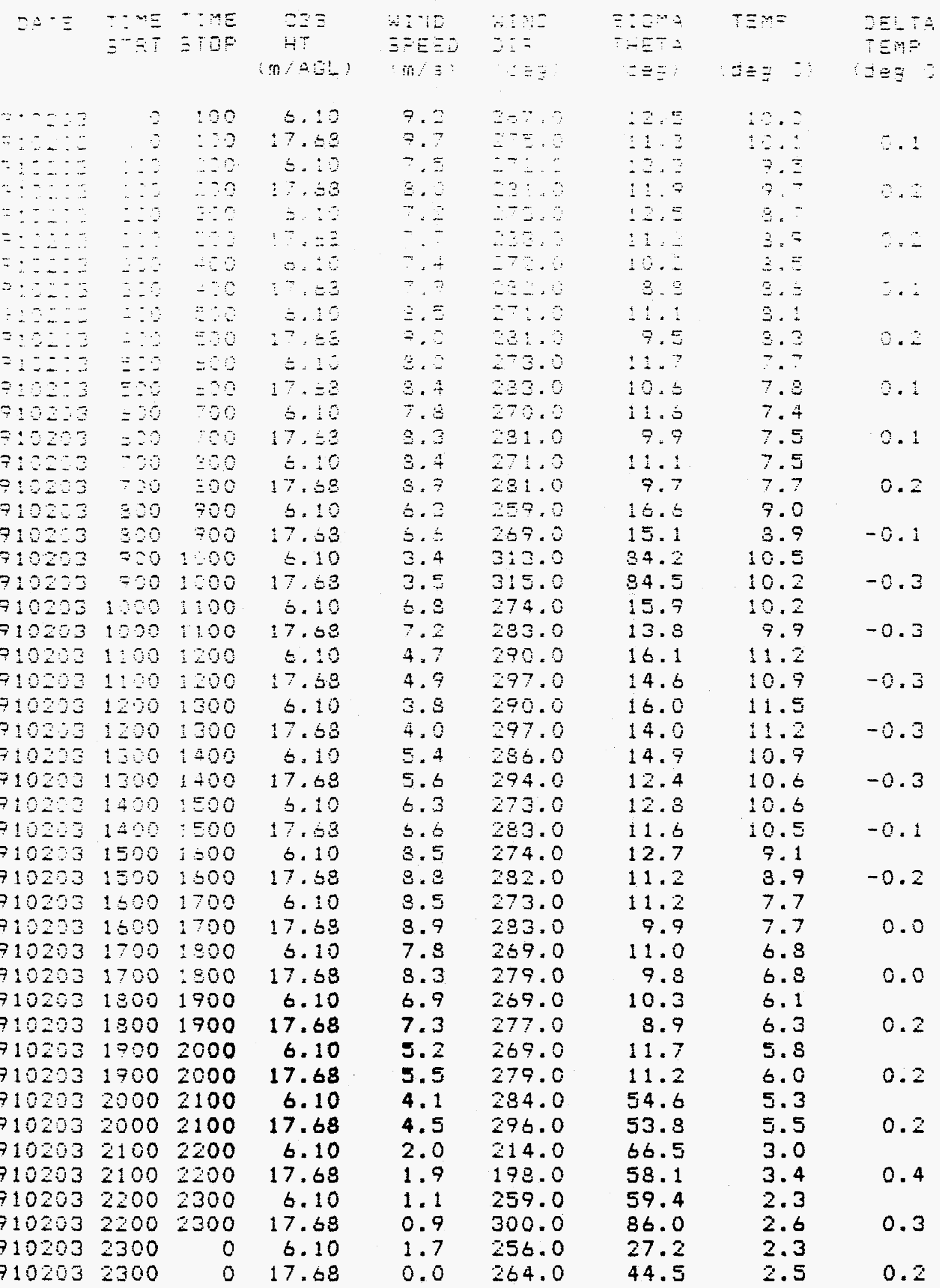




\begin{tabular}{|c|c|c|c|c|c|c|c|c|}
\hline $2:-\equiv$ & $\begin{array}{l}\cdots= \\
\equiv=\end{array}$ & $\begin{array}{l}\cdots= \\
\vdots: 0=\end{array}$ & $\begin{array}{c}2 E \equiv \\
-4 T \\
\because=2 !\end{array}$ & $\begin{array}{l}\because 5 \\
5 \% \Xi E D \\
\therefore ! \equiv \vdots\end{array}$ & $\begin{array}{l}x: 40 \\
\because: 5 \\
=\vdots \equiv\end{array}$ & 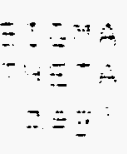 & 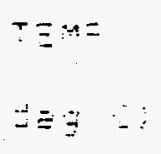 & $\begin{array}{l}z \equiv-1 \\
\vdots \equiv-1 \\
z=5\end{array}$ \\
\hline$=: \because:=$ & $?$ & $: \div$ & $5 .: 0$ & $Z=7$ & $\because=1, \because$ & $\therefore=$ & $3=$ & \\
\hline$=2$ & $\because$ & $\therefore 0$ & 17.53 & 1.5 & $\therefore=0.2$ & $2=$ & $3=$ & $\therefore .4$ \\
\hline$=\because \cdots$ & $\because$ & $\because 0$ & $=\ldots$ & 3.7 & $\therefore 7=2$ & 9 & $\Xi .9$ & \\
\hline$=$ & $\because$ & $\therefore 0$ & $\because \because . \pm$ & 4.8 & -35.8 & 7. & 4.2 & 2.4 \\
\hline$=$ & $\therefore$ & $\therefore=$ & 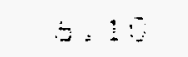 & $E . ?$ & $-67=5$ & $\therefore=$ & $\therefore=$ & \\
\hline$=: 2:$ & $\therefore \cdots$ & $\therefore \therefore$ & $\because=3$ & $\doteq .:$ & $\because 75,3$ & 9.7 & $\div$. & $\therefore$ \\
\hline$\because: \because=-$ & $\because \cdots$ & $\therefore$ & $=\therefore$ & $\because .8$ & $-35,0$ & $1:=$ & 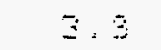 & \\
\hline 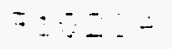 & $\therefore \because$ & $\therefore$ & $0^{+}: 8$ & 7.9 & $\therefore 7=5$ & 9.8 & $\overline{3} \cdot \bar{\gamma}$ & $3 .:$ \\
\hline$=: \therefore:$ & $\therefore:$ & $\therefore 0$ & $5=10$ & $\therefore=$ & $2=3,0$ & 10.1 & 3.5 & \\
\hline$=:-1$ & $\therefore \because$ & $\because 2$ & 17.33 & $\doteq .7$ & 254.0 & 9.3 & 3.7 & 0.1 \\
\hline $7: 02:=$ & $\because \therefore 2$ & $=\because$ & $=20$ & $\leq:$ & $\because=b .0$ & 51.1 & 2.5 & \\
\hline$=: 0: \div$ & $=20$ & $=-5$ & $17 . \leq 9$ & $\therefore$. & $-6 \pm .0$ & 10.3 & 3.7 & $C=$ \\
\hline 7: : & $\therefore 20$ & -90 & $5 .: 0$ & $=.2$ & 252.0 & 20.1 & 3.3 & \\
\hline $9102=2$ & $=20$ & -00 & $: 7: \leq 2$ & $\therefore=$ & 260.5 & 19.6 & 3.3 & 0.5 \\
\hline 91024 & $-\because$ & 40 & $5=10$ & $1 . \Xi$ & $z \leq 5.5$ & 74.4 & 3.4 & \\
\hline 91029 & 790 & 50 & $i 7=59$ & 2.4 & 334.0 & 84.5 & 3.9 & 0.5 \\
\hline 910201 & 500 & 700 & $t=10$ & $\therefore .0$ & 132.0 & 12.8 & 5.7 & \\
\hline $9: 0204$ & 50 & 900 & $\pm 7: \pm 0$ & 1.0 & 144.5 & 14.5 & $=.8$ & -0.1 \\
\hline$=1024$ & $=50$ & 1000 & 6.10 & $\therefore .5$ & 73.0 & 29.0 & 7.5 & \\
\hline 71904 & $=.0$ & 100 & 17.59 & $1=4$ & 103.0 & 31.0 & 7.1 & -0.2 \\
\hline $7102: 4$ & 1.00 & 100 & 6.10 & 2.4 & 105.0 & 34.6 & 7.5 & \\
\hline $7102=4$ & 1900 & $\therefore: 00$ & 17.59 & 2.4 & 115.0 & 36.1 & 7.1 & -0.4 \\
\hline 710204 & $1: 00$ & $=200$ & 6.10 & 3.4 & 108.0 & 46.5 & 7.3 & \\
\hline 910204 & $1: 00$ & $:-20$ & 17.59 & 3.3 & 118.0 & 44.9 & 7.0 & -0.3 \\
\hline $9102=4$ & $2=00$ & $: 300$ & 0.10 & 2.1 & 25.0 & 29.5 & 6.7 & \\
\hline 910204 & 1200 & 1300 & 17.68 & 1.7 & 32.0 & 28.0 & 6.3 & -0.4 \\
\hline 710204 & 1300 & 1400 & $t=10$ & 1.7 & 60.0 & 36.9 & 7.6 & \\
\hline 910204 & 1390 & 1400 & 17.68 & 1.6 & 55.0 & 37.4 & 7.3 & -0.3 \\
\hline 910204 & 1200 & 1500 & $5 .: 0$ & 2.1 & 52.0 & 23.3 & 7.0 & \\
\hline$=10204$ & 1200 & 1500 & $\pm 7=\leq 9$ & 1.7 & 58.0 & 21.9 & 6.8 & -0.2 \\
\hline 710204 & 1500 & $\therefore=00$ & 6.10 & 1.5 & 10.0 & 20.8 & 6.7 & \\
\hline 71029 & $1=00$ & $i \leq 00$ & 17.48 & 1.4 & 19.0 & 25.9 & 6.1 & -0.6 \\
\hline$=10204$ & 1000 & 1700 & 6.10 & 0.5 & 272.0 & 13.0 & 4.4 & \\
\hline 710205 & 1500 & 1700 & 17.68 & 0.1 & 346.0 & 19.8 & 4.3 & -0.1 \\
\hline 910204 & 1700 & 1300 & 5.10 & 1.6 & 234.0 & 17.0 & 3.5 & \\
\hline 710204 & 1700 & $\therefore 500$ & 17.68 & 0.0 & 195.0 & 69.2 & 3.7 & 0.2 \\
\hline 910204 & 1800 & 1700 & 6.10 & 3.7 & 217.0 & 7.3 & 4.7 & \\
\hline $\begin{array}{l}710204 \\
910204\end{array}$ & $\begin{array}{l}1300 \\
1900\end{array}$ & $\begin{array}{l}1700 \\
2000\end{array}$ & $\begin{array}{r}17.68 \\
6.10\end{array}$ & $\begin{array}{l}0.0 \\
3.2\end{array}$ & $\begin{array}{l}224.0 \\
202.0\end{array}$ & $\begin{array}{r}6.9 \\
12.6\end{array}$ & $\begin{array}{l}5.2 \\
4.2\end{array}$ & 0.5 \\
\hline 910204 & 1900 & 2000 & 17.68 & 2.4 & 210.0 & 15.0 & 4.7 & 0.5 \\
\hline 910204 & 2000 & 2100 & 6.10 & 2.6 & 257.0 & 46.0 & 3.3 & \\
\hline 910204 & 2000 & 2100 & 17.68 & 1.3 & 259.0 & 35.7 & 4.2 & 0.9 \\
\hline 910204 & 2100 & 2200 & 6.10 & 1.6 & 290.0 & 11.6 & 3.3 & \\
\hline 910204 & 2100 & 2200 & 17.58 & 0.0 & 320.0 & 15.0 & 3.7 & 0.4 \\
\hline 910204 & 2200 & 2300 & 6.10 & 0.8 & 291.0 & 13.7 & 3.1 & \\
\hline 910204 & 2200 & 2300 & 17.68 & 0.0 & 318.0 & 16.9 & 2.9 & -0.2 \\
\hline 910204 & 2300 & 0 & 6.10 & 0.7 & 278.0 & 14.8 & 2.8 & \\
\hline 910204 & 2300 & 0 & 17.68 & 0.0 & 332.0 & 16.9 & 2.6 & \\
\hline
\end{tabular}




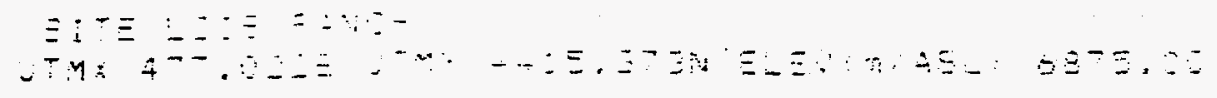

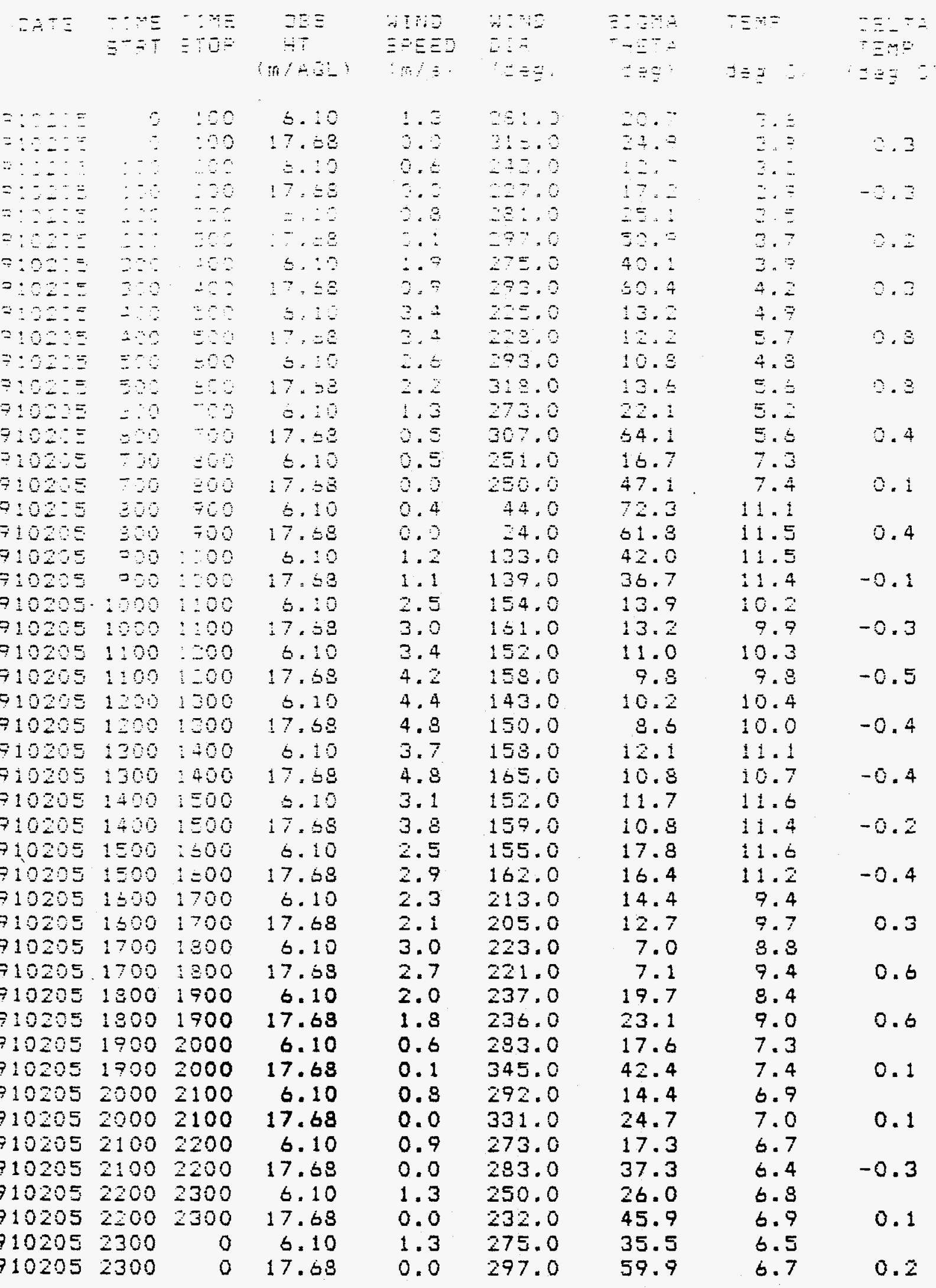


$\Xi \div \equiv-\therefore: \equiv=-\because Z$

\begin{tabular}{|c|c|c|c|c|c|c|c|}
\hline EATE & $\begin{array}{l}T: M E \text { ME } \\
\Xi: T \equiv T E F\end{array}$ & 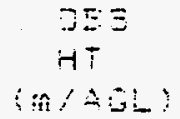 & 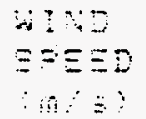 & 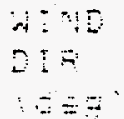 & 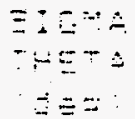 & $T \equiv M=$ & 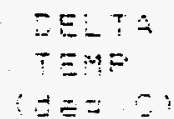 \\
\hline
\end{tabular}

\begin{tabular}{|c|c|c|c|c|c|c|c|c|}
\hline$\because 3 \div=$ & 3 & 100 & 5.10 & $i .3$ & $\Xi 3.8$ & $\therefore .3$ & $\vdots=$ & \\
\hline$\cdots=2=$ & 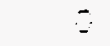 & $: 20$ & $17=\leq 3$ & 1.5 & 323. & 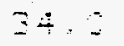 & $\because=$ & $\therefore .5$ \\
\hline$\because: \because \therefore=$ & $\therefore 5$ & $\because \because$ & $\leq .19$ & 2.6 & 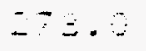 & 7. & 7.3 & \\
\hline$\because: \div=$ & $\therefore 0$ & $\therefore 5$ & $19=0$ & $\therefore, z$ & $-2=0.2$ & $1: .=$ & $E$ & $\therefore=$ \\
\hline$\because: \because:-$ & \begin{tabular}{l}
0 \\
\hdashline-1 \\
\hdashline-1
\end{tabular} & $\therefore-7$ & $\pm \quad \vdots$ & $\therefore:-$ & $-5=3$ & 7.5 & $=. \bar{Z}$ & \\
\hline$\because-3:=$ & $\because$ & $\because 0$ & $\therefore \quad=\overline{3}$ & $\therefore \therefore$ & 3.4 & $\overline{8} \cdot 2$ & $\bar{Z}=\check{Z}$ & 3 \\
\hline$=: 2=$ & $\because \because$ & $\therefore 9$ & $=: \because$ & $\therefore \bar{Y}$ & $\because 75.5$ & -7.3 & $=\ldots$ & \\
\hline 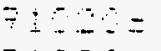 & $\because$ & $\therefore \because$ & $\therefore \quad \therefore$ & $\therefore=$ & $35=0$ & $30=$ & $=.5$ & $\therefore$ \\
\hline$=1: 2:=$ & $\div \therefore$ & $\therefore 8$ & $\doteq .15$ & $\therefore$ & 301.5 & $34 . i$ & 5.9 & \\
\hline$: 2:=$ & $\therefore$ & $\because 9$ & $\therefore 7.05$ & 3.5 & $3=4.6$ & 30.1 & $\overline{7} .0$ & 6.5 \\
\hline 1125 & $=5$ & $=0$ & $5=10$ & $\vdots=0$ & $3: 4.0$ & 53.8 & 5.3 & \\
\hline$: 2=$ & $=-9$ & $=0$ & $\because 7.60$ & $\therefore .7$ & $5=3.0$ & $\div 4.9$ & 5.7 & 0.4 \\
\hline $71020 \leq$ & $=90$ & $\because 0$ & 6.10 & $i .3$ & 322.0 & 24.7 & 4.3 & \\
\hline$=10205$ & $=20$ & 20 & $17=08$ & $-2=0$ & $35 \pm 0$ & 79.6 & 4.7 & 0.6 \\
\hline 71025 & -90 & 200 & $6 . \vdots 0$ & 1.1 & 357.0 & 30.0 & 3.9 & \\
\hline $7: 09$ & 70 & $=09$ & 17.93 & 1.1 & -0.0 & 26.2 & 4. & 0.3 \\
\hline 710205 & 59 & $=0$ & 5.10 & 3.1 & 5.0 & 17.3 & 4.7 & \\
\hline$=: 05$ & $=0$ & $=00$ & $17 . \leq 3$ & 2.3 & 7.5 & 17.7 & 4.7 & -0.2 \\
\hline$=10205$ & $=0$ & 100 & 5.10 & 4.7 & 2.0 & 10.7 & 4.6 & \\
\hline 910206 & 70 & $\div 00$ & 17.03 & 4.5 & 20.0 & 7.9 & 4.3 & -0.3 \\
\hline & 100 & $1: 00$ & 5.10 & 4.6 & 14.0 & 15.5 & 4.7 & \\
\hline $7: 0206$ & 1000 & \pm 100 & 17.63 & 4.6 & 2.0 & 14.3 & 4.7 & -0.5 \\
\hline
\end{tabular}


$\equiv:-\equiv: \equiv=2 \div:-$

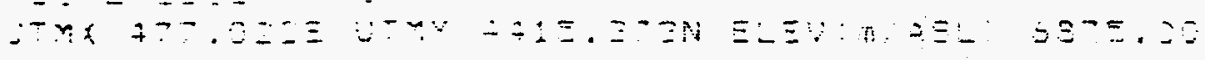

\begin{tabular}{|c|c|c|c|c|c|c|c|c|}
\hline$I S T=$ & $\begin{array}{l}T:-i= \\
\Xi \div=\bar{T}\end{array}$ & $\begin{array}{l}-\div E \\
3-0=\end{array}$ & $\begin{array}{c}200 \\
H T \\
n: C-y\end{array}$ & 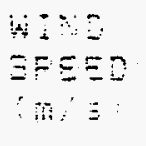 & $\begin{array}{l}\because \vdots 9 \\
\because= \\
=5\end{array}$ & 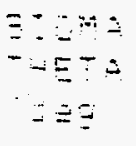 & $\operatorname{SE}$ & 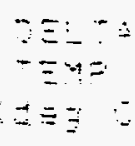 \\
\hline$=: Z=:$ & $\therefore=$ & $: 50$ & 5.10 & $\therefore .1$ & 17.5 & 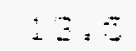 & $\equiv=$ & \\
\hline$=:-\cdots: \vdots$ & $\because \therefore$ & $: 0$ & $17 .=3$ & $z=$ & $-1 .=$ & $\therefore \because=0$ & $\Xi$. & -8.3 \\
\hline$\because \cdots$ & $1 \cdots$ & .90 & $s=:-$ & 3.1 & $\therefore$ & & 7. & \\
\hline$\therefore \because$ & $\therefore \therefore$ & -3 & 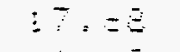 & $E=4$ & $\overline{7}, \overline{0}$ & $\because \therefore$ & 7. & $-\because$ \\
\hline$\therefore \therefore-\cdots$ & 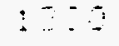 & $\therefore 9$ & $\leq \therefore$ & 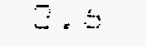 & $\therefore-2$ & $\therefore \dot{2} . \overrightarrow{2}$ & $\overline{7} .3$ & \\
\hline$\therefore-\therefore$ & $\therefore$ & $\therefore-$ & $\therefore \cdots=3$ & $\because \quad \div$ & $\because \because .9$ & $\overline{7}, \overline{7}$ & $=$. & - \\
\hline$=\vdots \cdots$ & $: \because:=$ & $\because 5$ & $\therefore \quad \therefore$ & $\therefore$ & $\therefore 4.5$ & $i 1.1$ & $7 \times$ & \\
\hline$=: \because 2-1$ & $\therefore \because$ & $:=1$ & $1= \pm$ & $\therefore=$ & $=2.5$ & 19.4 & $\exists=3$ & $-9,4$ \\
\hline$\therefore-2$ & $:=-\therefore$ & $\therefore \therefore$ & $=20$ & $\therefore$ & $6=2$ & 3.3 & 2.5 & \\
\hline 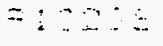 & $:=-9$ & $\therefore \therefore 6$ & $2:-3$ & $3: \therefore$ & 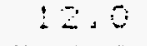 & 3.7 & $\because .3$ & $-3,2$ \\
\hline$=: 2=0$ & $1=0$ & $\therefore: 0$ & $5 .: 5$ & $\bar{L}=4$ & $3+7.0$ & 12.4 & 7.1 & \\
\hline $7: \cdots$ & $1=:$ & : ? & $: 7,=\Xi$ & $\because .5$ & $y=5=0$ & 11.3 & $?=1$ & 5.0 \\
\hline$=102: 3$ & $1-\cdots$ & $: \equiv 0$ & 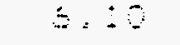 & $=4$ & 349.2 & 13.4 & $?=6$ & \\
\hline$=: 0: 2$ & $: 7:$ & $\therefore \therefore 0$ & $\because ?=3$ & $\therefore 7$ & $3=9.0$ & 12.5 & 7.3 & 3 \\
\hline $7: 920$ & $\therefore=-1$ & 170 & $\Xi . \vdots \square$ & $=4$ & 331.9 & 12.1 & $\leftrightarrows . ?$ & \\
\hline $7: 92$ & $\therefore \equiv$ & : 75 & 17.58 & $\ddot{\div}$ & 345.6 & 10.4 & $\bar{z}$ & 5.5 \\
\hline$=19-9$ & $i=-0$ & $\because-60$ & 5.10 & $=7$ & $3-4.0$ & $1=0$ & \pm .7 & \\
\hline 71020 & $i \overline{7}$ & $\therefore 0$ & 17,00 & 3.2 & 735.0 & 10.6 & 7.12 & 0.4 \\
\hline $9: 920$ & 9000 & $\because 100$ & 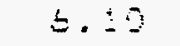 & 3.4 & 325.6 & 14.9 & $7 . \vdots$ & \\
\hline$=10=39$ & 2605 & $\because 100$ & $17=03$ & 3.5 & 333.0 & 13.4 & 7.3 & 0.2 \\
\hline 910200 & 2100 & $\because 200$ & $\leq 10$ & 3.5 & 342.0 & 11.7 & 6.9 & \\
\hline 910203 & $\because: 00$ & 2200 & 17.53 & 3.7 & 350.0 & 10.4 & 7.0 & 0.1 \\
\hline$=10-09$ & $2-00$ & $=200$ & $s=10$ & 3.9 & $341: 0$ & 12.0 & \pm .3 & \\
\hline $710-9$ & 2000 & $\because 300$ & 17.59 & 4.2 & 350.0 & 10.4 & 0.0 & 0.2 \\
\hline 710209 & $=300$ & 0 & 5.10 & 3.1 & 328.0 & 12.5 & 5.1 & \\
\hline 71029 & 2390 & 9 & $17=\leq ?$ & 3.5 & 338.0 & $11 \cdot 3$ & 5.3 & 0.2 \\
\hline
\end{tabular}




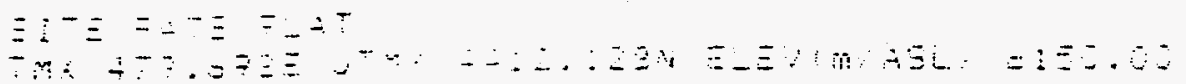

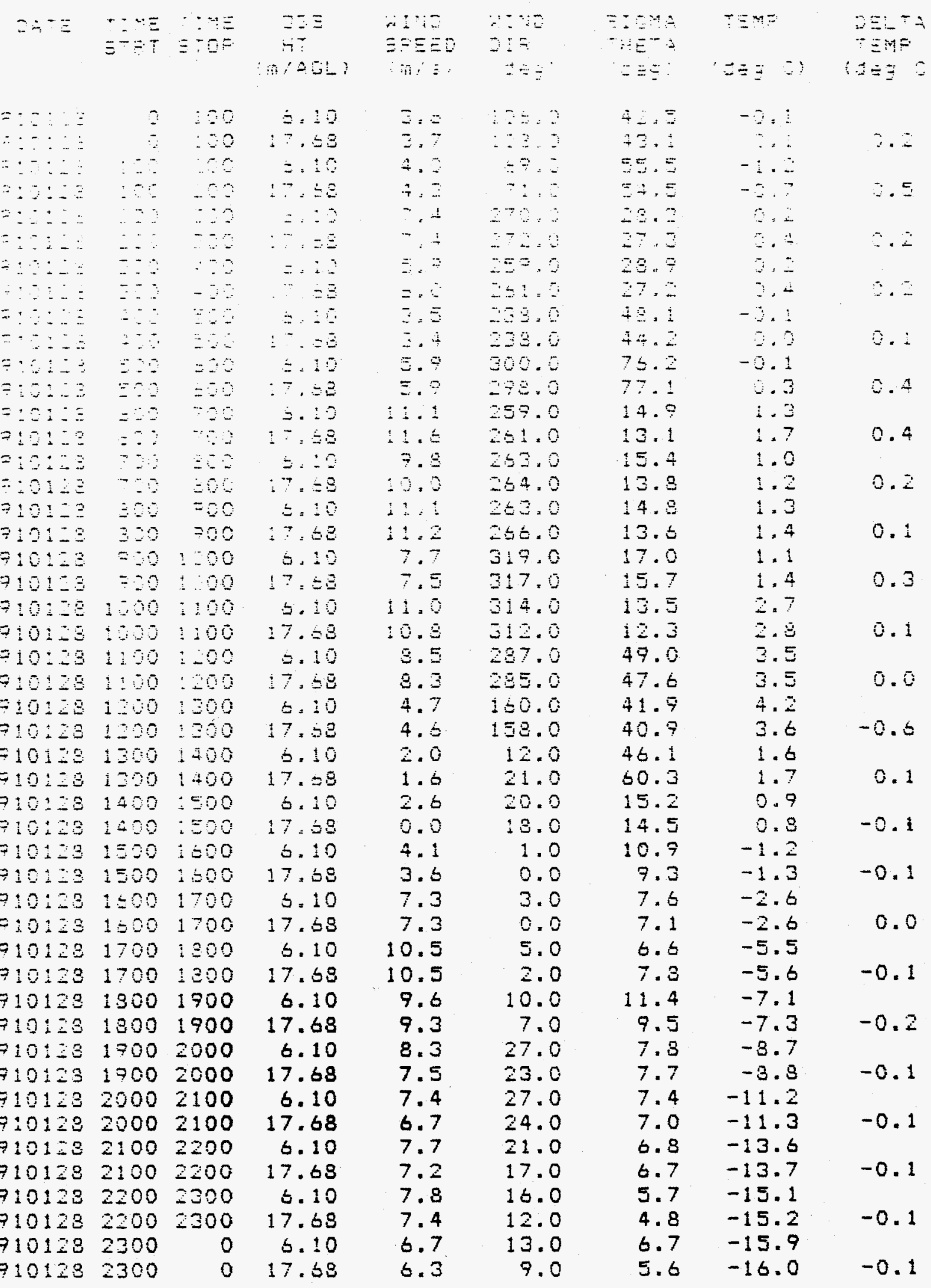




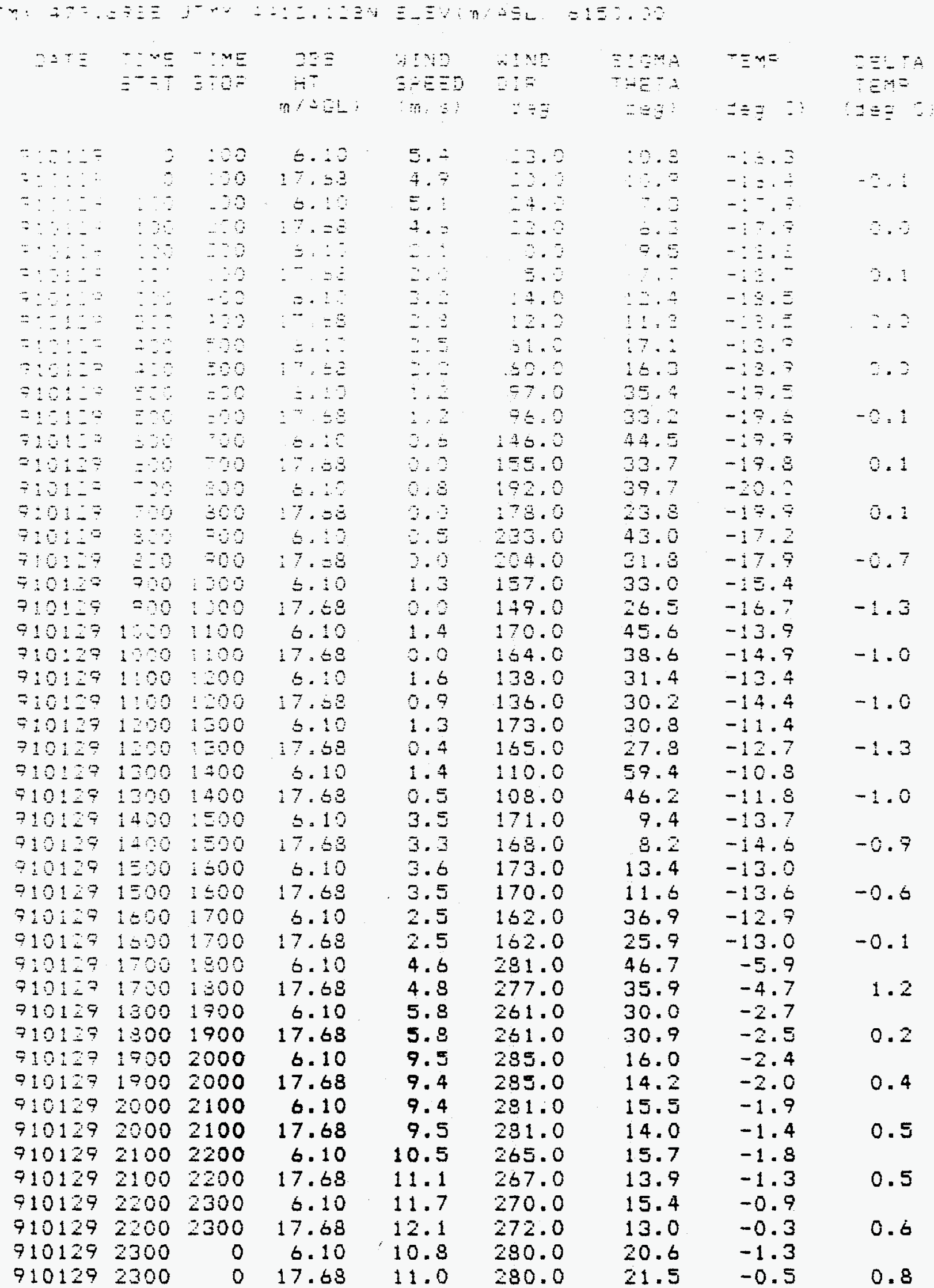




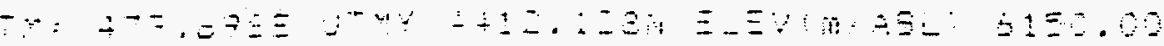

\begin{tabular}{|c|c|c|c|c|c|c|c|c|}
\hline 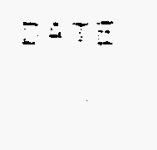 & $\begin{array}{l}\cdots n E \\
\vdots-n-\end{array}$ & git & 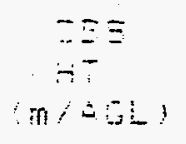 & $\begin{array}{l} \\
\equiv=\Xi \Xi 0 \\
\therefore\end{array}$ & 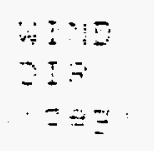 & 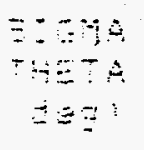 & $r \equiv w=$ & $\begin{array}{l}=\equiv-5 \\
-E M= \\
I \equiv \equiv\end{array}$ \\
\hline$\because::=$ & 8 & $\therefore 20$ &.$\pm: 0$ & 7.0 & $\therefore 3$. & $\vdots 0$ & $-! . \div$ & \\
\hline$=$ & 0 & $: 00$ & 17.53 & $\leq{ }^{-}$ & $\therefore 8.2$ & 12.0 & $-: .0$ & $\therefore .4$ \\
\hline$\because-$ & & $\therefore 20$ & $5 . \therefore$ & 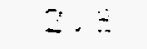 & 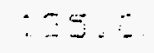 & $\therefore 2$. & $-\vdots . \cdot$ & \\
\hline$\because:$ & $:: 0$ & $\therefore: 0$ & $12=8$ & $\because \because$ & $: 45$. & $=0 \leqslant$ & -1.4 & 0.3 \\
\hline & $\therefore:$ & $\because 2$ & $\because \quad \therefore$ & 3. $:$ & $72=$ & $5=.5$ & -5.7 & \\
\hline$\because: \because$ & $\therefore \because$ & $\because 2$ & 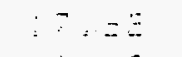 & $\therefore \quad$ & 32.0 & $5=5$ & -5.5 & 2.2 \\
\hline$\therefore \because$ & $\because \because$ & 40 & $\leq \therefore$ & $\therefore=$ & $\because z=0$ & $E 5: 5$ & -6.4 & \\
\hline$\therefore: \vdots:$ & $\because 20$ & 40 & $\because z=\vdots$ & 4,8 & 155.0 & 47.6 & $-\therefore=$ & $\bar{C}=$ \\
\hline $5:=2$ & $4 \because 2$ & 50 & $\leq: \therefore$ & 2.5 & $\therefore Z=0$ & 46.1 & -2.3 & \\
\hline $6:=$ & $+\because 0$ & 50 & $: 7=0$ & 3.3 & $1: 3.0$ & 44.9 & $-\mathbf{i}=3$ & $\sigma=$ \\
\hline$\therefore: 2:$ & $=25$ & $\therefore \therefore$ & $5: 10$ & $\because$. & $\because 55.0$ & 72.0 & -1.3 & \\
\hline$\therefore 2: 5$ & $\because 20$ & $=20$ & $: 7.53$ & 70 & $=0.0$ & 93.7 & -0.7 & 0.4 \\
\hline $0:=$ & $=: 8$ & -50 & $\leq .10$ & $: 8.4$ & 267.0 & 7.8 & -1.1 & \\
\hline $19=$ & $=0$ & 80 & $17:=0$ & $: \Xi .7$ & -39.0 & 6.5 & 0.1 & 1.2 \\
\hline$: 0: 1=$ & 38 & 220 & $\leq$. & $: 3 . i$ & 275.0 & 8.3 & -2.6 & \\
\hline $601=0$ & 250 & 50 & $i \vec{i}, 0 s$ & $1 \Xi .4$ & 295.0 & 7.0 & 0.5 & $i . i$ \\
\hline$: 0120$ & 820 & 700 & $=10$ & $: \leq .7$ & 297.0 & 7.3 & 1.0 & \\
\hline $9: 0130$ & 390 & 70 & $17=3$ & 17.2 & 297.0 & 3.1 & 2.0 & 1.0 \\
\hline 10132 & 720 & $: 90$ & 6.10 & $17=7$ & 288.0 & 8.3 & 2.2 & \\
\hline 0139 & $=90$ & 1000 & $17:=3$ & $: 7=7$ & -89.0 & 6.8 & $\therefore 3$ & 0.6 \\
\hline 25 & 1009 & 100 & $5:: 0$ & $: 7.7$ & $234=0$ & 7.1 & 3.7 & \\
\hline 10130 & 1000 & 1100 & 17.69 & $: 3=i$ & 205.0 & 7.3 & 4.0 & 0.3 \\
\hline 130 & 1100 & 1200 & 6.10 & $15=5$ & 276.0 & 10.9 & 4.5 & \\
\hline 10130 & 1100 & 1200 & 17.53 & $: 7.0$ & 278.0 & 3.6 & 4.7 & 0.2 \\
\hline 135 & 1200 & 1300 & 5.10 & $1 \Xi .0$ & 266.0 & 12.2 & 4.8 & \\
\hline 10130 & 1200 & 2300 & 17.58 & 15.4 & 259.0 & 10.9 & 4.8 & 0.0 \\
\hline 30 & 1300 & 1400 & 6.10 & 10.7 & 274.0 & 29.0 & 3.0 & \\
\hline$: 0: 30$ & 1300 & 1400 & $17=59$ & 50.8 & 276.0 & 28.2 & 6.0 & 0.0 \\
\hline 1019 & 1400 & 1500 & 5.10 & 6.9 & 253.0 & 26.7 & 6.7 & \\
\hline $10 \pm 70$ & 140 & 2500 & $17: 5$ & $\leq .3$ & 254.0 & 25.1 & 5.6 & -0.1 \\
\hline 10130 & 1500 & 1500 & 6.10 & 7.0 & 267.0 & 36.1 & 6.0 & \\
\hline 10130 & 1500 & 1500 & $17=68$ & $\leq .9$ & 270.0 & 33.4 & 5.7 & -0.1 \\
\hline 10130 & 1600 & 1700 & $b=10$ & 7.3 & 230.0 & 31.2 & 5.4 & \\
\hline 130 & 1500 & 1700 & 17.63 & 7.4 & 282.0 & 30.1 & 5.5 & 0.1 \\
\hline 130 & 1700 & 1300 & 6.10 & 13.9 & 286.0 & 10.7 & 4.6 & \\
\hline 120 & 1700 & 1300 & 17.68 & 14.3 & 287.0 & 9.3 & 5.0 & 0.4 \\
\hline 8130 & 1.800 & 1900 & 6.10 & 6.2 & 241.0 & 55.4 & 4.5 & \\
\hline 130 & 1900 & 1900 & 17.68 & 6.2 & 240.0 & 57.4 & 4.7 & 0.2 \\
\hline 130 & 1900 & 2000 & 6.10 & 4.4 & 240.0 & 66.7 & 4.5 & \\
\hline 130 & 1900 & 2000 & 17.68 & 4.4 & 240.0 & 67.5 & 4.7 & 0.2 \\
\hline 130 & 2000 & 2100 & 6.10 & 5.8 & 247.0 & 27.2 & 4.5 & \\
\hline 130 & 2000 & 2100 & 17.68 & 6.1 & 248.0 & 26.0 & 4.8 & 0.3 \\
\hline 130 & 2100 & 2200 & 6.10 & 4.4 & 263.0 & 62.7 & 4.7 & \\
\hline 130 & 2100 & 2200 & 17.68 & 4.4 & 259.0 & 63.8 & 4.8 & 0.1 \\
\hline 30 & 2200 & 2300 & 6.10 & 6.7 & 223.0 & 54.9 & 5.2 & \\
\hline 00 & 2200 & 2300 & 17.63 & 6.7 & 223.0 & 50.3 & 5.4 & 0.2 \\
\hline $\begin{array}{l}30 \\
30\end{array}$ & $\begin{array}{l}2300 \\
2300\end{array}$ & $\begin{array}{l}0 \\
0\end{array}$ & $\begin{array}{r}6.10 \\
17.63\end{array}$ & $\begin{array}{l}7.2 \\
7.5\end{array}$ & $\begin{array}{l}266.0 \\
254.0\end{array}$ & $\begin{array}{l}30.6 \\
82.9\end{array}$ & $\begin{array}{l}5.5 \\
5.8\end{array}$ & 0.3 \\
\hline & 2200 & & 16.00 & & & & J.0 & \\
\hline
\end{tabular}




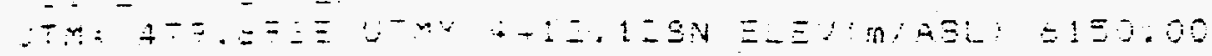

\begin{tabular}{|c|c|c|c|c|c|c|c|c|}
\hline$y \div=$ & 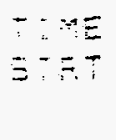 & $\begin{array}{l}-\because E \\
\text { Eัof }\end{array}$ & $\begin{array}{c}2 z \\
47 \\
201\end{array}$ & $\begin{array}{c}n \\
5=\Xi \Xi 0 \\
\therefore\end{array}$ & $\begin{array}{l}\therefore=0 \\
\therefore= \\
\therefore=5\end{array}$ & 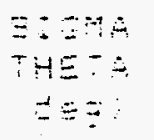 & 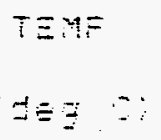 & 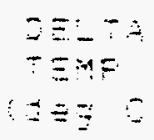 \\
\hline$=$ & 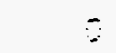 & 20 & $\doteq .10$ & $19 .:$ & 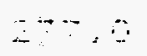 & 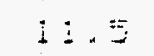 & $\therefore \ddot{z}$ & \\
\hline & $y$ & $: 20$ & $\div 7.53$ & $\because 2.3$ & $\therefore=0$ & 7.1 & $=$. & $\therefore .5$ \\
\hline$\because \cdots$ & $\therefore:$ & $\therefore 0$ & $3 .: 0$ & $\therefore 5$. & $\because 5=2$ & $\therefore .5$ & $\therefore$ & \\
\hline 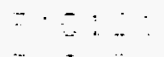 & $\because \because$ & 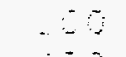 & $\therefore \because=3$ & $:=. \Xi$ & $-a$ & $\Xi . \overline{7}$ & $=. \equiv$ & $\therefore=$ \\
\hline$\because$ & $\because \because$ & $\because \because$ & $=\ldots$ & $\therefore \Xi . \vdots$ & $\cdots \div$ & $\therefore \ldots$ & $\Xi . \Xi$ & \\
\hline$\therefore:-$ & $\therefore$ & $\because \square$ & $a^{-\cdots}=-\varepsilon$ & $: \because$. & $\therefore--\sigma$ & 7.2 & 7. & $\therefore$. \\
\hline$=:$ & $\therefore \quad$ & $\therefore$ & $=. \therefore$ & $\therefore$ & $\therefore=5.0$ & $\therefore .5$ & S. & \\
\hline 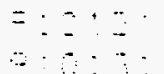 & $-\infty$ & $\because$ & $\vdots 7.3$ & $\therefore \therefore$ & $\because=0=0$ & 25.2 &.$\pm \leq$ & 3. \\
\hline$=$ & $\therefore 9$ & $\because 0$ & $=\therefore$ & $\because=$ & $2 \bar{Z}, 5$ & $6 \equiv . \Xi$ & $\Xi .-$ & \\
\hline$=: 3: z$ & $=0$ & $=0$ & $: \because: 3$ & ?- & 252.0 & $=\overline{7} .:$ & 5.5 & $\therefore=$ \\
\hline$=: 5: 5$ & $=: 0$ & $=62$ & $\Delta 19$ & $\therefore . Z$ & 274.5 & 12.3 & 5.7 & \\
\hline$\because: 0:=$ & $=20$ & $=-\infty$ & $: 7 .=3$ & $\therefore 2$. & $\because 76.0$ & 11.7 & 5.0 & 0.3 \\
\hline$\because: \because: Z:$ & $=-8$ & -9 & 6.10 & 20.5 & 257.0 & 13.5 & $\Xi .9$ & \\
\hline$=: 9121$ & $=-9$ & $\because 50$ & $\therefore 7=-2$ & $\therefore 9.9$ & 270 & 11.9 & 6.3 & 0.4 \\
\hline$=: 9:=$ & -8 & 80 & $=$. & $\because 2.3$ & 259.9 & 13.1 & \pm .2 & \\
\hline$=10: 31$ & -80 & $\Xi 5$ & $17=58$ & 13.2 & 270.0 & 11.2 & 5.7 & 0.5 \\
\hline $\bar{Y}: \bar{Z} \mathrm{I}$ & 30 & 700 & $5 .: 0$ & $1: 7$ & 27.0 & 11.2 & 6.7 & \\
\hline$=103$ & 30 & $=50$ & 37.50 & 12.1 & 272.0 & 3.9 & 7.0 & 0.3 \\
\hline $7: 5: 3:$ & $=5$ & 150 & 5.10 & 10.5 & $25 \Xi .0$ & 10.2 & 3.3 & \\
\hline$=10.31$ & $=-8$ & 100 & 17.03 & 11.0 & 256.0 & 5.4 & 3.5 & 0.3 \\
\hline $7: 5: 71$ & 125 & 1100 & $\Xi 10$ & 11.3 & 259.0 & 10.9 & 8.3 & \\
\hline $9: 013:$ & 1500 & 1100 & 17.28 & 11.5 & 260.0 & 7.2 & 3.5 & 0.2 \\
\hline $710: 9:$ & 1190 & $: 200$ & $5 .: 0$ & 10.7 & 259.0 & 14.7 & 10.5 & \\
\hline$=: 0: 3:$ & $1: 00$ & 1200 & 17.63 & $1 \div .3$ & 260.0 & 11.9 & 10.4 & -0.1 \\
\hline $7161=$ & 1200 & 1300 & 5.10 & 9.5 & 269.0 & 15.1 & 12.2 & \\
\hline$=1013:$ & 1200 & 1200 & 17.63 & 3.7 & 270.0 & 13.7 & 12.1 & -0.1 \\
\hline $91013:$ & 1200 & 1400 & 5.10 & 5.7 & 304.0 & 27.8 & 11.4 & \\
\hline 710131 & 1300 & $\$ 400$ & 17.68 & 5.8 & 302.0 & 25.7 & 11.8 & 0.4 \\
\hline $91013:$ & 1450 & 1500 & $=.10$ & 3.0 & 311.0 & 55.1 & 15.2 & \\
\hline $91013:$ & 1400 & $1 \equiv 00$ & 17.68 & 2.9 & 306.0 & 62.0 & 14.7 & -0.5 \\
\hline 910131 & 1500 & 1000 & 6.10 & 5.0 & 303.0 & 31.3 & 13.0 & \\
\hline $9: 0131$ & 1500 & $i \leq 00$ & 17.48 & 5.0 & 300.0 & 33.9 & 13.1 & 0.1 \\
\hline 710131 & \pm 000 & 1700 & 6.10 & 2.3 & 293.0 & 31.8 & 11.3 & \\
\hline $710: 3:$ & 1500 & 1700 & 17.68 & 2.5 & 259.0 & 37.1 & 11.6 & 0.3 \\
\hline 710131 & 1700 & 1800 & 6.10 & 1.1 & 220.0 & 55.3 & 8.1 & \\
\hline 910131 & 1700 & 1300 & 17.68 & 1.3 & 189.0 & 35.6 & 3.8 & 0.7 \\
\hline 710131 & 1300 & 1900 & 6.10 & 1.7 & 296.0 & 40.8 & 3.8 & \\
\hline 910131 & 1300 & 1900 & 17.68 & 1.4 & 281.0 & 47.4 & 7.1 & 0.3 \\
\hline 710131 & 1700 & 2000 & 6.10 & 6.1 & 282.0 & 18.9 & 8.2 & \\
\hline 710131 & 1900 & 2000 & 17.68 & 6.6 & 276.0 & 19.5 & 9.2 & 1.0 \\
\hline 710131 & 2000 & 2100 & 6.10 & 7.6 & 294.0 & 11.0 & 7.2 & \\
\hline 710131 & 2000 & 2100 & 17.68 & 7.2 & 296.0 & 9.0 & 7.7 & 2.7 \\
\hline 910131 & 2100 & 2200 & 6.10 & 6.3 & 293.0 & 10.5 & 7.6 & \\
\hline $91013 i$ & 2100 & 2200 & 17.68 & 5.6 & 280.0 & 10.8 & 9.5 & 1.9 \\
\hline 910131 & 2200 & 2300 & 6.10 & 5.1 & 290.0 & 17.1 & 8.1 & \\
\hline 910131 & 2200 & 2300 & 17.68 & 4.4 & 285.0 & 18.3 & 9.9 & 1.8 \\
\hline 910131 & 2300 & 0 & 6.10 & 4.8 & 302.0 & 12.4 & 9.2 & \\
\hline 910131 & 2300 & 0 & 17.63 & 3.9 & 299.0 & 13.3 & 10.1 & 0.9 \\
\hline
\end{tabular}




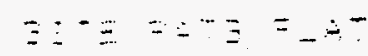

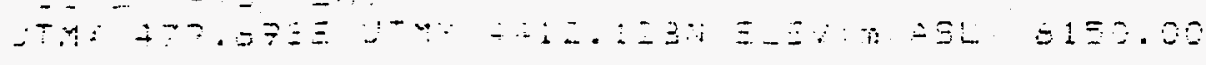

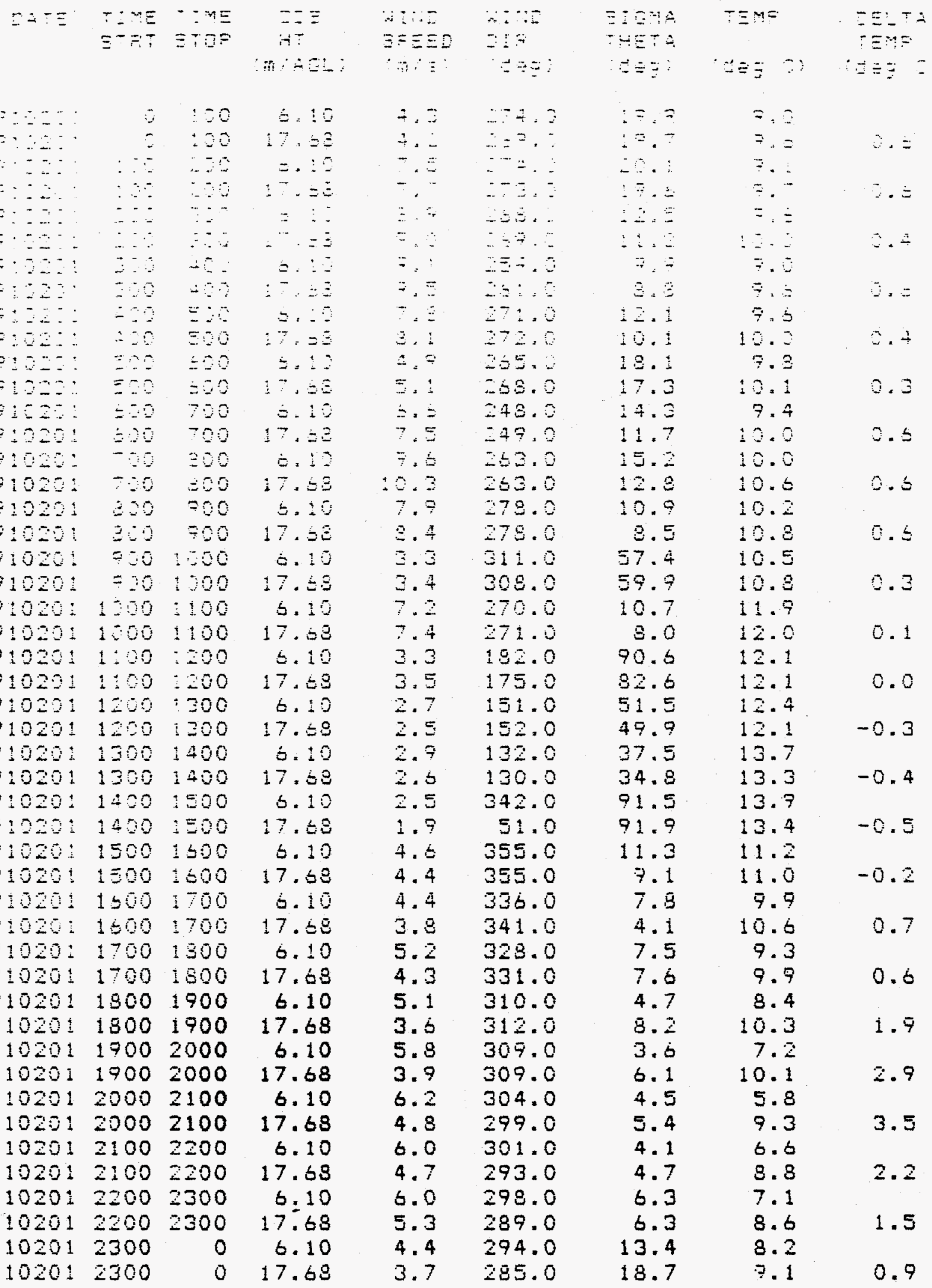




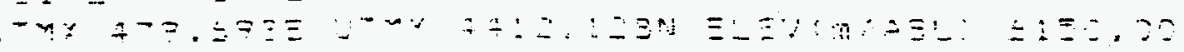

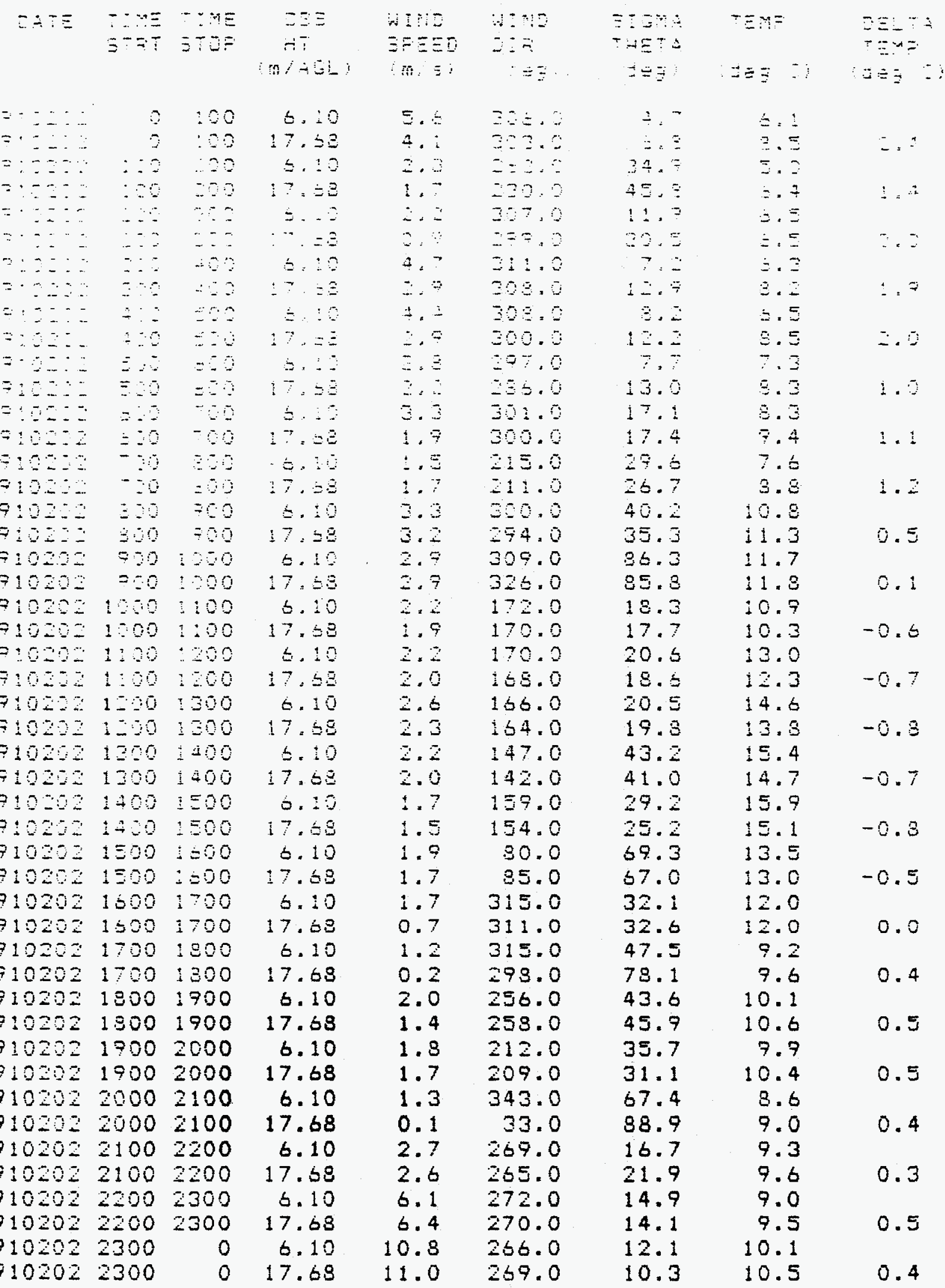




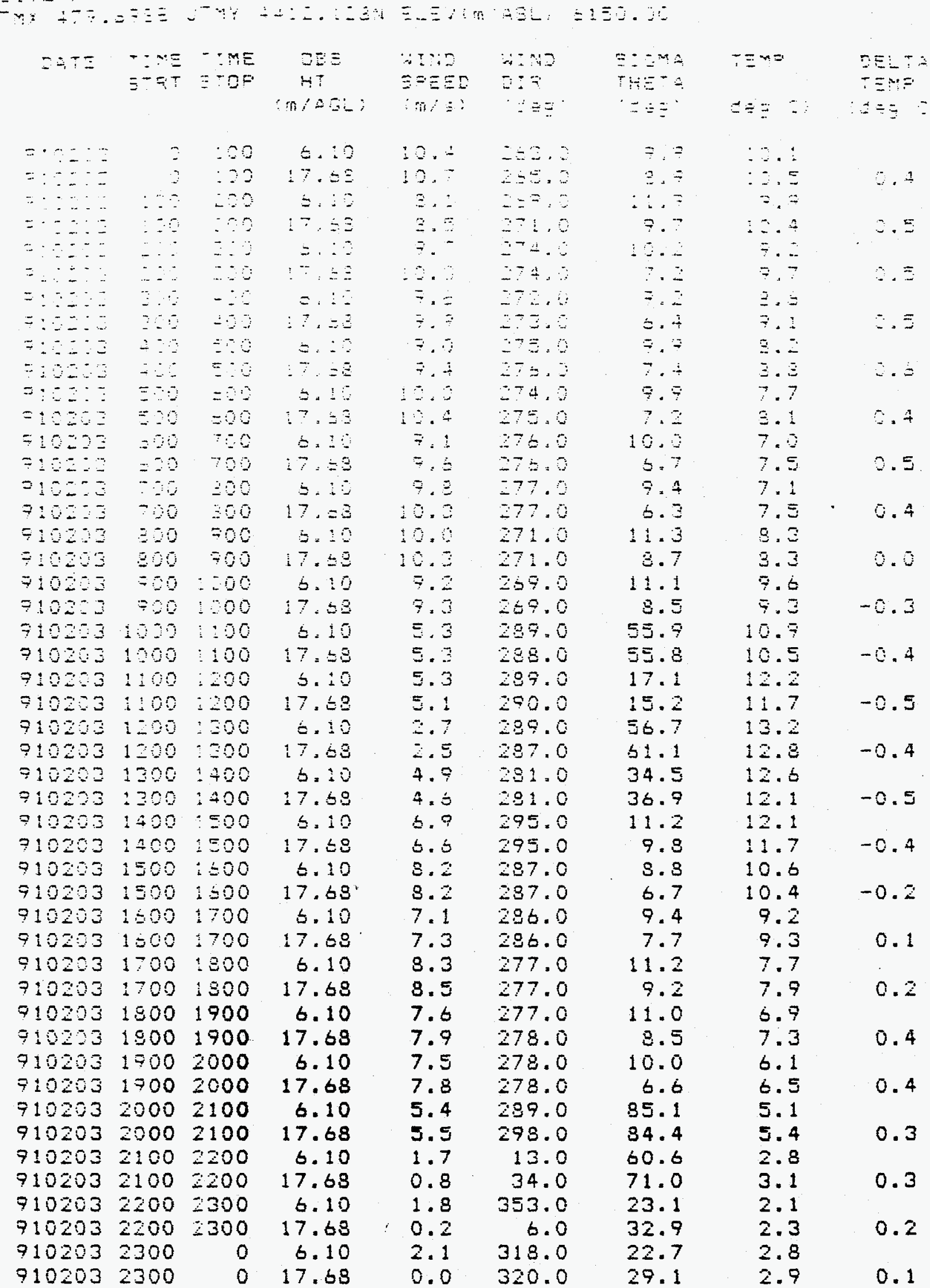



$\therefore$ क मे iil in

"a s.

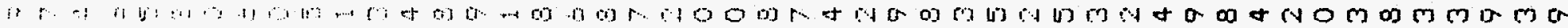

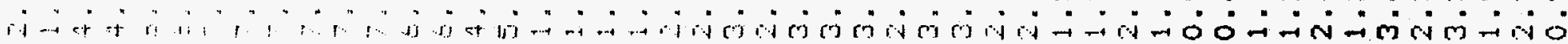

क $\operatorname{lin} x$

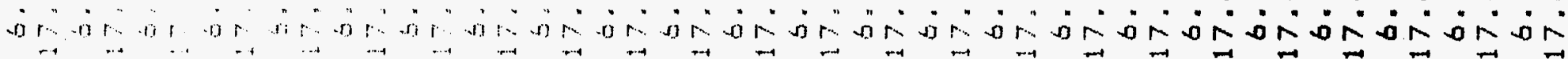

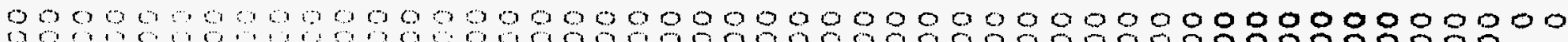
, a ........................ a a. i, ;

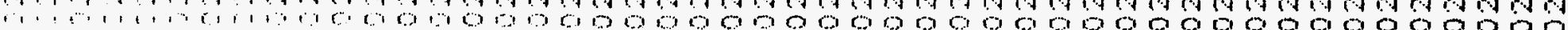

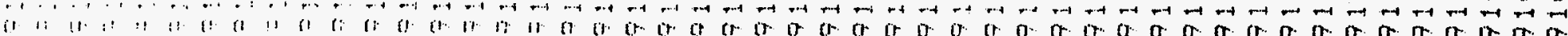




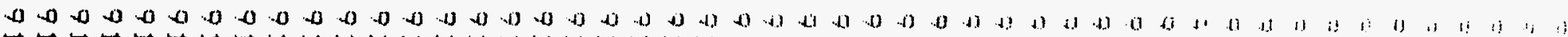

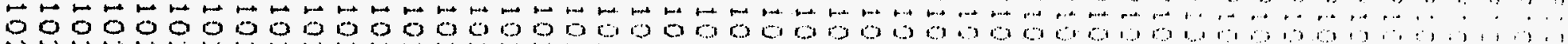

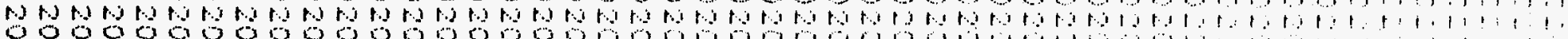

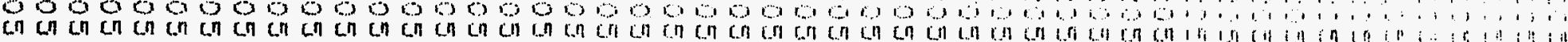

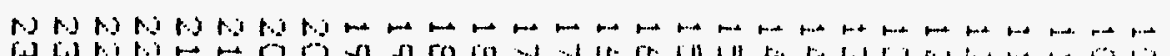

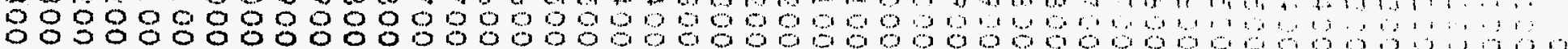

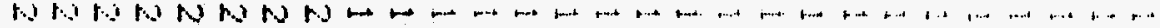

w W H

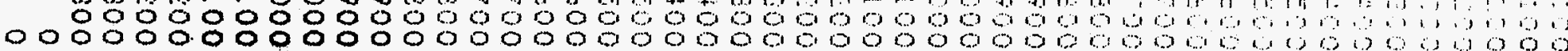

Faำ

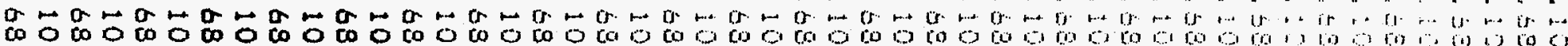

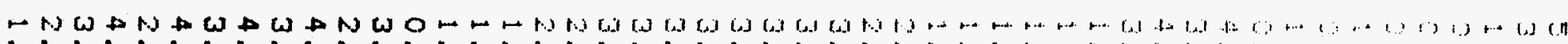

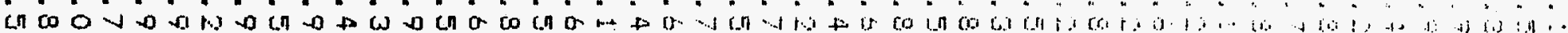

MpMGW

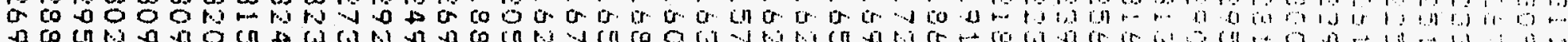

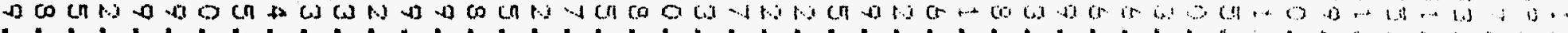

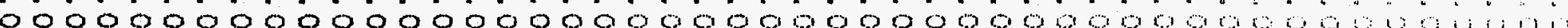

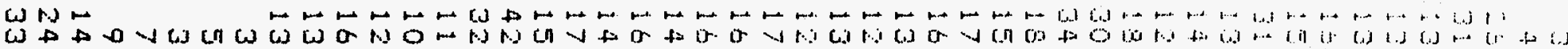

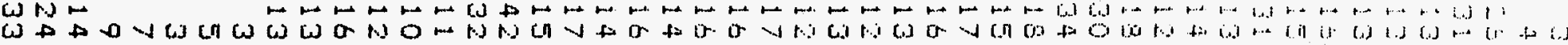
"

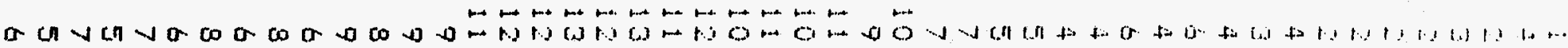

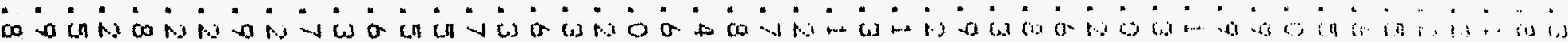

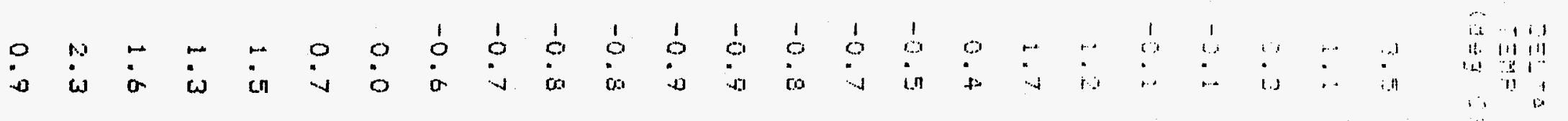




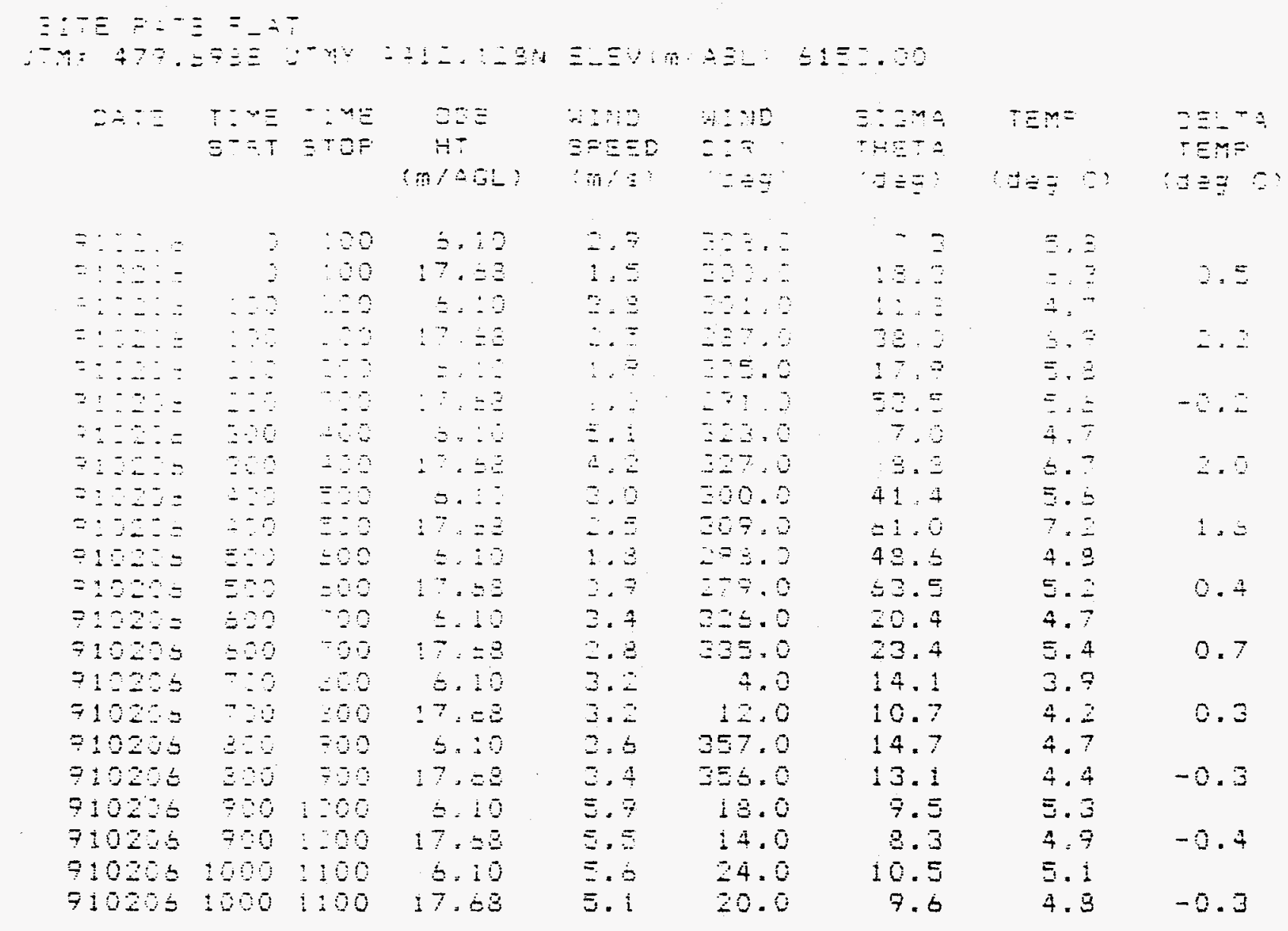




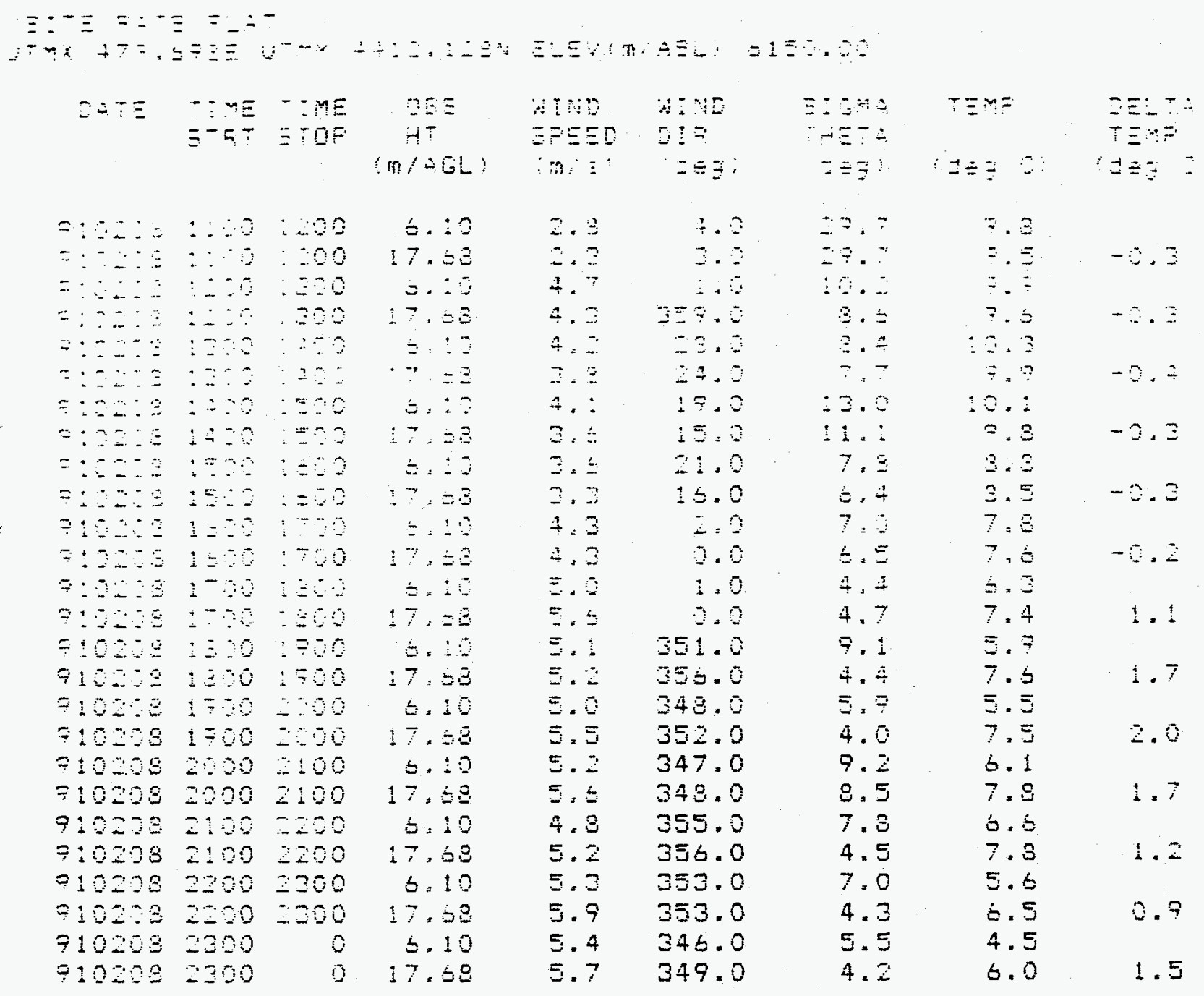


$\equiv: \equiv=-\therefore \equiv=\equiv+$

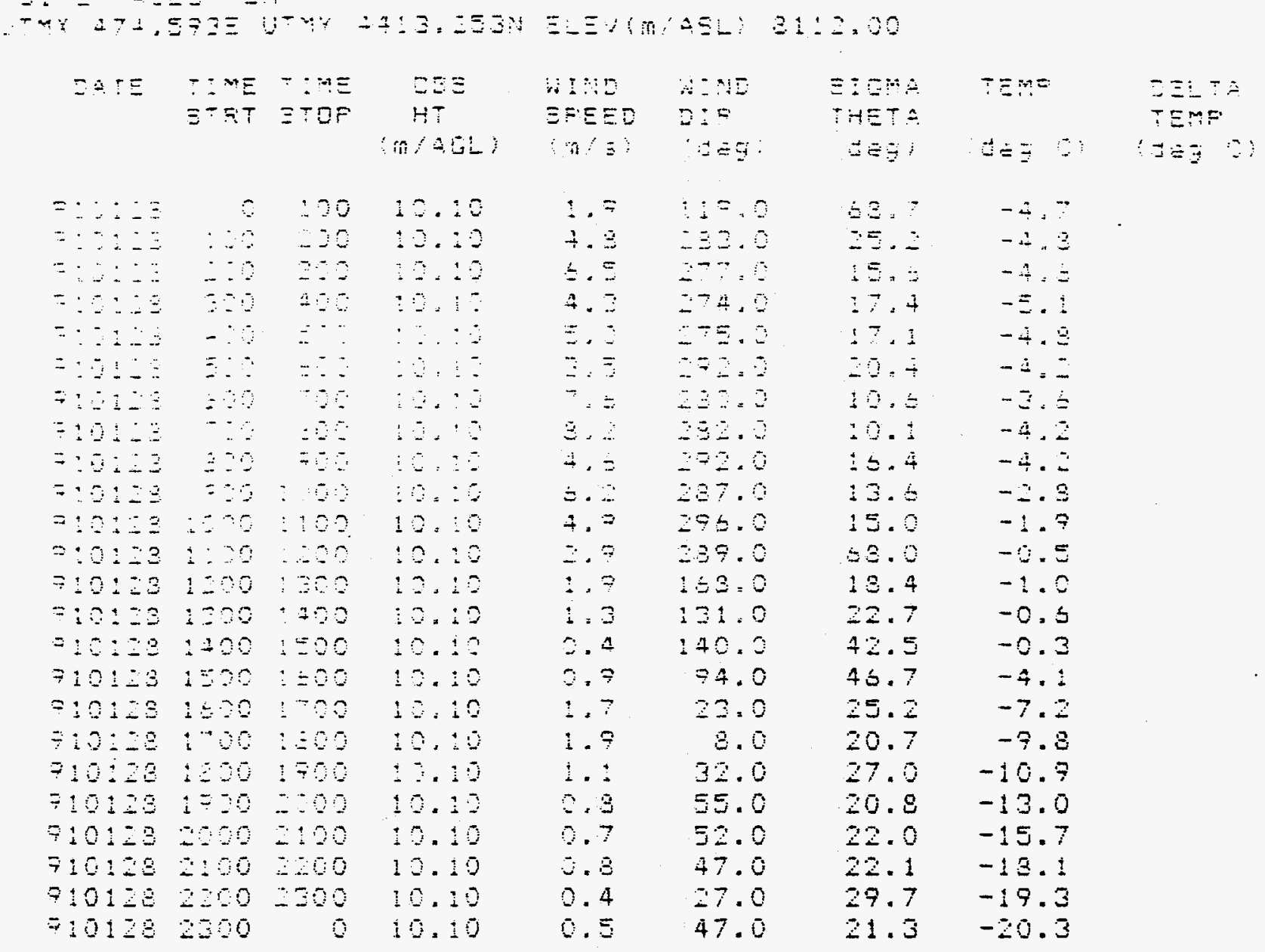


$\Xi:-\equiv=-\ldots \equiv \equiv \equiv$

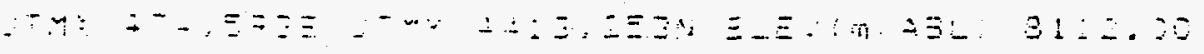

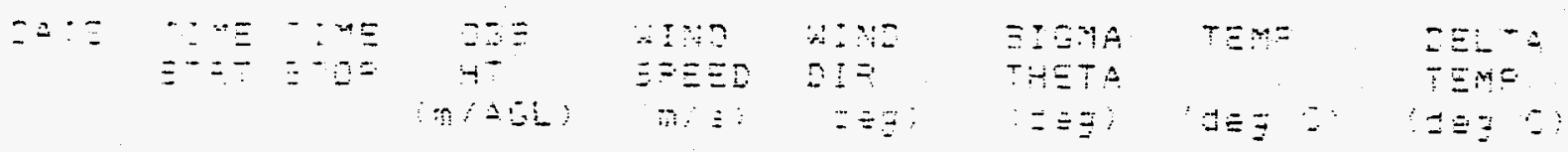

\begin{tabular}{|c|c|c|c|c|c|c|c|}
\hline$\because:::=$ & 3 & $\therefore \therefore$ & $: 2 .: 0$ & $0 .=$ & $7 \because .3$ & 23.3 & $-Z \therefore=$ \\
\hline$\therefore \div=$ & 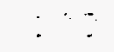 & $\because \div$ & 3.0 & $5=\overline{3}$ & $\because \therefore$ & $\because 2.5$ & $-2=$ \\
\hline$=-1-$ & $\therefore \quad$ & $\because \div$ & 10.5 & $0 .:$ & 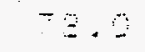 & $1=0$ & $-2=$ \\
\hline$\because \therefore:=$ & $\because \because$ & $\because \therefore$ & $\therefore 2: 5$ & $\because$ & 55 & $\div 5.5$ & $-\square$ \\
\hline$\because 5:=$ & $\therefore \therefore$ & $\therefore \because$ & $\because \because=$ & $\because \ddot{z}$ & $\therefore=2.3$ & $-4=$ & \\
\hline$\because \vdots \because \therefore=$ & $\because$ & $=-\because$ & $\therefore$ & $\because \vdots$ & $:-\overline{0}$ & 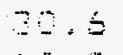 & \\
\hline$=: \div \vdots \div$ & $=\ldots$ & $\because$ & $\vdots 2=\vdots 2$ & $\because=\therefore$ & $i S 3.5$ & $1=2$ & $-z$ \\
\hline $5 \vdots-2=$ & -5 & $\therefore$ & $\because \quad \because$ & $\therefore=5$ & $179=0$ & $7 \div 3$ & \\
\hline$\therefore-1=$ & $\because$ & $=\because$ & 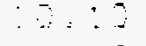 & $\because=$ & $2 B 1=9$ & 37.4 & $-i \leq$. \\
\hline$\vdots \div=$ & $\because \because$ & $: \quad \because$ & 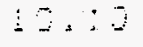 & $\because$. & $\therefore 7=6$ & 74.5 & -13 \\
\hline $6+9:=7$ & $\vdots:-$ & 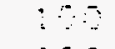 & 10,13 & $\therefore=$ & $=69.0$ & 23.1 & \\
\hline $2:=$ & $\because: \because$ & 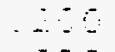 & $\because 9.9$ & -3 & 59.0 & $: 8.6$ & \\
\hline$\therefore \therefore=$ & $1 \because \cdots$ & 3 & $5+1$ & $z=$ & 29.0 & 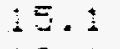 & \\
\hline $718 \div 7=$ & $\because \because 9$ & $\therefore \because$ & $1= \pm$ & $\therefore=$ & 290,0 & 18.5 & \\
\hline $9: 5:-=$ & $\div 0$ & $:=0$ & 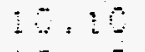 & 4.3 & $283 \times 0$ & 12.0 & \\
\hline $719: 57$ & $1=0$ & $\because=0$ & 15.15 & 2.3 & 275.0 & 20.1 & \\
\hline$=1 \hat{3}:-9$ & $\therefore=8$ & $\vdots \div 9$ & 10.10 & 3.5 & $2=2.0$ & 1 s. 1 & \\
\hline$=: 0127$ & 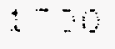 & .500 & $10=18$ & 4.3 & 275.0 & 5.5 & \\
\hline $710 \div 7$ & $:-20$ & $: 700$ & $i 0=i 9$ & 4.7 & 301.0 & 12.0 & \\
\hline $7101=7$ & $1=\div 0$ & $=00$ & $10: 20$ & $4: 7$ & $260=0$ & 13.2 & -6.8 \\
\hline$=1011=$ & $2=0$ & $=100$ & $10 .: 2$ & 5. & 301.0 & 13.0 & \\
\hline 27 & $2 \div 30$ & $=200$ & $10=10$ & $\dot{b .0}$ & 204.0 & 12.4 & -5 \\
\hline 89 & 200 & $=300$ & $10=10$ & 3.1 & 293.0 & 16.6 & - \\
\hline$=10127$ & $2=0$ & O & 10.10 & 5.7 & 299.0 & 13.4 & \\
\hline
\end{tabular}




\begin{tabular}{|c|c|c|c|c|c|c|c|c|}
\hline$\because--\equiv$ & $\begin{array}{l}U n E \\
\equiv=T\end{array}$ & $\begin{array}{l}\because n \equiv \\
\equiv T C P\end{array}$ & $\begin{array}{c}2=\Xi \\
H 5 \\
n=0-1\end{array}$ & $\begin{array}{l}2: 40 \\
\equiv=5 \equiv \\
: n: 5:\end{array}$ & 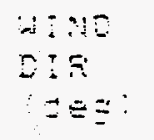 & 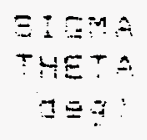 & $\begin{array}{l}T \equiv M= \\
\triangle \pm=Q\end{array}$ & 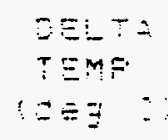 \\
\hline $\begin{array}{l}\cdots \\
\because \\
\cdots \\
\cdots\end{array}$ & 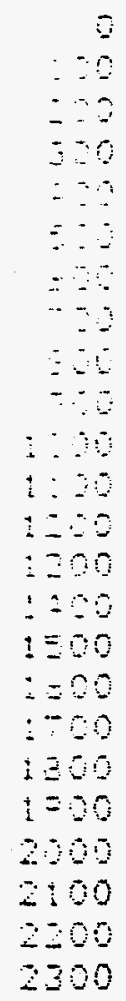 & 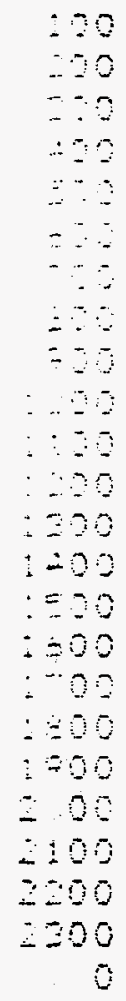 & 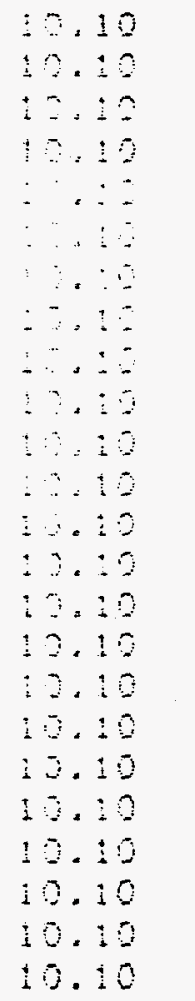 & 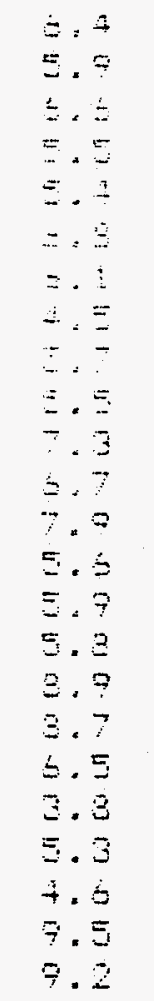 & 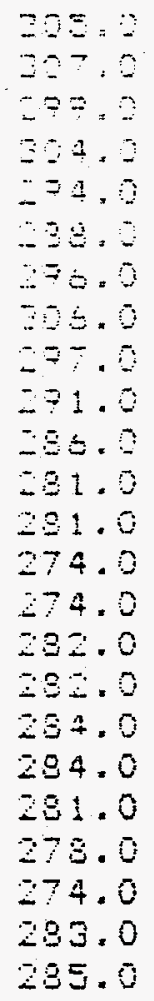 & $\begin{array}{l}1.1 .5 \\
13.5 \\
13.5 \\
15.9 \\
14.0 \\
12.9 \\
14.5 \\
15.9 \\
14.9 \\
14.7 \\
13.2 \\
13.9 \\
13.2 \\
21.6 \\
19.9 \\
17.0 \\
10.4 \\
10.1 \\
16.0 \\
27.0 \\
17.0 \\
21.5 \\
11.8 \\
12.1\end{array}$ & $\begin{array}{r}-5.4 \\
-5.4 \\
-5.1 \\
-4.4 \\
-4.5 \\
-5.5 \\
-5.4 \\
-3.5 \\
-2.0 \\
-2.1 \\
-1.1 \\
-0.3 \\
0.0 \\
0.9 \\
0.4 \\
0.1 \\
-0.1 \\
-0.5 \\
-0.2 \\
-0.4 \\
-0.4 \\
0.0 \\
1.1 \\
1.5\end{array}$ & \\
\hline
\end{tabular}




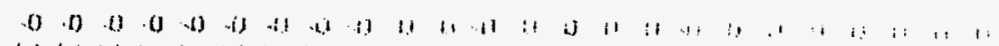

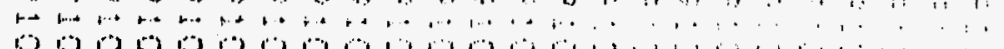
$1,1,1$

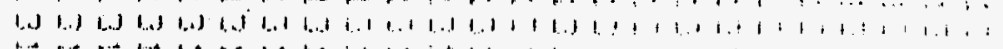

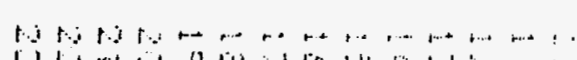

(1)

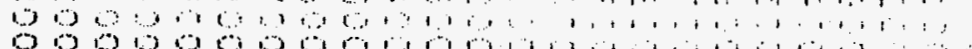

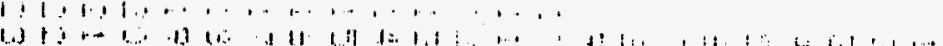

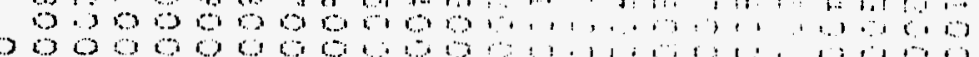

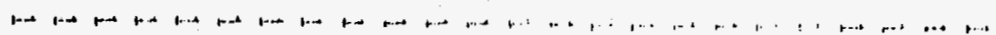

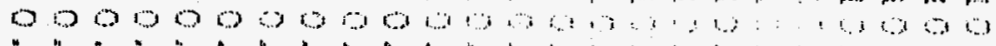

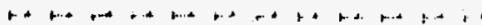

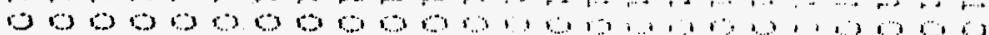

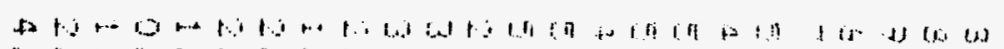

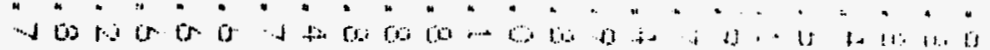

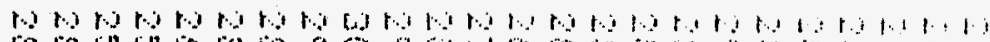

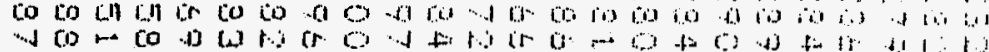

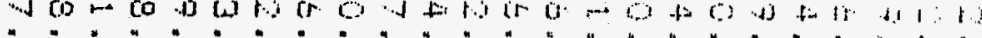

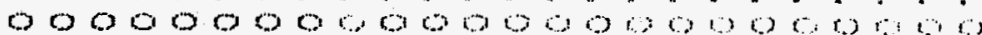

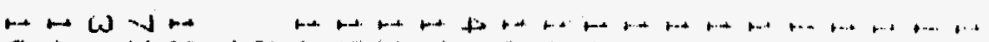

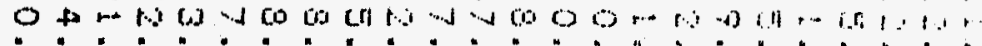

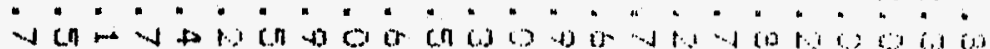

P t

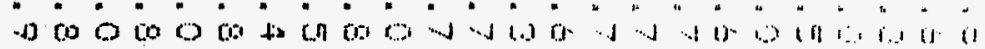




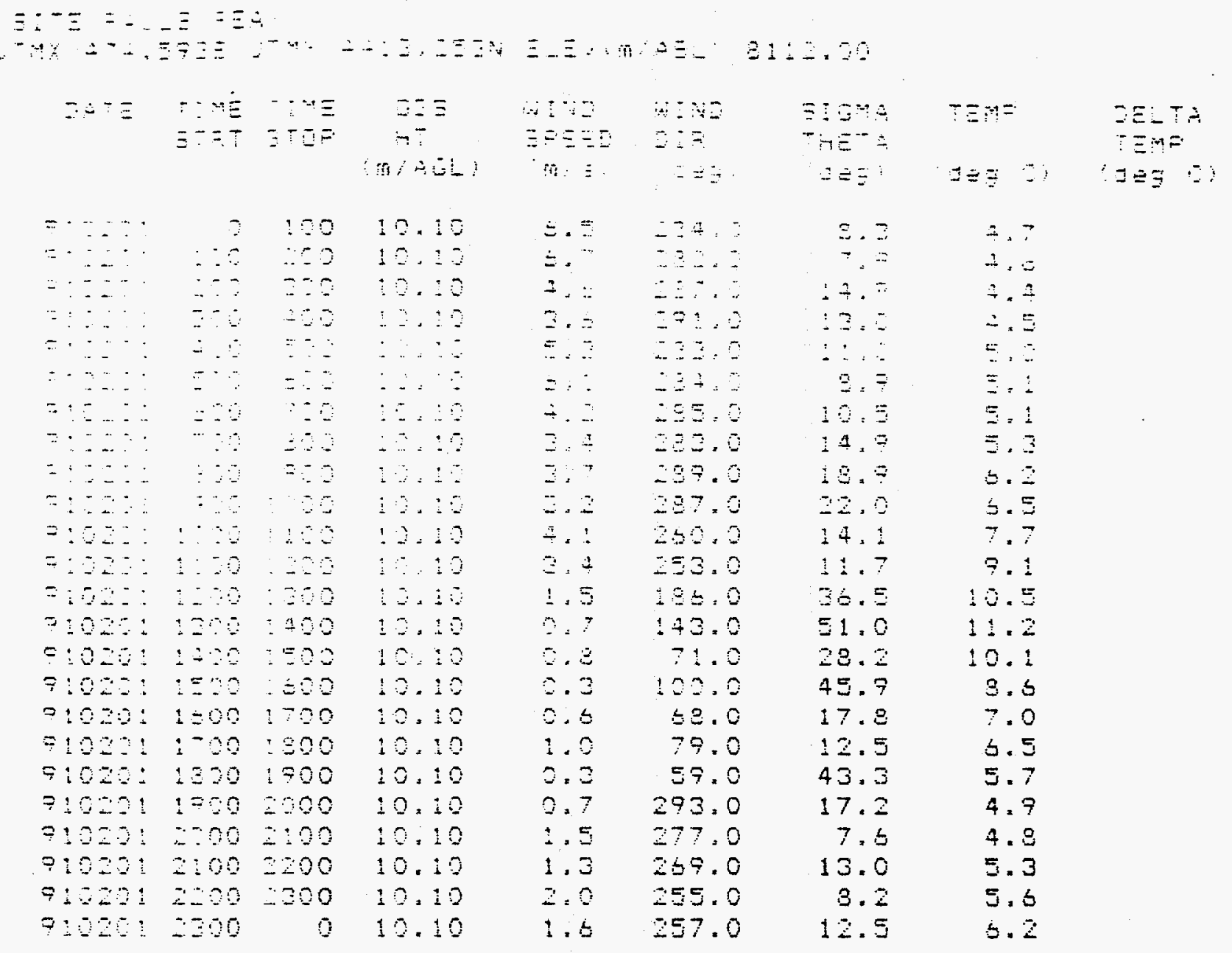


$\equiv:-\equiv=: \ldots \equiv \equiv \equiv$

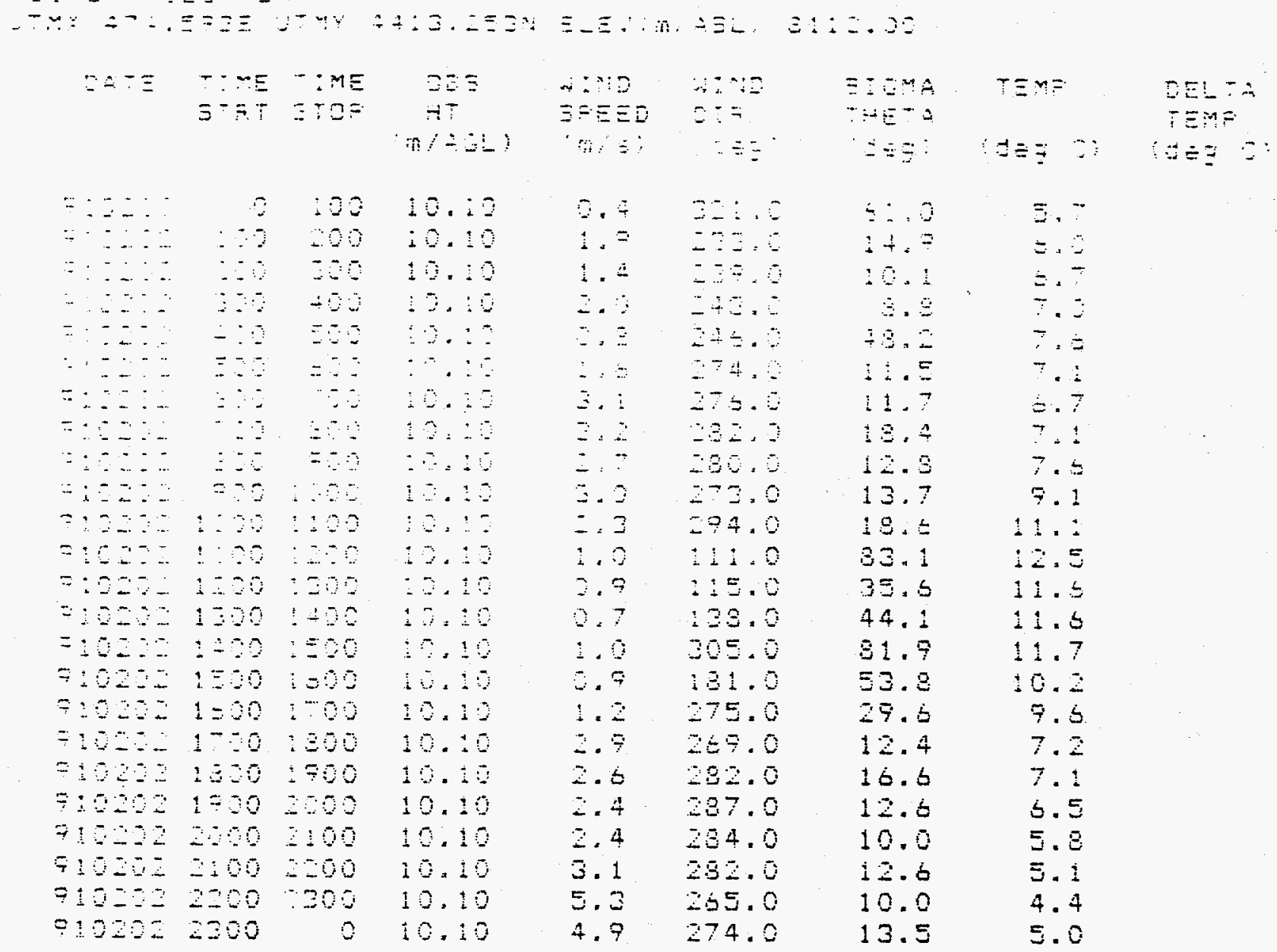




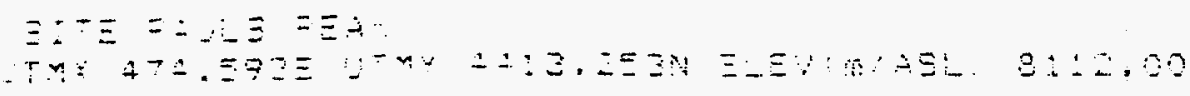

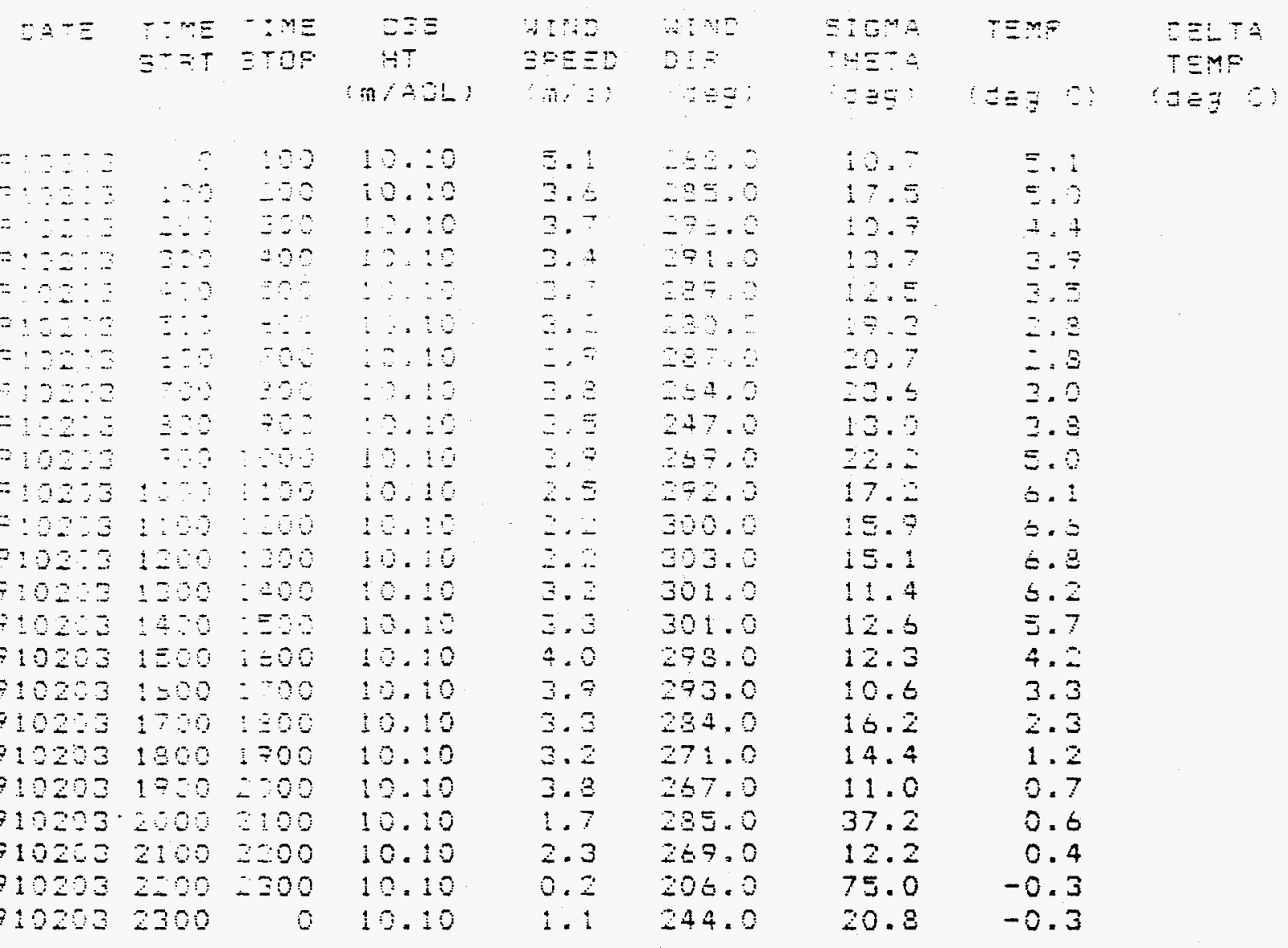


$\Xi:-\equiv=:-\vdots \equiv=\equiv$

-

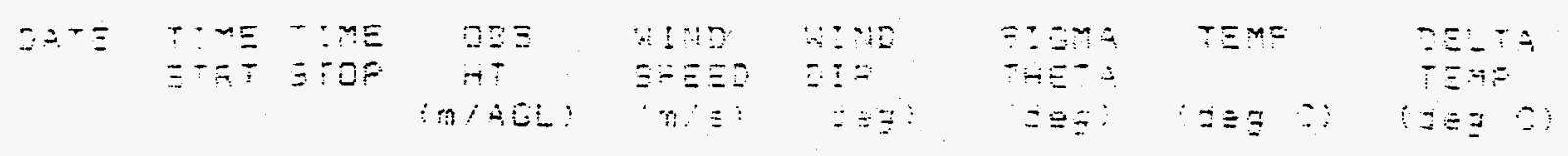

\begin{tabular}{|c|c|c|c|c|c|c|c|}
\hline$=\because \cdots:-$ & $\because$ & $: 0$ & 10.15 & $1 .=$ & $\therefore \Xi .0$ & $12=$ & $-\Xi: \Xi$ \\
\hline$\because \therefore \therefore$ & $\because$ & -20 & 15.25 & $\because \because$ & $\because 7.9$ & $\therefore 2,7$ & $-5 .=$ \\
\hline$=: \ldots \therefore$ & $\because \because$ & $2: 3$ & 19.10 & $2=$ & $\overline{-}=2=2$ & $\% .0$ & $-:=$ \\
\hline$\because \because \cdots$ & $=-$ & $\therefore \because$ & $13=13$ & $\therefore 3$ & $37:-8$ & $\therefore:$. & $-\vdots$ \\
\hline$\because: \therefore-1$ & $\div \because \because$ & $\because-3$ & $\therefore: \therefore$ & $\therefore$. & 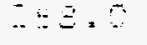 & $\pm \vdots:$ & - \\
\hline$\because: \because \div$ & $\because \ldots$ & $=\because$ & 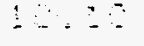 & $\therefore=$ & $\therefore=\ldots ?$ & 3.2 & \\
\hline$\because \div 2 \div$ & $=\because$ & $\because$ & 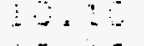 & $3=3$ & $\therefore=5$ & 7.7 & $-\vdots=$ \\
\hline$\because: 0:$ & $\because \because$ & $\because$ & $1: 10$ & $\therefore$ & 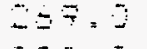 & $10+5$ & -5.7 \\
\hline$\because \therefore \therefore$ & $\equiv-$ & $\because-\because$ & $\because=\vdots$ & $\because+3$ & $2-1$ & $=7.1$ & $\therefore$ \\
\hline$= \pm-2$. & $=\because \therefore$ & $\vdots \quad \vdots$ & 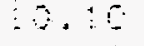 & $\because=$ & $: 11=5$ & $\ddot{-\Xi} \cdot \dot{1}$ & \\
\hline$=10:-1$ & $\therefore \because$ & $: 20$ & 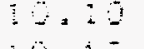 & $\therefore \leq$ & $: 17.9$ & -0.7 & \\
\hline$=\therefore-2$ & 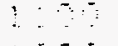 & $\ldots$ & $\div-1 \div$ & $\therefore .7$ & 105.0 & 16.6 & 3.1 \\
\hline$=1: 22$ & 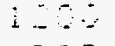 & $\therefore=5$ & $\because \div=15$ & $\therefore=$ & 110.0 & $=1.2$ & \\
\hline $71-24$ & 20 & 1400 & $-2=0$ & 1.3 & 135.0 & 21.9 & \\
\hline$\div 1 \because \ldots-$ & $1 \div \div$ & $:=0$ & 10.13 & 8.7 & 119.0 & 21.5 & 3.1 \\
\hline $9: 094$ & $1: \because 3$ & -50 & 10.10 & $\therefore 7$ & 147,0 & 23.1 & \\
\hline$=10=24$ & $\therefore=0$ & $\therefore 0$ & 10.20 & 0.7 & 159.0 & 15.1 & \\
\hline $7: 294$ & 170 & 1200 & 19.40 & 1.5 & 190.0 & 12.3 & 1.2 \\
\hline$\div 10=3$ & 130 & $:=0$ & $\div 2.20$ & 2.1 & 203.0 & 11.2 & 1.0 \\
\hline$=102=4$ & $i=20$ & -200 & 10.10 & $z=0$ & 229.0 & 15.5 & 1.0 \\
\hline $910=4$ & 200 & $=00$ & 10.10 & $\therefore i$ & $2 \leq 3.0$ & 11.3 & 0.9 \\
\hline 71024 & $\because: 00$ & $\therefore \therefore 0$ & 10.10 & $1 . \equiv$ & 265.0 & 8.8 & 0.9 \\
\hline $7: 0234$ & $2=00$ & $\therefore 300$ & 10.10 & $i .4$ & $=43.0$ & 10.2 & 4 \\
\hline$=10 \div 4$ & -300 & 0 & 10.10 & 0. & 269.0 & .2 & 1 \\
\hline
\end{tabular}




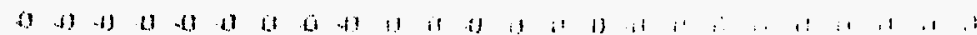

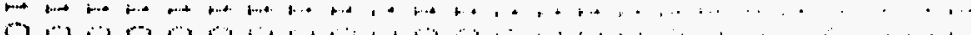
-

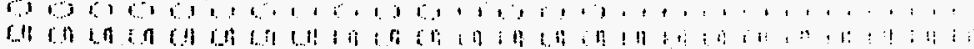

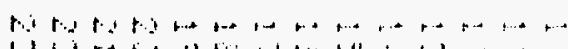

1 $1, \ldots, 1, \ldots, \ldots, \ldots$

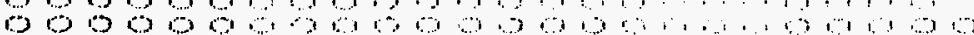

H

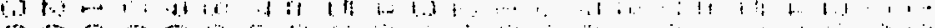

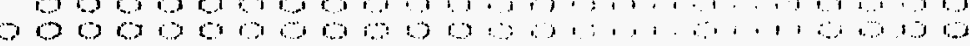

t.

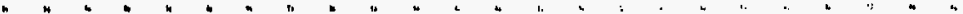

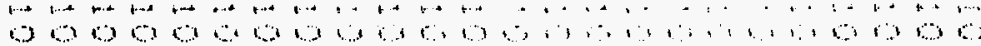

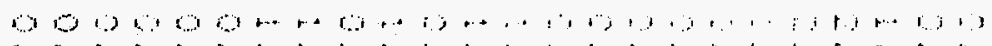
"म

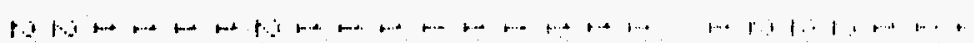

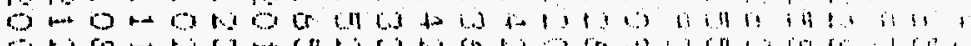

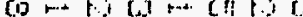

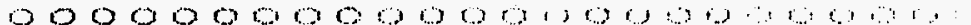

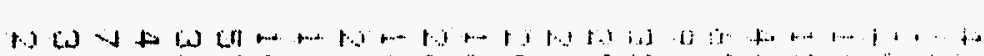

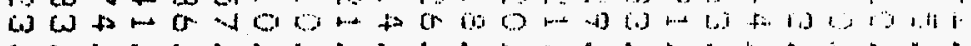

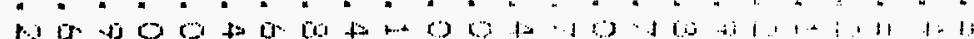

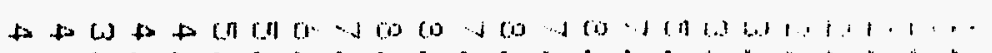
" 
$\equiv:-\therefore-\therefore \equiv \equiv \div$

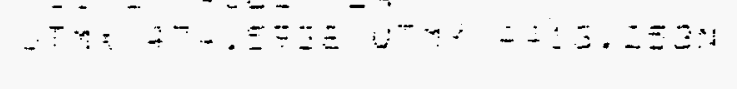

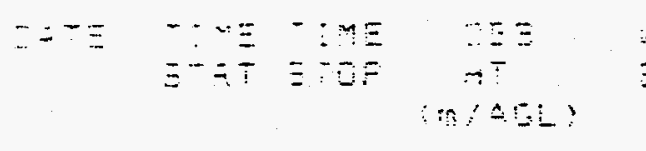

E- $\equiv$.

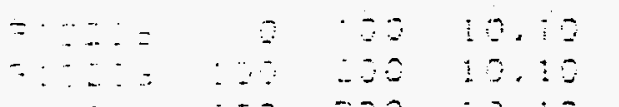

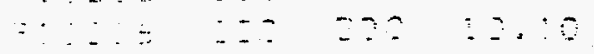

$\because:-\because=\quad \because \div \quad \therefore O$

$\because \because \cdots=$

ren

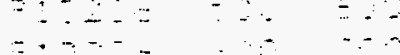

$\because \cdots=$

$=: 2:=$

$\therefore \quad 30$

$\therefore-\quad=2$

$=\because \cdots=$

$=\because \therefore=2 \therefore$

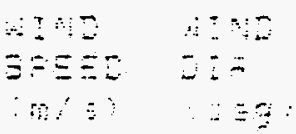

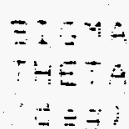

$x=n=$
$\therefore=z \quad \because$

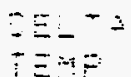

(2)

$-=5$

$=2$

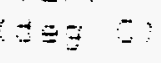

$\therefore 2 \quad 305.6$

0.

$\therefore, 3$

$\therefore$.

$\because$

$\therefore$

$\therefore:=$

$\therefore=$

$\therefore$

$\therefore=\therefore$

$\because: 0$

247.0

$\bar{\Sigma}=0$

$32=$

$\exists \div .2$

$=2.2$

20.5

4.8

$=0$

es:

49.9

73.3

18.4

$12 .=$

25.7

19.7

30.2

$97=$

27.7

20.7

$\div .3$

$4: \vdots$

$\therefore$.

$\therefore$

$\therefore=$

$-\therefore$

$0=5$

0.

0.3

0.0 


$$
\begin{aligned}
& \Xi:-\equiv=-\ldots \equiv \equiv \equiv \div
\end{aligned}
$$

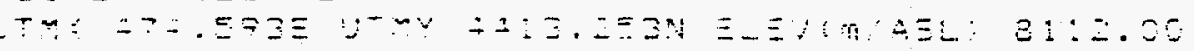

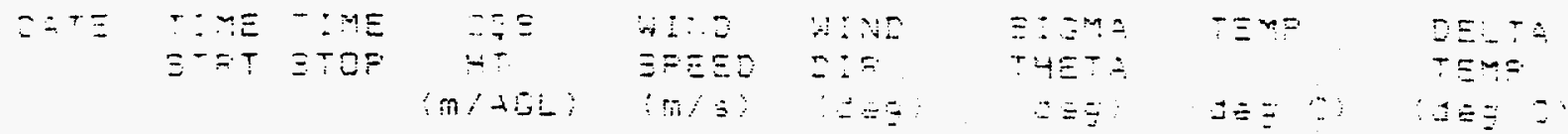

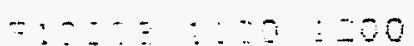

$$
\begin{aligned}
& 10.10 \quad 3.4 \quad 3+2= \\
& 0.3 \\
& \text { - } .9 .9 \\
& \therefore \\
& 7 ; \\
& \therefore= \\
& \therefore= \\
& \therefore .5 \\
& \text { 5. } \\
& \therefore= \\
& \because 5 \\
& \text { ․ } \\
& 3.1 \\
& -3=0 \\
& 5-5.0 \\
& 3+2 \\
& 25.5 \\
& 252.0 \\
& 559.0 \\
& 347.8 \\
& ==1 \\
& -3=7
\end{aligned}
$$

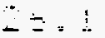

$$
\begin{aligned}
& =\therefore \\
& \because 4,=
\end{aligned}
$$

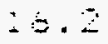

$$
\begin{aligned}
& \text { i. } 7 \\
& 10.7 \\
& 12 .: \\
& 16.7 \\
& 10.9 \\
& 11.7 \\
& \therefore: 7 \\
& 7=0 \\
& ? . \\
& \div . \\
& \therefore= \\
& \text { E: } \\
& 4 . \\
& 3.8 \\
& 3.7 \\
& 3.5 \\
& 2=7 \\
& \text { 2. }
\end{aligned}
$$

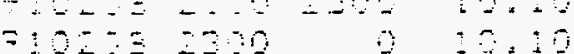


$\Xi-\Xi \cup n \div \equiv-\quad \div \leq \div=00$

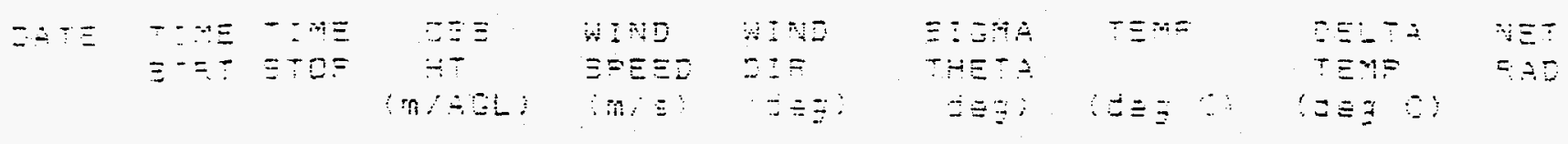

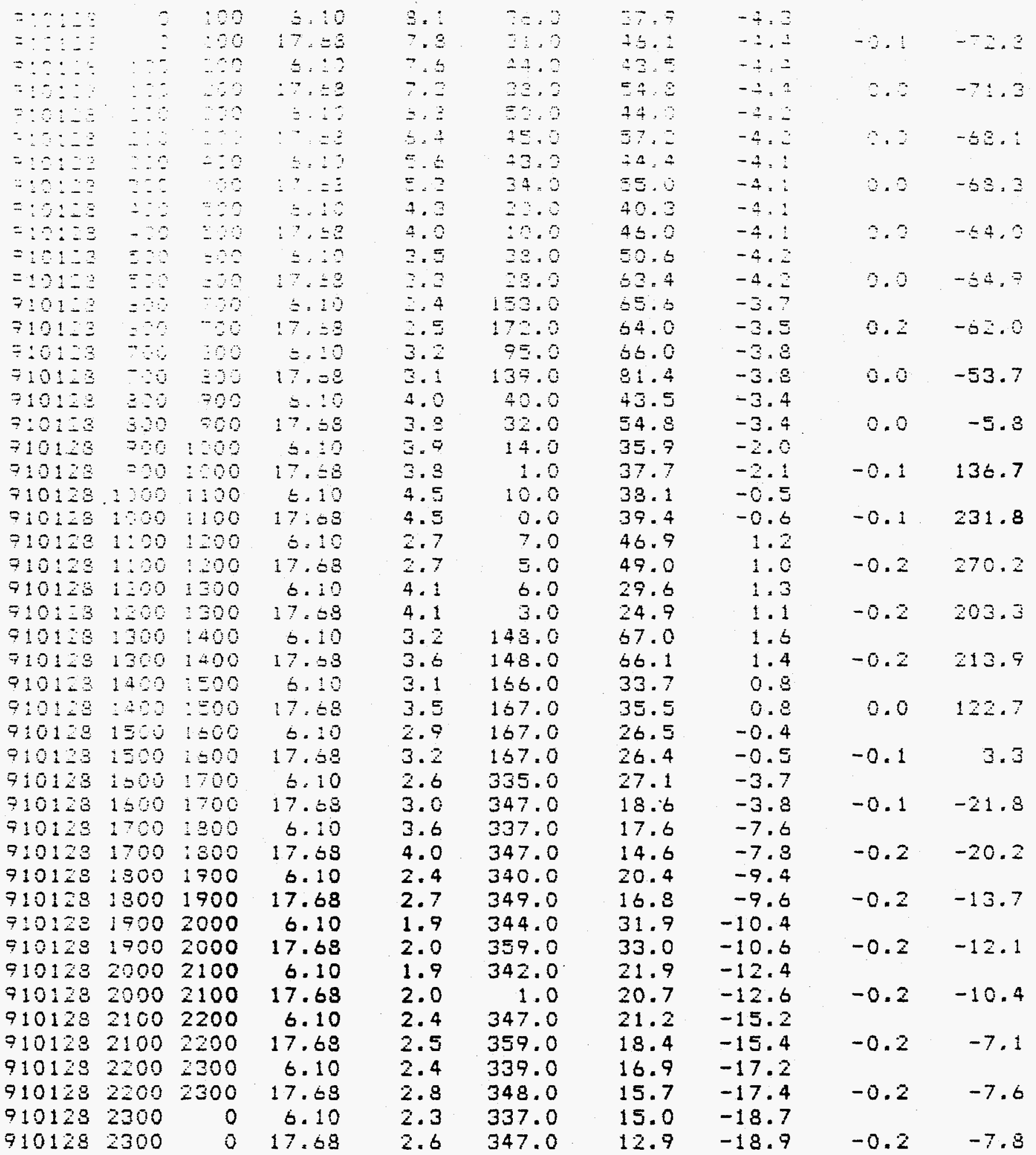




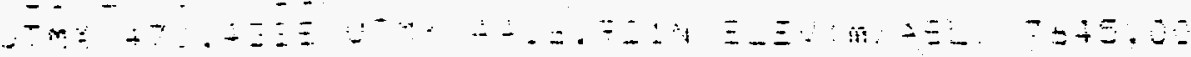

\begin{tabular}{|c|c|c|c|c|c|c|c|}
\hline$I: \div \bar{Z}$ & 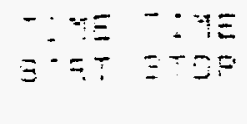 & 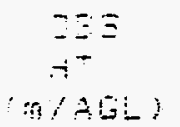 & $\begin{array}{l}\because \because 5 \\
\Xi=\Xi \Xi D \\
\because \because \vdots \vdots\end{array}$ & $\begin{array}{l}\therefore=5 \\
35 \\
2 \div 3\end{array}$ & 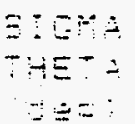 & $E=$ & 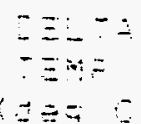 \\
\hline
\end{tabular}

\begin{tabular}{|c|c|c|c|c|c|c|c|c|c|}
\hline$=\cdots: \because$ & 3 & $: 00$ & $6 . \vdots 9$ & 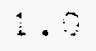 & $\Xi 4 \vdots .0$ & $i=$. & $-\vdots 7 . \vdots$ & & \\
\hline$\because \because:=$ & $\because$ & $: 02$ & 17.52 & $\vdots \ldots$ & $z=5 \times 2$ & $\vdots \cdots$ & $-: 7,=$ & $\therefore=$ & -9. \\
\hline$-\quad-:=$ & $\because$ & $\ldots$ & b.: & $1 .=$ & $3+9=$ & 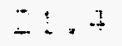 & 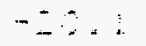 & & \\
\hline$\because \therefore \therefore=$ & $\because \because$ & $\therefore=$ & $: 7 .=3$ & $i:=$ & $y=3$ & $\therefore 1.5$ & $-\because 3$ & -2. & $-\vdots \vdots$ \\
\hline$\cdots: \cdots=$ & & $\because$ & 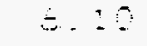 & $\therefore=\therefore$ & $= \pm 1,3$ & 17.5 & 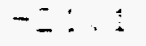 & & \\
\hline$\therefore \because: \therefore:$ & $\therefore \because$ & $\therefore=$ & $-\quad=3$ & 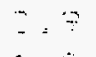 & $z=\div . Z$ & $\therefore=7$ & $-\div: 2$ & $-\because-$ & $-\cdots$ \\
\hline$\therefore:-:=$ & $\because \because$ & $\therefore$ & $=16$ & $\therefore=$ & 343.6 & $: \div, 2$ & $-2 \div=3$ & & \\
\hline$\therefore \quad \therefore=$ & $-\because$ & $\cdots$ & 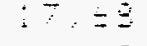 & $\therefore=$ & 342. & $14 \div$ & $-2:, 4$ & $-\because$. & $-E$ \\
\hline$\because: \therefore:=$ & $\therefore \therefore$ & $=\ldots$ & $\pm:$ & $\because \because$ & $=7 ?:$ & 42.9 & $-\because . \quad=$ & & \\
\hline $\bar{Z}: \therefore \vdots:=$ & $\therefore \because$ & $\because \because$ & $\therefore \quad . \quad \Xi$ & $\therefore=$ & 359.0 & 45.2 & - - & $-3 .=$ & -5 \\
\hline$\because:: \div \div$ & $=-\therefore$ & $\therefore 0$ & $\pm: \therefore$ & $:-7$ & $2 \overline{1}, \overline{0}$ & 14.3 & $-21=E$ & & \\
\hline$=\div 1=$ & 50 & $=-8$ & $\therefore 7:=\Xi$ & $\therefore=\dot{3}$ & $150=0$ & 14.5 & -91.9 & $-5=1$ & $-\vdots$. \\
\hline$=: 9120$ & $=\because 5$ & 0 & 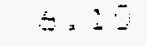 & $I=\bar{Z}$ & $i \div 4.5$ & 10.2 & $-\because=-$ & & \\
\hline$\because: 9: 5$ & $=3$ & 20 & 17.5 & $\therefore=$ & 152.0 & 9.2 & $-2=3$ & $-1 . i$ & -3 \\
\hline$=1919$ & 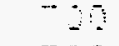 & $\therefore-1$ & $5=19$ & $\overline{7}=\overline{7}$ & $2-7.0$ & 75.3 & $-2-3$ & & \\
\hline $7: 9107$ & $\because 0$ & $=0$ & $: 7 .=3$ & 5.5 & $1=5.0$ & 36.5 & $-2=5$ & 0.3 & -12.6 \\
\hline$=1012 \%$ & $\therefore \square$ & $=00$ & 1.10 & $=4$ & 3.0 & 47.5 & $-\because=0$ & & \\
\hline$=10127$ & 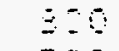 & $=00$ & 17.58 & 2.3 & 28.0 & 54.3 & -21.5 & 0.5 & -40.7 \\
\hline $7: 6197$ & $=\because 0$ & $:-09$ & 6.10 & 0.6 & 115.0 & 50.0 & -13.7 & & \\
\hline$=19127$ & $=-0$ & $: 0$ & 17.53 & 0.5 & 131.0 & 57.5 & -14.1 & -0.4 & 132 \\
\hline $710:=9$ & 100 & $: 10$ & 5.10 & 2.7 & 167.0 & 17.5 & -13.0 & & \\
\hline 710129 & $1 \% 6$ & 190 & 17.68 & $\because=5$ & 154.0 & 20.5 & -13.2 & -0.2 & 179.2 \\
\hline$=101=7$ & $1 \div 00$ & 1200 & 5.10 & $\because .8$ & 70.0 & 96.6 & -5.0 & & \\
\hline $7101=9$ & $i:-0$ & $: 20$ & $17=03$ & $\because=2$ & 37.0 & 92.9 & -5.5 & -6.5 & -97 \\
\hline $7101=9$ & $1=50$ & $: 00$ & $\leqq .: 0$ & 4.5 & 16.0 & 37.0 & -4.5 & & \\
\hline$=: 01: 7$ & 120 & $:=00$ & 17.58 & 4.5 & 7.0 & 39.5 & -4.7 & -0.4 & 12 \\
\hline $9: 0109$ & 1200 & $: 50$ & $5=5$ & 4.6 & 12.0 & 38.0 & -4.3 & & \\
\hline 710127 & 1.50 & 1400 & 17.53 & 4.5 & 8.0 & 37.1 & 4.5 & -0. & 31 \\
\hline 910127 & $: 400$ & $:=00$ & 5.10 & 4.2 & 35.0 & 43.1 & -4.5 & & \\
\hline$\Rightarrow: 01: 9$ & 1400 & $:=00$ & 17.68 & 3.9 & 29.0 & 52.4 & -4.6 & -0.1 & 71.9 \\
\hline 910127 & $:=00$ & 1500 & 6.10 & 4.8 & 31.0 & 41.3 & .2 & & \\
\hline$=101.7$ & $1=00$ & $i=00$ & 17.53 & 4.0 & 23.0 & 47.3 & -5.2 & 0.0 & 6.0 \\
\hline $9: 01: 9$ & $\pm=0$ & 1700 & 6.10 & 5.0 & 26.0 & 28.3 & -6.4 & & \\
\hline 910127 & $1=00$ & 170 & 17.68 & 4.8 & 20.0 & 33.7 & -6.3 & 0.1 & -65.8 \\
\hline 910127 & 170 & 1200 & 6.10 & 4.8 & 26.0 & 40.5 & -6.7 & & \\
\hline 010127 & 1790 & $: 300$ & 17.58 & 4.5 & 20.0 & 45.3 & -6.7 & 0.0 & -59.3 \\
\hline $9101=7$ & 1900 & 1900 & 5.10 & 5.4 & 34.0 & 33.0 & -6.9 & & \\
\hline $9: 0129$ & 1800 & 1700 & 17.68 & 5.1 & 32.0 & 45.4 & & 0.0 & -57.9 \\
\hline 710129 & 1900 & 2000 & 6.10 & 5.0 & 27.0 & 29.7 & -6.5 & & \\
\hline 910129 & 1700 & 2000 & 17.68 & 4.8 & 23.0 & 38.1 & -6.5 & 0.0 & -55.4 \\
\hline 910127 & 2000 & 2100 & 6.10 & 6.2 & 29.0 & 32.8 & -6.0 & & \\
\hline 710127 & 2000 & 2100 & 17.68 & 5.7 & 25.0 & 42.9 & -6.1 & -0.1 & -38.3 \\
\hline 910127 & 2100 & 2200 & 6.10 & 5.8 & 38.0 & 38.6 & -6.5 & & \\
\hline 910127 & 2100 & 2200 & 17.68 & 5.4 & 34.0 & 46.2 & -6.5 & 0.0 & -28.3 \\
\hline 910129 & 2200 & 2300 & 6.10 & 4.5 & 36.0 & & -5.9 & & \\
\hline 910129 & $2 \geq 00$ & 2300 & 17.63 & 4.3 & 31.0 & 52.8 & -6.0 & -0.1 & -36.0 \\
\hline 129 & 2300 & 0 & 6.10 & & 23.0 & 40.2 & -6.5 & & \\
\hline 910129 & 2300 & 0 & 17.68 & 5.9 & 17.0 & & -6.6 & -0.1 & -51.3 \\
\hline
\end{tabular}




\section{$\because: \div$}

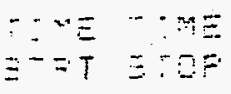

$$
\begin{aligned}
& \begin{array}{l}
3= \\
4=
\end{array} \\
& n \div=-1
\end{aligned}
$$

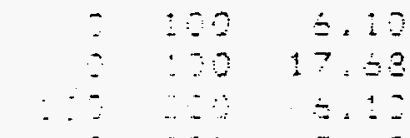

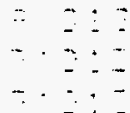

$\because \div:$

$\because:-:$

(2)

$=\div:=$

$-\div:=$

$710 \div$

$\because \div:=$

$\because: 0 \div=$

$=: 2:=$

$7: 0: 7$

$=0120$

$9: 913$

$910: 30$

$=: 0+5$

$=10: 30$

$7: 0: 39$ : :

710130 1:50 200

$710130150 \quad 1300$

$7: 013915001300$

$=! 0: 301300 \quad 1400$

$710: 01300 \quad 400$

$=101321490 \quad 5500$

$719.30: 400:=00$

$9101301500: 500$

$7101301300 \quad \div=00$

$7: 9: 50$ 1600 7700

$7101301500 \$ 700$

$=10151700: 300$

91013017001300

$710130 \quad 1300 \quad 1700$

Fio:3 13001900

71013019002000

$=10130 \quad 1900 \quad 2000$

91013020002100

$710130 \quad 2000 \quad 2100$

71013021002200

91013021002200

91013022002300

$910130 \quad 2200 \quad 2300$

9101302300

9101302300

$\vdots^{-}-0$

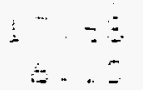

$i^{-\infty}:=$

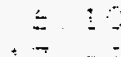

$\pm . \therefore$

$1 \because=3$

$\pm .10$

$17: 68$

6

$17=03$

$=2$.

17.33

6.5

17.93

‥

17.56

6.

17.50

6.50

$17 .=3$

6.10

17.53

6.10

17.59

6. 10

$17=: 3$

6.10

17.53

17.63

6. 10

17.68

6. 10

17.68

6.10

17.68

6.10

17.48

6.10

17.69

6.10

17.58
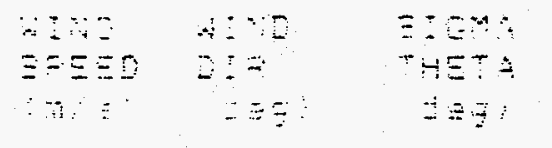
$\overline{1} \equiv$
$4=$

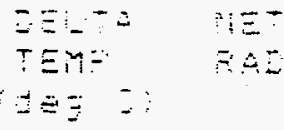

6.10

\section{$=$}

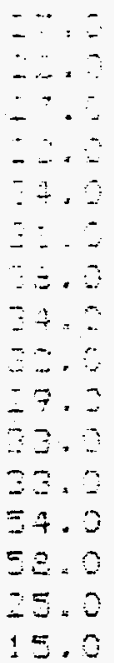

- $=0$

17.0

$27=0$

17.5

$\because 5.0$

$\because 1.0$

$\because 2.0$

17.0

20.0

11.0

34.0

23.0

23.0

17.0

41.0

33.0

37.0

$-4.0$

4.0

3.2

107.0

3.1

5.0

154.0

51.0

4. 6

7.6

32.0

29.0

7.0

8.5

7.9

8.2

7.7

6. 5

b. 2

6. 1

5.5

20.0

20.0

11.0

32.0

53.0

$4 \dot{0} 0$

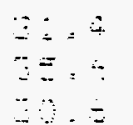

I?.

$\therefore=$

$\because=4$

$\because=9$

$\because \div$

ㄴ.

$\therefore$.

$\because=9$

43,4

49.3

58.5

36.5

40.5

3. 3.4

37.7

37.7

44.7

38.1

44.3

37.7

43.5

40.5

44.6

$=0.1$

55.0

42.7

49.3

44.0

55.9

58.4

70.0

71.4

33.5

50.4

77.5

32.6

40.0

26.6

28.0

28.6

30.7

49.0

59.9

52.4

66.1 $-\div=$

$-5=$
-5,

$-\therefore=$

$-=$

$-=$.

$-\div$.

$- \pm .3$

$-5.7$

-

$-5.1$

$-0.1$

$-5.5$

$-6.5$

$-6.0$

-b. 1

$-4.9$

$-4.5$

$-3.5$

$-3.5$

$-1.9$

$-2.0$

$-5.5$

$-0.6$

0.2

0.2

1.3

1. 2

1. 2

i. 1

0.5

0.4

0.3

0.4

$-0.3$

$-0.3$

$-0.1$

$-0.1$

0.2

0.1

0.4

0.4

0.3

0.2

0.8

0.7

0.7

0.6

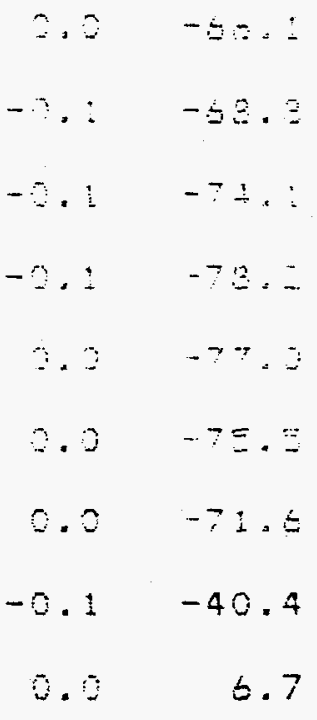

$-0.1125 .6$

$-0.1 \quad 207.2$

$-0.1 \quad \because 54.1$

$0.0 \quad-91.3$

$-0.1265 .9$

$-0.1 \quad 171.7$

$-0.1-45.3$

$0.1-54.3$

$0.0-68.9$

$0.0-56.8$

$-0.1-66.3$

$0.0 \quad-47.7$

$-0.1-39.0$

$-0.1 \quad-49.7$ 


\begin{tabular}{|c|c|c|c|c|c|c|c|c|c|}
\hline 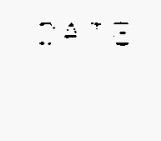 & $\begin{array}{l}\cdots \\
\Xi-=T\end{array}$ & $\begin{aligned} & := \\
7 & =0\end{aligned}$ & 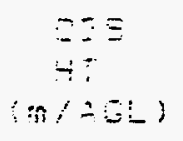 & 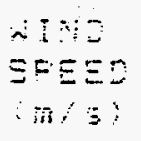 & 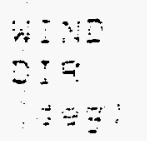 & 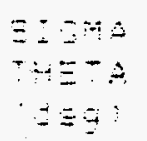 & $\therefore A B=0$ & 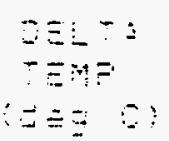 & $\begin{array}{l}\because \vdots \equiv T \\
\because \div 2\end{array}$ \\
\hline 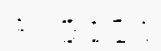 & 2 & $\because: 0$ & \pm .19 & 7.5 & 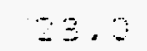 & $\because 5 .=$ & 5.3 & & \\
\hline$\because \because$ & $\because$ & $\therefore 2$ & $\vdots \div, \leq \Xi$ & 7.1 & $\because \quad:=$ & $\bar{\theta}=\bar{\gamma}$ & $\therefore \quad-$ & $-\because$ & -53.3 \\
\hline$\because: \because:$ & $\because \div$ & $\therefore$ & $\doteq .5$ & $7 .:$ & 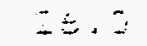 & $\because 3.2$ & $\therefore 7$ & & \\
\hline$\because: \because \cdots$ & $: \because$ & $\because 3$ & $\therefore \quad . \leq$ & $\doteq . \quad \vdots$ & $: \because .2$ & $\div:=$ & $2 . \dot{ }$ & -2. & -49.1 \\
\hline$\because: \because: \cdots$ & $\because \because$ & $\cdots$ & $\therefore \therefore$ & $\vdots .7$ & $\therefore \because z$ & $\div=.3$ & $\therefore 2$ & & \\
\hline$=: \because:=$ & $\therefore$ & $\because \because$ & $\because \because \vdots \Xi$ & $\leq$. & 32.2 & 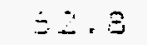 & $\vdots=A$ & $\because 2$ & -43.5 \\
\hline$=: \because: \cdots:$ & $\because=$ & $\therefore \because 0$ & $3,:$ & $=\ldots$ & 27.9 & $=E=E$ & $\therefore=:$ & & \\
\hline$\because: \because: \cdots$ & $\because \because$ & $\therefore \div$ & $\vdots \quad \vdots 3$ & $E$ & $=1,0$ & $\therefore 5.5$ & $!=1$ & 3.2 & $-4=\because$ \\
\hline$\because: \because:=$ & $\therefore \because$ & $=-9$ & 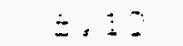 & $\div 7$ & 79.5 & $\therefore 9.7$ & 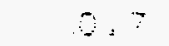 & & \\
\hline$=\because \because:-$ & $\because \because$ & 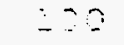 & $\because \because 2=2$ & $\div . ?$ & 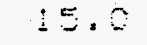 & 32.3 & 9.7 & 3.3 & -3.9 \\
\hline$\because: \because::$ & $=\because 5$ & $=3$ & $5: 12$ & $7=5$ & 13.9 & $4 i=0$ & 2.6 & & \\
\hline$=: 2:-$ & $=-\because$ & $=-$ & $: \because=5$ & 4.3 & \pm .0 & 43.2 & 0. & $\because 8$ & -40. \\
\hline$=\div \div \div$ & $=\because$ & -3 & $5: \therefore$ & 4.5 & 40.5 & 30.3 & 0.5 & & \\
\hline$\because: \because:=$ & $\therefore 2$ & $\therefore$ & $-\overline{-}=0$ & $4=4$ & 36.0 & 47.5 & 0.5 & 3.9 & -45.6 \\
\hline 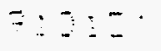 & -2 & $=50$ & $=\therefore 8$ & 5.3 & $71=0$ & 46.7 & 0.5 & & \\
\hline$\because: 8: \because:$ & $\because \because$ & $\because 3$ & $: 7.93$ & 3.1 & 75.0 & 57.7 & 0.7 & 0.1 & -35.1 \\
\hline $7: 9:=$ & $\vdots 0$ & $=2$ & 5.20 & 2.5 & 115.0 & $5 \overline{0} .7$ & 1.5 & & \\
\hline$=: 01.3:$ & $=-2$ & $=8$ & $i \div=\bar{\Xi}$ & 2.5 & 142.0 & 56.2 & 1.5 & 0.0 & -3.0 \\
\hline$=: 515$ & $=20$ & $\therefore: 0$ & $\doteq: 30$ & 2.1 & $4 b .0$ & 76.7 & 3.9 & & \\
\hline $9 \pm 12:$ & $=26$ & 1000 & $: 7=59$ & 2.1 & 35.0 & 84.6 & 4.1 & 0.2 & 27.0 \\
\hline $9 \pm 012:$ & 100 & 1100 & 5.10 & 3.7 & $\$ 5.0$ & $\$ 5.1$ & 4.1 & & \\
\hline $7: 01: 1$ & 150 & 100 & $17=08$ & 3.7 & $\doteqdot 1.0$ & 83.6 & 4.1 & 0.0 & 70.1 \\
\hline $910:=1$ & 1200 & $: 200$ & $\therefore 10$ & 5,3 & 14.0 & 35.0 & 5.4 & & \\
\hline $9101=$ & $: \div 0$ & $:=0$ & $17: 68$ & 5.0 & 3.0 & 37.1 & 5.4 & 0.0 & 297.1 \\
\hline $7 \div 01=2$ & $1-0$ & $1-00$ & 5.10 & 3.7 & $30 \times 0$ & 50.5 & 5.2 & & \\
\hline $70: 31$ & $1=0$ & $: 300$ & 17.63 & 3.8 & 23.0 & 56.4 & 8.0 & -0.2 & 414.5 \\
\hline $710:=$ & 230 & $: 400$ & 6.10 & 5.5 & 19.0 & 34.9 & 3.4 & & \\
\hline $7403:$ & 190 & 190 & $17.6 \overline{3}$ & 5.1 & 9.0 & 38.2 & 3.3 & -0.1 & 535.1 \\
\hline $710: 9$ & 140 & $: \pm 05$ & 5.10 & 4.9 & 3.0 & 29.8 & 7.0 & & \\
\hline $71012 \vdots$ & 140 & $1=90$ & 17.49 & 4.3 & 1.0 & 23.2 & 8.9 & -0.1 & 252.0 \\
\hline $51013:$ & 2500 & $i=00$ & 5.10 & 4.5 & 4.0 & 28.2 & 3.6 & & \\
\hline $9: 015:$ & $1=90$ & $:=00$ & 17.53 & 4.4 & 1.0 & 20.4 & 8.6 & 0.0 & 73.2 \\
\hline $7 \pm 0131$ & $1=00$ & 170 & 5.10 & 3.3 & 10.0 & 22.3 & 7.1 & & \\
\hline $71015:$ & $1=00$ & 1709 & 17.68 & 3.2 & 10.0 & 19.1 & 7.2 & 0.1 & -51.1 \\
\hline $7: 0131$ & 1700 & 1300 & 6.10 & 0.7 & 3.37 .0 & 57.4 & 1.0 & & \\
\hline $710: 31$ & 1700 & 1300 & 17.68 & 0.7 & 352.0 & 75.0 & 1.9 & 0.9 & -82.3 \\
\hline $71013:$ & 1900 & 1900 & 6.10 & 0.9 & 357.0 & 27.1 & -3.2 & & \\
\hline $\begin{array}{l}7 \pm 0131 \\
=1019\end{array}$ & $\begin{array}{l}1300 \\
1=00\end{array}$ & 1900 & 17.68 & 0.9 & 1.0 & 22.9 & -2.6 & 0.6 & -78.5 \\
\hline 910131 & $\begin{array}{l}1700 \\
1700\end{array}$ & $\begin{array}{l}2000 \\
2000\end{array}$ & $\begin{array}{r}6.10 \\
17.68\end{array}$ & $\begin{array}{l}1.0 \\
1.0\end{array}$ & 348.0 & $\begin{array}{l}28.6 \\
23.3\end{array}$ & $\begin{array}{l}-4 \cdot 5 \\
-37\end{array}$ & 0 & -777 \\
\hline 910131 & 2000 & $=100$ & 6.10 & 1.1 & 346.0 & $\begin{array}{l}-3.3 \\
31.9\end{array}$ & $\begin{array}{l}-3.7 \\
-5.6\end{array}$ & 0.8 & $-i$ \\
\hline $7: 0131$ & 2000 & 2100 & 17.68 & 1.0 & 1.0 & 22.1 & -4.2 & 1.4 & -73 \\
\hline 910131 & 2100 & 2200 & 6.10 & 1.7 & 5.0 & 34.5 & $-0 . \dot{0}$ & & \\
\hline 710131 & $2 \pm 00$ & $\because 200$ & 17.68 & 1.9 & 34.0 & 77.1 & 2.1 & 2.7 & -69.9 \\
\hline 910131 & 2200 & -300 & 6.10 & 1.8 & 359.0 & 69.5 & -1.2 & & \\
\hline 910131 & 2200 & 2300 & 17.68 & 1.7 & 19.0 & 65.1 & 0.6 & 1.8 & -65.1 \\
\hline 910131 & 2300 & 0 & 5.10 & 2.5 & 10.0 & 37.2 & 4.3 & & \\
\hline $91013 i$ & 2300 & 0 & 17.58 & 2.5 & 11.0 & 36.2 & 4.6 & & -70.5 \\
\hline
\end{tabular}


$\equiv:-\equiv=\cdots=-\cdots$

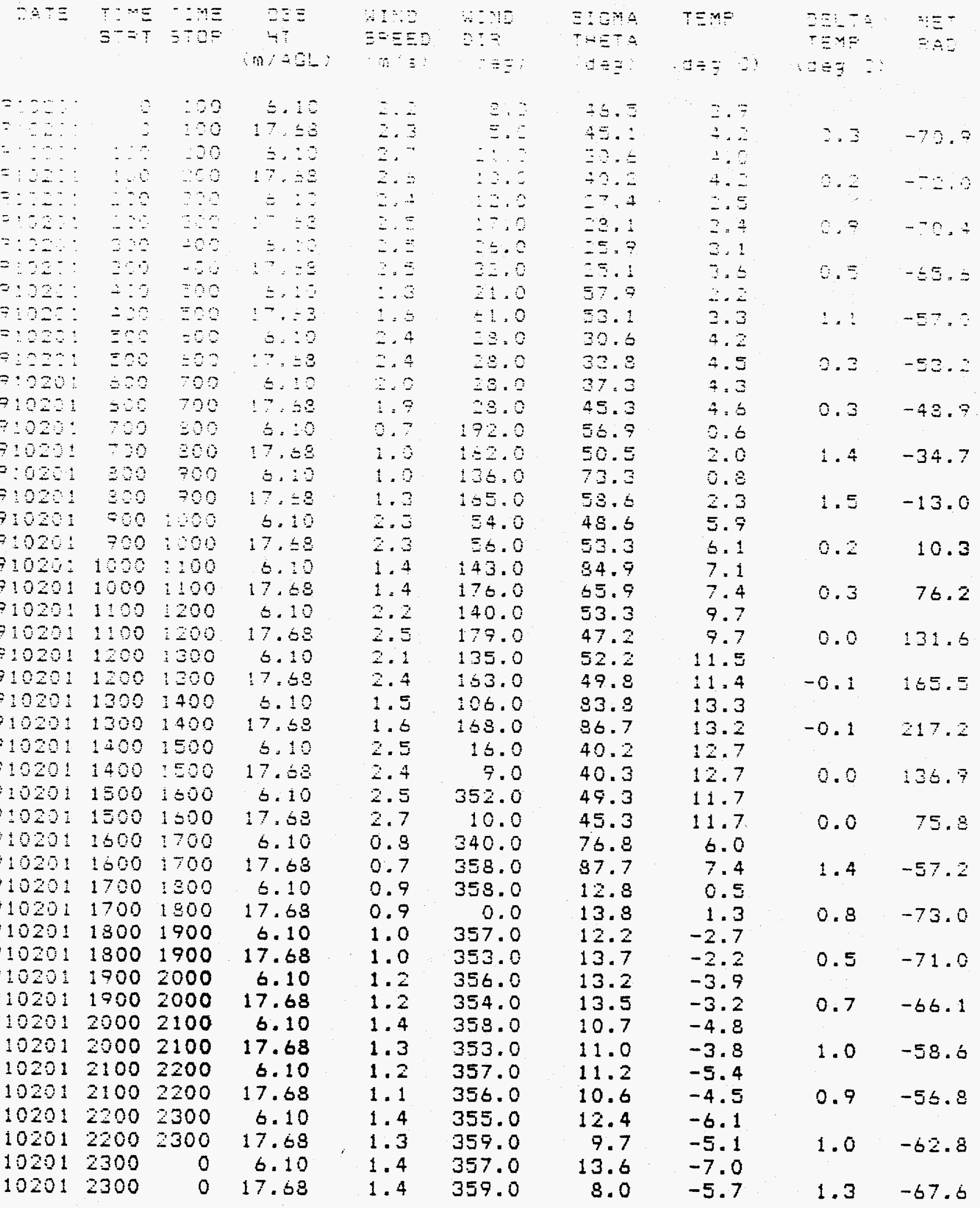




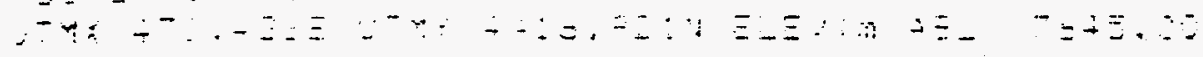

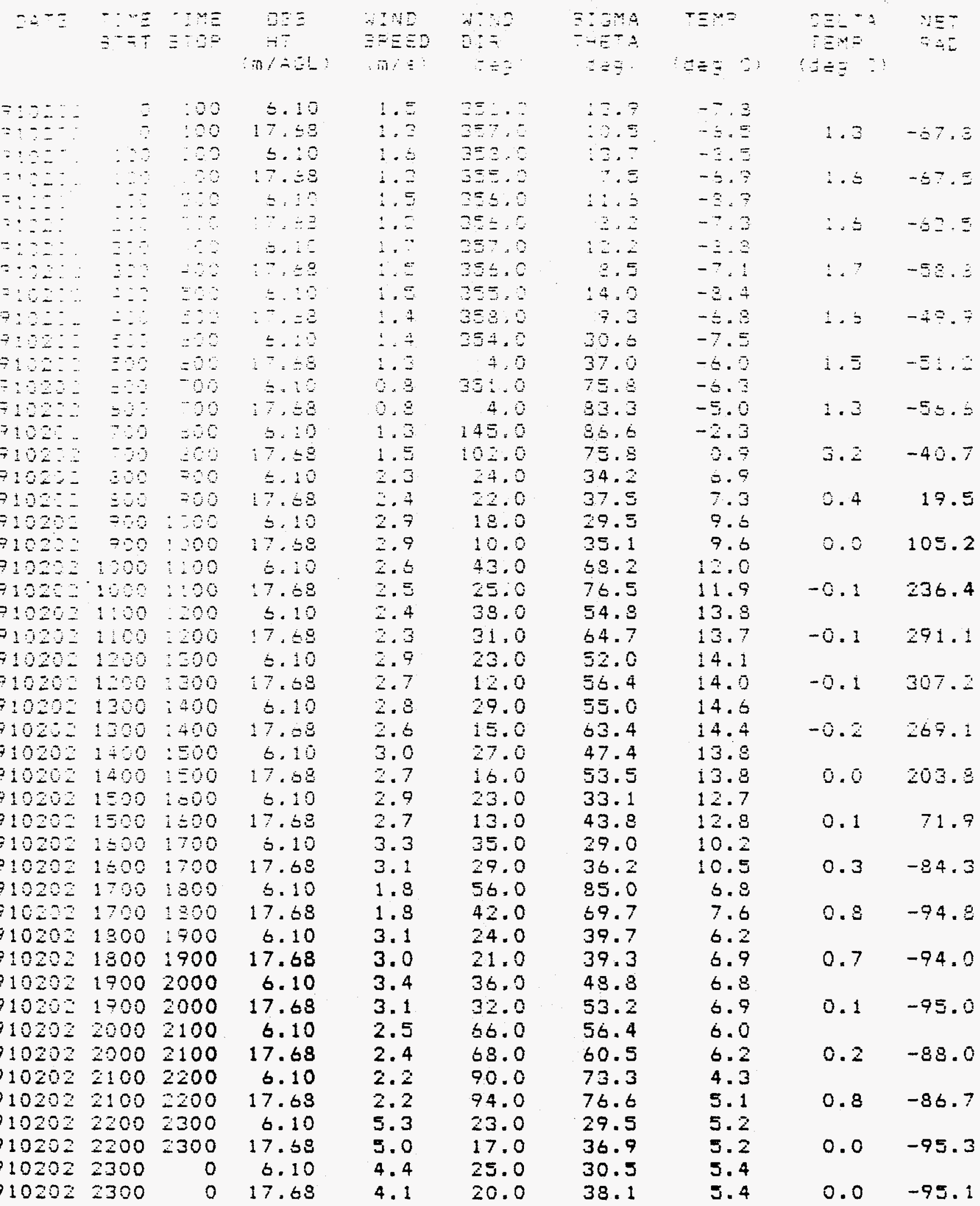




\begin{tabular}{|c|c|c|c|c|c|c|c|c|}
\hline $\begin{array}{l}\because \sim \equiv \\
\vdots=T\end{array}$ & $\begin{array}{l}-: D \\
\vdots T 0 F\end{array}$ & $\begin{array}{c}5 z \equiv \\
-4 T \\
7=-1\end{array}$ & 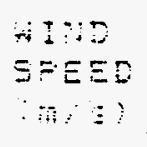 & $\begin{array}{l}A: 15 \\
2: 5 \\
=5\end{array}$ & 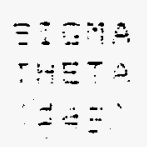 & 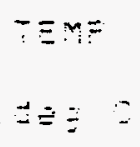 & 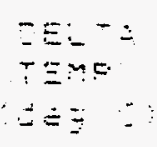 & $\begin{array}{l}1 \equiv- \\
P a 5\end{array}$ \\
\hline$\therefore=$ & $: 00$ & 6.10 & 4.2 & $\because \therefore$ & $\therefore:=$ & $\approx . \Xi$ & & \\
\hline$\because$ & $\because \because 8$ & 17.53 & 4.1 & $\because=:$ & 20.4 & $=.2$ & $\overline{5}$ & $-94 .=$ \\
\hline$\because$ & $\therefore$ & s.: & 4. & $=$. & $\therefore 9=$ & $\equiv .4$ & & \\
\hline$\because \because$ & 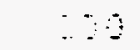 & $\therefore:=3$ & $\therefore: 1$ & 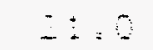 & $5 E \div$ & $\cong: 4$ & $\therefore 3$ & $-9=\div$ \\
\hline$\therefore=$ & $\therefore$ & $\div \therefore$ & $\equiv$. & $\because \ldots$ & $\therefore \ldots$ & $\therefore \ldots$ & & \\
\hline$\therefore$ & $\therefore$ & $\therefore=\equiv$ & $\div .7$ & -3 & 52 & $\therefore .4$ & 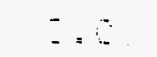 & -87. \\
\hline$\therefore 8$ & $\therefore$ & $=\ldots$ & $\equiv 4$ & $\because$ & -3 & 4.5 & & \\
\hline$=$ & $\therefore 0$ & $1-2=3$ & $4=7$ & $\therefore \div$ & 35. & 4.5 & & -87. \\
\hline$=-5$ & $\therefore 8$ & $b .5$ & $\equiv .5$ & $\therefore \therefore$ & 31.4 & $=7$ & & \\
\hline $82=8$ & $\because-0$ & $\therefore 7.6$ & 5. & $i 5.6$ & $3 ? .7$ & 3.7 & 5.5 & -37. \\
\hline $22: 2$ & $=20$ & $\leq .: 0$ & 5.7 & $=4.0$ & 27.5 & $3 .=$ & & \\
\hline$=\because 2$ & $\therefore 00$ & $: 7 . \leq 3$ & $E$. & 19.2 & 33.5 & $3 .=$ & 2.0 & $-3 \Xi$. \\
\hline$=: 0$ & 0 & $5: 5$ & 5.4 & $=2.0$ & 27.7 & $\therefore 9$ & & \\
\hline$=0$ & 100 & 17.53 & $\Xi .1$ & 24.0 & 34.5 & 2.7 & 2.0 & $-3 \leq .3$ \\
\hline-1 & $\therefore 80$ & $5: 10$ & 5.3 & 25.5 & $32=$ & 2.6 & & \\
\hline$=50$ & 50 & 17.53 & 4.9 & 21.0 & 39.0 & 2.5 & 0.0 & -73.5 \\
\hline 580 & 700 & $\doteq .5$ & 5.3 & 23.0 & 33.5 & 3.5 & & \\
\hline 300 & $=00$ & $: 7.53$ & 5.4 & 22.0 & 36.5 & 3.6 & 0.0 & 0.7 \\
\hline$=-0$ & $: 20$ & $\leq 10$ & 5.2 & 24.0 & 37.0 & 5.0 & & \\
\hline 790 & $\because 00$ & 17.53 & 4.8 & $=1.0$ & 43.7 & 4.7 & -0.1 & 165.7 \\
\hline 3150 & $: 190$ & $=.10$ & $\equiv .3$ & 17.0 & 35.5 & 5.7 & & \\
\hline 3100 & 1200 & 17.63 & 4.9 & 14.0 & 37.3 & 5.7 & -0.2 & 261.7 \\
\hline $31: 00$ & $\therefore 20$ & 6.10 & 4.3 & 15.0 & 35.0 & 6.7 & & \\
\hline$: 100$ & $\therefore=00$ & \pm 7.56 & 4.2 & 7.0 & 36.6 & 5.7 & -0.2 & 245.1 \\
\hline $1=00$ & $: 300$ & 5.10 & 3.7 & 20.0 & 30.3 & 7.1 & & \\
\hline 1200 & $: 200$ & 17.58 & 3.5 & 15.0 & 34.9 & 6.9 & -0.2 & 195.7 \\
\hline 31300 & $: 400$ & 6.10 & 4.6 & 22.0 & 34.7 & 7.0 & & \\
\hline 31250 & $: 400$ & 17.63 & 4.3 & 15.0 & 39.1 & 6.8 & -0.2 & 258.1 \\
\hline 1490 & $:=00$ & $s .10$ & 4.4 & 21.0 & 32.4 & 7.0 & & \\
\hline 1100 & $: \equiv 00$ & 17.58 & 4.0 & 17.0 & 33.2 & 5.8 & -0.2 & 212.3 \\
\hline 1500 & $\therefore=00$ & $=.10$ & 4.0 & 14.0 & 36.4 & 5.7 & & \\
\hline $1=00$ & $=00$ & 17.58 & 3.7 & 8.0 & 41.5 & 5.6 & -0.1 & 57.9 \\
\hline 1500 & 1700 & 6.10 & 5.1 & 24.0 & 32.7 & 4.1 & & \\
\hline $1=00$ & 1700 & 17.68 & 4.7 & 17.0 & 38.5 & 4.1 & 0.0 & -31.5 \\
\hline $1: 00$ & $\therefore 800$ & 6.10 & 4.5 & 23.0 & 30.3 & 2.8 & & \\
\hline 31700 & 1300 & 17.68 & 4.1 & 20.0 & 37.1 & 2.7 & -0.1 & -61.4 \\
\hline 1300 & 1900 & 6.10 & 4.3 & 29.0 & 24.2 & 1.8 & & \\
\hline $\begin{array}{l}1800 \\
1700\end{array}$ & $\begin{array}{l}1900 \\
2000\end{array}$ & $\begin{array}{r}17.68 \\
6.10\end{array}$ & $\begin{array}{l}3.9 \\
2.8\end{array}$ & $\begin{array}{l}28.0 \\
32.0\end{array}$ & $\begin{array}{l}31.4 \\
30.3\end{array}$ & $\begin{array}{l}1.8 \\
1.2\end{array}$ & 0.0 & -71.3 \\
\hline 1900 & 2000 & 17.68 & 2.6 & 32.0 & 43.3 & 1.2 & 0.0 & -64.9 \\
\hline 2000 & 2100 & 6.10 & 1.5 & 123.0 & 67.4 & 0.3 & & \\
\hline 2000 & 2100 & 17.68 & 1.5 & 131.0 & 78.5 & 0.6 & 0.3 & -55.0 \\
\hline 2100 & 2200 & 6.10 & 1.4 & 73.0 & 82.6 & 0.0 & & \\
\hline 2100 & 2200 & 17.68 & 1.4 & 80.0 & 84.0 & 0.6 & 0.6 & -50.2 \\
\hline 2200 & -300 & 6.10 & 2.2 & 43.0 & 37.9 & 0.9 & & \\
\hline 2200 & 2300 & 17.68 & 2.1 & 42.0 & 38.0 & 1.0 & 0.1 & -56.1 \\
\hline 2300 & 0 & 6.10 & 1.7 & 57.0 & 55.1 & 0.2 & & \\
\hline 2300 & 0 & 17.68 & 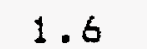 & 2.0 & 61.9 & 0.5 & 0.3 & -58 \\
\hline
\end{tabular}




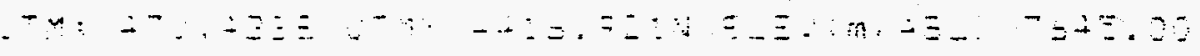

\begin{tabular}{|c|c|c|c|c|c|c|c|c|c|}
\hline$\because \div \equiv$ & $\begin{array}{l}\cdots \\
\vdots \\
\vdots\end{array}$ & $=$ & $\begin{array}{c}-3= \\
\cdots= \\
m=21\end{array}$ & 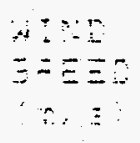 & 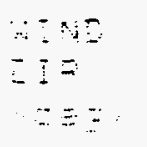 & 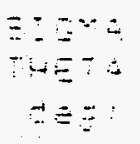 & 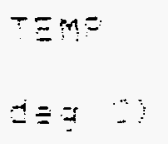 & 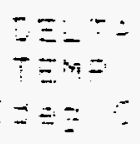 & 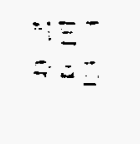 \\
\hline$\because: \therefore \therefore$ & $z$ & 8 & $\doteq . \vdots 0$ & こ & $\therefore=\square$ & $= \pm . i$ & $-\Xi . \Sigma$ & & \\
\hline$\therefore$ & & 20 & $: P=\leq 2$ & $\therefore$. & $\therefore \equiv$ & $=2 . \therefore$ & $-\therefore$ & $\therefore \Xi$ & $-\Xi 7, \therefore$ \\
\hline$\because \because$ & & $\therefore \because$ & $\leq .: 0$ & $\therefore .1$ & $\leq=\overline{-}$ & 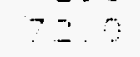 & $-2 .=$ & & \\
\hline$=: \because \therefore \quad \therefore$ & & $\therefore$ & $: \because .=3$ & $\therefore .5$ & $=3.2$ & 72.2 & $-\vdots$. & 3. & 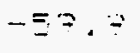 \\
\hline$\because: \therefore$ & & 2 & $=.: 0$ & 3. & $=3$ & $5 \pm .0$ & $\therefore i$ & & \\
\hline$\therefore: \because \because \therefore$ & $\therefore \therefore$ & $\because \because$ & 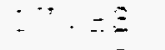 & $2=$ & 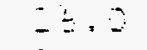 & $4: .9$ & -2. & $\therefore \therefore$ & $-5=$. \\
\hline$\because: \div: ?$ & $\because \because$ & $\because: \because$ & $\doteq::$ & $\therefore$ & $= \pm .0$ & $2:=$ & -0.2 & & \\
\hline$=\because \because 2:$ & $\therefore \therefore$ & $\therefore \because$ & $\therefore=3$ & $\Xi: \div$ & $\because \approx .3$ & 37.6 & -5.2 & 2. & $-5=$ \\
\hline$\because: \div-1$ & $\therefore \because$ & $\because \because$ & $\pm \therefore$ & 2. & $\therefore \therefore$ & 27.7 & -5.4 & & \\
\hline$=: 7:-2$ & $-\therefore$ & $\because: 3$ & $: 7:=3$ & $3=$ & 23.0 & 32.6 & -5.5 & -1. & -62.3 \\
\hline$=\because-\div$ & $=\because \because$ & $\therefore \div$ & $\doteq \therefore$ & $5: 5$ & $=7.0$ & $30: 5$ & -0.7 & & \\
\hline$=: 2: 3$ & $\Xi-$ & $=0$ & $\therefore-x=0$ & $3=4$ & $2 \div .0$ & 33.7 & -0.7 & 2.3 & $-72=$ \\
\hline 7.19 & $=-2$ & 5 & $\Delta .: 0$ & $\because$. & 79.0 & $=1.9$ & -0.5 & & \\
\hline$=: 0 \div$ & $=20$ & 30 & $17=5$ & $\therefore:$ & $7 \Xi .9$ & 50.7 & -8.5 & 0.2 & -67.5 \\
\hline $7: 024$ & -5 & $\because 5$ & $\therefore 19$ & $\therefore:$ & 347.0 & 53.1 & -3.3 & & \\
\hline $7: 024$ & -9 & 30 & $17=00$ & . & $1 i .0$ & 55.4 & -2.5 & 0.5 & -57.1 \\
\hline$=10204$ & $=0$ & $=00$ & $5 .: 0$ & 3.9 & $\therefore 0$ & 14.7 & -2.8 & & \\
\hline 710224 & $=20$ & 700 & $17 .=9$ & $0 . \div$ & 14.0 & 45.9 & -1.2 & 1.5 & 22.6 \\
\hline$?: 0204$ & 920 & $:: 00$ & $\leq .10$ & 1.3 & 77.0 & 101.6 & 3.4 & & \\
\hline 210204 & $=20$ & 1300 & 17.58 & 2.4 & 145.6 & 55.4 & 4.3 & 0.9 & 167.7 \\
\hline $7: 0204$ & $\therefore=90$ & 100 & 5.10 & 3.7 & 174.0 & 17.6 & 4.2 & & \\
\hline 710204 & 1000 & 1.00 & 17.59 & 4.2 & 172.0 & $i b . t$ & 3.9 & -0.3 & 260.2 \\
\hline 710204 & 1200 & $=200$ & $b=10$ & 2 & $179 \times 0$ & 16.4 & 4.4 & & \\
\hline $9: 0204$ & $1:-0$ & $: 200$ & 17.53 & 4.5 & 174.0 & 15.6 & 4.1 & -0.3 & 312.5 \\
\hline 910204 & 1260 & 1202 & $\leq .10$ & 4.5 & 173.0 & 18.7 & 4.5 & & \\
\hline 710204 & 120 & 200 & 17.58 & $=2$ & 170.0 & 16.0 & 4.2 & -0.3 & 32.2 \\
\hline 710204 & 1390 & 1400 & 0.10 & 5.2 & 167.0 & 14.7 & 4.2 & & \\
\hline 710204 & 1390 & 1400 & 17.58 & 5.9 & $1 \leq 5.0$ & 13.2 & 3.7 & -0.3 & $25 \% .4$ \\
\hline 710204 & 1400 & 1500 & $\leq .10$ & 5.4 & 133.0 & 12.1 & 4.2 & & \\
\hline 710204 & $1+50$ & $: 000$ & 17.58 & 5.0 & 162.0 & 7.1 & 4.0 & -0.2 & 223.0 \\
\hline 910204 & 1500 & $: 500$ & $=.10$ & 4.0 & 164.0 & 13.1 & 4.0 & & \\
\hline 710204 & $1 \equiv 30$ & 1600 & 17.63 & 4.4 & 152.0 & 11.5 & 4.0 & 0.0 & 101.5 \\
\hline 910204 & 1500 & 3700 & 6.10 & 3.7 & 150.0 & 12.3 & 2.8 & & \\
\hline 710204 & 1500 & 1700 & 17.68 & 4.2 & 159.0 & 9.7 & 3.0 & 0.2 & -50.9 \\
\hline 710204 & 1700 & 1000 & 6.10 & 0.5 & 123.0 & 86.3 & -0.5 & & \\
\hline 710204 & 1700 & $\$ 300$ & 17.68 & 0.7 & 111.0 & 83.9 & 0.1 & 0.6 & -73.5 \\
\hline 710204 & 1300 & 1900 & 6.10 & 0 & 359.0 & 14.1 & $-3 \cdot 5$ & & \\
\hline 910204 & 1800 & 1900 & 17.68 & 0. & 9.0 & 23.4 & -3.0 & 0.5 & -72.8 \\
\hline 710204 & 1700 & 2000 & 6.10 & 0.5 & 352.0 & 22.6 & -4.3 & & \\
\hline 910204 & 1900 & 2000 & 17.68 & 0.4 & 3.0 & 16.4 & -4.2 & 0.6 & -72.2 \\
\hline 710204 & 2000 & 2100 & 6.10 & 0.6 & 350.0 & 22.0 & -5.7 & & \\
\hline 910204 & 2000 & 2100 & 17.68 & 0.7 & 7.0 & 23.0 & -5.2 & 0.5 & -71.7 \\
\hline 710204 & 2100 & 2200 & 6.10 & 0.6 & 347.0 & 31.0 & -6.7 & & \\
\hline 910204 & 2100 & 2200 & 17.68 & 0.6 & 357.0 & 23.1 & -6.1 & 0.6 & -70.6 \\
\hline 710204 & 2200 & 2300 & 6.10 & 0.6 & 350.0 & 19.4 & -7.3 & & \\
\hline 910204 & 2200 & 2300 & 17.68 & 0.4 & 358.0 & 17.7 & -6.7 & 0.6 & -70.8 \\
\hline 910204 & 2300 & 0 & 6.10 & 1.0 & 347.0 & 15.6 & -7.6 & & \\
\hline 710204 & 2300 & 0 & 17.68 & 0.7 & 358.0 & 15.7 & -6.8 & 0.8 & $-71 \cdot 3$ \\
\hline
\end{tabular}




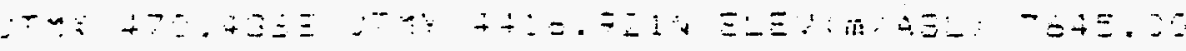

$5 \div 5$

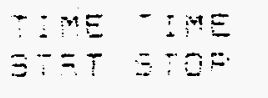
20
45
496 $\Xi F E \Xi$

in 1

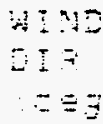

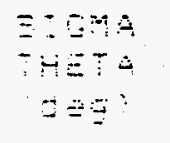

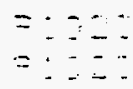

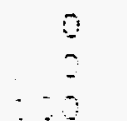

$: 00$

$\because \div$

$\dot{5}+20$

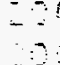

$=\therefore \div$

$=\because \because \cdots$

$=-\cdots$

$=\therefore \therefore$

$=: \therefore-$

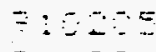

$=13=$

$=1-2=$

$=: 2 \div \equiv$

9102

$=105$

고은

$=1020$

$\because 10$

$\% 1025$

71025

$740 \div$

$715=$

P10 10

$7102=$

71055

710205

71020

710255

91020

$7102=$

710205

$0102=5$

$910=75$

71025

710205

919255

710205

910205

710205

910205

910205

710205

710205

910205

910205

910205

910205

9102052300
$1 \because:-8$

6.10

$: ?=53$

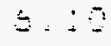

- $=5$

¿. $: 0$

$:-5$

o. 19

$\therefore ? .=9$

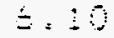

17.

$=.19$

17.59

$5 . \mathrm{i}$

17.39

5.10

17.59

2.10

$17: 59$

5.10

17.58

6.10

$17 . \leq 8$

b. 10

17.59

s. 10

17.08

$b=10$

17.53

6. 10

17.50

c. 10

17.69

6.10

17.68

6.10

17.68

6.10

17.68

6.10

17.68

6.10

17.68

6.10

17.68

6.10

17.68
7. 34.5

3.

0.

$\therefore$

2,4

$\therefore=$

$\therefore$

$\therefore=$

$\because=$

i. 5

8.5

$\therefore, 5$

.

0.5

0.5

i. 4

0.3

6. 2

1. 3

1.6

3.8

4. 1

4. 2

4.3

3.7

4.4

4.2

4.7

3.8

4. 3

4.4

5.0

3.1

3.9

0.6

0.8

1.1

1.0

1.1

1.2

1.2

1.2

1. 1

1.1

1.2

1.1

1.3

1.2 $z=2$.

$$
=
$$

$\because 0$

$\therefore-5$

$3+1.5$

7.0

$34 \div .0$

0.5

347.0

1.0

346.3

5.0

$4=0$

34.0

121.0

143.0

155.0

157.0

158.0

159.0

157.0

159.0

155.0

159.0

156.0

157.0

159.0

158.0

170.0

165.0

15.0

7.0

359.0

357.0

355.0

351.0

3.59 .0

353.0

358.0

358.0

357.0

359.0

356.0

357.0
$=4.7$
$=1.9$
$39=$
32.5
$=1.1$
42.7
+3.5

-

$44 .=$

-1.

39.3

28.6

43.6

34.9

43.3

34.4

23.5

48.8

55.6

40.1

9.1

5.1

10.3

4.5

11.0

5.9

1.

5.2

9.8

5.2

10.2

5.4

17.4

11.5

45.9

48.9

12.3

13.2

10.9

8.9

9.9

7.6

10.7

11.2

13.5

9.3

12.2

7.6

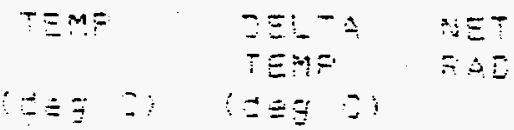

$$
\text { , }
$$

$-7=4$

$-2 . ?$

$-\div$.

- I =

$-\because .3$

-?. 8

$-8.7$

- ?.

$-3.4$

$-7.1$

$-8.5$

$-7.4$

$-3.3$

$-7$.

$-4.3$

$-2.7$

4.5

5.7

3.5

3. 2

9.7

3. 2

ㄱ. 1

3.6

8.7

S. 4

9.4

9. 1

3. 3

3.7

6.9

7.3

1.7

2.3

$-2.3$

$-1.8$

$-3.5$

$-3.0$

$-4.4$

$-3.5$

$-5.3$

$-4.4$ 
$\equiv:-\equiv=: \cdots=: \because \cdots$

\begin{tabular}{|c|c|c|c|c|c|c|c|c|c|}
\hline $5:=$ & 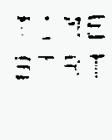 & $\begin{array}{l}-20 \\
\equiv+00\end{array}$ & 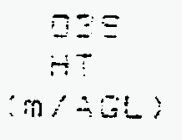 & $\begin{array}{l}\because \div \\
\Xi=\Xi \Xi 0 \\
\text { it } \vdots\end{array}$ & $\begin{array}{l}\because 15 \\
\therefore \div= \\
\because=\because\end{array}$ & 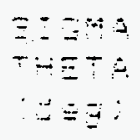 & $\Xi \Xi \ddot{z}=$ & 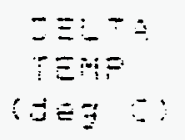 & $\begin{array}{l}\because \vdots \equiv- \\
\vdots=\bar{Z}\end{array}$ \\
\hline$=\vdots$ & $\Xi$ & 190 & 6.10 & 1.3 & $3=5$. & $1: \ldots$ & $-\therefore . \equiv$ & & \\
\hline 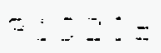 & $\because$ & 20 & 17.69 & $1 .:$ & $= \pm 3.0$ & 7.7 & $-\equiv .7$ & 2.9 & -57.2 \\
\hline$=: \therefore:=$ & $\therefore$ & -22 & $\leq .: 2$ & 1.4 & $3=7.2$ & $: 3.2$ & $-\leq . \because$ & & \\
\hline$=\div:=$ & $\because \because$ & 0 & $\because, 38$ & $\therefore 2$ & $\Xi \equiv \pm$. & $\therefore \Xi . ;$ & $-\Xi$. & $\therefore \%$ & -37.2 \\
\hline$=: \cdots=$ & & $\therefore 2$ & 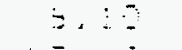 & $\therefore .3$ & $3=5.0$ & 14.0 & -7. & & \\
\hline$\because: \because z$ & $\therefore:$ & $\because$ & $\because-\quad-5$ & $\therefore=$ & $\Xi \equiv 2.0$ & 7.5 & $-\leq .:$ & $\therefore$ & $-=\bar{Z} . \overline{-}$ \\
\hline$=:-2=$ & & 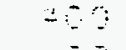 & 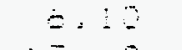 & $\therefore=$ & $z=0$ & $\therefore: \Xi$ & -7.4 & & \\
\hline$\because:: \div:=$ & & 1 & $\therefore \because=2$ & $\because=$ & $2=6.5$ & 3.7 & $-\div . \bar{Z}$ & $\therefore=1$ & 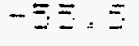 \\
\hline$=1: 2:=$ & & $\because 3$ & 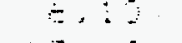 & $\therefore$ & $2=5.6$ & 22.7 & -7.4 & & \\
\hline$=1: 2=$ & $\therefore \because$ & $=0$ & $\therefore 7: 5$ & $\therefore z$ & $3=0,0$ & $7=9$ & -5.4 & 1.0 & $-53 \cdot=$ \\
\hline$=1:-2=$ & $\because \therefore$ & $=\because$ & 5.12 & $\therefore$ & 58.0 & $\therefore 4=\div$ & $-7 \cdot 6$ & & \\
\hline 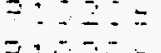 & $\because$ & $=6$ & $17=5$ & $\therefore=$ & $\begin{array}{l}3=0 \\
=\end{array}$ & 7.9 & $-\leq=0$ & 2.0 & -54, \\
\hline$=18$ & $\because 0$ & 3 & $==10$ & $1=3$ & $35 i=0$ & 19.3 & $-7= \pm$ & & \\
\hline $7:-2$ & -3 & -5 & $\because x=0$ & $\therefore$ & $=0 \times 9$ & 15.5 & $-5=$ & i.: & -54. \\
\hline $7: 0$ & -8 & $\because z$ & - & 1.5 & $3=3 \cdot 0$ & $12=2$ & -7 & & \\
\hline 910 & $\equiv 8$ & 30 & $1 \%=08$ & $=0$ & $\begin{array}{ll}3=0 \\
7=7\end{array}$ & $=1 \cdot 0$ & -0.2 & 37 & -4コ. \\
\hline $7: 0$ & 50 & $=00$ & 0.10 & $\therefore$ & $=0$ & $-1+0$ & $\begin{array}{r}4.5 \\
-3.0\end{array}$ & $\therefore=$ & \\
\hline 910 & $=-2$ & 100 & 5. 10 & 2.4 & 345.0 & 37.4 & 2.6 & 2.5 & 53. \\
\hline 71 & $\because 0$ & .200 & $: 7.63$ & 2.3 & 0.0 & 30.0 & 2.3 & -0.3 & 202.7 \\
\hline & $\because 0$ & $: 100$ & 5.10 & 2.4 & 347.0 & 51.5 & 3.3 & & \\
\hline 710 & 1000 & $: 100$ & 17.63 & 2.5 & 3.0 & 36.3 & 2.3 & -0.5 & 2 \\
\hline
\end{tabular}




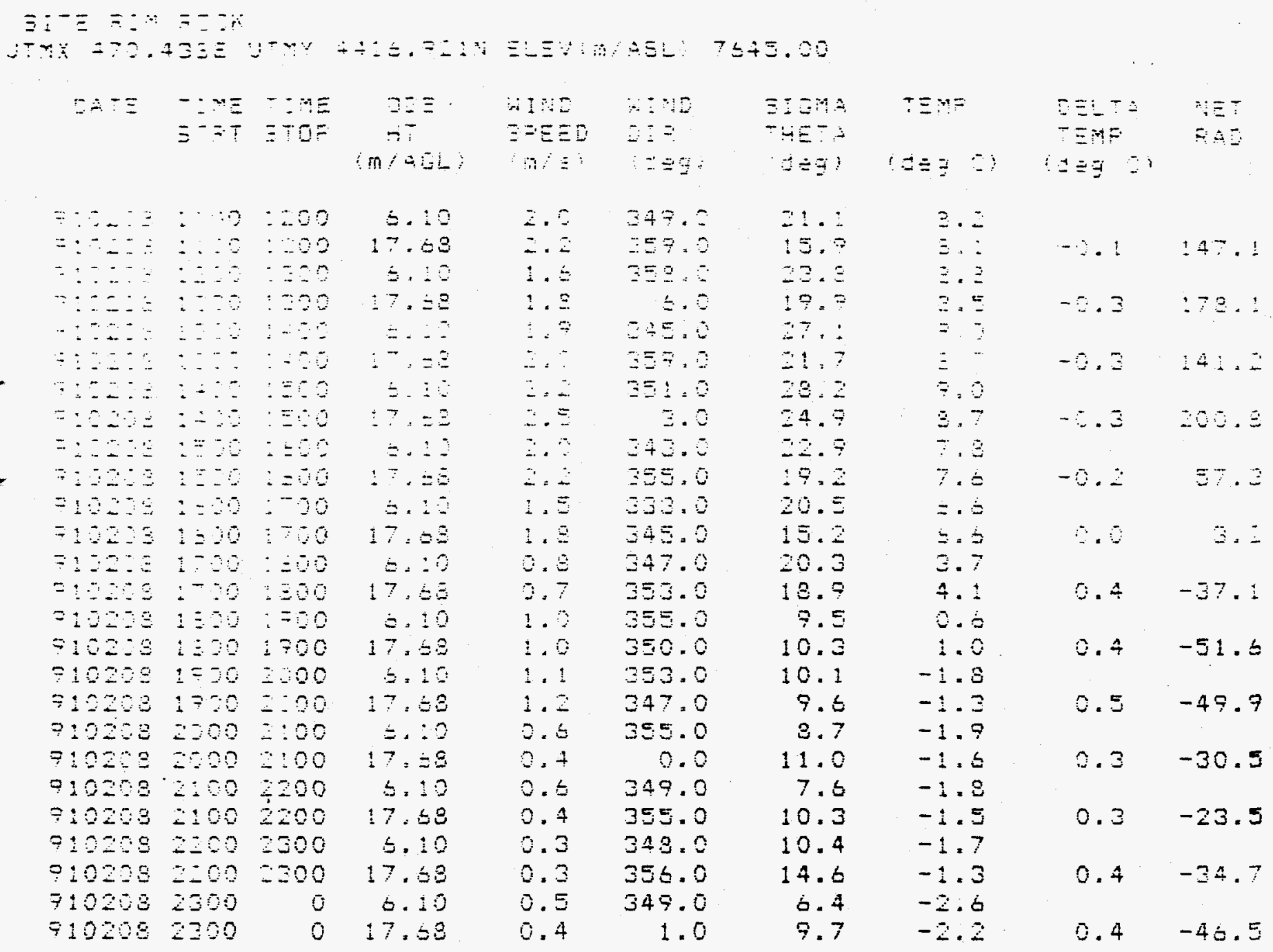




\begin{tabular}{|c|c|c|c|c|c|c|c|c|}
\hline 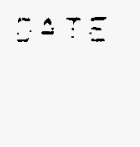 & 政 & $\begin{array}{l}\text { YME } \\
\text { EYJF }\end{array}$ & 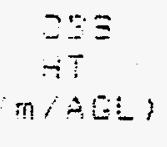 & 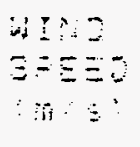 & $\begin{array}{c}4: y 5 \\
-25 \\
=5\end{array}$ & $\begin{aligned}= & :-4 \\
- & =-1 \\
& =-\end{aligned}$ & 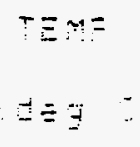 & $\begin{array}{c}20 \\
0 \\
0=5\end{array}$ \\
\hline$\therefore \therefore$ & 2 & $: 00$ & $=.10$ & 12.3 & -50.7 & 7. & -3.7 & \\
\hline 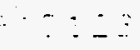 & $\because$ & $\therefore 0$ & 17.59 & $1 \div .2$ & $\therefore 69.0$ & $\therefore: 2$ & $-3=5$ & 2 \\
\hline$\therefore$ & $\therefore$ & $\therefore 0$ & $\leq .: 0$ & $\therefore=2$ & -5.0 & $\therefore \therefore$ & -3.2 & \\
\hline$\ldots$ & & 50 & $: 7 . \leq 0$ & $14 .:$ & $\because=2.0$ & $\therefore .9$ & -3.7 & $\therefore:$ \\
\hline$\therefore=3$ & $\therefore$ & $\because ?$ & $\therefore \quad \therefore$ & $\therefore:=$ & $=7: 0$ & $\because \theta$ & $-2=$ & \\
\hline$\cdots$ & $\because$ & $\cdots$ & $-\because \vdots$ & $\therefore$ & $-7 \vdots \quad=$ & $\therefore-7$ & $-9=2$ & $\therefore \therefore$ \\
\hline 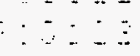 & $\because$ & $\therefore$ & $=\therefore$ & 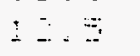 & $-7=0$ & $\therefore \because$ & -3.5 & \\
\hline 8 & 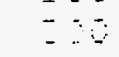 & $\therefore 0$ & $\therefore \quad \leq$ & $\therefore A$ & $2-5,2$ & $1 \therefore=$ & $-3=$ & 3 \\
\hline$\because \therefore-2$ & $-\cdots$ & $\because$ & $\therefore=\therefore$ & $\therefore=:$ & $=2 i .0$ & $\therefore \therefore$ & -3.5 & \\
\hline 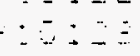 & $\therefore$ & $\because 8$ & $\therefore=\equiv$ & $: 3 . \div$ & $\because 3.0$ & $1 \leqslant .5$ & -3. & 0.4 \\
\hline$\therefore-三$ & $=\cdots$ & $=6$ & $\therefore$. & 20 & 265.0 & $\because Z$ & -3.3 & \\
\hline $8: 8$ & $\equiv 2$ & $=-\infty$ & $\therefore:=0$ & $\therefore .4$ & 206.0 & $1 \div .9$ & -7.7 & 0.4 \\
\hline$\therefore: 2$ & 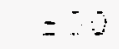 & $-\infty$ & $\therefore \therefore$ & $\because$. & $=20.0$ & $4 \therefore 6$ & $-3 .=$ & \\
\hline$: \div 2$ & $=-9$ & 700 & $\vdots ? .=8$ & $\therefore 2.5$ & $2 \leq 7.0$ & 35.5 & -3.4 & 0.2 \\
\hline$\therefore=3$ & $\because$ & 500 & 5.20 & 3. & 121.0 & $4 \therefore .7$ & -7.5 & \\
\hline$C: \div$ & --0 & 300 & $\therefore 7.68$ & 4.8 & $1: 5.0$ & $4 \because .5$ & -3.9 & 0.1 \\
\hline $5: 23$ & 230 & $=00$ & 5.10 & 4.3 & $1 \because 2=0$ & $2=.9$ & -7.3 & \\
\hline $01 \div 3$ & $\vdots \vdots$ & 700 & 17.50 & 5.8 & 122.0 & $2 \pi .2$ & -7.5 & -0.2 \\
\hline$\because 28$ & 790 & $=200$ & 5.10 & 3.2 & 221.0 & $7 \div .6$ & -5.1 & \\
\hline$: 23$ & 7.00 & $: 00$ & $i 7=\leq 8$ & $3: 9$ & 222.0 & 75.5 & -5.6 & -0.5 \\
\hline$i 23$ & 190 & 1100 & 5.10 & 4.6 & 235.6 & $2 \div .5$ & -4.4 & \\
\hline 2123 & 1000 & 1100 & 17.53 & 5.5 & 241.0 & $2: .4$ & -4.7 & -0.3 \\
\hline $2 \pm 28$ & $1: 50$ & $\therefore 200$ & $=10$ & $E: 0$ & 243.0 & 15.2 & -3.4 & \\
\hline$\therefore \therefore$ & $1: 50$ & $=200$ & $\div 7.58$ & 5. & 243.0 & 17.5 & -3.7 & -0.3 \\
\hline $2: 23$ & 1200 & $: 200$ & \pm .10 & 5.7 & $=46.0$ & 28.5 & -2.3 & \\
\hline $12=8$ & 1200 & 1200 & 17.5 & 6.6 & 250.0 & 1.0 & -2.5 & -0.2 \\
\hline$\therefore-3$ & 1300 & $: 400$ & 6.10 & 6.5 & 250.0 & 17.9 & -2.4 & \\
\hline 128 & 1500 & $: 400$ & $17 . \pm 3$ & 7.6 & 260.0 & 16.9 & -2.6 & -0.2 \\
\hline$\therefore=3$ & 1400 & 1000 & $5 .: 0$ & 5.3 & 270.0 & 21.9 & -1.4 & \\
\hline$\therefore 23$ & 1400 & $: 500$ & $17.6 \overline{8}$ & 6.5 & 274.0 & 20.6 & -1.6 & -0.2 \\
\hline 8 & $1=00$ & 1500 & 6.10 & 3.9 & 270.0 & $2 \because 5$ & -3.2 & \\
\hline 3 & $1=00$ & 1000 & 17.63 & 4. & 274.0 & 21.8 & -3.2 & 0.0 \\
\hline 23 & 1000 & 1700 & 5.10 & 1.8 & 259.0 & $1 \Xi .3$ & -4.1 & \\
\hline 29 & 1500 & 1700 & 17.53 & 2.4 & 258.0 & $1 \div .3$ & -0.3 & 3.8 \\
\hline 20 & 1700 & 1000 & $=.10$ & 3.3 & 81.0 & 72.4 & -6.8 & \\
\hline 23 & 1700 & $: 200$ & 17.68 & 4.3 & 87.0 & $7 \equiv: .4$ & -6.7 & 0.1 \\
\hline 23 & 1300 & 1700 & 6.10 & 2.5 & 101.0 & $1: .4$ & -9.6 & \\
\hline 8 & 1800 & 1900 & 17.68 & 3.1 & 101.0 & $\therefore .6$ & -9.7 & -0.1 \\
\hline 8 & 1900 & 2000 & 6.10 & 3.2 & 104.0 & 7.4 & -10.9 & \\
\hline 8 & $1=00$ & 2000 & 17.68 & 3.7 & 102.0 & 5.7 & -11.0 & -0.1 \\
\hline & 2000 & 2100 & 6.10 & 3.2 & 102.0 & 8.0 & -12.3 & \\
\hline & 2000 & 2100 & 17.68 & 3.8 & 102.0 & 5.9 & -12.4 & -0.1 \\
\hline & 2100 & 2200 & 6.10 & 3. & 103.0 & 7.9 & -14.6 & \\
\hline & 2100 & 2200 & 17.68 & 3. & 103.0 & 6.3 & -14.7 & -0.1 \\
\hline & 2200 & 2300 & 6.10 & 2. & 99.0 & 8.2 & -17.8 & \\
\hline & 2200 & 2300 & 17.68 & 3 & 100.0 & 6.3 & -17.9 & -0.1 \\
\hline & 23 & 0 & 6.10 & & .0 & 5.5 & -19.5 & \\
\hline & & 0 & 17.68 & 3 & 100.0 & 7.2 & -19.7 & -0. \\
\hline
\end{tabular}




\begin{tabular}{|c|c|c|c|c|c|c|c|c|}
\hline$\because \div-\equiv$ & $\begin{array}{l}\cdots \equiv \\
\Xi^{\prime}\end{array}$ & $\begin{array}{l}-y \equiv \\
\vdots 0\end{array}$ & $\begin{array}{c}\because=0 \\
4 \vdots \\
n=0-1\end{array}$ & $\begin{array}{c}\therefore \\
\equiv=E \equiv= \\
\vdots: \vdots\end{array}$ & 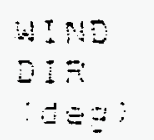 & 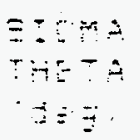 & $\sigma=\Xi \quad \because$ & 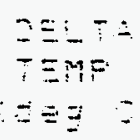 \\
\hline$\because \vdots: \because$ & 2 & $\because 8$ & $\doteq: 2$ & $z:=$ & $: 23: 3$ & $\therefore \Xi$ & $-\therefore z=$ & \\
\hline$\because:$ & $\because$ & $\therefore 0$ & $\div 7 . \leq 3$ & $\therefore .7$ & 53.3 & $\therefore \quad \therefore$ & $-z !$. & -3.2 \\
\hline$\therefore$ & & 3 & $\doteq .: 0$ & $\therefore$. & $=9.2$ & $\therefore$. & $-\because 2$. & \\
\hline$\because \ldots$ & $\therefore$ & $\because$ & $\because .=\theta$ & $\because$. & : : & $\therefore$. & $-\because \therefore$ & $-i .:$ \\
\hline$\therefore \therefore$ & $\therefore$ & $\because$ & $=::$ & $\therefore$. & $\div 5.2$ & 5.4 & $-\therefore:=$ & \\
\hline$\because=$ & & $\therefore$ & $=\vdots$ & $\therefore$ & 10.9 & $\therefore$ & 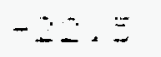 & $-3:$ \\
\hline$\because \therefore \ldots$ & $\because 9$ & $\because \because$ & $=.: \bar{z}$ & $\therefore$. & $: 0 E .0$ & 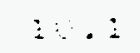 & 一シ. & \\
\hline$: \therefore=$ & & &.$-=8$ & $\therefore=$ & $3=$. & $\therefore .4$ & $-z=3$ & $-8:$ \\
\hline$\therefore:=$ & & $\therefore$ & $==8$ & $\therefore$ & 53.1 & $=0$ & -22.3 & \\
\hline$\therefore: 2=$ & $\div 20$ & $\because 2$ & $\because .=3$ & $\therefore$ & $9 \Xi .0$ & $2 \div .1$ & -23.4 & $-5 .:$ \\
\hline$\therefore \therefore=$ & & $\therefore 2$ & $\therefore: 2$ & $\therefore \therefore$ & 239.0 & $\leq: \ldots$ & -23.6 & \\
\hline $5:=$ & $=20$ & $\therefore=0$ & 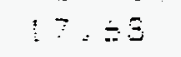 & $\because 2$ & 45.0 & 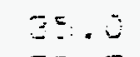 & -23.7 & -0.1 \\
\hline $0:-9$ & $=5$ & $\because$ & $=: 15$ & $\because . \because$ & 30.0 & 75 & -73.3 & \\
\hline $51=9$ & $=-0$ & $7, j$ & $17:=0$ & $\therefore$ & $2=9.0$ & $5: 5$ & -23.4 & -0.1 \\
\hline 2157 & -8 & 80 & $=-\therefore$ & 2.7 & 23.0 & $=-4$ & $-2=6$ & \\
\hline 0179 & -30 & $\Xi 50$ & $17=0$ & 5.5 & $2 \leq 4.6$ & 52.6 & -21.1 & 1.5 \\
\hline $51=0$ & 320 & $=90$ & $=10$ & $=.9$ & $2=0.0$ & $\because .3$ & -13.2 & \\
\hline 519 & $\vdots 90$ & $=09$ & $: 7,03$ & 7.9 & $25 i .0$ & $\div .3$ & $-1 j . i$ & 0.1 \\
\hline $51-7$ & $\div .0$ & 100 & 5.5 & 3.8 & 246.0 & 201 & -10.8 & \\
\hline $91=7$ & $=80$ & $: 90$ & $: 7.58$ & 4.4 & 250.9 & $2: 1$ & -11.1 & -0.3 \\
\hline $02-9$ & 100 & $1: 00$ & 0.10 & $\Xi .7$ & 2350 & $1 E .3$ & -10.5 & \\
\hline 129 & $1-00$ & $1: 00$ & 17.69 & 5.7 & 241.0 & $1: .0$ & -10.7 & $-\overline{0} . \overline{3}$ \\
\hline 129 & \pm 20 & $1=00$ & $b .10$ & 6.2 & 250.0 & 31.7 & -7.5 & \\
\hline $12 \%$ & $1: 0$ & $: 200$ & $17=53$ & 7.1 & $2 \Xi 4.0$ & 30.0 & -7.8 & -0.3 \\
\hline 127 & 1200 & 1200 & $=.10$ & 7.4 & 250.0 & $2: .3$ & -9.3 & \\
\hline 7 & $1=0$ & 1300 & 17,03 & 8.7 & $2=3.0$ & 19.7 & -10.1 & -0.3 \\
\hline 马 & 1300 & 1400 & 5.10 & 6.2 & 255.0 & $1 \div .6$ & -9.4 & \\
\hline 7 & 1300 & 1400 & 17.58 & 7.2 & 259.0 & $1 \div .2$ & -9.6 & -0.2 \\
\hline 7 & 1430 & $: 500$ & 5.10 & 5.2 & 247.0 & $2: .7$ & -9.3 & \\
\hline 9 & 1400 & $:=00$ & 17.68 & 7.3 & 250.0 & $1 \div .3$ & -7.6 & -0.3 \\
\hline 9 & $i=00$ & $i=00$ & 6.10 & 5.3 & 244.0 & $2 t .5$ & -9.3 & \\
\hline 9 & $1=90$ & 1500 & 17.58 & 6.3 & 247.0 & 23.6 & -7.5 & -0.2 \\
\hline 7 & 1500 & 1700 & 6.10 & 6.7 & 262.0 & $1: .3$ & -10.4 & \\
\hline & 1500 & 1700 & 17.63 & 7.3 & 262.0 & $1 \Xi .2$ & -10.4 & 0.0 \\
\hline & 1700 & 1300 & 6.10 & 9.2 & $2 \leq 2.0$ & 15.1 & -11.2 & \\
\hline & 1700 & $: 800$ & 17.68 & 10.6 & 262.0 & 13.0 & -11.0 & 0.2 \\
\hline & 1300 & 1700 & 6.10 & 11.0 & 265.0 & 10.5 & -11.9 & \\
\hline & 1800 & 1900 & 17.68 & 12.7 & 263.0 & 14.8 & -11.7 & 0.2 \\
\hline & 1700 & 2000 & 6.10 & 8.2 & 240.0 & 22.0 & -12.0 & \\
\hline & 1900 & 2000 & 17.68 & 9.9 & 251.0 & 21.1 & -11.9 & 0.1 \\
\hline & 2000 & 2100 & 6.10 & 13.5 & 263.0 & $\div .7$ & -11.7 & \\
\hline & 2000 & 2100 & 17.68 & 15.7 & 262.0 & 12.7 & -11.5 & 0.2 \\
\hline & 2100 & 2200 & 6.10 & 13.6 & 262.0 & 12.7 & -11.5 & \\
\hline & 2100 & 2200 & 17.68 & 15.8 & 261.0 & 17.3 & $-11 \cdot 3$ & 0.2 \\
\hline & 2200 & 2300 & 6.10 & 17.5 & 265.0 & 7.6 & -11.0 & \\
\hline & 2200 & 2300 & 17.68 & 19.9 & $2 \leq 1.0$ & 17.7 & -10.7 & 0.1 \\
\hline & 230 & 0 & 6.10 & 11.5 & 270.0 & 11.0 & -11.2 & \\
\hline & 2306 & 0 & 17.68 & 13.2 & 264.0 & 22.9 & -11.1 & 0.1 \\
\hline
\end{tabular}




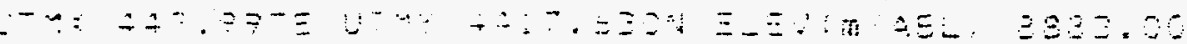

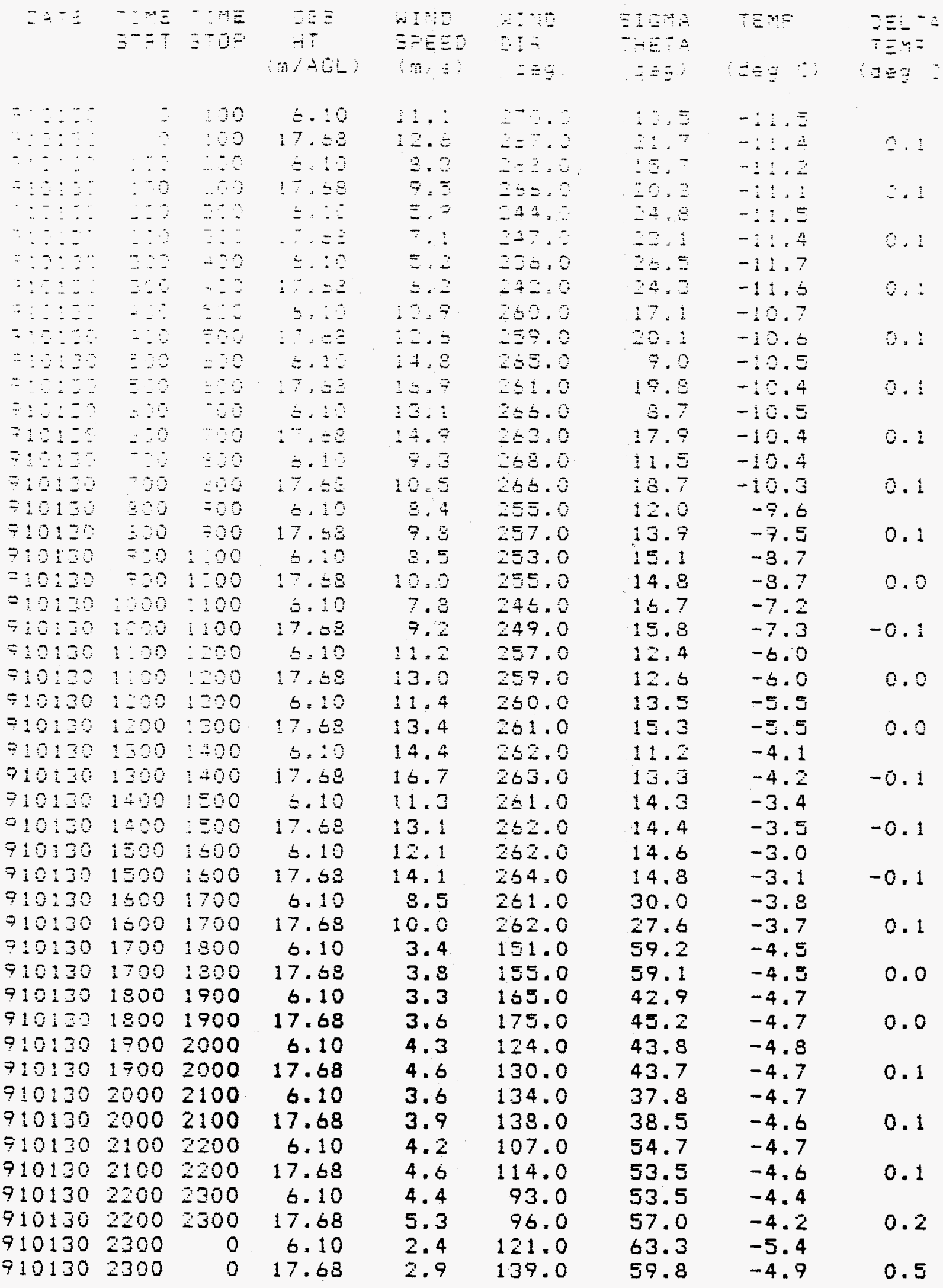




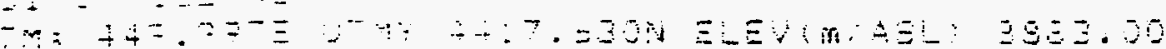

\begin{tabular}{|c|c|c|c|c|c|c|c|c|}
\hline$\because \therefore=$ & $\begin{array}{l}\because \cdots E \\
\vdots=\bar{T}\end{array}$ & $\begin{array}{l}-1= \\
=5\end{array}$ & $\begin{array}{l}\text { o: } \\
\mathrm{H}\end{array}$ & $\begin{array}{l}W D D \\
B=E D S\end{array}$ & \begin{tabular}{l}
$n: y=$ \\
\hdashline-2
\end{tabular} & 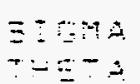 & $T E m=$ & 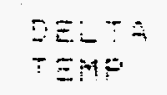 \\
\hline . & $\because$ & 2 & $=10$ & $1:=$ & $z==$ & $=0$ &,--- & \\
\hline$z$ & $\because$ & 20 & $17=03$ & 1.5 & 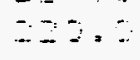 & $7=5$ & $-\therefore$ & $\vdots=\dot{1}$ \\
\hline & $\cdots$ & 28 & $\therefore .:$ & 2. & 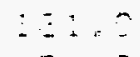 & $\because=$ & -5. & \\
\hline$\because$ & $\because 0$ & $\therefore 0$ & $:-1=3$ & $=4$ & $i=0$. & $=9.4$ & $-=.5$ & 27 \\
\hline & $\because \because$ & & $\therefore \quad \therefore$ & $\Xi=$ & $\therefore=$ & $3:=$ & $-\because \Xi=$ & \\
\hline & & $\therefore$ & $\because \because=5$ & 4. & $\because: 8$. & $\therefore=$ & $-=2$ & 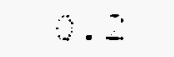 \\
\hline & - & $\therefore-5$ & $=20$ & $\div .4$ & $\therefore 0^{-}$ & 76.1 & $-Z .0$ & \\
\hline & $\because \therefore$ & $\therefore=$ & $: 7:=0$ & 3.9 & $1=5.0$ & $+4=$ & -1.7 & $\therefore$. \\
\hline & $-\cdots$ & $=-$ & $\doteq:$ & 4.3 & $230=$ & 41.0 & $-E .5$ & \\
\hline & $\therefore$ & $\equiv=0$ & $\therefore:=8$ & $\Xi .3$ & -30.0 & $i 5.9$ & $-5 . \vdots$ & $\therefore$. \\
\hline & $\therefore$ & $=8$ & $e: z$ & 7.5 & $25=0$ & 13.7 & -4.7 & \\
\hline & $\because$ & $=\therefore$ & $: 7=-5$ & 12.4 & $2=9.0$ & 15.7 & -4.7 & 12 \\
\hline 213 & $=0$ & -20 & $3 .: 0$ & 5. & 957.0 & $1 \Xi .1$ & -4.5 & \\
\hline $2: 2$ & $=\because$ & $=0$ & $\dot{i}= \pm 0$ & 7.0 & 250.5 & 17.5 & -4.3 & 0.2 \\
\hline & & 590 & $5 . \vdots 0$ & $\equiv .0$ & $2=4.0$ & 24.1 & -4.5 & \\
\hline 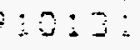 & -90 & $=03$ & $17=30$ & $b .1$ & 257.0 & 20.5 & -4.3 & 0.2 \\
\hline 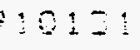 & $=80$ & 90 & $\dot{\theta}=0$ & 5.2 & 230.0 & 50.2 & -4.0 & \\
\hline$\therefore$ & 30 & $=00$ & 17.48 & 5.2 & 235.0 & 48.3 & -3.3 & 0.2 \\
\hline$\therefore 1=$ & $=8$ & $\therefore 00$ & 5.10 & 4.1 & 184.0 & 32.5 & -3.3 & \\
\hline 1019 & $=9$ & $\because 00$ & $17=-8$ & 4.5 & 172.0 & 30.3 & -3.3 & 0.0 \\
\hline $10: 5$ & $\because 95$ & 190 & $b .: 0$ & 3.0 & $2 \leq 2.0$ & 39.0 & -0.5 & \\
\hline$: 0: 3:$ & $: 590$ & $2: 00$ & 17.88 & 3.5 & $2=4.0$ & 36.4 & -0.7 & -0.2 \\
\hline$: 015$ & $1: 50$ & $:-5$ & $=.10$ & 1.5 & 317.0 & 67.5 & 3.5 & \\
\hline $1013:$ & $1: 90$ & 200 & $17: 53$ & 1.3 & 312.0 & 56.5 & 3.0 & -0.6 \\
\hline $10: 51$ & $: 20$ & $: 300$ & 5.10 & 2.1 & 195.0 & $b 1.5$ & 5.4 & \\
\hline $101=3$ & 1250 & $: 200$ & $17=5$ & 2.2 & 205.0 & 61.5 & 4.9 & -0.5 \\
\hline 20151 & 1320 & $: 400$ & 6.10 & 3.7 & 230.0 & 25.2 & 4.7 & \\
\hline $10 \pm=$ & 1300 & 1700 & 17.58 & 4.5 & 237.0 & 23.2 & 4.4 & -0.5 \\
\hline $10: 31$ & 1400 & 1500 & $=.10$ & 4.1 & 219.0 & 23.9 & 4.5 & \\
\hline 10132 & 140 & 1500 & 17.58 & 4.8 & 227.0 & $21 \cdot 1$ & 4.3 & -0.2 \\
\hline $1013 i$ & 100 & $i \leq 00$ & 5.10 & 3.5 & 200.0 & 21.0 & 4.1 & \\
\hline $1013=$ & $1=00$ & 1600 & 17.88 & 4.5 & 216.0 & 20.9 & 3.7 & -0.2 \\
\hline $1013:$ & $\therefore 00$ & $: 700$ & 5.10 & 2.4 & 3.0 & 43.1 & 3.3 & \\
\hline 10151 & $1=0$ & 1700 & 17.68 & 2.8 & 201.0 & 39.3 & $3 \cdot 3$ & 0.0 \\
\hline 10131 & 1700 & 1800 & 6.10 & 1.6 & 1.0 & 69.6 & 0.1 & \\
\hline 10131 & 1700 & $\therefore 800$ & 17.58 & 2.1 & 175.0 & 47.5 & 1.0 & 0.7 \\
\hline 10151 & 1300 & 1900 & 6.10 & 1.3 & 206.0 & 92.4 & -1.8 & \\
\hline 1013 & 1800 & 1900 & 17.68 & 1.9 & 193.0 & 73.9 & -0.5 & 1.3 \\
\hline 10131 & $1=00$ & 2000 & 6.10 & 1.1 & 56.0 & 72.8 & -4.2 & \\
\hline 10131 & 1700 & 2000 & 17.68 & 1.5 & 159.0 & 98.9 & -2.4 & 1.8 \\
\hline 10131 & 2000 & 2100 & 6.10 & 1.9 & 121.0 & 50.5 & -2.6 & \\
\hline 10131 & 2000 & 2100 & 17.68 & 2.3 & 142.0 & 50.9 & -1.4 & 1.2 \\
\hline 31 & 2100 & 2200 & 6.10 & 5.3 & 244.0 & 25.1 & -1.0 & \\
\hline 131 & 2100 & 2200 & 17.58 & 6.6 & 249.0 & 21.2 & -0.6 & 0.4 \\
\hline 13 & 2200 & 2300 & 6.10 & 9.2 & 34.0 & 11.8 & -0.7 & \\
\hline$\Delta$ & 2200 & 2300 & 17.58 & 12.1 & 7.0 & 11.3 & -0.4 & 0.3 \\
\hline 1 & 2300 & 0 & 6.10 & 10.1 & 5.0 & 11.8 & -0.4 & \\
\hline & 2300 & & 17.68 & 12.1 & 0 & 10 & -0.2 & 2 \\
\hline
\end{tabular}




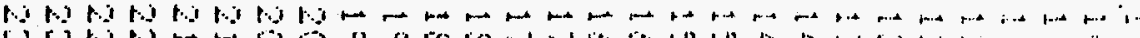

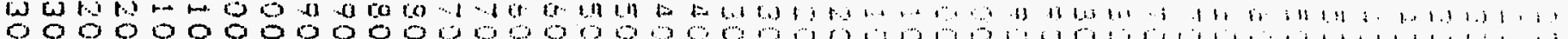

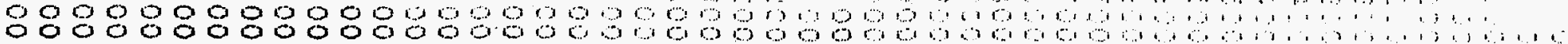

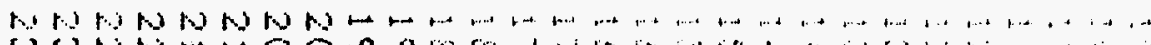

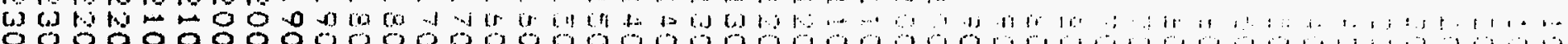

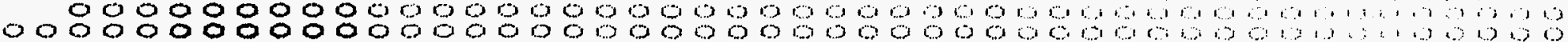
सू

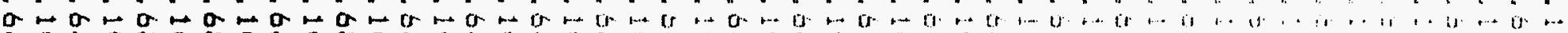

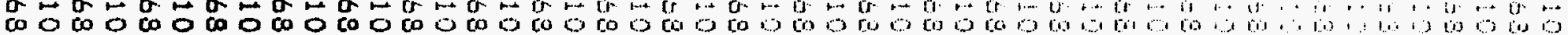

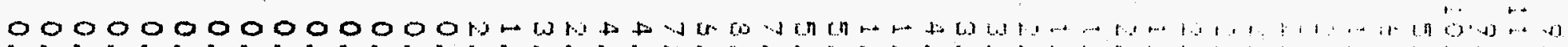

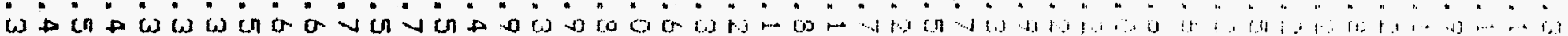

wo 0 o o c

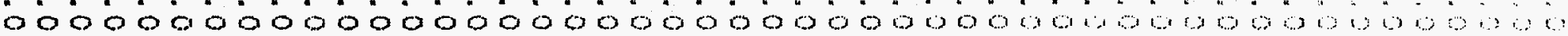

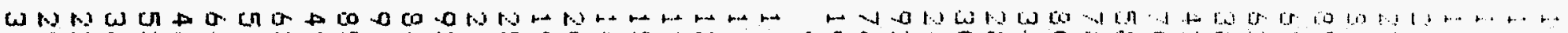

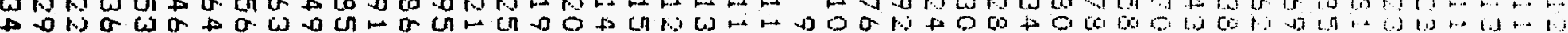
w $\begin{gathered}0 \\ 0\end{gathered}$

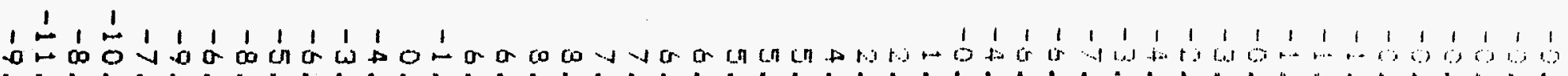

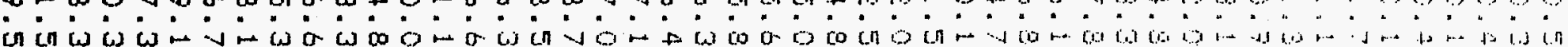

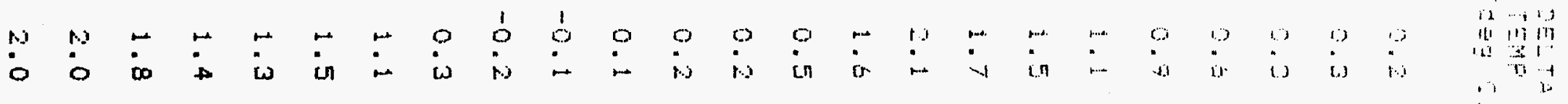




\begin{tabular}{|c|c|c|c|c|c|c|c|}
\hline $\begin{array}{l}\cdots:= \\
\vdots=7\end{array}$ & $\begin{array}{l}\because: \vdots \\
\Xi \because O F\end{array}$ & $\begin{array}{c}2 E \Xi \\
H 5 \\
m i=L\end{array}$ & 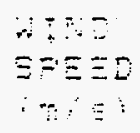 & $\begin{array}{l}\because 30 \\
\because:= \\
\vdots=5\end{array}$ & $\begin{array}{c}=:-1 \\
-1-E-2 \\
-E=\end{array}$ & $\checkmark \equiv \equiv$ & $\begin{array}{l}\text { ZE-TA } \\
T \equiv M F \\
\Xi \Xi Z \square\end{array}$ \\
\hline$=$ & $\because 20$ & 5.10 & $2: 3$ & $30=2$ & $4 \div=$ & $-\therefore=:$ & \\
\hline & .5 & $i \cdot . \pm 3$ & 2.2 & $\therefore=-.0$ & 50.9 & $-\because \because 5$ & $\therefore 3$ \\
\hline$\because \because$ & -20 & $\leq .: 2$ & 0.3 & $\because \because .9$ & $\pm 7, \pm$ & $-: Z, I$ & \\
\hline$\because \because$ & $\ldots 2$ & $\because \because \leq \geq$ & $\therefore: 2$ & $\therefore e ?=0$ & $: 3.7$ & $-\because !: \because$ & $\therefore=0$ \\
\hline$\because \because$ & $\because \because$ & $\therefore \quad \vdots$ & 5.5 & $-15=0$ & 22.5 & $-i \bar{i} \leqslant$ & \\
\hline$\therefore \because$ & $\because 2$ & $\therefore=\Sigma$ & $\therefore=$ & 220 & $\because 7=-$ & $-\vdots 1 \leq$ & $\therefore 0$ \\
\hline$\because z$ & $\therefore \therefore$ & $=: \because$ & $\therefore \equiv$ & $=4: 0$ & 45.7 & $-14,2$ & \\
\hline$\because$ & -23 & $\because:=8$ & $y=3$ & 296.2 & 2.5 & $-i:=7$ & $\therefore$ \\
\hline$\div$ & $=-8$ & $\leq .:-\bar{y}$ & $\because 4$ & $20 E .0$ & 33.7 & -14.3 & \\
\hline$\therefore \therefore$ & $\because \because$ & 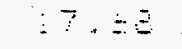 & $\therefore$. & 202.5 & 20.5 & 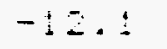 & $\therefore 2$ \\
\hline$\equiv$ & $=0$ & 5.20 & $\because 4$ & 309.5 & $\therefore 1.2$ & -12.5 & \\
\hline$=0$ & $=10$ & $: Y=-3$ & 3.5 & 293.0 & 43.4 & $- \pm:=4$ & 2.1 \\
\hline$=83$ & 30 & $5 .: 0$ & 9.7 & 320.0 & 52.5 & -12.3 & \\
\hline$=20$ & 90 & 17.58 & $5:$ & 335.6 & 73.3 & -10.4 & 2.7 \\
\hline 7.0 & $\equiv 0$ & $=.10$ & 2.3 & $3: 3.0$ & $b \leq .1$ & $-1: 2$ & \\
\hline$=50$ & 300 & $\because 7.58$ & 0.5 & 353.0 & 76.3 & -7.1 & 4.1 \\
\hline$\equiv 20$ & $=00$ & 5.10 & 3.5 & -73.0 & 76.5 & -1.4 & \\
\hline 300 & 70 & 17.53 & 4.3 & 263.0 & 75.6 & 1.1 & 2.5 \\
\hline 390 & $\because-00$ & 0.10 & 7.9 & 253.0 & 10.3 & 5.5 & \\
\hline$=90$ & $: 00$ & 17.53 & 7.5 & 250.0 & 7.2 & 5.7 & 0.2 \\
\hline$\because=190$ & 110 & $\$=20$ & $E .1$ & 242.0 & 14.7 & 6.9 & \\
\hline $02 \quad 1200$ & $: 200$ & 17.65 & 5.3 & 245.0 & 13.7 & 6.7 & 0.0 \\
\hline $021: 00$ & $: 00$ & 5.10 & 0.0 & 242.6 & 13.4 & 8.4 & \\
\hline $0=1: 00$ & $\div 20$ & 17.68 & 7.3 & 246.0 & $12 . \overline{3}$ & 3.3 & -0.1 \\
\hline $0=1220$ & $: 300$ & 5.10 & 7.8 & 247.0 & 10.5 & 8.7 & \\
\hline 001200 & $=300$ & 17.68 & 7.3 & 233.0 & 9.0 & 3.6 & -0.1 \\
\hline $2=1300$ & 1400 & 3.10 & 3.0 & 247.0 & 9.5 & 8.5 & \\
\hline $2=1300$ & 1400 & 17.68 & 7.7 & 252.0 & 3.6 & 3.4 & -0.1 \\
\hline$=1-90$ & 1500 & 0.10 & 7.7 & 250.0 & 8.7 & 7.7 & \\
\hline$\because 1200$ & 1500 & 17.63 & 9.3 & 254.0 & 7.5 & 7.7 & 0.0 \\
\hline $\begin{array}{l}=1500 \\
-1=00\end{array}$ & 1500 & 6.10 & 6.0 & 246.0 & 11.2 & 7.2 & \\
\hline $2=1500$ & $1=00$ & 17.59 & 7.4 & 250.0 & 10.1 & $7 \cdot 2$ & 0.0 \\
\hline $\begin{array}{l}i=00 \\
i=00\end{array}$ & $\begin{array}{r}1.00 \\
1700\end{array}$ & $\begin{array}{r}6.10 \\
-70\end{array}$ & 3.6 & 241.0 & 22.3 & $\begin{array}{l}5.7 \\
=.0\end{array}$ & \\
\hline $\begin{array}{ll}02 & 1500 \\
2 Z & 1700\end{array}$ & : & $\begin{array}{r}17.03 \\
6.10\end{array}$ & 4.5 & $\begin{array}{l}243.0 \\
307\end{array}$ & 19.1 & 3.9 & 0.2 \\
\hline$\therefore 1700$ & 1900 & 17.68 & $\begin{array}{l}1.0 \\
1.8\end{array}$ & 283.0 & $\begin{array}{l}36.7 \\
37.1\end{array}$ & 0.2 & 16 \\
\hline .021300 & 1900 & 6.10 & 0.5 & 34.0 & 70.9 & -3.7 & 1.6 \\
\hline $102 \quad 1300$ & 1700 & 17.68 & 0.5 & 303.0 & 87.1 & -2.2 & 1.5 \\
\hline 021900 & 2000 & 6.10 & 0.7 & 359.0 & 53.4 & -5.2 & 1.3 \\
\hline 021700 & 2000 & 17.68 & 0.5 & 333.0 & 51.5 & -2.6 & 2.6 \\
\hline 2000 & 2100 & 6.10 & 0.9 & 24.0 & 44.4 & -4.3 & \\
\hline 2000 & 2100 & 17.68 & 0.4 & 53.0 & 90.9 & -1.6 & 2.7 \\
\hline 022100 & 2200 & 6.10 & 0.9 & 354.0 & 46.0 & -5.1 & \\
\hline 2100 & 2200 & 17.68 & 1.2 & 309.0 & 54.2 & -2.4 & 2.7 \\
\hline $02 \quad 2200$ & 2300 & 6.10 & 4.9 & 233.0 & 26.4 & -0.7 & \\
\hline $02 \quad 2200$ & 2300 & 17.68 & 6.1 & 231.0 & 21.8 & 0.3 & 1.0 \\
\hline 2300 & 0 & 6.10 & 3.0 & 271.0 & 24.3 & 0.3 & \\
\hline 00 & 0 & 17.68 & 6.6 & 269.0 & 18.5 & 0.3 & 0.5 \\
\hline
\end{tabular}




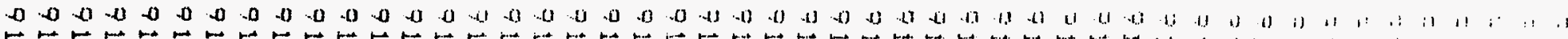

о

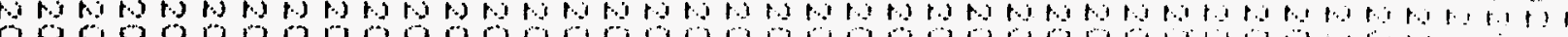

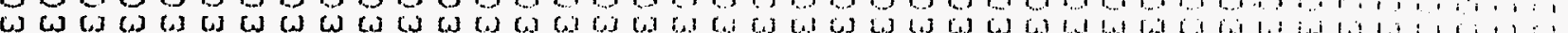

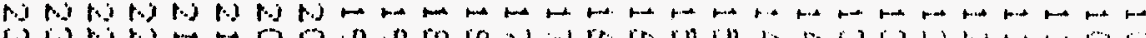

w

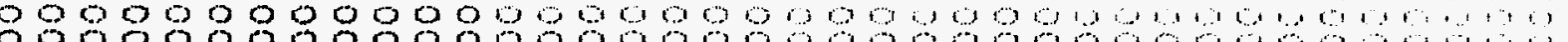

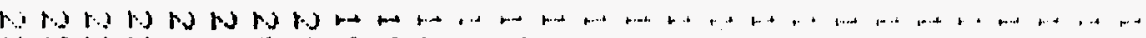

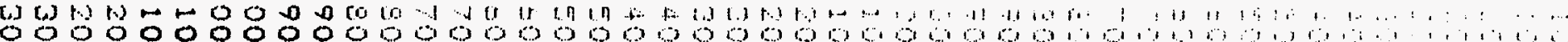

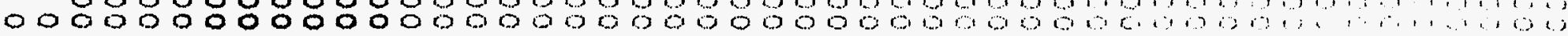

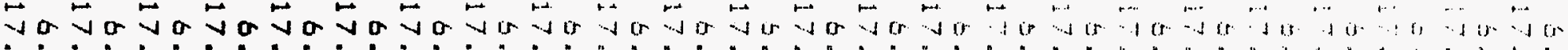

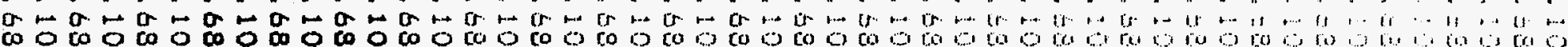

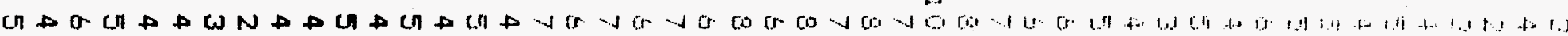

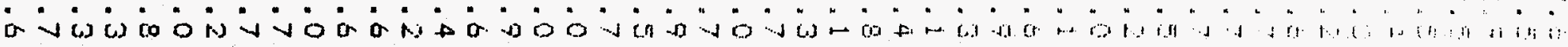

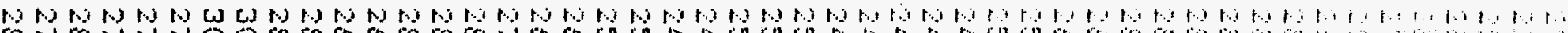

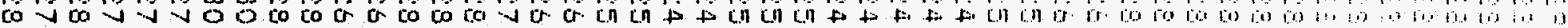
O

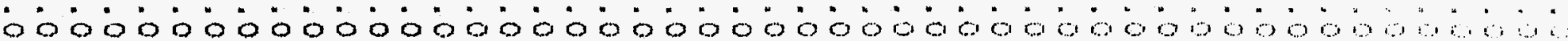

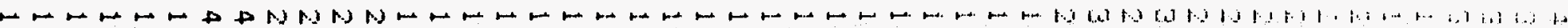

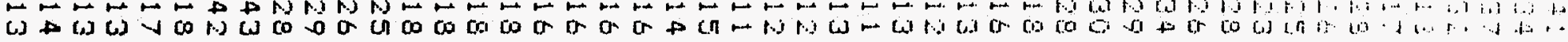

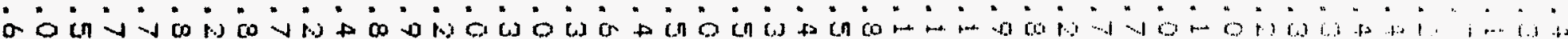

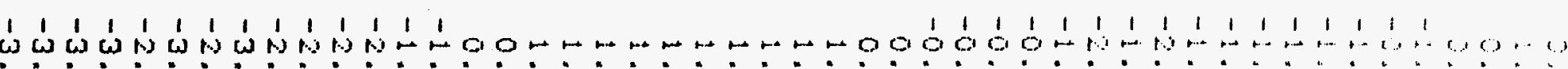

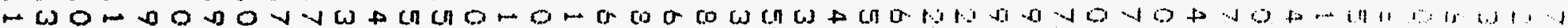

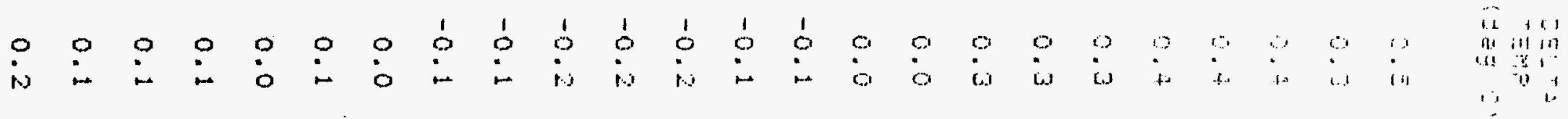




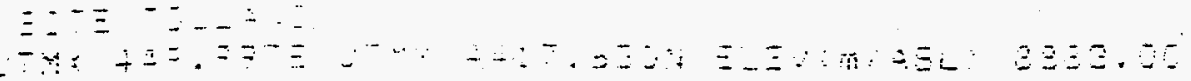

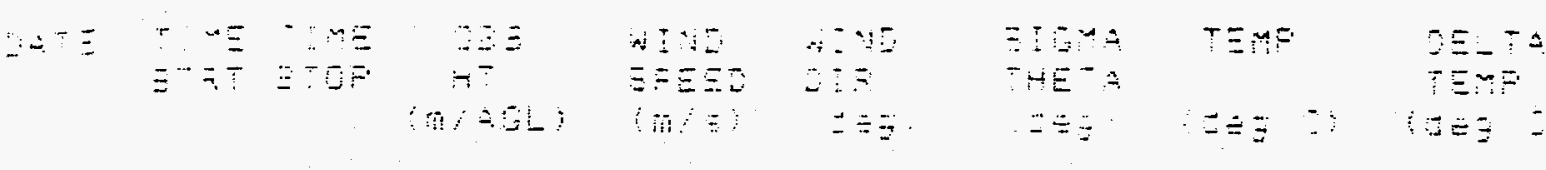

\begin{tabular}{|c|c|c|c|c|c|c|c|c|}
\hline$=:$ & $\because$ & 20 & 5.25 & E. & $\therefore \equiv \Xi .0$ & $\therefore \therefore=$ & $-3 .-$ & \\
\hline$\dot{-}$ & - & 0 & 17,52 & $\therefore:$ & 200.8 & $\therefore=8$ & -5.2 & $\sigma:$ \\
\hline$\because:$ & $\theta$ & $: 2:$ & $\leq .: 2$ & 5.7 & $=0 \pm .2$ & 12.6 & $-\Xi .9$ & \\
\hline . & $\therefore$ & $\because \because 3$ & $17=-2$ & $\leq=$ & 237.0 & $\therefore \rightarrow=$ & $-3=5$ & $\therefore=$ \\
\hline$\therefore$ & $-\therefore$ & $\because 9$ & $=\ldots$ & $\Xi:=$ & $\therefore 2.5$ & $\div 4.5$ & -3.9 & \\
\hline & $\therefore$ & & $\therefore \cdots$ & $\therefore \theta$ & 284,2 & $\therefore 4.2$ & $-2=8$ & $2+\vdots$ \\
\hline$\therefore-$. & $\therefore$ & $\therefore 0$ & $\therefore:$ & $\equiv$. & $=0.0$ & 17.1 & -4.3 & \\
\hline$\therefore \ldots$ & $\therefore$ & $\therefore 0$ & $\because \because=3$ & 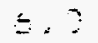 & 273.0 & 15.8 & $-4 . i$ & 9.2 \\
\hline$=: \cdots=$ & $\therefore \therefore$ & $\because \because$ & $\therefore .:$ & $\equiv .2$ & 2700 & 13.3 & -7.5 & \\
\hline$=\because \therefore-$ & $\therefore \because$ & $\because \because$ & $\because \because, \quad 3$ & $=.3$ & $\because 7 \leq .5$ & 22.2 & -4.4 & 9.2 \\
\hline$=:: 2:$ & $\Xi \because 2$ & $=\therefore$ & $=. \vdots 3$ & $\because 2$ & 705.9 & 24.7 & -4.7 & \\
\hline$=::=: 4$ & $=\because 8$ & $\therefore 2$ & $3^{-}=5$ & $4::$ & 503.0 & $=2.7$ & $-4 . \leq$ & 0.1 \\
\hline$=10 \%$ & $=0$ & $\because 7$ & $=.5$ & E. + & 278.0 & 15.5 & -4.5 & \\
\hline$=19294$ & $\therefore 0$ & 29 & $17:=3$ & $\doteq=4$ & 230.0 & 15.5 & -4.6 & 0.2 \\
\hline $7: 02: 2$ & $\because$ & $\therefore 8$ & 5.80 & $\therefore \Xi$ & 280.0 & 72.5 & -3.3 & \\
\hline $9: 02: 4$ & 70 & 200 & $17=03$ & 3.0 & 273.0 & 55.9 & -4.7 & 0.4 \\
\hline $7: 02: 1$ & 300 & $=00$ & $\leq 10$ & 1.0 & 104.0 & 17.0 & -3.9 & \\
\hline $7: 0254$ & $\equiv 20$ & $=00$ & 17.58 & 1.0 & 93.0 & 22.0 & -3.4 & 0.5 \\
\hline 10204 & $=20$ & 190 & $5 .: 0$ & 0.7 & 215.0 & 54.8 & 1.7 & \\
\hline$=1023 \%$ & $=20$ & 100 & $17: 53$ & 0.7 & $2=2.0$ & 40.5 & 1.2 & -0.5 \\
\hline $9: 02=4$ & $\because 200$ & $\therefore: 30$ & $b=10$ & 2.2 & $22 \leq .0$ & 22.9 & 2.3 & \\
\hline 810204 & 1000 & $: 50$ & $17=68$ & 2.6 & 232.0 & 20.6 & 2.4 & -0.4 \\
\hline 919204 & $1: 00$ & 200 & 5.10 & 4.7 & 250.0 & 11.0 & 2.4 & \\
\hline$=19204$ & $1:-0$ & .200 & 17.63 & $\Xi . \Xi$ & 255.0 & 10.6 & 2.2 & -0.2 \\
\hline 710204 & $1=00$ & $: 300$ & 5.10 & 7.4 & 247.0 & 9.7 & 1.4 & \\
\hline 71004 & 150 & 1300 & $17=53$ & 7.0 & 253.0 & 3.5 & 1.1 & -0.3 \\
\hline 710204 & 1300 & .400 & 5.10 & 6.4 & 251.0 & 11.9 & 1.6 & \\
\hline$=10254$ & 1300 & $\$ 200$ & $17 . \leq 3$ & 7.6 & 256.0 & 10.6 & 1.3 & -0.3 \\
\hline 910204 & 1400 & 1500 & 5.10 & 5.4 & 254.0 & 17.7 & 2.0 & \\
\hline 710204 & 1490 & 1000 & 17.53 & 5.3 & 258.0 & 16.2 & 1.3 & -0.2 \\
\hline 710204 & $1=00$ & 1600 & 6.10 & 5.8 & 252.0 & 1.1 & 1.7 & \\
\hline 710204 & $1=00$ & 1500 & $17=58$ & 7.0 & 257.0 & .9 & 1.5 & -0.2 \\
\hline 910204 & 1600 & 1700 & $s=10$ & 5.0 & 254.0 & 11.0 & 0.9 & \\
\hline 710204 & $1=00$ & 1700 & 17.58 & 6.1 & 253.0 & 7.2 & 1.0 & 0.1 \\
\hline$=10204$ & 1700 & 2300 & 5.10 & 4.8 & 273.0 & 12.1 & $-1 \cdot 3$ & \\
\hline 710204 & 1700 & 1000 & 17.68 & 5.9 & 273.0 & 10.6 & -0.6 & 0.7 \\
\hline 710204 & 1000 & 1700 & 6.10 & 2.7 & 286.0 & 14.4 & -1.9 & \\
\hline 710204 & 1300 & 1900 & 17.68 & 3.5 & 231.0 & 12.2 & $-1 \cdot 3$ & 0.6 \\
\hline 710204 & 1900 & 2000 & 6.10 & 1.4 & 323.0 & 28.7 & -3.1 & \\
\hline 910204 & 1900 & 2000 & 17.68 & 2.1 & 299.0 & 24.3 & -2.2 & 0.9 \\
\hline 910204 & 2000 & 2100 & 6.10 & 0.7 & 338.0 & 33.8 & -4.9 & \\
\hline 910204 & 2000 & 2100 & 17.68 & 1.1 & 305.0 & .1 & -3.4 & 1.5 \\
\hline 910204 & 2100 & 2200 & 6.10 & 0.5 & 18.0 & .9 & -7.1 & \\
\hline 910204 & 2100 & 2200 & 17.58 & 0.6 & 0.0 & 56.9 & -5.0 & 2.1 \\
\hline 910204 & 2200 & 2300 & 6.10 & 0.5 & 40.0 & 48.8 & -8.5 & \\
\hline 710204 & 2200 & 2300 & 17.68 & 0.6 & 83.0 & 50.0 & -6.9 & 1.6 \\
\hline $\begin{array}{l}910204 \\
910204\end{array}$ & 2300 & 0 & 6.10 & 0.5 & 44.0 & .6 & -9.2 & \\
\hline & 2300 & 0 & 17.68 & 0.6 & 51.0 & .5 & -6.9 & 2.3 \\
\hline
\end{tabular}




\begin{tabular}{|c|c|c|c|c|c|c|c|c|}
\hline$\therefore-\equiv$ & $\begin{array}{l}-\because \Omega E \\
\equiv-\Sigma T\end{array}$ & $\begin{array}{l}\because \cdots \\
\vdots \div 85\end{array}$ & $\begin{array}{c}2 E= \\
47 \\
\text { A } 25-1\end{array}$ & $\begin{array}{l}4:: 2 \\
\Xi F E \equiv 0 \\
n: \vdots\end{array}$ & 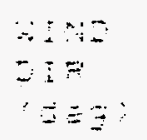 & 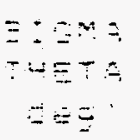 & 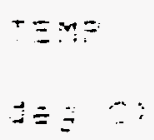 & 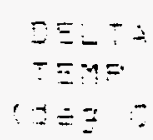 \\
\hline$\because \because z$ & $=$ & $\because 3$ & $\doteq: \therefore$ & $\therefore: \vdots$ & $=z \because z$ & $=4:$ & $-z,-$ & \\
\hline$\therefore=$ & $\because$ & $\therefore 0$ & $\because . \leq 3$ & $\therefore .9$ & 202.2 & $: 9$. & $-\vdots-$ & $\therefore 2$ \\
\hline$\cdots=$ & $\therefore \because$ & $\because: 2$ & $=. \therefore$ & $\approx \therefore$ & $\because \because \therefore$ & $2: 2$ & $-\vdots . ?$ & \\
\hline$\therefore=$ & $\therefore$ & $\therefore$ & $\therefore:=\Sigma$ & $\therefore 3$ & $\div 95$ & $=\div .=$ & -5 & $\therefore 5$ \\
\hline$\therefore$ & $\therefore:$ & $\because \vdots$ & $=\ldots$ & $\therefore$ : : & 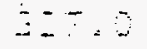 & $-\frac{3}{4}, 3$ & $-: .4$ & \\
\hline$\therefore:$ & $\therefore$ & -8 & $\therefore \quad=3$ & $\therefore \%$ & $\overline{-1} \because \overline{\mathrm{O}}$ & $\because 9 . \vdots$ & $-9=$ & $\therefore .5$ \\
\hline$\therefore$ & $\because \therefore$ & -3 & $=\therefore$ & $\therefore=$ & 854.2 & $=2 .=$ & -2.5 & \\
\hline$\because \cdots$ & $=\because$ & $\therefore:$ & $\therefore-=\vdots$ & $\therefore$ & $2-5.2$ & +2. & $-1=5$ & $\therefore .7$ \\
\hline$\because 2=$ & $\therefore: 3$ & $\therefore: 2$ & 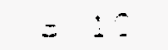 & $\therefore 8$ & 30.0 & $=2.5$ & -5.5 & \\
\hline$=$ & $\div \because$ & 8 & $1-=0$ & $\therefore .2$ & 9.0 & 50.3 & $-2,3$ & $\therefore .2$ \\
\hline 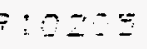 & $\Xi: 0$ & $=?$ & $=25$ & $\therefore= \pm$ & 8.0 & $\equiv i=8$ & $-5,7$ & \\
\hline$\approx=$ & $\Xi: 2$ & $=\Omega$ & $: \because:=3$ & $\therefore$ & $Z=5.0$ & $\equiv 0 . \Xi$ & $-\therefore .1$ & 2.6 \\
\hline $2 \div$ & $=: 0$ & $\because 8$ & \pm .10 & 0.8 & 40.0 & $\Xi 7.5$ & -5.7 & \\
\hline 6 & $=: 0$ & $\because 0$ & $17=0$ & 0.7 & $\because 3.0$ & 58.7 & -2.4 & 3.5 \\
\hline$\because=$ & $\because: 8$ & $=00$ & $=.: 0$ & 0.8 & 75.0 & 38.8 & -5.2 & \\
\hline$=$ & $-\because 0$ & $=90$ & $17 . \leq 9$ & 1.5 & $= \pm .0$ & 45.4 & -1.7 & 3.5 \\
\hline$=$ & $\equiv 20$ & $=09$ & $=.0$ & $\therefore .1$ & 237.0 & 91.3 & 2.1 & \\
\hline$=$ & 30 & 900 & $17:=3$ & $2 . \overline{7}$ & 2350 & 54.7 & 2.7 & 0.5 \\
\hline & $=-8$ & $: 00$ & $3 .: 0$ & 4.5 & 247.5 & 15.5 & 4.4 & \\
\hline 5 & $=50$ & $: 20$ & $i 7,63$ & $\Xi .5$ & $25-6$ & 14.6 & 4.3 & -0.1 \\
\hline & $1: 5$ & $: 200$ & 0.10 & 6.3 & $2=3.0$ & 11.4 & 5.3 & \\
\hline & $1 \div 0$ & 100 & 17.53 & 7.5 & 239.0 & 10.0 & $\Xi .2$ & -0.1 \\
\hline & $1: 00$ & 100 & $\Delta .10$ & 7.1 & $2=3.0$ & 11.2 & 5.0 & \\
\hline & $1: 00$ & $: 200$ & $17=03$ & 3.5 & 258.0 & 10.0 & 5.9 & -0.1 \\
\hline & $1 \div 0$ & $=200$ & 5.10 & 7.8 & 251.0 & 7.1 & 6.4 & \\
\hline 5 & $1=00$ & 1300 & 17.03 & 7.5 & 257.0 & 7.7 & 6.3 & -0.1 \\
\hline$\therefore$ & 1300 & 1400 & 0.10 & 8.0 & 259.0 & 9.7 & 6.5 & \\
\hline 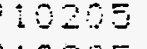 & 1200 & $1+00$ & 17.63 & 7.4 & 253.0 & S.6 & 5.4 & -0.1 \\
\hline $2=$ & 1400 & 1002 & 5.10 & 8.0 & 258.0 & 7.8 & 5.4 & \\
\hline$-1=$ & 140 & 1500 & $17=08$ & 7.5 & 263.0 & 3.2 & 6.4 & 0.0 \\
\hline$-6=$ & $1 \equiv 90$ & $=00$ & 0.10 & 5.7 & 253.0 & 10.2 & 0.6 & \\
\hline & $1=00$ & $1=00$ & 17.58 & 7.1 & 257.0 & 3.3 & $b . \dot{b}$ & 0.0 \\
\hline & 1500 & $: 700$ & 6.10 & 3.1 & 253.0 & 19.6 & 6.0 & \\
\hline & $1=00$ & 1700 & 17.58 & 3.9 & 264.0 & 16.5 & 6.1 & 0.1 \\
\hline & 1700 & 1800 & 0.10 & 0.3 & 334.0 & 65.1 & 0.7 & \\
\hline & 1700 & 1300 & 17.68 & 0.4 & 303.0 & 92.5 & 2.1 & 1.4 \\
\hline & 1300 & 1900 & 6.10 & 0.3 & 0.0 & 42.1 & -3.6 & \\
\hline & 1900 & 1900 & 17.68 & 0.4 & 314.0 & 74.8 & -2.3 & 1.3 \\
\hline & 1900 & 2000 & 6.10 & 0.3 & 289.0 & 38.3 & -5.6 & \\
\hline & 1700 & 2000 & 17.68 & 0.2 & 292.0 & 34.5 & -4.6 & 1.0 \\
\hline & 2000 & 2100 & 6.10 & 0.4 & 285.0 & 27.0 & -6.7 & \\
\hline & 2000 & 2100 & 17.68 & 0.3 & 282.0 & 23.2 & -5.6 & 1.3 \\
\hline & 2100 & 2200 & 6.10 & 0.5 & 284.0 & 23.5 & -7.3 & \\
\hline & 2100 & 2200 & 17.58 & 0.4 & 296.0 & 23.2 & -6.5 & 1.3 \\
\hline & 2200 & 2300 & 6.10 & 0.2 & 290.0 & 39.2 & -8.8 & \\
\hline & 2200 & 2300 & 17.68 & 0.3 & 291.0 & 15.5 & -7.2 & 1.6 \\
\hline & 2300 & 0 & 6.10 & 0.3 & 285.0 & 20.5 & -9.5 & \\
\hline & 2300 & 0 & 17.68 & 0.2 & 97.0 & 21.8 & -8.1 & 1.4 \\
\hline
\end{tabular}


$\equiv:-\equiv---2 \cdot=$

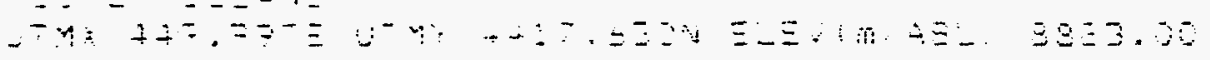

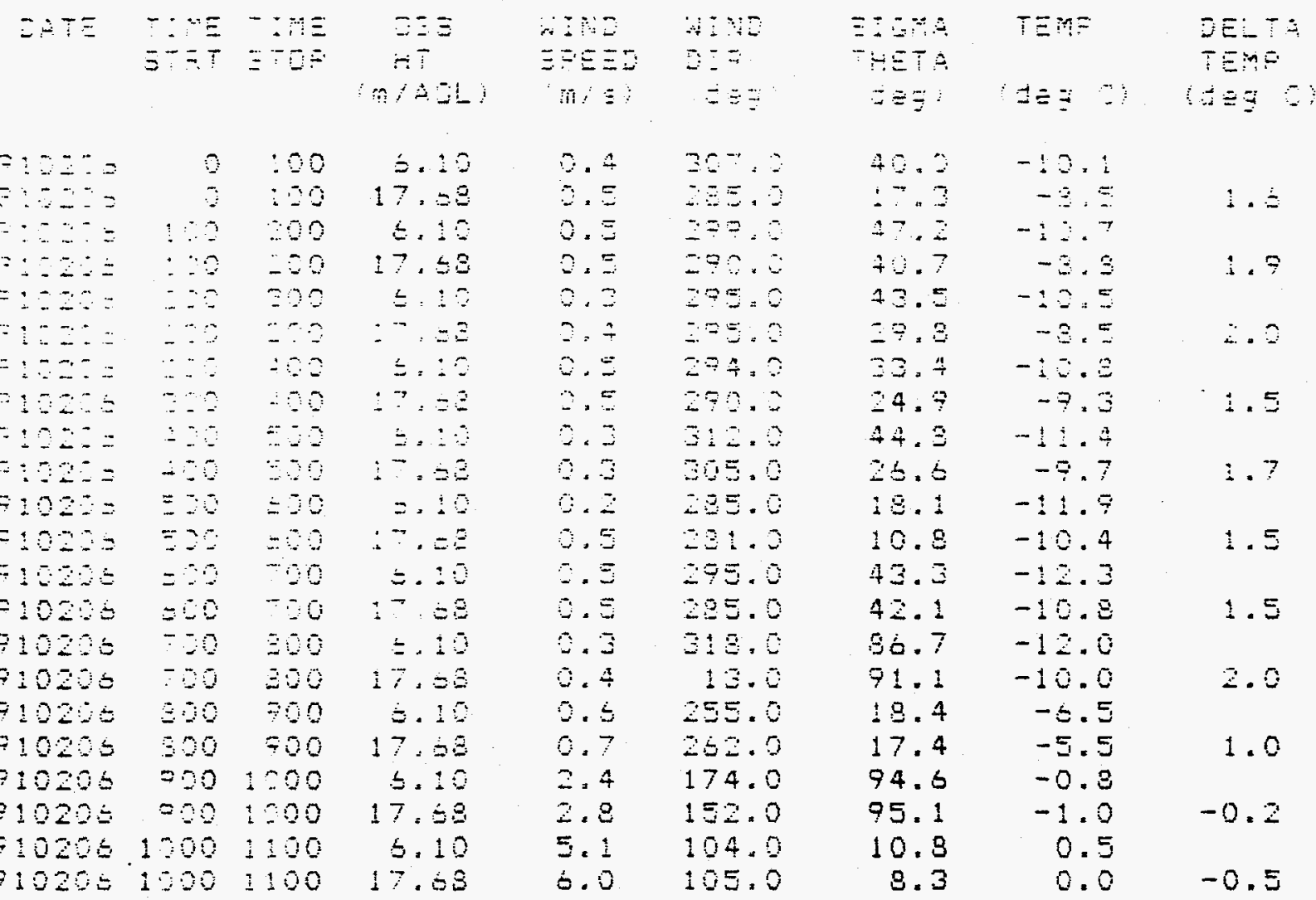




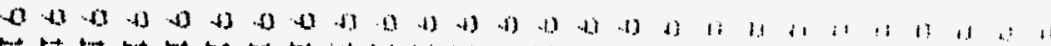

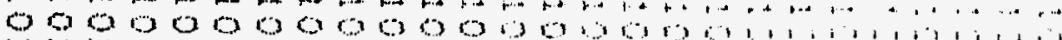

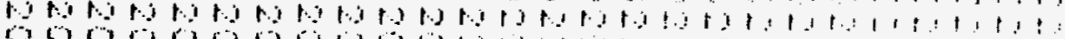

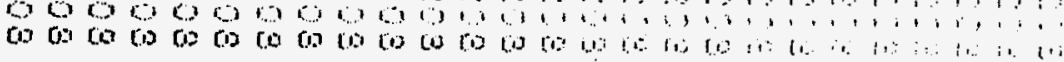

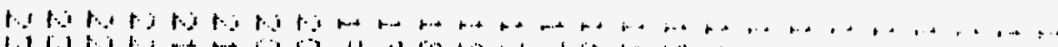

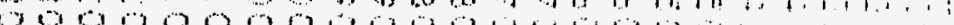

0000000000

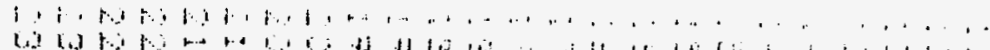

3.0.

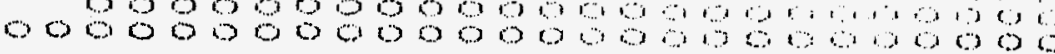

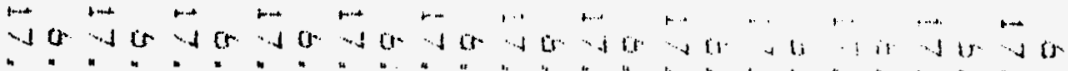

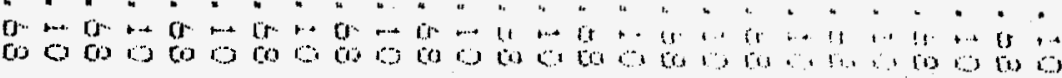

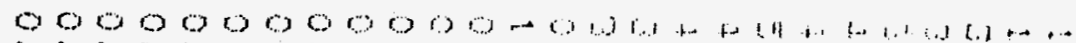

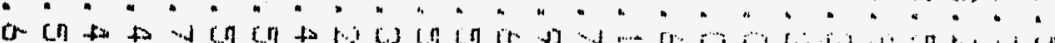

(a) [al $1 \%$ tas

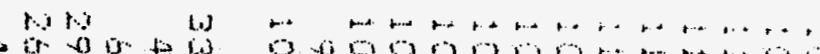

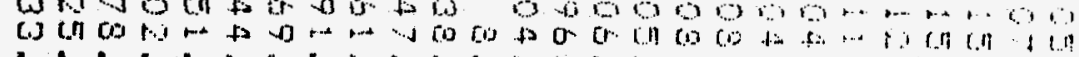

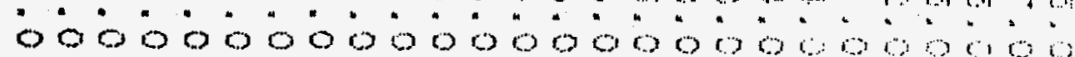

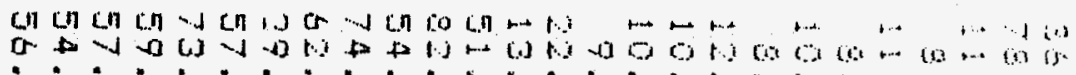

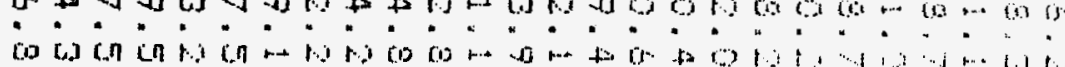

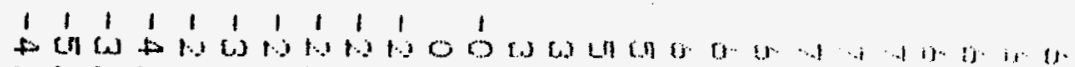
a

i.

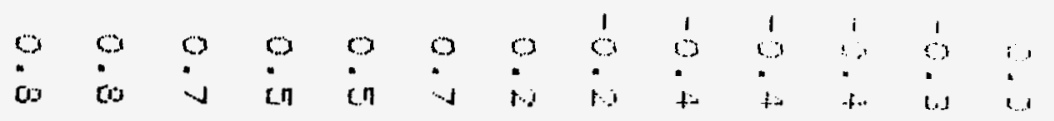

i1. $\cdots+1$ it i1 


\section{APPENDIX D}


LLNL Tower, LINDAS RANCH Jan, 1991

$476.30 \mathrm{~km} \mathrm{E}, 4413.98 \mathrm{~km} \mathrm{~N}$, Zone 13; Elev. $2003.8 \mathrm{~m} \mathrm{MSL}$
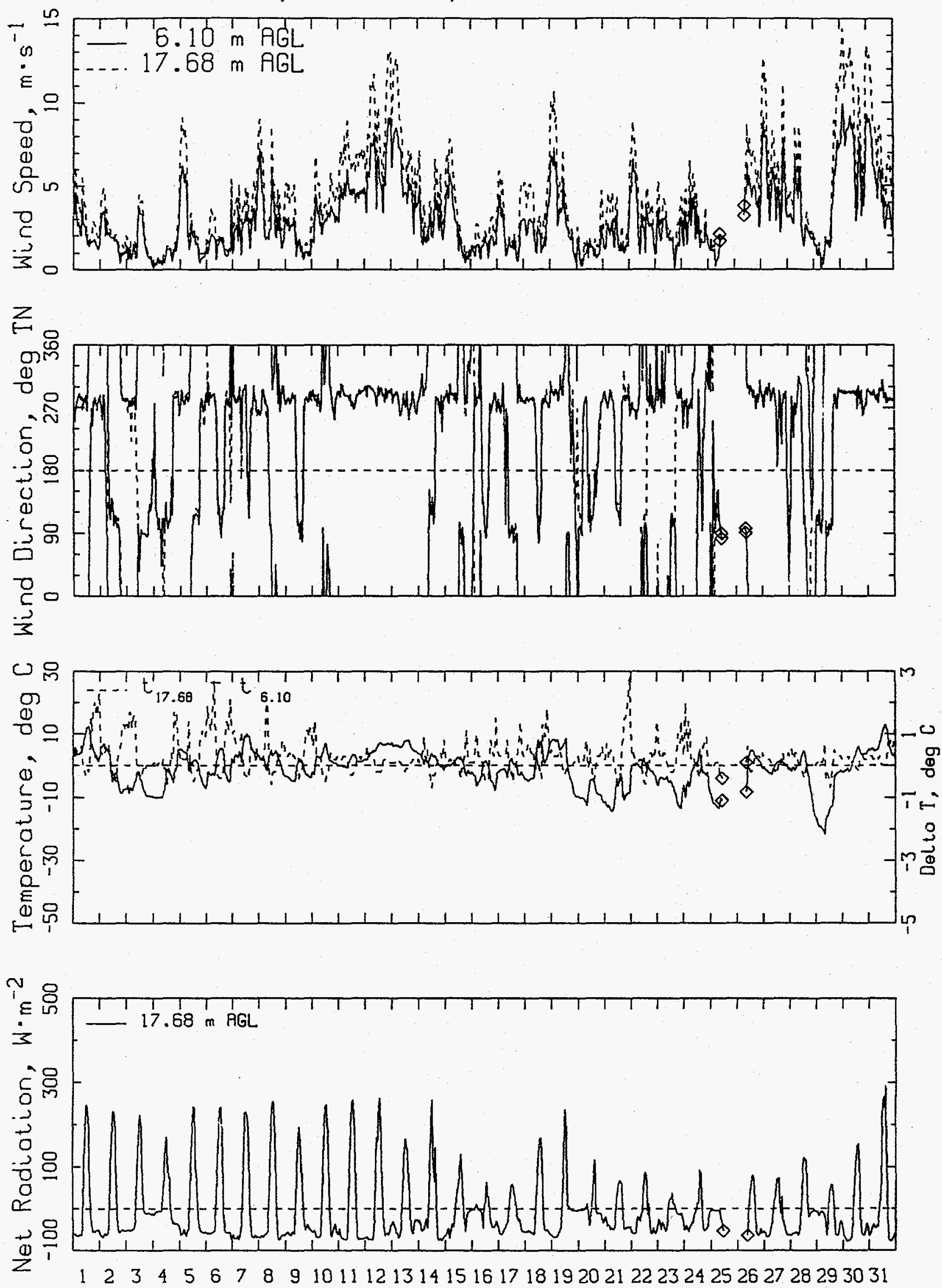

Day of Month

Rftowers $07 / 29 / 91$ 


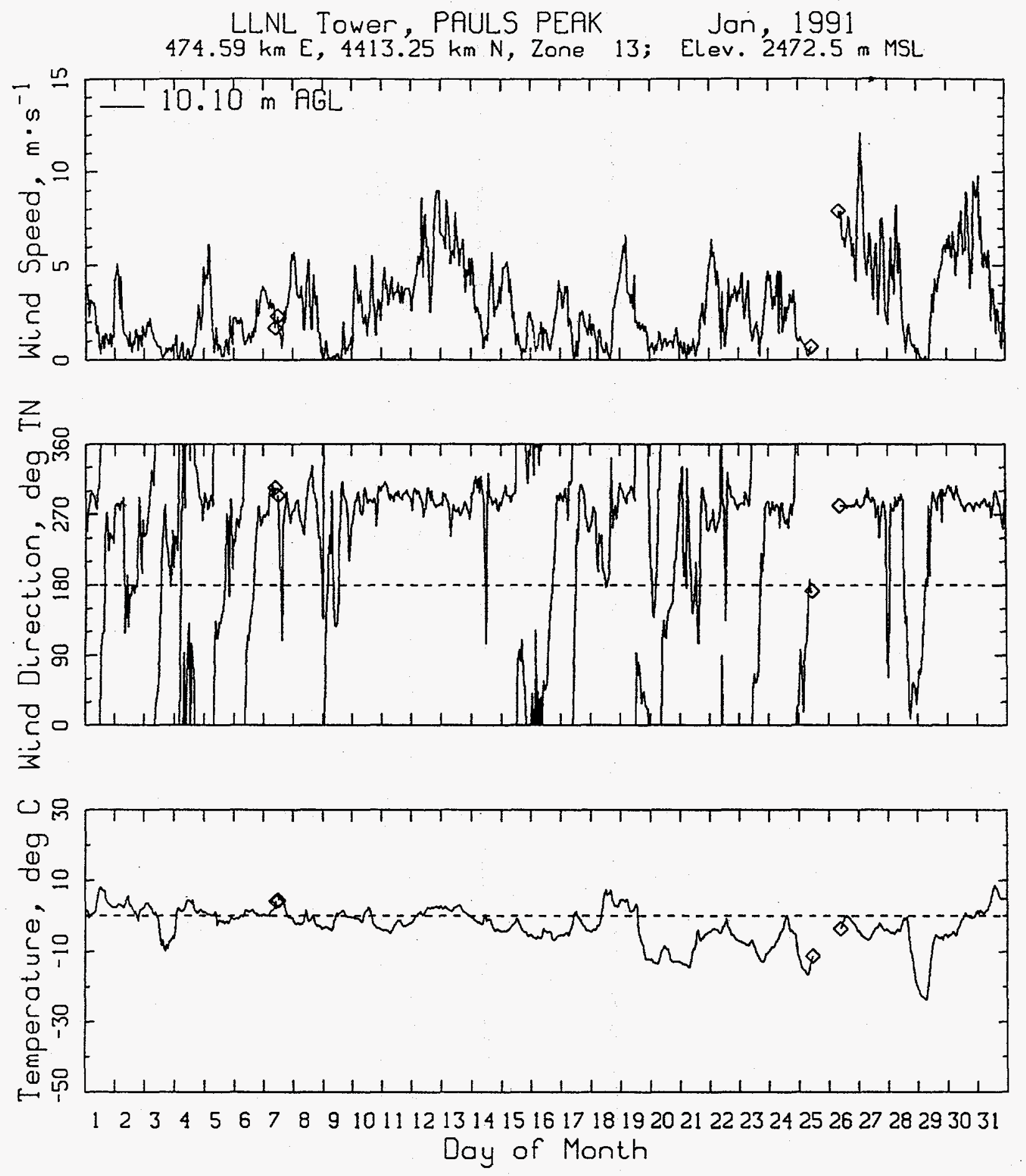

Rrtowers $07 / 29 / 91$ 
LLNL Tower, TOLLAND Jan, 1991 $450.00 \mathrm{~km} \mathrm{E}, 4417.63 \mathrm{~km} \mathrm{~N}$, Zone 13; Elev. $2707.5 \mathrm{~m} \mathrm{MSL}$
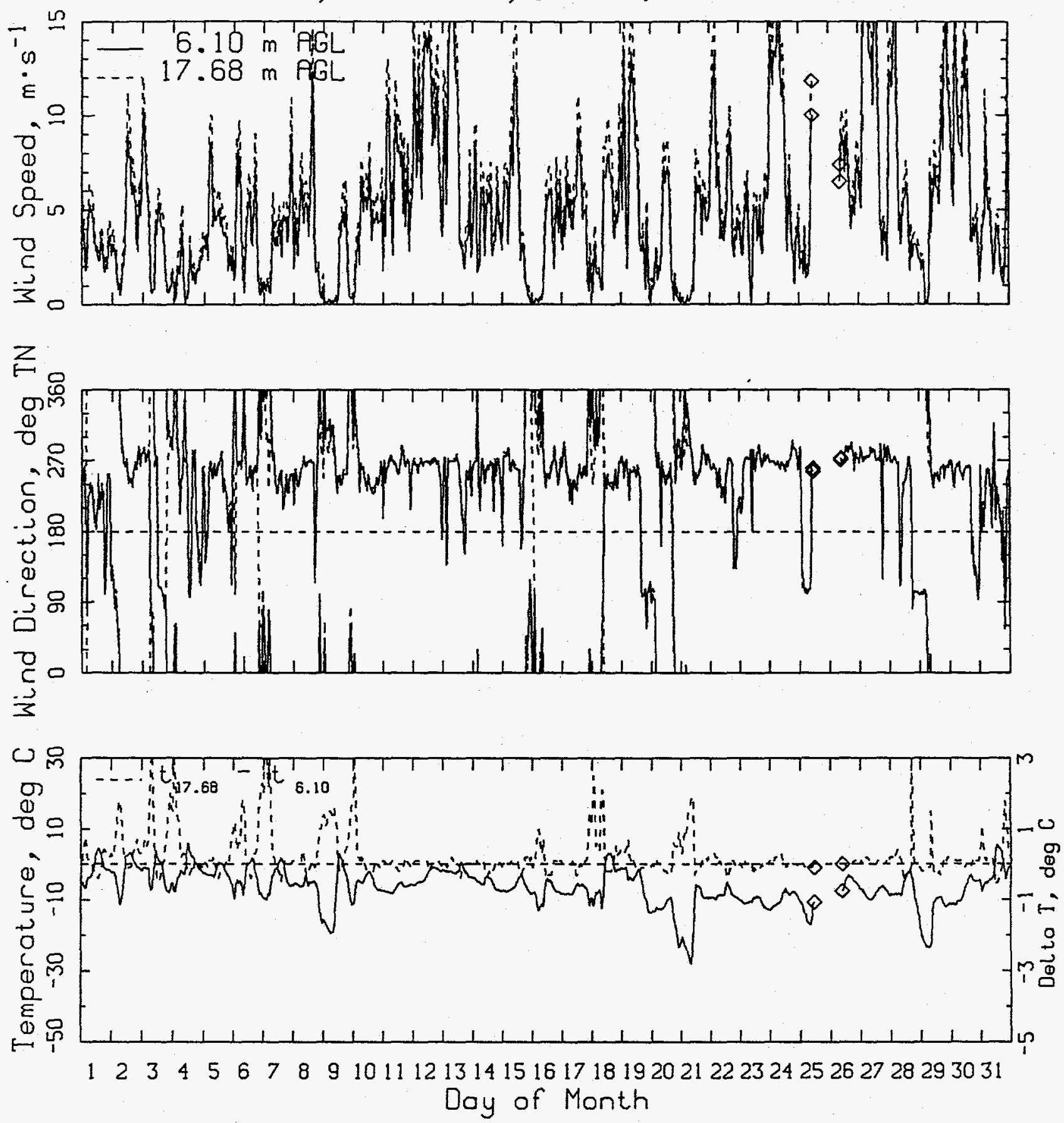
LLNL Tower, RIM ROCK Jan, 1991

$470.44 \mathrm{~km} \mathrm{E}, 4416.92 \mathrm{~km} \mathrm{~N}$, Zone 13; Elev. $2330.0 \mathrm{~m} \mathrm{MSL}$
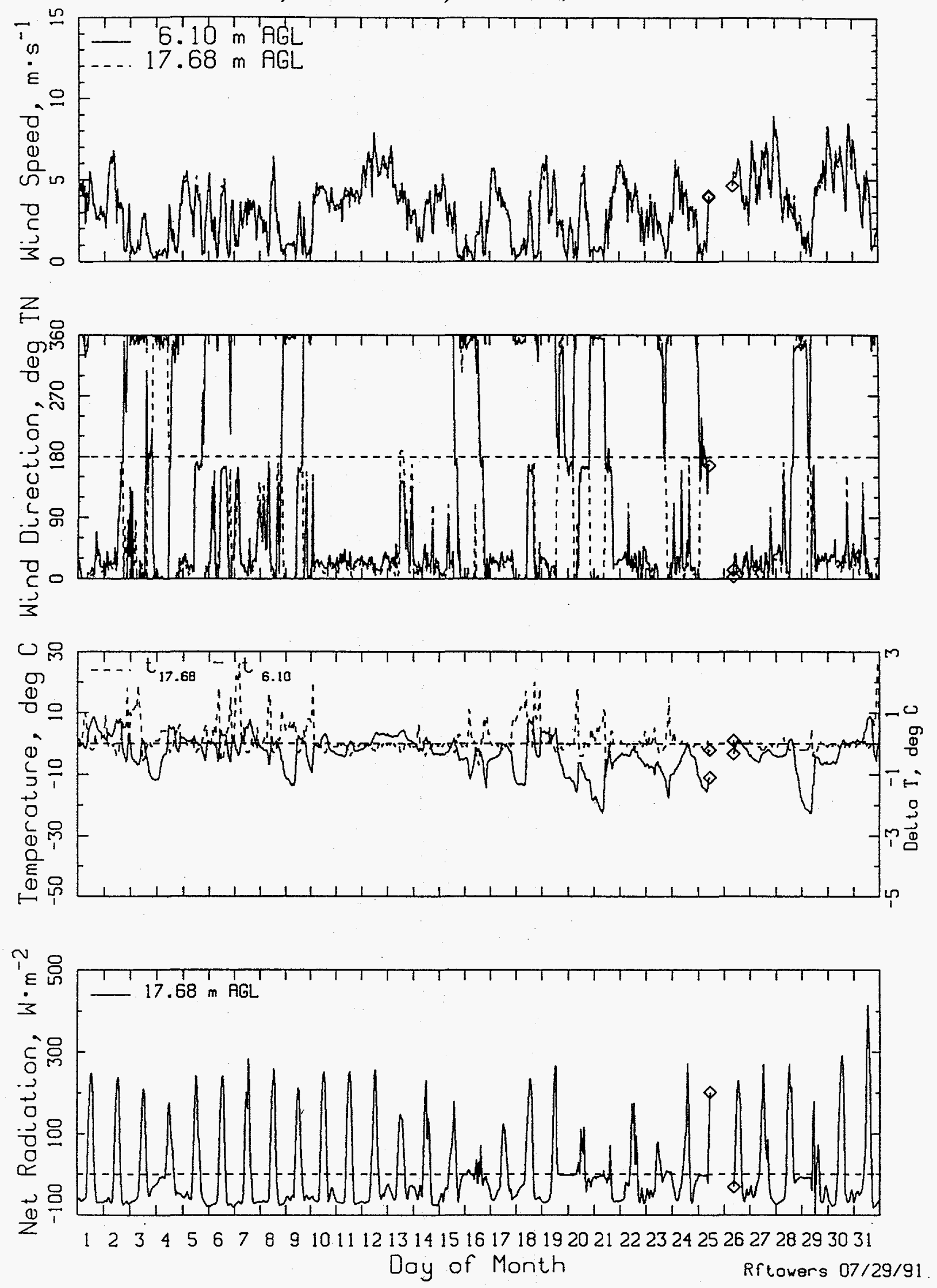

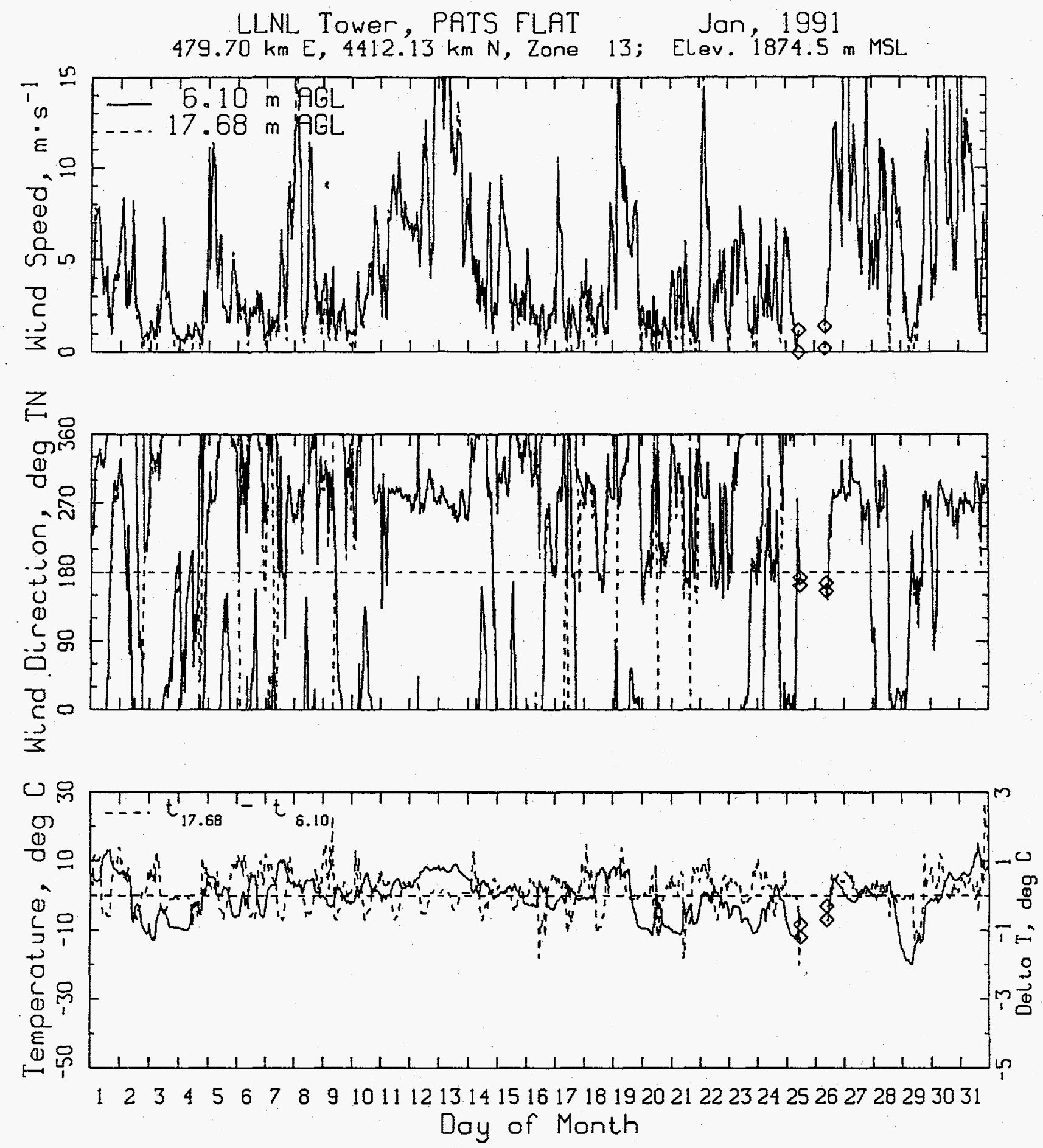
LLNL Tower, LOIS RANCH Jan, 1991

$477.02 \mathrm{~km} \mathrm{E}, 4415.37 \mathrm{~km} \mathrm{~N}$, Zone 13; Elev. $2095.5 \mathrm{~m} \mathrm{MSL}$
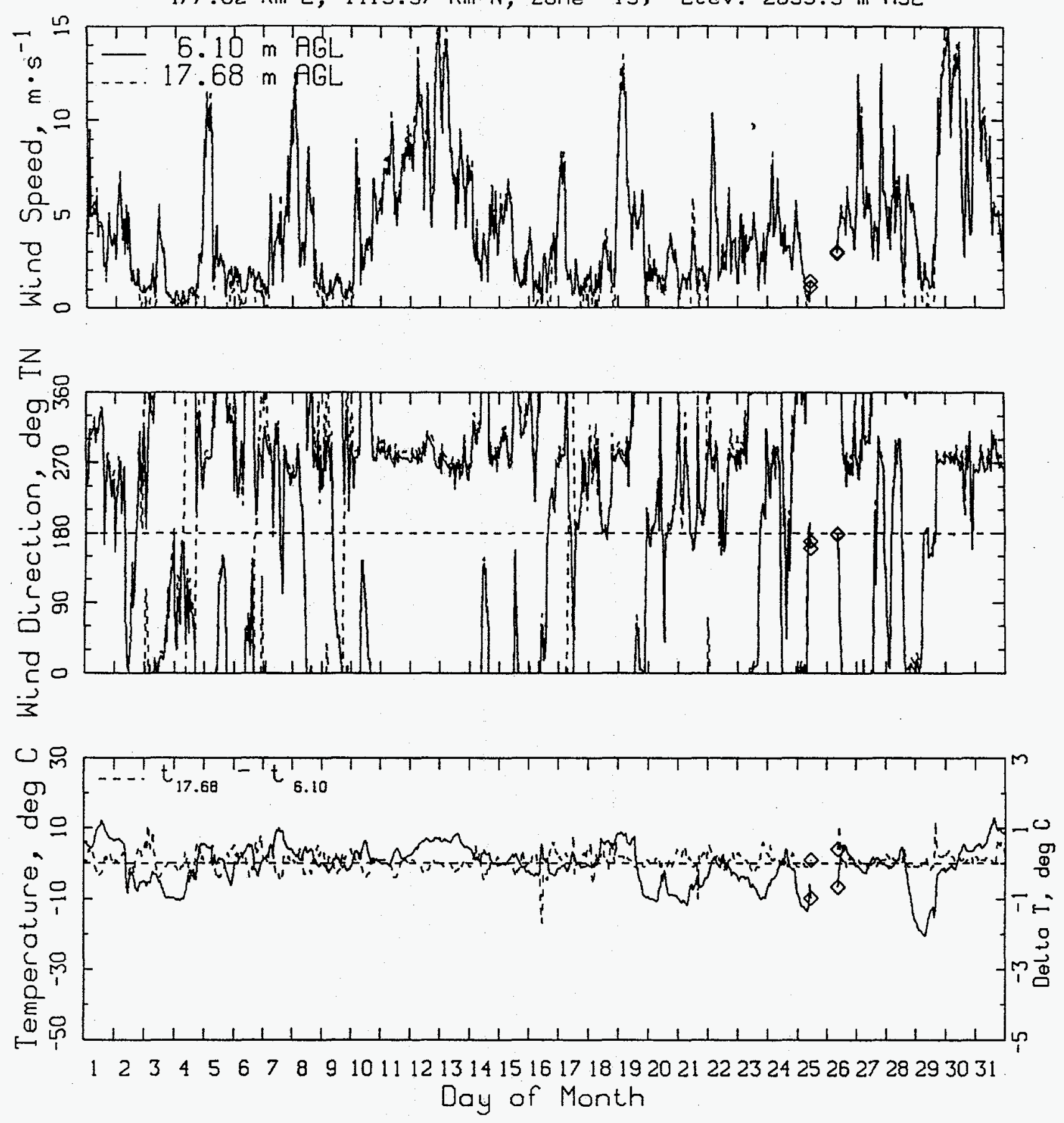
LLNL Tower, JIMS RANCH Jan, 1991 $473.21 \mathrm{~km} \mathrm{E}, 4416.54 \mathrm{~km} \mathrm{~N}$, Zone 13; Elev. 2029.4 m MSL
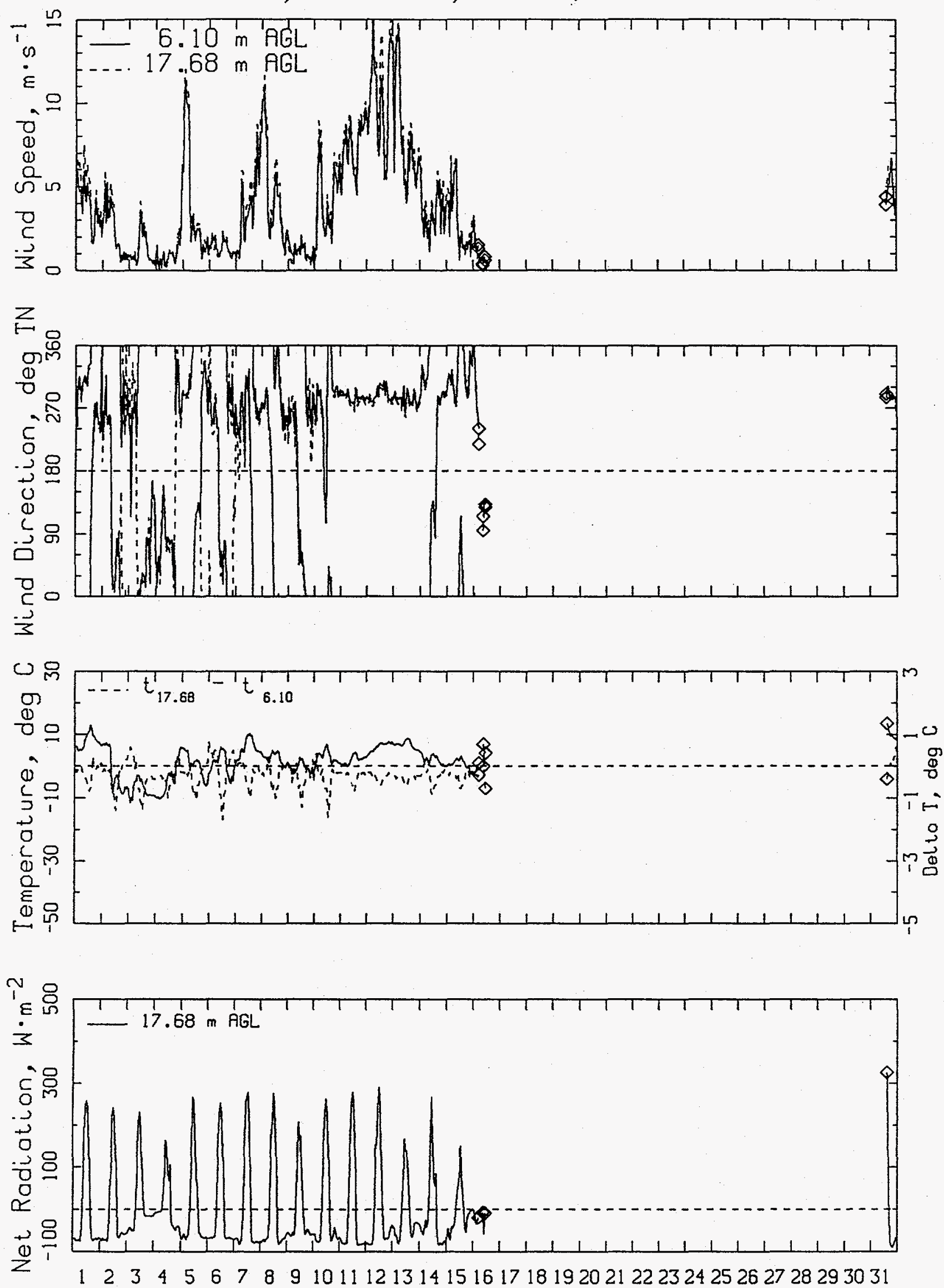
Doy of Month 

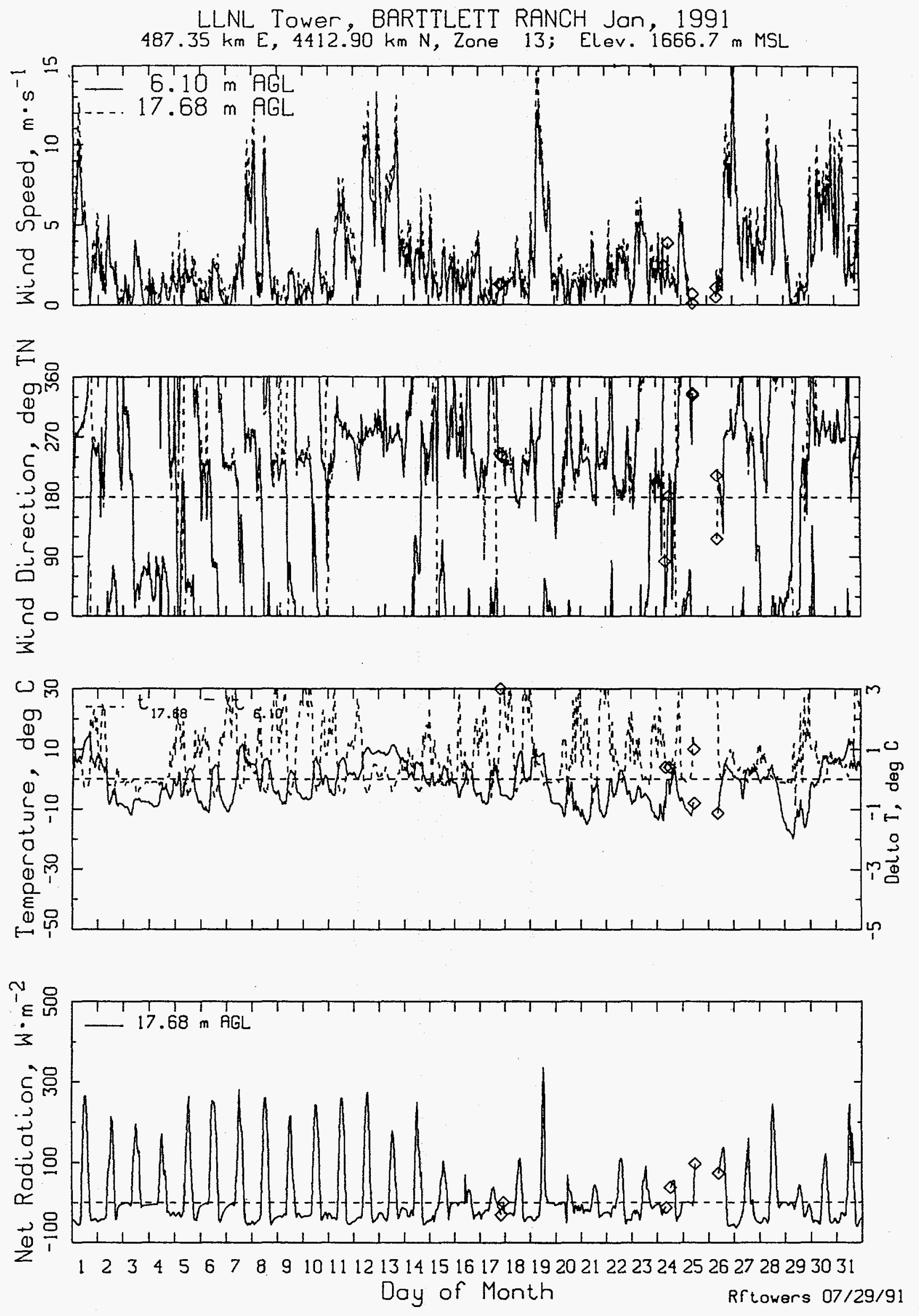
LLNL Tower, LINDAS RANCH Feb, 1991

$476.30 \mathrm{~km} \mathrm{E}, 4413.98 \mathrm{~km} \mathrm{~N}$, Zona 13; Elev. $2003.8 \mathrm{~m} \mathrm{MSL}$
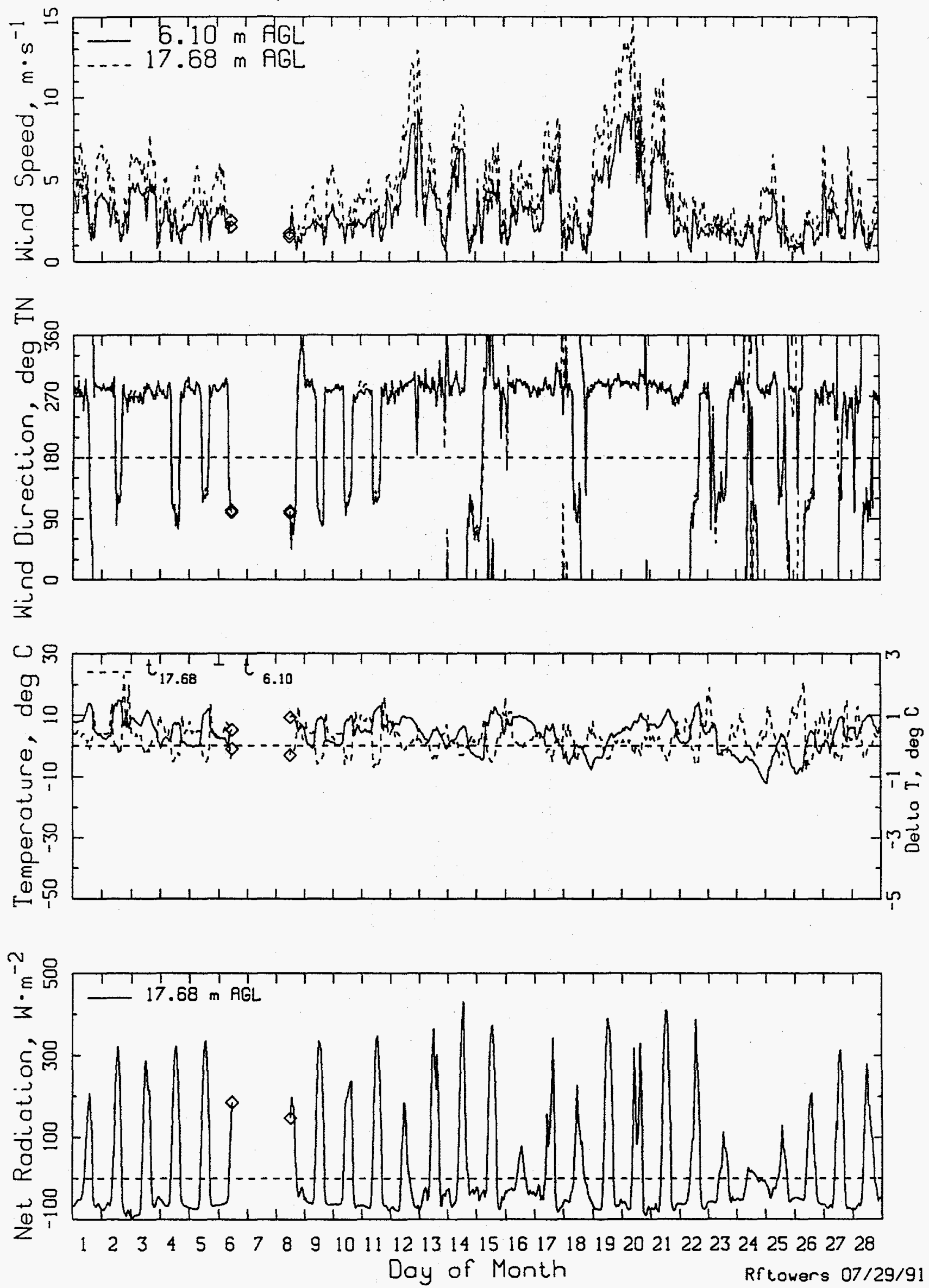
LLNL Tower, PAULS PEAK Feb, 1991

$474.59 \mathrm{kmE}, 4413.25 \mathrm{~km} \mathrm{~N}$, Zone 13; Elev. $2472.5 \mathrm{~m}$ MSL
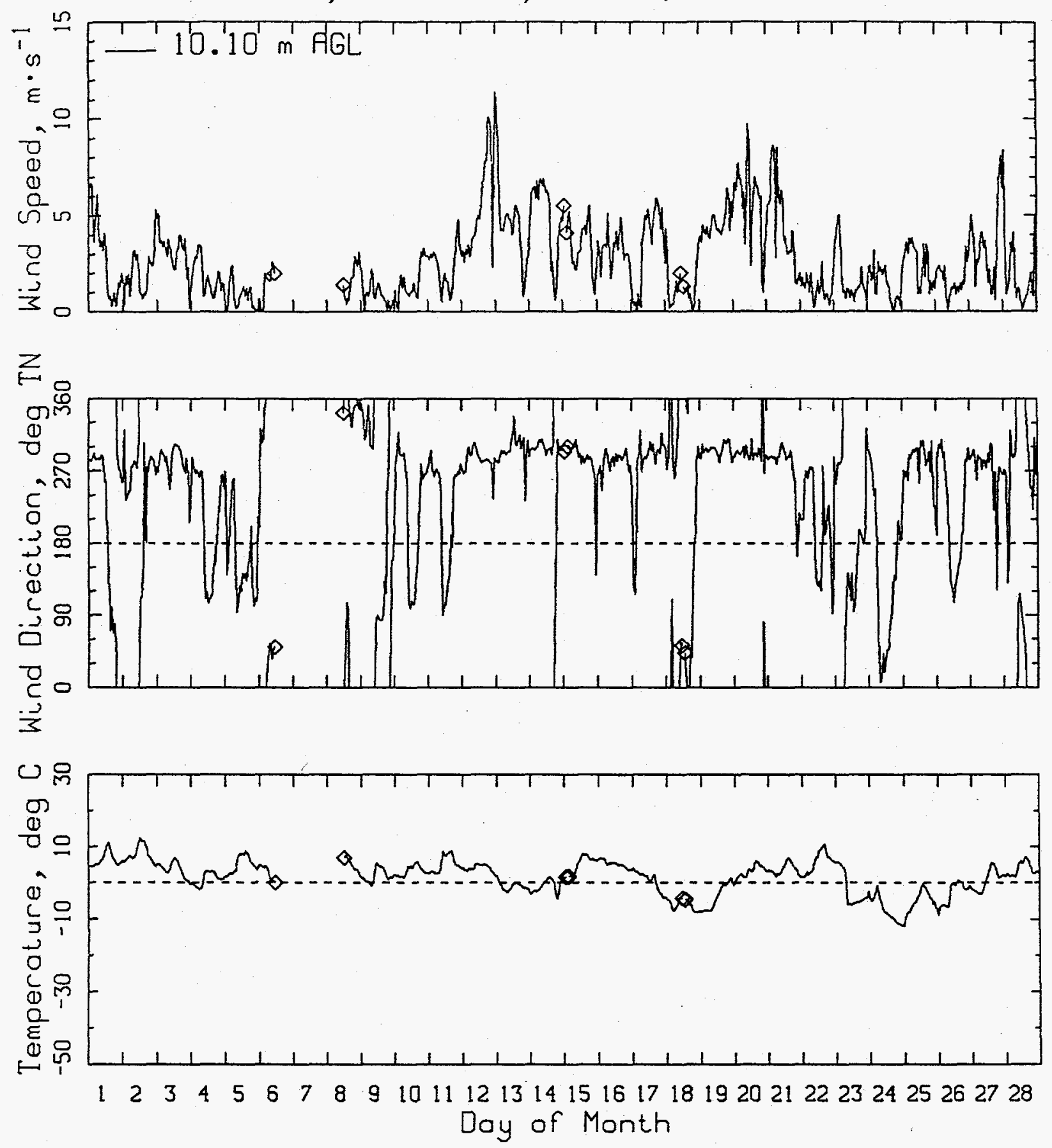
LLNL Tower, TOLLAND
$450.00 \mathrm{kmE}, 4417.63 \mathrm{~km} \mathrm{~N}$, Zone 13; Elev. $2707.5 \mathrm{~m} \mathrm{MSA}$
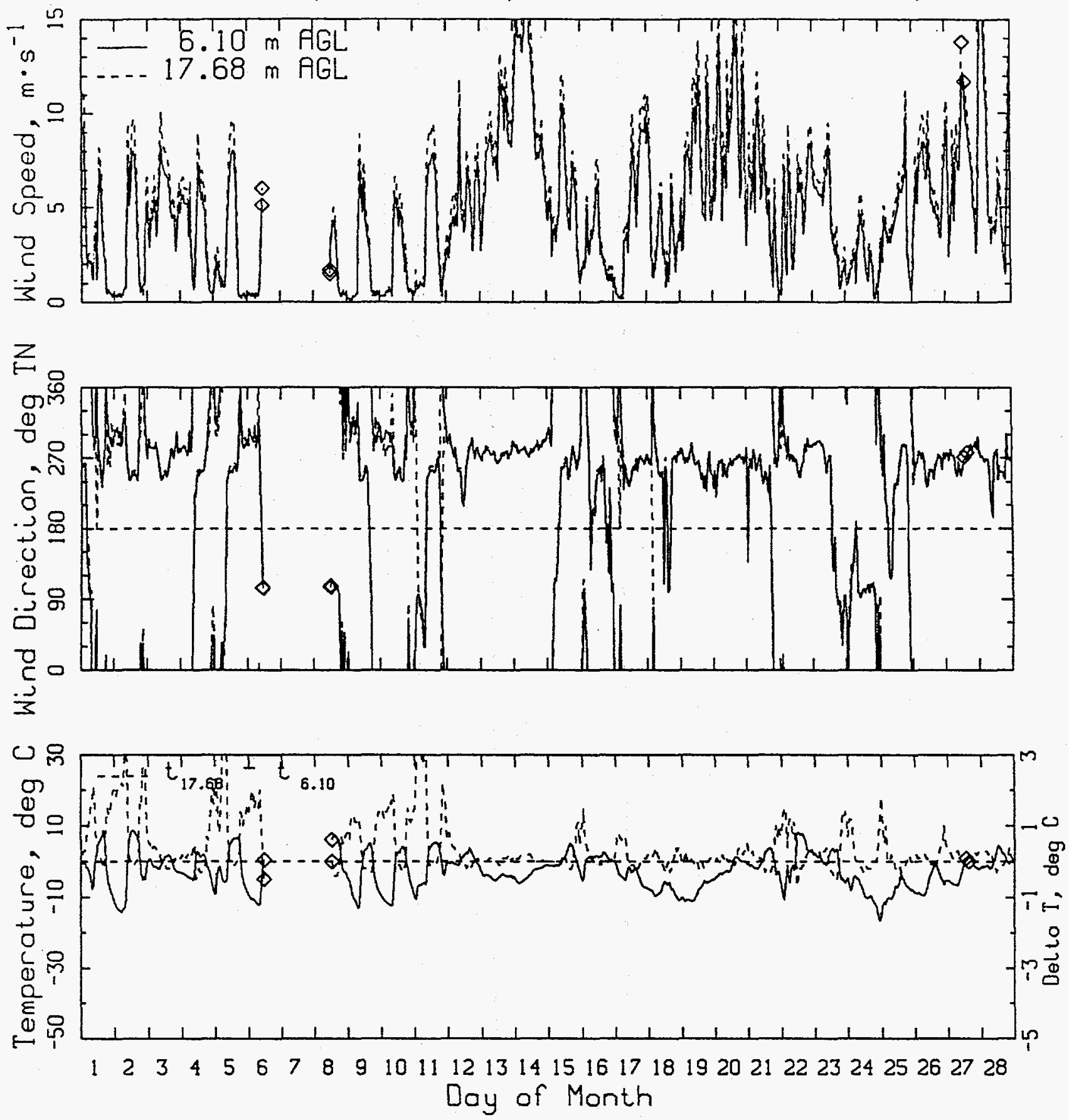
LLNL Tower, RIM ROCK Feb, 1991

$470.44 \mathrm{~km}$ E, $4416.92 \mathrm{~km} \mathrm{~N}$, Zone 13; Elev. $2330.0 \mathrm{~m} \mathrm{MSL}$
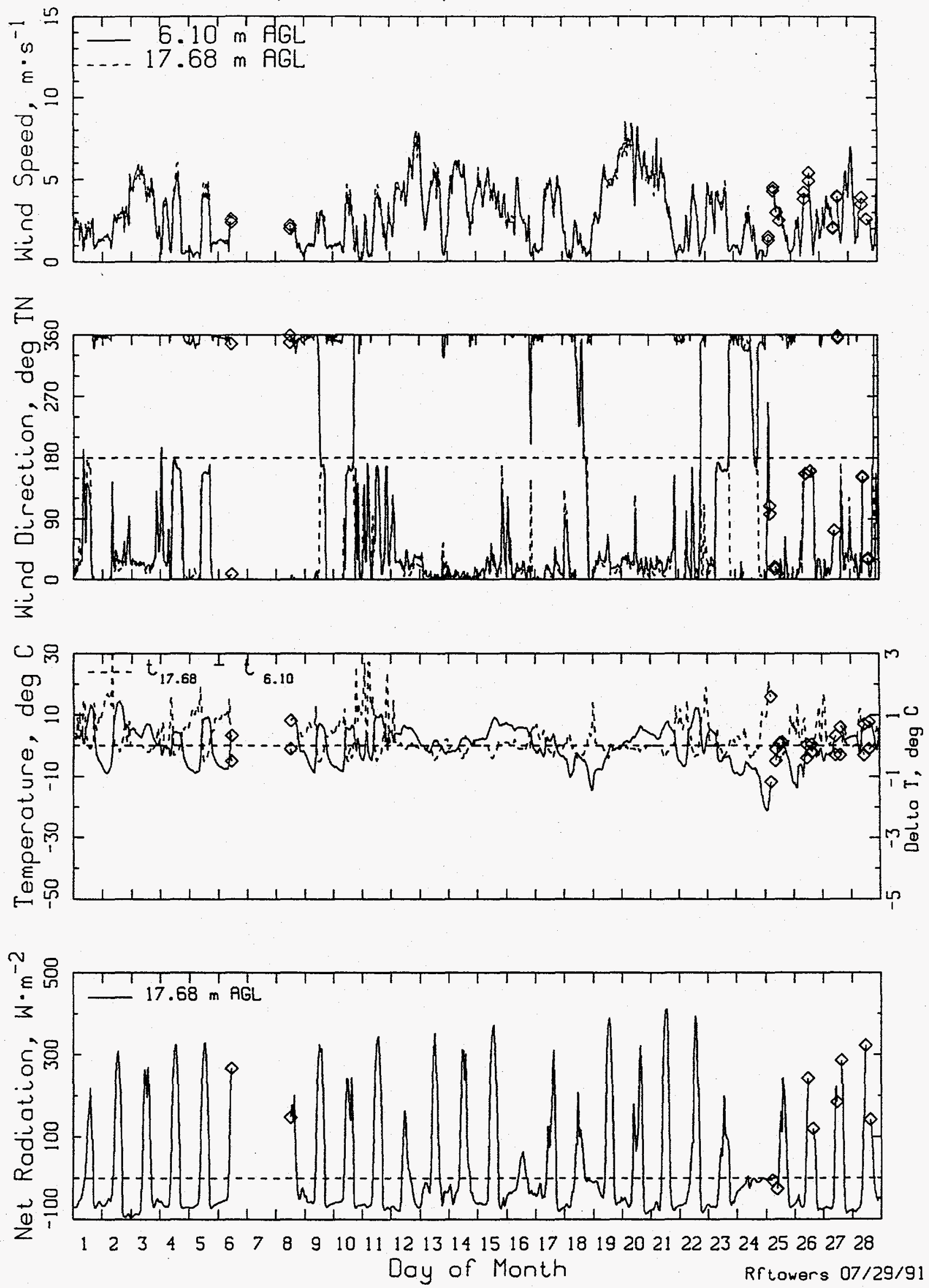


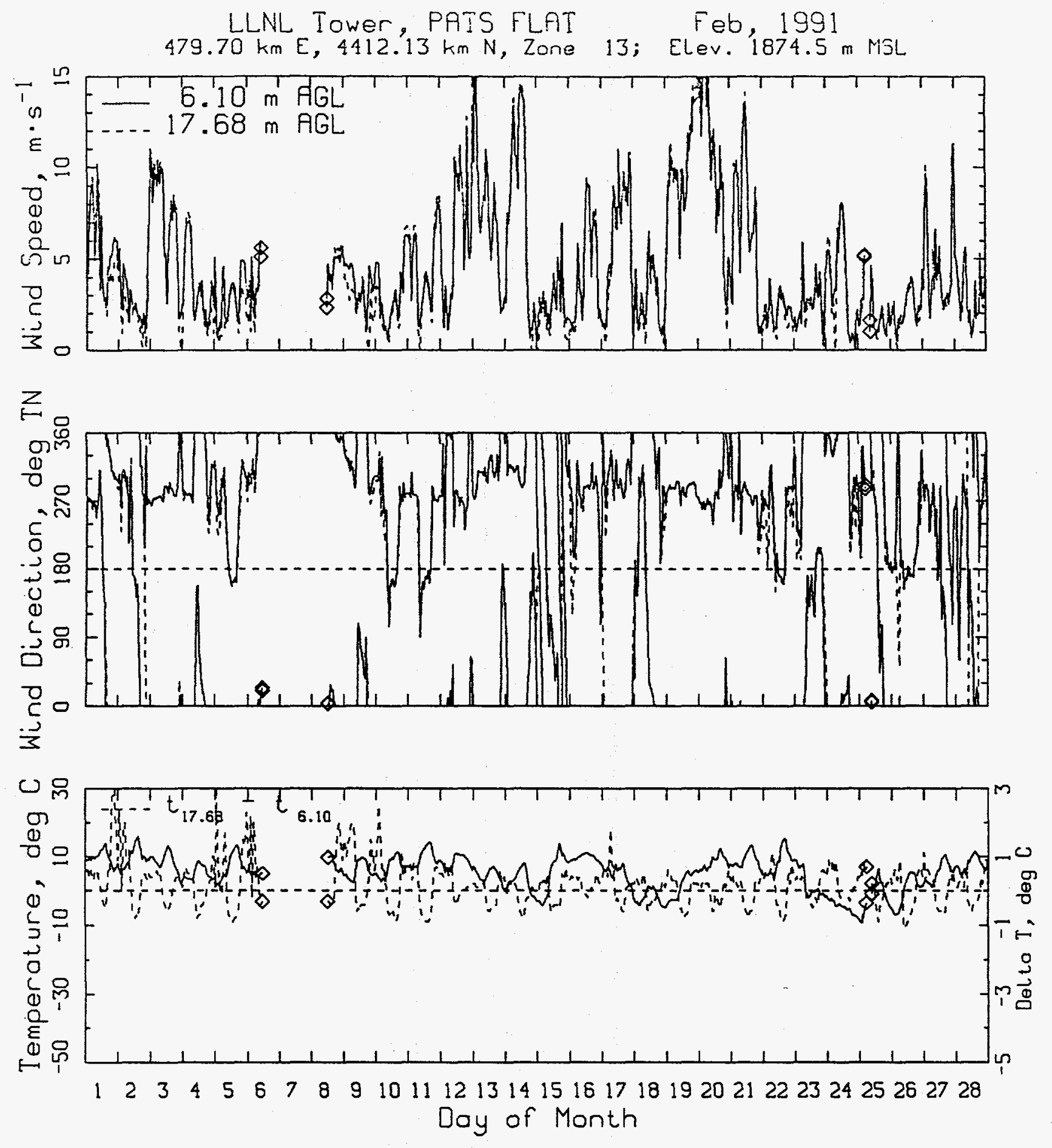



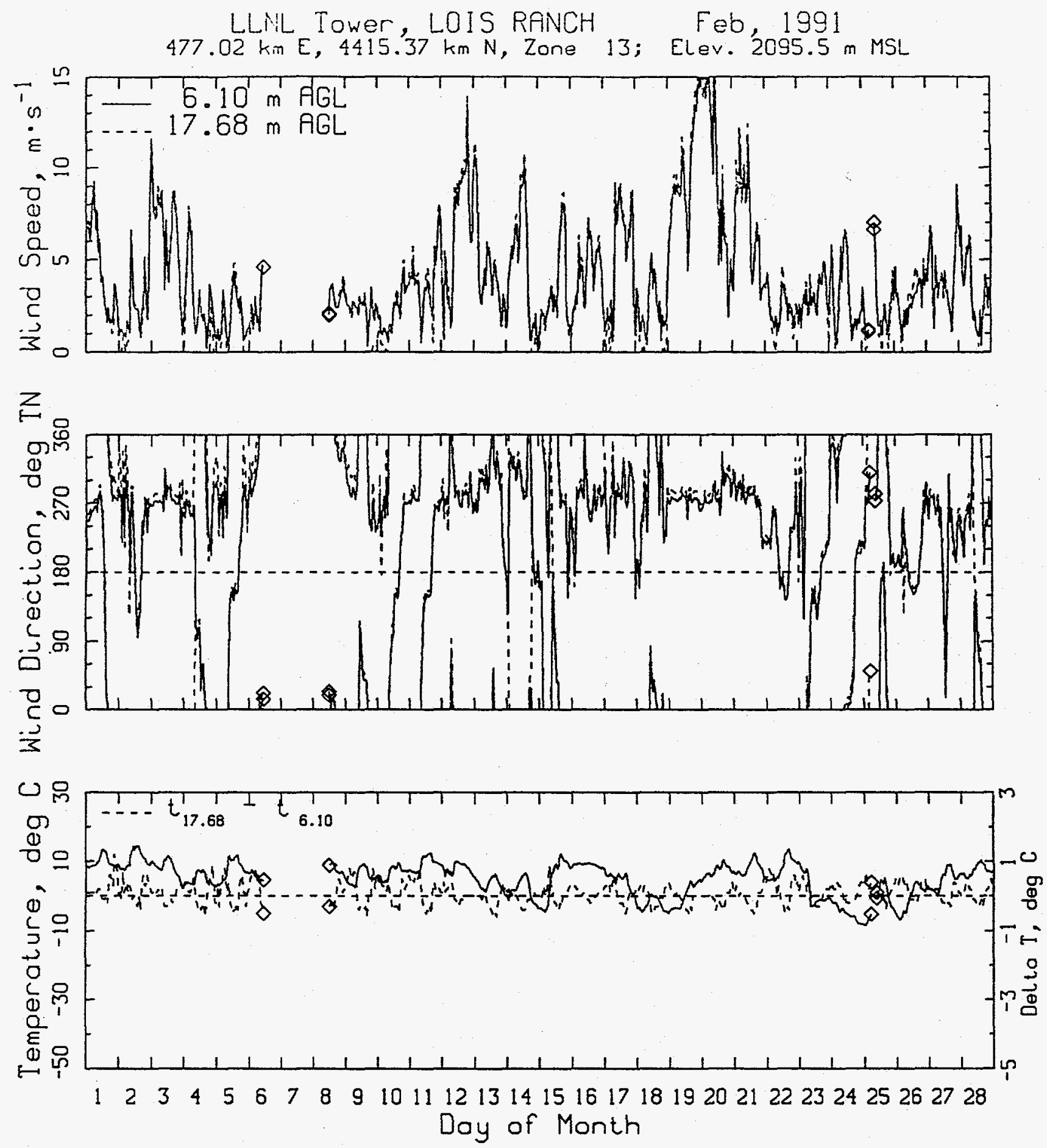
LLNL Tower, JIMS RANCH Fe', 1991

$473.21 \mathrm{~km} \mathrm{E}, 4416.54 \mathrm{~km} \mathrm{~N}$, Zone 13; Elev. $2029.4 \mathrm{~m} \mathrm{MSL}$
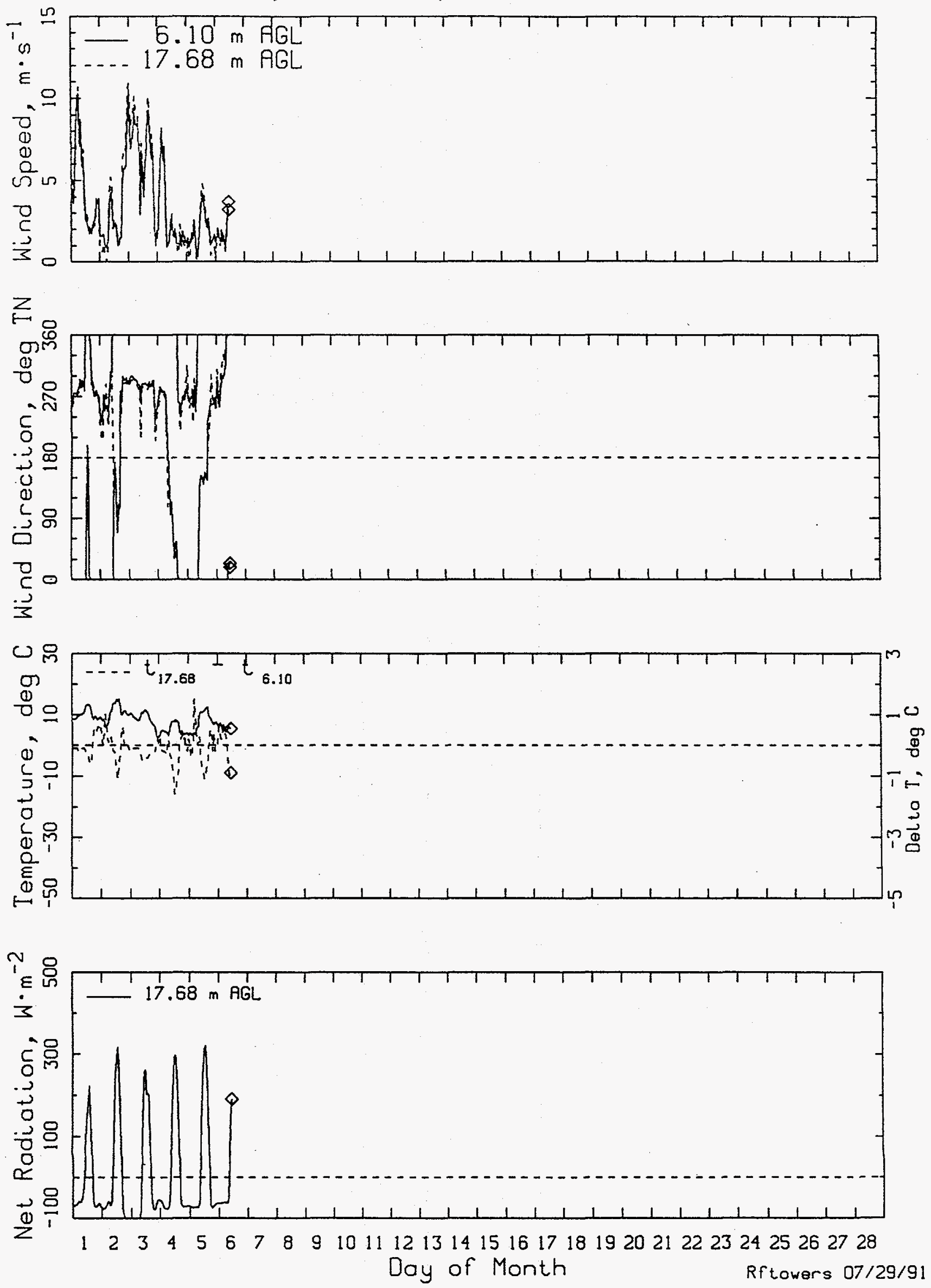

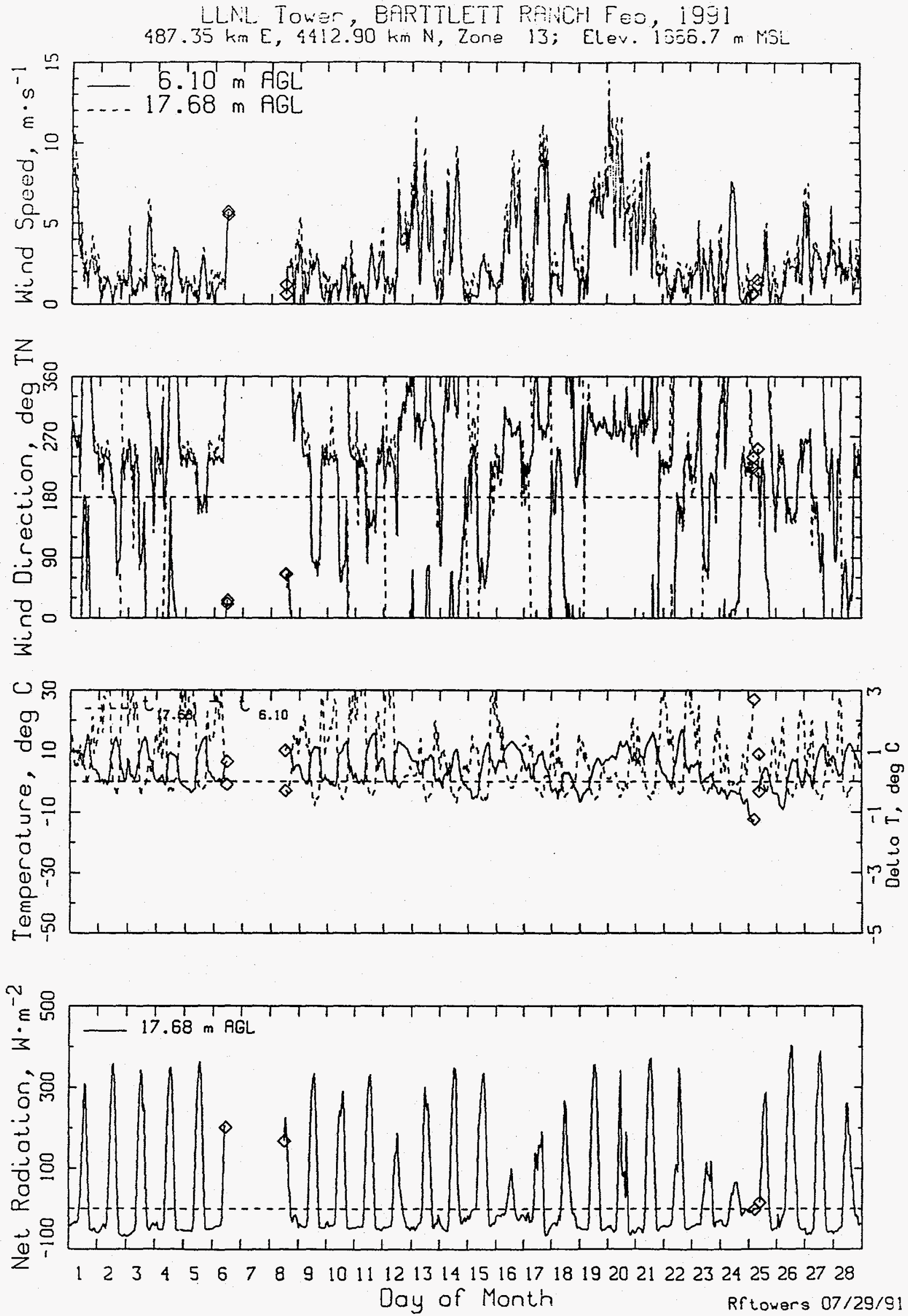